\title{
Long-Term Drug Therapy and Drug Holidays for Osteoporosis Fracture Prevention: A Systematic Review
}




\section{Comparative Effectiveness Review}

Number 218

\section{Long-Term Drug Therapy and Drug Holidays for Osteoporosis Fracture Prevention: A Systematic Review}

\section{Prepared for:}

Agency for Healthcare Research and Quality

U.S. Department of Health and Human Services

5600 Fishers Lane

Rockville, MD 20857

www.ahrq.gov

\section{Contract No. 290-2015-00008-I}

Prepared by:

Minnesota Evidence-based Practice Center

Minneapolis, MN

\section{Investigators:}

Howard A. Fink, M.D., M.P.H.

Roderick MacDonald, M.S.

Mary L. Forte, Ph.D., D.C.

Christina E. Rosebush, M.P.H.

Kristine E. Ensrud, M.D., M.P.H.

John T. Schousboe, M.D., Ph.D.

Victoria A. Nelson, M.Sc.

Kristen Ullman, M.P.H.

Mary Butler, Ph.D., M.B.A.

Carin M. Olson, M.D.

Brent C. Taylor, M.P.H., Ph.D.

Michelle Brasure, Ph.D., M.S.P.H., M.L.I.S.

Timothy J. Wilt, M.D., M.P.H.

AHRQ Publication No. 19-EHC016-EF

April 2019 


\section{Key Messages}

\section{Purpose of Review}

To summarize the effects of long-term osteoporosis drug treatment and of osteoporosis drug treatment discontinuation and holidays.

\section{Key Messages}

- Evidence on the effects of long-term osteoporosis drug treatment and drug continuation versus discontinuation is mostly limited to white, healthy, postmenopausal women.

- Long-term alendronate reduces radiographic vertebral and nonvertebral fractures in women with osteoporosis; long-term zoledronate reduces vertebral and nonvertebral fractures in women with osteopenia or osteoporosis.

- Long-term bisphosphonates may increase atypical femoral fractures and osteonecrosis of the jaw, although both are rare.

- In women with osteoporosis, long-term raloxifene reduces vertebral fractures, but not hip or nonvertebral fractures, and increases venous thromboembolism.

- Long-term oral hormone therapies reduce hip and clinical fractures but increase multiple serious harms.

- Evidence is insufficient about the effects of long-term denosumab, risedronate, ibandronate, teriparatide, and abaloparatide on fractures and harms.

- Continuing bisphosphonates after 3-5 years versus discontinuation reduces some measures of vertebral fractures, but not nonvertebral fractures. 
This report is based on research conducted by the Minnesota Evidence-based Practice Center (EPC) under contract to the Agency for Healthcare Research and Quality (AHRQ), Rockville, MD (Contract No. 290-2015-00008-I). The NIH Office of Disease Prevention commissioned and funded the report. The findings and conclusions in this document are those of the authors, who are responsible for its contents; the findings and conclusions do not necessarily represent the views of AHRQ or NIH. Therefore, no statement in this report should be construed as an official position of NIH, AHRQ, or the U.S. Department of Health and Human Services.

\section{None of the investigators have any affiliations or financial involvement that conflicts with the material presented in this report.}

The information in this report is intended to help healthcare decision makers-patients and clinicians, health system leaders, and policymakers, among others-make well-informed decisions and thereby improve the quality of healthcare services. This report is not intended to be a substitute for the application of clinical judgment. Anyone who makes decisions concerning the provision of clinical care should consider this report in the same way as any medical reference and in conjunction with all other pertinent information, i.e., in the context of available resources and circumstances presented by individual patients.

This report is made available to the public under the terms of a licensing agreement between the author and the Agency for Healthcare Research and Quality. This report may be used and reprinted without permission except those copyrighted materials that are clearly noted in the report. Further reproduction of those copyrighted materials is prohibited without the express permission of copyright holders.

AHRQ or U.S. Department of Health and Human Services endorsement of any derivative products that may be developed from this report, such as clinical practice guidelines, other quality enhancement tools, or reimbursement or coverage policies, may not be stated or implied.

This report may periodically be assessed for the currency of conclusions. If an assessment is done, the resulting surveillance report describing the methodology and findings will be found on the Effective Health Care Program website at www.effectivehealthcare.ahrq.gov. Search on the title of the report.

Persons using assistive technology may not be able to fully access information in this report. For assistance contact epc@ahrq.hhs.gov.

Suggested citation: Fink HA, MacDonald R, Forte ML, Rosebush CE, Ensrud KE, Schousboe JT, Nelson VA, Ullman K, Butler M, Olson CM, Taylor BC, Brasure M, Wilt TJ. Long-Term Drug Therapy and Drug Holidays for Osteoporosis Fracture Prevention: A Systematic Review. Comparative Effectiveness Review No. 218. (Prepared by the Minnesota Evidence-based Practice Center under Contract No. 290-2015-00008-I) AHRQ Publication No. 19-EHC016-EF. Rockville, MD: Agency for Healthcare Research and Quality; April 2019. Posted final reports are located on the Effective Health Care Program search page.

DOI: https://doi.org/10.23970/AHRQEPCCER218. 


\section{Preface}

The Agency for Healthcare Research and Quality (AHRQ), through its Evidence-based Practice Centers (EPCs), sponsors the development of systematic reviews to assist public- and private-sector organizations in their efforts to improve the quality of healthcare in the United States. These reviews provide comprehensive, science-based information on common, costly medical conditions, and new healthcare technologies and strategies.

Systematic reviews are the building blocks underlying evidence-based practice; they focus attention on the strength and limits of evidence from research studies about the effectiveness and safety of a clinical intervention. In the context of developing recommendations for practice, systematic reviews can help clarify whether assertions about the value of the intervention are based on strong evidence from clinical studies. For more information about AHRQ EPC systematic reviews, see www.effectivehealthcare.ahrq.gov/reference/purpose.cfm.

AHRQ expects that these systematic reviews will be helpful to health plans, providers, purchasers, government programs, and the healthcare system as a whole. Transparency and stakeholder input are essential to the Effective Health Care Program. Please visit the website (www.effectivehealthcare.ahrq.gov) to see draft research questions and reports or to join an email list to learn about new program products and opportunities for input.

If you have comments on this systematic review, they may be sent by mail to the Task Order Officers named below at: Agency for Healthcare Research and Quality, 5600 Fishers Lane, Rockville, MD 20857, or by email to epc@ahrq.hhs.gov.

Gopal Khanna, M.B.A.

Director

Agency for Healthcare Research and Quality

Stephanie Chang, M.D., M.P.H.

Director, Evidence-based Practice Program

Center for Evidence and Practice

Improvement

Agency for Healthcare Research and Quality
Arlene S. Bierman, M.D., M.S.

Director

Center for Evidence and Practice Improvement

Agency for Healthcare Research and Quality

Jill Huppert, M.D., M.P.H.

Task Order Officer

Center for Evidence and Practice

Improvement

Agency for Healthcare Research and Quality

Laura Pincock, Pharm.D., M.P.H.

Task Order Officer

Center for Evidence and Practice

Improvement

Agency for Healthcare Research and Quality 


\section{Acknowledgments}

We thank many individuals for their contributions to this project: Laura Pincock and Jill Huppert, our AHRQ Task Order Officers; Keisha Shropshire and Carrie Klabunde, of the National Institutes of Health (NIH) Office of Disease Prevention (ODP); and Lyndon Joseph, National Institute on Aging (NIA), and Faye Chen, National Institute of Arthritis and Musculoskeletal and Skin Diseases (NIAMS), the NIH nominators of this review topic. All provided support, direction, and editorial comments. We also thank Jeannine Ouellette and Cheryl Cole-Hill for their copy editorial help bringing the report to completion.

\section{Technical Expert Panel}

In designing the study questions and methodology at the outset of this report, the EPC consulted several technical and content experts. Broad expertise and perspectives were sought. Divergent and conflicted opinions are common and perceived as healthy scientific discourse that results in a thoughtful, relevant systematic review. Therefore, in the end, study questions, design, methodologic approaches, and/or conclusions do not necessarily represent the views of individual technical and content experts.

Technical Experts must disclose any financial conflicts of interest greater than \$5,000 and any other relevant business or professional conflicts of interest. Because of their unique clinical or content expertise, individuals with potential conflicts may be retained. The TOO and the EPC work to balance, manage, or mitigate any potential conflicts of interest identified.

The list of Technical Experts who provided input to this report follows:

Douglas C. Bauer, M.D.

Professor

University of California, San Francisco

School of Medicine

San Francisco, CA

Cynthia M. Boyd, M.D., M.P.H.*

Professor

Johns Hopkins School of Medicine

Baltimore, MD

Jane A. Cauley, Dr.P.H.*

Distinguished Professor of Epidemiology

University of Pittsburgh

Pittsburgh, PA

Angela M. Cheung, M.D., Ph.D.

Senior Scientist

Departments of Medicine and Medical

Imaging of University of Toronto

Toronto, Ontario, Canada

*Provided input on Draft Report
Carolyn J. Crandall, M.D., M.S., FACP*

Professor of Medicine

David Geffen School of Medicine at University of California, Los Angeles

Los Angeles, CA

Andrea Z. LaCroix, Ph.D.

Professor and Chief of Epidemiology

Family Medicine and Public Health at

University of California, San Diego

San Diego, CA

Eric S. Orwoll, M.D.

Professor of Medicine

Oregon Health \& Science University

Portland, OR 


\section{Peer Reviewers}

Prior to publication of the final evidence report, EPCs sought input from independent Peer Reviewers without financial conflicts of interest. However, the conclusions and synthesis of the scientific literature presented in this report do not necessarily represent the views of individual reviewers.

Peer Reviewers must disclose any financial conflicts of interest greater than $\$ 5,000$ and any other relevant business or professional conflicts of interest. Because of their unique clinical or content expertise, individuals with potential nonfinancial conflicts may be retained. The TOO and the EPC work to balance, manage, or mitigate any potential nonfinancial conflicts of interest identified.

The list of Peer Reviewers for this report follows:

Bart Clarke, M.D.

Division of Endocrinology and Metabolism

Mayo Clinic

Rochester, MN

Gail A. Greendale, M.D.

Professor of Medicine

David Geffen School of Medicine at

University of California, Los Angeles

Los Angeles, CA
Nancy Lane, M.D.

Director and Distinguished Professor

Center for Musculoskeletal Health and

Department of Internal Medicine

University of California at Davis, School of

Medicine

Sacramento, California

Jasvinder Singh, M.D., M.P.H.

Division of Rheumatology

University of Alabama

Birmingham, AL 


\section{Long-Term Drug Therapy and Drug Holidays for Osteoporosis Fracture Prevention: A Systematic Review}

\section{Structured Abstract}

Objective. To summarize the effects of long-term osteoporosis drug treatment (ODT) and ODT discontinuation and holidays on fractures and harms.

Data sources. MEDLINE ${ }^{\circledR}$, Embase ${ }^{\circledR}$, and Cochrane databases from 1995 to October 2018; ClinicalTrials.gov; bibliographies of relevant systematic reviews.

Review methods. We defined long-term ODT as $>3$ years and ODT holidays as discontinuation for $\geq 1$ year after $\geq 1$ year of use. Trials were used for incident fractures and harms, and controlled observational studies were included for additional harms. Two investigators rated risk of bias. For studies with low or medium risk of bias, one investigator extracted data and a second verified accuracy. Two investigators graded strength of evidence (SOE).

Results. Sixty-one English-language studies were included. In women with osteoporosis, 4 years of alendronate reduced clinical fractures (hazard ratio [HR] 0.64 [95\% confidence interval (CI) 0.50, 0.82]) (moderate SOE) and radiographic vertebral fractures (HR 0.50 [95\% CI 0.31, 0.82]) (moderate SOE), while 4 years of raloxifene reduced clinical vertebral fractures (relative risk 0.58 [95\% CI 0.43, 0.79]) (high SOE), but not hip (moderate SOE) or nonvertebral fractures (high SOE). In women with osteopenia or osteoporosis, 6 years of zoledronate reduced incident clinical fractures (HR 0.73 [95\% CI 0.60, 0.90]) (moderate SOE) and clinical vertebral fractures (HR 0.41 [95\% CI 0.22, 0.75]) (moderate SOE). In postmenopausal women with unknown osteoporosis or osteopenia status, both long-term oral estrogen and estrogen/progestin reduced clinical fractures (high SOE) and hip fractures (moderate SOE). After 3-5 years of prior treatment, continuation of zoledronate or alendronate versus drug holiday inconsistently reduced incident vertebral fracture outcomes (radiographic only for zoledronate [low SOE], clinical only for alendronate [moderate SOE]), but did not reduce nonvertebral fractures (low SOE). Hormone therapies increased cardiovascular events, mild cognitive impairment or dementia, and other harms. Observational studies showed that long-term bisphosphonates may increase atypical femoral fractures (AFF) (low SOE) and osteonecrosis of the jaw (low SOE in 2 comparisons, insufficient in 1).

Limitations. Most data were from white, healthy, postmenopausal women, limiting generalizability. Trials often had low power for incident clinical fractures. No trials compared active treatments, sequential treatments, or different durations of drug holidays. Harms and controls were inconsistently defined.

Conclusions. Long-term alendronate, zoledronate, and oral hormone therapy reduced nonvertebral fractures in older women, with oral hormone therapy also reducing hip fractures. While absolute reductions in typical fractures with long-term bisphosphonates are large relative to increases in AFF, reduced hip fracture risk with oral hormone therapy appears offset by increased risk of serious harms. Evidence is limited regarding ODT holidays for fractures and 
harms. Future research is needed, including randomized trials comparing ODT holiday durations and sequential treatments powered for clinical fractures, and controlled cohort studies of ODT holidays to estimate rare harms. 


\section{Contents}

Evidence Summary ......................................................................................................................... ES-1

Chapter 1. Introduction ...................................................................................................... 1

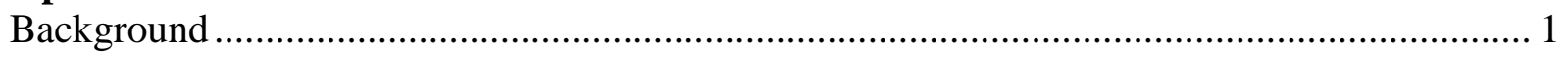

Scope and Key Questions ............................................................................................ 4

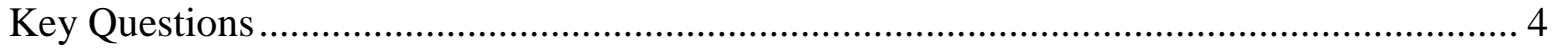

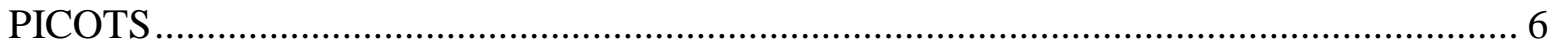

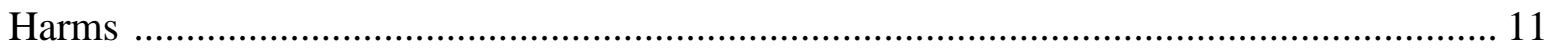

Analytic Framework …………………………......................................................... 12

Chapter 2. Methods ............................................................................................................................... 13

Topic Refinement and Review Protocol ............................................................................. 13

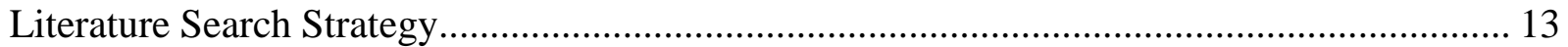

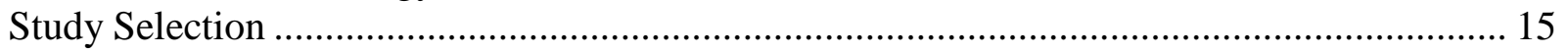

Risk of Bias Assessment ............................................................................................. 15

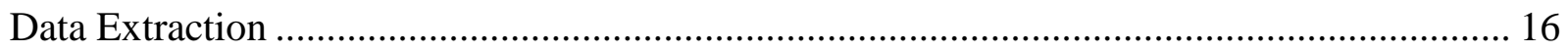

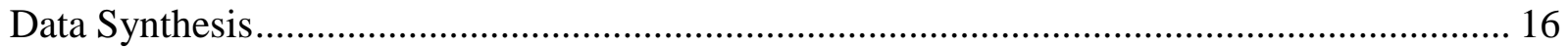

Strength of Evidence for Major Comparisons and Outcomes ................................................... 17

Applicability ....................................................................................................................... 17

Peer Review and Public Commentary .................................................................................. 18

Chapter 3. Search Results ................................................................................................................ 19

Chapter 4. Efficacy of Long-Term Osteoporosis Drug Treatment ........................................... 22

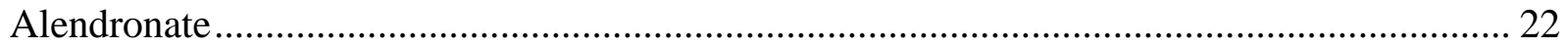

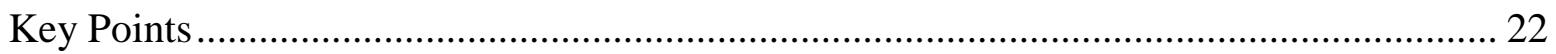

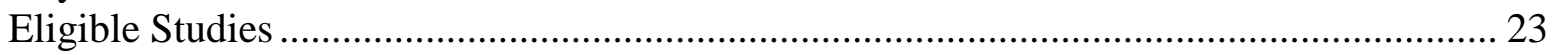

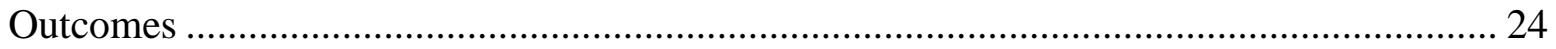

Variation in Long-Term Treatment Efficacy as a Function of Patient, Bone, or Osteoporosis

Drug Characteristics....................................................................................................... 25

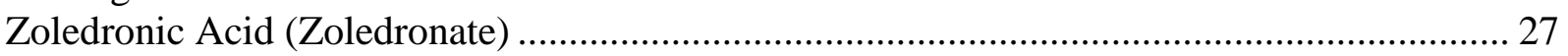

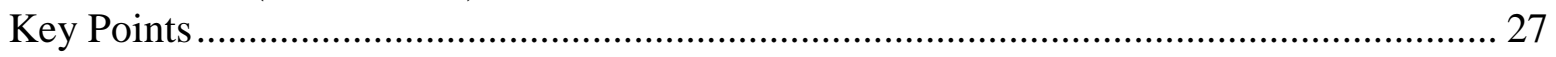

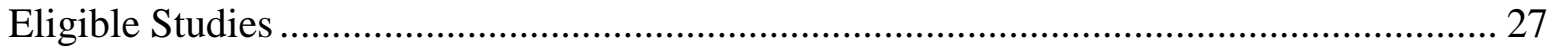

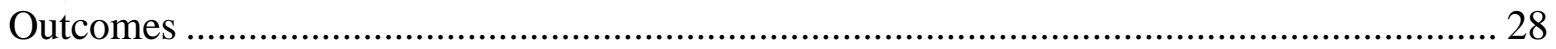

Variation in Long-Term Treatment Efficacy as a Function of Patient, Bone, or Osteoporosis

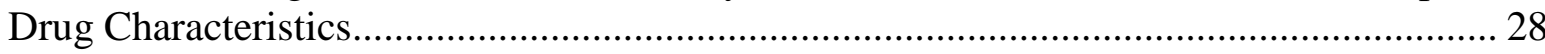

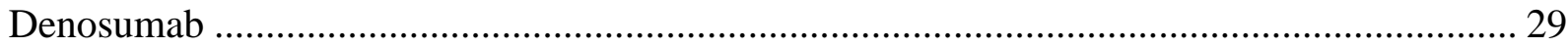

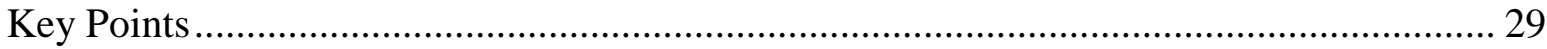

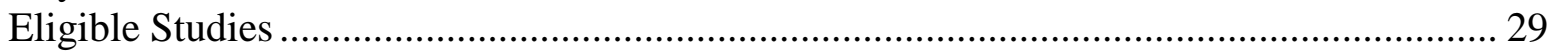

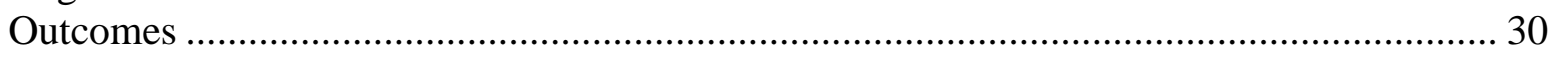

Variation in Long-Term Treatment Efficacy as a Function of Patient, Bone, or Osteoporosis

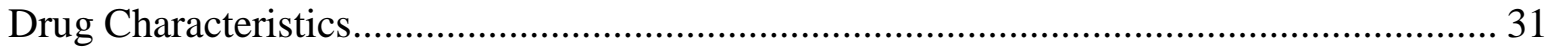

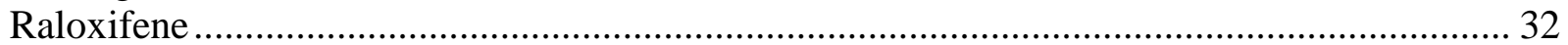

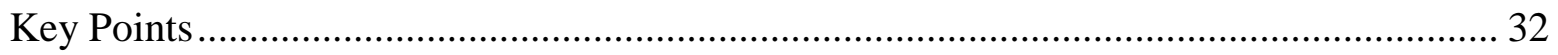

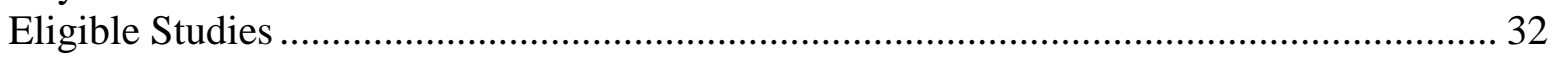

Outcomes ....................................................................................................................... 33

Variation in Long-Term Treatment Efficacy as a Function of Patient, Bone, or Osteoporosis

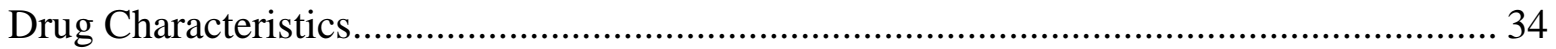

Hormone Therapy ……………………………………………………………………... 35 


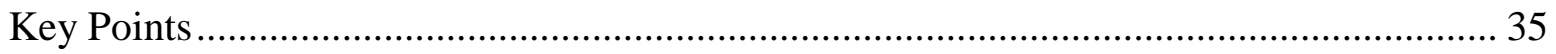

Eligible Studies …………………….......................................................................... 35

Outcomes .................................................................................................................. 37

Variation in Long-Term Treatment Efficacy as a Function of Patient, Bone, or Osteoporosis

Drug Characteristics.................................................................................................... 38

Other Drugs Investigated for Review ………………...................................................... 39

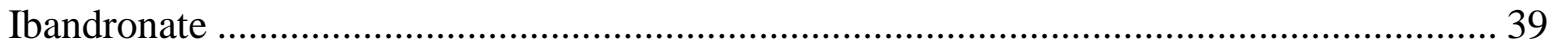

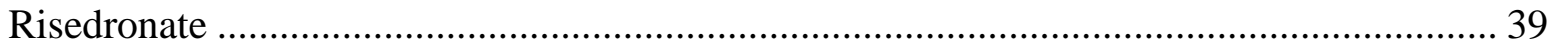

Chapter 5. Harms of Long-Term Drug Treatment ............................................................ 40

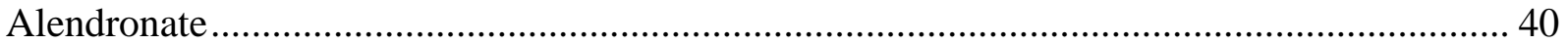

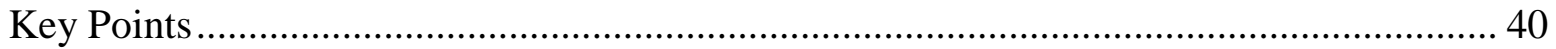

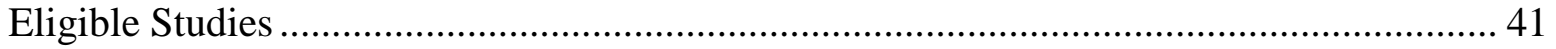

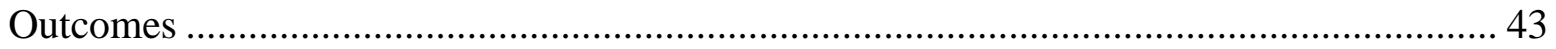

Variation in Long-Term Treatment Harms as a Function of Patient, Bone, or Osteoporosis

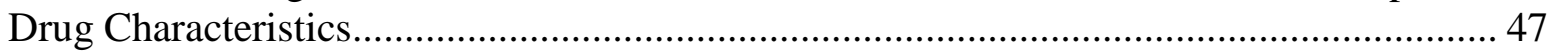

Zoledronic Acid (Zoledronate) ....................................................................................... 48

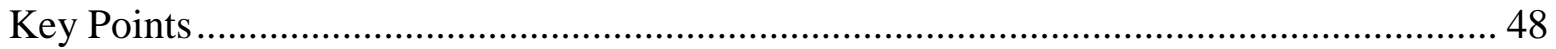

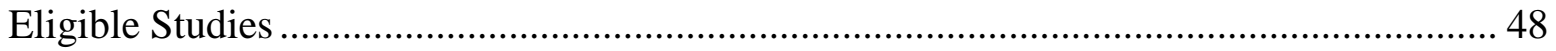

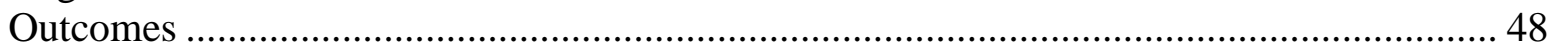

Variation in Long-Term Treatment Harms as a Function of Patient, Bone, or Osteoporosis

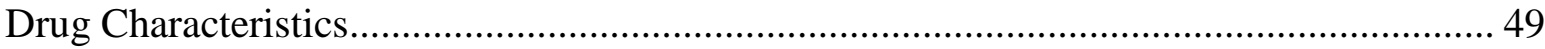

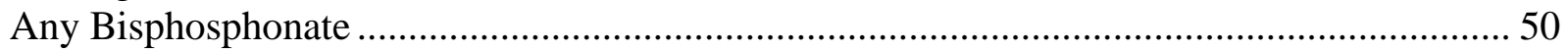

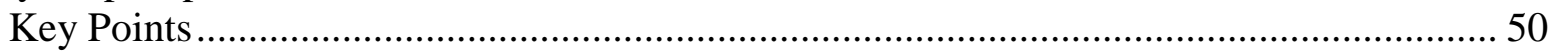

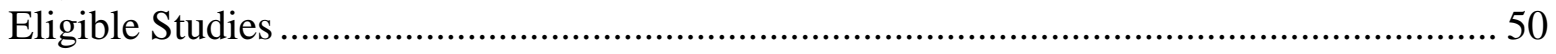

Outcomes ................................................................................................................... 52

Variation in Long-Term Treatment Harms as a Function of Patient, Bone, or Osteoporosis

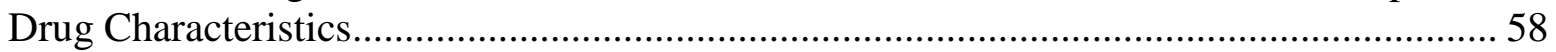

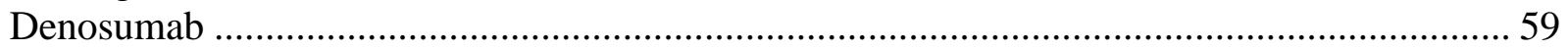

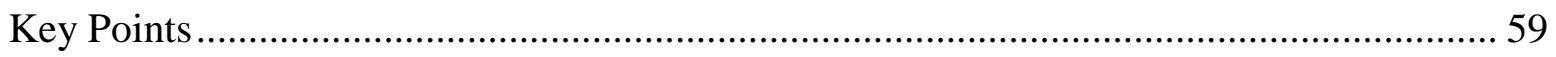

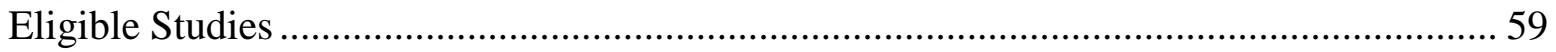

Outcomes ................................................................................................................. 59

Variation in Long-Term Treatment Harms as a Function of Patient, Bone, or Osteoporosis

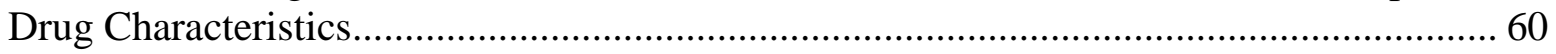

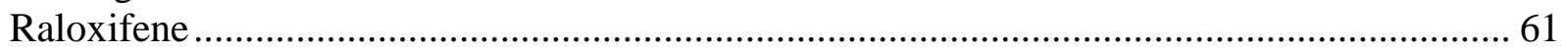

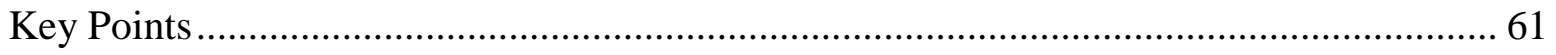

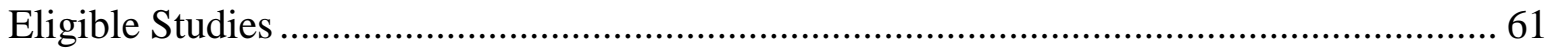

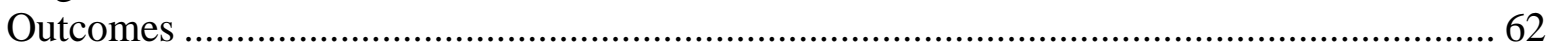

Variation in Long-Term Treatment Harms as a Function of Patient, Bone, or Osteoporosis

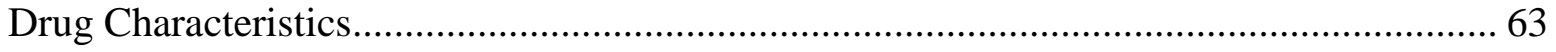

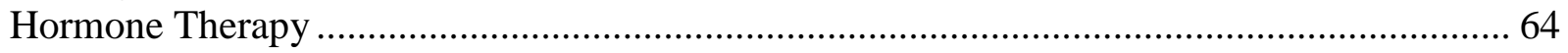

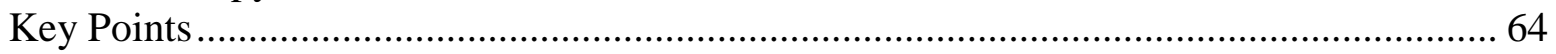

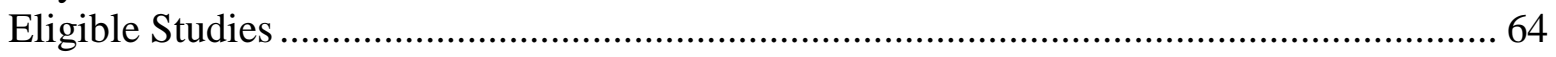

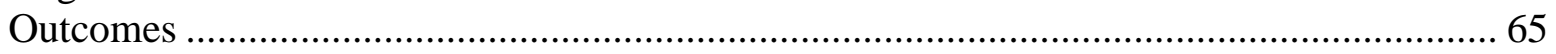

Variation in Long-Term Treatment Harms as a Function of Patient, Bone, or Osteoporosis

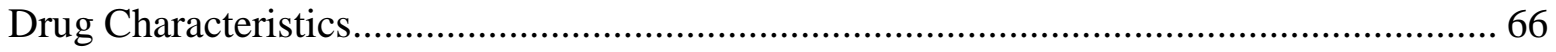

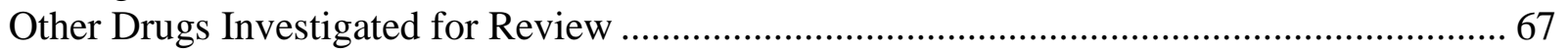




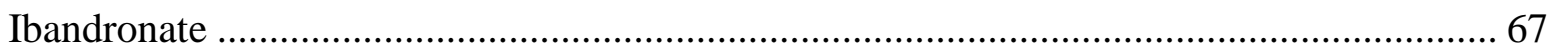

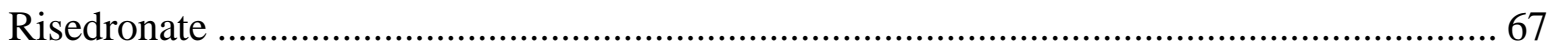

Chapter 6. Effects of Osteoporosis Drug Holidays ..................................................................6 68

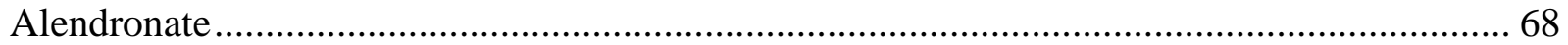

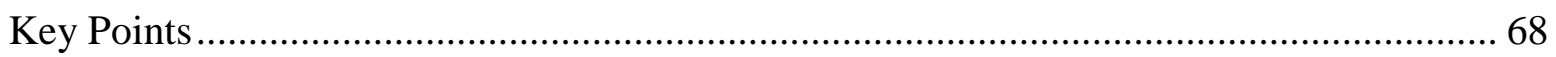

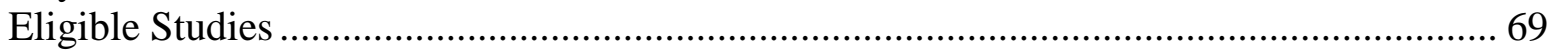

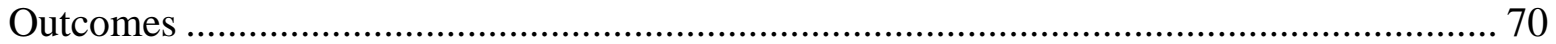

Variation in Effects of Drug Holiday as a Function of Patient, Bone, or Osteoporosis Drug

Characteristics............................................................................................................. 71

Zoledronic Acid (Zoledronate) ............................................................................................ 73

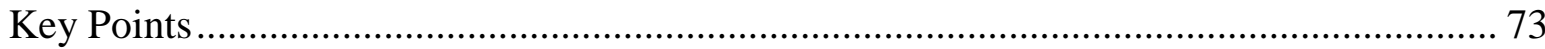

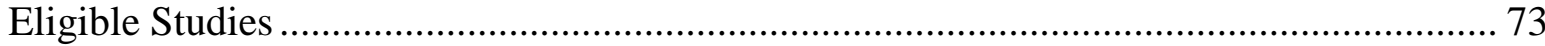

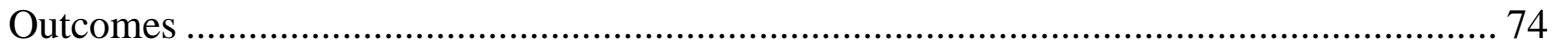

Variation in Effects of Drug Holiday as a Function of Patient, Bone, or Osteoporosis Drug

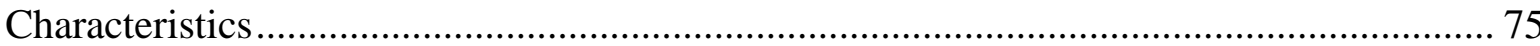

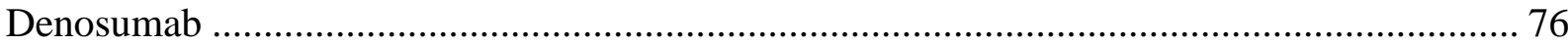

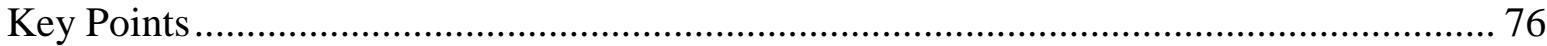

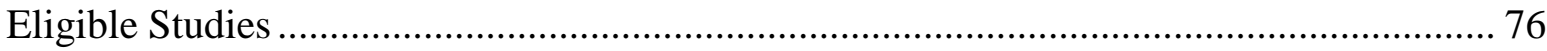

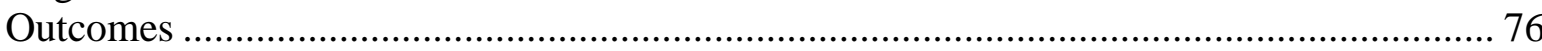

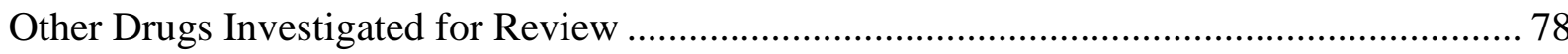

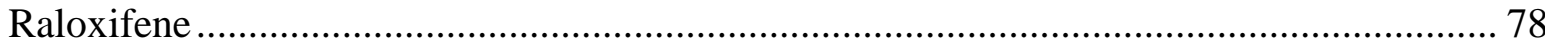

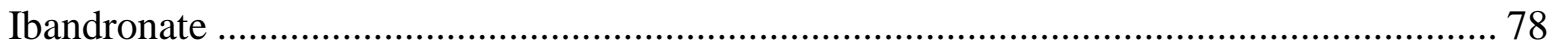

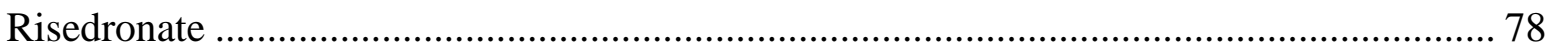

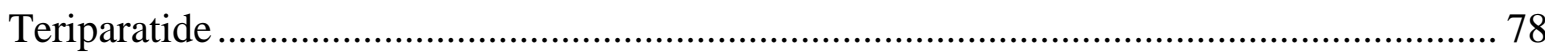

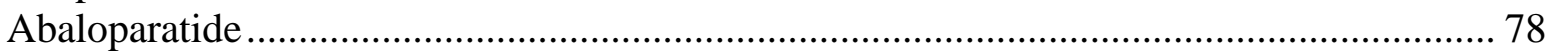

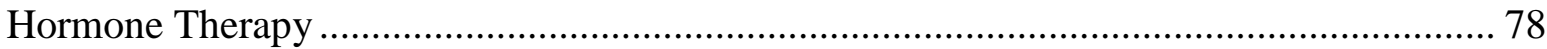

Chapter 7. Harms of Drug Holidays ................................................................................. 79

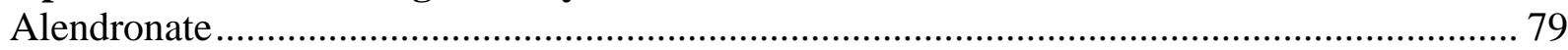

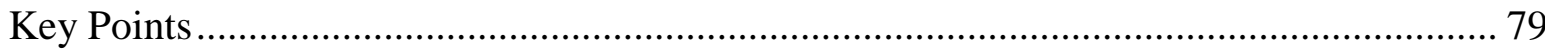

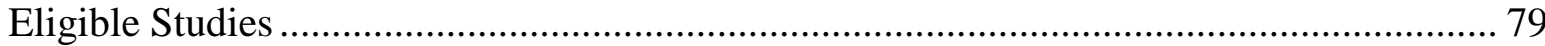

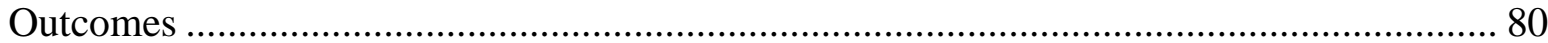

Variation in Harms During Drug Holiday as a Function of Patient, Bone, or Osteoporosis

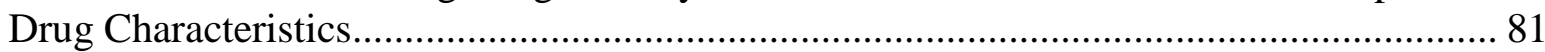

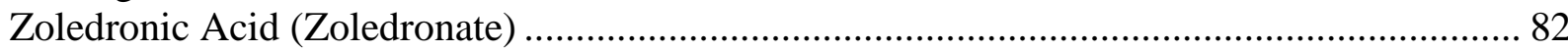

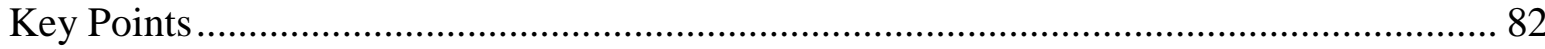

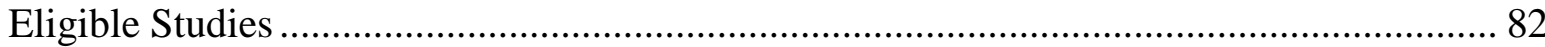

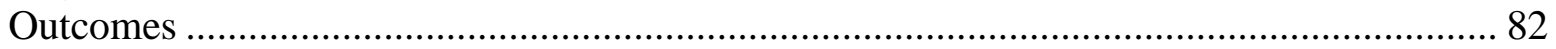

Variation in Harms During Drug Holiday as a Function of Patient, Bone, or Osteoporosis

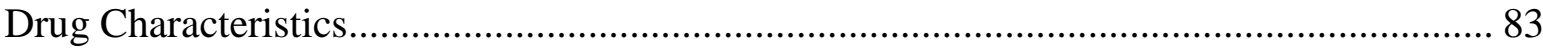

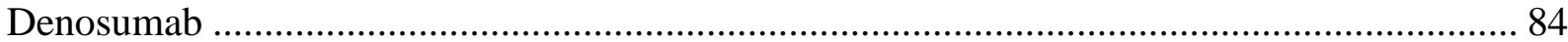

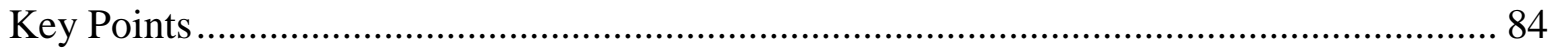

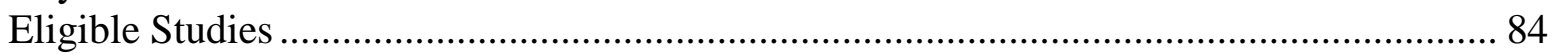

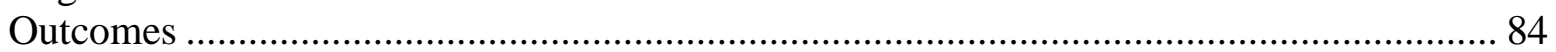

Variation in Harms During Drug Holiday as a Function of Patient, Bone, or Osteoporosis

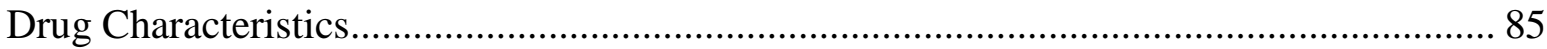

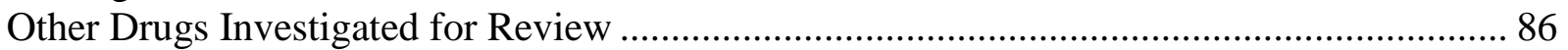




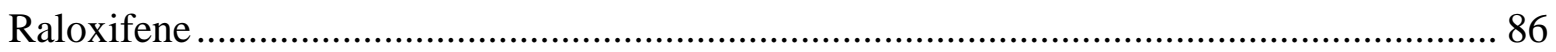

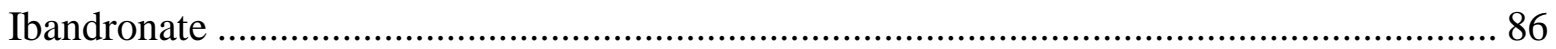

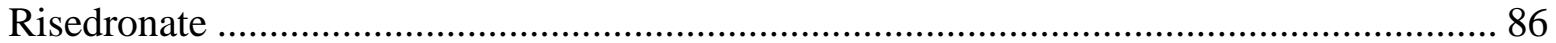

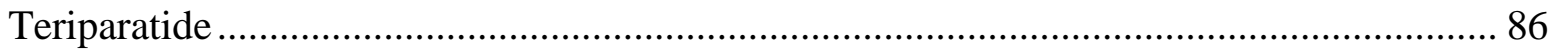

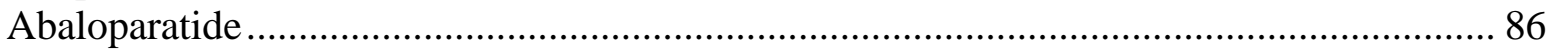

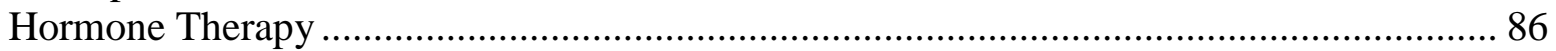

Chapter 8. Discussion ........................................................................................................................ 8 87

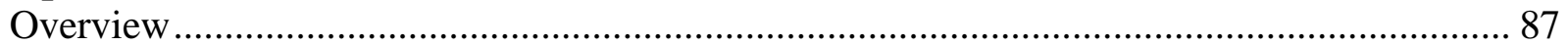

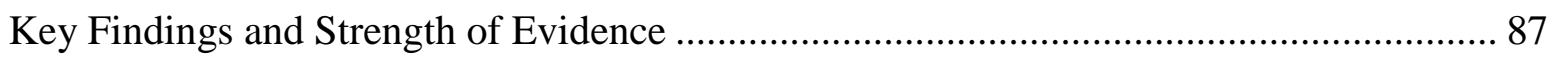

Applicability ....................................................................................................................... 91

Findings in Relation to What Is Already Known...................................................................... 91

Efficacy of Short-Term Osteoporosis Drug Treatment ......................................................... 91

Efficacy of Long-Term Osteoporosis Drug Treatment.............................................................. 91

Harms of Long-Term Osteoporosis Drug Treatment............................................................... 92

Effect of Osteoporosis Drug Treatment Holidays ................................................................. 93

Variation in Osteoporosis Drug Treatment Outcomes........................................................ 93

Limitations of the Comparative Effectiveness Review ............................................................. 94

Limitations of the Evidence Base ......................................................................................... 95

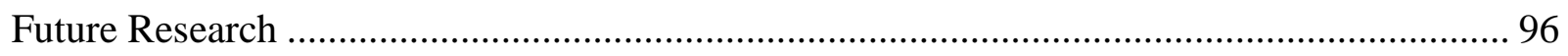

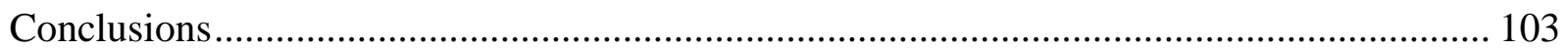

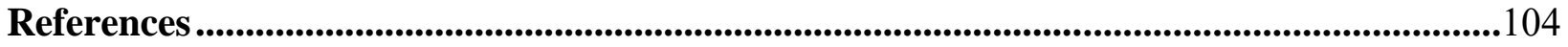

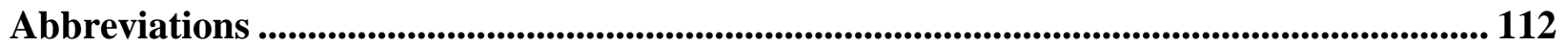

Tables

Table A. Evidence on efficacy of long-term ( $>3$ years) osteoporosis drug treatment............... ES-4

Table B. Evidence on harms of long-term ( $>3$ years) osteoporosis drug treatment .................. ES-8

Table C. Evidence on effects of osteoporosis drug continuation versus discontinuation on

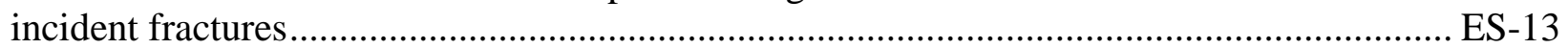

Table D. Evidence on harms of osteoporosis drug continuation versus discontinuation ....... ES-14

Table 1. U.S. FDA approved drugs used for osteoporosis treatment and prevention....................... 3

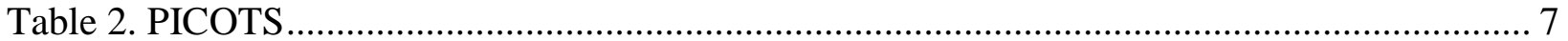

Table 3. Harms evaluated for possible association with long-term osteoporosis drug therapy.... 11

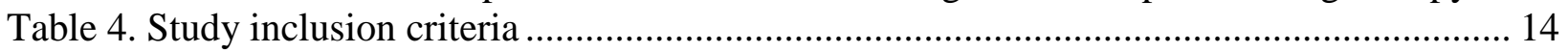

Table 5. Eligible publications that compared long-term osteoporosis drug use versus control or osteoporosis drug continuation versus discontinuation (placebo drug holiday) and reported on risk of incident fractures and/or harms ..................................................................................... 21

Table 6. Baseline participant characteristics from included alendronate long-term treatment

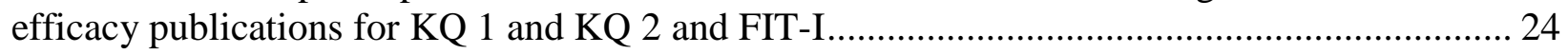

Table 7. Baseline characteristics of the zoledronate trial versus placebo........................................ 27

Table 8. Baseline characteristics of the denosumab trial versus placebo ....................................... 29

Table 9. Baseline participant characteristics from the MORE trial and CORE extension ............ 33

Table 10. Baseline characteristics of hormone therapy versus placebo/control RCTs.................. 36

Table 11. Estimated risk of ST/FS fracture, ONJ, or atrial fibrillation with alendronate use from controlled observational studies........................................................................................... 45

Table 12. Estimated risk of AFF or ST/FS fracture for any bisphosphonate use from

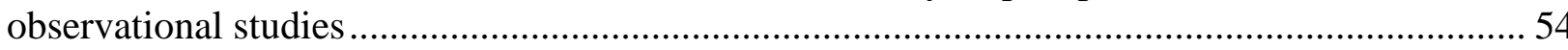


Table 13. Trial participant characteristics at the time of assignment to alendronate continuation versus discontinuation............................................................................................................ 70

Table 14. Participant characteristics at the baseline of the zoledronate continuation versus

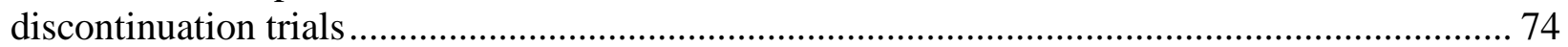

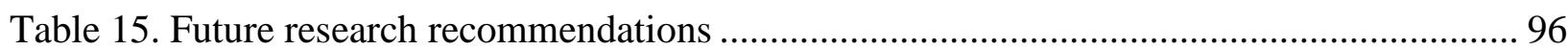

\section{Figures}

Figure 1. Analytic framework for long-term osteoporosis drug treatment and osteoporosis drug treatment holidays for fracture prevention.................................................................................. 12

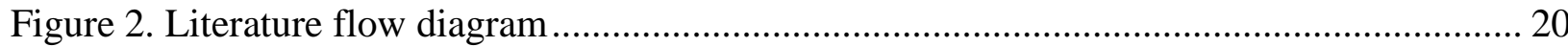

\section{Appendixes}

Appendix A. Search Strategy

Appendix B. Risk of Bias Assessment Decision Aid

Appendix C. Included References Risk of Bias Assessment

Appendix D. Evidence Tables

Appendix E. Excluded References 


\section{Evidence Summary}

\section{Introduction}

Osteoporosis is a skeletal disorder of low bone mass and microarchitectural deterioration of bone, leading to bone fragility and increased risk of fracture. ${ }^{1}$ Osteoporosis affects more than 10 million U.S. adults aged 50 years or older. ${ }^{2}$ About two million U.S. adults experience an osteoporotic or other low- or no-trauma fracture each year. ${ }^{3}$ These fractures frequently cause pain, disability, and impaired quality of life; ${ }^{4,5}$ and hip and clinical vertebral fractures, specifically, are associated with increased mortality. ${ }^{5,6}$ Because risk of most fractures rises steeply with age, and the population is aging, fracture burden is projected to increase in coming decades.

In short-term (18 to 36 months) randomized controlled trials (RCTs) for osteoporosis treatment, bisphosphonates (alendronate, zoledronate, risedronate, ibandronate), denosumab, teriparatide, and abaloparatide lower risk of nonvertebral fractures, clinical vertebral fractures (usually diagnosed in the community because of back pain, with study comparison of study and community radiographs), and radiographic vertebral fractures (identified in studies by comparing vertebral heights on scheduled serial vertebral radiographs; mostly unrecognized in the community). ${ }^{7,8}$ Several bisphosphonates (alendronate, zoledronate, risedronate) and denosumab also lower risk of hip fractures. ${ }^{7}$

Despite the evidence on the efficacy of short-term osteoporosis drug treatment for reducing fracture risk in appropriate patients, there is uncertainty about the balance of benefits and harms of long-term or continued treatment. A recent American College of Physicians clinical practice guideline recommended treatment of osteoporotic women with a bisphosphonate or denosumab for 5 years to reduce risk of hip and vertebral fractures, and suggested that high-risk patients may benefit from more than 5 years of treatment. ${ }^{8}$

However, concerns that long-term bisphosphonate persistence in bone might increase fracture risk by inhibiting normal repair of bone microdamage ${ }^{9,10}$ have led to the suggestion that bisphosphonate treatment be discontinued periodically. ${ }^{9}$ Several groups advocate bisphosphonate “drug holidays” to minimize harms while preserving as much fracture benefit as possible. But, there is no consensus about who should get them, when they should start, how long they should last, and the criteria for restarting treatment. ${ }^{9,11,12}$ By contrast, drug holidays are not recommended after denosumab, because bone loss increases rapidly after discontinuation, possibly increasing risk of radiographic vertebral fractures. ${ }^{13}$

Uncertainties about the most appropriate use of long-term osteoporosis drug treatment and of osteoporosis drug holidays led to scheduling a National Institutes of Health (NIH) Office of Disease Prevention (ODP) Pathways to Prevention (P2P) workshop. The goals of the workshop were to present an evidence-based synthesis of the pertinent research base, and to suggest future research to assist patients, clinicians, and other healthcare decision makers.

To further these aims, we conducted this systematic review to address the following questions: (1) What are the effects of long-term (>3 years) osteoporosis drug treatment versus control on risks of incident fractures and harms; (2) Do effects of long-term osteoporosis drug treatment vary as a function of patient, bone, or osteoporosis drug characteristics; (3) Among individuals receiving osteoporosis drug treatment to prevent fracture, what are the effects of continuing versus discontinuing treatment (i.e., osteoporosis drug holiday) on risks of incident fractures and harms; and (4) Do these outcomes of drug holidays vary as a function of patient, bone, or osteoporosis drug characteristics? 


\section{Methods}

The review was conducted following the Agency for Healthcare Research and Quality (AHRQ) methods guidance. The protocol is available at https://effectivehealthcare.ahrq.gov/topics/osteoporosis-fracture-prevention/research-protocol and is registered in PROSPERO. We detail our literature search strategy, study selection criteria, and data extraction and synthesis methods in the full report.

\section{Results}

We identified 8,356 unique publications through October 2018, of which 61 met eligibility criteria and were included in the review. Of 48 publications with low or medium risk of bias (ROB), there were 35 randomized or controlled clinical trials (9 unique studies) and 13 controlled observational studies (11 unique studies) (Appendix $\mathrm{C}$ of the full report). Most publications were based on three RCTs of alendronate, zoledronic acid, and raloxifene, respectively, and their extension studies.

All trials enrolled only postmenopausal women, with most limited to women with osteoporosis as defined by bone mineral density (BMD) and vertebral fracture history, and some including women with osteopenia. Observational studies included 84 to 100 percent women. Mean participant age was 72 years, with all but two studies reporting mean age $<80$ years. Most observational studies presumed participants had osteoporosis because of past fracture or use of osteoporosis drugs, but none reported BMD status.

\section{Long-Term Osteoporosis Drug Treatment}

\section{Efficacy}

Seven eligible placebo-controlled RCTs with low or medium ROB examined the effect of long-term treatment, one each for alendronate ${ }^{14}$ zoledronate, ${ }^{15}$ raloxifene,,${ }^{16,}{ }^{17}$ denosumab,${ }^{18}$ and estrogen, ${ }^{19}$ and two for estrogen/progestin. ${ }^{20,21}$

In women with osteopenia or osteoporosis by BMD, but with no past vertebral fracture, 4 years of alendronate versus placebo reduced incident radiographic vertebral fractures (hazard ratio [HR] 0.56 [95\% confidence interval (CI), 0.39, 0.80]) (high strength of evidence [SOE])(Table A), while absolute risk reductions for incident hip and nonvertebral fracture were small and not statistically significant (low SOE) ${ }^{14}$ In women with osteoporosis by BMD or past fracture, 4 years of raloxifene versus placebo reduced incident radiographic (relative risk [RR] $0.64[0.53,0.76]$ ) and clinical vertebral fractures (RR 0.58 [95\% CI 0.43, 0.79]) (both high $\mathrm{SOE}){ }^{16}$ However, raloxifene did not reduce incident hip or nonvertebral fracture (moderate and high SOE, respectively). In older women with osteopenia or osteoporosis, 6 years of zoledronate versus placebo reduced incident clinical fractures (HR 0.73 [95\% CI 0.60, 0.90]) (low SOE), incident nonvertebral fractures (HR 0.66 [95\% CI 0.51, 0.85]) (moderate SOE) and incident clinical vertebral fractures (HR 0.41 [95\% CI 0.22, 0.75]) (moderate SOE). ${ }^{15}$ Nonvertebral fractures appeared similarly reduced in the subset of women with osteopenia. In women with unknown osteoporosis or osteopenia status, incident clinical fractures (high SOE) and incident hip fractures (moderate SOE) both were reduced with hormone therapy compared to placebo, with 5.6 years of oral estrogen/progestin in women with an intact uterus, and with 7 years of unopposed oral estrogen in women with a hysterectomy. ${ }^{19}$ 
Evidence was insufficient to compare fracture risk between women on long-term denosumab versus placebo, ${ }^{18}$ and there were no data from eligible trials about the long-term fracture efficacy of sequential osteoporosis drug therapy (e.g., anabolic followed by anti-resorptive, or denosumab followed by bisphosphonate).

Alendronate, zoledronate, denosumab, and raloxifene for long-term treatment each increased hip and lumbar spine BMD compared to placebo.

\section{Variation in Efficacy as a Function of Patient, Bone, or Osteoporosis Drug Characteristics}

Efficacy of long-term alendronate appears to vary as a function of baseline BMD. ${ }^{14}$ Relative risk of incident clinical fractures was significantly reduced in women with osteoporotic BMD (femoral neck BMD $\leq-2.5$ (moderate SOE), but not in women with osteopenic BMD (femoral neck BMD -1.6 to >-2.5) (low SOE) (Table A; Appendix Table D8). In women with osteoporosis, relative risk of incident radiographic vertebral fracture with long-term alendronate was halved versus placebo (HR 0.50 [95\% CI 0.31, 0.82]) (moderate SOE). Although women with femoral neck BMD -2.5 to -2 had a similar relative reduction in these fractures versus placebo (HR 0.54 [95\% CI 0.28, 1.04]) (low SOE), a lower proportion of women with osteopenia had incident radiographic vertebral fractures, and results were not statistically significant. No tests of interaction were reported for these BMD stratified results.

In a post hoc analysis, women with osteoporosis had a reduced risk of incident hip fracture with long-term alendronate versus placebo, but women with osteopenia had no reduced risk (pvalue for interaction not reported). ${ }^{14}$ In additional post hoc analyses, some conducted in women with osteopenia, ${ }^{22,}{ }^{23}$ neither past nonvertebral fracture, ${ }^{23} 10$-year major osteoporotic fracture probability calculated with femoral neck BMD, ${ }^{24}$ nor pretreatment levels of bone turnover markers ${ }^{25}$ significantly modified the effect of long-term alendronate versus placebo on risk of any incident fracture outcome.

Neither age,${ }^{17}$ baseline BMD, ${ }^{17}$ nor baseline radiographic vertebral fracture ${ }^{16,17,26,27}$ modified the effect of long-term raloxifene versus placebo on risk of incident fractures. Two large trials of oral hormone therapy versus placebo in women with unknown osteoporosis or osteopenia status reported inconsistent findings about whether treatment effect on risk of incident hip and clinical fractures differed as a function of age or time since menopause. ${ }^{19,28}$ However, authors minimized their one significant interaction for age because of the many interactions examined. ${ }^{19}$ 


\begin{tabular}{|c|c|c|c|c|}
\hline $\begin{array}{l}\text { Comparison } \\
\text { \# Studies by Design } \\
\text { Treatment Duration }\end{array}$ & Participant Characteristics & $\begin{array}{l}\text { Incident } \\
\text { Fracture } \\
\text { Outcome } \\
\end{array}$ & $\begin{array}{l}\text { Relative and Absolute Risk Differences } \\
(95 \% \mathrm{Cl})\end{array}$ & $\begin{array}{l}\text { Strength of Evidence* } \\
\text { (Justification) }\end{array}$ \\
\hline \multirow{6}{*}{$\begin{array}{l}\text { Alendronate vS. } \\
\text { placebo } \\
1 \mathrm{RCT}^{14} \\
4 \mathrm{yr}\end{array}$} & \multirow[t]{4}{*}{$\begin{array}{l}\text { 4,432 PM women with osteopenia or } \\
\text { osteoporosis (T-score } \leq-1.6 \text { ) and no RVF }\end{array}$} & CF & $\begin{array}{l}\text { No difference: } \\
\mathrm{HR}=0.86[0.73,1.01] ; \mathrm{ARR}=-2[-4,0]\end{array}$ & Low (IM) \\
\hline & & NVF & $\begin{array}{l}\text { No difference: } \\
\mathrm{HR}=0.88[0.74,1.04] ; \mathrm{ARR}=-1[-3,0]\end{array}$ & $\begin{array}{l}\text { Low } \\
(\mathrm{IM})\end{array}$ \\
\hline & & Hip & $\begin{array}{l}\text { No difference: } \\
\mathrm{HR}=0.79[0.43,1.44] ; \mathrm{ARR}=-0.2[-0.8,0.4]\end{array}$ & Low \\
\hline & & RVF & $\begin{array}{l}\text { Lower risk: } \\
\mathrm{HR}=0.56[0.39,0.80] ; \mathrm{ARR}=-2[-3,-1]\end{array}$ & High \\
\hline & \multirow[t]{2}{*}{$\begin{array}{l}\text { 1,631 PM women with osteoporosis by } \\
\text { BMD (T-score } \leq-2.5 \text { ) and no RVF }\end{array}$} & $\mathrm{CF}$ & $\begin{array}{l}\text { Lower risk: } \\
\mathrm{HR}=0.64[0.50,0.82] ; A R R=-7[-10,-3]\end{array}$ & Moderate (RB) \\
\hline & & RVF & $\begin{array}{l}\text { Lower risk: } \\
\mathrm{HR}=0.50[0.31,0.82] ; \mathrm{ARR}=-3[-5,-1]\end{array}$ & Moderate (RB) \\
\hline \multirow{4}{*}{$\begin{array}{l}\text { Zoledronate vs. } \\
\text { placebo } \\
1 \mathrm{RCT}^{15} \\
6 \mathrm{yr}\end{array}$} & \multirow[t]{4}{*}{$\begin{array}{l}2,000 \text { PM women } \geq 65 \text { with osteoporosis or } \\
\text { osteopenia }\end{array}$} & CF & $\begin{array}{l}\text { Lower risk: } \\
\mathrm{HR}=0.73[0.60,0.90] ; \mathrm{ARR}=-5[-9,-2]\end{array}$ & $\begin{array}{l}\text { Moderate } \\
(\mathrm{IM})\end{array}$ \\
\hline & & NVF & $\begin{array}{l}\text { Lower risk: } \\
\mathrm{HR}=0.66[0.51,0.85] ; \mathrm{ARR}=-5[-8,-2]\end{array}$ & High \\
\hline & & Hip & $\begin{array}{l}\text { No difference: } \\
\mathrm{HR}=0.66[0.27,1.16] ; \mathrm{ARR}=-0.4[-1,0.5]\end{array}$ & $\begin{array}{l}\text { Low } \\
(\mathrm{h}-\mathrm{IM})\end{array}$ \\
\hline & & CVF & $\begin{array}{l}\text { Lower risk: } \\
\mathrm{HR}=0.41[0.22,0.75] ; \mathrm{ARR}=-2[-3,-1]\end{array}$ & $\begin{array}{l}\text { Moderate } \\
(\mathrm{IM})\end{array}$ \\
\hline $\begin{array}{l}\text { Denosumab** vs. } \\
\text { placebo } \\
1 \mathrm{RCT}^{18} \\
4 \mathrm{yr}\end{array}$ & $\begin{array}{l}365 \text { PM women with osteopenia or } \\
\text { osteoporosis by BMD }\end{array}$ & $\mathrm{CF}$ & $R R=0.97[0.40,2.35] ; A R R=-0.4[-10,9]$ & $\begin{array}{l}\text { Insufficient } \\
\text { (RB, IN, h-IM) }\end{array}$ \\
\hline \multirow{4}{*}{$\begin{array}{l}\text { Raloxifene vs. } \\
\text { placebo } \\
1 \text { RCT with } 1 \text { CCT } \\
\text { extension }^{16,17,26-37} \\
4 \text { to } 8 \mathrm{yr}\end{array}$} & \multirow[t]{4}{*}{$\begin{array}{l}6,828 \text { PM women with osteoporosis by } \\
\text { BMD or RVF }\end{array}$} & NVF & $\begin{array}{l}\text { No difference: } \\
4 \mathrm{yr}: \mathrm{RR}=0.93[0.81,1.06] \dagger ; \text { ARR NA } \\
8 \mathrm{yr}: \mathrm{HR}=1.00[0.82,1.21] \ddagger ; \text { ARR NA }\end{array}$ & $\begin{array}{l}4 \mathrm{yr}: \text { High } \\
8 \mathrm{yr}: \text { Moderate (RB) }\end{array}$ \\
\hline & & Hip & $\begin{array}{l}\text { No difference: } \\
4 \mathrm{yr}: \mathrm{RR}=0.97[0.62,1.52]+; \mathrm{ARR}=0[-0.6,0.5]\end{array}$ & $\begin{array}{l}\text { Moderate } \\
\text { (IM) }\end{array}$ \\
\hline & & CVF & $\begin{array}{l}\text { Lower risk: } \\
4 \mathrm{yr}: \mathrm{RR}=0.58[0.43,0.79] \neq ; \mathrm{ARR}=-2[-3,-1]\end{array}$ & High \\
\hline & & RVF & $\begin{array}{l}\text { Lower risk: } \\
4 \mathrm{yr}: \mathrm{RR}=0.64[0.53,0.76] \neq ; \mathrm{ARR}=-5[-6,-3]\end{array}$ & High \\
\hline Estrogen vs. placebo & 10,739 PM women with past hysterectomy & CF & $\begin{array}{l}\text { Lower risk: } \\
\mathrm{HR}=0.71[0.64,0.80] ; \mathrm{ARR}=-4[-5,-3]\end{array}$ & High \\
\hline
\end{tabular}




\begin{tabular}{|c|c|c|c|c|}
\hline $\begin{array}{l}\text { Comparison } \\
\text { \# Studies by Design } \\
\text { Treatment Duration }\end{array}$ & Participant Characteristics & $\begin{array}{l}\text { Incident } \\
\text { Fracture } \\
\text { Outcome }\end{array}$ & $\begin{array}{l}\text { Relative and Absolute Risk Differences } \\
(95 \% \mathrm{Cl})\end{array}$ & $\begin{array}{l}\text { Strength of Evidence* } \\
\text { (Justification) }\end{array}$ \\
\hline \multirow[t]{5}{*}{$\begin{array}{l}\text { 1 } \mathrm{RCT}^{19} \\
7.1 \mathrm{yr} \text { (mean) }\end{array}$} & & Hip & $\begin{array}{l}\text { Lower risk: } \\
\mathrm{HR}=0.65[0.45,0.94] ; \mathrm{ARR}=-0.5[-0.9,-0.08]\end{array}$ & $\begin{array}{l}\text { Moderate } \\
(\mathrm{IM})\end{array}$ \\
\hline & \multirow[t]{2}{*}{$\begin{array}{l}\text { 3,816 PM women with past hysterectomy } \\
\text { and past clinical fracture }\end{array}$} & $\mathrm{CF}$ & $\begin{array}{l}\text { Lower risk: } \\
\mathrm{HR}=0.73[0.62,0.86] ; \mathrm{ARR}=-5[-7,-2]\end{array}$ & $\begin{array}{l}\text { Low } \\
\text { (RB, IM) }\end{array}$ \\
\hline & & Hip & $\begin{array}{l}\text { Lower risk: } \\
\mathrm{HR}=0.55[0.32,0.94] ; \mathrm{ARR}=-1[-2,0]\end{array}$ & Low 1 (RB, IM) \\
\hline & $\begin{array}{l}53 \text { PM women with past hysterectomy and } \\
\text { osteoporosis by BMD }\end{array}$ & CF & $\mathrm{HR}=0.83[0.17,3.91] ;$ ARR NA & $\begin{array}{l}\text { Insufficient } \\
\text { (RB, h-IM) }\end{array}$ \\
\hline & $\begin{array}{l}363 \text { PM women with past hysterectomy } \\
\text { and osteopenia by BMD }\end{array}$ & $\mathrm{CF}$ & $\mathrm{HR}=0.83[0.49,1.40] ;$ ARR NA & $\begin{array}{l}\text { Insufficient } \\
\text { (RB, h-IM) }\end{array}$ \\
\hline \multirow{6}{*}{$\begin{array}{l}\text { Estrogen/progestin } \\
\text { vs. placebo } \\
1 \mathrm{RCT}^{21} \\
5.6 \mathrm{yr} \text { (mean) }\end{array}$} & \multirow[t]{3}{*}{ 16,608 PM women with intact uterus } & CF & $\begin{array}{l}\text { Lower risk: } \\
\mathrm{HR}=0.76[0.69,0.83] ; \mathrm{ARR}=-2.4[-3.3,-1.5]\end{array}$ & High \\
\hline & & Hip & $\begin{array}{l}\text { Lower risk: } \\
\mathrm{HR}=0.67[0.47,0.96] ; \mathrm{ARR}=-0.3[-0.6,-0.03]\end{array}$ & Moderate (IM) \\
\hline & & CVF & $\begin{array}{l}\text { Lower risk: } \\
\mathrm{HR}=0.65[0.46,0.92] ; \mathrm{ARR}=-0.3[-0.5,-0.02]\end{array}$ & Moderate (IM) \\
\hline & \multirow[t]{2}{*}{$\begin{array}{l}\text { 5,897 PM women with intact uterus and } \\
\text { past clinical fracture }\end{array}$} & CF & $\begin{array}{l}\text { Lower risk: } \\
\mathrm{HR}=0.78[0.68,0.91] ; \mathrm{ARR}=-3[-5,-1]\end{array}$ & $\begin{array}{l}\text { Low } \\
\text { (RB, IM) }\end{array}$ \\
\hline & & Hip & $\begin{array}{l}\text { No difference: } \\
\mathrm{HR}=0.77[0.48,1.22] ; \mathrm{ARR}=-0.3[-0.9,0.2]\end{array}$ & $\begin{array}{l}\text { Low } \\
\text { (RB, IM) }\end{array}$ \\
\hline & $\begin{array}{l}\text { PM women with intact uterus and } \\
\text { osteoporosis by BMD } \\
\text { n not reported }\end{array}$ & CF & $\mathrm{HR}=0.53[0.25,1.10] ;$ ARR NA & $\begin{array}{l}\text { Insufficient } \\
\text { (RB, h-IM) }\end{array}$ \\
\hline \multirow[b]{2}{*}{$\begin{array}{l}\text { Estrogen/progestin } \\
\text { vs. nonplacebo } \\
\text { control } \\
1 \mathrm{RCT}^{20} \\
4 \mathrm{yr}\end{array}$} & \multirow{2}{*}{$\begin{array}{l}36 \text { PM women with osteoporosis by BMD } \\
\text { (T-score } \leq-2 \text { ) and RVF }\end{array}$} & NVF & $\mathrm{RR}=0.93[0.06,13.5] ; \mathrm{ARR}=-0.5[-19,18]$ & Insufficient (RB, h-IM) \\
\hline & & RVF & $\mathrm{RR}=0.37[0.09,1.62] ; \mathrm{ARR}=-22[-53,8]$ & Insufficient (RB, h-IM) \\
\hline
\end{tabular}

Abbreviations: $\mathrm{ARR}=\mathrm{absolute}$ risk reduction; $\mathrm{BMD}=$ bone mineral density; $\mathrm{CCT}=$ controlled clinical trial; $\mathrm{CF}=\mathrm{clinical}$ fracture; $\mathrm{CI}=\mathrm{confidence}$ intervals; $\mathrm{CVF}=\mathrm{clinical}$ vertebral fracture; h-IM=highly imprecise; HR=hazard ratio; IM=imprecise; IN=indirect; NA=not available; NVF=nonvertebral fracture; PM=postmenopausal; RB=medium risk of bias; $\mathrm{RCT}=$ randomized controlled trial; $\mathrm{RR}=$ risk ratio: $\mathrm{RVF}=$ radiographic vertebral fracture;

*Definitions of terms for strength of evidence grades and domains ratings are detailed in the section of the main report titled, 'Strength of Evidence for Major Comparisons and Outcomes.’

†Results reported for raloxifene $60 \mathrm{mg} / \mathrm{d}$ and $120 \mathrm{mg} /$ day groups pooled together.

$\ddagger$ Results reported for raloxifene $60 \mathrm{mg} / \mathrm{d}$ dose group.

**Analyses pooled all participants initially assigned to denosumab, which included both those who received long-term and short-term denosumab. 


\section{Harms}

Due to few events, RCT data provided insufficient evidence about whether long-term alendronate or zoledronate increase risk of radiologically confirmed atypical femoral fracture (AFF), subtrochanteric or femoral shaft fractures without radiologically confirmed AFF features, or osteonecrosis of the jaw (ONJ).

Data from controlled, long-term observational studies suggest that alendronate and bisphosphonates as a class increase both radiologically confirmed AFF (low SOE), and subtrochanteric and femoral shaft fractures without radiologically confirmed with AFF features (low SOE) (Table B). Relative risks for these outcomes varied from 1 to $>100$ across studies, likely related to heterogeneity in designs. Relative risks appeared higher for radiologically confirmed AFF than for cases defined only by diagnostic codes. However, some AFF risk estimates were calculated using controls with subtrochanteric or femoral shaft fractures without AFF features. ${ }^{38,39}$ In those cases, risk estimates reflect the probability that a subtrochanteric or femoral shaft fracture will have AFF features, and not the relative risk of sustaining an AFF. ${ }^{40}$ Studies also differed in whether fractures cases were excluded for cancer and excess trauma; in whether current bisphosphonate use was compared to no use, limited past use, or nonbisphosphonate osteoporosis drug use; and in how they addressed potential confounding.

Few observational studies provided data about risk of ONJ. Different studies provided low and insufficient strength evidence, respectively, about whether long-term alendronate increases risk of ONJ. ${ }^{41-43}$ Relative risk estimates varied widely between studies, likely due to heterogeneity in case definitions, treatment control groups, and covariate modeling.

We found insufficient evidence about whether long-term zoledronate increases risk of AFF or ONJ. The single trial of long-term zoledronate versus placebo reported no cases of AFF or ONJ in either treatment group, ${ }^{15}$ and we identified no eligible observational studies that evaluated risk of these harms with long-term zoledronate.

Due to its pooling of results for both short- and long-term denosumab treatment, it was not possible to conclude anything about the risk of harms of long-term denosumab compared with placebo from the one study that met eligibility for this review. ${ }^{18}$ In long-term trials of oral hormone therapy, specifically estrogen/progestin and estrogen versus placebo in postmenopausal women with unknown osteoporosis or osteopenia status, risk was significantly increased for cardiovascular disease, and mild cognitive impairment or dementia. ${ }^{44-47}$ Risk for a composite outcome measure defined to weigh risk of incidence of any of several serious harms (coronary heart disease, invasive breast cancer, stroke, pulmonary embolism, endometrial cancer, colorectal cancer, or death due to other causes) versus hip fracture did not differ between estrogen/progestin and placebo or between estrogen and placebo. Results were similar regardless of participants' baseline fracture risk, indicating that risk of one or more of these harms offset the reduction in hip fractures even in participants at highest baseline fracture risk. In addition, estrogen/progestin was associated with an increased risk of invasive breast cancer. Long-term raloxifene versus placebo in treatment for osteoporosis significantly increased the risk of deep vein thrombosis ${ }^{16,28,35}$ and pulmonary embolism by about 3 to 4 -fold. ${ }^{16,27-29,} 35$ 


\section{Variation in Harms as a Function of Patient, Bone, or Osteoporosis Drug Characteristics}

We found little evidence about factors that modify risk of harms with long-term osteoporosis drug treatment. One study was inconclusive about whether relative risk for AFF associated with bisphosphonate use increased with age. ${ }^{38}$ Three controlled observational studies reported that $>5$ years of bisphosphonate use increased risk of subtrochanteric or femoral shaft fractures (ST/FSF) or radiologically confirmed AFF more than did 3-5 years of use. ${ }^{38,48,49}$ However, none of these studies reported tests for interaction by treatment duration. For long-term raloxifene versus placebo, one study reported that risk of deep venous thrombosis and pulmonary embolism did not vary as a function of baseline cardiovascular risk, ${ }^{32}$ and another that risk of incident stroke was lower with raloxifene versus placebo in women with increased cardiovascular risk. ${ }^{31}$ Trials of long-term oral hormone therapy evaluated whether risk of harms varied by a long list of patient characteristics. ${ }^{44-47}$ Though results suggested that risk of breast cancer with estrogen/progestin compared to placebo may be greater with increased duration of prior postmenopausal hormone use, this was the only significant result out of many examined, and may have been due to chance. Strength of evidence was not assessed for effect modifiers. 
Table B. Evidence on harms of long-term ( $>3$ years) osteoporosis drug treatment

\begin{tabular}{|c|c|c|c|c|}
\hline $\begin{array}{l}\text { Comparison } \\
\text { \# Studies by Design } \\
\text { Treatment Duration } \\
\end{array}$ & Participant Characteristics & Harms & $\begin{array}{l}\text { Relative and Absolute Risk Differences } \\
(95 \% \mathrm{Cl})\end{array}$ & $\begin{array}{l}\text { Strength of } \\
\text { Evidence* } \\
\text { (Justification) }\end{array}$ \\
\hline $\begin{array}{l}\text { Alendronate vs. } \\
\text { placebo } \\
1 \mathrm{RCT}^{50} \\
3 \text { to } 4.5 \mathrm{yr}\end{array}$ & $\begin{array}{l}6,459 \text { PM women with } \\
\text { osteopenia or osteoporosis (T- } \\
\text { score } \leq-1.6 \text { ) with or without } \\
\text { RVF }\end{array}$ & $\begin{array}{l}\text { ST or FS fracture DX with rare x-ray } \\
\text { review for confirmation of AFF } \\
\text { features ( } n=2 \text { cases) }\end{array}$ & $\begin{array}{l}\mathrm{HR}=1.03[0.06,16.46] ; \mathrm{ARR}=0[-0.09, \\
0.09]\end{array}$ & $\begin{array}{l}\text { Insufficient } \\
\text { (h-IM) }\end{array}$ \\
\hline \multirow{3}{*}{$\begin{array}{l}\text { Alendronate vs. no } \\
\text { osteoporosis drug } \\
\text { treatment } \\
2 \text { retrospective cohort } \\
\text { observational } \\
\text { studies }{ }^{41,51,52} \\
3.8 \text { yr (mean) and } \geq 6 \\
\text { yr }\end{array}$} & $\begin{array}{l}534 \text { adults } \geq 60 \text { yr with nonhip } \\
\text { fracture }(90 \% \text { women })\end{array}$ & $\begin{array}{l}\text { ST or FS fracture DX codes without } x- \\
\text { ray confirmation of AFF features ( } n=5 \\
\text { cases) }\end{array}$ & $\geq 6 \mathrm{yr} ; \mathrm{HR}=1.37[0.22,8.62] ;$ ARR NA & $\begin{array}{l}\text { Insufficient } \\
\text { (RB, h-IM) }\end{array}$ \\
\hline & \multirow[t]{2}{*}{$\begin{array}{l}220,360 \text { adults ( } 85 \% \text { women) } \\
\text { exposed to alendronate or no } \\
\text { osteoporosis drug general } \\
\text { population controls from } \\
\text { national database }\end{array}$} & $\begin{array}{l}\text { ST or FS fracture DX codes without } x- \\
\text { ray confirmation of AFF features } \\
\text { ( } n=309 \text { cases) }\end{array}$ & $\begin{array}{l}\text { Higher risk: } \\
\text { 3.8 yr; ST: } 0.17 \% \text { vs. } 0.06 \% ; \mathrm{HR}=2.41 \\
\text { [1.78, 3.27]; ARR=0.11 [0.08, } 0.15] \\
\text { 3.8 yr; FS: } 0.12 \% \text { vs. } 0.03 \% ; \mathrm{HR}=2.90 \\
\text { [1.97, 4.26]; ARR } 0.09(0.06,0.12) \\
\text { 3.8 yr: ST/FS: } 0.29 \% \text { vs. } 0.09 \% ; \text { ARR } 0.20 \\
(0.15,0.25)\end{array}$ & $\begin{array}{l}\text { Low } \\
\text { (RB, IM, LE) }\end{array}$ \\
\hline & & $\begin{array}{l}\text { ONJ DX codes without } x \text {-ray or } \\
\text { pathology review ( } n=28 \text { cases) }\end{array}$ & $\begin{array}{l}\text { Higher risk: } \\
3.8 \mathrm{yr} ; \mathrm{HR}=3.15[1.44,6.87] ; \text { ARR NA }\end{array}$ & $\begin{array}{l}\text { Low } \\
\text { (RB, IM, LE) }\end{array}$ \\
\hline $\begin{array}{l}\text { Alendronate vs. } \\
\text { raloxifene } \\
1 \text { retrospective cohort } \\
\text { observational study } \\
\sim 4 \text { yr (mean) }\end{array}$ & $\begin{array}{l}8,354 \text { women aged }>50 \mathrm{yr} \\
\text { from database of } 1 \text { hospital }\end{array}$ & $\begin{array}{l}\text { ONJ DX codes with } x \text {-ray and } \\
\text { pathology features ( } \mathrm{n}=40 \text { cases) }\end{array}$ & $\begin{array}{l}\text { Higher risk with alendronate: } \\
\mathrm{HR}=7.42[1.02,54.09] ; \text { ARR NA }\end{array}$ & $\begin{array}{l}\text { Low } \\
\text { (RB, IM, LE) }\end{array}$ \\
\hline $\begin{array}{l}\text { Alendronate vs. } \\
\text { raloxifene or } \\
\text { calcitonin } \\
1 \text { retrospective cohort } \\
\text { observational study }{ }^{42} \\
\text { Up to } 6 \text { yr }\end{array}$ & $\begin{array}{l}43,645 \text { adults aged } \geq 50 \mathrm{yr} \\
(84 \% \text { women) with recent hip } \\
\text { or vertebral fracture now on } \\
\text { osteoporosis drug treatment } \\
\text { from national database }\end{array}$ & $\begin{array}{l}\text { ONJ DX codes without } x \text {-ray or } \\
\text { pathology review ( } n=46 \text { cases) }\end{array}$ & $\mathrm{HR}=0.86[0.44,1.69] \dagger ;$ ARR NA & $\begin{array}{l}\text { Insufficient } \\
\text { (RB, h-IM) }\end{array}$ \\
\hline $\begin{array}{l}\text { Zolendronate vs. } \\
\text { placebo } \\
1 \mathrm{RCT}^{15} \\
6 \mathrm{yr} \\
\end{array}$ & $\begin{array}{l}2,000 \text { PM women } \geq 65 \text { with } \\
\text { osteoporosis or osteopenia }\end{array}$ & SAE & $\begin{array}{l}\text { No difference: } \\
\text { OR=0.84 }[0.70,1.00] ; \text { ARR }=-4[-9,0]\end{array}$ & $\begin{array}{l}\text { Low } \\
\text { (IM) }\end{array}$ \\
\hline $\begin{array}{l}\text { Bisphosphonate } \neq \text { vs. } \\
\text { no bisphosphonate } \\
3 \text { observational } \\
\text { studies }^{38,39,53} \\
\geq 3 \mathrm{yr}\end{array}$ & $\begin{array}{l}-2.8 \text { million (retrospective } \\
\text { cohort) and } 1,124 \text { (case- } \\
\text { control) adults aged } \geq 55 \mathrm{yr} \\
\text { from national database }(87 \% \\
\text { women cases and } 52 \% \\
\text { women controls in cohort }\end{array}$ & $\begin{array}{l}\text { AFF with radiologic features }(\mathrm{n}=172 \\
\text { cases) }\end{array}$ & $\begin{array}{l}\text { Higher risk: } \\
\text { Cohort } \\
\geq 4 \text { yr: } R R=126[55,288] \text {; ARR NA } \\
\text { Case-control } \\
\text { 3-4 yr: OR=40 [17, 91]; ARR=NA }\end{array}$ & $\begin{array}{l}\text { LoW } \\
\text { (RB, CO, LE) }\end{array}$ \\
\hline
\end{tabular}




\begin{tabular}{|c|c|c|c|c|}
\hline $\begin{array}{l}\text { Comparison } \\
\text { \# Studies by Design } \\
\text { Treatment Duration }\end{array}$ & Participant Characteristics & Harms & $\begin{array}{l}\text { Relative and Absolute Risk Differences } \\
(95 \% \mathrm{Cl})\end{array}$ & $\begin{array}{l}\text { Strength of } \\
\text { Evidence* }^{*} \\
\text { (Justification) }\end{array}$ \\
\hline & $\begin{array}{l}\text { analysis; } 86 \% \text { women in case- } \\
\text { control analysis) }\end{array}$ & & $\begin{array}{l}\text { 4-5 yr: OR=116 [58, 234]; ARR=NA } \\
>5 \text { yr: OR=93 [66, 132]; ARR=NA }\end{array}$ & \\
\hline & $\begin{array}{l}264 \text { women aged } \geq 65 \text { yr from } \\
\text { national primary practice } \\
\text { database (case-control) }\end{array}$ & $\begin{array}{l}\text { ST or FS fracture DX codes without } x- \\
\text { ray confirmation of AFF features } \\
\text { ( } n=44 \text { cases) }\end{array}$ & $\begin{array}{l}\text { Higher risk: } \\
>3 \text { yr: OR=9.46 }[2.17,41.3] ; A R R=N A\end{array}$ & $\begin{array}{l}\text { LoW } \\
\text { (RB, LE) }\end{array}$ \\
\hline & $\begin{array}{l}6,644 \text { women aged } \geq 50 \text { yr with } \\
\text { hip or femoral fracture from } 8 \\
\text { hospital medical records } \\
\text { databases (nested case- } \\
\text { control) }\end{array}$ & $\begin{array}{l}\text { AFF with radiologic features }(\mathrm{n}=196 \\
\text { cases) }\end{array}$ & $\begin{array}{l}\text { Higher risk: } \\
\text { Mean use } 5.2 \text { yr: OR=25.65 [10.74, } \\
61.28] ; \text { ARR=NA }\end{array}$ & $\begin{array}{l}\text { Low } \\
\text { (RB, LE) }\end{array}$ \\
\hline \multirow{2}{*}{$\begin{array}{l}\text { Current vs. past } \\
\text { bisphosphonates } \ddagger \\
2 \text { case-control } \\
\text { observational } \\
\text { studies }^{48,54} \\
\geq 3 \mathrm{yr}\end{array}$} & $\begin{array}{l}172 \text { PM women with } \geq 1 \mathrm{yr} \\
\text { bisphosphonate use from } 1 \\
\text { hospital database }\end{array}$ & $\begin{array}{l}\text { AFF with radiologic features }(n=43 \\
\text { cases) }\end{array}$ & $\begin{array}{l}\text { Higher risk with current bisphosphonate: } \\
\text { HR=3.36 [1.77, 11.91] to } 5.17 \text { [2.0, 13.36]; } \\
\text { ARR NA }\end{array}$ & $\begin{array}{l}\text { LoW } \\
\text { (RB, LE) }\end{array}$ \\
\hline & $\begin{array}{l}1,855 \text { women aged } \geq 68 \mathrm{yr} \\
\text { from a provincial database }\end{array}$ & $\begin{array}{l}\text { ST or FS fracture DX codes without } x- \\
\text { ray review ( } n=325 \text { cases) }\end{array}$ & $\begin{array}{l}\text { Higher risk with current bisphosphonate: } \\
3-5 \mathrm{yr}: \mathrm{OR}=1.59[0.80,3.15] ; \text { ARR=NA } \\
\geq 5 \mathrm{yr}: \mathrm{OR}=2.74[1.25,6.02] ; \mathrm{ARR}=\mathrm{NA}\end{array}$ & $\begin{array}{l}\text { Low } \\
(\mathrm{RB}, \mathrm{IM})\end{array}$ \\
\hline $\begin{array}{l}\text { Bisphosphonates } \dagger \\
\text { vs. pooled raloxifene } \\
\text { or calcitonin } \\
1 \text { retrospective cohort } \\
\text { observational study }{ }^{49} \\
\geq 3 \mathrm{yr}\end{array}$ & $\begin{array}{l}\text { 4,097 Medicare beneficiaries } \\
\text { ( } 97 \% \text { women) }\end{array}$ & $\begin{array}{l}\text { ST or FS fracture DX codes without } x- \\
\text { ray confirmation of AFF features } \\
\text { ( } n=34 \text { cases) }\end{array}$ & $\begin{array}{l}3-5 \text { yr: } \mathrm{HR}=1.20[0.55,2.61] ; A R R=0.1[- \\
0.3,0.5] \\
>5 \text { yr: } \mathrm{HR}=2.02[0.41,10.0] ; \mathrm{ARR}=0.1[- \\
0.1,0.4]\end{array}$ & $\begin{array}{l}\text { Insufficient } \\
\text { (RB, h-IM) }\end{array}$ \\
\hline $\begin{array}{l}\text { Denosumab†† vs. } \\
\text { placebo } \\
1 \mathrm{RCT}^{18} \\
4 \mathrm{yr}\end{array}$ & $\begin{array}{l}365 \text { PM women with } \\
\text { osteopenia or osteoporosis by } \\
\text { BMD }\end{array}$ & SAE & $\mathrm{RR}=1.64[0.69,3.88] ; \mathrm{ARR}=7[-3,17]$ & $\begin{array}{l}\text { Insufficient } \\
\text { (RB, IN, h-IM) }\end{array}$ \\
\hline $\begin{array}{l}\text { Raloxifene vs. } \\
\text { placebo } \\
1 \text { RCT with 1 CCT } \\
\text { extension }{ }^{16,17,26-37} \\
4 \text { to } 8 \text { yr }\end{array}$ & $\begin{array}{l}\text { 6,828 PM women with } \\
\text { osteoporosis by BMD or RVF }\end{array}$ & SAE & $\begin{array}{l}\text { No difference: } \\
8 \mathrm{yr}: \mathrm{RR}=0.93[0.86,1.00]^{\star \star} ; \mathrm{ARR}=-3[-6 \text {, } \\
\text { 0] }\end{array}$ & $\begin{array}{l}\text { Low } \\
(\mathrm{RB}, \mathrm{IM})\end{array}$ \\
\hline $\begin{array}{l}\text { Raloxifene vs. no } \\
\text { treatment } \\
1 \text { retrospective cohort } \\
\text { observational study }{ }^{41} \text {, }\end{array}$ & $\begin{array}{l}\text { 19,324 adults ( } 85 \% \text { women) } \\
\text { exposed to raloxifene or no } \\
\text { osteoporosis drug general } \\
\text { population controls from } \\
\text { national database }\end{array}$ & $\begin{array}{l}\text { ST or FS fracture DX codes without } x- \\
\text { ray confirmation of AFF features } \\
\text { ( } n=25 \text { cases) }\end{array}$ & $\begin{array}{l}\text { ST: HR=1.06 [0.34, 3.32]; ARR } 0.04[- \\
0.06,0.14] \\
\text { FS: HR=0.82 [0.21, 3.20]; ARR } 0.01[- \\
0.07,0.09]\end{array}$ & $\begin{array}{l}\text { Insufficient } \\
\text { (RB, h-IM) }\end{array}$ \\
\hline
\end{tabular}




\begin{tabular}{|l|l|l|l|}
\hline $\begin{array}{l}\text { Comparison } \\
\text { \# Studies by Design } \\
\text { Treatment Duration }\end{array}$ & Participant Characteristics & Harms & $\begin{array}{l}\text { Relative and Absolute Risk Differences } \\
\text { (95\% Cl) }\end{array}$ \\
\hline $\begin{array}{l}52 \\
3.8 \mathrm{yr} \text { (mean) }\end{array}$ & $\begin{array}{l}\text { Strength of } \\
\text { Evidence* } \\
\text { (Justification) }\end{array}$ \\
\hline
\end{tabular}

Abbreviations: AFF=atypical femoral fracture; $\mathrm{ARR}=$ absolute risk reduction; $\mathrm{BMD}=$ bone mineral density; $\mathrm{CCT}=$ controlled clinical trial; $\mathrm{CI}=\mathrm{confidence}$ intervals; $\mathrm{CO}=\mathrm{consistent;}$ $\mathrm{DX}=$ diagnosis; FS=femoral shaft; h-IM=highly imprecise; HR=hazard ratio; IM=imprecise; IN=indirect; LE=large effect; NA=not available (data not reported);

ONJ=osteonecrosis of the jaw; $\mathrm{OR}=$ odds ratio; $\mathrm{PM}=$ postmenopausal; $\mathrm{RB}=$ medium risk of bias; $\mathrm{RCT}=$ randomized controlled trial; $\mathrm{RR}=$ risk ratio: $\mathrm{RVF}=$ radiographic vertebral fracture; $\mathrm{SAE}=$ serious adverse event; $\mathrm{ST}=$ subtrochanteric

*Definitions of terms for strength of evidence grades and domains ratings are detailed in the section of the main report titled, 'Strength of Evidence for Major Comparisons and Outcomes.'

†Because the higher adjusted incidence rates in the alendronate group (0.15\%) compared with the raloxifene-calcitonin group (0.08\%) suggested a possibly increased risk, we manually recalculated the estimate of effect and found RR 1.20 (95\% CI 0.59, 2.56). Authors were contacted for clarification, but did not reply.

$\Varangle$ Included bisphosphonates varied by study. All studies included alendronate, risedronate, and one or more of the following: ibandronate, etidronate, and zoledronate.

**Results reported for raloxifene $60 \mathrm{mg} / \mathrm{d}$ dose group.

††Analyses pooled all participants initially assigned to denosumab, which included both those who received long-term and short-term denosumab. 


\section{Osteoporosis Drug Holidays}

\section{Effect}

In postmenopausal women who previously received 3-5 years of bisphosphonate, two trials compared continued versus discontinued alendronate for 5 more years, and one compared continued versus discontinued zoledronate for 3 more years. ${ }^{55-57}$ None found a reduction in incident nonvertebral fractures (Table C).

However, these trials collectively suggested a reduction in incident vertebral fractures. One enrolled women who previously received 5 years of alendronate in the active treatment arm of a trial for osteopenia or osteoporosis and a subsequent extension, and reported that alendronate continuation for 5 years versus placebo (drug holiday) reduced incident clinical vertebral fractures (HR 0.45 [95\% CI 0.24, 0.85]) (moderate SOE), but not incident radiographic vertebral fractures (HR 0.86 [95\% CI 0.60, 1.22]) (moderate SOE). ${ }^{55}$ A second trial enrolled women who previously received 3 years of zoledronate in the active treatment arm of a trial for osteoporosis, and reported that zoledronate continuation for 3 years versus placebo (drug holiday) reduced incident radiographic vertebral fractures (HR 0.51 [95\% CI 0.26, 0.95) (low SOE), but that evidence was insufficient about incident clinical vertebral fracture. ${ }^{58}$ In a third trial that enrolled women who previously received 5 years of alendronate in the active treatment arm of a trial for osteoporosis and a subsequent extension, and then nonrandomly assigned them to alendronate continuation for 2 years and 5 years versus placebo (drug holiday), evidence was insufficient to draw conclusions about differences in risk of incident clinical vertebral fractures or incident radiographic vertebral fractures. ${ }^{57-59}$ Similarly, we could not draw conclusions from a small, 4year denosumab dose-finding trial, because fracture results were pooled between the denosumab continuation and discontinuation treatment arms. ${ }^{18}$

In women who previously received 3-5 years of bisphosphonate treatment, continued bisphosphonate treatment for an additional 3-5 years was associated with stable or slightly decreased hip BMD, whereas women assigned to discontinue treatment (drug holiday) had significantly larger declines in hip BMD. A 4-year denosumab trial reported that compared to baseline, hip and spine BMD were most increased in women assigned denosumab for 4 years, back to pretreatment baseline in women assigned denosumab for 2 years followed by discontinuation for 2 years, and intermediately increased in women assigned denosumab for 2 years, placebo for 1 year, then denosumab for 1 year.

\section{Variation in Effect as a Function of Patient, Bone, or Osteoporosis Drug Characteristics}

In post hoc analyses, the effect of alendronate continuation versus discontinuation (drug holiday) on risk of incident clinical fractures, which did not differ overall, did not vary as a function of baseline BMD or radiographic vertebral fracture status. ${ }^{55,60}$ Further post hoc subgroup analyses suggested that in women without a prevalent radiographic vertebral fracture, continued alendronate versus discontinuation reduced risk of incident nonvertebral fractures in women with osteoporotic BMD but not in those with osteopenia. However, risk for incident nonvertebral fractures between alendronate continuation and discontinuation appeared not to differ in women with prevalent radiographic vertebral fractures. Further, risk of incident vertebral fractures appeared no different between alendronate continuation and discontinuation groups, regardless of baseline BMD or radiographic vertebral fracture status. The single positive 
outcome may have been due to chance. We found no evidence about possible modifiers of the effect of continuing any other osteoporosis drug treatment versus discontinuation on risk of incident fracture.

\section{Harms}

Trials of alendronate and zoledronate continuation versus discontinuation reported no difference between treatment groups in risk of serious adverse events (Table D). ${ }^{55,56,58-62}$ Too few cases of AFF with confirmed radiologic features, subtrochanteric or femoral shaft fractures without confirmed AFF features, or ONJ, occurred in these trials to draw conclusions about differences in their risk between treatment continuation and discontinuation groups. ${ }^{50,58}$ One retrospective cohort study reported that incidence of AFF was significantly higher in bisphosphonate users (99\% alendronate) who continued versus discontinued use $(0.15 \%$ vs. 0.03\%; estimated OR 6.03 [95\% CI 1.87, 19.42]). ${ }^{63}$ However, this analysis did not radiologically confirm AFF diagnoses and did not describe accounting for potentially confounding variables. Though atrial fibrillation appeared more frequently with zoledronate continuation versus discontinuation, the absolute number of events was low and possible differences between treatment groups were not statistically significant. ${ }^{58,59}$ It was not possible to draw conclusions about differences in harms between the denosumab continuation and discontinuation arms in one trial reporting because harms results for these two groups were pooled. ${ }^{18}$

\section{Variation in Harms as a Function of Patient, Bone, or Osteoporosis Drug Characteristics}

We found no evidence about whether the risk of harms between continuation of any osteoporosis drug treatment and discontinuation varied as a function of patient, bone or drug characteristics. 
Table C. Evidence on effects of osteoporosis drug continuation versus discontinuation* on incident fractures

\begin{tabular}{|c|c|c|c|c|}
\hline $\begin{array}{l}\text { Comparison } \\
\text { \# Studies by Design } \\
\text { Treatment Duration }\end{array}$ & Participant Characteristics & $\begin{array}{l}\text { Incident } \\
\text { Fracture } \\
\text { Outcome }\end{array}$ & $\begin{array}{l}\text { Relative and Absolute Risk Differences } \\
(95 \% \mathrm{Cl})\end{array}$ & Strength of Evidence $†$ \\
\hline \multirow{5}{*}{$\begin{array}{l}\text { Alendronate } \\
\text { continuation vs. } \\
\text { discontinuation } \\
\text { (AL x } 10 \text { yr vs. AL x } 5 \\
\text { yr followed by PBO x } \\
5 \text { yr) } \\
1 \text { RCT }^{55}\end{array}$} & \multirow{5}{*}{$\begin{array}{l}\text { 1,099 PM women previously received } \\
\text { alendronate } 5 \text { yr for osteopenia or } \\
\text { osteoporosis (T-score } \leq-1.6)\end{array}$} & CF & $\begin{array}{l}\text { No difference: } \\
\mathrm{RR}=0.93[0.71,1.21] ; \mathrm{ARR}=-1[-6,4]\end{array}$ & Moderate (IM) \\
\hline & & NVF & $\begin{array}{l}\text { No difference: } \\
\mathrm{RR}=1.00[0.76,1.32] ; \mathrm{ARR}=-0.1[-5,5]\end{array}$ & Moderate (IM) \\
\hline & & Hip & $R R=1.02[0.51,2.10] ; A R R=0[-2,2]$ & Insufficient (h-IM) \\
\hline & & CVF & $\begin{array}{l}\text { Lower risk with continuation: } \\
R R=0.45[0.24,0.85] ; A R R=-3[-5,-0.5]\end{array}$ & Moderate (IM) \\
\hline & & RVF & $\begin{array}{l}\text { No difference: } \\
\mathrm{RR}=0.86[0.60,1.22] ; \mathrm{ARR}=-1[-5,2]\end{array}$ & Moderate (IM) \\
\hline \multirow{3}{*}{$\begin{array}{l}\text { Alendronate } \\
\text { continuation vs. } \\
\text { discontinuation } \\
\text { (AL x } 7 \text { yr [A7] vs. AL } \\
\text { x } 5 \text { yr followed by } \\
\text { PBO x } 2 \text { yr [A5/P2]; } \\
\text { AL x } 10 \text { yr [A10] vs. } \\
\text { AL x } 7 \text { yr + PBO x } 3 \\
\text { yr [A7/P3]) } \\
1 \text { RCT }^{56,57}\end{array}$} & \multirow[t]{3}{*}{$\begin{array}{l}350 \text { PM women previously received } \\
\text { alendronate } 5 \text { yr for osteoporosis (T-score } \\
\leq-2.5)(n=350 \text { for } A 7 \text { vs. A5/P2; } n=247 \text { for } \\
\text { A10 vs. A7/P3) }\end{array}$} & NVF & $\begin{array}{l}\text { A7 vs. A5/P2: RR=0.87 }[0.40,1.91] ; A R R=- \\
1[-7,5] \\
\text { A10 vs. A7/P3: } R R=0.81[0.38,1.71] ; A R R= \\
-2[-11,6]\end{array}$ & $\begin{array}{l}\text { A7 vs. A5/P2: Insufficient } \\
\text { (h-IM) } \\
\text { A10 vs. A7/P3: Insufficient } \\
\text { (RB, h-IM) }\end{array}$ \\
\hline & & CVF & $\begin{array}{l}\text { A7 vs. A5/P2: RR= } 0.92[0.40,2.10] ; A R R=- \\
1[-6,5]\end{array}$ & Insufficient (h-IM) \\
\hline & & RVF & $\begin{array}{l}\text { AL10 vs. AL5/P5: RR=1.40 [0.52, 3.74]; } \\
\text { ARR=2.6 }[-4.6,9.9]\end{array}$ & Insufficient (RB, h-IM) \\
\hline $\begin{array}{l}\text { Zoledronate } \\
\text { continuation vs. } \\
\text { discontinuation } \\
(\mathrm{Z} \times 2 \text { yr vs. } \mathrm{Z} \times 1 \mathrm{yr} \\
\text { followed by PBO x } 1 \\
\mathrm{yr}) \\
1 \mathrm{RCT}^{61}\end{array}$ & 379 PM women with osteopenia & CF & $\mathrm{RR}=1.37[0.39,4.78] ; \mathrm{ARR}=1[-2,4]$ & Insufficient (h-IM) \\
\hline \multirow{6}{*}{$\begin{array}{l}\text { Zoledronate } \\
\text { continuation vs. } \\
\text { discontinuation }(Z \times 6 \\
\text { yr vs. Z x } 3 \text { yr } \\
\text { followed by PBO x } 3 \\
\text { yr) } \\
1 \mathrm{RCT}^{58}\end{array}$} & \multirow{6}{*}{$\begin{array}{l}\text { 1,233 PM women previously received } \\
\text { zoledronic acid } 3 \text { yr for osteoporosis by } \\
\text { BMD or RVF }\end{array}$} & $\mathrm{CF}$ & $\begin{array}{l}\text { No difference: } \\
\mathrm{HR}=1.04[0.71,1.54] \text {; ARR NA }\end{array}$ & Moderate (IM) \\
\hline & & NVF & $\begin{array}{l}\text { No difference: } \\
\mathrm{HR}=0.99[0.7,1.5] ; \mathrm{ARR}=-0.3[-3,3]\end{array}$ & Moderate (IM) \\
\hline & & Hip & $\mathrm{HR}=0.90[0.33,2.49] ; \mathrm{ARR}=-0.2[-1,1]$ & Insufficient (h-IM) \\
\hline & & CVF & $\mathrm{HR}=1.81[0.53,6.2] ;$ ARR NA & Insufficient (h-IM) \\
\hline & & RVF & $\begin{array}{l}\text { Lower risk with continuation: } \\
\text { OR=0.51 }[0.26,0.95] ; \text { ARR }=-3[-6,-1]\end{array}$ & Low (h-IM) \\
\hline & & CF & $\mathrm{HR}=1.11[0.45,2.73] ; \mathrm{ARR}=1[-7,10]$ & Insufficient (h-IM) \\
\hline
\end{tabular}




\begin{tabular}{|c|c|c|c|c|}
\hline $\begin{array}{l}\text { Comparison } \\
\text { \# Studies by Design } \\
\text { Treatment Duration }\end{array}$ & Participant Characteristics & $\begin{array}{l}\text { Incident } \\
\text { Fracture } \\
\text { Outcome }\end{array}$ & $\begin{array}{l}\text { Relative and Absolute Risk Differences } \\
(95 \% \mathrm{Cl})\end{array}$ & Strength of Evidence $\dagger$ \\
\hline $\begin{array}{l}\text { Zoledronate } \\
\text { continuation vs. } \\
\text { discontinuation } \\
(Z \times 9 \text { yr vs. Z x } 6 \text { yr } \\
\text { followed by PBO x } 3 \\
\text { yr) } \\
1 \text { RCT }^{59}\end{array}$ & $\begin{array}{l}190 \text { PM women previously received } \\
\text { zoledronic acid } 6 \text { yr for osteoporosis by } \\
\text { BMD or RVF }\end{array}$ & RVF & $\mathrm{OR}=0.58[0.13,2.55] ; \mathrm{ARR}=-2[-8,4]$ & Insufficient (h-IM) \\
\hline $\begin{array}{l}\text { Denosumab } \\
\text { continuation vs. } \\
\text { discontinuation ( } \mathrm{D} \times 4 \\
\text { yr vs. D x } 2 \text { yr } \\
\text { followed by PBO } \times 2 \\
\text { yr) } \\
1 \mathrm{RCT}^{18}\end{array}$ & $\begin{array}{l}314 \text { PM women with osteopenia or } \\
\text { osteoporosis by BMD }\end{array}$ & CF & No numerical data & Insufficient (no data) \\
\hline
\end{tabular}

Abbreviations: $\mathrm{AL}=$ alendronate; $\mathrm{ARR}=$ absolute risk reduction; $\mathrm{BMD}=$ bone mineral density; $\mathrm{CF}=\mathrm{clinical}$ fracture; $\mathrm{CI}=\mathrm{confidence}$ intervals; $\mathrm{CVF}=\mathrm{clinical}$ vertebral fracture; $\mathrm{D}=$ denosumab; h-IM=highly imprecise; $\mathrm{HR}=$ hazard ratio; $\mathrm{IM}=$ imprecise; $\mathrm{NA}=$ not available (no data reported); NVF=nonvertebral fracture; OR=odds ratio; $\mathrm{PBO}=$ placebo; $\mathrm{PM}=$ postmenopausal; RCT=randomized controlled trial; $\mathrm{RB}=$ medium risk of bias; $\mathrm{RR}=$ risk ratio: $\mathrm{RVF}=$ radiographic vertebral fracture; $\mathrm{Z}=$ =zoledronate

*Discontinuation $\geqq 1$ year after prior treatment $\geqq 1$ year.

†Definitions of terms for strength of evidence grades and domains ratings are detailed in the section of the main report titled, 'Strength of Evidence for Major Comparisons and Outcomes.'

Table D. Evidence on harms of osteoporosis drug continuation versus discontinuation*

\begin{tabular}{|c|c|c|c|c|}
\hline $\begin{array}{l}\text { Comparison } \\
\text { \# Studies by Design } \\
\text { Treatment Duration }\end{array}$ & $\begin{array}{l}\text { Participant } \\
\text { Characteristics }\end{array}$ & Harms & $\begin{array}{l}\text { Relative and Absolute Risk } \\
\text { Differences }(95 \% \mathrm{Cl})\end{array}$ & $\begin{array}{l}\text { Strength of } \\
\text { Evidence } \dagger \\
\text { (Justification) }\end{array}$ \\
\hline \multirow{3}{*}{$\begin{array}{l}\text { Alendronate continuation vs. } \\
\text { discontinuation } \\
(\mathrm{AL} \times 10 \mathrm{yr} \text { vs. AL x } 5 \mathrm{yr} \\
\text { followed by PBO x } 5 \mathrm{yr}) \\
1 \mathrm{RCT}^{55}\end{array}$} & \multirow{3}{*}{$\begin{array}{l}\text { 1,099 PM women } \\
\text { previously received } \\
\text { alendronate } 5 \text { yr for } \\
\text { osteopenia or osteoporosis } \\
\text { (T-score } \leq-1.6)\end{array}$} & SAE & $\begin{array}{l}\text { Stated as no difference, but no data } \\
\text { provided }\end{array}$ & $\begin{array}{l}\text { Insufficient } \\
\text { (no data) }\end{array}$ \\
\hline & & $\begin{array}{l}\text { Subtrochanteric or femoral shaft } \\
\text { fracture DX with rare } x \text {-ray review } \\
\text { ( } n=3 \text { cases) }\end{array}$ & $\begin{array}{l}\mathrm{HR}=1.33[0.12,14.67] ; \mathrm{ARR}=-0.1[-0.5, \\
0.7]\end{array}$ & $\begin{array}{l}\text { Insufficient } \\
\text { (h-IM) }\end{array}$ \\
\hline & & ONJ not defined ( $n=0$ cases) & No cases in either group & $\begin{array}{l}\text { Insufficient } \\
\text { (h-IM) }\end{array}$ \\
\hline
\end{tabular}




\begin{tabular}{|c|c|c|c|c|}
\hline $\begin{array}{l}\text { Comparison } \\
\text { \# Studies by Design } \\
\text { Treatment Duration }\end{array}$ & $\begin{array}{l}\text { Participant } \\
\text { Characteristics }\end{array}$ & Harms & $\begin{array}{l}\text { Relative and Absolute Risk } \\
\text { Differences }(95 \% \mathrm{Cl})\end{array}$ & $\begin{array}{l}\text { Strength of } \\
\text { Evidence } \dagger \\
\text { (Justification) }\end{array}$ \\
\hline $\begin{array}{l}\text { Alendronate continuation vs. } \\
\text { discontinuation } \\
\text { (AL x } 7 \mathrm{yr}[\mathrm{A} 7] \mathrm{vs} . \mathrm{AL} \times 5 \mathrm{yr} \\
\text { followed by PBO } \times 2 \mathrm{yr} \\
\text { [A5/P2]; } \\
\mathrm{AL} \times 10 \mathrm{yr}[\mathrm{A} 10] \mathrm{vs} . \mathrm{AL} \times 7 \mathrm{yr} \\
\left.+\mathrm{PBO}^{\circ} \times \mathrm{yr}[\mathrm{A} 7 / \mathrm{P} 3]\right) \\
1 \mathrm{RCT}^{56,57}\end{array}$ & $\begin{array}{l}350 \text { PM women previously } \\
\text { received alendronate } 5 \mathrm{yr} \\
\text { for osteoporosis (T-score } \\
\leq-2.5 \text { ) }\end{array}$ & SAE & $\begin{array}{l}\text { A7 vs. A5/P2: RR= } 1.05[0.57,1.96] ; \\
\text { ARR=1 }[-7,8] \\
\text { A10 vs. A7/P3: RR= } 1.21[0.75,1.96] \\
\text { ARR=5 }[-7,16]\end{array}$ & $\begin{array}{l}\text { A7 vs. A5/P2: } \\
\text { Insufficent } \\
\text { (h-IM) } \\
\text { A10 vs. A7/P3: } \\
\text { Insufficient } \\
\text { (RB, IM) }\end{array}$ \\
\hline $\begin{array}{l}\text { Bisphosphonate continuation } \\
\text { vs. discontinuation } \\
\text { (Continued BP } 3.5 \mathrm{yr} \text { [mean] } \\
\text { [persistent group] or } 4.1 \mathrm{yr} \\
\text { [mean] [nonpersistent group] } \\
\text { vs. BP holiday } 3.1 \text { yr [mean]) } \\
1 \text { retrospective cohort } \\
\text { observational study }{ }^{63}\end{array}$ & $\begin{array}{l}39,502 \text { women aged } \geq 45 \\
\text { yr with } \geq 3 \text { yr of prior } \geq 50 \% \\
\text { adherent BP use }(99 \% \\
\text { alendronate })\end{array}$ & "AFF" (not defined) (n=47 cases) & $\begin{array}{l}\text { Higher risk with bisphosphonate } \\
\text { (alendronate) continuation: } \\
\text { Pooled continuation groups } 0.15 \% \\
(44 / 28005) \text { vs. discontinuation } 0.03 \% \\
(3 / 11497) \\
\text { OR=6.03 }[1.87,19.42] ; \text { ARR }=0.13[0.08 \text {, } \\
0.19]\end{array}$ & $\begin{array}{l}\text { Low } \\
\text { (RB, IM, LE) }\end{array}$ \\
\hline \multirow{2}{*}{$\begin{array}{l}\text { Zoledronate continuation vs. } \\
\text { discontinuation } \\
(Z \times 2 \text { yr vs. } Z \times 1 \text { yr followed } \\
\text { by PBO x } 1 \text { yr) } \\
1 \mathrm{RCT}^{61}\end{array}$} & \multirow[t]{2}{*}{$\begin{array}{l}379 \text { PM women with } \\
\text { osteopenia }\end{array}$} & SAE & $\begin{array}{l}\text { No difference: } \\
\mathrm{RR}=0.91[0.50,1.67] ; \mathrm{ARR}=-1[-7,5]\end{array}$ & Low (h-IM) \\
\hline & & ONJ ( $n=0$ cases) & No cases occurred & $\begin{array}{l}\text { Insufficient (h- } \\
\text { IM) }\end{array}$ \\
\hline \multirow{3}{*}{$\begin{array}{l}\text { Zoledronate continuation vs. } \\
\text { discontinuation }(Z \times 6 \text { yr vs. } Z \\
\times 3 \text { yr followed by PBO x } 3 \text { yr) } \\
1 \mathrm{RCT}^{58}\end{array}$} & \multirow{3}{*}{$\begin{array}{l}\text { 1,233 PM women } \\
\text { previously received } \\
\text { zoledronic acid } 3 \text { yr for } \\
\text { osteoporosis by BMD or } \\
\text { RVF }\end{array}$} & SAE & $\begin{array}{l}\text { No difference: } \\
\mathrm{RR}=1.14[0.96,1.36] ; \mathrm{ARR}=4[-1,9]\end{array}$ & Low (IM) \\
\hline & & AFF not defined ( $n=0$ cases) & No cases occurred & $\begin{array}{l}\text { Insufficient (h- } \\
\text { IM) }\end{array}$ \\
\hline & & $\begin{array}{l}\text { ONJ (exposed jaw bone }>6 \text { wks) } \\
(n=1 \text { case) }\end{array}$ & $\begin{array}{l}\text { One case occurred (in continuation } \\
\text { group) }\end{array}$ & $\begin{array}{l}\text { Insufficient (h- } \\
\text { IM) }\end{array}$ \\
\hline \multirow{3}{*}{$\begin{array}{l}\text { Zoledronate continuation vs. } \\
\text { discontinuation } \\
(\mathrm{Z} \times 9 \mathrm{yr} \text { vs. } \mathrm{Z} \times 6 \text { yr followed } \\
\text { by PBO x } 3 \mathrm{yr}) \\
1 \mathrm{RCT}^{59}\end{array}$} & \multirow{3}{*}{$\begin{array}{l}190 \text { PM women previously } \\
\text { received zoledronic acid } 6 \\
\text { yr for osteoporosis by BMD } \\
\text { or RVF }\end{array}$} & SAE & $\begin{array}{l}\text { No difference: } \\
\mathrm{RR}=0.86[0.54,1.36] ; \mathrm{ARR}=-3[-16,9]\end{array}$ & $\begin{array}{l}\text { Low } \\
\text { (IM) }\end{array}$ \\
\hline & & $\begin{array}{l}\text { AFF with radiologic features }(n=0 \\
\text { cases) }\end{array}$ & No cases occurred & $\begin{array}{l}\text { Insufficient (h- } \\
\text { IM) }\end{array}$ \\
\hline & & $\begin{array}{l}\text { ONJ (exposed jaw bone }>6 \\
\text { weeks) ( } n=0 \text { cases) }\end{array}$ & No cases occurred & $\begin{array}{l}\text { Insufficient (h- } \\
\text { IM) }\end{array}$ \\
\hline $\begin{array}{l}\text { Denosumab continuation vs. } \\
\text { discontinuation }(\mathrm{D} \times 4 \mathrm{yr} \text { vs. D } \\
\times 2 \mathrm{yr} \text { followed by PBO } \times 2 \mathrm{yr}) \\
1 \mathrm{RCT}^{18}\end{array}$ & $\begin{array}{l}314 \text { PM women with } \\
\text { osteopenia or osteoporosis } \\
\text { by BMD }\end{array}$ & SAE & No numerical data & $\begin{array}{l}\text { Insufficient } \\
\text { (no data) }\end{array}$ \\
\hline
\end{tabular}


Abbreviations: $\mathrm{AFF}=$ atypical femoral fracture; $\mathrm{AL}=$ alendronate; $\mathrm{ARR}=$ absolute risk reduction; $\mathrm{BMD}=\mathrm{bone}$ mineral density; $\mathrm{BP}=\mathrm{bisphosphonate;} \mathrm{CI}=\mathrm{confidence}$ intervals; $\mathrm{D}=$ denosumab; $\mathrm{DX}=$ diagnosis; h-IM=highly imprecise; $\mathrm{HR}=$ hazard ratio; $\mathrm{IM}=$ =imprecise; $\mathrm{LE}=$ large effects; $\mathrm{ONJ}=$ osteonecrosis of the jaw; OR=odds ratio; $\mathrm{PBO}=$ placebo;

$\mathrm{PM}=$ postmenopausal; $\mathrm{RCT}=$ randomized controlled trial; $\mathrm{RB}=$ medium risk of bias; $\mathrm{RR}=$ risk ratio: $\mathrm{SAE}=$ serious adverse event; $\mathrm{Z}=$ zoledronate

*Discontinuation $\geqq 1$ year after prior treatment $\geqq 1$ year.

†Definitions of terms for strength of evidence grades and domains ratings are detailed in the section of the main report titled, 'Strength of Evidence for Major Comparisons and Outcomes.’ 


\section{Discussion}

In long-term placebo-controlled trials, alendronate for 4 years reduced incident radiographic vertebral and nonvertebral fractures in women with osteoporosis, and zoledronate for 6 years reduced vertebral and nonvertebral fractures in women with osteopenia or osteoporosis. Observational studies suggested that long-term treatment with bisphosphonates as a class increased risk of AFF, ST/FSF, and ONJ, though these adverse events were rare. In women with osteoporosis, long-term raloxifene for 4 years reduced incident vertebral fractures, but not hip or nonvertebral fractures; long-term raloxifene also increased risk of deep vein thrombosis and pulmonary embolism. In women with unknown osteoporosis or osteopenia status, oral hormone therapy for 5-7 years reduced incident clinical and hip fractures compared with placebo, but increased risk of cardiovascular disease and cognitive impairment. Trials also showed that continuation of zoledronate or alendronate after 3-5 years of prior treatment versus discontinuation reduced some vertebral fracture outcomes but not others, did not reduce nonvertebral fractures, and observational data suggested that continuation of bisphosphonates as a class may increase risk of ST/FSF compared with discontinuation.

Whereas long-term treatment with alendronate reduced risk of incident clinical fractures compared with placebo in women with osteoporosis, it did not reduce fracture risk in women with osteopenia. Otherwise, risk of fracture with long-term alendronate versus placebo did not vary by history of prior fracture, World Health Organization Fracture Risk Assessment Tool (FRAX ${ }^{\circledR}$ ) score, or pretreatment levels of bone turnover markers. Risk of incident fracture between long-term raloxifene and placebo did not vary as a function of age, baseline BMD, or history of prior fracture. Reduction in incident clinical fracture with oral hormone therapy compared with placebo appeared possibly greater in women aged 60-79 years than in younger women, though similar results were not found for hip fracture, suggesting possible chance findings. We found no information about possible modifiers of fracture risk with long-term zoledronate treatment.

Our findings have several clinical implications. In women with osteoporosis, indications for long-term raloxifene may be limited, because it only reduces vertebral fractures, while both longterm alendronate and zoledronate also reduced nonvertebral fractures. While the effects of longterm alendronate and zoledronate appear roughly similar in older women with osteoporosis, only long-term zoledronate also reduced nonvertebral fractures in women with osteopenia. It is unclear if these possibly discrepant findings are explained in part by differences in study populations (e.g., the zoledronate population was older). Unfortunately, there are no eligible long-term trials that directly compare alendronate and zoledronate in older women with osteopenia. While oral hormone therapies for 5-7 years lowered both clinical and hip fractures in women not selected to be at high fracture risk, and might be expected to have larger effects in those with osteoporosis, because fracture benefits were offset by risk of serious harms, these agents are not likely to be a viable option for long-term osteoporosis treatment. However, it is unknown whether a lower dose or different route of administration of hormone therapy would have a more favorable balance of fracture benefits to harms. In patients who have completed 3-5 years of bisphosphonate treatment, continued alendronate or zoledronate versus discontinuation each reduced one of two measures of incident vertebral fracture, but did not reduce nonvertebral fractures. Observational data suggested that long-term bisphosphonates increase risk of AFF, that risk likely increases with longer duration of treatment, and that these events are rare. Estimating the relative balance between benefits and harms, for every 1,000 women with osteoporosis 
treated with alendronate for 4 years or with osteopenia or osteoporosis treated with zoledronate for 6 years compared with placebo, approximately 50 to 70 more will avoid an incident clinical fracture, while an additional 2 will experience a ST/FSF. Since only a minority of ST/FSF meet AFF criteria, the absolute number of additional AFF would be expected to be smaller.

Analogously, for every 1,000 women previously treated for osteopenia or osteoporosis with 3 to 5 years of alendronate or zoledronate who continue bisphosphonate treatment another 3 to 5 years, compared with discontinuation, approximately 30 more will avoid an incident vertebral fracture, while an additional 1 will experience a ST/FSF. However, the inconsistency of the vertebral fracture results, the uncertainty around the outcome risk estimates, and the fact that relatively few ST/FSF meet AFF criteria, suggest that the ratio of these fracture benefits to AFF harms with bisphosphonate continuation could be either substantially larger or smaller.

Evidence appeared less robust for ONJ, but suggested long-term bisphosphonate treatment also may increase risk of this outcome. Data from eligible studies did not identify clear patient, bone or drug characteristics that modify likelihood of fracture benefits or harms with long-term or continuing osteoporosis drug treatment.

\section{Limitations}

The available data limit this review in several ways. First, there were few unique trials of long-term osteoporosis drug treatment or of drug discontinuation, and only one trial that included a treatment arm involving osteoporosis drug discontinuation and subsequent osteoporosis drug resumption. We often identified only one trial for a given treatment comparison. Second, only two trials were designed with incident fracture as the primary outcome. Consequently, many studies had few incident clinical fractures, especially for hip fractures, and statistical power often was low to precisely estimate differences in their risk between treatment interventions. Third, all trials were conducted in generally health, usually white, postmenopausal women, limiting their generalizability. Further, most of the trials were conducted in populations selected for osteoporosis or osteopenia by BMD or radiographic vertebral fracture criteria. Generalizability of results to populations who have other reasons for heightened fracture risk is unknown. Fourth, observational studies investigating the association between treatment and risk of AFF or ONJ had marked methodologic differences that likely affected the specificity of these outcomes and the associated risk estimates. Major differences included the definitions of the cases (e.g., whether or not fractures were defined using American Society for Bone and Mineral Research [ASBMR] radiographic AFF features) and noncase controls, drug therapy exposure and control groups, and adjustment for possible confounding. Fifth, reporting on harms was sparse and inconsistent between studies, limiting confidence around harms risk estimates, and raising concerns about possible reporting bias. Sixth, few studies reported information about possible effect modifiers of drug treatment outcomes. These analyses were almost entirely post hoc, often did not test for interactions between potential effect modifiers, treatment assignment, and treatment outcomes, and did not test for multiple testing, raising the likelihood of type 1 errors. Seventh, there were no eligible long-term fracture trials for several U.S. Food and Drug Administration (FDA) approved osteoporosis drugs, including risedronate, ibandronate, teriparatide, and abaloparatide; long-term fracture data for denosumab came from only one small trial in which the pooling of fracture data from different intervention groups made interpretation impossible. There also were no eligible trials of sequential treatment, such as with an anabolic followed by an anti-resorptive, or denosumab followed by bisphosphonate. Finally, there were no usable data comparing different durations of osteoporosis drug holidays. 


\section{Research Needs}

Future trials of long-term osteoporosis drug treatment and osteoporosis drug continuation versus discontinuation should be designed with adequate statistical power to assess risks of clinical fracture endpoints, including hip fractures, the fracture type with the greatest risk of morbidity and mortality. Broader trial samples that include men, nonwhite women, more adults with comorbidities, and adults aged 80 years and older are needed to improve generalizability. Future long-term trials should evaluate sequential osteoporosis drug treatment, including comparisons of anabolic therapy followed by antiresorptive therapy, and denosumab followed by bisphosphonate therapy, with both compared with continuous long-term antiresorptive therapy. Trials should compare continuous long-term osteoporosis drug treatment to different osteoporosis drug holiday durations, with or without restarting osteoporosis drug therapy, and possibly with repeating cycles of osteoporosis drug therapy alternating with drug holidays. Future studies should systematically collect, analyze and report harms data. Randomized trials will continue to have limited statistical power to estimate the risk of rare treatment harms such as AFF and ONJ, so observational studies will be essential for examining these outcomes. These observational studies should use consensus case definitions, ${ }^{64,65}$ standard non-case and exposure controls, cohort designs to estimate incidence rates, and adequate statistical adjustment to reduce the effects of confounding by indication and selection bias. Observational studies may provide insights about the benefits and harms of drug holidays of different durations and about patient and treatment characteristics that predict which patients are likely to benefit or be harmed by treatment continuation versus discontinuation. ${ }^{66,67}$ Future trials and observational studies should pre-specify analyses to investigate possible effect modifiers of benefits and harms of long-term osteoporosis drug treatment and drug holiday outcomes. Among other factors, these should include age, and BMD and bone markers before and during long-term treatment or drug holidays. Patient-level data from osteoporosis drug trials on the associations of early treatment changes in BMD and bone turnover markers with risk of incident fractures may improve understanding of the potential use and limitations of these measures as surrogates for incident fracture. ${ }^{68-70}$

\section{Conclusions}

Only alendronate, zoledronate, and oral hormone therapy reduced nonvertebral fractures with long-term treatment. However, for all these agents, these fracture benefits were limited to mostly older, postmenopausal women. They were further limited to women with osteopenia or osteoporosis for zoledronate, and to women with osteoporosis for alendronate. Absolute reductions in clinical fractures with long-term bisphosphonates appeared far greater than absolute increases in risk of AFF and ONJ with these treatments. However, reductions in hip fracture with long-term oral hormone therapy appear offset by risk of serious harms. In patients with prior osteoporosis drug treatment, continued treatment appeared to reduce vertebral fractures but not nonvertebral fractures, and may increase risk of AFF. While fracture benefits of continued osteoporosis drug treatment versus drug holiday numerically appeared to outweigh these risks, the more limited morbidity prevented and greater uncertainty about the outcome measures and risk estimates require further investigation to better inform clinical decisions about continuing treatment. This research should include examination of how these benefits and risks vary as a function of patient, bone, and drug treatment characteristics (e.g., age, sex, comorbidity, pre-drug holiday BMD, duration of prior osteoporosis drug treatment). Future 
modeling studies also may incorporate probabilities of experiencing fracture-related morbidity to help patients weigh trade-offs of treatment more easily. 


\section{References}

1. Consensus development conference: diagnosis, prophylaxis, and treatment of osteoporosis. Am J Med. 1993 Jun;94(6):646-50. PMID: 8506892.

2. Wright NC, Looker AC, Saag KG, et al. The recent prevalence of osteoporosis and low bone mass in the United States based on bone mineral density at the femoral neck or lumbar spine. J Bone Miner Res. 2014 Nov;29(11):2520-6. doi: 10.1002/jbmr.2269. PMID: 24771492.

3. Burge R, Dawson-Hughes B, Solomon DH, et al. Incidence and economic burden of osteoporosis-related fractures in the United States, 2005-2025. J Bone Miner Res. 2007 Mar;22(3):465-75. doi: 10.1359/jbmr.061113. PMID: 17144789.

4. Johnell O, Kanis JA. An estimate of the worldwide prevalence and disability associated with osteoporotic fractures. Osteoporos Int. 2006 Dec;17(12):1726-33. doi: 10.1007/s00198-006-0172-4. PMID: 16983459.

5. Schousboe JT. Epidemiology of Vertebral Fractures. J Clin Densitom. 2016 JanMar;19(1):8-22. doi: 10.1016/j.jocd.2015.08.004. PMID: 26349789.

6. Katsoulis M, Benetou V, Karapetyan T, et al. Excess mortality after hip fracture in elderly persons from Europe and the USA: the CHANCES project. J Intern Med. 2017 Mar;281(3):300-10. doi: 10.1111/joim.12586. PMID: 28093824.

7. Miller PD, Hattersley G, Riis BJ, et al. Effect of Abaloparatide vs Placebo on New Vertebral Fractures in Postmenopausal Women With Osteoporosis: A Randomized Clinical Trial. JAMA. 2016 Aug

16;316(7):722-33. doi:

10.1001/jama.2016.11136. PMID:

27533157.
8. Qaseem A, Forciea MA, McLean RM, et al. Treatment of Low Bone Density or Osteoporosis to Prevent Fractures in Men and Women: A Clinical Practice Guideline Update From the American College of Physicians. Ann Intern Med. 2017 Jun 6;166(11):818-39. doi: 10.7326/M15-1361. PMID: 28492856.

9. Adler RA, El-Hajj Fuleihan G, Bauer DC, et al. Managing Osteoporosis in Patients on Long-Term Bisphosphonate Treatment: Report of a Task Force of the American Society for Bone and Mineral Research. J Bone Miner Res. 2016 Jan;31(1):16-35. doi: 10.1002/jbmr.2708. PMID: 26350171.

10. Sellmeyer DE. Atypical fractures as a potential complication of long-term bisphosphonate therapy. JAMA. 2010 Oct 6;304(13):1480-4. doi: 10.1001/jama.2010.1360. PMID: 20924014.

11. Compston J, Bowring C, Cooper A, et al. Diagnosis and management of osteoporosis in postmenopausal women and older men in the UK: National Osteoporosis Guideline Group (NOGG) update 2013. Maturitas. 2013 Aug;75(4):392-6. doi: 10.1016/j.maturitas.2013.05.013. PMID: 23810490.

12. Watts NB, Bilezikian JP, Camacho PM, et al. American Association of Clinical Endocrinologists Medical Guidelines for Clinical Practice for the diagnosis and treatment of postmenopausal osteoporosis: executive summary of recommendations. Endocr Pract. 2010 Nov-Dec;16(6):1016-9. PMID: 21216723.

13. Cummings SR, Ferrari S, Eastell R, et al. Vertebral Fractures After Discontinuation of Denosumab: A Post Hoc Analysis of the Randomized Placebo-Controlled FREEDOM Trial and Its Extension. J Bone Miner Res. 2018 Feb;33(2):190-8. doi: 10.1002/jbmr.3337. PMID: 29105841. 
14. Cummings SR, Black DM, Thompson DE, et al. Effect of alendronate on risk of fracture in women with low bone density but without vertebral fractures: results from the Fracture Intervention Trial. JAMA. 1998 Dec 23-30;280(24):2077-82. PMID: 9875874.

15. Reid IR, Horne AM, Mihov B, et al. Fracture prevention with zoledronate in older women with osteopenia. New England Journal of Medicine. 2018. 379(25):24072416.

16. Delmas PD, Ensrud KE, Adachi JD, et al. Efficacy of raloxifene on vertebral fracture risk reduction in postmenopausal women with osteoporosis: four-year results from a randomized clinical trial. J Clin Endocrinol Metab. 2002 Aug;87(8):3609-17. doi: 10.1210/jcem.87.8.8750. PMID: 12161484.

17. Siris ES, Harris ST, Eastell R, et al. Skeletal effects of raloxifene after 8 years: results from the continuing outcomes relevant to Evista (CORE) study. J Bone Miner Res. 2005 Sep;20(9):1514-24. doi: 10.1359/JBMR.050509. PMID: 16059623.

18. Miller PD, Bolognese MA, Lewiecki EM, et al. Effect of denosumab on bone density and turnover in postmenopausal women with low bone mass after long-term continued, discontinued, and restarting of therapy: a randomized blinded phase 2 clinical trial. Bone. 2008 Aug;43(2):222-9. doi: 10.1016/j.bone.2008.04.007. PMID: 18539106.

19. Jackson RD, Wactawski-Wende J, LaCroix $\mathrm{AZ}$, et al. Effects of conjugated equine estrogen on risk of fractures and BMD in postmenopausal women with hysterectomy: results from the women's health initiative randomized trial. J Bone Miner Res. 2006 Jun;21(6):817-28. doi: 10.1359/jbmr.060312. PMID: 16753012.

20. Wimalawansa S, J. A four-year randomized controlled trial of hormone replacement and bisphosphonate, alone or in combination, in women with postmenopausal osteoporosis. American Journal of Medicine. 1998 Mar;104(3):219-26. PMID: 9552083.
21. Cauley JA, Robbins J, Chen Z, et al. Effects of estrogen plus progestin on risk of fracture and bone mineral density: the Women's Health Initiative randomized trial. JAMA. 2003 Oct 1;290(13):1729-38. doi: 10.1001/jama.290.13.1729. PMID: 14519707.

22. Quandt SA, Thompson DE, Schneider DL, et al. Effect of alendronate on vertebral fracture risk in women with bone mineral density $\mathrm{T}$ scores of-1.6 to -2.5 at the femoral neck: the Fracture Intervention Trial. Mayo Clin Proc. 2005 Mar;80(3):343-9. PMID: 15757015.

23. Ryder KM, Cummings SR, Palermo L, et al. Does a history of non-vertebral fracture identify women without osteoporosis for treatment? J Gen Intern Med. 2008 Aug;23(8):1177-81. doi: 10.1007/s11606008-0622-0. PMID: 18459010.

24. Donaldson MG, Palermo L, Ensrud KE, et al. Effect of alendronate for reducing fracture by FRAX score and femoral neck bone mineral density: the Fracture Intervention Trial. J Bone Miner Res. 2012 Aug;27(8):1804-10. doi: 10.1002/jbmr.1625. PMID: 22492479.

25. Bauer DC, Garnero P, Hochberg MC, et al. Pretreatment levels of bone turnover and the antifracture efficacy of alendronate: the fracture intervention trial. J Bone Miner Res. 2006 Feb;21(2):292-9. doi: 10.1359/JBMR.051018. PMID: 16418785.

26. Johnell O, Cauley JA, Kulkarni PM, et al. Raloxifene reduces risk of vertebral fractures [corrected] in postmenopausal women regardless of prior hormone therapy. J Fam Pract. 2004 Oct;53(10):789-96. PMID: 15469774.

27. Sontag A, Wan X, Krege JH. Benefits and risks of raloxifene by vertebral fracture status. Curr Med Res Opin. 2010 Jan;26(1):71-6. doi: 10.1185/03007990903427082. PMID: 19908937.

28. Cauley JA, Norton L, Lippman ME, et al. Continued breast cancer risk reduction in postmenopausal women treated with raloxifene: 4-year results from the MORE trial. Multiple outcomes of raloxifene evaluation. Breast Cancer Res Treat. 2001 Jan;65(2):125-34. PMID: 11261828. 
29. Barrett-Connor E, Cauley JA, Kulkarni PM, et al. Risk-benefit profile for raloxifene: 4year data From the Multiple Outcomes of Raloxifene Evaluation (MORE) randomized trial. J Bone Miner Res. 2004

Aug;19(8):1270-5. doi:

10.1359/JBMR.040406. PMID: 15231013.

30. Barrett-Connor E, Cox DA, Song J, et al. Raloxifene and risk for stroke based on the framingham stroke risk score. Am J Med. 2009 Aug;122(8):754-61. doi: 10.1016/j.amjmed.2009.01.033. PMID: 19540454.

31. Barrett-Connor E, Grady D, Sashegyi A, et al. Raloxifene and cardiovascular events in osteoporotic postmenopausal women: fouryear results from the MORE (Multiple Outcomes of Raloxifene Evaluation) randomized trial. JAMA. 2002 Feb 20;287(7):847-57. PMID: 11851576.

32. Duvernoy CS, Kulkarni PM, Dowsett SA, et al. Vascular events in the Multiple Outcomes of Raloxifene Evaluation (MORE) trial: incidence, patient characteristics, and effect of raloxifene. Menopause. 2005 Jul-Aug;12(4):444-52. doi:

10.1097/01.GME.0000151653.02620.89. PMID: 16037760.

33. Ensrud K, Genazzani AR, Geiger MJ, et al. Effect of raloxifene on cardiovascular adverse events in postmenopausal women with osteoporosis. Am J Cardiol. 2006 Feb 15;97(4):520-7. doi:

10.1016/j.amjcard.2005.09.083. PMID: 16461049.

34. Grady D, Cauley JA, Stock JL, et al. Effect of Raloxifene on all-cause mortality. Am J Med. 2010 May;123(5):469 e1-7. doi: 10.1016/j.amjmed.2009.12.018. PMID: 20399327.

35. Grady D, Ettinger B, Moscarelli E, et al. Safety and adverse effects associated with raloxifene: multiple outcomes of raloxifene evaluation. Obstet Gynecol. 2004

Oct;104(4):837-44. doi:

10.1097/01.AOG.0000137349.79204.b8. PMID: 15458908.
36. Martino S, Cauley JA, Barrett-Connor E, et al. Continuing outcomes relevant to Evista: breast cancer incidence in postmenopausal osteoporotic women in a randomized trial of raloxifene. J Natl Cancer Inst. 2004 Dec 1;96(23):1751-61. doi: 10.1093/jnci/djh319. PMID: 15572757.

37. Martino S, Disch D, Dowsett SA, et al. Safety assessment of raloxifene over eight years in a clinical trial setting. Curr Med Res Opin. 2005 Sep;21(9):1441-52. doi: 10.1185/030079905X61839. PMID: 16197663.

38. Schilcher J, Koeppen V, Aspenberg P, et al. Risk of atypical femoral fracture during and after bisphosphonate use. Acta Orthop. 2015 Feb;86(1):100-7. doi: 10.3109/17453674.2015.1004149. PMID: 25582459.

39. Lim SJ, Yeo I, Yoon PW, et al. Incidence, risk factors, and fracture healing of atypical femoral fractures: a multicenter case-control study. Osteoporosis International. 2018. doi: 10.1007/s00198-018-4640-4. PMID: 623239244.

40. Black DM, Abrahamsen B, Bouxsein ML, et al. Atypical Femur Fractures-Review of epidemiology, relationship to bisphosphonates, prevention and clinical management. 2018.

41. Vestergaard P, Schwartz K, Rejnmark L, et al. Oral bisphosphonate use increases the risk for inflammatory jaw disease: a cohort study. J Oral Maxillofac Surg. 2012 Apr;70(4):821-9. doi: 10.1016/j.joms.2011.02.093. PMID: 21764202.

42. Lin TC, Yang CY, Kao Yang YH, et al. Incidence and risk of osteonecrosis of the jaw among the Taiwan osteoporosis population. Osteoporos Int. 2014 May;25(5):1503-11. doi: 10.1007/s00198014-2624-6. PMID: 24515577.

43. Chiu WY, Chien JY, Yang WS, et al. The risk of osteonecrosis of the jaws in Taiwanese osteoporotic patients treated with oral alendronate or raloxifene. J Clin Endocrinol Metab. 2014 Aug;99(8):272935. doi: 10.1210/jc.2013-4119. PMID: 24758181. 
44. Shumaker SA, Legault C, Rapp SR, et al. Estrogen plus progestin and the incidence of dementia and mild cognitive impairment in postmenopausal women: the Women's Health Initiative Memory Study: a randomized controlled trial. JAMA. 2003 May 28;289(20):2651-62. doi: 10.1001/jama.289.20.2651. PMID: 12771112.

45. Shumaker SA, Legault C, Kuller L, et al. Conjugated equine estrogens and incidence of probable dementia and mild cognitive impairment in postmenopausal women: Women's Health Initiative Memory Study. JAMA. 2004 Jun 23;291(24):2947-58. doi: 10.1001/jama.291.24.2947. PMID: 15213206.

46. Rossouw JE, Anderson GL, Prentice RL, et al. Risks and benefits of estrogen plus progestin in healthy postmenopausal women: principal results From the Women's Health Initiative randomized controlled trial. JAMA. 2002 Jul 17;288(3):321-33. PMID: 12117397.

47. Anderson GL, Limacher M, Assaf AR, et al. Effects of conjugated equine estrogen in postmenopausal women with hysterectomy: the Women's Health Initiative randomized controlled trial. JAMA. 2004 Apr

14;291(14):1701-12. doi:

10.1001/jama.291.14.1701. PMID: 15082697.

48. Park-Wyllie LY, Mamdani MM, Juurlink DN, et al. Bisphosphonate use and the risk of subtrochanteric or femoral shaft fractures in older women. JAMA. $2011 \mathrm{Feb}$ 23;305(8):783-9. doi: 10.1001/jama.2011.190. PMID: 21343577.

49. Kim SY, Schneeweiss S, Katz JN, et al. Oral bisphosphonates and risk of subtrochanteric or diaphyseal femur fractures in a population-based cohort. J Bone Miner Res. 2011 May;26(5):993-1001. doi: 10.1002/jbmr.288. PMID: 21542002.
50. Black D, M. Kelly, M, P. Genant, H, K. Palermo, L, Eastell, R, Bucci, Rechtweg C, Cauley, J, Leung, P, C. Boonen, S, Santora, A, de, Papp A, Bauer, D, C. Fracture Intervention Trial, Steering Committee, Horizon Pivotal Fracture Trial Steering, Committee. Bisphosphonates and fractures of the subtrochanteric or diaphyseal femur. New England Journal of Medicine. 2010 May 13;362(19):1761-71. PMID: 20335571.

51. Abrahamsen B, Eiken P, Eastell R. Subtrochanteric and diaphyseal femur fractures in patients treated with alendronate: a register-based national cohort study. J Bone Miner Res. 2009

Jun;24(6):1095-102. doi: 10.1359/jbmr.081247. PMID: 19113931.

52. Vestergaard P, Schwartz F, Rejnmark L, et al. Risk of femoral shaft and subtrochanteric fractures among users of bisphosphonates and raloxifene. Osteoporos Int. 2011 Mar;22(3):993-1001. doi: 10.1007/s00198010-1512-y. PMID: 21165600.

53. Erviti J, Alonso A, Oliva B, et al. Oral bisphosphonates are associated with increased risk of subtrochanteric and diaphyseal fractures in elderly women: a nested case-control study. BMJ Open. 2013 Jan 30;3(1)doi: 10.1136/bmjopen-2012002091. PMID: 23370011.

54. Koh J, H., Myong J, P., Yoo J, et al. Predisposing factors associated with atypical femur fracture among postmenopausal Korean women receiving bisphosphonate therapy: 8 years' experience in a single center. Osteoporosis International. 201701 Nov;28(11):3251-9. PMID: 617716961.

55. Black DM, Schwartz AV, Ensrud KE, et al. Effects of continuing or stopping alendronate after 5 years of treatment: the Fracture Intervention Trial Long-term Extension (FLEX): a randomized trial. JAMA. 2006 Dec 27;296(24):2927-38. doi: 10.1001/jama.296.24.2927. PMID: 17190893. 
56. Bone HG, Hosking D, Devogelaer JP, et al. Ten years' experience with alendronate for osteoporosis in postmenopausal women. $\mathrm{N}$ Engl J Med. 2004 Mar 18;350(12):1189-99. doi: 10.1056/NEJMoa030897. PMID: 15028823.

57. Tonino RP, Meunier PJ, Emkey R, et al. Skeletal benefits of alendronate: 7-year treatment of postmenopausal osteoporotic women. Phase III Osteoporosis Treatment Study Group. J Clin Endocrinol Metab. 2000 Sep;85(9):3109-15. doi: 10.1210/jcem.85.9.6777. PMID: 10999794.

58. Black DM, Reid IR, Boonen S, et al. The effect of 3 versus 6 years of zoledronic acid treatment of osteoporosis: a randomized extension to the HORIZON-Pivotal Fracture Trial (PFT). J Bone Miner Res. 2012 Feb;27(2):243-54. doi: 10.1002/jbmr.1494. PMID: 22161728.

59. Black DM, Reid IR, Cauley JA, et al. The effect of 6 versus 9 years of zoledronic acid treatment in osteoporosis: a randomized second extension to the HORIZON-Pivotal Fracture Trial (PFT). J Bone Miner Res. 2015 May;30(5):934-44. doi: 10.1002/jbmr.2442. PMID: 25545380.

60. Schwartz AV, Bauer DC, Cummings SR, et al. Efficacy of continued alendronate for fractures in women with and without prevalent vertebral fracture: the FLEX trial. J Bone Miner Res. 2010 May;25(5):976-82. doi: 10.1002/jbmr.11. PMID: 20200926.

61. McClung M, Miller P, Recknor C, et al. Zoledronic acid for the prevention of bone loss in postmenopausal women with low bone mass: a randomized controlled trial. Obstetrics \& Gynecology. 2009;114(5):9991007.

62. Ensrud KE, Barrett-Connor EL, Schwartz A, et al. Randomized trial of effect of alendronate continuation versus discontinuation in women with low BMD: results from the Fracture Intervention Trial long-term extension. J Bone Miner Res. 2004 Aug;19(8):1259-69. doi: 10.1359/JBMR.040326. PMID: 15231012.

63. Adams AL, Adams JL, Raebel MA, et al. Bisphosphonate Drug Holiday and Fracture Risk: A Population-Based Cohort Study. Journal of Bone and Mineral Research. 2018 PMID: 622353500.
64. Khan AA, Morrison A, Hanley DA, et al. Diagnosis and management of osteonecrosis of the jaw: a systematic review and international consensus. J Bone Miner Res. 2015 Jan;30(1):3-23. doi: 10.1002/jbmr.2405. PMID: 25414052.

65. Shane E, Burr D, Abrahamsen B, et al. Atypical subtrochanteric and diaphyseal femoral fractures: second report of a task force of the American Society for Bone and Mineral Research. J Bone Miner Res. 2014 Jan;29(1):1-23. doi: 10.1002/jbmr.1998. PMID: 23712442.

66. Adams AL LB, Ryan DS, Geiger EJ, Dell RM, Black DM. Do Drug Holidays Reduce Atypical Femur Fracture Risk?: Results from the Southern California Osteoporosis Cohort Study (SOCS). American Society for Bone and Mineral Research Annual Meeting, September 28-October 1, Montréal, Québec, Canada. 2018.

67. Curtis J CR, Li Z, Arora T, Saag K, Wright N, Daigle S, Kilgore M, Delzell E. The Impact of Bisphosphonate Drug Holidays on Fracture Rates. American Society for Bone and Mineral Research Annual Meeting, September 28-October 1, Montréal, Québec, Canada. 2018.

68. Bauer DC. Change in Bone Turnover as a Surrogate for Fracture Outcomes: A Novel Individual-level Analysis of Pooled Antiresorptive Trials from the FNIH Bone Quality Study [abstract]. American Society for Bone and Mineral Research Annual Meeting, September 28-October 1, Montréal, Québec, Canada. 2018.

69. Bauer DC, Black DM, Bouxsein ML, et al. Treatment-Related Changes in Bone Turnover and Fracture Risk Reduction in Clinical Trials of Anti-Resorptive Drugs: A Meta-Regression. Journal of Bone and Mineral Research. 2018;33(4):634-42.

70. Black D. Change in BMD as a Surrogate for Fracture Risk Reduction in Osteoporosis Trials: Results from Pooled, Individual-level Patient Data from the FNIH Bone Quality Project [abstract]. American Society for Bone and Mineral Research Annual Meeting, September 28-October 1, Montréal, Québec, Canada. 2018. 


\section{Chapter 1. Introduction}

\section{Background}

Osteoporosis is a skeletal disorder of low bone mass and microarchitectural deterioration of bone, leading to bone fragility and increased risk of fracture. ${ }^{1}$ In 1994, a World Health Organization (WHO) Study Group operationally defined osteoporosis in women as femoral neck bone mineral density (BMD) equal to or lower than 2.5 standard deviations below the mean BMD of young white women. ${ }^{2}$ Considering BMD at either the femoral neck or lumbar spine, and extrapolating this definition to men and nonwhite women, osteoporosis affects more than 10 million U.S. adults aged 50 years or older. ${ }^{3}$ About 2 million U.S. adults experience an osteoporotic or other low- or no-trauma fracture each year, including about 300,000 with hip fractures. ${ }^{4}$ Clinically recognized fractures at many skeletal sites cause pain, disability, and impaired quality of life. Hip and clinical vertebral fractures, specifically, are associated with an increased risk of mortality. ${ }^{5} 6$ Incident radiographic vertebral fractures, a commonly reported outcome in drug treatment trials, are defined by quantifying vertebral height losses between baseline and scheduled follow-up radiographs. These are clinically diagnosed in about 15-25\% of cases, ${ }^{7-12}$ usually because of back pain. Even in individuals whose new radiographic vertebral fracture is not diagnosed, new or worsened back pain is more common than in individuals without new radiographic vertebral fractures. ${ }^{12,13}$ Because risk of most fractures rises steeply with age, and the population is aging, fracture burden is projected to increase in coming decades.

In short-term (18 to 36 months) randomized controlled trials for osteoporosis treatment, U.S. Food and Drug Administration approved (Table 1) oral and intravenous bisphosphonates (alendronate, zoledronate, risedronate, ibandronate), denosumab, teriparatide, and abaloparatide have lowered risk of both vertebral and nonvertebral fractures; and several bisphosphonates (alendronate, zoledronate, risedronate) and denosumab also have lowered risk of hip fractures. ${ }^{14}$, ${ }^{15}$ Despite the evidence on the efficacy of short-term osteoporosis drug treatment for reducing fracture risk, there are limits to this evidence. Evidence on fracture protection is predominately from studies of postmenopausal women with osteoporosis defined by low BMD or by the presence of vertebral fractures found on screening x-rays. Data are far more limited in postmenopausal women without osteoporosis, even in those with heightened fracture risk because of low bone mass (i.e., osteopenia) combined with other risk factors, such as falls or a high WHO Fracture Risk Assessment Tool [FRAX $\left.{ }^{\circledR}\right]^{16}$ score. ${ }^{17}$ Short-term osteoporosis drug treatment also may be associated with side effects. Oral bisphosphonates increase risk of upper gastrointestinal symptoms and IV bisphosphonates may increase risk of atrial fibrillation; denosumab increases risk of infection; teriparatide increases risk of hypercalcemia; raloxifene increases risk of hot flashes; and both raloxifene and estrogen increase risk of venous thromboembolism and stroke. ${ }^{14}$ Observational studies suggest that bisphosphonates and denosumab are associated with rare atypical femoral fractures (AFF) and osteonecrosis of the jaw. $^{18}$

Compared to what is known about the effects of short-term osteoporosis drug treatment, the benefits and harms of long-term (>3 years) osteoporosis drug treatment are far less clear, including among individuals with osteopenia, though heightened risk of AFF is a concern. ${ }^{19}$ Because of sparse data on fracture outcomes from long-term trials and the high cost of trials adequately powered to evaluate incident fracture outcomes, many investigators have sought to identify appropriate surrogate endpoints for fractures. Early treatment changes in BMD and, to a lesser extent, in bone turnover markers, ${ }^{20}$ may predict short-term risk of incident fractures. It is 
unclear, however, whether changes in these measures will predict a high enough proportion of the anti-fracture efficacy of osteoporosis drug treatments to be considered adequate fracture surrogate outcomes. Further, it needs to be established whether changes in these measures will predict long-term nonvertebral fracture risk with drug treatment, including within individuals.

Beyond any overall effect of long-term osteoporosis drug treatment, there may be important differences in both fracture efficacy and risk of harms based on patient, bone, and osteoporosis drug characteristics (i.e., possible effect modifiers). Better understanding of these factors may allow prescribers and patients to make more informed treatment decisions to maximize benefit (e.g., reduced fracture risk) while minimizing harms.

Uncertainty about the benefits of long-term bisphosphonate use, coupled with concerns that long-term bisphosphonate persistence in bone might increase fracture risk by inhibiting normal repair of bone microdamage, ${ }^{21,22}$ have led to the suggestion to periodically discontinue bisphosphonate therapy. ${ }^{22}$ Though several groups advocate bisphosphonate "drug holidays” as a strategy to preserve as much fracture benefit as possible while minimizing harms, there is no consensus about who should get them, when they should start, how long they should last, how they should be monitored, or the criteria for restarting therapy. ${ }^{22-24}$ In contrast to bisphosphonates, drug holidays are not recommended after denosumab therapy; bone turnover and bone loss increase rapidly after denosumab discontinuation, and case series and post hoc data suggest a possible small post-treatment increase in risk of radiographic vertebral fractures. ${ }^{25}$

Uncertainties about the most appropriate use of long-term osteoporosis drug treatment and of osteoporosis drug holidays spurred the scheduling of a National Institutes of Health Office of Disease Prevention Pathways to Prevention program workshop on this topic. The goal of the workshop was to present an evidence-based synthesis of the pertinent research base, identify research gaps, and suggest future research needs to assist patients, clinicians, and other healthcare decision makers. To inform this workshop and clinicians and osteoporosis researchers more generally, we conducted this systematic review to address four questions. First, what are the effects of long-term osteoporosis drug therapy (>3 years) versus control on risk for incident clinical fractures, incident radiographic vertebral fractures, change in BMD, and harms? Second, do the effects of long-term osteoporosis drug treatment on these outcomes vary as a function of patient, bone, or osteoporosis drug characteristics? Third, among individuals receiving osteoporosis drug treatment to prevent fracture, what are the effects of continuing versus at least temporarily discontinuing treatment on risks for incident fractures and harms? Fourth, do outcomes of osteoporosis drug treatment continuation versus discontinuation vary as a function of patient, bone, or osteoporosis drug characteristics? These questions were refined and formalized as the Key Questions listed below. 
Table 1. U.S. FDA approved drugs used for osteoporosis treatment and prevention

\begin{tabular}{|c|c|c|c|c|c|}
\hline Drug Class & Drug Name & $\begin{array}{l}\text { Delivery } \\
\text { Route }\end{array}$ & $\begin{array}{l}\text { Dosing } \\
\text { Frequency }\end{array}$ & $\begin{array}{l}\text { Long- } \\
\text { Term }\end{array}$ & Mechanism in Bone \\
\hline Bisphosphonate & Alendronate & Oral & $\begin{array}{l}\text { Daily, } \\
\text { weekly }\end{array}$ & $\begin{array}{l}>3 \\
\text { years }\end{array}$ & $\begin{array}{l}\text { Common mechanism for nitrogen } \\
\text { bisphosphonates: preferentially } \\
\text { adhere to bone surface at sites of high } \\
\text { bone turnover, there inhibiting a } \\
\text { specific osteoclast intracellular } \\
\text { enzyme, causing osteoclast death and } \\
\text { reduced bone resorption; bind to bone } \\
\text { mineral and continue to be } \\
\text { systemically released for years after } \\
\text { drug discontinuation, continuing some } \\
\text { suppression of bone turnover and } \\
\text { reduction of bone resorption }\end{array}$ \\
\hline Bisphosphonate & Ibandronate & Oral, IV & $\begin{array}{l}\text { Daily, } \\
\text { weekly, } \\
\text { monthly }\end{array}$ & $\begin{array}{l}>3 \\
\text { years }\end{array}$ & $\begin{array}{l}\text { Common mechanism for nitrogen } \\
\text { bisphosphonates }\end{array}$ \\
\hline Bisphosphonate & Risedronate & Oral & $\begin{array}{l}\text { Daily, } \\
\text { weekly }\end{array}$ & $\begin{array}{l}>3 \\
\text { years }\end{array}$ & $\begin{array}{l}\text { Common mechanism for nitrogen } \\
\text { bisphosphonates }\end{array}$ \\
\hline Bisphosphonate & Zoledronate & IV & $\begin{array}{l}\text { Every } 12 \text { to } \\
18 \text { months }\end{array}$ & $\begin{array}{l}>3 \\
\text { years }\end{array}$ & $\begin{array}{l}\text { Common mechanism for nitrogen } \\
\text { bisphosphonates }\end{array}$ \\
\hline Biologic & Denosumab & SC & 6 months & $\begin{array}{l}>3 \\
\text { years }\end{array}$ & $\begin{array}{l}\text { A monoclonal antibody to RANKL that } \\
\text { binds it and prevents its interaction } \\
\text { with RANK on osteoclasts, inhibiting } \\
\text { osteoclast formation, function, and } \\
\text { survival, and thereby decreasing bone } \\
\text { resorption; suppression of bone } \\
\text { turnover and resorption is rapidly } \\
\text { reversed after drug discontinuation }\end{array}$ \\
\hline $\begin{array}{l}\text { PTH related } \\
\text { anabolic }\end{array}$ & $\begin{array}{l}\text { Teriparatide } \\
\text { (recombinant } \\
\text { PTH) }\end{array}$ & SC & Daily & $\begin{array}{l}\text { Not } \\
\text { used }>2 \\
\text { years }\end{array}$ & $\begin{array}{l}\text { Binds specifically to PTH receptors. } \\
\text { With intermittent (i.e., once daily) } \\
\text { rather than continuous exposure, } \\
\text { stimulates bone formation more than } \\
\text { bone resorption, leading to net } \\
\text { anabolic effects on bone }\end{array}$ \\
\hline $\begin{array}{l}\text { PTH related } \\
\text { anabolic }\end{array}$ & $\begin{array}{l}\text { Abaloparatide } \\
\text { (PTH analog) }\end{array}$ & SC & Daily & $\begin{array}{l}\text { Not } \\
\text { used }>2 \\
\text { years }\end{array}$ & $\begin{array}{l}\text { In bone, acts as agonist at PTH } \\
\text { receptors. Daily rather than } \\
\text { continuous exposure stimulates bone } \\
\text { formation more than bone resorption, } \\
\text { leading to net anabolic effects on } \\
\text { bone }\end{array}$ \\
\hline SERM & Raloxifene & Oral & Daily & $\begin{array}{l}>3 \\
\text { years }\end{array}$ & $\begin{array}{l}\text { Binds to nuclear estrogen receptors in } \\
\text { bone and other tissues, acting as } \\
\text { agonist in some tissues and } \\
\text { antagonist in others. Is agonist in } \\
\text { bone, decreasing bone resorption. }\end{array}$ \\
\hline $\begin{array}{l}\text { Estrogen and } \\
\text { Estrogen/ } \\
\text { Progestin } \\
\text { combination } \\
\text { products }\end{array}$ & Multiple* & $\begin{array}{l}\text { Oral, } \\
\text { transdermal, } \\
\text { transvaginal }\end{array}$ & Daily & $\begin{array}{l}>3 \\
\text { years }\end{array}$ & $\begin{array}{l}\text { Estrogens bind estrogen receptors } \alpha \\
\text { and } ß, \text { acting as agonists in estrogen- } \\
\text { sensitive tissues. In bone, they } \\
\text { decrease bone resorption. Progestins } \\
\text { may decrease nuclear estrogen } \\
\text { receptors and suppress epithelial DNA } \\
\text { synthesis in endometrial tissue. }\end{array}$ \\
\hline
\end{tabular}




\begin{tabular}{|l|l|l|l|l|l|}
\hline Drug Class & Drug Name & $\begin{array}{l}\text { Delivery } \\
\text { Route }\end{array}$ & $\begin{array}{l}\text { Dosing } \\
\text { Frequency }\end{array}$ & $\begin{array}{l}\text { Long- } \\
\text { Term }\end{array}$ & Mechanism in Bone \\
\hline $\begin{array}{l}\text { Estrogen with } \\
\text { SERM }\end{array}$ & $\begin{array}{l}\text { Conjugated } \\
\text { estrogens/ } \\
\text { bazedoxifene* }\end{array}$ & Oral & Daily & $\begin{array}{l}>3 \\
\text { years }\end{array}$ & $\begin{array}{l}\text { Estrogen mechanism is as detailed } \\
\text { above. Bazedoxifene binds estrogen } \\
\text { receptors, acting as an agonist in } \\
\text { some tissues (e.g., bone, resulting in } \\
\text { decreased bone resorption) and as an } \\
\text { antagonist in other tissues (e.g., } \\
\text { uterus, decreasing endometrial } \\
\text { hyperplasia). }\end{array}$ \\
\hline
\end{tabular}

Abbreviations: FDA=Food and Drug Administration; IV=intravenous; PTH=parathyroid hormone, RANK=receptor activator of nuclear factor kappa-B; RANKL=receptor activator of nuclear factor kappa-B ligand; SC=subcutaneous; SERM=selective estrogen receptor modulator

*Food and Drug Administration approved for osteoporosis prevention, but not for osteoporosis treatment.

\section{Scope and Key Questions}

\section{Key Questions}

Key Question 1. Among men and postmenopausal women aged $\geq 50$ years with osteoporosis ${ }^{a}$ or osteopenia/low bone mass, ${ }^{b}$ what is the efficacy of long-term (>3 years) osteoporosis drug therapy in reducing risk of incident fracture and on change in BMD?

Key Question 2. Among men and postmenopausal women aged $\geq 50$ years with osteoporosis ${ }^{\mathrm{a}}$ or osteopenia/low bone mass, ${ }^{\mathrm{b}}$ does efficacy of longterm osteoporosis drug therapy for reducing risk of incident fracture vary as a function of patient, bone, or osteoporosis drug characteristics? ${ }^{c}$

Key Question 3. Among men and postmenopausal women aged $\geq 50$ years with osteoporosis ${ }^{\mathrm{a}}$ or osteopenia/low bone mass, ${ }^{\mathrm{b}}$ what is the risk of harms associated with long-term (>3 years) osteoporosis drug therapy?

Key Question 4. Among men and postmenopausal women aged $\geq 50$ years with osteoporosis ${ }^{\mathrm{a}}$ or osteopenia/low bone mass, ${ }^{\mathrm{b}}$ does the risk of harms associated with long-term (>3 years) osteoporosis drug therapy vary as a function of patient, bone, or osteoporosis drug characteristics? ${ }^{\mathrm{C}}$

Key Question 5. Among men and postmenopausal women aged $\geq 50$ years currently receiving drug therapy ( $\geq 1$ year) started for osteoporosis ${ }^{a}$ or osteopenia/low bone mass ${ }^{b}$ to prevent fracture, what is the effect of osteoporosis drug treatment holidays ( $\geq 1$ year) on incident fracture risk and change in BMD? 
Key Question 6. Among men and postmenopausal women aged $\geq 50$ years currently receiving drug therapy ( $\geq 1$ year) started for osteoporosis ${ }^{\mathrm{a}}$ or osteopenia/low bone mass ${ }^{b}$ to prevent fracture, does the effect of osteoporosis drug treatment holidays ( $\geq 1$ year) on incident fracture risk vary as a function of patient, bone, or osteoporosis drug characteristics?c

Key Question 7. Among men and postmenopausal women aged $\geq 50$ years currently receiving drug therapy ( $\geq 1$ year) started for osteoporosis ${ }^{\mathrm{a}}$ or osteopenia/low bone mass ${ }^{b}$ to prevent fracture, what is the risk of harms of osteoporosis drug treatment holidays ( $\geq 1$ year)?

Key Question 8. Among men and postmenopausal women aged $\geq 50$ years currently receiving drug therapy started for osteoporosis ${ }^{\mathrm{a}}$ or osteopenia/low bone mass ${ }^{b}$ to prevent fracture, does risk of harms associated with osteoporosis drug treatment holidays vary as a function of patient, bone, or osteoporosis drug characteristics?c

\footnotetext{
${ }^{a}$ Osteoporosis defined by hip or lumbar spine dual-energy x-ray absorptiometry (DXA) BMD T-score <-2.5, past clinical hip or vertebral fracture, or prevalent radiographic vertebral fracture.

bosteopenia/low bone mass defined by hip or lumbar spine DXA BMD T-score $<-1.0$ and $>-2.5$.

'Patient characteristics (age, sex, race, osteoporosis status ${ }^{\mathrm{a}}$, fracture history [clinical fractures, radiographic vertebral fractures], calculated fracture risk [e.g. FRAX ${ }^{\circledR}$ ], comorbid conditions); Bone characteristics (BMD, biomarkers); Osteoporosis drug characteristics (dose, frequency, treatment duration, delivery route).
} 


\section{PICOTS}

Table 2 outlines the populations, interventions, comparisons, outcomes, timing, and settings (PICOTS) eligible for the present review. 


\section{Table 2. PICOTS}

\begin{tabular}{|c|c|c|c|c|c|c|}
\hline KQ & Population & Intervention & Comparator & Health Outcomes and Harms & Timing & Setting \\
\hline $\begin{array}{l}\text { KQ 1: } \\
\text { Long- } \\
\text { term } \\
\text { treatment } \\
\text { efficacy }\end{array}$ & $\begin{array}{l}\text { Men and PM women } \\
\text { aged } \geq 50 \text { years with } \\
\text { osteoporosis* or } \\
\text { osteopenia/low bone } \\
\text { mass } † \text { being studied } \\
\text { for fracture prevention } \\
\text { treatment. }\end{array}$ & $\begin{array}{l}\text { Osteoporosis drug treatment (see } \\
\text { Table 1) }\end{array}$ & $\begin{array}{l}\text { Placebo, } \\
\text { active control }\end{array}$ & $\begin{array}{l}\text { Final: } \\
\text { Incident clinical fracture (any, } \\
\text { nonvertebral, hip, vertebral, } \\
\text { nonhip nonvertebral, MOF) } \\
\text { Intermediate: } \\
\text { Primary: Incident radiographic } \\
\text { vertebral fracture } \\
\text { Secondary: DXA BMD change }\end{array}$ & $>3$ years & Any \\
\hline $\begin{array}{l}\text { KQ 2: } \\
\text { Effect } \\
\text { modifiers } \\
\text { of long- } \\
\text { term } \\
\text { treatment } \\
\text { efficacy }\end{array}$ & $\begin{array}{l}\text { Men and PM women } \\
\text { aged } \geq 50 \text { years with } \\
\text { osteoporosis* or } \\
\text { osteopenia/low bone } \\
\text { mass } † \text { being studied } \\
\text { for fracture prevention } \\
\text { treatment. }\end{array}$ & $\begin{array}{l}\text { Possible effect modifiers of incident } \\
\text { fractures with long-term treatment: } \\
\text { Patient characteristics: pretreatment } \\
\text { age (and years since menopause } \\
\text { for estrogen-related treatments), } \\
\text { race, sex, comorbid conditions (DM, } \\
\text { CKD, CVD), osteoporosis status } \\
\text { (osteoporosis*, low bone mass, } \\
\text { normal), fracture history (clinical } \\
\text { fractures, radiographic vertebral } \\
\text { fractures), calculated pre-treatment } \\
\text { fracture risk (e.g., FRAX }{ }^{\circledR} \text { ) } \\
\text { Bone characteristics: pretreatment } \\
\text { and early treatment (e.g. } 1 \text { year) } \\
\text { imaging (L-spine, total hip \& femoral } \\
\text { neck DXA BMD) and biochemical } \\
\text { markers of bone turnover (CTX, } \\
\text { NTX, P1NP, BSAP) } \\
\text { Osteoporosis drug characteristics: } \\
\text { dose, frequency, treatment duration, } \\
\text { delivery route }\end{array}$ & $\begin{array}{l}\text { Placebo, } \\
\text { active control }\end{array}$ & $\begin{array}{l}\text { Final: } \\
\text { Incident clinical fracture (any, } \\
\text { nonvertebral, hip, vertebral, } \\
\text { nonhip nonvertebral, MOF) } \\
\text { Intermediate: } \\
\text { Primary: Incident radiographic } \\
\text { vertebral fracture }\end{array}$ & $>3$ years & Any \\
\hline
\end{tabular}




\begin{tabular}{|c|c|c|c|c|c|c|}
\hline KQ & Population & Intervention & Comparator & Health Outcomes and Harms & Timing & Setting \\
\hline $\begin{array}{l}\mathrm{KQ} 3: \\
\text { Long- } \\
\text { term } \\
\text { treatment } \\
\text { harms }\end{array}$ & $\begin{array}{l}\text { Men and PM women } \\
\text { aged } \geq 50 \text { years with } \\
\text { osteoporosis* or } \\
\text { osteopenia/low bone } \\
\text { mass } † \text { being studied } \\
\text { for fracture prevention } \\
\text { treatment. } \\
\text { For rare harms only: } \\
\text { Men and PM women } \\
\text { aged } \geq 50 \text { years being } \\
\text { studied for fracture } \\
\text { prevention treatment } \\
\text { regardless of baseline } \\
\text { BMD. }\end{array}$ & $\begin{array}{l}\text { Osteoporosis drug treatment (see } \\
\text { Table 1) }\end{array}$ & $\begin{array}{l}\text { Placebo, no } \\
\text { treatment, } \\
\text { active control }\end{array}$ & $\begin{array}{l}\text { See Table } 3 \text { below for possible } \\
\text { harms outcomes evaluated }\end{array}$ & $>3$ years & Any \\
\hline $\begin{array}{l}\text { KQ 4: } \\
\text { Effect } \\
\text { modifiers } \\
\text { of long- } \\
\text { term } \\
\text { treatment } \\
\text { harms }\end{array}$ & $\begin{array}{l}\text { Men and PM women } \\
\text { aged } \geq 50 \text { years with } \\
\text { osteoporosis* or } \\
\text { osteopenia/low bone } \\
\text { mass } \dagger \text { being studied } \\
\text { for fracture prevention } \\
\text { treatment. } \\
\text { For rare harms only: } \\
\text { Men and PM women } \\
\text { aged } \geq 50 \text { years being } \\
\text { studied for fracture } \\
\text { prevention treatment } \\
\text { regardless of baseline } \\
\text { BMD. }\end{array}$ & $\begin{array}{l}\text { List of possible effect modifiers of } \\
\text { harms with long-term treatment is } \\
\text { the same as the possible effect } \\
\text { modifiers of incident fractures with } \\
\text { long-term treatment detailed above } \\
\text { for KQ } 2 \text {. }\end{array}$ & $\begin{array}{l}\text { Placebo, no } \\
\text { treatment, } \\
\text { active control }\end{array}$ & $\begin{array}{l}\text { See Table } 3 \text { below for possible } \\
\text { harms outcomes evaluated }\end{array}$ & $>3$ years & Any \\
\hline
\end{tabular}




\begin{tabular}{|c|c|c|c|c|c|c|}
\hline KQ & Population & Intervention & Comparator & Health Outcomes and Harms & Timing & Setting \\
\hline $\begin{array}{l}\text { KQ 5: } \\
\text { Effect of } \\
\text { drug } \\
\text { treatment } \\
\text { holidays }\end{array}$ & $\begin{array}{l}\text { Men and PM women } \\
\text { aged } \geq 50 \text { years with } \\
\text { osteoporosis* or } \\
\text { osteopenia/low bone } \\
\text { mass currently } \\
\text { receiving osteoporosis } \\
\text { drug therapy for } \\
\text { fracture prevention. }\end{array}$ & $\begin{array}{l}\text { Osteoporosis drug treatment } \\
\text { discontinuation (placebo drug } \\
\text { holiday) for } \geq 1 \text { year after } \geq 1 \text { year of } \\
\text { prior osteoporosis drug treatment }\end{array}$ & $\begin{array}{l}\text { Continued } \\
\text { osteoporosis } \\
\text { drug } \\
\text { treatment } \\
\text { after } \geq 1 \text { year } \\
\text { of prior } \\
\text { osteoporosis } \\
\text { drug } \\
\text { treatment }\end{array}$ & $\begin{array}{l}\text { Final: } \\
\text { Incident clinical fracture (any, } \\
\text { nonvertebral, hip, vertebral, } \\
\text { nonhip nonvertebral, MOF) } \\
\text { Intermediate: } \\
\text { Primary: Incident radiographic } \\
\text { vertebral fracture } \\
\text { Secondary: DXA BMD change }\end{array}$ & $\begin{array}{l}\geq 1 \text { year } \\
\text { osteoporosis } \\
\text { drug } \\
\text { discontinuation } \\
\text { after } \geq 1 \text { year of } \\
\text { prior } \\
\text { osteoporosis } \\
\text { drug treatment }\end{array}$ & Any \\
\hline $\begin{array}{l}\text { KQ 6: } \\
\text { Effect } \\
\text { modifiers } \\
\text { of effect } \\
\text { of drug } \\
\text { treatment } \\
\text { holidays }\end{array}$ & $\begin{array}{l}\text { Men and PM women } \\
\text { aged } \geq 50 \text { years with } \\
\text { osteoporosis* or } \\
\text { osteopenia/low bone } \\
\text { mass currently } \\
\text { receiving osteoporosis } \\
\text { drug therapy for } \\
\text { fracture prevention. }\end{array}$ & $\begin{array}{l}\text { Possible effect modifiers of incident } \\
\text { fractures with osteoporosis drug } \\
\text { treatment holidays: } \\
\text { Patient characteristics: age, sex, } \\
\text { race, osteoporosis status }{ }^{\star} \text {, fracture } \\
\text { history [clinical fractures, } \\
\text { radiographic vertebral fractures], } \\
\text { calculated fracture risk [e.g. } \\
\text { FRAX®], comorbid conditions } \\
\text { Bone characteristics: BMD and } \\
\text { biomarkers of bone turnover, } \\
\text { including both measures prior to the } \\
\text { drug holiday and during the drug } \\
\text { holiday. } \\
\text { Osteoporosis drug characteristics: } \\
\text { pre-drug holiday agent/class, time } \\
\text { between pre-holiday drug initiation } \\
\text { and start of drug holiday, duration of } \\
\text { drug holiday, post-drug holiday } \\
\text { agent/class }\end{array}$ & $\begin{array}{l}\text { Continued } \\
\text { osteoporosis } \\
\text { drug } \\
\text { treatment } \\
\text { after } \geq 1 \text { year } \\
\text { prior } \\
\text { osteoporosis } \\
\text { drug } \\
\text { treatment }\end{array}$ & $\begin{array}{l}\text { Final: } \\
\text { Incident clinical fracture (any, } \\
\text { nonvertebral, hip, vertebral, } \\
\text { nonhip nonvertebral, MOF) } \\
\text { Intermediate: } \\
\text { Primary: Incident radiographic } \\
\text { vertebral fracture }\end{array}$ & $\begin{array}{l}\geq 1 \text { year } \\
\text { osteoporosis } \\
\text { drug } \\
\text { discontinuation } \\
\text { after } \geq 1 \text { year of } \\
\text { prior } \\
\text { osteoporosis } \\
\text { drug treatment }\end{array}$ & Any \\
\hline
\end{tabular}




\begin{tabular}{|c|c|c|c|c|c|c|}
\hline KQ & Population & Intervention & Comparator & Health Outcomes and Harms & Timing & Setting \\
\hline $\begin{array}{l}\text { KQ 7: } \\
\text { Harms of } \\
\text { drug } \\
\text { treatment } \\
\text { holidays }\end{array}$ & $\begin{array}{l}\text { Men and PM women } \\
\text { aged } \geq 50 \text { years with } \\
\text { osteoporosis* or } \\
\text { osteopenia/low bone } \\
\text { mass currently } \\
\text { receiving osteoporosis } \\
\text { drug therapy for } \\
\text { fracture prevention. }\end{array}$ & $\begin{array}{l}\text { Osteoporosis drug treatment } \\
\text { discontinuation (placebo drug } \\
\text { holiday) for } \geq 1 \text { year after } \geq 1 \text { year of } \\
\text { prior osteoporosis drug treatment }\end{array}$ & $\begin{array}{l}\text { Continued } \\
\text { osteoporosis } \\
\text { drug } \\
\text { treatment } \\
\text { after } \geq 1 \text { year } \\
\text { of prior } \\
\text { osteoporosis } \\
\text { drug } \\
\text { treatment }\end{array}$ & $\begin{array}{l}\text { See Table } 3 \text { below for possible } \\
\text { harms outcomes evaluated }\end{array}$ & $\begin{array}{l}\geq 1 \text { year } \\
\text { osteoporosis } \\
\text { drug } \\
\text { discontinuation } \\
\text { after } \geq 1 \text { year of } \\
\text { prior } \\
\text { osteoporosis } \\
\text { drug treatment }\end{array}$ & Any \\
\hline $\begin{array}{l}\text { KQ 8: } \\
\text { Effect } \\
\text { modifiers } \\
\text { of harms } \\
\text { of drug } \\
\text { treatment } \\
\text { holidays }\end{array}$ & $\begin{array}{l}\text { Men and PM women } \\
\text { aged } \geq 50 \text { years with } \\
\text { osteoporosis* or } \\
\text { osteopenia/low bone } \\
\text { mass currently } \\
\text { receiving osteoporosis } \\
\text { drug therapy for } \\
\text { fracture prevention. }\end{array}$ & $\begin{array}{l}\text { List of possible effect modifiers of } \\
\text { harms with osteoporosis drug } \\
\text { treatment holidays is the same as } \\
\text { the possible effect modifiers of } \\
\text { incident fractures with drug holidays } \\
\text { detailed above for KQ } 6 \text {. }\end{array}$ & $\begin{array}{l}\text { Continued } \\
\text { osteoporosis } \\
\text { drug } \\
\text { treatment } \\
\text { after } \geq 1 \text { year } \\
\text { prior } \\
\text { osteoporosis } \\
\text { drug } \\
\text { treatment }\end{array}$ & $\begin{array}{l}\text { See Table } 3 \text { below for possible } \\
\text { harms outcomes evaluated }\end{array}$ & $\begin{array}{l}\geq 1 \text { year } \\
\text { osteoporosis } \\
\text { drug } \\
\text { discontinuation } \\
\text { after } \geq 1 \text { year } \\
\text { prior } \\
\text { osteoporosis } \\
\text { drug treatment }\end{array}$ & Any \\
\hline
\end{tabular}

Abbreviations: $\mathrm{BMD}=$ bone mineral density; $\mathrm{BSAP}=$ bone specific alkaline phosphatase; $\mathrm{CKD}=$ chronic kidney disease; $\mathrm{CTX}=\mathrm{C}$-terminal telopeptide; $\mathrm{CVD}=$ cardiovascular disease; $\mathrm{DM}=$ diabetes mellitus; DXA=dual x-ray absorptiometry; FRAX=World Health Organization (WHO) Fracture Risk Assessment Tool; KQ=Key Question; MOF=major

osteoporotic fracture; NTX=N-terminal telopeptide; P1NP=procollagen I intact N-terminal; PICOTS=populations, interventions, comparators, outcomes, timing, and settings/study design; PM=postmenopausal; RCT=randomized controlled trial

*Osteoporosis defined by hip or lumbar spine DXA BMD T-score of -2.5 or worse, past clinical hip or vertebral fracture, or prevalent radiographic vertebral fracture. †Osteopenia/low bone mass defined by hip or lumbar spine DXA BMD T-score $<-1.0$ and $>-2.5$. 


\section{Harms}

Table 3 summarizes the harms that were evaluated by this review for possible association with long-term osteoporosis drug therapy.

Table 3. Harms evaluated for possible association with long-term osteoporosis drug therapy

\begin{tabular}{|c|c|}
\hline Drug Class & Harms Outcomes Evaluated \\
\hline Bisphosphonates & $\begin{array}{l}\text { ONJ*, AFF*, atrial fibrillation*, heart attack, musculoskeletal pain, upper GI intolerance, } \\
\text { esophageal cancer }\end{array}$ \\
\hline Biologic (Denosumab) & $\begin{array}{l}\mathrm{ONJ}^{\star}, \mathrm{AFF}^{*} \text {, atrial fibrillation*, heart attack, musculoskeletal pain, upper GI intolerance, } \\
\text { esophageal cancer, infection, fracture after stopping therapy }\end{array}$ \\
\hline $\begin{array}{l}\text { PTH related anabolic } \\
\text { (recombinant PTH and } \\
\text { PTH analog) }\end{array}$ & $\begin{array}{l}\text { Hypercalcemia, hypercalciuria, osteosarcoma, fracture after stopping therapy, upper GI } \\
\text { intolerance }\end{array}$ \\
\hline SERM & $\begin{array}{l}\text { Stroke, venous thromboembolic disease (PE, DVT), hot flashes, mild cognitive } \\
\text { impairment, dementia, mortality }\end{array}$ \\
\hline $\begin{array}{l}\text { Estrogen, Estrogen with } \\
\text { Progestin, Estrogen } \\
\text { with SERM }\end{array}$ & $\begin{array}{l}\text { Cardiovascular disease (heart attack, stroke), venous thromboembolic disease (PE, } \\
\text { DVT), cancer (breast, ovarian, endometrial), mild cognitive impairment, dementia, } \\
\text { mortality }\end{array}$ \\
\hline
\end{tabular}

Abbreviations: AFF=atypical femoral fracture; DVT=deep venous thrombosis; GI=gastrointestinal; ONJ=osteonecrosis of the jaw; PE=pulmonary embolism; PTH=parathyroid hormone; SERM=selective estrogen receptor modulator

*Investigated as rare harms for this report. Some studies reported subtrochanteric/femoral shaft fractures without radiological AFF features as a proxy for AFF. 


\section{Analytic Framework}

Figure 1 outlines the analytic framework used to guide the present review.

Figure 1. Analytic framework for long-term osteoporosis drug treatment and osteoporosis drug treatment holidays for fracture prevention

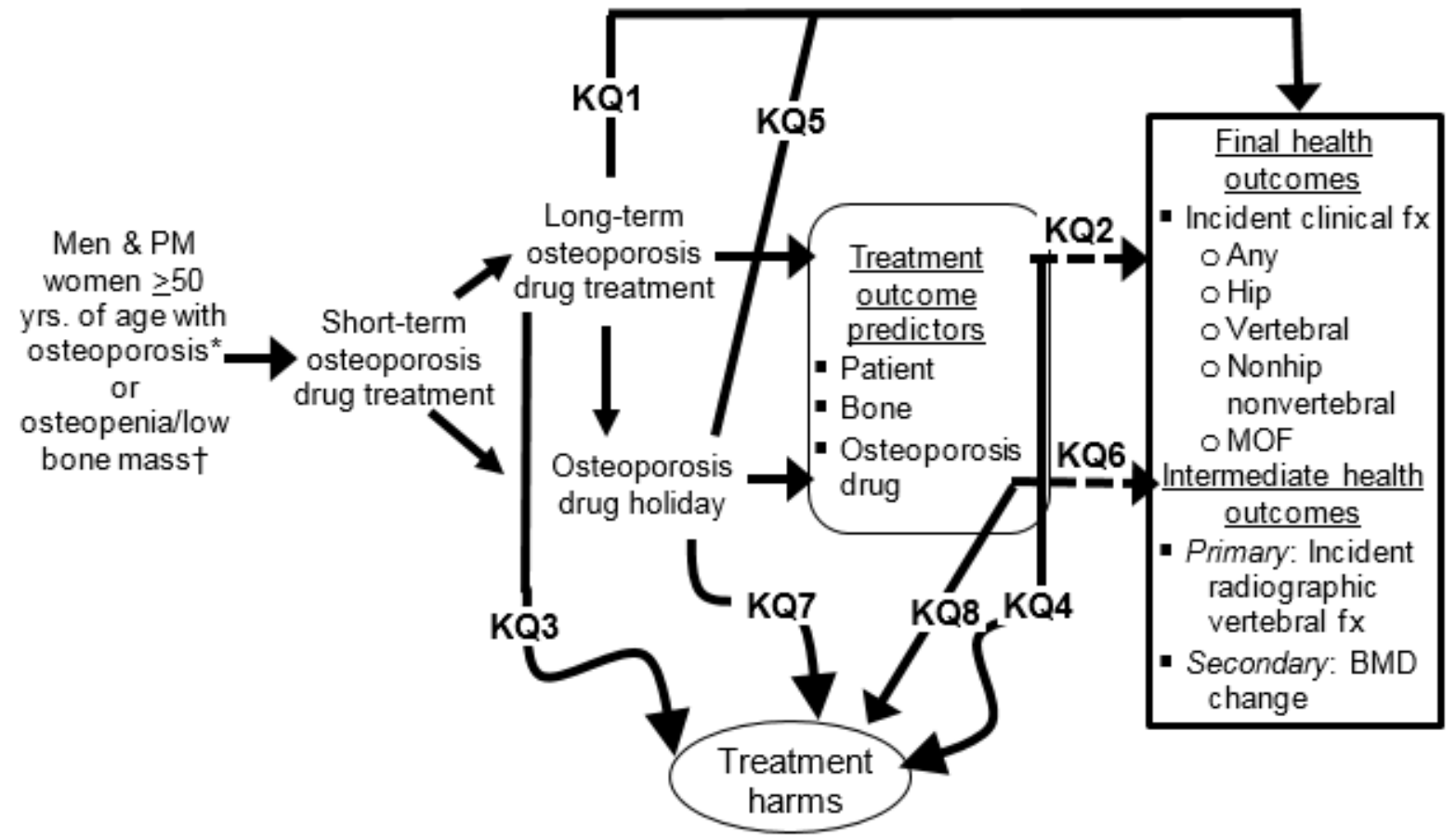

Abbreviations: $\mathrm{BMD}=$ =bone mineral density; $\mathrm{fx}=$ fracture; $\mathrm{KQ}=$ key question; $\mathrm{MOF}=$ major osteoporotic fracture;

$\mathrm{PM}=$ postmenopausal; yrs=years

*Osteoporosis defined by hip or lumbar spine dual-energy x-ray absorptiometry (DXA) BMD T-score <-2.5, past clinical hip or vertebral fracture, or prevalent radiographic vertebral fracture

†Osteopenia/low bone mass defined by hip or lumbar spine DXA BMD T-score $<-1.0$ and $>-2.5$ 


\section{Chapter 2. Methods}

This comparative effectiveness review follows methods suggested in the Agency for Healthcare Research and Quality (AHRQ) Methods Guide for Effectiveness and Comparative Effectiveness Reviews (http://www.effectivehealthcare.ahrq.gov/methodsguide.cfm); certain methods map to the Preferred Reporting Items for Systematic Reviews and Meta-Analyses (PRISMA) checklist. ${ }^{26}$

\section{Topic Refinement and Review Protocol}

The National Institutes of Health (NIH) Office of Disease Prevention (ODP) Working Group, which included individuals from the ODP, National Institute on Aging and National Institute of Arthritis and Musculoskeletal and Skin Diseases, developed the first version of questions for this review. Questions were refined in collaboration with the NIH ODP Working Group, a NIH Content Area Expert Group, and a Technical Expert Panel. The resulting Key Questions (KQs) were incorporated into the final protocol, which was posted April 12, 2018 at https://effectivehealthcare.ahrq.gov/topics/osteoporosis-fracture-prevention/research-protocol and registered in PROSPERO.

\section{Literature Search Strategy}

We searched Ovid Medline ${ }^{\circledR}$, Ovid Embase ${ }^{\circledR}$, and the Cochrane Central Register of Controlled Trials (CENTRAL) to identify previous randomized controlled trials (RCTs), controlled clinical trials, and controlled observational studies published and indexed in bibliographic databases. Our search strategy, Appendix A, included relevant medical subject headings and natural language terms for the concepts of osteoporosis and drug treatment. These concepts were combined with validated filters to select study designs. Dates for the search algorithm were 1995 to June 2018. We supplemented our searches with backward citation searches of relevant systematic reviews published from 2012 and onward. We updated our electronic database search through October 2018 while the draft report was under peer review.

We searched ClinicalTrials.gov to identify additional relevant completed and ongoing studies. AHRQ also opened a Supplemental Evidence and Data for Systematic Reviews (SEADs) portal to solicit pharmaceutical manufacturer protocols with additional information about published or unpublished drug studies. Grey literature search results were used to identify studies, outcomes, and analyses not reported in the published literature to assess publication and reporting bias and inform future research needs.

We reviewed studies relevant to inclusion criteria based on our population, intervention, comparators, timing, and settings (PICOTS) framework outlined in Table 4. 
Table 4. Study inclusion criteria

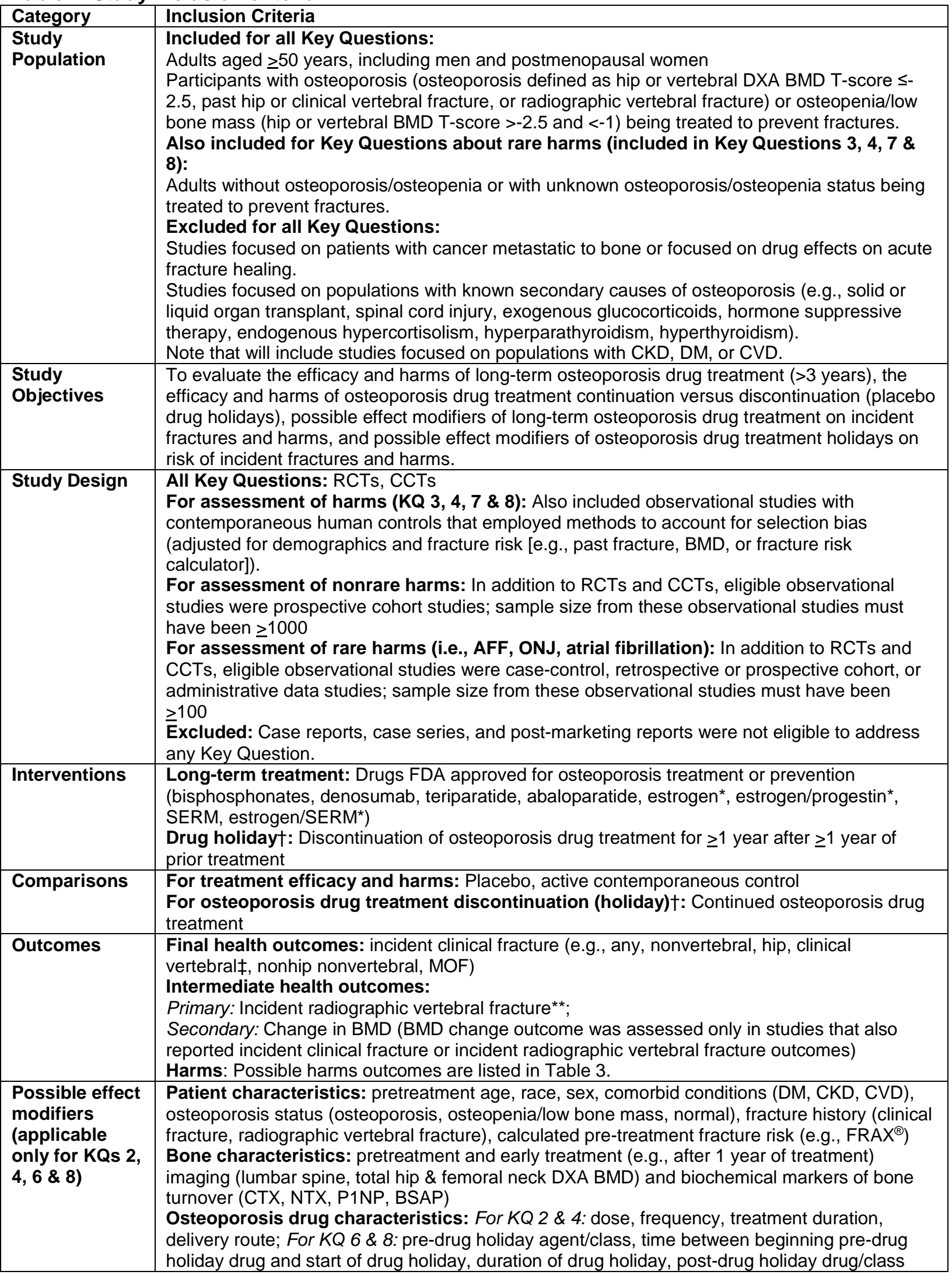




\begin{tabular}{|l|l|}
\hline Category & Inclusion Criteria \\
\hline Timing & $\begin{array}{l}\text { Long-term osteoporosis drug treatment: treatment duration }>3 \text { years. } \\
\text { Osteoporosis drug treatment holidays: treatment discontinuation } \geq 1 \text { year after prior } \\
\text { osteoporosis drug treatment } \geq 1 \text { year }\end{array}$ \\
\hline Setting & Any \\
\hline $\begin{array}{l}\text { Publication } \\
\text { type }\end{array}$ & $\begin{array}{l}\text { Published in full text in peer reviewed journals. } \\
\text { Used systematic reviews and eligible studies to identify additional references. } \\
\text { Data were supplemented by grey literature if it included sufficient information to assess } \\
\text { eligibility and risk of bias. }\end{array}$ \\
\hline $\begin{array}{l}\text { Publication } \\
\text { language }\end{array}$ & English \\
\hline
\end{tabular}

Abbreviations: AFF=Atypical femoral fracture; BMD=bone mineral density; BSAP=bone-specific alkaline phosphatase; $\mathrm{CCT}=$ controlled clinical trial; $\mathrm{CKD}=$ chronic kidney disease; $\mathrm{CTX}=\mathrm{C}$-terminal telopeptide; $\mathrm{CVD}=$ cardiovascular disease; DM=diabetes mellitus; DXA=dual x-ray absorptiometry; FDA=U.S. Food and Drug Administration; FRAX=World Health Organization (WHO) Fracture Risk Assessment Tool; KQ=Key Question; MOF=major osteoporotic fracture; NTX=N-terminal telopeptide; ONJ=osteonecrosis of the jaw; P1NP=procollagen I intact N-terminal; RCT=randomized controlled trial

*FDA approved for osteoporosis prevention, but not for osteoporosis treatment. †Because all trials of osteoporosis drug continuation versus discontinuation (drug holiday) framed and reported results for the comparison of the drug continuation intervention versus the drug discontinuation reference group, we elected to not flip and recalculate all the results of these studies, but presented drug holiday comparisons as continued treatment versus the reference of discontinued treatment.

¥Clinical vertebral fractures are usually diagnosed in the community because of back pain, with study confirmation by comparing baseline study radiograph and subsequent community radiographs.

**Radiographic vertebral fractures are identified in studies by comparing vertebral heights on scheduled serial vertebral radiographs; mostly, these are unrecognized in the community.

\section{Study Selection}

We screened titles and abstracts of all references identified from our bibliographic database search, references from relevant systematic reviews, and grey literature. Studies considered possibly eligible based on our inclusion criteria by at least one of two independent reviewers were flagged for full text screening. Then, two independent reviewers screened the full text publications to determine if inclusion criteria were met. Differences in screening decisions were resolved by discussion between investigators, and, if necessary, consultation with a third investigator or team consensus. For studies excluded at the full text review stage, reasons for ineligibility were documented. Reviewers regularly met to discuss inclusion criteria and ensure consistency between reviewers.

\section{Risk of Bias Assessment}

Based on AHRQ guidance, ${ }^{27}$ we assessed risk of bias (ROB) of eligible studies in their design, analysis and reporting. ROB was assessed by two independent investigators guided by a modified instrument we created for a previous AHRQ review (Appendix B). Differences in ROB decisions were resolved via reviewer discussion and, if necessary, team consensus. We selected items most relevant in assessing ROB for this topic, including: (1) participant selection (adequacy of randomization method); (2) attrition/incomplete outcome data (loss to follow-up), (3) detection bias (outcome assessor masking, outcome measurement quality); (4) performance bias (intention to treat analysis, adjustment for potential confounding variables, participant masking to treatment assignment); and (5) reporting bias (selective outcomes reporting). Summary ROB assessments for each study were classified as low, medium, or high based upon the collective ROB inherent in each domain and the reviewers' collective informed opinion that the study estimate represented what the study would have found had it been perfectly designed, conducted, analyzed, and reported (Appendix C). 
ROB was assessed separately for the following outcomes: incident clinical fractures (any, nonvertebral, hip, vertebral, nonhip nonvertebral, major osteoporotic fracture [MOF]), incident radiographic vertebral fractures, atypical femoral fracture (AFF) with confirmed radiological features, subtrochanteric/femoral shaft fractures, osteonecrosis of the jaw (ONJ), and incident fracture after stopping osteoporosis drug treatment (rebound fractures). Differences in ROB ratings between investigators were resolved by discussion, and, if necessary, team consensus. Screening and ROB assessment were performed in Distiller (DistillerSR, Evidence Partners, Ottawa, Canada).

\section{Data Extraction}

For studies meeting inclusion criteria, one investigator extracted relevant data into extraction forms created in Microsoft Word. Evidence tables were reviewed and verified for accuracy by a second investigator.

Limited data were extracted from studies with high ROB: author, publication year, study design, intervention, types of efficacy outcomes, and whether adverse effects were reported (Appendix Table D7). For studies with low or medium ROB, we extracted additional information: participant inclusion criteria, setting, participant baseline characteristics (age, race, sex, comorbid conditions [diabetes mellitus (DM), chronic kidney disease (CKD), cardiovascular disease (CVD)], osteoporosis status (i.e., osteoporosis versus osteopenia/low bone mass versus normal, including how the study defined its eligible population), fracture history, calculator estimated fracture risk, bone mineral density (BMD), bone turnover marker levels (C-terminal telopeptide [CTX], N-terminal telopeptide [NTX], procollagen I intact N-terminal [P1NP], bonespecific alkaline phosphatase [BSAP]), intervention details (drug class, name, dose and delivery route), control intervention details, follow-up duration, study funding, and results of efficacy outcomes and adverse effects (Appendix D).

\section{Data Synthesis}

Results were organized first by KQ, then treatment comparison, then treatment outcome (incident clinical fractures, incident radiographic vertebral fractures, change in BMD, harms). Studies that compared continuous long-term treatment with continuous control were included in the long-term osteoporosis drug treatment section of the report. Studies that compared osteoporosis drug continuation versus discontinuation were included in the drug holiday section of the report, even if the total duration of treatment in the continuation group was $>3$ years.

For studies with low and moderate risk of bias, we summarized results in evidence tables. Because there were few treatment-outcome comparisons with more than one eligible study and those had substantial clinical and methodological heterogeneity (participant population, intervention, outcome measures), we judged that statistical pooling was inappropriate and conducted qualitative synthesis.

When applicable data were available within individual studies or across multiple studies (i.e., tests of interaction, stratified results, special population), we evaluated a priori selected possible effect modifiers of osteoporosis drug treatment and osteoporosis drug treatment holidays on efficacy and harms outcomes (i.e., age, race, sex, comorbid conditions [DM, CKD, CVD], osteoporosis status, fracture history, calculated estimated fracture risk, BMD, CTX, NTX, P1NP, BSAP, drug dose, frequency, treatment duration and delivery route, pre-drug holiday agent/class, time between osteoporosis drug initiation and start of drug holiday, duration of drug holiday, post-drug holiday agent/class). 


\section{Strength of Evidence for Major Comparisons and Outcomes}

The overall strength of evidence (SOE) was evaluated by two independent reviewers. Differences were resolved by consultation between investigators, and, if necessary, consultation with a third investigator. SOE was rated individually for the following efficacy outcomes: any incident clinical fracture, incident nonvertebral fracture, incident hip fracture, incident clinical vertebral fracture, incident nonhip nonvertebral fracture, incident MOF, and incident radiographic vertebral fractures. Strength of evidence also was graded for the following harms: serious adverse events, AFF with confirmed radiologic features, subtrochanteric/femoral shaft fractures without confirmed radiologic AFF features, ONJ, and incident clinical fracture after stopping osteoporosis drug treatment (rebound fractures).

SOE ratings were based on five required domains: (1) study limitations; (2) directness; (3) consistency; (4) precision; and (5) reporting bias. ${ }^{28}$ Study limitations were rated as low, medium, or high, based on the collective ROB of the individual studies pertaining to an evidence base. Directness was rated as direct or indirect, based on the presence or absence of a single, direct link between intervention and outcome. Consistency was rated as consistent or inconsistent, based on the similarity of the direction and size of an effect between studies, or unknown when there was only a single study. Precision was rated as precise, imprecise, or highly imprecise, based on the degree of certainty around an estimate or a small number of fracture or harm events. ${ }^{29}$ An imprecise estimate was one for which the confidence interval was wide enough to include clinically distinct conclusions or there were few events, and a highly imprecise estimate was one for which confidence intervals were wide and there were few events, or the confidence intervals were wide enough to include both a large risk reduction and a large risk increase. Other factors considered in assessing strength of evidence included dose-response relationships, the presence of confounders, and strength of association.

Based on these factors, the overall evidence for each outcome (Appendix D) was rated as: ${ }^{28}$

High: Very confident that estimate of effect lies close to true effect. Few or no deficiencies in body of evidence, findings believed to be stable.

Moderate: Moderately confident that estimate of effect lies close to true effect. Some deficiencies in body of evidence; findings likely to be stable, but some doubt.

Low: Limited confidence that estimate of effect lies close to true effect; major or numerous deficiencies in body of evidence. Additional evidence necessary before concluding that findings are stable or that estimate of effect is close to true effect.

Insufficient: No evidence, unable to estimate an effect, or no confidence in estimate of effect. No evidence is available or the body of evidence precludes judgment.

An overall rating of high SOE would be assigned when included studies were RCTs with low risk of bias, and the results were consistent, direct, and precise.

\section{Applicability}

Applicability of findings was determined according to the PICOTS framework. Study characteristics that affected applicability included, but were not limited to, the population (age, race, sex, presence or lack of comorbidities, country from which the study participants were enrolled), narrow eligibility criteria, and patient and intervention characteristics potentially associated with treatment response that were different than those described by population studies. ${ }^{30}$ 


\section{Peer Review and Public Commentary}

AHRQ and NIH staff and an AHRQ associate editor reviewed the first draft of this report. After we revised the report based on these reviews, experts in osteoporosis, primary care, geriatrics, and systematic reviews were invited to provide external peer review. After we revised the report again based on these peer reviews, the next draft was posted on the AHRQ website for 4 weeks to elicit public comment. We then revised the report again based on these public comments, and documented all public comments, our responses and revisions in a disposition of comments report that we submitted with the final report for posting on the Effective Health Care website. 


\section{Chapter 3. Search Results}

We identified 8,356 unique citations (Figure 2) from 1995 to October 2018 from bibliographic databases addressing osteoporosis and drug treatment. An initial title and abstract review excluded 7,026 publications that were not related to relevant drug treatments for patients with osteoporosis. Full texts of 1,330 publications from the electronic database searches and five publications identified by hand search were reviewed to determine final inclusion. Appendix E provides a list of publications excluded at full text review. We identified 61 unique references eligible for inclusion, which are summarized in Table 5. Risk of bias (ROB) assessments for these references can be found in Appendix C.

Of 48 publications with low or medium ROB, there were 35 randomized or controlled clinical trials (9 unique studies) and 13 controlled observational studies (11 unique studies) (Appendix C). Most publications were based on three randomized controlled trials of alendronate, zoledronic acid, and raloxifene, respectively, and their extension studies.

All trials enrolled only postmenopausal women, with most limited to women with osteoporosis as defined by bone mineral density (BMD) and vertebral fracture history, and a few also including women with osteopenia. The observational studies included between 84 to 100 percent women. Mean participant age was 72 years, with all but two studies reporting a mean age of $<80$ years. Most of the observational studies presumed that participants had osteoporosis because of past fracture or their use of osteoporosis medications, but none reported BMD status. 
Figure 2. Literature flow diagram

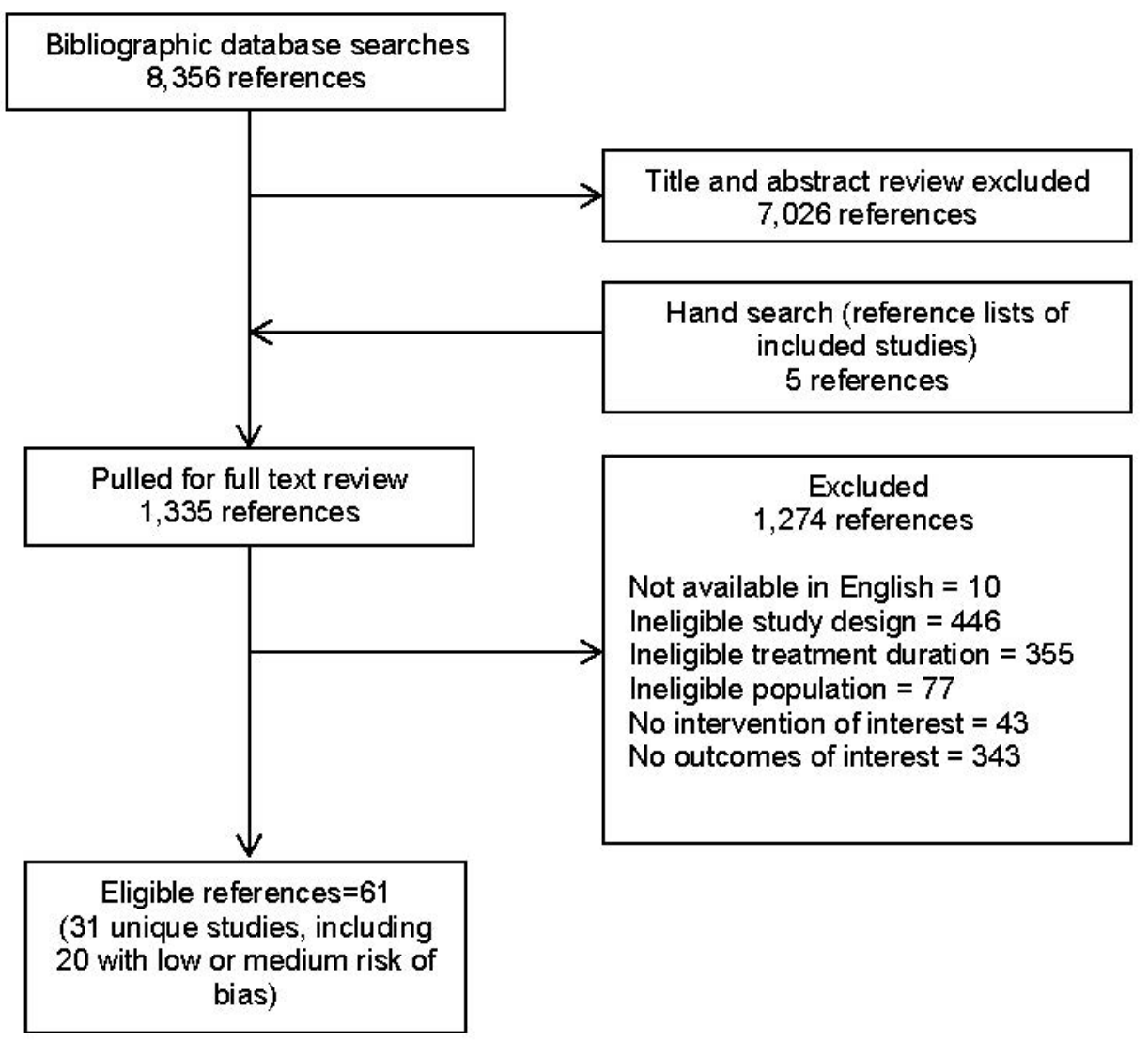


Table 5. Eligible publications that compared long-term osteoporosis drug use versus control or osteoporosis drug continuation versus discontinuation (placebo drug holiday) and reported on risk of incident fractures and/or harms

\begin{tabular}{|c|c|c|c|}
\hline $\begin{array}{l}\text { Drug name } \\
\text { (Drug class) }\end{array}$ & $\begin{array}{c}\text { Low or Medium ROB } \\
\text { Publications } \\
\text { (Analyzed) }\end{array}$ & $\begin{array}{c}\text { High ROB } \\
\text { Publications } \\
\text { (Not Analyzed) }\end{array}$ & $\begin{array}{c}\text { Total Number of } \\
\text { Publications }\end{array}$ \\
\hline $\begin{array}{l}\text { Alendronate } \\
\text { (Bisphosphonate) }\end{array}$ & $\begin{array}{c}13 \text { RCTs or CCTs } \\
\text { FIT/FLEX) } \\
4 \text { observational }{ }^{44-47}\end{array}$ & $\begin{array}{c}2 \mathrm{RCTS}^{48,49}(\mathrm{FIT}) \\
4 \text { observational }^{50-53}\end{array}$ & $\begin{array}{l}23 \text { publications: } \\
15 \text { RCTs or CCTs } \\
8 \text { observational } \\
\end{array}$ \\
\hline $\begin{array}{l}\text { Zoledronic acid } \\
\text { (Bisphosphonate) }\end{array}$ & $\begin{array}{c}4 \mathrm{RCTs}^{54-57} \text { ( } 2 \mathrm{HORIZON} \\
\text { extensions) }\end{array}$ & $\begin{array}{c}1 \mathrm{RCT}^{58} \text { (HORIZON } \\
\text { extension) } \\
\end{array}$ & $\begin{array}{l}5 \text { publications: } \\
5 \text { RCTs }\end{array}$ \\
\hline $\begin{array}{l}\text { Risedronate } \\
\text { (Bisphosphonate) }\end{array}$ & 0 & $2 \mathrm{RCTs}^{59,60}$ & $\begin{array}{l}2 \text { publications: } \\
2 \text { RCTs }\end{array}$ \\
\hline $\begin{array}{l}\text { Ibandronate } \\
\text { (Bisphosphonate) }\end{array}$ & 0 & $1 \mathrm{RCT}^{61}$ & $\begin{array}{c}1 \text { publication: } \\
1 \mathrm{RCT}\end{array}$ \\
\hline $\begin{array}{l}\text { Any bisphosphonate } \\
\text { (studies reported results as a } \\
\text { class) }\end{array}$ & 6 observational ${ }^{62-67}$ & 2 observational 68,69 & $\begin{array}{l}8 \text { publications: } \\
8 \text { observational }\end{array}$ \\
\hline $\begin{array}{l}\text { Denosumab } \\
\text { (Biologic) }\end{array}$ & $1 \mathrm{RCT}^{70}$ & $1 \mathrm{RCT}^{71}$ & $\begin{array}{l}2 \text { publications: } \\
2 \text { RCTs }\end{array}$ \\
\hline $\begin{array}{l}\text { Teriparatide } \\
\text { (recombinant PTH anabolic) }\end{array}$ & 0 & 0 & 0 \\
\hline $\begin{array}{l}\text { Abaloparatide } \\
\text { (PTH analog anabolic) }\end{array}$ & 0 & 0 & 0 \\
\hline $\begin{array}{l}\text { Raloxifene } \\
\text { (SERM) }\end{array}$ & $\begin{array}{c}14 \text { RCTs or CCTs }{ }^{72-85}(14 \\
\text { MORE/CORE) }\end{array}$ & 0 & $\begin{array}{l}14 \text { publications: } \\
14 \text { RCTs or CCTs }\end{array}$ \\
\hline $\begin{array}{l}\text { Estrogen and Estrogen/ Progestin } \\
\text { Combination products }\end{array}$ & $3 \mathrm{RCT}^{86-88}$ & 0 & $\begin{array}{l}3 \text { publications: } \\
\text { 3 RCTs }\end{array}$ \\
\hline $\begin{array}{l}\text { Conjugated estrogens/ } \\
\text { bazedoxifene* } \\
\text { (Estrogen with SERM) }\end{array}$ & 0 & 0 & 0 \\
\hline $\begin{array}{l}\text { Pooled osteoporosis drugs } \\
\text { bisphosphonates, raloxifene }\end{array}$ & 3 observational ${ }^{89-91}$ & 0 & $\begin{array}{l}3 \text { publications: } \\
3 \text { observational }\end{array}$ \\
\hline Total number of publications & $\begin{array}{l}48 \text { publications: } \\
35 \text { RCTs or CCTs } \\
13 \text { observational }\end{array}$ & $\begin{array}{c}13 \text { publications: } \\
7 \text { RCTs } \\
6 \text { observational }\end{array}$ & $\begin{array}{l}61 \text { publications: } \\
42 \text { RCTs or CCTs } \\
19 \text { observational }\end{array}$ \\
\hline
\end{tabular}

Abbreviations: CCT=Controlled clinical trial; CORE=Continuing Outcomes Relevant to Evista; FIT=Fracture Intervention Trial; FLEX=Fracture Intervention Trial Long Term Extension; HORIZON=Health Outcomes and Reduced Incidence with Zoledronic Acid Once Yearly trial; MORE=Multiple Outcomes of Raloxifene Evaluation; PTH=parathyroid hormone; RCT=randomized controlled trials; ROB=risk of bias; SERM=selective estrogen receptor modulator

*Approved by the Food and Drug Administration for osteoporosis prevention, but not for osteoporosis treatment. 


\section{Chapter 4. Efficacy of Long-Term Osteoporosis Drug Treatment}

Chapter 4 reviews the evidence from eligible studies that address Key Questions 1 and 2. Key Question 1 examines the efficacy of long-term ( $>3$ years) osteoporosis drug treatment versus inactive control (e.g., placebo, no treatment) or a different active treatment in reducing risk of incident fractures and on change in bone mineral density (BMD). Key Question 2 examines whether efficacy of long-term osteoporosis drug treatment versus control in reducing risk of incident fractures varies as a function of patient, bone, or drug characteristics.

We report the literature on the effect of continuing osteoporosis drug treatment versus discontinuing it on risk of incident fractures and on change in BMD in the drug holiday section of the report (Chapter 6, Key Question 5), regardless of the duration of drug treatment before allocation to continuation or discontinuation.

This chapter is organized by drug, with Key Question 1 and Key Question 2 discussed sequentially within each drug subsection. Drug versus placebo or no treatment comparisons are listed first, followed by any comparisons of drug versus another active drug treatment. We provide an overview at the start of specific drug subsections when the literature base was complex. Summary statements about the included studies are below; individual study details are provided in the report tables and appendixes.

\section{Alendronate}

\section{Key Points}

\section{Key Question 1}

- In postmenopausal women with either osteoporosis or osteopenia (femoral neck [FN] BMD T-score $\leq-1.6$ ) but without a baseline vertebral fracture, between alendronate versus placebo for 4 years:

o Alendronate was associated with no difference in risk of incident clinical fractures (low strength of evidence [SOE]), incident nonvertebral fractures (low SOE), or incident hip fractures (low SOE).

o Alendronate was associated with a lower risk of incident radiographic vertebral fractures (high SOE).

\section{Key Question 2}

- In postmenopausal women with either osteoporosis or osteopenia and but without a baseline vertebral fracture, the effect of alendronate treatment versus placebo for 4 years varied by baseline BMD:

o In women with FN-BMD T-score $<-2.5$ (osteoporosis), alendronate was associated with a lower risk of both incident clinical fractures (moderate SOE) and incident radiographic vertebral fractures (moderate SOE).

0 In women with FN-BMD T-score -2 to -2.5 (osteopenia), alendronate was not associated with a lower risk of incident clinical fractures (low SOE) and may not lower risk of incident radiographic vertebral fractures (low SOE).

o In women with FN-BMD T-score -1.6 to -2 (osteopenia), alendronate was not associated with a lower risk of incident clinical fractures (low SOE), but evidence 
was insufficient to draw conclusions about risk of incident radiographic vertebral fractures.

- In postmenopausal women with baseline FN-BMD -1.6 to > -2.5 (osteopenia), the effect of alendronate treatment versus placebo for 4 years:

o On risk of incident nonvertebral fracture, was not modified by history of prior nonvertebral fracture.

o On risk of incident clinical and radiographic vertebral fracture, was not modified by presence of baseline radiographic vertebral fracture.

o On risks of incident clinical fracture, nonvertebral fracture, major osteoporotic fracture, and radiographic vertebral fracture, was not modified by baseline World Health Organization Fracture Risk Assessment Tool (FRAX) score.

- In postmenopausal women, whether with osteoporosis (baseline vertebral fracture or FNBMD $<-2.5$ ) or not (no baseline vertebral fracture and FN-BMD -1.6 to $>-2.5$ ), there was no consistent modification of the effect of treatment with alendronate versus placebo for 4 years by pretreatment levels of bone turnover markers.

\section{Eligible Studies}

Nine eligible publications of three unique studies compared long-term alendronate treatment versus placebo, no treatment or a different active treatment, and reported on risk of incident fractures. We rated four of these studies as having high risk of bias (ROB) ${ }^{48-51}$ and extracted only limited data (Appendix D). For the remaining five studies with low or medium ROB, ${ }^{2}, 35,36,39,40$ additional information was extracted in evidence tables and SOE summary tables, available in Appendix D.

All five publications with low or medium ROB were from the Fracture Intervention Trial (FIT), in which participants were randomized to alendronate versus placebo. No eligible randomized controlled trials (RCTs) reporting incident fracture compared long-term alendronate versus a different active treatment. Trials that compared the efficacy of long-term alendronate versus shorter term alendronate on risk of incident fractures are reported under Key Question 5 about osteoporosis drug treatment holidays in Chapter 6 of this report.

FIT was conducted in the U.S. and enrolled postmenopausal women aged 55-80 years with osteoporosis or osteopenia/low bone mass (FN-BMD $\leq 0.68 \mathrm{~g} / \mathrm{cm}^{2}[\mathrm{~T}$ score $\left.\leq-1.6]\right) .{ }^{35,92}$ FIT excluded women with recent or severe peptic ulcers, dyspepsia that required daily treatment and significant renal dysfunction. Prior osteoporosis drug treatment was restricted. All participants were randomized to alendronate $5 \mathrm{mg}$ daily versus placebo, with the alendronate dose changed to $10 \mathrm{mg}$ daily at the 24-month clinic visit based on emerging evidence from other trials.

FIT was comprised of two parallel RCTs. FIT-I enrolled 2027 women with baseline radiographic vertebral fracture, had a mean treatment duration of 2.9 years, and was excluded from this review because treatment duration did not exceed three years as required to define long-term treatment for this review. ${ }^{92}$ FIT-II enrolled 4432 women without baseline radiographic vertebral fracture, had a mean treatment duration of 4 years, was rated as low ROB and was included in this review. ${ }^{35}$ There also were multiple reports of post hoc analyses of FIT-II or pooled analyses of FIT-I plus FIT-II. We included the pooled reports if either mean or median treatment duration was reported as greater than 3 years, or, if neither mean nor median was reported, if more than 50 percent of the pooled sample came from FIT-II and the article otherwise met inclusion criteria. Four publications met these criteria, were rated as medium ROB and were included in this review. ${ }^{32,36,39,40}$ 
Baseline participant characteristics for the FIT-II RCT ${ }^{35}$ and the four related reports ${ }^{32,36,39,40}$ are listed in Table 6 (below) and Appendix Table D2. The mean age of FIT-II women was 68 years and most women were of white race (97\%). Thirty-seven percent of the FIT-II sample had osteoporosis at baseline (FN-BMD T score <-2.5).

Although the 2.9-year FIT-I RCT was excluded from this review of long-term treatment, its baseline participant characteristics are included in Table 6 for comparison, since all or part of FIT-I was pooled in three included reports that contained FIT-II data. ${ }^{32,36,39}$

Table 6. Baseline participant characteristics from included alendronate long-term treatment efficacy publications for KQ 1 and KQ 2 and FIT-I

\begin{tabular}{|c|c|c|c|c|c|c|}
\hline & $\begin{array}{c}\text { Cummings } \\
1998^{35} \\
\text { FIT-II }\end{array}$ & $\begin{array}{c}\text { Ryder } \\
\mathbf{2 0 0 8}^{40} \\
\text { FIT-II } \\
\text { subgroup }\end{array}$ & $\begin{array}{c}\text { Donaldson } \\
\mathbf{2 0 1 2}^{36} \\
\text { pooled } \\
\text { FIT-I \& II }\end{array}$ & $\begin{array}{c}\text { Quandt } \\
2005^{39} \\
\text { pooled FIT-I } \\
\text { \& II subset }\end{array}$ & $\begin{array}{l}\text { Bauer } \\
2006^{32} \\
\text { pooled } \\
\text { FIT-I \& II }\end{array}$ & $\begin{array}{c}\text { FIT-I }^{92} \\
\text { (excluded) }\end{array}$ \\
\hline Sample N & 4432 & 2785 & 6459 & 3737 & 6186 & 2027 \\
\hline $\mathrm{N}(\%)$ from FIT-II & $4432(100)$ & $2785(100)$ & 4432 (68.6) & $2797(74.8)$ & NR & 0 \\
\hline$\%$ of FIT-II sample & $100 \%$ & $62.8 \%$ & $100 \%$ & $63.1 \%$ & ND & 0 \\
\hline Mean follow-up & $4.2 \mathrm{yr}$ & $4.2 \mathrm{yr}$ & $4 \mathrm{yr}^{\star}$ & $3-4.5 \mathrm{yr}$ & $3.2 \mathrm{yr}$ & $2.9 \mathrm{yr}$ \\
\hline Mean age (yr) & 68 & 66 & 68 & 68 & 69 & 71 \\
\hline Age <65 yr (\%) & 34 & 38 & NR & NR & NR & 16 \\
\hline Age 65-74 yr (\%) & 53 & 52 & NR & NR & NR & 57 \\
\hline Age 75-81 yr (\%) & 13 & 11 & NR & NR & NR & 27 \\
\hline Female (\%) & 100 & 1010 & 100 & 100 & 100 & 100 \\
\hline White race (\%) & 97 & NR & NR & NR & NR & 97 \\
\hline Baseline RVF (\%) & 0 & 0 & 31 & 25 & 31 & 100 \\
\hline $\begin{array}{l}\% \text { Prior fracture since } \\
\text { age } \geq 45 \mathrm{yr}\end{array}$ & 36 & 32 & 44 & 37 & NR & 58 \\
\hline Enrolled T-score & $\leq-1.6$ & -1.0 to -2.5 & $\leq-1.6$ & -1.6 to -2.5 & $\leq-1.6$ & $\leq-1.6$ \\
\hline Comorbid conditions & NR & NR & NR & NR & NR & NR \\
\hline
\end{tabular}

Abbreviations: FIT=Fracture Intervention Trial; $\mathrm{NR}=$ not reported for this study subsample; $\mathrm{RVF}=$ radiographic vertebral fracture; yr=year

*Calculated by Evidence-based Practice Center

\section{Outcomes}

All evidence on the long-term efficacy of alendronate versus placebo on risk of incident fractures and change in BMD comes from the single, eligible, low ROB FIT-II RCT, described in detail above. ${ }^{35}$

\section{Incident Clinical Fractures}

In the FIT-II RCT, alendronate for 4 years versus placebo did not significantly reduce risk of incident clinical fracture (12.3\% vs. 14.1\%; hazard ratio [HR] 0.86 [95\% confidence intervals (CI) $0.73,1.01]$ ) (low SOE), incident nonvertebral fracture (11.8\% vs. 13.3\%; HR 0.88 [95\% CI $0.74,1.04]$ ) (low SOE), or incident hip fracture (0.9\% vs. 1.1\%, HR 0.79 [95\% CI 0.43, 1.44]) (low SOE). ${ }^{35}$

\section{Incident Radiographic Vertebral Fractures}

Compared with placebo, alendronate was associated with a significantly lower risk of incident radiographic vertebral fracture (2.1\% vs. 3.8\%; HR 0.56 [95\% CI 0.39, 0.80]) (high SOE). 


\section{Change in BMD}

Alendronate for four years was associated with statistically significant increases in BMD compared with placebo, ranging from 3.4 percent for total hip (TH) BMD to 8.3 percent for lumbar spine (LS)-BMD ( $<<0.001$ for all between-group differences).

\section{Variation in Long-Term Treatment Efficacy as a Function of Patient, Bone, or Osteoporosis Drug Characteristics}

We looked at whether the efficacy of long-term alendronate treatment versus placebo or inactive control on risk of incident fracture varies as a function of patient, bone, or drug characteristics. Eligible studies included evidence for the following possible effect modifiers.

\section{Baseline BMD}

In a FIT-II subgroup analysis planned before treatment assignments were unmasked, the effect of long-term alendronate versus placebo on risks of any incident clinical fracture and incident radiographic vertebral fracture varied by baseline BMD. ${ }^{35}$ While risk of incident clinical fracture was significantly lower with four years of alendronate versus placebo in women with a baseline FN-BMD T-score of <-2.5 (13.1\% vs. 19.6\%; HR 0.64 [95\% CI 0.50, 0.82]), there was no difference between treatment groups in women with FN-BMD T-scores between -2.5 to -2.0 or between -2.0 to -1.6 (p-value for interaction 0.01 ). Risk of incident radiographic vertebral fracture was significantly lower with four years of alendronate versus placebo in women with a baseline FN-BMD T-score of <-2.5 (2.9\% vs. 5.8\%; HR 0.50 [95\% CI 0.31, 0.82]). The reduction in risk appeared similar in magnitude in the FN-BMD T-score -2.5 to -2 subgroup (1.9\% vs. 3.6\%; HR 0.54 [95\% CI 0.28, 1.04]), but was not statistically significant, likely in part to there being fewer women in this BMD subgroup with radiographic vertebral fractures. There appeared to be no difference between alendronate versus placebo on risk of incident radiographic vertebral fractures in women with FN-BMD T-scores between -2.0 to -1.6 (1.3\% vs. 1.5\%; HR $0.82[0.33,2.07])$ (p-value for interaction was not reported) (Appendix D3).

In a post hoc FIT-II analysis, alendronate was associated with a significantly lower risk of incident hip fracture in women with baseline FN-BMD T-scores of $<-2.5$ (HR 0.44 [95\% CI 0.18, 0.97]), but not in those with baseline FN-BMD T-scores >-2.5 (HR 1.84 [95\% CI 0.70, 5.36]) (p-value for interaction not reported).

\section{Prior Fracture}

In a post hoc analysis of the subgroup of FIT-II women with FN-BMD T-score between -1.6 and -2.5 (medium ROB), the effect of 4 years of alendronate versus placebo on risk of incident nonvertebral fracture was insignificant overall, and not different between individuals with and without a prior nonvertebral fracture after age 45 (p-value for interaction 0.37) (Appendix D3). ${ }^{40}$

In a post hoc analysis that pooled FIT-I and FIT-II women with FN-BMD T-scores between 1.6 to -2.5 (medium ROB), the effect of four years of alendronate versus placebo on risks of incident clinical and radiographic vertebral fracture, which favored alendronate overall, was not significantly different between individuals with and without a radiographic vertebral fracture at study baseline (p-value for interactions 0.44 and 0.54, respectively) (Appendix D3). ${ }^{39}$ 


\section{Combined Baseline BMD and Prevalent Radiographic Vertebral Fracture Status}

In a post hoc analysis that pooled data from all FIT-I and FIT-II participants (medium ROB), the effect of four years of alendronate versus placebo on risk of incident fractures was evaluated as a function of baseline osteoporotic status. ${ }^{32}$ Participants were categorized at baseline as either osteoporotic (FN-BMD $\leq-2.5$ or baseline radiographic vertebral fracture) or not osteoporotic (FN-BMD > -2.5 and no baseline radiographic vertebral fracture). In osteoporotic women, alendronate lowered risk of both incident nonvertebral fracture (HR 0.69 [95\% CI 0.58, 0.83]) and incident radiographic vertebral fracture (HR 0.50 [95\% CI 0.39, 0.65]) compared with placebo. However, in nonosteoporotic women, there was no between-treatment difference in risk of either fracture outcome. No test for interaction was reported.

\section{FRAX Score}

In a post hoc analysis that pooled data from all FIT-I and FIT-II participants (medium ROB), the effect of four years of alendronate versus placebo on risk of incident clinical fracture, nonvertebral fracture, major osteoporotic fracture (MOF), and radiographic vertebral fracture were not significantly different between individuals with higher versus lower FRAX scores (Appendix Table D3) ${ }^{36}$ For every 10 percent increase in the FRAX 10-year probability of MOF as calculated with FN-BMD, the p-values for the interaction of FRAX score on the association of treatment assignment and these fracture outcomes were $0.38,0.61,0.42$ and 0.88 , respectively. These p-values indicate that the associations between treatment and fracture did not differ by baseline FRAX score. Results were similar when FRAX scores for 10-year probability of MOF were modeled in tertiles and as $\geq 20$ percent versus $<20$ percent.

\section{Bone Turnover Markers}

One post hoc analysis of pooled data from all FIT-I and FIT-II participants (medium ROB) evaluated whether pretreatment levels of several bone turnover markers modified the effect of four years of alendronate versus placebo on risk of incident fractures. Participants were stratified as osteoporotic (FN-BMD T-score $\leq-2.5$ or prevalent radiographic vertebral fracture) or not osteoporotic (FN-BMD T-score $>-2.5$ and no prevalent radiographic vertebral fracture). ${ }^{32}$

In osteoporotic women, the effect of alendronate versus placebo on risk of incident nonvertebral fracture was significantly greater in women with higher versus lower pretreatment levels of procollagen I intact N-terminal (P1NP) (p for interaction 0.02). However, the effect of treatment on risk of incident nonvertebral fracture did not vary significantly as a function of pretreatment levels of bone-specific alkaline phosphatase (BSAP) or C-terminal telopeptide (CTX) (p for interaction not significant). Further, there were no significant interactions with any of the three bone turnover markers on the effect of treatment group on risk of incident radiographic vertebral fracture.

In nonosteoporotic women, the association between treatment group assignment and risk of either incident nonvertebral fracture or incident radiographic vertebral fracture did not vary as a function of pretreatment levels of P1NP, BSAP or CTX (interactions not significant). 


\section{Zoledronic Acid (Zoledronate)}

\section{Key Points}

\section{Key Question 1}

- In postmenopausal women with osteopenia in at least one hip (BMD T-score between 1.0 to -2.5) (a minority of women met at least one criterion for osteoporosis), between zoledronate for 6 years versus placebo:

o Zoledronate was associated with a lower risk of incident clinical fractures (moderate SOE), incident nonvertebral fractures (high SOE), and incident clinical vertebral fractures (moderate SOE).

o There appeared to be no difference in risk of incident hip fractures (low SOE).

\section{Key Question 2}

- Evidence was insufficient to draw conclusions about whether differences in risk of incident fractures between long-term zoledronate and placebo varied as a function of patient, bone or drug characteristics.

\section{Eligible Studies}

One eligible publication of one RCT compared long-term zoledronate treatment versus placebo and reported on risk of incident fracture (Table 7). ${ }^{57}$ Details of this study with low ROB were extracted in evidence tables, summary SOE assessments (Appendix D), and ROB assessments (Appendix C).

This single-site RCT randomized 2000 postmenopausal women aged $\geq 65$ years to zoledronate $5 \mathrm{mg}$ intravenously every 18 months for 6 years versus injections with saline placebo. Women were eligible if they had osteopenia at either hip (T-score between -1.0 and 2.5), even if they had osteoporosis $(\mathrm{T}<-2.5)$ at the other hip or spine (8\% of the participants) or had a baseline radiographic vertebral fracture (13\% of participants). Women were excluded if their LS-BMD T-score was worse than -3.0.

Table 7. Baseline characteristics of the zoledronate trial versus placebo

\begin{tabular}{|l|l|}
\hline Characteristic & Mean or \% \\
\hline Number randomized & 2000 \\
\hline Age, mean years & 71 \\
\hline Gender, woman & $100 \%$ \\
\hline Race, white (European) & $95 \%$ \\
\hline Race, East Asian & $2 \%$ \\
\hline Women with a T-score <-2.5 (considered osteoporotic) & $8 \%$ \\
\hline Mean FN-BMD T-score & -1.64 \\
\hline Mean LS-BMD T-score & -0.89 \\
\hline Mean hip BMD T-score* & -1.26 \\
\hline
\end{tabular}




\begin{tabular}{|l|l|}
\hline History of nonvertebral fracture since age $\geq 45 \mathrm{yr}$ & $24 \%$ \\
\hline Prevalent radiographic vertebral fracture & $13 \%$ \\
\hline Comorbid conditions & NR \\
\hline
\end{tabular}

Abbreviations: BMD=bone mineral density; FN=femoral neck; LS =lumbar spine; NR=not reported

*Study did not specify whether reported baseline hip BMD was from total hip or femoral neck.

\section{Outcomes}

\section{Incident Clinical Fractures}

The primary trial endpoint was incident fragility fracture, which it defined as a clinical or radiographic vertebral fracture or any nonvertebral fracture excepting fractures of the toes, metatarsals, metacarpals, fingers, skull, facial bones, or mandible. Incident fractures of primary interest for this review were considered secondary endpoints.

Zoledronate for 6 years versus placebo significantly reduced the risk of incident clinical fracture (16.3\% vs. 21.4\%; HR 0.73 [95\% CI 0.60, 0.90]) (moderate SOE), incident nonvertebral fracture (10.1\% vs. 14.8\%; HR 0.66 [95\% CI 0.51, 0.85]) (high SOE), and incident clinical vertebral fracture (1.4\% vs. 3.4\%; HR 0.41 [95\% CI 0.22, 0.75]) (moderate SOE). Zoledronate did not significantly reduce risk of incident hip fracture compared with placebo (0.8\% vs. $1.2 \%$, HR 0.66 [95\% CI 0.27, 1.16]) (low SOE).

\section{Incident Radiographic Vertebral Fractures}

No information was reported about risk of incident radiographic vertebral fractures.

\section{Change in BMD}

Change in BMD was a secondary endpoint and results were displayed graphically. Compared with placebo, there were significant improvements in BMD with zoledronate over the 6-year treatment period. Mean change from baseline in LS-BMD increased approximately 7 percent with zoledronate versus a 1 percent decrease for placebo. Mean change in TH-BMD improved approximately 4 percent in the zoledronate group compared with a decrease of nearly 4 percent in the placebo group.

\section{Variation in Long-Term Treatment Efficacy as a Function of Patient, Bone, or Osteoporosis Drug Characteristics}

No eligible studies evaluated whether the efficacy of long-term zoledronate versus placebo or other inactive control on risk of incident clinical fracture varied as a function of patient, bone, or drug characteristics. 


\section{Denosumab}

\section{Key Points}

\section{Key Question 1}

- In postmenopausal women with BMD T score $<-1.8$ (includes both osteopenia and osteoporosis), evidence was insufficient to draw conclusions about differences between 4 years of denosumab therapy versus placebo on risk of incident clinical fractures.

- There was no evidence from eligible trials about the efficacy of long-term denosumab versus placebo on risk of incident radiographic vertebral fractures.

\section{Key Question 2}

- Evidence was insufficient to draw conclusions about whether differences in risk of incident fractures between long-term denosumab and placebo or other inactive treatment vary as a function of patient, bone or drug characteristics.

\section{Eligible Studies}

One eligible publication of one study compared long-term denosumab treatment versus placebo, no treatment or a different active treatment, and reported on risk of incident fracture (Table 8).$^{70}$ Details of this study with medium ROB were extracted in evidence tables, summary SOE assessments (Appendix D), and ROB assessments (Appendix C).

This multisite trial randomized 365 postmenopausal women with LS-BMD T-score of -1.8 to -4.0 or proximal femur T-score of -1.8 to -3.5 to placebo $(n=46)$ versus one of seven intravenous denosumab dosing regimens $(n=319)$ for 2 years. These dosing regimens included three that were lower and two that were higher than the current U.S. Food and Drug Administration (FDA) recommended denosumab dosing. After 2 years, individuals were nonrandomly assigned to treatment groups for the following 2 years. Those initially assigned placebo remained assigned to placebo. Five of the groups assigned denosumab, including three of the low dose groups, were assigned to denosumab 60 mg every 6 months for 2 years $(n=231)$, one of the original high dose denosumab groups was switched to placebo for 2 years $(n=47)$, and the last denosumab group was switched to placebo for 1 year before starting denosumab $60 \mathrm{mg}$ every 6 months for 1 year $(n=41)$.

Table 8. Baseline characteristics of the denosumab trial versus placebo

\begin{tabular}{|l|l|}
\hline Characteristic & Mean or \% \\
\hline Number randomized & 365 \\
\hline Age, mean years & 62.4 \\
\hline Gender, woman & $100 \%$ \\
\hline Race, white & $86 \%$ \\
\hline Race, Hispanic & $11 \%$ \\
\hline Mean LS-BMD T-score & -2.16 \\
\hline
\end{tabular}




\begin{tabular}{|l|l|}
\hline Mean hip BMD T-score* & -1.42 \\
\hline History of clinical fracture & NR \\
\hline Comorbid conditions & NR \\
\hline
\end{tabular}

Abbreviations: BMD=bone mineral density; LS-BMD=lumbar spine bone mineral density; NR=not reported

*Study did not specify whether reported baseline hip BMD was from total hip or femoral neck.

\section{Outcomes}

\section{Incident Clinical Fractures}

In this trial, clinical and osteoporotic fractures were considered neither primary or secondary outcomes, but rather were identified during routine adverse event reporting. ${ }^{70}$ It is unclear whether incident fracture reports were confirmed by radiographic reports. Further, incidence of fractures was reported collectively for all women initially assigned to denosumab $(n=314)$, with no separate results provided for those assigned to denosumab for four years, those assigned to denosumab for two years followed by placebo for 2 years, or those assigned to denosumab for 2 years followed by placebo for 1 year and then denosumab again for 1 year. Through 4 years, incident clinical fractures occurred in $10.5 \%$ of 314 individuals originally assigned to denosumab (the discrepancy of this denominator from the 319 the study reported were initially assigned denosumab was not explained) and $10.9 \%$ of those originally assigned placebo (risk ratio [RR] 0.97 [95\% CI 0.40, 2.35]). Incident osteoporotic fracture (any clinical fracture excluding those of the phalanges, face or those caused by severe trauma) occurred in $7.0 \%$ of the participants originally assigned denosumab vs. 8.7\% in the original placebo group (RR 0.81 [95\% CI 0.29, 2.23]). Because of inclusion in the denosumab group of some participants with low initial doses and others who were assigned only to short-term treatment, evidence is insufficient to draw conclusions about the efficacy of long-term use of FDA approved dosing of denosumab versus placebo for prevention of incident fractures.

\section{Incident Radiographic Vertebral Fractures}

No information was reported about risk of incident radiographic vertebral fractures.

\section{Change in BMD}

The primary study endpoint was mean percentage change from baseline in dual-energy $x$-ray absorptiometry (DXA) LS-BMD and TH-BMD. Unlike for fracture outcomes, BMD results were reported separately for participants assigned to denosumab for four years, denosumab for 2 years followed by placebo for 2 years, denosumab for 2 years followed by placebo for one year followed by denosumab retreatment for one year, and placebo for 4 years. Among the original 277 participants in the continuous denosumab and placebo groups, 66 percent $(n=182)$ completed the 4-year trial. Mean change in LS-BMD from baseline ranged from 9.4 percent to 11.8 percent in the continuous denosumab group compared with -2.4 percent for the placebo group (betweengroup $\mathrm{p}<0.001$ ). Mean change in TH-BMD from baseline ranged from 4.0 percent to 6.1 percent in the continuous denosumab group compared with -3.5 percent for the placebo group (betweengroup $\mathrm{p}<0.001)$. 


\section{Variation in Long-Term Treatment Efficacy as a Function of Patient, Bone, or Osteoporosis Drug Characteristics}

No eligible trials evaluated whether the efficacy of long-term denosumab versus placebo or other inactive control on risk of incident fractures varied as a function of patient, bone or drug characteristics. 


\section{Raloxifene}

\section{Key Points}

\section{Key Question 1}

- In postmenopausal women with osteoporosis, between raloxifene treatment and placebo for 4 years:

o There was no significant difference between treatments in risk of incident nonvertebral fracture (high SOE) or incident hip fracture (moderate SOE).

o Raloxifene was associated with a lower risk of incident clinical vertebral fracture (high SOE) and incident radiographic vertebral fracture (high SOE).

- In postmenopausal women with osteoporosis, between raloxifene treatment and placebo for 8 years:

o There was no significant difference between treatments in risk of incident nonvertebral fracture (moderate SOE).

o Evidence was insufficient to draw conclusions about risk of incident hip, incident clinical vertebral or incident radiographic vertebral fractures.

\section{Key Question 2}

- Associations between raloxifene and placebo with risk of incident fractures did not appear to vary as a function of patient, bone, or drug characteristics, though SOE for these associations was not assessed.

\section{Eligible Studies}

Five eligible publications of one unique study compared long-term raloxifene treatment versus placebo and reported on risk of incident fractures (Table 9). ${ }^{72,76,81,84,85}$ All studies had low or medium ROB. Appendix D provides detailed evidence tables and SOE assessments. Summary ROB assessments can be found in Appendix C.

All these publications were from the Multiple Outcomes of Raloxifene Evaluation (MORE) trial and its extension, the Continuing Outcomes Relevant to Evista (CORE) study.

MORE was a multinational RCT that compared raloxifene $60 \mathrm{mg} /$ day and $120 \mathrm{mg} /$ day to placebo in 7705 postmenopausal women with osteoporosis (LS-BMD or FN-BMD T-score $\leq-$ 2.5, or "low BMD” [undefined] with $\geq 1$ moderate or $\geq 2$ mild baseline radiographic vertebral fractures, or $\geq 2$ moderate baseline radiographic vertebral fractures) for 3 years with a 1-year extension phase. ${ }^{9}$ During the extension phase, participants remained blinded to their originally assigned treatment, but were allowed to use other bone-active agents as clinically indicated. For the initial 3-year treatment phase, the primary outcome was incident radiographic vertebral fracture, defined as a new or worsened vertebral deformity on at least two of three types of independent radiologic assessments during scheduled study radiographs. Secondary endpoints included incident clinical vertebral fracture identified on study radiographs performed to evaluate back pain suggestive of fracture, and incident self-reported nonvertebral fracture confirmed by nonstudy radiographic reports.

CORE was a 4-year follow-up to the MORE RCT conducted at 130 of the 180 MORE study sites. ${ }^{82}$ Of the 6511 participants enrolled at those 130 MORE sites, 4011 chose to enroll in 
CORE. This nonrandomized controlled clinical trial assigned individuals who had been randomized to either raloxifene $60 \mathrm{mg} /$ day or $120 \mathrm{mg} /$ day in MORE to take raloxifene 60 $\mathrm{mg}$ /day in CORE. Women who had been randomized to placebo in MORE were assigned to continue placebo in CORE. Most CORE participants had an approximately 1-year interval between the end of the MORE extension and the beginning of CORE, during which they received no study drug. As during the MORE extension phase, CORE participants were allowed to take other bone-active agents as clinically indicated. All 4011 CORE enrollees were followed according to their MORE treatment assignment (raloxifene or placebo), including the 20 percent who did not take study medication during CORE, either by choice or due to contraindication. CORE participants and investigators remained blinded to MORE and CORE treatment assignment. Incident nonvertebral fracture was a secondary endpoint in CORE, and was selfreported by participants and confirmed by radiologic report.

Baseline characteristics for the MORE and CORE populations are described in detail in Appendix Table D2. The four reports from MORE had low ROB, ${ }^{72,76,81,85}$ and the single report from CORE had medium ROB for fracture outcomes but high ROB for BMD outcomes (available in $<10 \%$ of CORE population). ${ }^{84}$

Table 9. Baseline participant characteristics from the MORE trial and CORE extension

\begin{tabular}{|l|l|l|}
\hline Characteristic, mean, or $\%$ & MORE & CORE* $^{*}$ \\
\hline Number enrolled and randomized & 7705 & 4011 \\
\hline Age, mean & 66 & 66 \\
\hline Years post-menopausal & 19 & 18 \\
\hline Gender, woman & $100 \%$ & $100 \%$ \\
\hline Race, white & $96 \%$ & $96 \%$ \\
\hline Mean FN-BMD T-score & -2.33 & -2.32 \\
\hline Mean LS-BMD T-score & -2.57 & -2.59 \\
\hline History of clinical fracture & NR & NR \\
\hline Prevalent radiographic vertebral fracture & $37 \%$ & $36 \%$ \\
\hline Cardiovascular disease & $3 \%$ & $2 \%$ \\
\hline Chronic kidney disease & NR & NR \\
\hline Diabetes & $5 \%$ & $3 \%$ \\
\hline
\end{tabular}

Abbreviations: BMD=bone mineral density; CORE= Continuing Outcomes Relevant to Evista; FN=femoral neck; LS=lumbar spine; MORE=Multiple Outcomes of Raloxifene Evaluation; NR=not reported

*Characteristics available only from MORE baseline

\section{Outcomes}

\section{Incident Clinical Fractures}

In analyses in which results for women allocated to raloxifene $60 \mathrm{mg} / \mathrm{day}$ and $120 \mathrm{mg} / \mathrm{day}$ were pooled and compared with placebo through 4 years of follow-up, there was no difference between treatment groups in risk of incident nonvertebral fracture (12\% vs. 11\%; RR 0.93 [95\% 
CI 0.81, 1.06]) (high SOE) or incident hip fracture (1\% vs. 1\%; RR 0.97 [95\% CI 0.62, 1.52]) (moderate SOE) ${ }^{72,76}$ However, raloxifene $60 \mathrm{mg} /$ day was associated with a reduced four-year risk of incident clinical vertebral fracture versus placebo ( $2 \%$ vs. 4\%; RR 0.58 [95\% CI 0.43, 0.79]) (high SOE) ${ }^{85}$

Through 8 years of follow-up, there was no difference between raloxifene and placebo in incidence of any nonvertebral fracture (HR 1.00 [95\% CI 0.82, 1.21]) (moderate SOE) or in the incidence of a pooled subset of nonvertebral fractures (clavicle, humerus, wrist, pelvis, hip, or lower leg) (HR 1.01 [95\% CI 0.81, 1.26]). ${ }^{84}$

\section{Incident Radiographic Vertebral Fractures}

Through 4 years of follow-up, raloxifene reduced the risk of incident radiographic vertebral fracture versus placebo at both $60 \mathrm{mg} /$ day ( $8 \%$ vs. $12 \%$ as estimated from figure 2; RR 0.64 [95\% CI 0.53, 0.76]) and $120 \mathrm{mg} /$ day doses (7\% vs. 12\%; RR 0.57 [95\% CI 0.48, 0.69]) (high $\mathrm{SOE}) .^{76}$ No data were reported for incident radiographic vertebral fractures through eight years.

\section{Change in BMD}

Changes in LS-BMD and FN-BMD through four years were significantly greater compared with placebo with both raloxifene $60 \mathrm{mg} /$ day (2.6\% for LS-BMD and $2.1 \%$ for FN-BMD) and raloxifene $120 \mathrm{mg}$ /day (2.5\% for LS-BMD and $2.3 \%$ for FN-BMD) ( $<<0.001$ for all comparisons). ${ }^{76}$ No data were reported for BMD changes through 8 years.

\section{Variation in Long-Term Treatment Efficacy as a Function of Patient, Bone, or Osteoporosis Drug Characteristics}

We looked at whether the efficacy of long-term raloxifene therapy versus inactive control on risk of incident fracture varies as a function of patient, bone, and/or drug characteristics. Eligible studies included evidence for the following possible effect modifiers.

\section{Age}

In the CORE extension population, the relative risk for incident nonvertebral fracture between the raloxifene $60 \mathrm{mg} /$ day and placebo groups through 8 years did not differ as a function of participant age at MORE baseline (p for interaction $\geq 0.10$ ).

\section{Baseline BMD}

In the CORE extension population, the relative risk for incident nonvertebral fracture between the raloxifene $60 \mathrm{mg} /$ day and placebo groups through 8 years did not differ as a function of participant BMD (site not specified) at MORE baseline ( $\mathrm{p}$ for interaction $\geq 0.10$ ).

\section{Prior Fracture}

In the MORE population, risks of incident radiographic vertebral fracture and clinical vertebral fracture during 4-year follow-up for raloxifene $60 \mathrm{mg} /$ day $^{76,81,85}$ or raloxifene 120

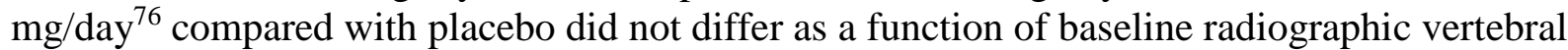
fracture status. During 8-year follow-up from the start of MORE to the end of CORE, the effect of raloxifene versus placebo on risk of incident nonvertebral fracture risk was insignificant overall, insignificant within women who had a baseline radiographic vertebral fracture, and insignificant in women who did not have a baseline radiographic vertebral fracture. Nevertheless, 
the p-value for interaction by baseline radiographic vertebral fracture status was reported at $<0.10{ }^{84}$

\section{Hormone Therapy}

\section{Key Points}

\section{Key Question 1}

- In postmenopausal women with unknown osteoporosis or osteopenia status, and with prior hysterectomy, 7 years of unopposed, high dose oral estrogen versus placebo lowered risk of both incident clinical fractures (high SOE) and incident hip fractures (moderate SOE).

- In postmenopausal women with unknown osteoporosis or osteopenia status, and with an intact uterus, 5.6 years of high dose oral estrogen plus progestin versus placebo lowered risk of both incident clinical fractures (high SOE) and incident hip fractures (moderate SOE).

- In postmenopausal women with past clinical fracture, but unknown BMD or vertebral fracture status, and with prior hysterectomy, 7 years of unopposed, high dose oral estrogen versus placebo lowered risk of both incident clinical fractures (moderate SOE) and incident hip fractures (low SOE).

- In postmenopausal women with past clinical fracture, but unknown BMD or vertebral fracture status, and with an intact uterus, 5.6 years of high dose oral estrogen plus progestin lowered risk of incident clinical fractures (low SOE), but was not associated with lower risk of incident hip fractures (low SOE).

\section{Key Question 2}

- Evidence was insufficient to draw conclusions about whether differences in risk of incident fractures between long-term, high dose unopposed oral estrogen or estrogen plus progestin versus placebo or other inactive treatment vary as a function of patient, bone or drug characteristics.

\section{Eligible Studies}

We identified 3 eligible RCTs that compared hormone therapy versus placebo ${ }^{86,87}$ or nonplacebo control ${ }^{88}$ and reported long-term results for incident fracture (Table 10). Appendix D provides detailed evidence tables and SOE assessments. Summary ROB assessments can be found in Appendix C.

Two of these trials, from the U.S.-based Women's Health Initiative (WHI), had low ROB and enrolled participants without regard to whether they had osteoporosis or osteopenia. The first WHI trial randomized 16,608 postmenopausal women with an intact uterus to an estrogen/progestin combination (oral conjugated estrogen $0.625 \mathrm{mg} /$ day plus medroxyprogesterone $2.5 \mathrm{mg} /$ day) versus placebo for a mean treatment duration of 5.6 years. ${ }^{86}$ Thirty-nine percent of women reported a history of past clinical fracture. Baseline vertebral radiographs were not collected and baseline DXA BMD was measured in 6 percent of participants (1024 women enrolled at 3 of 40 clinical sites). The second WHI trial randomized 10,739 postmenopausal women with a history of hysterectomy to unopposed oral estrogen 
(conjugated estrogen $0.625 \mathrm{mg} /$ day) versus placebo for a mean treatment duration of 7.1 years. Thirty-nine percent of women reported a history of past clinical fracture. Baseline vertebral radiographs were not collected and baseline DXA BMD was measured in 9 percent of participants (938 women enrolled at 3 of 40 clinical sites). Participants in both WHI trials were allowed to co-enroll in an RCT of calcium plus vitamin D supplementation versus placebo. Fracture efficacy results for these two trials were analyzed only for individuals who reported a past clinical fracture and in those with measured baseline BMD. A third, unrelated trial, which had medium ROB, enrolled 72 postmenopausal women with osteoporosis, defined as 1 to 4 baseline radiographic vertebral fractures and DXA LS-BMD T-score $\leq-2.0$. Of the 72 women enrolled in this study, 36 were randomized to etidronate alone or to a combination of etidronate plus conjugated estrogen/progestin. Because etidronate is not FDA approved, these treatment groups were not analyzed. The remaining 36 women were randomized to an oral conjugated estrogen/progestin combination (conjugated estrogen $0.625 \mathrm{mg} /$ day plus norgestrel $150 \mathrm{mg}$ for 12 days each month) versus nonplacebo control for 4 years. All participants received daily supplemental calcium $1000 \mathrm{mg}$ and vitamin D 400 units. The country where the trial was performed was not identified.

Table 10. Baseline characteristics of hormone therapy versus placebo/control RCTs

\begin{tabular}{|c|c|c|c|}
\hline Characteristic & $\begin{array}{c}\text { Cauley } 2003^{86} \\
\text { WHI }\end{array}$ & $\begin{array}{c}\text { Jackson } 2006^{87} \\
\text { WHI }\end{array}$ & $\begin{array}{c}\text { Wimalawansa } \\
1998^{88}\end{array}$ \\
\hline Treatment intervention & $\begin{array}{c}\text { Oral Estrogen }+ \\
\text { Progestin vs. PBO }\end{array}$ & Oral Estrogen vs. PBO & $\begin{array}{l}\text { Oral Estrogen + } \\
\text { Progestin vs. non- } \\
\text { PBO control }\end{array}$ \\
\hline Treatment duration & $5.6 \mathrm{yr}$ & $7.1 \mathrm{yr}$ & $4 \mathrm{yr}$ \\
\hline Number randomized & $\begin{array}{c}16,608 \\
\text { (BMD measured in } \\
6 \%[n=1024])\end{array}$ & $\begin{array}{c}10,739 \text { (BMD measured } \\
\text { in } 9 \%[n=938])\end{array}$ & $36^{*}$ \\
\hline Age, mean years & 63 & 64 & 65 \\
\hline Gender, woman & $100 \%$ & $100 \%$ & $100 \%$ \\
\hline Race, white & $84 \%$ & $75 \%$ & $100 \%$ \\
\hline Time since menopause & $\begin{array}{c}<10 \mathrm{yr}=36 \% \\
10-19 \mathrm{yr}=40 \% \\
\geq 20 \mathrm{yr}=24 \%\end{array}$ & $\begin{array}{c}\text { Mean } 19 \mathrm{yr} \\
<10 \mathrm{yr}=18 \% \\
10-19 \mathrm{yr}=32 \% \\
>20 \mathrm{yr}=50 \%\end{array}$ & Median $15 \mathrm{yr}$ \\
\hline Mean LS-BMD T-score & -1.3 & -1.2 & -2.1 \\
\hline Mean FN-BMD T-score & NR & NR & -1.6 \\
\hline Mean TH-BMD T-score & -0.9 & -0.8 & $\overline{N R}$ \\
\hline History of clinical fracture & $39 \%$ & $39 \%$ & NR \\
\hline $\begin{array}{l}\text { Number of baseline RVF per } \\
\text { participant }\end{array}$ & $\overline{N R}$ & NR & 2.3 \\
\hline Cardiovascular disease & $\begin{array}{c}\mathrm{MI}=2 \% \\
\text { Stroke }=0.8 \%\end{array}$ & $\begin{array}{c}\mathrm{MI}=3 \% \\
\text { Stroke }=1.6 \%\end{array}$ & NR \\
\hline Chronic kidney disease & NR & NR & NR \\
\hline Diabetes & $4 \% 0^{93}$ & $8 \%{ }^{93}$ & NR \\
\hline
\end{tabular}

Abbreviations: BMD=bone mineral density; FN=femoral neck; LS=lumbar spine; MI=myocardial infarction; NR=not reported; PBO=placebo; RCT=randomized controlled trial; RVF=radiographic vertebral fractures; TH=total hip; WHI=Women's Health Initiative

*Excludes the 36 women randomized to etidronate or to combined etidronate plus estrogen/progestin. 


\section{Outcomes}

\section{Incident Clinical Fractures}

In the first WHI trial, in women with unknown osteoporosis or osteopenia status and an intact uterus, those randomized to oral estrogen/progestin for 5.6 years versus placebo had a lower risk of both incident clinical fracture (8.6\% vs. 11.1\%; HR=0.76 [95\% CI 0.69, 0.83]) (high SOE) and incident hip fracture ( $0.61 \%$ vs. $0.90 \%$; HR $=0.67$ [95\% CI 0.47, 0.96]) (moderate SOE). Among the subset of women with a history of prior clinical fracture, those randomized to estrogen/progestin versus placebo had a lower risk of incident clinical fracture (11\% vs. $14 \%$; $\mathrm{HR}=0.78$ [95\% CI 0.68, 0.91]) (low SOE), but no statistically significant difference in risk of incident hip fracture (1.1\% vs. $1.4 \%$; HR $=0.77$ [95\% CI 0.48, 1.22]) (low SOE) ${ }^{86}$ In the subset of women who completed baseline BMD testing and were found to have osteoporosis by baseline DXA BMD T-score $<-2.5$ at any hip or vertebral site, evidence was insufficient to draw conclusions about a difference in risk of any incident clinical fracture between the estrogen/progestin and placebo groups ( $\mathrm{HR}=0.53$ [95\% CI 0.25, 1.10]).

In the second WHI trial, in women with unknown osteoporosis or osteopenia status and a past hysterectomy, those randomized to oral estrogen for 7.1 years versus placebo had a lower risk of both incident clinical fracture (10\% vs. 14\%; HR=0.71 [95\% CI 0.64, 0.80]) (high SOE) and incident hip fracture ( $0.87 \%$ vs. $1.3 \%$; HR $=0.65$ [95\% CI 0.45, 0.94]) (moderate SOE). Among the subset of women with a history of prior clinical fracture (36\% of participants), those randomized to estrogen versus placebo for 7.1 years had a lower risk of both incident clinical fracture (14\% vs. 19\%; HR=0.73 [95\% CI 0.62, 0.86]) (low SOE) and incident hip fracture $\left(1.0 \%\right.$ vs. $1.9 \%$; HR $=0.55$ [95\% CI 0.32, 0.94]) (low SOE) ${ }^{87}$ In the subset of women who completed baseline BMD testing and were found to have osteoporosis $(n=53)$ or osteopenia $(\mathrm{n}=363)$, evidence was insufficient to draw conclusions about differences in risk of any incident clinical fracture between treatment groups ( $\mathrm{HR}=0.83$ [95\% CI 0.17, 3.91] and $\mathrm{HR}=0.83$ [95\% CI $0.49,1.40]$, respectively).

In the small non-WHI trial, incident fractures were reported as secondary endpoints. ${ }^{88}$ Over its 4-year trial period, one participant each in the estrogen/progestin and control groups had an incident nonvertebral fracture ( $7 \%$ vs. $7 \%$; RR=0.93 [95\% CI 0.06, 13.5]) (insufficient SOE).

\section{Incident Radiographic Vertebral Fractures}

Results for incident radiographic vertebral fractures were not reported in the WHI trials. In the non-WHI trial, incident radiographic vertebral fractures occurred in 13 percent of women randomized to estrogen/progestin versus 36 percent in the control group (RR=0.37 [95\% CI 0.09, 1.62]) (insufficient SOE).

\section{Change in BMD}

In both WHI trials, change in BMD was a secondary endpoint, available only in the small minority of women with BMD measurements $(<10 \%)$. Results for change in BMD between baseline and follow-up were reported together for all women with baseline BMD, but not separately by baseline BMD category (e.g., osteoporosis or osteopenia). Three-year BMD follow-up was $82-83 \%$ in one of these trials ${ }^{87}$ and was not reported in the other trial. ${ }^{86}$ Compared to the placebo groups, individuals in both studies assigned to the hormone groups experienced an approximately $3.5 \%$ greater increase in TH-BMD and 4-5\% greater increase in LS-BMD between baseline and follow-up at 3 years. 
In the small, non-WHI trial, the primary study endpoints were mean percentage change from baseline in DXA LS-BMD and TH-BMD. Among the 36 participants randomized to estrogen/progestin versus placebo, 81 percent $(n=29)$ completed the 4-year trial. Participants randomized to estrogen/progestin had a statistically significantly larger increase in LS-BMD during the 4-year follow-up period compared with the control, 7 percent vs. -2.5 percent respectively (mean difference [MD] 9.5\% [95\% CI 7.0, 12.0]). Results also favored the estrogen/progestin group for change in TH-BMD (4.8\% vs. -4.4\%; MD 9.2\% [95\% CI 6.8, 11.6]).

\section{Variation in Long-Term Treatment Efficacy as a Function of Patient, Bone, or Osteoporosis Drug Characteristics}

In both WHI trials, the effect of treatment assignment between hormone therapy and placebo on incident fracture risk in the women with unknown osteoporosis or osteopenia status was evaluated as a function of different patient characteristics in more than 40 tests for interaction.

In both trials, the effects of treatment assignment on risk of incident clinical fracture and incident hip fracture were evaluated as a function of age, years since menopause, body mass index, smoking status, fall history, total calcium intake, parental fracture history, history of prior hormone therapy, race/ethnicity, fracture history, and summary fracture risk score. In the trial that compared estrogen/progestin versus placebo, results also were examined as a function of osteoporotic BMD in the small subset of participants with available baseline BMD measures. In the trial that compared unopposed estrogen versus placebo, results also were examined as a function of history of oophorectomy.

Nearly all tests of interaction were not statistically significant ( $>>0.05)$, with the following exceptions: the reduction in risk of incident hip fracture with estrogen/progestin versus placebo appeared greater in women with total calcium intakes of more than $1200 \mathrm{mg} /$ day compared to lower intakes $(\mathrm{p}=0.02)$; the reduction in risk of incident hip fracture with unopposed estrogen versus placebo appeared greater in women at least 20 years since menopause compared to women with fewer years since menopause $(p=0.05)$; the reduction in risk of incident clinical fracture with unopposed estrogen versus placebo appeared greater in women aged 60-69 and 7079 compared with women 50-59 years $(\mathrm{p}=0.03)$, and greater in women with high or moderate versus low baseline summary fracture risk scores $(\mathrm{p}=0.04)$. In addition, reduction in risk of incident clinical fracture with unopposed estrogen versus placebo, though not statistically significantly different by years since menopause $(\mathrm{p}=0.06)$, showed a trend similar to that for hip fractures, with risk appearing numerically lower in women 60-69 and 70-79 compared to that in women aged 50-59 years. 


\section{Other Drugs Investigated for Review}

\section{Ibandronate}

No eligible studies of long-term treatment with ibandronate versus inactive control (e.g., placebo, no treatment) or a different active treatment reported on risk of incident fracture or on whether risk of incident fracture between treatments varied as a function of patient, bone, or ibandronate or control characteristics.

\section{Risedronate}

Two eligible studies of long-term treatment with risedronate versus inactive control (e.g., placebo, no treatment) or a different active treatment reported on risk of incident fracture. ${ }^{59,60}$ These studies were rated high ROB and only limited data were extracted (Appendix Table D7). We identified no eligible studies that compared risedronate with control, and assessed whether risk of incident fracture between treatments varied as a function of patient, bone, or risedronate or control characteristics. 


\section{Chapter 5. Harms of Long-Term Drug Treatment}

Chapter 5 reviews the evidence from eligible studies that addresses Key Questions 3 and 4. Key Question 3 addresses the harms of long-term ( $>3$ years) osteoporosis drug treatment versus placebo, no treatment or a different active treatment. Key Question 4 addresses whether risk of harms varies by patient, bone, or drug characteristics.

We report the literature on the effect of continuing osteoporosis drug treatment versus discontinuing it on risk of harms in the drug holiday section of the report (Chapter 7, Key Question 7), regardless of the duration of drug treatment before allocation to continuation or discontinuation.

This chapter is organized by drug, with Key Questions 3 and 4 discussed sequentially within each drug subsection. Drug versus placebo or no treatment comparisons are listed first, followed by any comparisons of drug versus another active drug treatment. We provide an overview at the start of specific drug subsections when the literature base was complex. Summary statements about the included studies are below; individual study details are provided in Appendix D.

\section{Alendronate}

\section{Key Points}

\section{Key Question 3}

- In randomized controlled trials (RCT):

o In postmenopausal women with femoral neck (FN) bone mineral density (BMD)

T-score $\leq-1.6$ and without baseline vertebral fracture, between alendronate and placebo for 4 years:

- There was no difference in risk of upper gastrointestinal (GI) events, mortality (low strength of evidence [SOE]), or hospitalization due to adverse events (low SOE).

o In postmenopausal women with FN-BMD T-score $\leq-1.6$, between alendronate and placebo for 3 to 4.5 years:

- Evidence was insufficient to draw conclusions about differences in risk of atypical femoral fracture (AFF) with confirmed radiologic features or subtrochanteric or femoral shaft fractures without confirmed radiologic AFF features.

- In observational studies:

o Between alendronate and no osteoporosis medication use for up to 11 years:

- Risk of subtrochanteric or femoral shaft fracture without radiologic confirmation of AFF features appeared higher with alendronate versus no osteoporosis drug prescription in one cohort study (low SOE), but evidence was insufficient to draw conclusions about differences in risk between alendronate and no treatment in a second cohort study.

- Risk of osteonecrosis of the jaw (ONJ) appeared higher with alendronate versus no osteoporosis drug prescription in one cohort study (low SOE).

- There appeared to be no difference in risk of atrial fibrillation, though SOE was not graded. 
o Evidence was insufficient to draw conclusions about differences in risk of ONJ with alendronate versus raloxifene or calcitonin.

\section{Key Question 4}

- In postmenopausal women with FN-BMD T-score $\leq-1.6$, between alendronate and placebo for 3.2 to 4 years:

o Risk of upper gastrointestinal adverse events did not appear to differ as a function of age, prior history of upper GI tract disease, renal function or use of nonsteroidal anti-inflammatory drugs (NSAIDs).

- Risk of coronary heart disease, cerebrovascular disease, cancer and mortality did not appear to differ as a function of renal function.

- We found no evidence about whether risk of AFF, subtrochanteric or femoral shaft fracture without confirmed radiologic AFF features, ONJ, or atrial fibrillation between alendronate and control varies as a function of patient, bone or osteoporosis drug treatment factors.

\section{Eligible Studies}

Fourteen eligible publications of eleven unique studies compared long-term alendronate treatment versus placebo, no treatment or a different active treatment, and reported on risk of harms. We rated four of these studies as having high $\mathrm{ROB}^{47,50-52}$ and extracted only limited data (Appendix Table D7). For the remaining ten studies with low or medium ROB, there were four eligible publications from one unique RCT with low ${ }^{35,43}$ and medium ${ }^{31,38} \mathrm{ROB}$, respectively, and six observational studies with medium ROB. ${ }^{44-46,89-91}$ We extracted additional information from these studies in evidence tables, SOE summary tables (Appendix D) and ROB summary tables (Appendix C). Summary data of rare harms from controlled observational studies can be found in Table 11.

The eligible RCT reports were based on the Fracture Intervention Trial (FIT), which excluded women with recent or recurrent GI ulcers, significant upper GI tract bleeding in the past five years, esophageal or gastric varices, or use of daily medication for dyspepsia. In the FIT-II RCT, risks of mortality, any adverse event leading to hospitalization, and upper GI events (included abdominal pain, esophagitis, esophageal ulcer, acid regurgitation/reflux, and other) were compared between women randomized for 4 years to alendronate versus placebo. ${ }^{35}$ In a second FIT report, a pooled analysis of FIT-I and FIT-II participants, risk was compared between alendronate and placebo for a mean follow-up of 3.8 years for upper GI tract adverse events, diagnoses and symptoms. ${ }^{31}$ The third FIT report pooled participants from FIT-I and FIT-II and estimated risk of incident subtrochanteric or femoral shaft fracture. ${ }^{43}$ Self-reported fractures were centrally confirmed by review of community radiographic reports. For reports noting subtrochanteric or femoral shaft fractures, outside radiographs were sought but rarely available. Descriptions in the radiographic reports of atypical features were recorded. Pathologic fractures, periprosthetic fractures, and fractures due to excess trauma were excluded. Another pooled analysis of FIT-I and FIT-II participants reported on risk of GI events, coronary heart disease, cerebrovascular disease, cancer and mortality as a function of baseline renal function. ${ }^{38}$ None of the reports provided information about the risk of ONJ or atrial fibrillation.

Four observational studies used Danish national data to examine risk of subtrochanteric and femoral shaft fracture, ${ }^{44,89}$ inflammatory jaw events possibly attributable to $\mathrm{ONJ},{ }^{91}$ or atrial fibrillation ${ }^{90}$ with long-term alendronate. In the earliest of these studies, among men and women 
$\geq 51$ years of age who experienced a hospital treated nonhip fracture, 178 individuals adherent with alendronate for $>6$ years (i.e., medication possession ratio $>80 \%$ ) were matched 2:1 with age, sex, and same index fracture site controls who were not exposed to bisphosphonate treatment. ${ }^{44}$ Subtrochanteric and femoral shaft fractures were identified by International Classification of Diseases (ICD)-10 diagnosis codes, without radiologic review for AFF features or exclusions for excess trauma or cancer. The other three Danish studies evaluated 55,090 patients who received an alendronate prescription during an 11-year period. For each exposed participant, three age and gender matched controls were randomly selected from the entire Danish population who had not been prescribed an osteoporosis medication. Mean treatment follow-up was 3.8 years for all osteoporosis medications collectively. Though time on treatment was not reported for alendronate specifically, 53 percent of treated patients were prescribed alendronate (another $38 \%$ were prescribed etidronate). One of these studies reported on risk of any inflammatory jaw event as defined by diagnostic codes that included osteomyelitis, osteitis, osteoradionecrosis, periostitis and sequestrum, without a requirement that coding exceeded one encounter, and without review of radiology and pathology records. ${ }^{91}$ In the second of these reports, the primary outcome was subtrochanteric or femoral shaft fracture as defined by ICD8 and ICD10 diagnostic codes, without review of radiology records and without exclusions for trauma or cancer. ${ }^{89}$ For the third report, the primary outcome was atrial fibrillation or flutter defined as a recorded incident leading to hospitalization or an outpatient contact. ${ }^{90}$ Participant mean age was 71 years and no information was reported about baseline BMD or prior fracture history.

One retrospective cohort study conducted in Taiwan reported risk of ONJ in 6485 osteoporotic women treated with alendronate versus that in 1869 osteoporotic women using raloxifene. ${ }^{45} \mathrm{ONJ}$ was defined as exposed maxillofacial bone for more than 8 weeks meeting the following criteria: qualifying ICD-9 diagnostic code, at least one dentistry visit claim, radiographic documentation of ill-defined lytic lesions of the jawbone, and pathology records documenting sequestra or osteomyelitis. Participants were excluded for having head and neck cancer before or during the alendronate treatment period, a history of radiotherapy to the jaws, or previously receiving other antiresorptive treatment. Alendronate treatment duration ranged up to 11 years, but mean duration was about 1.8 years, including a mean duration of about 4 years in those diagnosed with ONJ. Women were 50 years of age or older (mean 70 years). Sixteen percent of participants in both treatment groups had diabetes. Women treated with alendronate more often were chronic users of glucocorticoids. The study did not report baseline BMD or prior fracture history.

A second retrospective cohort study conducted in Taiwan reported risk of ONJ for alendronate versus raloxifene or calcitonin in men and women who had been started on osteoporosis medication following a recent clinical vertebral or hip fracture. ${ }^{46} \mathrm{ONJ}$ was defined as meeting the following criteria: qualifying ICD-9 diagnostic code for at least three consecutive visits over at least 8 weeks, and documentation of receipt of appropriate broad-spectrum oral antibiotics. Participants were excluded for a history of radiation to the jaws. Radiology and pathology records were not reviewed. Participants were 50 years of age or older (mean 74 years). About 24 percent had diabetes. In the original cohort $(n=43,645)$, the alendronate treated group differed from the raloxifene/calcitonin treated group in less often being female (79\% vs. 87\%), more often having had a hip fracture as their pre-treatment fracture (29\% vs. $15 \%$ ), and more often having baseline gingival or periodontal disease (27\% vs. $23 \%)$. The study did not report 
baseline BMD. Baseline prognostic variables were similar between treatment groups in the propensity-matched cohort $(\mathrm{n}=32,006)$.

Baseline characteristics for both observational studies are reported in Appendix Table D1.

\section{Outcomes}

\section{Serious Adverse Events}

In the FIT-II RCT, although serious adverse events as defined by the U.S. Food and Drug Administration (FDA) were not reported, there was no difference between women assigned to long-term alendronate versus placebo in risk of mortality $(1.7 \%$ vs. $1.8 \%$; hazard ratio [HR] 0.92 [95\% confidence interval [CI] 0.59, 1.45]) (low SOE) or adverse events leading to hospitalization (29\% vs. 27\%; relative hazard [RH] 1.09 [95\% CI 0.98, 1.22]) (low SOE). ${ }^{35}$

\section{Upper GI Tract Adverse Events}

In the FIT-II RCT, alendronate for 4.2 years versus placebo did not significantly increase risk of any upper GI tract event (47.2\% vs. 47.5\%; RH 1.00 [95\% CI 0.92, 1.09]), or of abdominal pain, esophageal ulcer, or acid regurgitation/reflux (Appendix D4) ${ }^{35}$ Risk of esophagitis was $0.9 \%$ in the alendronate group versus $0.5 \%$ in the placebo group (RH 1.90 [95\% CI 0.90, 4.26]).

In a pooled analysis of FIT-I and FIT-II data, alendronate was not associated with an increased risk of any upper GI tract adverse event (47.5\% vs. 46.2\%; RR 1.02 [95\% CI 0.95, $1.10]) .{ }^{31}$ There also was no between group difference in risk of dyspepsia (18.2\% vs. 19.1\%; RR 0.94 [95\% CI 0.84, 1.05]), abdominal pain, nausea, vomiting, or serious upper GI tract adverse event (RR 1.10 [95\% CI 0.77, 1.56]). (Appendix Table D4).

\section{Subtrochanteric and Femoral Shaft Fractures}

No eligible long-term alendronate study reported information about risk of radiologically confirmed AFF. Three eligible studies provided conflicting evidence about the association of long-term alendronate use with risk of incident subtrochanteric or femoral shaft fracture. ${ }^{43,44,89}$ In a secondary analysis of pooled FIT-I and FIT-II RCT data, one woman each in the alendronate and placebo groups had a subtrochanteric or femoral shaft fracture $(0.031 \%$ vs. $0.031 \%$; HR 1.03 [95\% CI 0.06, 16.46] (insufficient SOE). ${ }^{43}$ One retrospective cohort study reported five cases of subtrochanteric or femoral shaft fracture in the combined groups of patients highly adherent with alendronate $>6$ years $(n=178)$ and a no treatment group $(n=356)$, and provided insufficient strength evidence to draw conclusions about a difference in risk between groups (HR 1.37 [95\%

CI 0.22, 8.62]). ${ }^{44} \mathrm{~A}$ second retrospective cohort study reported that alendronate prescription for a mean of approximately 3.8 years compared with no osteoporosis drug prescription was associated with an increased risk of subsequent subtrochanteric fracture $(0.017 \%$ vs. $0.006 \%$; HR 2.41 [95\% CI 1.78, 3.27) (low SOE) and femoral shaft fracture (0.012\% vs. 0.003\%; HR 2.90 $[95 \%$ CI $1.97,4.26]$ ) (low SOE). ${ }^{89}$

\section{Osteonecrosis of the Jaw-Defined by Diagnostic Codes Plus Clinical Confirmation}

One retrospective cohort study reported higher risk with alendronate versus raloxifene for ONJ defined by diagnostic codes for an inflammatory jaw condition, plus radiologic and pathologic confirmation (HR 7.42; 95\% CI 1.02, 54.09) (low SOE). ${ }^{45}$ A second study, that defined ONJ based on recurrent and persistent plus receipt of appropriate antibiotics reported 
that risk for alendronate versus raloxifene or calcitonin was HR 0.86 (95\% CI 0.44, 1.69) (insufficient SOE) ${ }^{46}$. Because the higher adjusted incidence rates in the alendronate group $(0.15 \%)$ compared with the raloxifene-calcitonin group $(0.08 \%)$ suggested a risk estimated higher than 1.0, we manually recalculated the estimate of effect and found RR 1.20 (95\% CI 0.59, 2.56). Authors were contacted for clarification, but did not reply.

\section{Osteonecrosis of the Jaw-Defined by Diagnostic Codes Only}

One retrospective cohort study reported an increased risk of any inflammatory jaw event defined only by diagnostic codes in participants exposed to approximately 3.8 years of alendronate exposure versus no osteoporosis drug prescription (0.03\% vs. 0.01\%; HR 3.15 [95\% CI 1.44, 6.87]) (low SOE). ${ }^{91}$

\section{Atrial Fibrillation}

One eligible study reported that alendronate prescription for a mean of approximately 3.8 years compared with no osteoporosis drug prescription was not associated with risk of subsequent atrial fibrillation or flutter (1.3\% vs. 1.0\%; HR 1.04 [95\% CI 0.98, 1.10]). ${ }^{90}$ 
Table 11. Estimated risk of ST/FS fracture, ${ }^{*}$ ONJ, or atrial fibrillation with alendronate use from controlled observational studies

\begin{tabular}{|c|c|c|c|c|c|c|c|}
\hline Outcome & $\begin{array}{c}\text { Author, } \\
\text { Year } \\
\text { Country } \\
\text { Risk of bias }\end{array}$ & $\begin{array}{c}\text { Study } \\
\text { Design } \\
\text { (n=\# Cases; } \\
\mathrm{N}=\# \\
\text { Evaluated) }\end{array}$ & $\begin{array}{c}\text { Population } \\
\text { Age (mean) } \\
\text { Gender (\%) } \\
\text { Comorbid } \\
\text { Conditions } \\
(\%) \\
\end{array}$ & $\begin{array}{l}\text { ST/FS fracture, ONJ, or } \\
\text { Atrial Fibrillation Case } \\
\text { Definitions }\end{array}$ & $\begin{array}{l}\text { Alendronate } \\
\text { Treatment } \\
\text { Duration }\end{array}$ & $\begin{array}{l}\text { Treatment Control } \\
\text { Group }\end{array}$ & $\begin{array}{c}\text { Results for Alendronate vs. } \\
\text { Control }(95 \% \mathrm{Cl}) \\
\text { Model Covariates }\end{array}$ \\
\hline \multirow[t]{2}{*}{ ST/FS fracture } & $\begin{array}{l}\text { Abrahamsen } \\
2009^{44} \\
\text { Denmark } \\
\text { Medium }\end{array}$ & $\begin{array}{l}\text { Retrospective } \\
\text { cohort } \\
n=5 \\
N=534\end{array}$ & $\begin{array}{l}\text { Age } 73 \\
\text { Female } 90 \% \\
\text { Comorbid } \\
\text { conditions } \\
\text { NR }\end{array}$ & $\begin{array}{l}\text { ST/FS cases: ST/FS } \\
\text { fractures defined by ICD10 } \\
\text { diagnostic codes. No } \\
\text { radiology records were } \\
\text { reviewed and there were no } \\
\text { exclusions for trauma or } \\
\text { cancer. }\end{array}$ & $\begin{array}{l}>6 \text { years (mean } \\
\text { not reported) }\end{array}$ & $\begin{array}{l}\text { No prescription for } \\
\text { bisphosphonate }\end{array}$ & $\begin{array}{l}\text { HR=1.37 }(0.22,8.62) \\
\text { Age, sex, number of } \\
\text { comedications, oral glucocorticoid } \\
\text { use, Charlson comorbidity index. }\end{array}$ \\
\hline & $\begin{array}{l}\text { Vestergaard } \\
2011^{89} \\
\text { Denmark } \\
\text { Medium }\end{array}$ & $\begin{array}{l}\text { Retrospective } \\
\text { cohort } \\
n=309 \\
N=220,360\end{array}$ & $\begin{array}{l}\text { Age } 71 \\
\text { Female 85\% } \\
\text { CHF 2\% } \\
\text { DM 2\% } \\
\text { HTN 34\% }\end{array}$ & $\begin{array}{l}\text { ST/FS cases: ST/FS } \\
\text { fractures defined by ICD8 } \\
\text { and ICD10 diagnostic codes. } \\
\text { No radiology records were } \\
\text { reviewed and there were no } \\
\text { exclusions for trauma or } \\
\text { cancer. }\end{array}$ & $\begin{array}{l}\text { Range } 0-11 \text { years } \\
\text { Mean duration } \\
\sim 3.8 \text { years }\end{array}$ & $\begin{array}{l}\text { No prescription for } \\
\text { osteoporosis drug }\end{array}$ & $\begin{array}{l}\text { Subtrochanteric: } 0.17 \% \text { alendronate } \\
\text { vs. 0.06\% control; HR 2.41 (1.78, } \\
\text { 3.27); ARR 0.11 (0.08, 0.15) } \\
\text { Femoral shaft: } 0.12 \% \text { alendronate } \\
\text { vs. 0.03\% control; HR 2.90 (1.97, } \\
\text { 4.26); ARR } 0.09 \text { (0.06, 0.12) } \\
\text { ST/FS: } 0.29 \% \text { alendronate vs. } \\
0.09 \% \text { control; ARR } 0.20 \text { (0.15, } \\
0.25) \\
\text { History of past fracture, systemic } \\
\text { hormone use, systemic } \\
\text { corticosteroid use, alcoholism }\end{array}$ \\
\hline \multirow[t]{2}{*}{$\mathrm{ONJ}$} & $\begin{array}{l}\text { Vestergaard } \\
2012^{91} \\
\text { Denmark } \\
\text { Medium }\end{array}$ & $\begin{array}{l}\text { Retrospective } \\
\text { cohort } \\
n=28 \\
N=220,360\end{array}$ & $\begin{array}{l}\text { Age 71 } \\
\text { Female 85\% } \\
\text { CHF 2\% } \\
\text { DM 2\% } \\
\text { HTN 34\% }\end{array}$ & $\begin{array}{l}\text { ONJ case: Diagnosis codes } \\
\text { for inflammatory jaw event } \\
\text { including osteomyelitis, } \\
\text { osteitis, osteoradionecrosis, } \\
\text { periostitis and sequestrum. } \\
\text { Radiology and pathology } \\
\text { records not reviewed. }\end{array}$ & $\begin{array}{l}\text { Range } 0-11 \text { years } \\
\text { Mean duration } \\
\sim 3.8 \text { years }\end{array}$ & $\begin{array}{l}\text { No prescription for } \\
\text { osteoporosis drug }\end{array}$ & $\begin{array}{l}0.03 \% \text { alendronate vs. } 0.01 \% \\
\text { control; HR } 3.15 \text { (1.44, } 6.87) \\
\text { Diabetes, Sjogren's syndrome, } \\
\text { chemotherapy, irradiation, } \\
\text { alcoholism, use of systemic } \\
\text { corticosteroids, prior jaw events }\end{array}$ \\
\hline & $\begin{array}{l}\text { Chiu } 2014^{45} \\
\text { Taiwan } \\
\text { Medium }\end{array}$ & $\begin{array}{l}\text { Retrospective } \\
\text { cohort } \\
n=40 \\
N=8,354\end{array}$ & $\begin{array}{l}\text { Age } 72 \\
\text { Female 88\% } \\
\text { CKD 2\% } \\
\text { DM 16\% } \\
\text { HTN 44\% }\end{array}$ & $\begin{array}{l}\text { ONJ case: ICD9 diagnosis } \\
\text { codes for inflammatory } \\
\text { dental condition, claim for } \geq 1 \\
\text { dentistry visit, radiographic } \\
\text { confirmation, pathologic } \\
\text { confirmation. }\end{array}$ & $\begin{array}{l}\text { Range } 0-11 \text { years } \\
\text { Mean duration } \\
\sim 1.8 \text { years }(\sim 4.0 \\
\text { years in ONJ } \\
\text { group) }\end{array}$ & Raloxifene & $\begin{array}{l}\text { HR } 7.42(1.02,54.09) \\
\text { Reported "adjusting for other } \\
\text { possible contributions from other } \\
\text { variables," but specific covariates } \\
\text { included in the model are unclear. }\end{array}$ \\
\hline
\end{tabular}




\begin{tabular}{|c|c|c|c|c|c|c|c|}
\hline Outcome & $\begin{array}{c}\text { Author, } \\
\text { Year } \\
\text { Country } \\
\text { Risk of bias }\end{array}$ & $\begin{array}{c}\text { Study } \\
\text { Design } \\
\text { (n=\# Cases; } \\
\mathrm{N}=\# \\
\text { Evaluated) }\end{array}$ & $\begin{array}{c}\text { Population } \\
\text { Age (mean) } \\
\text { Gender (\%) } \\
\text { Comorbid } \\
\text { Conditions } \\
(\%) \\
\end{array}$ & $\begin{array}{c}\text { ST/FS fracture, ONJ, or } \\
\text { Atrial Fibrillation Case } \\
\text { Definitions }\end{array}$ & $\begin{array}{l}\text { Alendronate } \\
\text { Treatment } \\
\text { Duration }\end{array}$ & $\begin{array}{c}\text { Treatment Control } \\
\text { Group }\end{array}$ & $\begin{array}{l}\text { Results for Alendronate vs. } \\
\text { Control }(95 \% \mathrm{Cl}) \\
\text { Model Covariates }\end{array}$ \\
\hline & $\begin{array}{l}\text { Lin } 2014^{46} \\
\text { Taiwan } \\
\text { Medium }\end{array}$ & $\begin{array}{l}\text { Retrospective } \\
\text { cohort } \\
\text { Unmatched } \\
\text { cohort: } \\
\mathrm{n}=46 \\
\mathrm{~N}=43,645 \\
\text { Propensity } \\
\text { matched } \\
\text { cohort: } \\
\mathrm{n}=37 \\
\mathrm{~N}=32,006\end{array}$ & $\begin{array}{l}\text { Age } 74 \\
\text { Female 84\% } \\
\text { CKD 3\% } \\
\text { DM 24\% }\end{array}$ & $\begin{array}{l}\text { ONJ case: ICD9 diagnostic } \\
\text { codes from at least } 3 \\
\text { consecutive visits over at } \\
\text { least } 8 \text { weeks, and receipt of } \\
\text { appropriate broad-spectrum } \\
\text { antibiotics. }\end{array}$ & $\begin{array}{l}\text { Up to } 6 \text { years } \\
\text { (mean and } \\
\text { median not } \\
\text { reported) }\end{array}$ & $\begin{array}{l}\text { Non- } \\
\text { bisphosphonate } \\
\text { osteoporosis } \\
\text { medication (e.g. } \\
\text { raloxifene or } \\
\text { calcitonin) }\end{array}$ & $\begin{array}{l}\text { Propensity matched cohort: } 0.15 \% \\
\text { alendronate vs. 0.08\% control; HR } \\
0.86(0.44,1.69) \dagger \\
\text { Age, gender, year, fracture type, } \\
\text { fracture history, fall history, } \\
\text { comorbidities (DM, hyperlipidemia, } \\
\text { pancreatitis, gingival and } \\
\text { periodontal diseases, other } \\
\text { diseases and conditions of teeth } \\
\text { and supporting structures, } \\
\text { dentoalveolar surgery, rheumatoid } \\
\text { arthritis, systemic lupus } \\
\text { erythematosus, renal disease, } \\
\text { HTN, Alzheimer's disease), } \\
\text { and comedications (antiepileptics, } \\
\beta \text {-blockers, benzodiazepines, } \\
\text { glucocorticoids, hormone therapy, } \\
\text { COX-2 agents, SSRI, thyroid } \\
\text { drugs, and sleep/hypnotic agents). }\end{array}$ \\
\hline Atrial fibrillation & $\begin{array}{l}\text { Vestergaard } \\
2010^{90} \\
\text { Denmark } \\
\text { Medium }\end{array}$ & $\begin{array}{l}\text { Retrospective } \\
\text { cohort } \\
n=2,364 \\
N=220,360\end{array}$ & $\begin{array}{l}\text { Age } 71 \\
\text { Female } 85 \% \\
\text { CHF 2\% } \\
\text { DM 2\% } \\
\text { HTN 34\% }\end{array}$ & $\begin{array}{l}\text { Atrial fibrillation case: } \\
\text { recorded incident leading to } \\
\text { hospitalization or an } \\
\text { outpatient contact }\end{array}$ & $\begin{array}{l}\text { Range } 0-11 \text { years } \\
\text { Mean duration } \\
\sim 3.8 \text { years }\end{array}$ & $\begin{array}{l}\text { No prescription for } \\
\text { osteoporosis drug }\end{array}$ & $\begin{array}{l}\text { 1.3\% alendronate vs. } 1.0 \% \text { control; } \\
\text { HR } 1.04(0.98,1.10) \\
\text { Prior atrial fibrillation, heart valve } \\
\text { disease, heart failure, } \\
\text { hyperthyroidism, diuretic use, other } \\
\text { cardiovascular drug use, COPD, } \\
\text { COPD medication use, alcoholism }\end{array}$ \\
\hline
\end{tabular}

Abbreviations: ARR=absolute risk reduction; $\mathrm{CHF}=$ chronic heart failure; $\mathrm{CI}=$ confidence interval; $\mathrm{CKD}=$ chronic kidney disease; $\mathrm{COPD}=\mathrm{chronic}$ obstructive pulmonary disease; $\mathrm{COX}=$ cyclooxygenase; $\mathrm{DM}=$ diabetes mellitus; $\mathrm{HR}=$ adjusted hazard ratio; HTN=hypertension; $\mathrm{ICD}=\mathrm{International} \mathrm{Classification} \mathrm{of} \mathrm{Diseases;} \mathrm{N}=$ number; ONJ=osteonecrosis of the jaw; SSRI=selective serotonin reuptake inhibitors; ST/FS= Subtrochanteric/femoral shaft

*No studies reported results for atypical femoral fractures meeting American Society for Bone and Mineral Reasearch radiographic criteria.

†Because the higher adjusted incidence rates in the alendronate group compared with the raloxifene-calcitonin group (0.15\% vs. $0.08 \%)$ suggested a possibly increased risk, we manually recalculated the estimate of effect and found risk ratio 1.20 (95\% CI 0.59, 2.56). Authors were contacted for clarification, but did not reply. 


\section{Variation in Long-Term Treatment Harms as a Function of Patient, Bone, or Osteoporosis Drug Characteristics}

We looked at whether the effect of long-term alendronate therapy versus control (placebo, no treatment, or active control) on risk of harms varies as a function of patient, bone, and/or drug characteristics. Two eligible studies with medium risk of bias included evidence for the following possible effect modifiers of long-term alendronate treatment harms: ${ }^{31,38}$

\section{Upper Gastrointestinal Tract Adverse Events}

A post hoc pooled analysis of FIT-I and FIT-II data ${ }^{31}$ reported that the risk of alendronate versus placebo on upper GI adverse events did not differ as a function of age, current use of NSAIDs, or history of an upper GI tract disease that was not an exclusion criterion for study participation (e.g., peptic ulcer, reflux esophagitis). No data were provided to support these statements for most upper GI tract adverse events. However, in a manuscript figure, unadjusted rates for esophageal adverse events and gastroduodenal perforations, ulcerations and bleeding appeared visually similar across age categories (55-65, 66-70, 71-75 and 76-85 years). Also, unadjusted RR between alendronate and placebo groups for these outcomes were similar and 95 percent confidence intervals were widely overlapping between strata defined based on prior history of a nonexclusionary upper GI tract disease and current NSAID use (Appendix Table D4). A second post hoc pooled analysis of FIT-I and FIT-II data reported that renal function (estimated glomerular filtration rate [eGFR] $<45 \mathrm{ml} / \mathrm{min}$ vs. $\geq 45 \mathrm{ml} / \mathrm{min}$ based on the Cockcroft Gault formula) did not modify the effect of alendronate versus placebo on risk of GI events, coronary heart disease, cerebrovascular disease, cancer, or mortality. ${ }^{38}$ 


\section{Zoledronic Acid (Zoledronate)}

\section{Key Points}

\section{Key Question 3}

- All data on long-term zoledronate treatment harms were from a single RCT. There were no eligible observational studies that compared long-term zoledronate versus no treatment or another control, let alone any that reported on risk of harms.

- In mostly osteopenic postmenopausal women (one hip BMD T-score -1 to -2.5, but including a minority of women with osteoporosis by BMD or baseline vertebral fracture), between zoledronate and placebo for 6 years:

o There was no difference in risk of serious adverse events (low SOE)

o Evidence was insufficient to draw conclusions about differences in risk of AFF or ONJ (no cases of either AFF or ONJ occurred during the trial).

\section{Key Question 4}

- Evidence was insufficient to draw conclusions about whether differences in risk of harms between long-term zoledronate and placebo or a different active treatment vary as a function of patient, bone or drug characteristics.

\section{Eligible Studies}

One eligible RCT reported on risk of harms of long-term zoledronate treatment versus placebo. ${ }^{57}$ No eligible observational studies addressed risk of harms of long-term zoledronate treatment.

Described in detail previously, the single eligible trial enrolled 2000 postmenopausal women, aged $\geq 65$ years, most of whom had osteopenia (all had a T-score -1.0 to -2.5 in at least one hip). However, some participants met criteria for osteoporosis (8\% had a BMD T-score $<-2.5$ at their other hip or the spine, and $13 \%$ had a baseline radiographic vertebral fracture). Participants were randomized to intravenous zoledronate $5 \mathrm{mg}$ every 18 months $(\mathrm{n}=1000)$ versus placebo $(\mathrm{n}=1000)$. Treatment duration was 6 years. The trial was rated with low ROB. Study details were extracted in evidence tables, a ROB assessment table (Appendix C), and a SOE assessments summary table (Appendix D).

Reports of eligible studies that compared zoledronate continuation versus discontinuation (placebo drug holiday) and reported on risk of harms are reviewed in Chapter 7 of this report.

\section{Outcomes}

\section{Serious Adverse Events}

Risk of any serious adverse event was 40.0 percent with zoledronate treatment versus 44.3 percent with placebo, a result that was not statistically significant (odds ratio [OR] 0.84 [95\% CI $0.70,1.00] ; \mathrm{p}=0.052$ ) (low SOE). 


\section{Cardiovascular Events}

Risk of a cardiovascular event, including sudden death, myocardial infarction, coronaryartery revascularization, or stroke, was not significantly different between women allocated to zoledronate versus placebo (5.3\% vs. 6.9\%; OR 0.76 [95\% CI 0.52, 1.09]). Individually, risk of sudden death, myocardial infarction, or stroke was not statistically significantly different between groups.

\section{Mortality}

Risk of all-cause mortality was not statistically significantly different between the zoledronate and placebo groups (2.7\% vs. $4.1 \%$; OR 0.65 [95\% CI 0.40, 1.05]). Sudden death was reported for 3 women in the zoledronate and one in the placebo group.

\section{Atypical Femoral Fracture}

The trial reported that no cases of AFF occurred in either treatment group.

\section{Osteonecrosis of the Jaw}

The trial reported that no cases of ONJ occurred in either treatment group.

\section{Variation in Long-Term Treatment Harms as a Function of Patient, Bone, or Osteoporosis Drug Characteristics}

We identified no eligible study that compared long-term zoledronate treatment versus placebo, no treatment or a different active treatment, and assessed whether risk of harms varied as a function of patient, bone or drug characteristics. 


\section{Any Bisphosphonate}

\section{Key Points}

\section{Key Question 3}

- All data on harms associated with unspecified bisphosphonate treatment were from observational studies. There were no randomized long-term trials that compared any bisphosphonate versus placebo, let alone any that reported on risk of harms.

- There was higher risk of AFF with confirmed radiologic features with long-term use of any bisphosphonate versus no bisphosphonate use (low SOE) or versus past bisphosphonate use (low SOE).

- There was higher risk of subtrochanteric/femoral shaft fractures without radiologic AFF features with long-term use of any bisphosphonate versus no bisphosphonate use (low SOE).

- Evidence was insufficient to draw conclusions about differences in risk of subtrochanteric or femoral shaft fractures with long-term use of any bisphosphonate versus raloxifene or calcitonin use.

- There was no evidence from eligible studies about risk of ONJ between long-term use of any bisphosphonate versus control.

\section{Key Question 4}

- Risks of radiologically confirmed AFF and of subtrochanteric or femoral shaft fracture without radiologic confirmation of AFF features appeared greater with increased duration of long-term bisphosphonate use versus control, though SOE for these association were not graded.

\section{Eligible Studies}

Eight eligible publications of eight unique observational studies compared long-term treatment with any bisphosphonate (reported results for bisphosphonates collectively) versus no bisphosphonate treatment, past bisphosphonate treatment or active control and reported on risk of harms. We assessed two studies as high $\mathrm{ROB}^{68,69}$ and extracted limited data (Appendix Table D7). The remaining six studies had medium ROB. ${ }^{62-67}$ Appendix D provides detailed evidence tables and summary SOE assessments; ROB assessments are provided in Appendix C.

Three studies compared the effect of long-term use of any bisphosphonate versus no osteoporosis drug use on risk of harms. The first of these studies, conducted in Sweden, performed both retrospective cohort and case-control analyses. ${ }^{66}$ Participants were men or women aged $\geq 55$ years, had a diagnosis code for either subtrochanteric or femoral shaft fracture during a three-year period and no associated excessive trauma or pathological fracture. All had their radiographs reviewed for American Society for Bone and Mineral Research (ASBMR) criteria. ${ }^{19}$ Those whose fractures met ASBMR criteria were categorized as having AFF and those whose fractures did not meet ASBMR criteria were categorized as non-AFF controls. In the cohort analysis, the age and sex-stratified risk of AFF was estimated for participants who received bisphosphonates (per the Swedish Prescription Register) compared with that in those who did not. Osteoporosis status was not reported. In the case-control analysis, the multivariable- 
adjusted odds of AFF versus non-AFF subtrochanteric or femoral shaft fractures were compared between individuals with long-term bisphosphonate use and those with no osteoporosis drug use. The second of these studies was a nested case-control study conducted in Spain that estimated risk of subtrochanteric or femoral shaft fracture compared with no hip fracture. ${ }^{62}$ The study population consisted of women aged 65 years or older (mean 82 years) identified in a national primary practice research database over a 3-year study period. Subtrochanteric or femoral shaft fracture cases were identified using diagnostic codes, with exclusions for excessive trauma and cancer, but no review of radiographs for AFF features. Each case was matched by age and calendar year of enrollment to five controls with no history of hip fracture. Information on bisphosphonate use before the fracture or a corresponding index date was obtained from the research database. Osteoporosis status was not reported. The third of these studies was a multicenter case-control study conducted in South Korea ${ }^{67}$ Participants were women aged $>50$ years with a diagnosis code for either subtrochanteric or femoral shaft fracture during a four-year period, and no associated femoral neck, intertrochanteric, distal femoral, high-energy, prosthetic or pathologic fracture. All participants had radiographs reviewed; those whose met ASBMR criteria were categorized as having AFF and those whose subtrochanteric or femoral shaft fractures were judged not to have AFF radiologic features were categorized as non-AFF controls. Risk of AFF was estimated for those with a history of bisphosphonate use (per medical record chart review; mean duration 5.2 years) compared with those without history of bisphosphonate use. Osteoporosis or osteopenia rates were higher in the AFF group (85\%) than the control group $(18 \%)$.

Two studies compared long-term bisphosphonate use to past bisphosphonate use. The first of these was a nested case-control study conducted in Canada that estimated risk with use of any bisphosphonate for $\geq 5$ years versus that with past use for $<100$ days. ${ }^{65}$ Cases were defined using diagnostic codes for subtrochanteric or femoral shaft fracture, with exclusions for excessive trauma and cancer/pathological fracture, but no review of radiographs for AFF features. Each case was matched to five controls who had not been hospitalized with a subtrochanteric or femoral shaft fracture. The study population consisted of women aged 68 years or older (median age 83 years) included in a provincial research database over a 5-year period who began treatment with a bisphosphonate within the past 3 years. No results were reported for baseline BMD. About 70 percent of cases and 24 percent of controls reported an osteoporotic fracture in the preceding 5 years. The second of these studies was a retrospective, nested case-control study conducted in postmenopausal women in Korea. ${ }^{64}$ It compared AFF risk between women with current use of any bisphosphonate for $\geq 5$ years versus that in women with past use for $\geq 1$ year that was stopped between 6 months and 5 years prior to the study start date. Participants were identified through electronic hospital records over an 8-year study period. AFF cases were defined using diagnostic codes and radiologic confirmation of AFF features. Cases were excluded for excessive trauma or cancer. Mean participant age was 68 years, mean pre-fracture (or most recent) dual-energy x-ray absorptiometry (DXA) BMD T-score at the total hip and femoral neck were -1.33 and -1.10 , respectively. Women with AFF were matched by age and sex to three controls without AFF.

The fifth study compared long-term use of any bisphosphonate versus long-term raloxifene or calcitonin use. This retrospective cohort study, conducted in the United States, estimated risk of subtrochanteric or femoral shaft fracture with use of any bisphosphonate for $>3$ years versus that with long-term use of raloxifene or calcitonin. ${ }^{63}$ Cases were defined using diagnosis codes for subtrochanteric or femoral shaft fracture, without exclusions for trauma or cancer/pathologic 
fracture, and no review of radiographs for AFF features. Study participants were Medicare beneficiaries, mean age was 80 years, and 97 percent of participants were women. Individuals were presumed to have osteoporosis, though only 10 to 15 percent had a prior history of hip or vertebral fracture and no results were reported for baseline BMD. The bisphosphonate and raloxifene/calcitonin groups were propensity score-matched one-to-one.

\section{Outcomes}

All data on harms associated with unspecified bisphosphonate treatment were from observational studies.

\section{Atypical Femoral Fractures}

Three controlled observational studies each provided low strength evidence that long-term bisphosphonate use was associated with a significantly increased risk of radiologically confirmed AFF.

Two studies compared risk of long-term bisphosphonate use versus no bisphosphonate use. In one of these studies, long-term ( $\geq 3$ years) bisphosphonate use was associated with increased risk of AFF in both retrospective cohort (RR 126 [95\% CI 55, 288]) and case-control analyses (range of OR 40 [95\% CI 17, 91] to 116 [95\% CI 58, 234]). ${ }^{66}$ In the second of these studies, bisphosphonate use for 5.2 years was associated with increased risk of AFF (OR 25.65 [95\% CI $10.74,61.28]) .{ }^{67}$ In this latter study, 85 percent of the bisphosphonate group had osteoporosis or osteopenia (defined as FN-BMD T-score <-2.0) compared to 18 percent in the control group. This difference in population may be evidence of confounding by indication.

One study reported on risk of radiologically confirmed AFF for current versus past bisphosphonate use, and risk was significantly increased in all reported models (HR 3.36 to $5.17)^{64}$ (Table 12).

\section{Subtrochanteric/Femoral Shaft Fractures}

The relative risk of subtrochanteric or femoral shaft fractures without radiologically confirmed AFF features in individuals who received long-term bisphosphonate treatment compared with controls varied between three studies reporting these results (Table 12). Compared to no use of bisphosphonates, risk of subtrochanteric or femoral shaft fractures was significantly increased with use for $>3$ years (OR 9.46 [95\% CI 2.17, 41.3]). ${ }^{62}$ Compared to minimal past bisphosphonate use (<100 days), risk of subtrochanteric or femoral shaft fractures was not significantly increased with 3-5 years of bisphosphonate use (OR 1.59 [95\% 0.80, $3.15]){ }^{65}$ but was increased with $\geq 5$ years of bisphosphonate use (OR 2.74 [95\% CI 1.25, 6.02]). ${ }^{65}$ Compared to raloxifene or calcitonin use, risk of subtrochanteric or femoral shaft fractures was not increased with either 3-5 years or $>5$ years of bisphosphonate use. ${ }^{63}$

Risks for AFF and subtrochanteric or femoral shaft fractures varied substantially between studies. This variation may have been attributable to several differences in study design. First, studies differed in case definition, in particular whether radiological confirmation for AFF features was required. Studies that didn't require confirmation of these radiologic features necessarily included many typical osteoporotic femur fractures as cases. This would have had the effect of overestimating the absolute risk of AFF, and could have underestimated the relative risk of AFF if long-term bisphosphonate treatment lowered risk of typical osteoporotic femur fractures in these study populations. Studies also used different non-AFF control groups, with some using no hip fracture and others using subtrochanteric or femoral shaft fractures with 
radiographs that did not have AFF features. If long-term bisphosphonates lowered risk of these typical osteoporotic femoral fractures, use of this latter control group could have increased the estimated risk of AFF independently of any direct effect on AFF risk. Studies also differed in terms of the duration of bisphosphonate exposure, the nonbisphosphonate control group (no bisphosphonate use, past bisphosphonate use, other osteoporosis drug use), case control versus retrospective cohort design, and which covariables were included in adjusted statistical models.

The impact of these factors in isolation was difficult to discern because they generally were not compared within single studies and studies differed in multiple ways. Nevertheless, the two studies of any long-term bisphosphonate use versus no use that defined AFF using ASBMR task force criteria, excluded pathological fractures, and compared risk of being a radiologically confirmed AFF case to that of being a subtrochanteric or femoral shaft fracture without radiologic AFF features had the highest estimated risks. These ranged from OR 40 to 116 and RR 126 in one study, ${ }^{66}$ to OR 25.65 in the second study. ${ }^{67}$ The single study that compared risk of radiologically confirmed AFF to no history of AFF, while still excluding pathologic fractures, reported smaller, but still significantly increased risks, with HR ranging from 3.36 to $5.17 .{ }^{64}$ For the two studies that didn't evaluate radiographs for AFF features, but excluded cases for trauma or cancer, risk estimates ranged from OR 1.59 to $2.74^{65}$ and OR 9.46. ${ }^{62}$ By comparison, the single study that neither evaluated radiographs for AFF features, nor excluded cases for trauma or cancer, found no increase in risk. ${ }^{63}$ Further, the three studies that compared long-term bisphosphonate use to no use reported the highest relative estimates of AFF or subtrochanteric or femoral shaft fracture risk, ranging from OR 9.46, ${ }^{62}$ to RR 40 and 126 (range of estimates in this study due to different models and follow-up times), ${ }^{66}$ and RR 25.65. ${ }^{67}$ By comparison, the studies that compared long-term bisphosphonate use to past use ${ }^{64,65}$ reported intermediately increased risk of AFF, and the study that compared long-term bisphosphonate use to long-term raloxifene or calcitonin use reported no increased risk of subtrochanteric or femoral shaft fracture. ${ }^{63}$

\section{Osteonecrosis of the Jaw}

No eligible studies compared long-term treatment with bisphosphonates as a class versus a control group and reported on risk of ONJ. 
Table 12. Estimated risk of AFF* or ST/FS fracture $\dagger$ for any bisphosphonate use from observational studies

\begin{tabular}{|c|c|c|c|c|c|c|c|}
\hline Outcome & $\begin{array}{l}\text { Author, } \\
\text { Year } \\
\text { Country } \\
\text { Risk of } \\
\text { Bias }\end{array}$ & Study Design & $\begin{array}{c}\text { Population } \\
\text { Age (mean) } \\
\text { Gender (\%) } \\
\text { Comorbid } \\
\text { Conditions } \\
(\%)\end{array}$ & Case and Control Definitions & $\begin{array}{l}\text { BP } \\
\text { Treatmen } \\
\text { t Duration }\end{array}$ & $\begin{array}{l}\text { Treatment } \\
\text { Control } \\
\text { Group }\end{array}$ & $\begin{array}{l}\text { RR or OR }(95 \% \mathrm{Cl}) \\
\text { Model Covariates }\end{array}$ \\
\hline \multirow[t]{4}{*}{$\mathrm{AFF}^{*}$} & \multirow[t]{4}{*}{$\begin{array}{l}\text { Schilcher, } \\
2015^{66} \\
\text { Sweden } \\
\text { Medium }\end{array}$} & $\begin{array}{l}\text { Retrospective cohort } \\
\text { ( } n \sim 98,200 \text { BP users, } \\
n \sim 2.8 \text { million non-BP } \\
\text { users) }\end{array}$ & $\begin{array}{l}\text { Age NR } \\
\text { Female 87\% } \\
\text { Comorbid } \\
\text { conditions } \\
\text { NR }\end{array}$ & $\begin{array}{l}\text { AFF case ( } n=172 \text { total, } n=27 \text { with } \geq 4 \text { years BP use): } \\
\text { Diagnosis code for subtrochanteric or femoral shaft } \\
\text { fracture, exclusions for excessive trauma or } \\
\text { pathological fracture, met ASBMR radiographic criteria } \\
\text { for AFF. } \\
\text { Non-AFF control: Diagnosis code for subtrochanteric } \\
\text { or femoral shaft fracture, exclusion for excessive } \\
\text { trauma or pathological fracture, did not meet ASBMR } \\
\text { radiographic criteria for AFF. }\end{array}$ & $\geq 4$ years & $\begin{array}{l}\text { Non-use of } \\
\text { BP }\end{array}$ & $\begin{array}{l}\text { RR } 126(55,288) \\
\text { (women only) } \\
\text { Age }\end{array}$ \\
\hline & & Case-control & $\begin{array}{l}\text { Age 81 } \\
\text { Female 83\% } \\
\text { CKD 39\% } \\
\text { CVD 58\% } \\
\text { DM 14\% }\end{array}$ & $\begin{array}{l}\text { AFF case }(n=172 \text { total, } n=17 \text { with } 3-4 \text { years of } B P \\
\text { use): Same as above } \\
\text { Non-AFF control }(n=952) \text { : Same as above }\end{array}$ & $3-4$ years & $\begin{array}{l}\text { Same as } \\
\text { above }\end{array}$ & $\begin{array}{l}\text { OR } 40(17,91) \\
\text { Age, sex, cortisone } \\
\text { use, Charlson's } \\
\text { comorbidity index }\end{array}$ \\
\hline & & Case-control & See above & $\begin{array}{l}\text { AFF case ( } n=172 \text { total, } n=16 \text { with } 4-5 \text { years of BP } \\
\text { use): Same as above } \\
\text { Non-AFF control }(n=952) \text { : Same as above }\end{array}$ & $4-5$ years & $\begin{array}{l}\text { Same as } \\
\text { above }\end{array}$ & $\begin{array}{l}\text { OR } 116(58,234) \\
\text { Age, sex, cortisone } \\
\text { use, Charlson's } \\
\text { comorbidity index }\end{array}$ \\
\hline & & Case-control & See above & $\begin{array}{l}\text { AFF case }(n=172 \text { total, } n=11 \text { with }>5 \text { years of BP } \\
\text { use): Same as above } \\
\text { Non-AFF control }(n=952): \text { Same as above }\end{array}$ & $>5$ years & $\begin{array}{l}\text { Same as } \\
\text { above }\end{array}$ & $\begin{array}{l}\text { OR } 93(66,132) \\
\text { Age, sex, cortisone } \\
\text { use, Charlson's } \\
\text { comorbidity index }\end{array}$ \\
\hline
\end{tabular}




\begin{tabular}{|c|c|c|c|c|c|c|c|}
\hline Outcome & $\begin{array}{l}\text { Author, } \\
\text { Year } \\
\text { Country } \\
\text { Risk of } \\
\text { Bias }\end{array}$ & Study Design & $\begin{array}{c}\text { Population } \\
\text { Age (mean) } \\
\text { Gender (\%) } \\
\text { Comorbid } \\
\text { Conditions } \\
(\%)\end{array}$ & Case and Control Definitions & $\begin{array}{l}\text { BP } \\
\text { Treatmen } \\
\text { t Duration }\end{array}$ & $\begin{array}{c}\text { Treatment } \\
\text { Control } \\
\text { Group }\end{array}$ & $\begin{array}{l}\text { RR or OR }(95 \% \mathrm{Cl}) \\
\text { Model Covariates }\end{array}$ \\
\hline & $\begin{array}{l}\text { Koh, } \\
2017^{64} \\
\text { Korea } \\
\text { Medium }\end{array}$ & Case-control & $\begin{array}{l}\text { Age } 68 \\
\text { Female } \\
100 \% \\
\text { CKD NR } \\
\text { CVD NR } \\
\text { DM 13\% }\end{array}$ & $\begin{array}{l}\text { AFF case }(n=43) \text { : Diagnosis codes for subtrochanteric } \\
\text { and femoral shaft fracture, exclusions for } \\
\text { cancer/pathologic fracture, met ASBMR radiographic } \\
\text { criteria for AFF. } \\
\text { Non-AFF control }(n=129) \text { : No history of AFF. }\end{array}$ & $\geq 5$ years & $\begin{array}{l}\text { No BP use } \geq 6 \\
\text { months to } 5 \\
\text { years after } \geq 1 \\
\text { year of BP } \\
\text { treatment }\end{array}$ & $\begin{array}{l}\text { Model 1: HR 5.17 } \\
\text { (2.0, 13.36) } \\
\text { Model 2: HR } 4.37 \\
\text { (1.68, 11.41) } \\
\text { Model 3: HR } 3.36 \\
\text { (1.77, 11.91) } \\
\text { Model 1: BMI, } \\
\text { continued } \\
\text { bisphosphonate, } \\
\text { long-term } \\
\text { glucocorticoid use } \\
\text { Model 2: BMI, } \\
\text { continued } \\
\text { bisphosphonate, } \\
\text { rheumatoid arthritis } \\
\text { Model 3: BMI, } \\
\text { continued } \\
\text { bisphosphonate, } \\
\text { disease-modifying } \\
\text { anti-rheumatic drugs }\end{array}$ \\
\hline & $\begin{array}{l}\text { Lim, } \\
2018^{67} \\
\text { South } \\
\text { Korea } \\
\text { Medium }\end{array}$ & Case-control & $\begin{array}{l}\text { Age } 72 \\
\text { Female } \\
100 \% \\
\text { DM 20\% } \\
\text { CKD NR } \\
\text { CVD 10\% }\end{array}$ & $\begin{array}{l}\text { AFF case }(n=196) \text { : Diagnosis codes for } \\
\text { subtrochanteric and femoral shaft fracture, exclusions } \\
\text { for cancer/pathologic fracture, met ASBMR } \\
\text { radiographic criteria for AFF. } \\
\text { Non-AFF control ( } n=96) \text { : Diagnosis code for } \\
\text { subtrochanteric or femoral shaft fracture, exclusion for } \\
\text { excessive trauma or pathological fracture, did not meet } \\
\text { ASBMR radiographic criteria for AFF. }\end{array}$ & $\begin{array}{l}\text { Mean } 5.2 \\
\text { years } \\
\text { (range 1- } \\
17 \text { years) }\end{array}$ & $\begin{array}{l}\text { Non-use of } \\
\text { BP }\end{array}$ & $\begin{array}{l}\text { OR 25.65 (10.74, } \\
61.28) \\
\text { Model covariates not } \\
\text { reported }\end{array}$ \\
\hline
\end{tabular}




\begin{tabular}{|c|c|c|c|c|c|c|c|}
\hline Outcome & $\begin{array}{l}\text { Author, } \\
\text { Year } \\
\text { Country } \\
\text { Risk of } \\
\text { Bias }\end{array}$ & Study Design & $\begin{array}{l}\text { Population } \\
\text { Age (mean) } \\
\text { Gender (\%) } \\
\text { Comorbid } \\
\text { Conditions } \\
\quad(\%)\end{array}$ & Case and Control Definitions & $\begin{array}{l}\text { BP } \\
\text { Treatmen } \\
\text { t Duration }\end{array}$ & $\begin{array}{l}\text { Treatment } \\
\text { Control } \\
\text { Group }\end{array}$ & $\begin{array}{l}\text { RR or OR }(95 \% \mathrm{Cl}) \\
\text { Model Covariates }\end{array}$ \\
\hline \multirow[t]{4}{*}{$\begin{array}{l}\text { ST/FS } \\
\text { fracture } †\end{array}$} & $\begin{array}{l}\text { Erviti, } \\
2013^{62} \\
\text { Spain } \\
\text { Medium }\end{array}$ & Case-control & $\begin{array}{l}\text { Age } 82 \\
\text { Female } \\
100 \% \\
\text { CKD 6\% } \\
\text { CVD NR } \\
\text { DM 21\% }\end{array}$ & $\begin{array}{l}\text { STIFS cases }(n=44) \text { : Diagnosis codes for } \\
\text { subtrochanteric and femoral shaft fracture with } \\
\text { exclusions for excessive trauma and cancer, but no } \\
\text { review of radiographs. } \\
\text { Non-ST/FS fracture control }(n=220) \text { : No hip fracture. }\end{array}$ & $>3$ years & $\begin{array}{l}\text { Non-use of } \\
\text { BP }\end{array}$ & $\begin{array}{l}\text { OR } 9.46(2.17,41.3) \\
\text { Age, calendar year of } \\
\text { enrollment in primary } \\
\text { care database, } \\
\text { smoking, alcoholism, } \\
\text { BMI, previous } \\
\text { fracture, } \\
\text { comorbidities, other } \\
\text { medications } \\
\text { (including raloxifene, } \\
\text { hormone therapy) }\end{array}$ \\
\hline & \multirow[t]{2}{*}{$\begin{array}{l}\text { Park- } \\
\text { Wyllie, } \\
2011^{65} \\
\text { Canada } \\
\text { Medium }\end{array}$} & Case-control & $\begin{array}{l}\text { Age } 83 \\
\text { Female } \\
100 \% \\
\text { CHF } 6 \% \\
\text { CKD NR } \\
\text { DM } 7 \%\end{array}$ & $\begin{array}{l}\text { ST/FS cases }(n=204) \text { : Hospitalized with diagnosis } \\
\text { codes for first subtrochanteric or femoral shaft fracture, } \\
\text { with exclusions including for excessive trauma, } \\
\text { cancer/pathologic fracture, and current use of non-BP } \\
\text { osteoporosis medications, but no review of } \\
\text { radiographs. } \\
\text { Non-ST/FS fracture control ( } n=1070) \text { : Not } \\
\text { hospitalized with subtrochanteric or femoral shaft } \\
\text { fracture. }\end{array}$ & $3-5$ years & $\begin{array}{l}<100 \text { total } \\
\text { days of past } \\
\text { BP use }\end{array}$ & $\begin{array}{l}\text { OR } 1.59(0.80,3.15) \\
\text { Socioeconomic } \\
\text { status, } \\
\text { comedications, drug } \\
\text { count, comorbidities, } \\
\text { recent medical visits, } \\
\text { prior fall, prior } \\
\text { osteoporotic fracture, } \\
\text { BMD test past } 5 \\
\text { years }\end{array}$ \\
\hline & & Case-control & See above & $\begin{array}{l}\text { ST/FS cases }(n=121) \text { : Same as above. } \\
\text { Non-ST/FS fracture control }(n=460) \text { : Same as } \\
\text { above. }\end{array}$ & $\geq 5$ years & $\begin{array}{l}\text { Same as } \\
\text { above }\end{array}$ & $\begin{array}{l}\text { OR } 2.74(1.25,6.02) \\
\text { Socioeconomic } \\
\text { status, } \\
\text { comedications, drug } \\
\text { count, comorbidities, } \\
\text { recent medical visits, } \\
\text { prior fall, prior } \\
\text { osteoporotic fracture, } \\
\text { BMD test past } 5 \\
\text { years }\end{array}$ \\
\hline & $\begin{array}{l}\text { Kim, } \\
2011^{63} \\
\text { US } \\
\text { Medium }\end{array}$ & $\begin{array}{l}\text { Retrospective cohort } \\
\text { ( } n=2,591 \text { BP users, } \\
n=2,309 \text { raloxifene } \\
\text { or calcitonin users) }\end{array}$ & $\begin{array}{l}\text { Age } 80 \\
\text { Female } 97 \% \\
\text { CHF 22\% } \\
\text { CKD 3\% } \\
\text { DM 26\% } \\
\text { HTN } 67 \%\end{array}$ & $\begin{array}{l}\text { ST/FS cases }(n=26) \text { : Diagnosis codes for } \\
\text { subtrochanteric and femoral shaft fracture, but no } \\
\text { exclusions for trauma or cancer/pathologic fracture, } \\
\text { and no review of radiographs. } \\
\text { Non-ST/FS fracture control: Not specified. }\end{array}$ & 3-5 years & $\begin{array}{l}\text { Raloxifene or } \\
\text { calcitonin }\end{array}$ & $\begin{array}{l}\text { HR } 1.20(0.55,2.61) \\
\text { Propensity score- } \\
\text { matched by } \\
\text { demographics, } \\
\text { healthcare utilization, } \\
\text { comorbidities, and } \\
\text { other medications }\end{array}$ \\
\hline
\end{tabular}




\begin{tabular}{|c|c|c|c|c|c|c|c|}
\hline Outcome & $\begin{array}{l}\text { Author, } \\
\text { Year } \\
\text { Country } \\
\text { Risk of } \\
\text { Bias }\end{array}$ & Study Design & $\begin{array}{c}\text { Population } \\
\text { Age (mean) } \\
\text { Gender (\%) } \\
\text { Comorbid } \\
\text { Conditions } \\
(\%)\end{array}$ & Case and Control Definitions & $\begin{array}{l}\text { BP } \\
\text { Treatmen } \\
\text { t Duration }\end{array}$ & $\begin{array}{c}\text { Treatment } \\
\text { Control } \\
\text { Group }\end{array}$ & $\begin{array}{l}\text { RR or OR }(95 \% \mathrm{Cl}) \\
\text { Model Covariates }\end{array}$ \\
\hline & & $\begin{array}{l}\text { Retrospective cohort } \\
\text { ( } n=2,371 \text { BP users, } \\
n=1,726 \text { raloxifene } \\
\text { or calcitonin users) }\end{array}$ & See above & $\begin{array}{l}\text { ST/FS cases }(n=8): \text { Same as above. } \\
\text { Non-ST/FS fracture control: Not specified. }\end{array}$ & $>5$ years & $\begin{array}{l}\text { Same as } \\
\text { above }\end{array}$ & $\begin{array}{l}\text { HR } 2.02(0.41,10.0) \\
\text { Propensity score- } \\
\text { matched by } \\
\text { demographics, } \\
\text { healthcare utilization, } \\
\text { comorbidities, and } \\
\text { other medications }\end{array}$ \\
\hline
\end{tabular}

Abbreviations: AFF=atypical femoral fracture; ASBMR=American Society for Bone and Mineral Research; BMI=body mass index; BP=bisphosphonates; CHF=chronic heart failure; $\mathrm{CI}=$ confidence interval; $\mathrm{CKD}=$ chronic kidney disease; $\mathrm{CVD}=$ cardiovascular disease; $\mathrm{DM}=$ =diabetes mellitus; $\mathrm{HR}=$ =adjusted hazard ratio; $\mathrm{HTN}=$ hypertension; $\mathrm{N}=$ =number; $\mathrm{OR}=$ odds ratio; $\mathrm{RR}=$ risk ratio; $\mathrm{ST} / \mathrm{FS}=$ subtrochanteric/femoral shaft

*Atypical femoral fractures must have been defined as meeting ASBMR radiographic criteria for AFF.

†Subtrochanteric/femoral shaft fractures were fractures of these sites not stated as meeting ASBMR radiographic criteria for AFF. 


\section{Variation in Long-Term Treatment Harms as a Function of Patient, Bone, or Osteoporosis Drug Characteristics}

We looked at whether the harms of long-term bisphosphonate therapy versus control vary as a function of patient, bone, and/or drug characteristics. Eligible studies included evidence for the following potential effect modifiers:

\section{Age}

A Swedish retrospective cohort study reported risk of radiologically confirmed AFF with long-term bisphosphonate use versus no bisphosphonate use as a function of age in women only. ${ }^{66}$ The age-adjusted risk of AFF for bisphosphonate treatment compared with no use of osteoporosis drugs appeared possibly greater in women $\geq 80$ years of age (OR 163 [95\% CI 39, 687]) compared to that in women <80 years of age (OR 100 [95\% CI 40, 253]). However, authors did not report results for a test of interaction.

\section{Bisphosphonate Treatment Duration}

Three studies each reported risk estimates for AFF or subtrochanteric or femoral shaft fractures between long-term bisphosphonate use and control for multiple treatment duration strata. Collectively they suggested that in populations with $>3$ years of bisphosphonate use, risk of these fractures may be higher with longer treatment duration.

One study reported on risk of AFF ${ }^{66}$ It found that compared with no osteoporosis drug use, risk of AFF with radiologically confirmed AFF features with long-term bisphosphonate use appeared greater with bisphosphonate use of either 4 to 5 years (OR 116 [95\% CI 58, 234]) or >5 years (OR 93 [95\% CI 66, 132]) than with use for 3 to 4 years (OR 40 [95\% CI 17, 91]).

Two studies reported on risk of subtrochanteric or femoral shaft fractures. The first of these reported that compared with past bisphosphonate use for $<100$ days, risk of subtrochanteric or femoral shaft fracture was not significantly increased with 3 to 5 years of bisphosphonate use (OR 1.59 [95\% CI 0.80, 3.15]), but was increased with $>5$ years of bisphosphonate use (OR 2.74 [95\% CI 1.25, 6.02]). ${ }^{65}$ The second study reported risk for subtrochanteric or femoral shaft fractures with long-term bisphosphonate use versus raloxifene or calcitonin use. ${ }^{63}$ Risk appeared possibly higher with $>5$ years use (HR 2.02 [95\% CI 0.41, 10.0]) than with 3 to 5 years use (HR 1.20 [95\% CI 0.55, 2.61]), though neither result was statistically significant. Confidence intervals overlapped in all studies and none tested for an interaction as a function of treatment duration. 


\section{Denosumab}

\section{Key Points}

\section{Key Question 3}

- In postmenopausal women with osteoporosis, between long-term denosumab versus placebo, trial evidence was insufficient to draw conclusions about differences in risk of on-treatment serious adverse events, AFF, or ONJ, or post-treatment fracture risk.

- No controlled observational studies compared long-term treatment with denosumab versus no treatment or another control, let alone reported on risk of harms such as AFF, ONJ, or post-treatment fracture risk.

\section{Key Question 4}

- We found no evidence about whether differences in risk of harms between long-term denosumab and placebo or other control vary as a function of patient, bone or drug characteristics.

\section{Eligible Studies}

Two eligible publications of two trials and no eligible observational studies compared longterm treatment with denosumab versus placebo, no treatment or another active treatment and reported on risk of harms. One publication was rated high $\mathrm{ROB}^{71}$ and only limited data were extracted (Appendix Table D7). The remaining publication, ${ }^{70}$ which had medium ROB, was extracted in detailed evidence tables, SOE assessments (Appendix D), and summary ROB assessments (Appendix C).

Described in detail previously, this trial randomized postmenopausal women with osteoporosis or low bone mass/osteopenia to placebo $(n=46)$ versus one of seven intravenous denosumab dosing regimens $(n=319)$ for 2 years. These dosing regimens included three that were lower and two that were higher than the current FDA recommended denosumab osteoporosis dosing. After 2 years, individuals were nonrandomly assigned to treatment groups for the following 2 years. Those initially assigned placebo remained assigned to placebo. Five of the groups assigned denosumab, including three of the low dose groups, were assigned to denosumab 60 mg every 6 months for 2 years $(n=231)$, one of the original high dose denosumab groups was switched to placebo for 2 years $(\mathrm{n}=47)$, and the last denosumab group was switched to placebo for 1 year before starting denosumab $60 \mathrm{mg}$ every 6 months for 1 year $(\mathrm{n}=41)$.

\section{Outcomes}

\section{Serious Adverse Events}

Through 4 years, risk of any serious adverse event was 17.8 percent in the women initially assigned to one of the denosumab groups versus 10.9 percent in the women originally assigned to placebo (RR 1.64 [95\% CI 0.69, 3.88]). However, because harms results were reported collectively for all women initially assigned to denosumab, regardless of starting dose or whether they continued denosumab for 4 years, 2 years, or stopped it for 1 year before restarting it for 1 
year, no direct comparison between 4 years of continuous denosumab and placebo groups was possible.

\section{Cardiovascular Events}

One fatal cardiovascular event (stroke) was reported in the collective denosumab group.

\section{Mortality}

There were four deaths (1.3\%) in the collective denosumab group and none in the placebo group.

\section{Atypical Femoral Fracture}

No information was reported about incidence of AFF.

\section{Osteonecrosis of the Jaw}

No information was reported about incidence of ONJ.

\section{Post-Treatment Fractures}

Authors stated that no increase in fracture incidence was observed among the small number of patients who discontinued denosumab treatment, but reported no numerical data comparing results between women assigned to continue denosumab and those assigned to discontinue it.

\section{Variation in Long-Term Treatment Harms as a Function of Patient, Bone, or Osteoporosis Drug Characteristics}

We identified no eligible studies that compared long-term denosumab treatment versus placebo, no treatment or a different active treatment, and assessed whether risk of harms varied as a function of patient, bone or drug characteristics. 


\section{Raloxifene}

\section{Key Points}

\section{Key Question 3}

- In postmenopausal women with osteoporosis, between raloxifene and placebo:

o There was no difference in risk of serious adverse events at 8 years (low SOE).

o There was a higher risk of deep venous thrombosis at 4 years and of pulmonary embolism at 8 years.

- In postmenopausal women with osteoporosis, evidence was insufficient to draw conclusions about differences between long-term raloxifene and either placebo or no osteoporosis drug treatment for risk of AFF or ONJ.

\section{Key Question 4}

- Evidence was insufficient to draw conclusions about whether differences in risk of harms between long-term raloxifene and placebo, no osteoporosis drug treatment or a different active treatment vary as a function of patient, bone, or drug characteristics.

\section{Eligible Studies}

Twelve eligible publications from one RCT and its extension, ${ }^{72-80,82,83,85}$ and three eligible publications from one observational study, ${ }^{89-91}$ compared long-term raloxifene treatment with placebo, no treatment or a different active treatment, and reported on risk of harms. All studies had low or medium ROB, and information from these studies was extracted in evidence tables, SOE summary tables (Appendix D) and ROB summary tables (Appendix C).

Eight reports from the Multiple Outcomes of Raloxifene Evaluation (MORE) RCT with low ROB compared raloxifene to placebo through 4 years. Two compared raloxifene $60 \mathrm{mg} /$ day to placebo ( $\mathrm{n}=5114$ to 5133$)^{77,85}$, three compared a pooled raloxifene group $(60 \mathrm{mg} /$ day and 120 $\mathrm{mg} /$ day) to placebo ( $\mathrm{n}=7617$ to 7705$)^{72,73,80}$, and three separately compared raloxifene 60 $\mathrm{mg}$ /day and $120 \mathrm{mg} /$ day to placebo ( $\mathrm{n}=6828$ to 7705$)$. $^{74-76}$ Four medium ROB studies from the 4 year Continuing Outcomes Relevant to Evista (CORE) extension compared raloxifene 60 $\mathrm{mg} /$ day to placebo through 8 years ( $\mathrm{n}=4011$ to 5133). Details of the MORE and CORE study designs are reported above.

The three reports from one observational study compared raloxifene (doses not specified) to no osteoporosis treatment. ${ }^{89-91}$ The study sample was drawn from the Danish general population and consisted of 4,831 individuals who filled a prescription for raloxifene and 14,493 age and gender matched controls. Mean age was 64 years. Participants generally were healthy with few comorbidities. In a larger study sample, that also included individuals who received other osteoporotic drugs and their matched controls, 85 percent of participants were women and mean followup was 3.8 years. 


\section{Outcomes}

\section{Serious Adverse Events}

Among women who enrolled in CORE, there was no difference in risk of serious adverse events between raloxifene and placebo during the four years of CORE follow-up (years 5-8 from their MORE enrollment; $23 \%$ vs. $25 \%$, $\mathrm{p}=0.22$ ). ${ }^{82}$ Similarly, among CORE enrollees, there was no difference in risk of treatment-emergent serious adverse events between raloxifene and placebo during their combined 8 years of follow-up in MORE and CORE (42\% vs. 46\%; RR $0.93[95 \%$ CI $0.86,1.00]$ ) (low SOE). ${ }^{83}$

\section{Venous Thromboembolism}

After 4 years in the MORE trial, which excluded individuals with a stroke or venous thromboembolic event in the last 10 years, incidence of deep vein thrombosis (DVT) during 4 years of follow-up was higher in the pooled raloxifene group (60 mg/day and $120 \mathrm{mg} /$ day) compared with placebo. ${ }^{75,76,80}$ However, estimates of effect between publications ranged slightly from RR $2.8\left(95 \%\right.$ CI 1.3, 5.9) ${ }^{76}$ to RR 3.1 (95\% CI 1.4, 6.9) ${ }^{80}$ Five MORE papers suggested that raloxifene (all but one paper ${ }^{85}$ pooled the $60 \mathrm{mg} /$ day and $120 \mathrm{mg} /$ day doses) was associated with an increased risk of pulmonary embolism versus placebo. However, results only were statistically significant in one paper (RR 4.5 [95\% CI 1.1, 19.5]), ${ }^{80}$ and were borderline significant in the others. ${ }^{72,75,76,85}$ The percentage of participants with pulmonary embolism varied between reports ( $0.31 \%$ to $0.43 \%$ for raloxifene and $0.08 \%$ to $0.23 \%$ for placebo). Through 8 years, among women who participated in the CORE extension $(\mathrm{n}=4011)$, there was no difference between raloxifene $60 \mathrm{mg}$ /day and placebo in risk of DVT, ${ }^{78,82,83}$ but risk of pulmonary embolism was higher in the raloxifene group compared with placebo.

\section{Hot Flashes}

Through 8 years, among women who participated in the CORE extension $(n=4011)$, risk of hot flashes was higher in the raloxifene group compared with placebo. ${ }^{78,82,83}$

\section{Cardiovascular Disease}

Through eight years, among women who participated in the CORE extension ( $\mathrm{n}=4011)$, there was no difference between raloxifene $60 \mathrm{mg} /$ day and placebo in risk of stroke ${ }^{78,82,83}$ or cardiovascular mortality. ${ }^{78}$

\section{Mortality}

Risk of all-cause mortality was not different between treatment groups when considering follow-up only during the CORE extension period. ${ }^{82,83}$ However, mortality was lower with raloxifene versus placebo when considering only the raloxifene group originally randomized to $60 \mathrm{mg} /$ day and considering follow-up through both the MORE and CORE phases (HR 0.68 [95\% CI 0.46, 0.99]). ${ }^{79}$

\section{Subtrochanteric or Femoral Shaft Fractures}

In data from one observational study, compared with age and gender-matched controls who received no osteoporosis treatment, evidence was insufficient about whether individuals treated with raloxifene had a difference in risk of either subtrochanteric (HR 1.06 [95\% CI 0.34, 3.32]) 
or femoral shaft (HR 0.82 [95\% CI 0.21, 3.20]) fracture. ${ }^{89}$ No data were reported about AFF in either the MORE trial or CORE extension.

\section{Osteonecrosis of the Jaw-Defined by Diagnostic Codes Only}

In data from one observational study, compared with age and gender-matched controls who received no osteoporosis treatment, evidence was insufficient about whether individuals treated with raloxifene had a difference in risk of any inflammatory jaw event as defined only by diagnostic codes (none of 4,831 and two of 14,493 participants in the raloxifene and no osteoporosis treatment groups, respectively). ${ }^{91}$ No data were reported about ONJ in either the MORE trial or CORE extension.

\section{Atrial Fibrillation}

In multivariable-adjusted data from one observational study, compared with age and gendermatched controls who received no osteoporosis treatment, individuals treated with raloxifene appeared to have no increased risk of atrial fibrillation (OR 0.82 [95\% CI 0.60, 1.13]), though SOE was not assessed. ${ }^{90}$ No data were reported about atrial fibrillation in either the MORE trial or CORE extension.

\section{Variation in Long-Term Treatment Harms as a Function of Patient, Bone, or Osteoporosis Drug Characteristics}

We looked at whether the harms of long-term raloxifene therapy versus placebo, no treatment or a different active treatment vary as a function of patient, bone, and/or drug characteristics. Eligible studies included evidence for the following effect potential modifiers.

\section{Baseline Risk for Cardiovascular Disease}

In one publication, though risk of incident stroke was no different between raloxifene and placebo overall, risk was lower versus placebo in the subgroup of women at increased baseline cardiovascular risk (RR 0.38 [95\% CI 0.15, 0.94]). ${ }^{74}$ A second publication found that risk of venous thromboembolism, mostly comprised of DVT or pulmonary embolism, was not statistically significantly different between raloxifene $60 \mathrm{mg} /$ day and placebo in the overall study population (HR 1.86 [95\% CI 0.97, 3.56]) or in a subgroup at increased cardiovascular risk (HR 1.32 [95\% CI 0.37, 4.69]). ${ }^{77}$

\section{Prior Fracture}

In one publication, the effect of raloxifene $60 \mathrm{mg} /$ day versus placebo on risk of stroke, pulmonary embolism, DVT, hot flashes and mortality did not differ as a function of baseline radiographic vertebral fracture status. ${ }^{85}$

\section{Raloxifene Dose and Treatment Duration}

One publication reported that there was no significant difference in risk of DVT or pulmonary embolism between the raloxifene $60 \mathrm{mg} /$ day and $120 \mathrm{mg} /$ day groups, and that risks of venous thromboembolism and DVT (but not of pulmonary embolism) were higher in the pooled raloxifene groups versus placebo through 2 years of treatment but not in later years. ${ }^{80}$ 


\section{Hormone Therapy}

\section{Key Points}

\section{Key Question 3}

- In postmenopausal women with unknown osteoporosis or osteopenia status:

o In those with a prior hysterectomy, compared with placebo, 7 years of oral, high dose conjugated estrogen was associated with an increased risk for stroke, deep venous thrombosis, the composite outcome of cardiovascular disease, and the composite outcome of mild cognitive impairment or probable dementia.

o In those with an intact uterus, compared with placebo, 5.6 years of oral, high dose estrogen plus progestin was associated with an increased risk for stroke, coronary heart disease, deep venous thrombosis, pulmonary embolism, invasive breast cancer, probable dementia, the composite outcome of cardiovascular disease, and the composite outcome of mild cognitive impairment or probable dementia.

- In postmenopausal women with past clinical fracture or osteoporosis by BMD, evidence was insufficient to draw conclusions about any differences in risk for long-term harms between estrogen or estrogen/progestin therapy and control.

\section{Key Question 4}

- The association of estrogen plus progestin versus placebo on risk of breast cancer appeared greater in women with increased duration of prior postmenopausal hormone use.

- All other reported tests of interaction for whether the risk of harms associated with either estrogen alone or estrogen plus progestin versus placebo differed by participant characteristic were not statistically significant.

\section{Eligible Studies}

As detailed earlier, two large Women's Health Initiative (WHI) trials randomized postmenopausal women aged 50 to 79 years with unknown osteoporosis or osteopenia status to long-term hormone therapy versus placebo and reported harms. One trial assigned 16,608 women with an intact uterus to oral estrogen (conjugated estrogen $0.625 \mathrm{mg} /$ day) plus progestin (medroxyprogesterone $2.5 \mathrm{mg} /$ day) versus placebo for 5.6 years. ${ }^{94}$ The other allocated 10,739 women with a prior hysterectomy to oral estrogen (conjugated estrogen $0.625 \mathrm{mg} /$ day) versus placebo for 7 years. ${ }^{95}$ Two additional WHI reports provided details on risk of cognitive diagnoses with hormone therapy versus placebo. ${ }^{96,97}$ An additional, 4-year, non-WHI trial, described in greater detail earlier, randomized 36 postmenopausal women with osteoporosis or osteopenia to estrogen/progestin versus placebo. ${ }^{88}$ 


\section{Outcomes}

\section{Serious Adverse Events}

Incidence of serious adverse events was not reported. Only the small non-WHI trial reported on withdrawals due to adverse events (or to other medical problems), which it stated were not different at 4 years between the estrogen/progestin and control groups (17\% vs. $17 \%$, $\mathrm{p}=1.0)$.

The WHI trial comparing oral estrogen plus progestin versus placebo reported that during an average 5.6 year follow-up period, women with unknown osteoporosis or osteopenia status who were randomized to estrogen plus progestin had a higher risk of stroke (HR 1.41 [95\% CI 1.07, 1.85]), coronary heart disease (HR 1.29 [95\% CI 1.02, 1.63]), deep venous thrombosis (HR 2.07 [95\% CI 1.49, 2.87]), pulmonary embolism (HR 2.13 [95\% CI 1.39, 3.25]), invasive breast cancer (HR 1.26 [95\% CI 1.00, 1.59]), probable dementia (HR 2.05 [95\% CI 1.21, 3.48]), the composite outcome of cardiovascular disease (HR 1.22 [95\% CI 1.09, 1.36]), and the composite outcome of mild cognitive impairment or probable dementia (HR 1.44 [95\% CI 1.04, 1.99]). ${ }^{94,97}$ However, there was a lower risk of colorectal cancer (HR 0.63 [95\% CI 0.43, 0.92]).

The WHI trial comparing oral estrogen versus placebo reported that during an average 7 year follow-up period, women with unknown osteoporosis or osteopenia status who were randomized to estrogen had a higher risk of stroke (HR 1.39 [95\% CI 1.10, 1.77]), deep venous thrombosis (HR 1.47 [95\% CI 1.04, 2.08]), the composite outcome of cardiovascular disease (HR 1.12 [95\% CI 1.01, 1.24]), and the composite outcome of mild cognitive impairment or probable dementia (HR 1.38 [95\% CI 1.01, 1.89]). ${ }^{95,}{ }^{96}$ However, there was no significant difference between treatment groups in risk of coronary heart disease (HR 0.91 [95\% CI 0.75, 1.12]), pulmonary embolism (HR 1.34 [95\% CI 0.87, 2.06]), invasive breast cancer (HR 0.77 [95\% CI 0.59, 1.01]), colorectal cancer (HR 1.08 [95\% CI 0.75, 1.55]), or probable dementia (HR 1.49 [95\% CI 0.83, 2.66]).

To weigh the risk of serious harms against reduction in hip fractures, both WHI trials reported results for a global index, defined as first occurrence of coronary heart disease, invasive breast cancer, stroke, pulmonary embolism, endometrial cancer, colorectal cancer, hip fracture, or death due to other causes. These studies then reported the hazard ratio for the global index outcome in tertiles of study participants stratified by their baseline risk of incident fracture. For estrogen plus progestin compared with placebo, the risk for the global index outcome in women at the lowest, intermediate, and highest fracture risk was HR 1.20 (95\% CI 0.93, 1.58), HR 1.23 (95\% CI, 1.04, 1.46), and HR 1.03 (95\% CI, 0.88, 1.24), respectively. ${ }^{86}$ For estrogen compared with placebo, the risk for the global index outcome in women at the lowest, intermediate, and highest fracture risk was HR 0.81 (95\% CI 0.62, 1.05), HR 1.09 (95\% CI, 0.92, 1.30), and HR 1.04 (95\% CI, 0.88, 1.23), respectively. ${ }^{87}$

\section{Mortality}

In the WHI trial comparing oral estrogen plus progestin versus placebo, during an average 5.6-year follow-up period, hormone therapy was not associated with an increased risk of allcause mortality (HR 0.98 [95\% CI 0.82, 1.18]). ${ }^{94}$ Similarly, in the WHI trial comparing oral estrogen versus placebo, during an average 7-year follow-up period, hormone therapy was not associated with an increased risk of all-cause mortality (HR 1.04 [95\% CI 0.88, 1.22]). ${ }^{95}$ In the small non-WHI trial conducted in women with osteoporosis or osteopenia, no deaths were reported for either the estrogen/progestin or the control groups. ${ }^{88}$ 


\section{ONJ or AFF}

No information was reported about incidence of ONJ or AFF.

\section{Variation in Long-Term Treatment Harms as a Function of Patient, Bone, or Osteoporosis Drug Characteristics}

In the WHI trial that compared oral estrogen plus progestin versus placebo, ${ }^{94,} 97$ the association of treatment assignment with risk of breast cancer appeared greater with increased duration of prior postmenopausal hormone use. However, the association of treatment with invasive breast cancer did not differ by age, race/ethnicity, family history, parity, age at first birth, body mass index, or Gail-model risk score. The association of treatment group with risk of coronary heart disease did not differ based on history of prior coronary heart disease. The association of treatment group with risk of coronary heart disease, stroke, or venous thromboembolism did not significantly differ by age, race/ethnicity, body mass index, prior hormone use, smoking status, blood pressure, diabetes, aspirin use, or statin use. Last, the associations of estrogen alone and estrogen plus progestin versus placebo on risk for dementia did not significantly differ by any of 13 dementia risk factors evaluated ( $\mathrm{p}$ for all tests for interaction $>0.05$ ).

In the WHI trial that compared oral estrogen versus placebo, ${ }^{95,} 96$ the association of treatment assignment with risk of coronary heart disease, stroke, venous thromboembolism, invasive breast cancer, and mortality did not significantly differ by age, race/ethnicity, or body mass index.

We identified no eligible studies in women selected for osteoporosis or osteopenia that compared estrogen or estrogen/progestin with control, and assessed whether risk of harms varied as a function of patient, bone or drug characteristics. 


\section{Other Drugs Investigated for Review}

\section{Ibandronate}

One eligible study reported on harms with long-term treatment with ibandronate versus placebo, no treatment, or another active treatment. ${ }^{61}$ This study was rated high ROB and only limited data were extracted (Appendix Table D7). We identified no eligible studies that compared ibandronate with control, and assessed whether risk of harms varied as a function of patient, bone or drug characteristics.

\section{Risedronate}

Two eligible studies of long-term treatment with risedronate versus placebo, no treatment, or a different active treatment reported on harms. ${ }^{59,60}$ These studies were rated high ROB and only limited data were extracted (Appendix Table D7). We identified no eligible studies that compared risedronate with control, and assessed whether risk of harms varied as a function of patient, bone or drug characteristics 


\section{Chapter 6. Effects of Osteoporosis Drug Holidays}

Chapter 6 discusses Key Questions 5 and 6. Key Question 5 addresses the effects of osteoporosis drug treatment continuation versus discontinuation (placebo drug holiday) on risk of incident fracture. Key Question 6 examines whether the effect on risk of fracture varies by patient, bone, or drug characteristics.

\section{Alendronate}

\section{Key Points}

\section{Key Question 5}

- In postmenopausal women who had received 5 years of alendronate for osteopenia or osteoporosis (femoral neck [FN] bone mineral density [BMD] T-score $\leq-1.6$ ), alendronate continuation for 5 more years versus alendronate discontinuation was associated with:

o Lower risk of incident clinical vertebral fractures (moderate strength of evidence [SOE]).

o No difference in risk of incident clinical fractures (moderate strength of evidence [SOE]), incident nonvertebral fractures (moderate SOE), or incident radiographic vertebral fractures (low SOE).

o Insufficient evidence about incident hip fractures.

- In postmenopausal women who had received 5 years of alendronate for osteoporosis (lumbar spine [LS]-BMD T-score $\leq-2.5$ ):

o Between alendronate continuation for 2 more years versus alendronate discontinuation, evidence was insufficient to draw conclusions about differences in risk of incident nonvertebral fractures or incident clinical vertebral fractures.

o Between alendronate continuation for 5 more years versus alendronate continuation for 2 more years followed by discontinuation, evidence was insufficient to draw conclusions about differences in risk of incident nonvertebral fracture during follow-up years 8-10.

\section{Key Question 6}

- In postmenopausal women who had received 5 years of alendronate for FN-BMD T-score $\leq-1.6$ (osteopenia or osteoporosis):

0 The effect of alendronate continuation for 5 more years versus alendronate discontinuation on risk of incident nonvertebral fracture and incident clinical fracture did not appear to vary as a function of baseline BMD or prevalence of baseline radiographic vertebral fracture.

o Among those with a baseline radiographic vertebral fracture, the effect of alendronate continuation for 5 more years versus alendronate discontinuation on risk of incident nonvertebral fracture, incident clinical fracture and incident radiographic vertebral fracture did not appear to vary as a function of baseline BMD.

0 Among those without a baseline radiographic vertebral fracture, the effect of alendronate continuation for 5 more years versus alendronate discontinuation: 
- On risk of incident nonvertebral fracture, appeared to vary as a function of baseline BMD.

- On risk of incident clinical fracture and incident radiographic vertebral fracture, did not appear to vary as a function of baseline BMD.

\section{Eligible Studies}

Four eligible publications of two unique studies compared alendronate continuation versus discontinuation and reported on risk of incident fractures. We rated three of these publications as having low risk of bias (ROB) ${ }^{33,41,42}$ and one as having medium ROB. ${ }^{34}$ We extracted detailed information from these studies in evidence tables, SOE summary tables (Appendix D), and ROB summary tables (Appendix C).

Two low ROB publications were from the Fracture Intervention Trial Long Term Extension (FLEX), an extension of the previously described U.S.-based Fracture Intervention Trial (FIT) study ${ }^{33,41}$ Eligibility in FLEX was limited to women assigned to the alendronate treatment arm in either FIT-I or FIT-II ( $\mathrm{n}=3236$ total from both studies) who received at least 3 years (mean 5.0 years) of alendronate during blinded follow-up in FIT (mean 3.8 years) and a subsequent openlabel period (mean 1.9 years). Further, they must have had a total hip (TH)-BMD T-score at FLEX baseline that was $\geq-3.5$ and no worse than at their FIT baseline. The 1099 FLEX enrollees were randomized to continuation of alendronate $10 \mathrm{mg} /$ day for 5 years versus discontinuation (placebo drug holiday) (Table 13). Change in TH-BMD was the primary efficacy outcome and incident fracture was a secondary outcome. At FLEX baseline, mean participant age was 73 years, 60 percent of participants reported a clinical fracture since age 45, 34 percent had a prevalent radiographic vertebral fracture, and 78 percent were taking alendronate (Appendix D5). ${ }^{33}$ Though 29 percent of women had FN-BMD T-scores of $\leq-2.5$, mean FN-BMD T-scores were in the osteopenic/low bone mass range.

Two publications ${ }^{34,42}$ were extensions of a pair of nearly identical dose-ranging randomized controlled trials (RCT), one conducted in the U.S. and the other in several non-U.S. countries. ${ }^{98}$ Together, these studies enrolled 994 postmenopausal women aged 45-80 years with LS-BMD Tscore $\leq-2.5$. Women were randomized to one of three alendronate regimens ( $5 \mathrm{mg} /$ day for 3 years, $10 \mathrm{mg} /$ day for 3 years, or $20 \mathrm{mg} /$ day for 2 years followed by $5 \mathrm{mg} /$ day for 1 year) or placebo. All results for the two RCTs were pooled. At baseline, mean age was 64 years and 21 percent of participants had a prevalent radiographic vertebral fracture. Among the 598 women initially assigned alendronate, 439 participated in an extension in which they continued their original alendronate regimen for 2 years ( $5 \mathrm{mg} / \mathrm{day}, 10 \mathrm{mg} /$ day, and continuing $5 \mathrm{mg} /$ day for the third group). Though all women knew they were receiving alendronate, they and the investigators were blinded to their dose regimen. After a total of 5 years from the beginning of the study, 350 of these women entered another 2-year extension, in which women originally assigned alendronate $5 \mathrm{mg} /$ day and $10 \mathrm{mg} /$ day continued these regimens and the group originally assigned alendronate $20 \mathrm{mg}$ /day and later changed to $5 \mathrm{mg} /$ day was switched to blinded placebo. ${ }^{42}$ This study was rated as having low ROB. Although participants remained blinded to their treatment assignments, these assignments were not random. In a final extension, 247 women continued these blinded treatment assignments an additional 3 years. ${ }^{34}$ This last extension study was rated as having medium ROB. Change in LS-BMD was the primary efficacy outcome. Secondary outcomes included change in FN-BMD. Incident fractures were reported as safety outcomes. Authors did not report participant characteristics from the baseline of the extension studies. 
Table 13. Trial participant characteristics at the time of assignment to alendronate continuation versus discontinuation

\begin{tabular}{|c|c|c|c|c|}
\hline Characteristic & FLEX 32,40 & $\begin{array}{l}\text { Main Study* } \\
\text { (Excluded) })^{92}\end{array}$ & $\begin{array}{l}\text { Extension 2* } \\
\text { (Included) }^{41}\end{array}$ & $\begin{array}{l}\text { Extension 3* } \\
\text { (Included) }\end{array}$ \\
\hline $\begin{array}{l}\text { Number enrolled and } \\
\text { randomized }\end{array}$ & $N=1099$ & $N=994$ & $N=350$ & $N=247$ \\
\hline Age, mean years & 73 & 64 & 63 & 63 \\
\hline Gender, women & $100 \%$ & $100 \%$ & $100 \%$ & $100 \%$ \\
\hline Race, white & $97 \%$ & $87 \%$ & NR & NR \\
\hline Mean FN-BMD $\left(\mathrm{g} / \mathrm{cm}^{2}\right)$ & 0.61 & $\begin{array}{l}\text { Hologic } 0.60 \\
\text { Lunar } 0.73 \\
\text { Norland } 0.66\end{array}$ & NR & NR \\
\hline Mean LS-BMD $\left(\mathrm{g} / \mathrm{cm}^{2}\right)$ & 0.90 & $\begin{array}{c}\text { Hologic } 0.71 \\
\text { Lunar } 0.81 \\
\text { Norland } 0.67\end{array}$ & 0.71 & $\begin{array}{c}\text { Hologic or Norland } \\
0.70 \\
\text { Lunar } 0.81\end{array}$ \\
\hline Mean TH-BMD $\left(\mathrm{g} / \mathrm{cm}^{2}\right)$ & 0.73 & NR & NR & NR \\
\hline FN-BMD T-score $\leq-2.5$ & $29 \%$ & NR & NR & NR \\
\hline LS-BMD T-score $\leq-2.5$ & NR & $100 \%$ & NR & NR \\
\hline TH-BMD T-score $\geq-3.5$ & $100 \%$ & NR & NR & NR \\
\hline Prior fracture $\geq 45$ years & $60 \%$ & NR & NR & NR \\
\hline $\begin{array}{l}\text { Prevalent radiographic } \\
\text { vertebral fracture }\end{array}$ & $34 \%$ & $21 \%$ & $21 \%$ & $25 \%$ \\
\hline Comorbid conditions & NR & NR & NR & NR \\
\hline
\end{tabular}

Abbreviations: $\mathrm{BMD}=$ =bone mineral density; FLEX=Fracture Intervention Trial Long-Term Extension; FN=femoral neck; LS=lumbar spine; NR=not reported; TH=total hip

*Dose ranging study

\section{Outcomes}

\section{Incident Clinical Fractures}

In FLEX, whose participants previously were treated with alendronate for a mean of five years, women randomized to continued alendronate for 5 years versus discontinuation (placebo drug holiday) had reduced risk of incident clinical vertebral fracture (2.4\% vs. 5.3\%; risk ratio [RR]0.45 [95\% confidence interval (CI) 0.24, 0.85]) (moderate SOE). However, there was no difference between continuation and drug holiday in risk of any incident clinical fracture (19.9\% vs. 21.3\%; RR 0.93 [95\% CI 0.71, 1.21]) (moderate SOE), or nonvertebral fracture (18.9\% vs. 19.0\%; RR 1.00 [95\% CI 0.76, 1.32]) (moderate SOE). Evidence was insufficient to draw conclusions about differences in risk of hip fracture (3.0\% vs. 3.0\%; RR 1.02 [95\% CI 0.51, $2.10])^{33}$

In the dose-ranging extension study, in results pooling both alendronate continuation arms versus the discontinuation arm, for the year 6-7 extension, evidence was insufficient for differences between treatment groups for both incident nonvertebral fracture (RR 0.87 [95\% CI 
0.40, 1.91]) and incident clinical vertebral fracture (RR 0.92 [95\% CI 0.40, 2.10]). ${ }^{42}$ For the year 8-10 extension, evidence was insufficient about whether incidence of nonvertebral fracture differed between the pooled alendronate continuation and discontinuation groups (RR 0.81 [95\% CI 0.38, 1.71]). ${ }^{34}$

\section{Incident Radiographic Vertebral Fractures}

In FLEX, continued alendronate versus discontinuation (placebo) did not reduce risk of incident radiographic vertebral fractures (9.8\% vs. 11.3\%; RR 0.86 [95\% CI 0.60, 1.22]) (low SOE). ${ }^{33}$ In the dose-ranging extension study, continued alendronate versus discontinuation did not reduce risk of incident radiographic vertebral fracture between years 6-10, whether considering the $5 \mathrm{mg}$ /day and $10 \mathrm{mg} /$ day alendronate continuation groups together (RR 1.40 [95\% CI 0.52, 3.74]) or separately (calculated by Evidence-based Practice Center). ${ }^{34}$

\section{Bone Mineral Density}

In FLEX, during the 5-year follow-up on continued alendronate versus discontinuation (placebo), though both treatment groups experienced a mean decline in TH-BMD, this BMD loss was significantly smaller in those assigned to continue alendronate $(-1.02 \%$ vs. $-3.38 \%$; mean difference [MD] 2.36 [95\% CI 1.81, 2.90]). ${ }^{33}$ Results for other hip sites showed essentially no change in BMD in women assigned alendronate for an additional five years versus a significantly greater 2 to 3 percent decline from FLEX baseline in those randomized to discontinuation. Although both treatment groups had an increase in LS-BMD during FLEX, the gain was significantly greater in the alendronate continuation group (MD 3.74 [95\% CI 3.03, 4.45]). Authors stated that sensitivity analyses in which data were not carried forward to replace missing values yielded similar results.

The dose-ranging extension studies ${ }^{34,42}$ did not report between treatment group differences in BMD change, but did report results within groups. For study years 6-10, the discontinuation group experienced a mean decline of approximately 2 percent in TH-BMD and FN-BMD and no change in LS-BMD. By comparison, during this period neither TH-BMD or FN-BMD significantly changed while LS-BMD increased in both continuing alendronate dose groups. ${ }^{34}$

\section{Variation in Effects of Drug Holiday as a Function of Patient, Bone, or Osteoporosis Drug Characteristics}

We looked at whether the effects of alendronate continuation versus discontinuation on risk of incident fracture varies as a function of patient, bone, and/or drug characteristics. Eligible studies included evidence for the following potential effect modifiers:

\section{Baseline BMD}

One post hoc FLEX analysis reported that risk of incident nonvertebral fracture and incident clinical vertebral fracture, neither of which differed between the alendronate continuation and discontinuation groups overall, also did not differ as a function of baseline FN-BMD (interaction p-values 0.40 and 0.72 , respectively). ${ }^{33}$ A second post hoc FLEX analysis stratified further and reported that within the subset of women with a prevalent radiographic vertebral fracture at FLEX baseline, the effect of continued alendronate versus discontinuation on risk of incident nonvertebral fracture, clinical vertebral fracture and radiographic vertebral fracture did not differ as a function of baseline FN-BMD. ${ }^{41}$ By comparison, in women without a prevalent radiographic 
vertebral fracture at FLEX baseline, the effect of continued alendronate versus discontinuation on risk of incident nonvertebral fracture differed as a function of baseline FN-BMD (interaction $\mathrm{p}$-value 0.019). These results suggested that in women without a prevalent radiographic vertebral fracture at baseline, compared to women with higher baseline FN-BMD, women with the lowest FN-BMD levels may have greater reduction in risk of incident nonvertebral fracture with continued versus discontinued alendronate. However, neither the effect of continued versus discontinued alendronate on risk of incident clinical vertebral fracture nor incident radiographic vertebral fracture appeared to differ as a function of baseline FN-BMD (interactions not significant).

\section{History of Prior Fracture}

One post hoc FLEX analysis reported that risk of incident nonvertebral fracture between alendronate continuation and discontinuation did not differ as a function of prevalent radiographic vertebral fracture (interaction p-value 0.23). ${ }^{33}$ Similarly, risk of incident clinical vertebral fracture neither differed with treatment overall, nor as a function of prevalent radiographic vertebral fracture (interaction p-value 0.86 ). 


\section{Zoledronic Acid (Zoledronate)}

\section{Key Points}

\section{Key Question 5}

- In postmenopausal women with osteopenia, between zoledronate continued for 2 years and zoledronate stopped after 1 year and switched to placebo for 1 year:

o Evidence was insufficient to draw conclusions about differences in risk of incident clinical fractures.

- In postmenopausal women with osteoporosis, between zoledronate continued for 6 years and zoledronate stopped after 3 years and switched to placebo for 3 years:

o There was a lower risk of incident radiographic vertebral fractures (low SOE).

o There was no difference in risk of incident clinical fractures (moderate SOE) or incident nonvertebral fractures (moderate SOE).

o Evidence was insufficient to draw conclusions about differences in risk of incident clinical vertebral fractures or risk of incident hip fractures.

- In postmenopausal women with osteoporosis, between zoledronate continued for 9 years and zoledronate stopped after 6 years and switched to placebo for 3 years:

0 Evidence was insufficient to draw conclusions about differences in risk of incident clinical fractures or incident radiographic vertebral fractures.

\section{Key Question 6}

- We identified no evidence about whether differences in risk of incident fractures between zoledronate continuation and discontinuation vary as a function of patient, bone or drug characteristics.

\section{Eligible Studies}

Four eligible publications of two unique RCTs compared continuation of zoledronate versus discontinuation of zoledronate (placebo drug holiday) and reported on risk of incident fractures. We rated one of these studies as having high $\mathrm{ROB}^{58}$ and extracted only limited data (Appendix Table D7). The remaining three publications of two unique RCTs all were rated as having low ROB. ${ }^{54-56}$ We extracted additional information from these studies in evidence tables and SOE summary tables (Appendix D).

The earliest study, by McClung and colleagues, enrolled postmenopausal women with osteopenia (LS-BMD $<-1$ and $>-2.5$, FN-BMD $>-2.5$, no grade two or three baseline radiographic vertebral fracture, and zero to one grade one baseline radiographic vertebral fracture). ${ }^{56}$ Participants were randomized to zoledronate $5 \mathrm{mg}$ once annually for 2 years (Z2) versus zoledronate $5 \mathrm{mg}$ once annually for 1 year followed by 1 year of placebo (Z1/P1). The other two publications were extensions of the Health Outcomes and Reduced Incidence with Zoledronic Acid Once Yearly-Pivotal Fracture Trial (HORIZON), an international, multisite trial of 7765 postmenopausal women with osteoporosis (FN-BMD T-score $\leq-2.5$, or FN-BMD Tscore $\leq-1.5$ with at least two mild or one moderate baseline radiographic vertebral fracture). ${ }^{99}$ This trial randomized participants to zoledronate $5 \mathrm{mg}$ once annually for 3 years versus placebo. Following the initial 3-year trial period, women initially randomized to zoledronate were 
randomized to continue zoledronate 3 more years for a total of 6 years (Z6) versus placebo for three more years (Z3/P3). ${ }^{54}$ A second extension was conducted in which women randomized to zoledronate for 6 years were then randomized to remain on zoledronate 3 more years for a total of 9 years (Z9) or switch to placebo for 3 years (Z6/P3). ${ }^{55}$ Table 14 summarizes baseline participant characteristics of the zoledronate continuation versus discontinuation trials.

Table 14. Participant characteristics at the baseline of the zoledronate continuation versus discontinuation trials

\begin{tabular}{|c|c|c|c|}
\hline Characteristic & $\begin{array}{l}\text { HORIZON, First } \\
\text { Extension }^{54}\end{array}$ & $\begin{array}{l}\text { HORIZON, Second } \\
\text { Extension }^{55}\end{array}$ & McClung $^{56}$ \\
\hline Number enrolled and randomized & $N=1233$ & $\mathrm{~N}=190$ & $N=379$ \\
\hline Age, mean years & 76 & 78 & 60 \\
\hline Gender, women & $100 \%$ & NR & $100 \%$ \\
\hline Race, white & NR (mostly European) & NR (mostly European) & $93 \%$ \\
\hline Mean FN-BMD T-score & -2.57 & -2.44 & -1.42 \\
\hline Mean TH-BMD T-score & $-2.07^{\star}$ & $-1.98^{*}$ & NR \\
\hline FN-BMD T-score $\leq-2.5$ & $55 \%$ & $45 \%$ & $0 \%$ \\
\hline FN-BMD T-score $>-2.5$ to -1.5 & $41 \%$ & $47 \%$ & NR \\
\hline FN-BMD T-score >-1.5 & $4 \%$ & $7 \%$ & NR \\
\hline History of clinical fracture & NR & NR & NR \\
\hline $\begin{array}{l}\text { Prevalent radiographic vertebral } \\
\text { fracture, none }\end{array}$ & $39 \%$ & $44 \%$ & NR \\
\hline $\begin{array}{l}\text { Prevalent radiographic vertebral } \\
\text { fracture, one }\end{array}$ & $28 \%$ & $28 \%$ & NR \\
\hline $\begin{array}{l}\text { Prevalent radiographic vertebral } \\
\text { fracture, } \geq 2\end{array}$ & $33 \%$ & $28 \%$ & $0 \%$ \\
\hline Comorbid conditions & NR & NR & NR \\
\hline
\end{tabular}

Abbreviations: BMD=bone mineral density; FN-BMD=femoral neck bone mineral density; HORIZON= Health Outcomes and Reduced Incidence with Zoledronic Acid Once Yearly-Pivotal Fracture Trial; NR=not reported; TH-BMD=total hip bone mineral density

*Calculated by Evidence-based Practice Center.

\section{Outcomes}

\section{Incident Clinical Fractures}

In the McClung trial, incident fractures were neither a primary nor secondary endpoint, but were reported as adverse events. ${ }^{56}$ Incidence of fractures was not separately reported for the second year of the trial, during the time when treatment groups were receiving different treatments, but only for the 2-year trial period as a whole. During this 2-year period, evidence was insufficient about whether risk of any incident clinical fracture differed between the Z2 and Z1/P1 groups (3.0\% vs. 2.2\%; RR=1.37; 95\% CI 0.39, 4.78). 
Incident fractures were secondary endpoints in both HORIZON zoledronate extension RCTs. During the first extension study (years 4-6), there were no differences between the Z6 and Z3/P3 treatment groups in risk of any incident clinical fractures (HR 1.04 [95\% CI 0.71, 1.54]) (moderate SOE) or incident nonvertebral fractures (7.6\% vs. 8.2\%; HR 0.99 [0.7, 1.5]) (moderate $\mathrm{SOE}$ ). However, evidence was insufficient about whether risks between groups differed for incident hip fracture (1.3\% vs. 1.4\%; HR=0.90 [95\% CI 0.33, 2.49]), or incident clinical vertebral fracture (HR 1.81 [95\% CI 0.53, 6.2]). ${ }^{54}$

During the second HORIZON extension period (years 7-9), 190 women enrolled and few incident fractures occurred. Evidence was insufficient about differences between the Z9 and Z6/P3 treatment groups in risk of incident clinical fractures (11\% vs. 9\%; HR 1.11 [95\% CI 0.45, 2.73]). ${ }^{55}$

\section{Incident Radiographic Vertebral Fractures}

In the first HORIZON extension study (years 4-6), risk of incident radiographic vertebral fracture was lower in the Z6 group versus the Z3/P3 group (3.0\% vs. 6.2\%; OR 0.51 [95\% CI $0.26,0.95]$ ) (low SOE). During the second HORIZON extension (years 7-9), there were few incident radiographic vertebral fractures and evidence was insufficient about whether risk differed between the Z9 and Z6/P3 treatment groups (3.2\% vs. 5.3\%; OR 0.58 [95\% CI 0.13, $2.55]) .^{55}$

\section{Change in BMD}

The primary study endpoints in the McClung trial and the first and second HORIZON extension studies were change in LS-BMD, FN-BMD, and TH-BMD, respectively. Study results are reported here, but strength of evidence was not assessed for BMD outcomes in this report.

In the McClung trial, compared with the Z1/P1 group, the Z2 group had a small but statistically significantly larger increase in LS-BMD during the 2-year follow-up period (5.2\% vs. 4.4\%; MD 0.76\% [95\% CI 0.70, 0.82]). Small but statistically significant differences in THBMD and FN-BMD also favored the Z2 group.

In the first HORIZON extension, 75 percent $(\mathrm{n}=921)$ of participants randomized at year 3 completed follow-up BMD measures at year 6. During this interval, BMD levels in the Z3/P3 group regressed slightly but did not fall below pretreatment levels, while BMD levels in the Z6 group remained constant. This resulted in a small but statistically significant difference between treatment groups in change in FN-BMD that favored the Z6 group (MD 1.04\% [95\% CI 0.43, 1.65]). Results similarly favored the Z6 group for change in TH-BMD (MD $1.22 \%$ [95\% CI $0.75,1.70])$. In the second HORIZON extension, 72 percent $(n=136)$ of participants randomized at year 6 completed follow-up BMD measures at year 9 . Mean changes from year 6 to year 9 in FN-BMD and TH-BMD were similar in the Z9 and Z6/P3 groups.

\section{Variation in Effects of Drug Holiday as a Function of Patient, Bone, or Osteoporosis Drug Characteristics}

We identified no eligible trials that compared zoledronate continuation versus discontinuation (placebo drug holiday) and assessed whether risk of incident fracture varied as a function of patient, bone or drug characteristics. 


\section{Denosumab}

\section{Key Points}

\section{Key Question 5}

- Evidence was insufficient to draw conclusions about whether risk of incident clinical fractures differed between denosumab continuation and discontinuation (placebo drug holiday).

- Evidence was insufficient to draw conclusions about whether placebo following prior denosumab treatment was associated with rapid bone loss compared to continued denosumab treatment.

\section{Key Question 6}

- We identified no evidence about whether differences in risk of incident fractures between denosumab continuation and discontinuation vary as a function of patient, bone or drug characteristics.

\section{Eligible Studies}

One eligible publication of one RCT compared denosumab continuation versus discontinuation (placebo drug holiday) and reported on risk of fractures. ${ }^{70}$ Data from this study, which had medium ROB, were extracted in detailed evidence tables, SOE assessments (Appendix D), and summary ROB assessments (Appendix C).

Described in detail previously, this trial randomized postmenopausal women with osteoporosis or low bone mass/osteopenia to placebo $(n=46)$ versus one of seven denosumab regimens $(n=319)$ for 2 years. These dosing regimens included three that were lower and two that were higher than the current U.S. Food and Drug Administration recommended denosumab osteoporosis dosing. After 2 years, individuals were nonrandomly assigned to treatment groups for the following 2 years. Those individuals initially assigned placebo remained assigned to placebo. Five of the groups assigned denosumab, including three of the low dose groups, were assigned to denosumab 60 mg every 6 months for 2 years $(n=231)$, one of the original high dose denosumab groups was switched to placebo for 2 years $(n=47)$, and the last denosumab group was switched to placebo for 1 year before starting denosumab $60 \mathrm{mg}$ every 6 months for 1 year $(\mathrm{n}=41)$.

\section{Outcomes}

\section{Incident Clinical Fractures}

Incident fractures were not reported as primary or secondary outcomes, but only as adverse events. Authors reported that during 4 years of follow-up, among women originally randomized to denosumab, 10.5 percent had an incident clinical fracture and 7.0 percent had an incident osteoporotic fracture (any clinical fracture excluding those of the phalanges, face or those caused by severe trauma). Authors stated that no increase in fracture incidence was observed among the small number of patients who discontinued denosumab treatment, but reported no numerical data 
comparing results between women assigned to continue denosumab and those assigned to discontinue it.

\section{Incident Radiographic Vertebral Fractures}

No information was reported about risk of incident radiographic vertebral fractures.

\section{Change in BMD}

Among women assigned 4 years of denosumab, LS-BMD increased 9.4 to 11.8 percent from baseline and TH-BMD increased 4.0 to 6.1 percent from baseline. In women who received denosumab for 2 years, followed by placebo for 1 year, and then 1 year of denosumab, the increase in LS-BMD and TH-BMD between baseline and year 4 were 9.0 percent and 3.9 percent, respectively. In women randomized to denosumab for 2 years followed by 2 years of placebo, authors reported no numerical data, but stated that BMD after 4 years returned to near its pre-denosumab treatment baseline but was greater than that in women assigned 4 years of placebo. Authors did not report changes in BMD during the placebo periods following prior denosumab.

\section{Variation in Effects of Drug Holiday as a Function of Patient, Bone, or Osteoporosis Drug Characteristics}

We identified no eligible trials that compared denosumab continuation versus discontinuation (placebo drug holiday) and assessed whether the risk of incident fracture varied as a function of patient, bone, or drug characteristics. 


\section{Other Drugs Investigated for Review}

\section{Raloxifene}

We identified no eligible studies that compared raloxifene continuation with discontinuation and reported on risk of incident fracture, or whether differences in risk of incident fractures between raloxifene continuation and discontinuation (placebo drug holiday) vary as a function of patient, bone, or drug characteristics.

\section{Ibandronate}

We identified no eligible studies that compared ibandronate continuation with discontinuation and reported on risk of incident fracture, or whether differences in risk of incident fractures between ibandronate continuation and discontinuation (placebo drug holiday) vary as a function of patient, bone, or drug characteristics.

\section{Risedronate}

We identified no eligible studies that compared risedronate continuation with discontinuation and reported on risk of incident fracture, or whether differences in risk of incident fractures between risedronate continuation and discontinuation (placebo drug holiday) vary as a function of patient, bone, or drug characteristics.

\section{Teriparatide}

We identified no eligible studies that compared teriparatide continuation with discontinuation and reported on risk of incident fracture, or whether differences in risk of incident fractures between teriparatide continuation and discontinuation (placebo drug holiday) vary as a function of patient, bone, or drug characteristics.

\section{Abaloparatide}

We identified no eligible studies that compared abaloparatide continuation with discontinuation and reported on risk of incident fracture, or whether differences in risk of incident fractures between abaloparatide continuation and discontinuation (placebo drug holiday) vary as a function of patient, bone, or drug characteristics.

\section{Hormone Therapy}

We identified no eligible studies that compared hormone therapy continuation with discontinuation and reported on risk of incident fracture, or whether differences in risk of incident fractures between hormone therapy continuation and discontinuation (placebo drug holiday) vary as a function of patient, bone, or drug characteristics. 


\section{Chapter 7. Harms of Drug Holidays}

Chapter 7 discusses Key Questions 7 and 8. Key Question 7 addresses the effect of osteoporosis drug treatment continuation versus discontinuation (placebo drug holiday) on risk of harms. Key Question 8 examines whether the effect on risk of harms varies by patient, bone or drug characteristics.

\section{Alendronate}

\section{Key Points}

\section{Key Question 7}

- In postmenopausal women who had received 5 years of alendronate for femoral neck (FN)-bone mineral density (BMD) T-score $\leq-1.6$ (osteopenia or osteoporosis):

o Between alendronate continuation for 3 more years or 5 more years versus discontinuation (placebo drug holiday), evidence was insufficient to draw conclusions about differences in risk of serious adverse events.

o Between alendronate continuation for 5 more years versus discontinuation, evidence was insufficient to draw conclusions about differences in risk of osteonecrosis of the jaw (ONJ), atypical femoral fracture(AFF), or subtrochanteric or femoral shaft fracture.

- In postmenopausal women who had received 5 years of alendronate for lumbar spine (LS)-BMD T-score $\leq-2.5$ :

o Between alendronate continuation for 2 years versus discontinuation, there was no difference in risk for serious adverse events (low strength of evidence [SOE]).

o Between alendronate continuation for 5 years versus alendronate continuation for 3 more years followed by discontinuation, evidence was insufficient to draw conclusions about differences in risk of serious adverse events.

o Between alendronate continuation up to 5 years versus discontinuation, evidence was insufficient to draw conclusions about differences in risk of ONJ, AFF, or subtrochanteric or femoral shaft fracture.

\section{Key Question 8}

- We identified no evidence about whether the effect of alendronate continuation versus discontinuation on harms varied as a function of patient, bone or drug characteristics.

\section{Eligible Studies}

Six eligible publications of three unique studies compared alendronate continuation versus discontinuation and reported on risk of harms. We rated three of these publications as having low risk of bias (ROB) $)^{33,37,43}$ and three as having medium ROB. ${ }^{34,42,53}$ We extracted detailed information from these studies in evidence tables, SOE summary tables (Appendix D) and ROB summary tables (Appendix C).

As described in detail above, these studies enrolled postmenopausal women who had osteoporosis $^{34,42}$ or a mix of osteoporosis and osteopenia/low bone mass ${ }^{33,41,43}$ when they had been randomly assigned to the alendronate arm of a placebo controlled randomized controlled 
trial (RCT) 5 years earlier. After 5 years of alendronate, the Fracture Intervention Trial Long-

Term Extension (FLEX) study randomized 1099 women to continuation versus discontinuation ${ }^{33}$,

41,43 and the other study nonrandomly assigned two of three alendronate dose ranging groups to continued alendronate and the third alendronate group to placebo. ${ }^{34,42}$

\section{Outcomes}

\section{Serious Adverse Events}

In FLEX, authors reported that during 5 years of follow-up there were no significant differences between the alendronate discontinuation and continuation groups in risk of serious adverse events or mortality, but no numerical data were provided. ${ }^{33}$ In an interim report, after 3 years of FLEX follow-up, serious adverse events data were not reported, and there was no between-group difference in risk of mortality or adverse event causing hospitalization. ${ }^{37}$ In the earlier dose-ranging study, during the year 6-7 extension, risks of any serious adverse event appeared similar between continuation and discontinuation groups (11.9\% vs. $11.3 \%$; risk ratio [RR] 1.05 [95\% confidence interval [CI] 0.57, 1.96]) (low SOE). ${ }^{42}$ Evidence was insufficient about whether risk of serious adverse events differed between alendronate continuation and discontinuation groups during the year 8-10 extension (RR 1.21 [95\% CI 0.75, 1.96]). ${ }^{34}$

\section{Upper Gastrointestinal Adverse Events}

Participants randomized to alendronate continuation for 5 years versus discontinuation in FLEX did not appear to differ in risk of upper gastrointestinal (GI) tract adverse events, but no numerical data were provided. ${ }^{33}$ However, in results reported after 3 years of FLEX follow-up, the discontinuation group had a higher risk of any upper GI event (36\% vs. $30 \%, \mathrm{p}=0.04$ ). ${ }^{37}$ In the earlier dose-ranging study, risk of any upper GI event appeared similar between continuation and discontinuation groups during the year 6-7 extension (15.9\% and $17.2 \%$ in the two continuation groups vs. $18.3 \%$ in the discontinuation group). ${ }^{42}$ During the year 8-10 extension of the dose ranging study, authors again reported that risk of upper GI events did not differ between treatment groups $(24.1 \%, 14.1 \%$ and $27.9 \%$ in discontinuation, alendronate $5 \mathrm{mg} /$ day and alendronate $10 \mathrm{mg}$ /day groups, respectively). ${ }^{34}$

\section{Subtrochanteric or Femoral Shaft Fracture}

In a secondary analysis of FLEX data, investigators reviewed radiology reports and medical records for all hip and femur fractures to identify subtrochanteric and femoral shaft fractures. Pathologic, periprosthetic, and high-energy trauma fractures were excluded. Radiographs were rarely available to review for atypical features, though when atypical features were described in the report, these were recorded. Two women in the alendronate continuation group and one in the discontinuation group had a subtrochanteric or femoral shaft fracture $(0.030 \%$ vs. $0.023 \%$; hazard ratio [HR] 1.33 [95\% CI 0.12, 14.67] (insufficient SOE). ${ }^{43}$

A retrospective cohort study including women at least 45 years of age and with $\geq 3$ years of bisphosphonate use, compared the incidence of osteoporosis-related fractures in those who had discontinued use of bisphosphonates for $\geq 1$ year (drug holiday group) to those who continued bisphosphonate use..$^{53}$ Alendronate accounted for 99 percent of the bisphosphonate use among the cohort. Continual use was defined as persistent (use with $\geq 50 \%$ adherence) or non-persistent (use with $<50 \%$ adherence). Fractures were identified using International Classification of Disease (ICD) $9^{\text {th }}$ Edition diagnosis codes only, with no documentation that radiographs were 
reviewed for features of atypical femoral fractures. Incidence of subtrochanteric or femoral shaft fractures was rare, with $44(0.15 \%)$ in the combined persistent and non-persistent continual use groups combined and three $(0.03 \%)$ in the drug holiday group (odds ratio [OR] 6.03 [95\% CI 1.87, 19.42]) (low SOE).

\section{Osteonecrosis of the Jaw}

In FLEX, authors reported that there were no cases of ONJ. ${ }^{33}$

\section{Atrial Fibrillation}

No eligible publication reported information about incidence of atrial fibrillation.

\section{Variation in Harms During Drug Holiday as a Function of Patient, Bone, or Osteoporosis Drug Characteristics}

We identified no eligible studies that assessed whether the risk of harms between alendronate continuation and discontinuation vary as a function of patient, bone or drug characteristics. 


\section{Zoledronic Acid (Zoledronate)}

\section{Key Points}

\section{Key Question 7}

- In postmenopausal women with osteopenia:

o Between zoledronate for 2 years versus zoledronate for 1 year followed by placebo for 1 year, there was no difference in risk of serious adverse events (moderate SOE).

- In postmenopausal women with osteoporosis:

o Between zoledronate for 6 years versus zoledronate for 3 years followed by placebo for 3 years, there was no difference in risk of serious adverse events (low SOE).

o Between zoledronate for 9 years versus zoledronate for 6 years followed by placebo for 3 years, there was no difference in risk of serious adverse events (low SOE).

- Evidence was insufficient to draw conclusions about differences in risk of AFF or ONJ between zoledronate continuation and discontinuation.

\section{Key Question 8}

- We identified no eligible studies that assessed whether differences in risk of harms between zoledronate continuation and discontinuation vary as a function of patient, bone or drug characteristics.

\section{Eligible Studies}

Three eligible publications of two unique RCTs and no observational studies compared continuation of zoledronate treatment with discontinuation (placebo drug holiday) and reported on risk of harms. All were rated as having low ROB and were described in detail previously. ${ }^{54-56}$ Appendix D provides detailed evidence tables and SOE tables; and Appendix C provides summary ROB assessments for these studies.

In the McClung trial, 379 postmenopausal women with osteopenia were randomized to zoledronate for two years versus zoledronate for one year followed by placebo for one year. ${ }^{56}$ In the first Health Outcomes and Reduced Incidence with Zoledronic Acid Once Yearly-Pivotal Fracture Trial (HORIZON) trial extension, 1233 postmenopausal women who previously had been randomized to receive zoledronate for 3 years for osteoporosis were randomized to receive zoledronate for another 3 years versus placebo. ${ }^{54}$ In the second HORIZON trial extension, 190 women who had been randomized to zoledronate in the first extension were randomized to receive zoledronate for another 3 years versus placebo. ${ }^{55}$

\section{Outcomes}

\section{Serious Adverse Events}

In the McClung trial, risk of harms was not separately reported for the second year of the trial, during which the groups were on different treatments, but only for the 2-year trial period as 
a whole. During the entire 2-year period, risk of serious adverse events was not significantly different between participants assigned to zoledronate continuation versus discontinuation (9.4\% vs. $10.6 \%$; RR 0.91 [95\% CI 0.50, 1.67]) (moderate SOE). ${ }^{56}$ One death due to sepsis was reported in the continuation group. In the HORIZON trial extensions, there also was no difference in risk of serious adverse events between the zoledronate continuation and discontinuation groups, neither during years 4 to 6 (31\% vs. 27\%; RR 1.14 [95\% CI 0.96, 1.36]) (low SOE) nor during years 7 to 9 (26\% vs. 30\%; RR 0.86 [95\% CI 0.54, 1.36]) (low SOE). ${ }^{54,55}$

\section{Atypical Femoral Fractures}

Both HORIZON extension studies reported that no cases of AFF occurred in either the zoledronate continuation or discontinuation group during follow-up, ${ }^{54,55}$ with documentation that radiographs were reviewed for possible AFF features in just one of these reports ${ }^{55}$ (insufficient SOE for both comparisons).

\section{Osteonecrosis of the Jaw}

The McClung trial reported that no cases of ONJ occurred in either treatment group (insufficient SOE). ${ }^{56}$ In the HORIZON extension studies, one case of ONJ was reported in the zoledronate continuation group and none in the zoledronate discontinuation group during years 4 to 6 (insufficient SOE), ${ }^{54}$ and no cases of ONJ occurred in either group during years 7 to 9 (insufficient SOE). ${ }^{55}$

\section{Atrial Fibrillation}

The McClung trial reported that no cases of atrial fibrillation occurred in either the zoledronate continuation or discontinuation group. In the HORIZON extension studies, incidence of atrial fibrillation was not statistically significantly different between the continuation and discontinuation groups during years 4 to $6(3.4 \% \text { vs. } 2.1 \%, p=0.17)^{54}$ or between years 7 to 9 (5.4\% vs. $1.1 \%, \mathrm{p}=0.11){ }^{55}$

\section{Variation in Harms During Drug Holiday as a Function of Patient, Bone, or Osteoporosis Drug Characteristics}

We identified no eligible studies that compared zoledronate continuation with discontinuation (placebo drug holiday) and assessed whether risk of harms varied as a function of patient, bone, or drug characteristics. 


\section{Denosumab}

\section{Key Points}

\section{Key Question 7}

- In postmenopausal women with osteoporosis or osteopenia, between denosumab continuation and discontinuation (placebo drug holiday):

O Evidence was insufficient to draw conclusions about differences in risk of harms, including for risk of AFF, ONJ, and fractures after denosumab discontinuation.

\section{Key Question 8}

- We identified no evidence about whether differences in risk of harms between denosumab continuation and discontinuation vary as a function of patient, bone or drug characteristics.

\section{Eligible Studies}

One eligible publication of one RCT compared denosumab continuation versus discontinuation (placebo drug holiday) and reported on risk of harms. ${ }^{70}$ Appendix D provides detailed evidence tables and strength of evidence tables; and Appendix C provides summary ROB assessments for this study.

Described in detail previously, this trial randomized postmenopausal women with osteoporosis or low bone mass/osteopenia to placebo $(n=46)$ versus one of seven denosumab regimens $(\mathrm{n}=319)$ for 2 years. These dosing regimens included three that were lower and two that were higher than the current U.S. Food and Drug Administration recommended denosumab dosing. After 2 years, individuals initially assigned placebo remained assigned to placebo. Five of the groups assigned denosumab, including three of the low dose groups, were assigned to denosumab 60 mg every 6 months for 2 years $(n=231)$, one of the original high dose denosumab groups was switched to placebo for 2 years $(n=47)$, and the last denosumab group was switched to placebo for 1 year before starting denosumab $60 \mathrm{mg}$ every 6 months for 1 year $(\mathrm{n}=41)$.

\section{Outcomes}

\section{Serious Adverse Events}

Through 4 years, risk of any serious adverse event was 17.8 percent in the women initially assigned to one of the denosumab groups. However, because harms results were reported collectively for all women initially assigned to denosumab, regardless of whether they continued denosumab for 4 years, 2 years, or stopped it for 1 year before restarting it for 1 year, no direct comparison between denosumab continuation and discontinuation groups was possible.

\section{Atypical Femoral Fracture}

No information was reported about incidence of AFF.

\section{Osteonecrosis of the Jaw}

No information was reported about incidence of ONJ. 


\section{Post-Treatment Fractures}

Authors stated that no increase in fracture incidence was observed among the small number of patients who discontinued denosumab treatment, but reported no numerical data comparing results between women assigned to continue denosumab and those assigned to discontinue it.

\section{Variation in Harms During Drug Holiday as a Function of Patient, Bone, or Osteoporosis Drug Characteristics}

We identified no eligible studies that compared denosumab continuation with discontinuation (placebo drug holiday) and assessed whether risk of harms vary as a function of patient, bone or drug characteristics. 


\section{Other Drugs Investigated for Review}

\section{Raloxifene}

We identified no eligible studies that compared raloxifene continuation with discontinuation and reported on risk of harms, or whether differences in risk of harms between raloxifene continuation and discontinuation (placebo drug holiday) vary as a function of patient, bone, or drug characteristics.

\section{Ibandronate}

We identified no eligible studies that compared ibandronate continuation with discontinuation and reported on risk of harms, or whether differences in risk of harms between ibandronate continuation and discontinuation (placebo drug holiday) vary as a function of patient, bone, or drug characteristics.

\section{Risedronate}

We identified no eligible studies that compared risedronate continuation with discontinuation and reported on risk of harms, or whether differences in risk of harms between risedronate continuation and discontinuation (placebo drug holiday) vary as a function of patient, bone, or drug characteristics.

\section{Teriparatide}

We identified no eligible studies that compared teriparatide continuation with discontinuation and reported on risk of harms, or whether differences in risk of harms between teriparatide continuation and discontinuation (placebo drug holiday) vary as a function of patient, bone, or drug characteristics.

\section{Abaloparatide}

We identified no eligible studies that compared abaloparatide continuation with discontinuation and reported on risk of harms, or whether differences in risk of harms between abaloparatide continuation and discontinuation (placebo drug holiday) vary as a function of patient, bone, or drug characteristics.

\section{Hormone Therapy}

We identified no eligible studies that compared hormone therapy continuation with discontinuation and reported on risk of harms, or whether differences in risk of harms between hormone therapy continuation and discontinuation (placebo drug holiday) vary as a function of patient, bone, or drug characteristics. 


\section{Chapter 8. Discussion}

\section{Overview}

Prior systematic reviews have addressed the well-established randomized controlled trial (RCT) evidence on the efficacy of numerous U.S. Food and Drug Administration (FDA) approved medications for short-term reduction in risk of vertebral and nonvertebral fractures, and of bisphosphonates and denosumab for short-term reduction in risk of hip fractures. ${ }^{15,18,100}$ This literature was not the focus of the current report.

Rather, in the current systematic review, we examined evidence pertaining to the clinical question of how long to treat with osteoporosis drugs in patients without prior treatment and what to do after completing a course of short-term osteoporosis drug treatment. More specifically, we evaluated the evidence on the benefits and harms of longer-term osteoporosis drug treatment ( $>3$ years) and the benefits and harms of osteoporosis drug holidays. We defined osteoporosis drug holidays as discontinuation of treatment for at least 1 year after at least 1 year of prior treatment. We also examined whether the effects of these interventions vary as a function of patient, bone, or osteoporosis drug characteristics.

We limited the review of efficacy to RCTs and controlled clinical trials (CCT). For harms, we also evaluated observational studies, requiring contemporaneous control groups to limit bias. We analyzed data only from studies judged to have low or medium risk of bias (ROB). We graded strength of evidence (SOE) for the most clinically important, patient-centered outcomes of incident clinical fractures, incident radiographic vertebral fractures, serious adverse events (SAEs), atypical femoral fractures (AFF), osteonecrosis of the jaw (ONJ), and post-treatment fractures.

\section{Key Findings and Strength of Evidence}

\section{Key Question 1: Efficacy of Long-Term Osteoporosis Drug Treatment}

There were few eligible trials of long-term (>3 years) osteoporosis drug treatment versus placebo. All participants in these studies were postmenopausal women.

In women with osteoporosis, we found high strength evidence that both alendronate and raloxifene for 4 years reduced the risk of radiographic vertebral fractures ( $\sim 35-45 \%$ relative risk reduction [RRR] and 2-5\% absolute risk reduction [ARR]), and that raloxifene reduced risk of clinical vertebral fractures ( 40\% RRR and $2 \%$ ARR). However, neither significantly reduced risk of any type of nonvertebral fracture. In women with osteopenia or osteoporosis, we found high strength evidence that zoledronate for 6 years versus placebo reduced the risk of incident nonvertebral fracture ( 35\% RRR and 5\% ARR) and incident clinical vertebral fracture ( $60 \%$ RRR and 2\% ARR), and moderate strength evidence that zoledronate reduced risk of incident clinical fracture ( $25 \%$ RRR and 5\% ARR). In women with unknown osteoporosis or osteopenia status, we found that both oral estrogen/progestin (5.6 years in those with an intact uterus) and unopposed oral estrogen (7 years in those with prior hysterectomy) versus placebo reduced the risk of both incident clinical fracture ( $\sim 25-30 \%$ RRR, high SOE) and incident hip fracture ( 35\% RRR, moderate SOE). Evidence was insufficient about efficacy of other treatments for long-term fracture prevention. 


\section{Key Question 2: Variation in Efficacy of Long-Term Osteoporosis Drug Treatment as a Function of Patient, Bone, or Osteoporosis Drug Treatment Characteristics}

Efficacy of long-term alendronate appears to vary as a function of baseline bone mineral density (BMD). Between long-term alendronate and placebo, there was a significant 35 percent RRR and 6-7\% ARR in incident clinical fractures among women with osteoporosis, but not in women with osteopenia. Similarly, women with osteoporosis had a 55 percent RRR and 1 percent ARR in incident hip fracture with long-term alendronate versus placebo, but women with higher BMD had no decreased risk. For incident radiographic vertebral fractures, the pattern was different. Women with osteoporosis had a significant 50 percent RRR and 3 percent ARR in incident radiographic vertebral fractures. By comparison, women with an osteopenic femoral neck (FN)BMD T-score of -2.5 to -2 had a similar 45 percent RRR in incident radiographic vertebral fractures with long-term alendronate versus placebo, but experienced fewer events, confidence intervals were wider, and results were not statistically significant. Neither past nonvertebral fracture, World Health Organization Fracture Assessment Tool (FRAX) 10-year major osteoporotic fracture probability calculated with BMD, nor pretreatment levels of bone turnover markers modified the effect of long-term alendronate versus placebo on risk of any incident fracture outcome. Neither age, baseline BMD nor baseline radiographic vertebral fracture modified the effect of long-term raloxifene versus placebo on risk of incident fractures. Few of several tests for interaction suggested that the effect of oral estrogen/progestin or unopposed oral estrogen versus placebo on risk of incident clinical fracture or incident hip fracture varied by patient characteristics, though results suggested a possibly greater benefit in women aged 60-79 years or more than 20 years since menopause compared to younger women or those with more recent menopause. Strength of evidence was not assessed for effect modifiers. We found no other evidence from eligible studies about whether efficacy of long-term treatment with any other osteoporosis drugs varied by patient, bone or drug characteristics.

\section{Key Question 3: Harms of Long-Term Osteoporosis Drug Treatment}

RCT data provided insufficient evidence about whether long-term alendronate, zoledronate, or bisphosphonates as a class increase risk of radiologically confirmed AFF, subtrochanteric or femoral shaft fractures without confirmed AFF features, or ONJ; too few events were observed in eligible trials.

Data from controlled, long-term observational studies suggested that alendronate and bisphosphonates as a class increase risk of both radiologically confirmed AFF (low SOE), and subtrochanteric and femoral shaft fractures that have not been radiologically confirmed to have AFF features (low SOE). Relative risk estimates for these outcomes varied widely across studies, likely related to heterogeneity in study designs. Magnitudes of relative risk appeared higher in studies that defined cases as radiologically confirmed AFF than in those that relied only on diagnostic codes without more specific radiologic confirmation for AFF features. However, some of these AFF risk estimates, including from one recently published study, ${ }^{67}$ may have been biased because they used a control group of patients with subtrochanteric or femoral shaft fractures without AFF features. Results from these studies reflect the probability that a subtrochanteric or femoral shaft fracture, if it occurs, will have AFF features, not the relative risk of sustaining an AFF per se. ${ }^{101}$ Further, because there were no cohort studies that reported cases of radiologically confirmed AFF compared to nonfracture controls, it was not possible from eligible studies to estimate population incidence of AFF attributable to alendronate or 
bisphosphonates as a class. Several long-term observational studies of alendronate collectively suggested an association with increased risk of ONJ (insufficient to low SOE). However, as with AFF, the magnitude of relative risk estimates varied widely between studies, likely due to heterogeneity in case definitions, treatment control groups, and covariate modeling. ONJ incidence rates from eligible cohort studies also varied widely. We found insufficient evidence about whether long-term zoledronate increases risk of AFF or ONJ. The single trial of long-term zoledronate versus placebo reported no cases of AFF or ONJ in either treatment group, and we identified no eligible observational studies that evaluate the risk of these harms with long-term zoledronate versus any control group.

Due to authors' pooling of short- and long-term results, it was not possible to conclude anything about the risk of harms between long-term denosumab and placebo from the one study that met eligibility for this review. The long-term trials of estrogen/progestin and estrogen versus placebo in postmenopausal women with unknown osteoporosis or osteopenia status identified a host of increased harms risks, including stroke, deep venous thrombosis, cardiovascular disease, and the composite outcome of mild cognitive impairment or probable dementia. In addition, estrogen/progestin was associated with an increased risk of coronary heart disease, pulmonary embolism, and invasive breast cancer. Long-term raloxifene versus placebo significantly increased the risk of deep vein thrombosis (DVT) and pulmonary embolism (PE).

\section{Key Question 4: Variation in Harms of Long-Term Osteoporosis Drug Treatment as a Function of Patient, Bone, or Osteoporosis Drug Treatment Characteristics}

We found little evidence about factors that modify risks of harms with long-term osteoporosis drug treatment. One study was inconclusive about whether relative risk for AFF associated with bisphosphonate use increased with age. Several observational studies suggested that $>5$ years of bisphosphonate use increased risk of subtrochanteric or femoral shaft fractures, or radiologically confirmed AFF, more versus a control exposure than did 3 to 5 years of use. For long-term raloxifene versus placebo, one study reported that risk of DVT and PE did not vary as a function of baseline cardiovascular risk and another that risk of incident stroke was lower with raloxifene versus placebo in the subgroup of women at increased baseline cardiovascular risk. Trials of long-term hormone therapy evaluated whether risk of harms varied by a long list of patient characteristics. Though results suggested that risk of breast cancer with estrogen/progestin compared to placebo may be greater with increased duration of prior postmenopausal hormone use, this was the only statistically significant result out of many tests of interaction, and may have been attributable to chance. Strength of evidence was not assessed for effect modifiers.

\section{Key Question 5: Effects of Osteoporosis Drug Treatment Holidays}

RCT data showed that continuation of alendronate for 10 years versus 5 years and continuation of zoledronate for 6 years versus 3 years each lowered risk of one of two measures of incident vertebral fracture, but did not lower risk of any type of nonvertebral fracture. Data were insufficient about whether there is a difference in risk of fractures between zoledronate for 9 years versus 6 years. All these findings were reported in prior systematic reviews. Data from two additional trials, neither recently published, were insufficient to draw conclusions about differences in fracture risk between continuation versus discontinuation of zoledronate for 2 years versus 1 year, or for denosumab 4 years versus either 2 years followed only by placebo or 
2 years followed by placebo and later denosumab resumption. We did not consider observational studies for evidence about risk of fractures with osteoporosis drug treatment holidays due to concerns about confounding by indication.

\section{Key Question 6: Variation in Effect of Osteoporosis Drug Treatment Holidays as a Function of Patient, Bone, or Osteoporosis Drug Treatment Characteristics,}

The little published data about whether the effect of osteoporosis drug continuation versus discontinuation on risk of incident fracture varied by patient, bone or drug characteristics was available only for alendronate. A post hoc subgroup analysis suggested that while alendronate continuation versus discontinuation did not reduce risk of any nonvertebral fracture overall, in the subset of women without a prevalent radiographic vertebral fracture, those with osteoporotic FN-BMD may be more likely than those with osteopenic FN-BMD to have a reduced risk of incident nonvertebral fracture. However, risk of other incident fractures with continued versus discontinued treatment in this patient subgroup did not differ by BMD. In the absence of consistent findings, it is possible this outcome was due to chance. Strength of evidence was not assessed for effect modifiers. We found no evidence about possible effect modifiers of continuing any other osteoporosis drug treatment versus discontinuation on risk of incident fracture.

\section{Key Question 7: Harms During Osteoporosis Drug Treatment Holidays}

Trials of alendronate and zoledronate continuation versus discontinuation found no difference in risk of serious adverse events (insufficient to low SOE). Atrial fibrillation appeared more frequent with zoledronic acid continuation versus discontinuation in both extension studies, but events were uncommon and possible differences between treatment groups were not statistically significant. Too few cases of ONJ occurred in these trials to draw conclusions and studies either provided no information about AFF or reported too few events to draw conclusions about differences in risk of these outcomes between treatment groups (insufficient SOE). For denosumab, one trial assigned postmenopausal women to continuous denosumab treatment for 4 years versus treatment for 2 years followed by placebo versus treatment for 2 years followed by 1 year of placebo and retreatment for 1 year. Authors stated that no increase in fracture incidence was observed among the few women who discontinued denosumab treatment, but reported no numerical data comparing post-treatment fractures risk with fracture risk in women assigned to continue denosumab, those assigned continuous placebo, and those assigned to restart denosumab after a placebo interval.

\section{Key Question 8: Variation in Harms During Osteoporosis Drug Treatment Holidays as a Function of Patient, Bone, or Osteoporosis Drug Treatment Characteristics}

We found no evidence about whether patient, bone or drug characteristics modify the risk of harms between continuation of any osteoporosis drug treatment and discontinuation (placebo drug holiday). 


\section{Applicability}

It was the aim of the review to apply to the general population of adults aged $\geq 50$ years with osteoporosis or osteopenia/low bone mass. Studies that focused on adults $<50$ years of age or on individuals with known secondary causes of osteoporosis (e.g., exogenous glucocorticoids) were excluded. Studies that focused on patients with cancer metastatic to bone or acute fracture healing also were excluded. Review findings may not apply to these populations.

Trial inclusion criteria also limited the generalizability of the review findings. All trials were restricted to postmenopausal women, only a small proportion of participants were among the oldest old (e.g., $\geq 80$ years), and participants generally had low levels of comorbidity. Therefore, the applicability of review findings to younger women, men, the oldest old, and patients with multiple comorbid conditions common in primary care settings is unknown. The evidence on the efficacy of long-term alendronate, denosumab, raloxifene, and hormone therapy versus placebo on risk of incident fracture came almost entirely from trials conducted entirely in the U.S., whereas the long-term zoledronate trial was conducted at a single site in New Zealand. So, the applicability of results to other geographic settings is uncertain. Trial populations often were selected to be low-risk for specific treatment harms (e.g., upper gastrointestinal adverse events for alendronate and venous thromboembolism for raloxifene), so the risk of harms reported in trials may be lower than what would be expected in clinical populations typically treated with these agents. Because most extension studies in the review enrolled only a minority of the individuals who participated in the preceding randomized study phase, their participants may be even less representative of the general osteoporosis population than were original trial enrollees.

\section{Findings in Relation to What Is Already Known}

\section{Efficacy of Short-Term Osteoporosis Drug Treatment}

As reported in a 2014 Agency for Healthcare Research and Quality (AHRQ) review, ${ }^{100}$ a related 2017 American College of Physicians Clinical Practice Guideline, ${ }^{14}$ and a subsequently published trial of abaloparatide, ${ }^{15}$ there is well-established evidence from short-term RCTs (18 to 36 months) in postmenopausal women with osteoporosis that alendronate, risedronate, ibandronate, zoledronate, denosumab, teriparatide, ${ }^{15}$ abaloparatide, and raloxifene lower risk of incident vertebral fractures. All these agents except ibandronate and raloxifene also lower shortterm risk of incident nonvertebral fractures, and alendronate, zoledronate, and denosumab also lower short-term risk of incident hip fractures. RCT evidence on short-term fracture protection in men comes primarily from a single 2-year trial of zoledronate in men with osteoporosis that showed a reduction in risk of incident radiologic vertebral fractures.

The current review did not address efficacy of short-term osteoporosis drug treatment or evaluate possible effect modifiers of short-term treatment outcomes.

\section{Efficacy of Long-Term Osteoporosis Drug Treatment}

Long-term placebo-controlled trials of osteoporosis drugs versus placebo address the question of whether a long-term course of osteoporosis drug treatments lowers risk of fractures compared to a long-term placebo. They do not directly address the question of whether patients who have completed short-term osteoporosis drug treatment will lower their risk of fractures by continuing that treatment. 
The few long-term ( $>3$ year duration), placebo-controlled trials that were included in the prior AHRQ review on osteoporosis drug treatments, and were eligible for the current review and reported interpretable data evaluated alendronate, raloxifene, estrogen/progestin, and unopposed estrogen, respectively. A 4-year alendronate trial in postmenopausal women with osteopenia or osteoporosis, and without a baseline radiographic vertebral fracture, showed a reduction in risk of incident radiographic vertebral fractures and, in the subgroup of women with osteoporosis, also showed a reduction in risk of incident clinical fractures. A 4-year raloxifene trial in postmenopausal women with osteoporosis showed a reduction in clinical vertebral fractures and radiographic vertebral fractures but no reduction in nonvertebral fractures. Two long-term trials in postmenopausal women with unknown osteoporosis or osteopenia status that compared unopposed oral estrogen for 7 years and oral estrogen/progestin for 5.6 years, respectively, versus placebo, reported reduced risks in both incident hip and incident clinical fractures.

Findings from the recently published 6-year RCT comparing zoledronate versus placebo in older women with osteopenia or osteoporosis are a substantial addition to this prior evidence base on the efficacy of long-term osteoporosis drug treatment versus long-term placebo. Zoledronate treatment was associated with significant reductions in risk of both nonvertebral fractures and clinical vertebral fractures. Where reported, the magnitudes of the relative reductions in fracture risk with zoledronate versus placebo look similar between the overall study population and in the osteopenic subset. In contrast, our review found no eligible studies with interpretable trial data on the long-term efficacy of denosumab versus placebo for fracture reduction, and no placebo-controlled trial data on the efficacy of long-term treatment with any sequential osteoporosis drug treatment regimen (e.g., anabolic followed by antiresorptive, denosumab followed by bisphosphonate). Further, we found no eligible long-term trials directly comparing different active osteoporosis drug treatment regimens.

\section{Harms of Long-Term Osteoporosis Drug Treatment}

While the prior AHRQ review reported a significant 10 percent relative increase in risk Tf mild upper gastrointestinal events with bisphosphonates versus placebo, the current review of long-term treatment found no difference in risk. The prior review reported a statistically significant 1.6-fold increase in thromboembolic events with raloxifene compared to placebo, but the current review, deriving long-term data from just the Multiple Outcomes of Raloxifene Evaluation (MORE) trial and follow-up Continuing Outcomes Relevant to Evista (CORE) study found a large magnitude, but borderline statistically significant 3 to 4 -fold increase in risk of DVT and PE. A 2013 meta-analysis estimated that bisphosphonate exposure was associated with a significant increase in risk of subtrochanteric and femoral shaft fractures and AFF considered as a pooled outcome (risk ratio [RR] 1.70 [95\% CI 1.22, 2.37]). ${ }^{102}$ In stratified analysis, that meta-analysis reported that risk in studies that used the American Society for Bone and Mineral Research (ASBMR) criteria to define AFF was RR 11.78 (95\% CI 0.39, 359.69) compared to RR 1.62 (95\% CI 1.18, 2.22) for studies that used the outcome of subtrochanteric or femoral shaft fractures defined primarily by diagnosis codes.

In the current review, we included new studies that examined the association of long-term bisphosphonate treatment with AFF as defined by ASBMR criteria. ${ }^{64,66,67}$ However, study methodology still varies in other ways that likely affected their risk estimates (e.g., selection of exposure control, selection of fracture control, model covariables). In addition, whereas prior reviews have estimated an incidence rate of 0.8 AFFs per 10,000 women treated with 3 years of 
bisphosphonate treatment, ${ }^{103}$ we found that it was not possible from the eligible studies in the current review to estimate a population incidence rate of AFF with long-term treatment. Prior reviews have reported a more than 2-fold increased risk of ONJ with bisphosphonates used for osteoporosis, but also important differences in ONJ case definitions. ${ }^{100}$ We found these same challenges when limiting our review to studies reporting long-term osteoporosis drug treatment. For example, long-term studies differed regarding whether they relied only on diagnostic codes, whether they required that clinical findings were persistent, whether they required pathologic or radiologic diagnostic confirmation, and whether they excluded cases that may have been caused by other factors, such as local radiation.

\section{Effect of Osteoporosis Drug Treatment Holidays}

Most patients diagnosed with osteoporosis (for whom short-term osteoporosis drug trial results are applicable) remain at elevated risk for fractures at the completion of three years of treatment, raising the question of how long patients should be treated with drugs to prevent fracture.

The results of the largest trials of osteoporosis drug treatment continuation versus discontinuation, detailed in the current review, also were described in the 2014 AHRQ review. In one trial, postmenopausal women randomized to alendronate for 10 years versus 5 years had a lower risk of clinical vertebral fractures but no reduction in risk of radiographic vertebral fractures or in any other type of clinical fractures. In a second trial, postmenopausal women randomized to continue zoledronate for a total of 6 years versus 3 years had a lower risk of radiographic vertebral fractures but no difference in risk of any type of clinical vertebral fracture or of any nonvertebral fracture. An older trial of long-term alendronate continuation versus discontinuation reported no reduction in incident clinical fracture or incident radiographic vertebral fractures with longer versus shorter alendronate treatment.

The explanation for the variability in risk of incident vertebral fractures between these longterm bisphosphonate trials is unclear. The studies used comparable definitions and ascertainment methods. The magnitude of relative risk reductions in clinical vertebral fracture with continued alendronate in the Fracture Intervention Trial Long Term Extension (FLEX) trial and in risk of incident radiographic vertebral fracture with continued zoledronate in the Health Outcomes and Reduced Incidence with Zoledronic Acid Once Yearly (HORIZON) extension are large. However, because they are based on a small absolute number of vertebral fractures and results aren't corroborated by all other vertebral fracture results within the same or other trials, our confidence in these findings is somewhat limited. The current review did not identify additional trials that addressed this issue. More studies are needed to clarify the effects of bisphosphonate continuation versus discontinuation on risk of incident clinical and radiographic vertebral fractures.

\section{Variation in Osteoporosis Drug Treatment Outcomes}

The current review highlights the relative paucity of information available to help target which patients are most and least likely to experience reductions in risk of incident fractures or increases in risk of harms from long-term osteoporosis drug treatment or continuing versus discontinuation of osteoporosis drug treatment. Because so few trials examined the effects of long-term or continuing osteoporosis drug treatment on fracture risk and harms, opportunities to investigate factors that predict long-term trial treatment outcomes have been limited. Opportunities to independently validate them in a different trial dataset have been rare. 
Validation is important for guiding clinical decision making because nearly all subgroup analyses and tests for interaction in these studies were conducted post hoc, often when overall results were negative, and therefore are prone to type I error.

Because of sparse data on fracture prevention from long-term treatment trials and the high cost of trials adequately powered to evaluate incident fracture outcomes, many investigators have sought to identify appropriate surrogate endpoints. Some have advocated using change in BMD during treatment as an important clinical outcome. However, we found no trials that reported on whether differences in risk of incident fractures with long-term treatment varied as a function of changes in BMD during that treatment. By comparison, we found one post hoc, subgroup analysis that suggested that in women without a prevalent radiographic vertebral fracture, those with lower FN-BMD may have a greater reduction in risk of incident nonvertebral fracture with continued versus discontinued alendronate. These results were not corroborated by differences in risk of any other fracture type examined, or in women with prevalent radiographic vertebral fractures, and this may have been a chance finding. Though preliminary data from the Foundation for the National Institutes of Health (FNIH) Bone Quality project suggest that early treatment changes in BMD and, to a lesser extent, bone formation markers, may predict the antifracture efficacy of antiresorptive osteoporosis drugs, ${ }^{20,104,105}$ the applicability of these data to long-term treatment is unknown. We found no data about whether any patient, bone or drug characteristics modify the effect of drug holidays on risk of harms.

\section{Limitations of the Comparative Effectiveness Review}

Possible limitations of the review methodology include that information about possible effect modifiers of osteoporosis drug treatment outcomes was not always well described in study statistical analysis sections and was variably reported in tables, figures and narrative text. Therefore, it is possible that some information on effect modifiers that was relevant for the review was overlooked. For this review, we excluded non-English language studies based primarily on concerns about available resources. However, as the literature in this field is almost exclusively published in English-language, and we solicited recommendations for additional studies that might meet eligibility criteria from an National Institute of Health (NIH) Office of Disease Prevention Working Group, an NIH Content Area Expert Group, and a Technical Expert Panel, it is unlikely that important studies were missed. Because we extracted information on change in BMD only from eligible studies that also reported incident fracture outcomes, we likely missed some studies that reported change in BMD. This may have caused a selection bias for this outcome.

Strengths of the review methodology include its emphasis on patient-important efficacy outcomes, primarily incident clinical fractures and secondarily incident radiographic vertebral fractures and harms. Another strength is that SOE was assessed for patient-important outcomes of incident fracture and harms and not for change in BMD. A further strength is that this review required observational studies of AFF, ONJ or atrial fibrillation to have an exposure control group. Although uncontrolled studies might have provided information about incidence of these harms, they would not account for the nonzero risk of these harms in comparable populations not receiving these medications, and likely would have overestimated the risk attributable to these treatments. 


\section{Limitations of the Evidence Base}

The first limitation of the evidence base was the small number of eligible, placebo-controlled long-term trials, most of which were designed with BMD change rather than incident fracture as the primary outcome. There were no long-term trials of denosumab that provided usable data on the risk of incident fractures. There also were no long-term trials of either teriparatide or abaloparatide, both of which have product labeling recommending against lifetime use for more than 2 years. There were few eligible trials of osteoporosis drug continuation versus discontinuation, none of which designated incident fracture as the primary outcome. Consequently, for many incident clinical fracture outcomes, there were relatively few events, and risk estimates were imprecise, limiting strength of evidence. Harms reporting in published studies seemed inconsistent and incomplete despite their likely standard collection and reporting to the pharmaceutical manufacturers and the U.S. FDA. This raised concerns about possible reporting bias. Because there were not multiple long-term or drug holiday studies with comparable populations, interventions, and outcomes, consistency of findings could not be determined. The maximum duration of these trials was another limitation. Though three continuation versus discontinuation studies lasted 9-10 years, two had too few remaining participants by that time to draw meaningful conclusions about the risk of incident clinical fractures.

Another limitation is that studies used different definitions and methods for ascertaining outcomes. For example, incident clinical vertebral fractures in Fracture Intervention Trial (FIT) and FLEX required initial recognition and imaging in the community that was then confirmed by study comparison of community and study vertebral radiographs. In contrast, in MORE and CORE, community recognition was not required and incident clinical vertebral fractures were identified when study participants reported back pain at scheduled study visits, which prompted investigators to order a study vertebral radiograph. Outcome definitions for observational studies that evaluated risk of AFF, ONJ and atrial fibrillation were far more variable. For AFF, studies differed in whether cases were defined solely based on diagnosis codes, whether cases were excluded for trauma or cancer, and whether radiologic review for AFF features was required. These differences likely affected specificity. Studies that compared risk of AFF to that of subtrochanteric or femoral shaft fractures may have provided an exaggerated estimate of the risk of AFF with treatment, instead estimating the risk that patients who have subtrochanteric or femoral shaft fractures have AFF features. To the extent individual osteoporosis drugs differ in their effects on the risk of femur fractures associated with excess trauma, cancer, and with and without radiologic AFF features, these differences also could have affected risk estimates. Still other differences in study design could have affected risk estimates. Examples include differences in treatment control group (e.g., no bisphosphonate use, past bisphosphonate use, raloxifene or calcitonin use), and in the covariables included in the adjusted statistical models. Since individual studies did not evaluate the impact of these different analytic approaches on results, and studies often differed in multiple ways, the differences in the results between these studies were difficult to interpret. These issues apply similarly to the outcome of ONJ. For example, studies that examined the risk of ONJ with long-term treatment differed regarding whether they defined cases only based on diagnostic codes, whether they required that clinical findings were persistent, whether they required pathologic or radiologic diagnostic confirmation, and whether they excluded cases that may have been caused by other factors, such as local radiation. 
The evidence base also was limited in that few studies evaluated possible effect modifiers on risks for incident fracture and harms with long-term osteoporosis drug treatment. Even when these were investigated, nearly all analyses were conducted post hoc, tests of interaction were not always reported, and results were not adjusted for testing of multiple outcomes, raising the likelihood of type 1 errors. We found virtually no data examining the effect of age on differences between treatments in risk for incident fractures and harms. This means that the literature did not provide evidence to inform clinical decisions about whether there is an optimal age to start osteoporosis drug treatment or to stop it.

Inconsistency of results reported by different publications from the same study was another limitation of the evidence base. For example, multiple MORE and CORE study publications reported modestly different risks of DVT and PE associated with long-term raloxifene that were difficult to reconcile, even after considering variation in their analytic cohorts and follow-up periods.

\section{Future Research}

Table 15 summarizes the areas needing future research based on the gaps identified in this review.

Table 15. Future research recommendations

\begin{tabular}{|c|c|c|}
\hline Key Question & Research Gap & Future Research Recommendations \\
\hline \multirow[t]{3}{*}{ General } & $\begin{array}{l}\text { All eligible RCTs of long-term } \\
\text { osteoporosis drug treatment and of } \\
\text { osteoporosis drug continuation versus } \\
\text { discontinuation were conducted in } \\
\text { postmenopausal women and most were } \\
\text { conducted in the U.S. None included } \\
\text { men and few were multinational. }\end{array}$ & $\begin{array}{l}\text { Future RCTs of long-term osteoporosis } \\
\text { drug treatment and of osteoporosis drug } \\
\text { continuation versus discontinuation } \\
\text { should include men and be conducted in a } \\
\text { variety of geographic regions. }\end{array}$ \\
\hline & $\begin{array}{l}\text { Published trials of long-term } \\
\text { osteoporosis drug treatment and of } \\
\text { osteoporosis drug continuation versus } \\
\text { discontinuation have been conducted in } \\
\text { populations that were generally healthy. } \\
\text { Drug treatment benefits and harms in } \\
\text { these populations may not be } \\
\text { generalizable to many of the patients } \\
\text { with osteoporosis in clinical settings, } \\
\text { due to their higher prevalence of } \\
\text { comorbidities. }\end{array}$ & $\begin{array}{l}\text { Future trials of long-term osteoporosis } \\
\text { drug treatment and of osteoporosis drug } \\
\text { continuation versus discontinuation } \\
\text { should be performed in more diverse } \\
\text { populations, including those with multiple } \\
\text { comorbidities. }\end{array}$ \\
\hline & $\begin{array}{l}\text { In spite of the availability of these } \\
\text { effective short-term treatment options to } \\
\text { reduce fracture risk, few men and } \\
\text { women with osteoporosis receive these } \\
\text { treatments and these numbers are } \\
\text { declining. }{ }^{106} \text { Among older U.S. adults } \\
\text { with hip fractures, not treating with } \\
\text { osteoporosis drugs has been estimated } \\
\text { to increase risk of future nonvertebral } \\
\text { fractures. }{ }^{106} \text { Even among patients who } \\
\text { start osteoporosis drug treatment, } \\
\text { adherence and persistence with } \\
\text { treatment are poor. }{ }^{107}\end{array}$ & $\begin{array}{l}\text { Though potential undertreatment of } \\
\text { appropriate patients with osteoporosis } \\
\text { drugs was outside the scope of this } \\
\text { review, future research should examine } \\
\text { how patients and clinicians make } \\
\text { decisions about these treatments. }{ }^{108}\end{array}$ \\
\hline
\end{tabular}




\begin{tabular}{|c|c|c|}
\hline Key Question & Research Gap & Future Research Recommendations \\
\hline \multirow{4}{*}{$\begin{array}{l}\text { KQ1: Among men and } \\
\text { postmenopausal women } \\
\text { aged } \geq 50 \text { years with } \\
\text { osteoporosis* or } \\
\text { osteopenia/low bone } \\
\text { mass, } † \text { what is the } \\
\text { efficacy of long-term (>3 } \\
\text { years) osteoporosis drug } \\
\text { therapy for reducing risk } \\
\text { of incident fracture and on } \\
\text { change in BMD? }\end{array}$} & $\begin{array}{l}\text { Eligible long-term osteoporosis drug } \\
\text { treatment trials have had relatively few } \\
\text { fracture events at longer follow-up } \\
\text { times, resulting in imprecise estimates } \\
\text { of incident fracture risk. This limits } \\
\text { confidence that reported estimates of } \\
\text { risk represent true effects. }\end{array}$ & $\begin{array}{l}\text { Future RCTs of long-term osteoporosis } \\
\text { drug treatment should be designed with } \\
\text { adequate power to compare risk of clinical } \\
\text { fracture endpoints. }\end{array}$ \\
\hline & $\begin{array}{l}\text { There were no eligible long-term } \\
\text { placebo-controlled trials with adequate } \\
\text { power to assess risk of clinical fracture } \\
\text { endpoints that compared long-term } \\
\text { ibandronate, risedronate, or denosumab } \\
\text { with placebo. }\end{array}$ & $\begin{array}{l}\text { Future RCTs with adequate power to } \\
\text { compare risk of clinical fracture endpoints } \\
\text { should be conducted for osteoporosis } \\
\text { drugs that don't yet have established } \\
\text { long-term fracture prevention efficacy, } \\
\text { including ibandronate, risedronate, and } \\
\text { denosumab. }\end{array}$ \\
\hline & $\begin{array}{l}\text { There were no eligible long-term trials } \\
\text { of sequential treatment with adequate } \\
\text { power to assess risk of clinical fracture } \\
\text { endpoints. }\end{array}$ & $\begin{array}{l}\text { Future RCTs with adequate power to } \\
\text { compare risk of clinical fracture endpoints } \\
\text { should be conducted to compare } \\
\text { sequential therapy, including denosumab } \\
\text { followed by bisphosphonate therapy, and } \\
\text { anabolic therapy (e.g. teriparatide or } \\
\text { abaloparatide). Studies should include a } \\
\text { continuous long-term antiresorptive } \\
\text { treatment control group. }\end{array}$ \\
\hline & $\begin{array}{l}\text { There were no eligible long-term } \\
\text { placebo-controlled trials with adequate } \\
\text { power to assess risk of clinical fracture } \\
\text { endpoints that compared long-term } \\
\text { terparatide or abaloparatide with } \\
\text { placebo, likely related to associations of } \\
\text { these treatments with osteosarcoma in } \\
\text { animals. }\end{array}$ & $\begin{array}{l}\text { Future RCTs with adequate power to } \\
\text { compare risk of clinical fracture endpoints } \\
\text { should be conducted to compare anabolic } \\
\text { therapy (e.g. teriparatide or abaloparatide) } \\
\text { followed by antiresorptive therapy versus } \\
\text { antiresorptive therapy alone. }\end{array}$ \\
\hline
\end{tabular}




\begin{tabular}{|c|c|c|}
\hline Key Question & Research Gap & Future Research Recommendations \\
\hline & $\begin{array}{l}\text { Future long-term placebo-controlled } \\
\text { osteoporosis drug trials and } \\
\text { comparative effectiveness trials will be } \\
\text { expensive and time consuming. } \\
\text { Adequate surrogates for incident } \\
\text { fracture are not established. }\end{array}$ & $\begin{array}{l}\text { Analyses should clarify the extent to } \\
\text { which individual and combinations of } \\
\text { potential surrogate markers (e.g., } \\
\text { changes in BMD, bone turnover markers, } \\
\text { and/or QCT measures) account for } \\
\text { differences in risk of incident fractures } \\
\text { between long-term osteoporosis drug } \\
\text { treatment and placebo. The Foundation } \\
\text { for the NIH (FNIH) Bone Quality Project, a } \\
\text { collaboration of Pharma, the federal } \\
\text { government, academia, and an } \\
\text { osteoporosis specialty society, is } \\
\text { evaluating pooled patient-level data from } \\
\text { 140,000 participants in osteoporosis drug } \\
\text { trials to address these questions. A } \\
\text { recently published FNIH meta-regression } \\
\text { reported that early treatment changes in } \\
\text { bone formation markers (but not bone } \\
\text { resorption markers) explain a modest } \\
\text { percentage of the effect of treatment on } \\
\text { incident vertebral fractures, but not on } \\
\text { nonvertebral fractures. Preliminary data } \\
\text { suggest that early changes in BMD } \\
\text { explain a larger percentage of the } \\
\text { treatment effect than do changes in bone } \\
\text { markers, and do so for the effect of } \\
\text { treatment on both vertebral and } \\
\text { nonvertebral fractures. } \\
\text { Observational studies may be faster and } \\
\text { cheaper for estimating fracture risk with } \\
\text { long-term osteoporosis drug treatments, } \\
\text { but are prone to bias. These biases may } \\
\text { be reduced by use of methods to account } \\
\text { for treatment selection, but confounding } \\
\text { by indication cannot entirely be eliminated } \\
\text { and these studies should be interpreted } \\
\text { cautiously to avoid false inferences. }\end{array}$ \\
\hline & $\begin{array}{l}\text { Past placebo-controlled trials of long- } \\
\text { term osteoporosis drug treatment that } \\
\text { included different arms for different } \\
\text { regimens of the osteoporosis drug, } \\
\text { including continuous use, but did not } \\
\text { report results separately for the different } \\
\text { osteoporosis drug regimens did not } \\
\text { allow direct comparisons of the effects } \\
\text { of continuous long-term treatment of } \\
\text { these agents versus placebo on risk of } \\
\text { incident fracture. }\end{array}$ & $\begin{array}{l}\text { Such past placebo-controlled trials of } \\
\text { long-term osteoporosis drug treatment } \\
\text { that included a continuous treatment arm } \\
\text { should separately compare results for the } \\
\text { different osteoporosis regimens to } \\
\text { placebo. }\end{array}$ \\
\hline & $\begin{array}{l}\text { Given strong evidence that several FDA } \\
\text { approved medications reduce short- } \\
\text { term risk (18 months to } 3 \text { years) of new } \\
\text { vertebral and nonvertebral fractures in } \\
\text { postmenopausal women with } \\
\text { osteoporosis, it may not be ethical to } \\
\text { randomize postmenopausal women } \\
\text { with osteoporosis to placebo in future } \\
\text { long-term fracture prevention trials. }\end{array}$ & $\begin{array}{l}\text { Future placebo-controlled trials of long- } \\
\text { term osteoporosis drug treatment may } \\
\text { need to target patient populations different } \\
\text { than those included in the short-term trials } \\
\text { (e.g., not postmenopausal women) and/or } \\
\text { who decline or have contraindications to } \\
\text { medications with documented short-term } \\
\text { nonvertebral fracture protection and/or } \\
\text { allow participants in both treatment } \\
\text { groups to also take other osteoporosis } \\
\text { drugs during the trial. }\end{array}$ \\
\hline
\end{tabular}




\begin{tabular}{|c|c|c|}
\hline Key Question & Research Gap & Future Research Recommendations \\
\hline \multirow[t]{3}{*}{$\begin{array}{l}\text { KQ2: Among men and } \\
\text { postmenopausal women } \\
\text { aged } \geq 50 \text { years with } \\
\text { osteoporosis* or } \\
\text { osteopenia/low bone } \\
\text { mass, } † \text { does efficacy of } \\
\text { long-term (>3 years) } \\
\text { osteoporosis drug therapy } \\
\text { for reducing risk of } \\
\text { incident fracture vary as a } \\
\text { function of patient, bone, } \\
\text { or osteoporosis drug } \\
\text { characteristics? }\end{array}$} & $\begin{array}{l}\text { Many long-term osteoporosis drug } \\
\text { treatment trials were conducted in } \\
\text { populations that were not diverse. Such } \\
\text { study populations may include little } \\
\text { variability to facilitate analyses } \\
\text { regarding whether treatment results } \\
\text { vary as a function of patient } \\
\text { characteristics. } \\
\text { Guidelines are recommending } \\
\text { pharmacologic fracture prevention } \\
\text { therapy for individuals with elevated } \\
\text { estimated fracture risk even in the } \\
\text { absence of trials showing fracture } \\
\text { reduction with treatment of this } \\
\text { population. }\end{array}$ & $\begin{array}{l}\text { Future trials of long-term osteoporosis } \\
\text { drug treatment should include populations } \\
\text { who have not been included in prior trials, } \\
\text { such as men, individuals who are frail and } \\
\text { have multiple comorbidities, nonwhite } \\
\text { women, and the oldest old. This would be } \\
\text { not just to improve the applicability of the } \\
\text { evidence base, but to facilitate analyses } \\
\text { regarding whether treatment results vary } \\
\text { as a function of patient characteristics. } \\
\text { Future studies should be conducted in } \\
\text { populations who have high fracture risk in } \\
\text { the absence of osteoporosis by either } \\
\text { BMD, past hip fracture, or prevalent } \\
\text { vertebral fracture criteria. }\end{array}$ \\
\hline & $\begin{array}{l}\text { Relatively few analyses have evaluated } \\
\text { possible effect modification of patient, } \\
\text { bone or osteoporosis drug } \\
\text { characteristics on the efficacy of long- } \\
\text { term osteoporosis drug treatment for } \\
\text { preventing fractures. }\end{array}$ & $\begin{array}{l}\text { Future studies should investigate the } \\
\text { possible effect modification of } \\
\text { characteristics that could help guide } \\
\text { clinical decision making about which } \\
\text { patients are most and least likely to } \\
\text { experience reductions in fracture risk with } \\
\text { long-term treatment. }\end{array}$ \\
\hline & $\begin{array}{l}\text { Most published analyses that have } \\
\text { examined possible effect modifiers of } \\
\text { long-term treatment efficacy have been } \\
\text { post hoc. Statistical power to detect } \\
\text { significant interactions and significant } \\
\text { associations within subgroups have } \\
\text { been limited. A few trials have } \\
\text { examined many factors in multiple } \\
\text { publications. There has been minimal } \\
\text { independent evaluation of the same } \\
\text { factors in different trials. This could } \\
\text { increase risk of type I errors. }\end{array}$ & $\begin{array}{l}\text { Future studies should specify analysis } \\
\text { plans to investigate possible effect } \\
\text { modification a priori. Such analyses } \\
\text { should be replicated in different trial } \\
\text { datasets. }\end{array}$ \\
\hline \multirow[t]{2}{*}{$\begin{array}{l}\text { KQ3: Among men and } \\
\text { postmenopausal women } \\
\text { aged } \geq 50 \text { years with } \\
\text { osteoporosis* or } \\
\text { osteopenia/low bone } \\
\text { mass, } † \text { what is the risk of } \\
\text { harms associated with } \\
\text { long-term (>3 years) } \\
\text { osteoporosis drug } \\
\text { therapy? }\end{array}$} & $\begin{array}{l}\text { Harms reporting in published long-term } \\
\text { osteoporosis drug trials have not always } \\
\text { appeared systematic and sometimes } \\
\text { has not been quantitative, raising the } \\
\text { risk of reporting bias and preventing } \\
\text { pooling of harms data across multiple } \\
\text { trials. Rare harms potentially associated } \\
\text { with antiresporptive agents sometimes } \\
\text { have not been mentioned. }\end{array}$ & $\begin{array}{l}\text { Future long-term osteoporosis drug trials } \\
\text { should systematically collect, analyze and } \\
\text { report harms data, including both potential } \\
\text { harms common across agents and } \\
\text { required by the FDA (SAEs), drug-specific } \\
\text { adverse effect, and rare harms of concern } \\
\text { (e.g., AFF, ONJ). Results should be } \\
\text { reported including numerators and } \\
\text { denominators to facilitate interpretations } \\
\text { about absolute risk and potential pooling } \\
\text { across studies. }\end{array}$ \\
\hline & $\begin{array}{l}\text { Published observational studies that } \\
\text { evaluated risk of AFF with long-term } \\
\text { osteoporosis drug treatment often have } \\
\text { used definitions of AFF that may not } \\
\text { distinguish AFF from typical femoral } \\
\text { fractures or from femoral fractures } \\
\text { caused by excess trauma, cancer or } \\
\text { other pathological conditions, potentially } \\
\text { biasing estimates of the risk of AFF with } \\
\text { these long-term treatments. }\end{array}$ & $\begin{array}{l}\text { Future observational studies investigating } \\
\text { risk of AFF with long-term antiresorptive } \\
\text { osteoporosis drugs should use standard } \\
\text { definitions for AFF, including appropriate } \\
\text { diagnostic coding, exclusions for excess } \\
\text { trauma, cancer and other pathologic } \\
\text { fractures, and radiologic confirmation that } \\
\text { fractures have ASBMR task force criteria } \\
\text { for AFF. }\end{array}$ \\
\hline
\end{tabular}




\begin{tabular}{|c|c|c|}
\hline Key Question & Research Gap & Future Research Recommendations \\
\hline & $\begin{array}{l}\text { Published observational studies that } \\
\text { evaluated risk of ONJ with long-term } \\
\text { osteoporosis drug treatment often have } \\
\text { used definitions of ONJ that may not } \\
\text { distinguish ONJ from unrelated jaw } \\
\text { conditions. }\end{array}$ & $\begin{array}{l}\text { Future observational studies investigating } \\
\text { risk of ONJ with long-term antiresorptive } \\
\text { osteoporosis drugs should use standard } \\
\text { definitions for ONJ, as per ASBMR Task } \\
\text { Force criteria, including appropriate } \\
\text { diagnostic coding, documented } \\
\text { persistence of exposed bone in the } \\
\text { maxillofacial region persistent for } \geq 8 \\
\text { weeks after recognition by a healthcare } \\
\text { provider, and no history of craniofacial } \\
\text { radiation. }\end{array}$ \\
\hline & $\begin{array}{l}\text { Most observational studies that have } \\
\text { evaluated the association of } \\
\text { osteoporosis drug treatment with rare } \\
\text { harms are case-control studies. Though } \\
\text { this study design is efficient for } \\
\text { estimating relative risks of the rare } \\
\text { harms associated with these drug } \\
\text { exposures, they cannot be used to } \\
\text { estimate the absolute risk of these } \\
\text { harms. }\end{array}$ & $\begin{array}{l}\text { Future retrospective and prospective } \\
\text { cohort studies should be conducted to } \\
\text { estimate the risk of rare harms associated } \\
\text { with antiresorptive osteoporosis drug } \\
\text { treatment to best place these risks in the } \\
\text { context of the effects of these treatments } \\
\text { on other potentially beneficial and harmful } \\
\text { outcomes. }\end{array}$ \\
\hline & $\begin{array}{l}\text { Accelerated bone turnover and rapid } \\
\text { bone loss occur after denosumab } \\
\text { discontinuation. Case series and a large } \\
\text { prospective study using a virtual } \\
\text { placebo in lieu of a control group } \\
\text { suggest that risk of multiple } \\
\text { radiographic vertebral fractures is } \\
\text { increased after denosumab } \\
\text { discontinuation. It is uncertain whether } \\
\text { this risk of post-denosumab vertebral } \\
\text { fractures appears increased only } \\
\text { because of removal of an effective } \\
\text { treatment or it truly is increased } \\
\text { compared to if the patients never had } \\
\text { received denosumab. The magnitude } \\
\text { and duration of risk of post-denosumab } \\
\text { vertebral and nonvertebral fractures are } \\
\text { unclear. The efficacy of different post- } \\
\text { denosumab antiresorptive treatment } \\
\text { regimens for fracture prevention are } \\
\text { unknown. } 109\end{array}$ & $\begin{array}{l}\text { Future research should: (1) better } \\
\text { estimate the magnitude and duration of } \\
\text { risk for post-denosumab vertebral and } \\
\text { nonvertebral fractures; (2) clarify whether } \\
\text { post-denosumab fracture risk only } \\
\text { appears increased because of removal of } \\
\text { an effective treatment or truly is increased } \\
\text { compared to if patients had not received } \\
\text { denosumab at all; and (3) evaluate the } \\
\text { efficacy of different post-denosumab } \\
\text { antiresorptive regimens for fracture } \\
\text { prevention. }{ }^{09}\end{array}$ \\
\hline
\end{tabular}




\begin{tabular}{|c|c|c|}
\hline Key Question & Research Gap & Future Research Recommendations \\
\hline $\begin{array}{l}\text { KQ4: Among men and } \\
\text { postmenopausal women } \\
\text { aged } \geq 50 \text { years with } \\
\text { osteoporosis* or } \\
\text { osteopenia/low bone } \\
\text { mass, } † \text { does the risk of } \\
\text { harms associated with } \\
\text { long-term (>3 years) } \\
\text { osteoporosis drug therapy } \\
\text { vary as a function of } \\
\text { patient, bone, or } \\
\text { osteoporosis drug } \\
\text { characteristics? }\end{array}$ & $\begin{array}{l}\text { There are minimal data from completed } \\
\text { trials or observational studies examining } \\
\text { possible effect modifiers of long-term } \\
\text { osteoporosis drug treatment harms. }\end{array}$ & $\begin{array}{l}\text { Ongoing or completed trials of long-term } \\
\text { osteoporosis drug treatment versus } \\
\text { placebo that are collecting or have } \\
\text { collected harms should perform post hoc } \\
\text { exploratory analyses of possible effect } \\
\text { modifiers of harms to generate } \\
\text { hypotheses to test in future studies. } \\
\text { Studies should use common methods to } \\
\text { enable pooling of results and/or validation } \\
\text { of findings. } \\
\text { Future trials and prospective cohort } \\
\text { studies that compare long-term } \\
\text { osteoporosis drug treatment versus } \\
\text { placebo and systematically collect harms } \\
\text { outcomes, should plan a priori to examine } \\
\text { possible effect modifiers of harms } \\
\text { outcomes. } \\
\text { Future research should investigate } \\
\text { whether findings of extended DXA femur } \\
\text { scans may independently predict risk of } \\
\text { atypical femoral fracture in patients } \\
\text { currently receiving bisphosphonate or } \\
\text { denosumab treatment for osteoporosis. }\end{array}$ \\
\hline \multirow{2}{*}{$\begin{array}{l}\text { KQ5: Among men and } \\
\text { postmenopausal women } \\
\text { aged } \geq 50 \text { years currently } \\
\text { receiving drug therapy } \\
\text { started for osteoporosis* } \\
\text { or osteopenia/low bone } \\
\text { mass to prevent fracture, } \\
\text { what is the effect of } \\
\text { osteoporosis drug } \\
\text { treatment holidays on } \\
\text { incident fracture risk and } \\
\text { improving BMD? }\end{array}$} & $\begin{array}{l}\text { Past trials of osteoporosis drug } \\
\text { treatment continuation versus } \\
\text { discontinuation have been too small to } \\
\text { be adequately powered to evaluate the } \\
\text { effects on incident clinical fracture } \\
\text { outcomes. }\end{array}$ & $\begin{array}{l}\text { Larger trials are needed that compare } \\
\text { osteoporosis drug treatment continuation } \\
\text { versus discontinuation and are powered } \\
\text { to look at incident clinical fracture } \\
\text { outcomes. }\end{array}$ \\
\hline & $\begin{array}{l}\text { No published trials have directly } \\
\text { compared different durations of } \\
\text { osteoporosis drug treatment holidays } \\
\text { with or without restarting osteoporosis } \\
\text { drug treatment and reported on } \\
\text { differences in incident fracture risk } \\
\text { between these groups. }\end{array}$ & $\begin{array}{l}\text { Past or ongoing trials (RCTs or CCTs) } \\
\text { that assigned participants to different } \\
\text { durations of osteoporosis drug treatment } \\
\text { holidays with or without restarting } \\
\text { osteoporosis drug treatment should report } \\
\text { their incident fracture data in a manner } \\
\text { which allows direct comparison of these } \\
\text { fracture outcomes between these different } \\
\text { treatment groups, including comparison } \\
\text { with a continuous osteoporosis drug } \\
\text { treatment if it is available. } \\
\text { New trials should be conducted that } \\
\text { assign participants (RCTs preferable, but } \\
\text { CCTs also would be informative) to } \\
\text { different durations of osteoporosis drug } \\
\text { treatment holidays with or without } \\
\text { restarting osteoporosis drug treatment } \\
\text { and compare risk of incident fractures } \\
\text { between treatment groups. The trials also } \\
\text { should include an arm of continuous } \\
\text { osteoporosis drug treatment (i.e., drug } \\
\text { holiday duration of zero). RCTs are } \\
\text { preferable, but CCTs also would be } \\
\text { informative. }\end{array}$ \\
\hline
\end{tabular}




\begin{tabular}{|c|c|c|}
\hline Key Question & Research Gap & Future Research Recommendations \\
\hline \multirow{2}{*}{$\begin{array}{l}\text { KQ6: Among men and } \\
\text { postmenopausal women } \\
\text { aged } \geq 50 \text { years currently } \\
\text { receiving drug therapy } \\
\text { started for osteoporosis* } \\
\text { or osteopenia/low bone } \\
\text { mass } † \text { to prevent fracture, } \\
\text { does the effect of } \\
\text { osteoporosis drug } \\
\text { treatment holidays on } \\
\text { incident fracture risk vary } \\
\text { as a function of patient, } \\
\text { bone, or osteoporosis } \\
\text { drug characteristics? }\end{array}$} & $\begin{array}{l}\text { Past trials of osteoporosis drug } \\
\text { treatment continuation versus } \\
\text { discontinuation have been inadequately } \\
\text { powered to evaluate for possible effect } \\
\text { modification of treatment effects on risk } \\
\text { of incident clinical fracture outcomes. }\end{array}$ & $\begin{array}{l}\text { Larger trials are needed that compare } \\
\text { osteoporosis drug treatment continuation } \\
\text { versus discontinuation and are powered } \\
\text { to look at incident clinical fracture } \\
\text { outcomes and evaluate whether } \\
\text { differences in incident fracture risk vary as } \\
\text { a function of a priori identified patient, } \\
\text { bone or osteoporosis drug characteristics. }\end{array}$ \\
\hline & $\begin{array}{l}\text { No published trials have directly } \\
\text { compared different durations of } \\
\text { osteoporosis drug treatment holidays } \\
\text { with or without restarting osteoporosis } \\
\text { drug treatment, reported on differences } \\
\text { in incident fracture risk between these } \\
\text { groups, and evaluated whether } \\
\text { differences in incident fracture risk vary } \\
\text { as a function of patient, bone or } \\
\text { osteoporosis drug characteristics. }\end{array}$ & $\begin{array}{l}\text { New trials that compare osteoporosis drug } \\
\text { continuation versus discontinuation (as } \\
\text { detailed in KQ5 section) and } \\
\text { systematically collect incident fracture } \\
\text { outcomes, should plan a priori to examine } \\
\text { possible effect modifiers of incident } \\
\text { fracture risk. Factors to explore as } \\
\text { possible effect modifiers include age, } \\
\text { BMD before and during the drug holiday, } \\
\text { and levels of bone turnover markers } \\
\text { before and during the drug holiday. }\end{array}$ \\
\hline $\begin{array}{l}\text { KQ7: Among men and } \\
\text { postmenopausal women } \\
\text { aged } \geq 50 \text { years currently } \\
\text { receiving drug therapy } \\
\text { started for osteoporosis* } \\
\text { or osteopenia/low bone } \\
\text { mass } † \text { to prevent fracture, } \\
\text { what is the risk of harms } \\
\text { of osteoporosis drug } \\
\text { treatment holidays? }\end{array}$ & $\begin{array}{l}\text { No published trials have directly } \\
\text { compared different durations of } \\
\text { osteoporosis drug treatment holidays } \\
\text { with or without restarting osteoporosis } \\
\text { drug treatment and reported on } \\
\text { differences in risk of harms between } \\
\text { these groups. }\end{array}$ & $\begin{array}{l}\text { New trials should be conducted that } \\
\text { assign participants (RCTs preferable, but } \\
\text { CCTs also would be informative) to } \\
\text { different durations of osteoporosis drug } \\
\text { treatment holidays with or without } \\
\text { restarting osteoporosis drug treatment. } \\
\text { Studies even could alternate multiple } \\
\text { cycles of active treatment and } \\
\text { discontinuation. These trials should } \\
\text { systematically collect, analyze and report } \\
\text { risk of harms between treatment groups, } \\
\text { including both common and rare harms. } \\
\text { The trials also should include an arm of } \\
\text { continuous osteoporosis drug treatment } \\
\text { (i.e., drug holiday duration of zero). }\end{array}$ \\
\hline \multirow{2}{*}{$\begin{array}{l}\text { KQ8: Among men and } \\
\text { postmenopausal women } \\
\text { aged } \geq 50 \text { years currently } \\
\text { receiving drug therapy } \\
\text { started for osteoporosis* } \\
\text { or osteopenia/low bone } \\
\text { mass } † \text { to prevent fracture, } \\
\text { does risk of harms } \\
\text { associated with } \\
\text { osteoporosis drug } \\
\text { treatment holidays vary as } \\
\text { a function of patient, } \\
\text { bone, or osteoporosis } \\
\text { drug characteristics? }\end{array}$} & $\begin{array}{l}\text { We found limited evidence from eligible } \\
\text { studies about whether patient, bone or } \\
\text { osteoporosis drug characteristics } \\
\text { modify risk for harms associated with } \\
\text { osteoporosis drug continuation versus } \\
\text { discontinuation. }\end{array}$ & $\begin{array}{l}\text { New trials that compare osteoporosis drug } \\
\text { continuation versus discontinuation (as } \\
\text { detailed in KQ7 section) and } \\
\text { systematically collect harms outcomes, } \\
\text { should plan a priori to analyze and report } \\
\text { possible effect modifiers of harms } \\
\text { outcomes. }\end{array}$ \\
\hline & $\begin{array}{l}\text { Ongoing or completed trials of } \\
\text { osteoporosis drug continuation versus } \\
\text { discontinuation that are in the process } \\
\text { of collecting or already have collected } \\
\text { harms did not plan a priori to evaluate } \\
\text { possible effect modification of treatment } \\
\text { harms. }\end{array}$ & $\begin{array}{l}\text { Ongoing or completed trials of } \\
\text { osteoporosis drug continuation versus } \\
\text { discontinuation that are collecting or have } \\
\text { collected harms should perform post hoc } \\
\text { exploratory analyses of possible effect } \\
\text { modifiers of harms to generate } \\
\text { hypotheses to test in future studies, with } \\
\text { possible coordination between different } \\
\text { ongoing or completed studies to use } \\
\text { common methods and/or to validate } \\
\text { findings. }\end{array}$ \\
\hline
\end{tabular}

Abbreviations: AFF=atypical femoral fracture; ASBMR=American Society of Bone and Mineral Research; BMD=bone mineral density; CCT=controlled clinical trials; FDA=U.S. Food and Drug Administration; HRpQCT=high-resolution peripheral quantitative computed tomography; $\mathrm{ONJ}=$ osteonecrosis of the jaw; RCT=randomized clinical trials; $\mathrm{SAE}=$ serious adverse event.

*Osteoporosis defined by hip or lumbar spine dual-energy x-ray absorptiometry (DXA) BMD T-score <-2.5, past clinical hip or vertebral fracture, or prevalent radiographic vertebral fracture

†Osteopenia/low bone mass defined by hip or lumbar spine DXA BMD T-score $<-1.0$ and $>-2.5$ 


\section{Conclusions}

Only alendronate, zoledronate, and oral hormone therapy reduced nonvertebral fractures with long-term treatment. However, for all these agents, these fracture benefits were limited to mostly older, postmenopausal women. They were further limited to women with osteopenia or osteoporosis for zoledronate, and to women with osteoporosis for alendronate. Absolute reductions in clinical fractures with long-term bisphosphonates appeared far greater than absolute increases in risk of AFF and ONJ with these treatments. However, reductions in hip fracture with long-term oral hormone therapy appear offset by risk of serious harms. In patients with prior osteoporosis drug treatment, continued treatment appeared to reduce vertebral fractures but not nonvertebral fractures, and may increase risk of AFF. While fracture benefits of continued osteoporosis drug treatment versus drug holiday numerically appeared to outweigh these risks, the more limited morbidity prevented and greater uncertainty about the outcome measures and risk estimates require further investigation to better inform clinical decisions about continuing treatment. This research should include examination of how these benefits and risks vary as a function of patient, bone, and drug treatment characteristics (e.g., age, sex, comorbidity, pre-drug holiday BMD, duration of prior osteoporosis drug treatment). Further, incorporating fracture morbidity in a decision model possibly would help patients weigh their trade-offs more easily. 


\section{References}

1. Consensus development conference: diagnosis, prophylaxis, and treatment of osteoporosis. Am J Med. 1993 Jun;94(6):646-50. PMID: 8506892.

2. Assessment of fracture risk and its application to screening for postmenopausal osteoporosis. Report of a WHO Study Group. World Health Organ Tech Rep Ser. 1994;843:1-129. PMID: 7941614.

3. Wright NC, Looker AC, Saag KG, et al. The recent prevalence of osteoporosis and low bone mass in the United States based on bone mineral density at the femoral neck or lumbar spine. J Bone Miner Res. 2014 Nov;29(11):2520-6. doi: 10.1002/jbmr.2269. PMID: 24771492.

4. Burge R, Dawson-Hughes B, Solomon DH, et al. Incidence and economic burden of osteoporosis-related fractures in the United States, 2005-2025. J Bone Miner Res. 2007 Mar;22(3):465-75. doi: 10.1359/jbmr.061113. PMID: 17144789.

5. Katsoulis M, Benetou V, Karapetyan T, et al. Excess mortality after hip fracture in elderly persons from Europe and the USA: the CHANCES project. J Intern Med. 2017 Mar;281(3):300-10. doi: 10.1111/joim.12586. PMID: 28093824.

6. Schousboe JT. Epidemiology of Vertebral Fractures. J Clin Densitom. 2016 JanMar;19(1):8-22. doi: 10.1016/j.jocd.2015.08.004. PMID: 26349789.

7. Cooper C, Atkinson EJ, O'Fallon WM, et al. Incidence of clinically diagnosed vertebral fractures: a population-based study in Rochester, Minnesota, 1985-1989. J Bone Miner Res. 1992 Feb;7(2):221-7. doi: 10.1002/jbmr.5650070214. PMID: 1570766.

8. Ensrud KE, Blackwell TL, Fink HA, et al. What Proportion of Incident Radiographic Vertebral Fractures in Older Men Is Clinically Diagnosed and Vice Versa: A Prospective Study. J Bone Miner Res. 2016 Aug;31(8):1500-3. doi: 10.1002/jbmr.2831. PMID: 26969847.
9.

Ettinger B, Black DM, Mitlak BH, et al. Reduction of vertebral fracture risk in postmenopausal women with osteoporosis treated with raloxifene: results from a 3-year randomized clinical trial. Multiple Outcomes of Raloxifene Evaluation (MORE) Investigators. JAMA. 1999 Aug 18;282(7):637-45. PMID: 10517716.

10. Fink HA, Milavetz DL, Palermo L, et al. What proportion of incident radiographic vertebral deformities is clinically diagnosed and vice versa? J Bone Miner Res. 2005;20(7):1216-22.

11. Maricic M, Adachi JD, Sarkar S, et al. Early effects of raloxifene on clinical vertebral fractures at 12 months in postmenopausal women with osteoporosis. Archives of internal medicine. 2002;162(10):1140-3.

12. Nevitt MC, Ettinger B, Black DM, et al. The association of radiographically detected vertebral fractures with back pain and function: a prospective study. Ann Intern Med. 1998 May 15;128(10):793-800. PMID: 9599190.

13. Fink HA, Litwack-Harrison S, Ensrud KE, et al. Association of Incident, Clinically Undiagnosed Radiographic Vertebral Fractures With Follow-Up Back Pain Symptoms in Older Men: the Osteoporotic Fractures in Men (MrOS) Study. J Bone Miner Res. 2017 Nov;32(11):2263-8. doi: 10.1002/jbmr.3215. PMID: 28880398.

14. Qaseem A, Forciea MA, McLean RM, et al. Treatment of Low Bone Density or Osteoporosis to Prevent Fractures in Men and Women: A Clinical Practice Guideline Update from the American College of Physicians. Ann Intern Med. 2017 May 09doi: 10.7326/M15-1361. PMID: 28492856.

15. Miller PD, Hattersley G, Riis BJ, et al. Effect of Abaloparatide vs Placebo on New Vertebral Fractures in Postmenopausal Women With Osteoporosis: A Randomized Clinical Trial. JAMA. 2016 Aug

16;316(7):722-33. doi: 10.1001/jama.2016.11136. PMID: 27533157. 
16. FRAX WHO Fracture Risk Assessment Tool.https://www.sheffield.ac.uk/FRAX/ind ex.aspx. Accessed on February 27, 2018.

17. McClung MR, Geusens P, Miller PD, et al. Effect of risedronate on the risk of hip fracture in elderly women. Hip Intervention Program Study Group. N Engl J Med. 2001 Feb 1;344(5):333-40. doi: 10.1056/NEJM200102013440503. PMID: 11172164.

18. Qaseem A, Forciea MA, McLean RM, et al. Treatment of Low Bone Density or Osteoporosis to Prevent Fractures in Men and Women: A Clinical Practice Guideline Update From the American College of Physicians. Ann Intern Med. 2017 Jun 6;166(11):818-39. doi: 10.7326/M15-1361. PMID: 28492856.

19. Shane E, Burr D, Abrahamsen B, et al. Atypical subtrochanteric and diaphyseal femoral fractures: second report of a task force of the American Society for Bone and Mineral Research. J Bone Miner Res. 2014 Jan;29(1):1-23. doi: 10.1002/jbmr.1998. PMID: 23712442.

20. Bauer DC, Black DM, Bouxsein ML, et al. Treatment-Related Changes in Bone Turnover and Fracture Risk Reduction in Clinical Trials of Anti-Resorptive Drugs: A Meta-Regression. J Bone Miner Res. 2018 Apr;33(4):634-42. doi: 10.1002/jbmr.3355. PMID: 29318649.

21. Sellmeyer DE. Atypical fractures as a potential complication of long-term bisphosphonate therapy. JAMA. 2010 Oct 6;304(13):1480-4. doi: 10.1001/jama.2010.1360. PMID: 20924014.

22. Adler RA, El-Hajj Fuleihan G, Bauer DC, et al. Managing Osteoporosis in Patients on Long-Term Bisphosphonate Treatment: Report of a Task Force of the American Society for Bone and Mineral Research. J Bone Miner Res. 2016 Jan;31(1):16-35. doi: 10.1002/jbmr.2708. PMID: 26350171.
23. Watts NB, Bilezikian JP, Camacho PM, et al. American Association of Clinical Endocrinologists Medical Guidelines for Clinical Practice for the diagnosis and treatment of postmenopausal osteoporosis: executive summary of recommendations. Endocr Pract. 2010 Nov-Dec;16(6):1016-9. PMID: 21216723.

24. Compston J, Bowring C, Cooper A, et al. Diagnosis and management of osteoporosis in postmenopausal women and older men in the UK: National Osteoporosis Guideline Group (NOGG) update 2013. Maturitas. 2013 Aug;75(4):392-6. doi: 10.1016/j.maturitas.2013.05.013. PMID: 23810490.

25. Cummings SR, Ferrari S, Eastell R, et al. Vertebral Fractures After Discontinuation of Denosumab: A Post Hoc Analysis of the Randomized Placebo-Controlled FREEDOM Trial and Its Extension. J Bone Miner Res. 2018 Feb;33(2):190-8. doi: 10.1002/jbmr.3337. PMID: 29105841.

26. Moher D, Liberati A, Tetzlaff J, et al. Preferred reporting items for systematic reviews and meta-analyses: the PRISMA statement. Journal of Clinical Epidemiology. 2009 Oct;62(10):1006-12. doi: 10.1016/j.jclinepi.2009.06.005. PMID: 19631508.

27. Viswanath B, Jose SP, Squassina A, et al. Cellular models to study bipolar disorder: A systematic review. Journal of Affective Disorders. 201511 Jun;184:36-50. doi: http://dx.doi.org/10.1016/j.jad.2015.05.037. PMID: 2015111614.

28. Berkman ND, Lohr K, Ansari M, et al. Grading the strength of a body of evidence when assessing health care interventions for the effective health care program of the Agency for Healthcare Research and Quality: An update. . Rockville, MD: Agency for Healthcare Research and Quality; 2013. (vol. AHRQ Publication No. 13(14)-EHC 130-EF).

29. Guyatt GH, Oxman AD, Kunz R, et al. GRADE guidelines 6. Rating the quality of evidence--imprecision. J Clin Epidemiol. 2011 Dec;64(12):1283-93. doi: 10.1016/j.jclinepi.2011.01.012. PMID: 21839614. 
30. Atkins D, Chang S, Gartlehner G, et al. Assessing the applicability of studies when comparing medical interventions Agency for Healthcare Research and Quality. January 2011.

31. Bauer DC, Black D, Ensrud K, et al. Upper gastrointestinal tract safety profile of alendronate: the fracture intervention trial. Arch Intern Med. 2000 Feb 28;160(4):51725. PMID: 10695692.

32. Bauer DC, Garnero P, Hochberg MC, et al. Pretreatment levels of bone turnover and the antifracture efficacy of alendronate: the fracture intervention trial. J Bone Miner Res. 2006 Feb;21(2):292-9. doi:

10.1359/JBMR.051018. PMID: 16418785.

33. Black DM, Schwartz AV, Ensrud KE, et al. Effects of continuing or stopping alendronate after 5 years of treatment: the Fracture Intervention Trial Long-term Extension (FLEX): a randomized trial. JAMA. 2006 Dec 27;296(24):2927-38. doi: 10.1001/jama.296.24.2927. PMID: 17190893.

34. Bone HG, Hosking D, Devogelaer JP, et al. Ten years' experience with alendronate for osteoporosis in postmenopausal women. $\mathrm{N}$ Engl J Med. 2004 Mar 18;350(12):1189-99. doi: 10.1056/NEJMoa030897. PMID: 15028823.

35. Cummings SR, Black DM, Thompson DE, et al. Effect of alendronate on risk of fracture in women with low bone density but without vertebral fractures: results from the Fracture Intervention Trial. JAMA. 1998 Dec 23-30;280(24):2077-82. PMID: 9875874.

36. Donaldson MG, Palermo L, Ensrud KE, et al. Effect of alendronate for reducing fracture by FRAX score and femoral neck bone mineral density: the Fracture Intervention Trial. J Bone Miner Res. 2012 Aug;27(8):1804-10. doi: 10.1002/jbmr.1625. PMID: 22492479.

37. Ensrud KE, Barrett-Connor EL, Schwartz A, et al. Randomized trial of effect of alendronate continuation versus discontinuation in women with low BMD: results from the Fracture Intervention Trial long-term extension. J Bone Miner Res. 2004 Aug;19(8):1259-69. doi: 10.1359/JBMR.040326. PMID: 15231012.
38. Jamal SA, Bauer DC, Ensrud KE, et al. Alendronate treatment in women with normal to severely impaired renal function: an analysis of the fracture intervention trial. J Bone Miner Res. 2007 Apr;22(4):503-8. doi: 10.1359/jbmr.070112. PMID: 17243862 .

39. Quandt SA, Thompson DE, Schneider DL, et al. Effect of alendronate on vertebral fracture risk in women with bone mineral density $\mathrm{T}$ scores of-1.6 to -2.5 at the femoral neck: the Fracture Intervention Trial. Mayo Clin Proc. 2005 Mar;80(3):343-9. PMID: 15757015.

40. Ryder KM, Cummings SR, Palermo L, et al. Does a history of non-vertebral fracture identify women without osteoporosis for treatment? J Gen Intern Med. 2008 Aug;23(8):1177-81. doi: 10.1007/s11606008-0622-0. PMID: 18459010.

41. Schwartz AV, Bauer DC, Cummings SR, et al. Efficacy of continued alendronate for fractures in women with and without prevalent vertebral fracture: the FLEX trial. J Bone Miner Res. 2010 May;25(5):976-82. doi: 10.1002/jbmr.11. PMID: 20200926.

42. Tonino RP, Meunier PJ, Emkey R, et al. Skeletal benefits of alendronate: 7-year treatment of postmenopausal osteoporotic women. Phase III Osteoporosis Treatment Study Group. J Clin Endocrinol Metab. 2000 Sep;85(9):3109-15. doi: 10.1210/jcem.85.9.6777. PMID: 10999794.

43. Black DM, Kelly MP, Genant HK, et al. Bisphosphonates and fractures of the subtrochanteric or diaphyseal femur. N Engl J Med. 2010 May 13;362(19):1761-71. doi: 10.1056/NEJMoa1001086. PMID: 20335571.

44. Abrahamsen B, Eiken P, Eastell R. Subtrochanteric and diaphyseal femur fractures in patients treated with alendronate: a register-based national cohort study. J Bone Miner Res. 2009 Jun;24(6):1095-102. doi: 10.1359/jbmr.081247. PMID: 19113931. 
45. Chiu WY, Chien JY, Yang WS, et al. The risk of osteonecrosis of the jaws in Taiwanese osteoporotic patients treated with oral alendronate or raloxifene. J Clin Endocrinol Metab. 2014 Aug;99(8):272935. doi: 10.1210/jc.2013-4119. PMID: 24758181.

46. Lin TC, Yang CY, Kao Yang YH, et al. Incidence and risk of osteonecrosis of the jaw among the Taiwan osteoporosis population. Osteoporos Int. 2014 May;25(5):1503-11. doi: 10.1007/s00198014-2624-6. PMID: 24515577.

47. Chiu WY, Yang WS, Chien JY, et al. The influence of alendronate and tooth extraction on the incidence of osteonecrosis of the jaw among osteoporotic subjects. PLoS ONE. 2018 April;13 (4) PMID: 621788516.

48. Chapurlat RD, Palermo L, Ramsay P, et al. Risk of fracture among women who lose bone density during treatment with alendronate. The Fracture Intervention Trial. Osteoporos Int. 2005 Jul;16(7):842-8. doi: 10.1007/s00198-004-1770-7. PMID: 15580479 .

49. Nevitt MC, Ross PD, Palermo L, et al. Association of prevalent vertebral fractures, bone density, and alendronate treatment with incident vertebral fractures: effect of number and spinal location of fractures. The Fracture Intervention Trial Research Group. Bone. 1999 Nov;25(5):613-9. PMID: 10574584.

50. Abrahamsen B, Eiken P, Eastell R. Cumulative alendronate dose and the longterm absolute risk of subtrochanteric and diaphyseal femur fractures: a register-based national cohort analysis. J Clin Endocrinol Metab. 2010 Dec;95(12):5258-65. doi: 10.1210/jc.2010-1571. PMID: 20843943.

51. Abrahamsen B, Eiken P, Prieto-Alhambra D, et al. Risk of hip, subtrochanteric, and femoral shaft fractures among mid and long term users of alendronate: nationwide cohort and nested case-control study. BMJ. 2016 Jun 28;353:i3365. doi: 10.1136/bmj.i3365. PMID: 27353596.
52. Wang CC, Lu HT, Dusetzina SB, et al. The Association Between Long-Term Bisphosphonate Use and the Risk of Fracture Among Women Aged 50 or Older with Osteoporosis. J Womens Health (Larchmt). 2016 Jul;25(7):738-46. doi: 10.1089/jwh.2015.5617. PMID: 27096405.

53. Adams AL, Adams JL, Raebel MA, et al. Bisphosphonate Drug Holiday and Fracture Risk: A Population-Based Cohort Study. J Bone Miner Res. 2018 Jul;33(7):1252-9. doi: 10.1002/jbmr.3420. PMID: 29529334.

54. Black DM, Reid IR, Boonen S, et al. The effect of 3 versus 6 years of zoledronic acid treatment of osteoporosis: a randomized extension to the HORIZON-Pivotal Fracture Trial (PFT). J Bone Miner Res. 2012 Feb;27(2):243-54. doi: 10.1002/jbmr.1494. PMID: 22161728.

55. Black DM, Reid IR, Cauley JA, et al. The effect of 6 versus 9 years of zoledronic acid treatment in osteoporosis: a randomized second extension to the HORIZON-Pivotal Fracture Trial (PFT). J Bone Miner Res. 2015 May;30(5):934-44. doi: 10.1002/jbmr.2442. PMID: 25545380.

56. McClung M, Miller P, Recknor C, et al. Zoledronic acid for the prevention of bone loss in postmenopausal women with low bone mass: a randomized controlled trial. Obstet Gynecol. 2009 Nov;114(5):9991007. doi: 0.1097/AOG.0b013e3181bdce0a. PMID: 20168099.

57. Reid IR, Horne AM, Mihov B, et al. Fracture Prevention with Zoledronate in Older Women with Osteopenia. N Engl J Med. 2018 Dec 20;379(25):2407-16. doi: 10.1056/NEJMoa1808082. PMID: 30575489.

58. Cosman F, Cauley JA, Eastell R, et al. Reassessment of fracture risk in women after 3 years of treatment with zoledronic acid: when is it reasonable to discontinue treatment? J Clin Endocrinol Metab. 2014 Dec;99(12):4546-54. doi: 10.1210/jc.20141971. PMID: 25215556.

59. Sorensen OH, Crawford GM, Mulder H, et al. Long-term efficacy of risedronate: a 5year placebo-controlled clinical experience. Bone. 2003 Feb;32(2):120-6. PMID: 12633783. 
60. Ste-Marie LG, Sod E, Johnson T, et al. Five years of treatment with risedronate and its effects on bone safety in women with postmenopausal osteoporosis. Calcif Tissue Int. 2004 Dec;75(6):469-76. doi: 10.1007/s00223-004-0039-7. PMID: 15478000.

61. Miller PD, Recker RR, Reginster JY, et al. Efficacy of monthly oral ibandronate is sustained over 5 years: the MOBILE longterm extension study. Osteoporos Int. 2012 Jun;23(6):1747-56. doi: 10.1007/s00198011-1773-0. PMID: 21953471.

62. Erviti J, Alonso A, Oliva B, et al. Oral bisphosphonates are associated with increased risk of subtrochanteric and diaphyseal fractures in elderly women: a nested case-control study. BMJ Open. 2013 Jan 30;3(1)doi: 10.1136/bmjopen-2012002091. PMID: 23370011.

63. Kim SY, Schneeweiss S, Katz JN, et al. Oral bisphosphonates and risk of subtrochanteric or diaphyseal femur fractures in a population-based cohort. J Bone Miner Res. 2011 May;26(5):993-1001. doi: 10.1002/jbmr.288. PMID: 21542002.

64. Koh J, H., Myong J, P., Yoo J, et al. Predisposing factors associated with atypical femur fracture among postmenopausal Korean women receiving bisphosphonate therapy: 8 years' experience in a single center. Osteoporosis International. 201701 Nov;28(11):3251-9. PMID: 617716961.

65. Park-Wyllie LY, Mamdani MM, Juurlink DN, et al. Bisphosphonate use and the risk of subtrochanteric or femoral shaft fractures in older women. JAMA. 2011 Feb 23;305(8):783-9. doi: 10.1001/jama.2011.190. PMID: 21343577.

66. Schilcher J, Koeppen V, Aspenberg P, et al. Risk of atypical femoral fracture during and after bisphosphonate use. Acta Orthop. 2015 Feb;86(1):100-7. doi: 10.3109/17453674.2015.1004149. PMID: 25582459.

67. Lim SJ, Yeo I, Yoon PW, et al. Incidence, risk factors, and fracture healing of atypical femoral fractures: a multicenter case-control study. Osteoporos Int. 2018 Nov;29(11):2427-35. doi: 10.1007/s00198018-4640-4. PMID: 30039251.
68. Meier RP, Perneger TV, Stern R, et al. Increasing occurrence of atypical femoral fractures associated with bisphosphonate use. Arch Intern Med. 2012 Jun 25;172(12):930-6. doi: 10.1001/archinternmed.2012.1796. PMID: 22732749.

69. Lee YK, Ahn S, Kim KM, et al. Incidence Rate of Atypical Femoral Fracture after Bisphosphonates Treatment in Korea. J Korean Med Sci. 2018 Jan 29;33(5):e38. doi: 10.3346/jkms.2018.33.e38. PMID: 29349947.

70. Miller PD, Bolognese MA, Lewiecki EM, et al. Effect of denosumab on bone density and turnover in postmenopausal women with low bone mass after long-term continued, discontinued, and restarting of therapy: a randomized blinded phase 2 clinical trial. Bone. 2008 Aug;43(2):222-9. doi: 10.1016/j.bone.2008.04.007. PMID: 18539106.

71. Leder BZ, Tsai JN, Uihlein AV, et al. Denosumab and teriparatide transitions in postmenopausal osteoporosis (the DATASwitch study): extension of a randomised controlled trial. Lancet. 2015 Sep 19;386(9999):1147-55. doi: 10.1016/S01406736(15)61120-5. PMID: 26144908.

72. Barrett-Connor E, Cauley JA, Kulkarni PM, et al. Risk-benefit profile for raloxifene: 4year data From the Multiple Outcomes of Raloxifene Evaluation (MORE) randomized trial. J Bone Miner Res. 2004

Aug;19(8):1270-5. doi: 10.1359/JBMR.040406. PMID: 15231013.

73. Barrett-Connor E, Cox DA, Song J, et al. Raloxifene and risk for stroke based on the framingham stroke risk score. Am J Med. 2009 Aug;122(8):754-61. doi: 10.1016/j.amjmed.2009.01.033. PMID: 19540454.

74. Barrett-Connor E, Grady D, Sashegyi A, et al. Raloxifene and cardiovascular events in osteoporotic postmenopausal women: fouryear results from the MORE (Multiple Outcomes of Raloxifene Evaluation) randomized trial. JAMA. 2002 Feb 20;287(7):847-57. PMID: 11851576. 
75. Cauley JA, Norton L, Lippman ME, et al. Continued breast cancer risk reduction in postmenopausal women treated with raloxifene: 4-year results from the MORE trial. Multiple outcomes of raloxifene evaluation. Breast Cancer Res Treat. 2001 Jan;65(2):125-34. PMID: 11261828.

76. Delmas PD, Ensrud KE, Adachi JD, et al. Efficacy of raloxifene on vertebral fracture risk reduction in postmenopausal women with osteoporosis: four-year results from a randomized clinical trial. J Clin Endocrinol Metab. 2002 Aug;87(8):3609-17. doi: 10.1210/jcem.87.8.8750. PMID: 12161484.

77. Duvernoy CS, Kulkarni PM, Dowsett SA, et al. Vascular events in the Multiple Outcomes of Raloxifene Evaluation (MORE) trial: incidence, patient characteristics, and effect of raloxifene. Menopause. 2005 Jul-Aug;12(4):444-52. doi:

10.1097/01.GME.0000151653.02620.89. PMID: 16037760.

78. Ensrud K, Genazzani AR, Geiger MJ, et al. Effect of raloxifene on cardiovascular adverse events in postmenopausal women with osteoporosis. Am J Cardiol. 2006 Feb 15;97(4):520-7. doi:

10.1016/j.amjcard.2005.09.083. PMID: 16461049 .

79. Grady D, Cauley JA, Stock JL, et al. Effect of Raloxifene on all-cause mortality. Am J Med. 2010 May;123(5):469 e1-7. doi: 10.1016/j.amjmed.2009.12.018. PMID: 20399327.

80. Grady D, Ettinger B, Moscarelli E, et al. Safety and adverse effects associated with raloxifene: multiple outcomes of raloxifene evaluation. Obstet Gynecol. 2004

Oct;104(4):837-44. doi:

10.1097/01.AOG.0000137349.79204.b8. PMID: 15458908.

81. Johnell O, Cauley JA, Kulkarni PM, et al. Raloxifene reduces risk of vertebral fractures [corrected] in postmenopausal women regardless of prior hormone therapy. J Fam Pract. 2004 Oct;53(10):789-96. PMID: 15469774.
82. Martino S, Cauley JA, Barrett-Connor E, et al. Continuing outcomes relevant to Evista: breast cancer incidence in postmenopausal osteoporotic women in a randomized trial of raloxifene. J Natl Cancer Inst. 2004 Dec 1;96(23):1751-61. doi: 10.1093/jnci/djh319. PMID: 15572757.

83. Martino S, Disch D, Dowsett SA, et al. Safety assessment of raloxifene over eight years in a clinical trial setting. Curr Med Res Opin. 2005 Sep;21(9):1441-52. doi: 10.1185/030079905X61839. PMID: 16197663.

84. Siris ES, Harris ST, Eastell R, et al. Skeletal effects of raloxifene after 8 years: results from the continuing outcomes relevant to Evista (CORE) study. J Bone Miner Res. 2005 Sep;20(9):1514-24. doi: 10.1359/JBMR.050509. PMID: 16059623.

85. Sontag A, Wan X, Krege JH. Benefits and risks of raloxifene by vertebral fracture status. Curr Med Res Opin. 2010 Jan;26(1):71-6. doi: 10.1185/03007990903427082. PMID: 19908937.

86. Cauley JA, Robbins J, Chen Z, et al. Effects of estrogen plus progestin on risk of fracture and bone mineral density: the Women's Health Initiative randomized trial. JAMA. 2003 Oct 1;290(13):1729-38. doi: 10.1001/jama.290.13.1729. PMID: 14519707.

87. Jackson RD, Wactawski-Wende J, LaCroix AZ, et al. Effects of conjugated equine estrogen on risk of fractures and BMD in postmenopausal women with hysterectomy: results from the women's health initiative randomized trial. J Bone Miner Res. 2006 Jun;21(6):817-28. doi: 10.1359/jbmr.060312. PMID: 16753012.

88. Wimalawansa SJ. A four-year randomized controlled trial of hormone replacement and bisphosphonate, alone or in combination, in women with postmenopausal osteoporosis. Am J Med. 1998 Mar;104(3):219-26. PMID: 9552083.

89. Vestergaard P, Schwartz F, Rejnmark L, et al. Risk of femoral shaft and subtrochanteric fractures among users of bisphosphonates and raloxifene. Osteoporos Int. 2011 Mar;22(3):993-1001. doi: 10.1007/s00198010-1512-y. PMID: 21165600. 
90. Vestergaard P, Schwartz K, Pinholt EM, et al. Risk of atrial fibrillation associated with use of bisphosphonates and other drugs against osteoporosis: a cohort study. Calcif Tissue Int. 2010 May;86(5):335-42. doi: 10.1007/s00223-010-9349-0. PMID: 20309678.

91. Vestergaard P, Schwartz K, Rejnmark L, et al. Oral bisphosphonate use increases the risk for inflammatory jaw disease: a cohort study. J Oral Maxillofac Surg. 2012

Apr;70(4):821-9. doi: 10.1016/j.joms.2011.02.093. PMID: 21764202.

92. Black DM, Cummings SR, Karpf DB, et al. Randomised trial of effect of alendronate on risk of fracture in women with existing vertebral fractures. Fracture Intervention Trial Research Group. Lancet. 1996 Dec 7;348(9041):1535-41. PMID: 8950879.

93. Stefanick ML, Cochrane BB, Hsia J, et al. The Women's Health Initiative postmenopausal hormone trials: overview and baseline characteristics of participants. Ann Epidemiol. 2003 Oct;13(9 Suppl):S7886. PMID: 14575940.

94. Rossouw JE, Anderson GL, Prentice RL, et al. Risks and benefits of estrogen plus progestin in healthy postmenopausal women: principal results From the Women's Health Initiative randomized controlled trial. JAMA. 2002 Jul 17;288(3):321-33. PMID: 12117397.

95. Anderson GL, Limacher M, Assaf AR, et al. Effects of conjugated equine estrogen in postmenopausal women with hysterectomy: the Women's Health Initiative randomized controlled trial. JAMA. 2004 Apr 14;291(14):1701-12. doi: 10.1001/jama.291.14.1701. PMID: 15082697.

96. Shumaker SA, Legault C, Kuller L, et al. Conjugated equine estrogens and incidence of probable dementia and mild cognitive impairment in postmenopausal women: Women's Health Initiative Memory Study. JAMA. 2004 Jun 23;291(24):2947-58. doi: 10.1001/jama.291.24.2947. PMID: 15213206.
97. Shumaker SA, Legault C, Rapp SR, et al. Estrogen Plus Progestin and the Incidence of Dementia and Mild Cognitive Impairment in Postmenopausal Women. Jama. 2003 May 28;289(20):2651-62. doi: 10.1001/jama.289.20.2651. PMID: 12771112.

98. Liberman UA, Weiss SR, Broll J, et al. Effect of oral alendronate on bone mineral density and the incidence of fractures in postmenopausal osteoporosis. The Alendronate Phase III Osteoporosis Treatment Study Group. N Engl J Med. 1995 Nov 30;333(22):1437-43. doi: 10.1056/NEJM199511303332201. PMID: 7477143.

99. Black DM, Delmas PD, Eastell R, et al. Once-yearly zoledronic acid for treatment of postmenopausal osteoporosis. N Engl J Med. 2007 May 3;356(18):1809-22. doi: 10.1056/NEJMoa067312. PMID: 17476007.

100. Crandall CJ, Newberry SJ, Diamant A, et al. Comparative effectiveness of pharmacologic treatments to prevent fractures: an updated systematic review. Ann Intern Med. 2014 Nov 18;161(10):711-23. doi: 10.7326/M140317. PMID: 25199883.

101. Black D, Abrahamsen B, Bouxsein M, et al. Atypical Femur Fractures-Review of epidemiology, relationship to bisphosphonates, prevention and clinical management. Endocrine Reviews. 2018;40(2):333-68.

102. Gedmintas L, Solomon DH, Kim SC. Bisphosphonates and risk of subtrochanteric, femoral shaft, and atypical femur fracture: a systematic review and meta-analysis. J Bone Miner Res. 2013 Aug;28(8):1729-37. doi: 10.1002/jbmr.1893. PMID: 23408697.

103. Black DM, Rosen CJ. Postmenopausal osteoporosis. New England Journal of Medicine 2016;374(3):254-62.

104. Bauer DC. Change in Bone Turnover as a Surrogate for Fracture Outcomes: A Novel Individual-level Analysis of Pooled Antiresorptive Trials from the FNIH Bone Quality Study. American Society for Bone and Mineral Research Annual Meeting, September 28-October 1, Montréal, Québec, Canada. 2018; Abstract \#1058. 
105. Black D. Change in BMD as a Surrogate for Fracture Risk Reduction in Osteoporosis Trials: Results from Pooled, Individual-level Patient Data from the FNIH Bone Quality

Project [abstract]. American Society for

Bone and Mineral Research Annual

Meeting, September 28-October 1, Montréal, Québec, Canada. 2018; Abstract \#1070.

106. Desai RJ, Mahesri M, Abdia Y, et al. Association of osteoporosis medication use after hip fracture with prevention of subsequent nonvertebral fractures: an instrumental variable analysis. JAMA Network Open. 2018;1(3):e180826-e.

107. Chodick G, Moser SS, Goldshtein I. Nonadherence with bisphosphonates among patients with osteoporosis: impact on fracture risk and healthcare cost. Expert Rev Pharmacoecon Outcomes Res. 2016 Jun;16(3):359-70. doi: 10.1586/14737167.2016.1171145. PMID: 27015247.

108. Khosla S, Cauley JA, Compston J, et al. Addressing the Crisis in the Treatment of Osteoporosis: A Path Forward. J Bone Miner Res. 2017 Mar;32(3):424-30. doi: 10.1002/jbmr.3074. PMID: 28099754.

109. Tsourdi E, Zillikens MC. Certainties and Uncertainties About Denosumab

Discontinuation. Calcif Tissue Int. 2018 Jul;103(1):1-4. doi: 10.1007/s00223-0180436-y. PMID: 29926143. 


\section{Abbreviations}

\begin{tabular}{|c|c|}
\hline $\mathrm{AE}$ & Adverse event \\
\hline AFF & Atypical femoral fracture \\
\hline AFib & Atrial fibrillation \\
\hline aHR & Adjusted hazard ratio \\
\hline AHRQ & Agency for Healthcare Research and Quality \\
\hline ALP & Alkaline phosphatase \\
\hline $\mathrm{aOR}$ & Adjusted odds ratio \\
\hline ASBMR & American Society for Bone and Mineral Research \\
\hline BSAP & Bone specific alkaline phosphatase \\
\hline BMD & Bone mineral density \\
\hline BMI & Body mass index $\left(\mathrm{kg} / \mathrm{m}^{2}\right)$ \\
\hline CCT & Controlled clinical trial \\
\hline CENTRAL & Cochrane Central Register of Controlled Trials \\
\hline CER & Comparative Effectiveness Review \\
\hline CFx & Clinical fracture \\
\hline $\mathrm{CHF}$ & Congestive heart failure \\
\hline CI & Confidence interval \\
\hline CKD & Chronic kidney disease \\
\hline CORE & Continuing Outcomes Relevant to Evista \\
\hline COX & cyclooxygenase \\
\hline CTX & C-terminal telopeptide of type 1 collagen \\
\hline CVD & Cardiovascular disease \\
\hline CVFx & Clinical vertebral fracture \\
\hline DM & Diabetes mellitus \\
\hline DVT & Deep vein thrombosis \\
\hline DXA & Dual-energy x-ray absorptiometry \\
\hline eGFR & Estimated glomerular filtration rate \\
\hline EHR & Electronic health record \\
\hline EPC & Evidence-Based Practice Center \\
\hline FDA & U.S. Food and Drug Administration \\
\hline FIT & Fracture Intervention Trial \\
\hline FLEX & Fracture Intervention Trial Long-Term Extension \\
\hline FN & Femoral neck \\
\hline FN-BMD & Femoral neck bone mineral density \\
\hline FRAX & World Health Organization Fracture Risk Assessment Tool \\
\hline $\mathrm{Fx}$ & Fracture \\
\hline GRADE & Grading of Recommendations Assessment, Development, and Evaluation \\
\hline GI & Gastrointestinal \\
\hline $\mathrm{g} / \mathrm{cm}^{2}$ & Grams per centimeter squared \\
\hline HORIZON & Health Outcomes and Reduced Incidence with Zoledronic Acid Once Yearly Trial \\
\hline HR & Hazard ratio \\
\hline ICD & International Classification of Diseases \\
\hline ITT & Intention to treat \\
\hline IV & Intravenous \\
\hline KQ & Key Question \\
\hline
\end{tabular}




\begin{tabular}{|c|c|}
\hline LS & Lumbar spine \\
\hline LS-BMD & Lumbar spine bone mineral density \\
\hline MD & Mean difference \\
\hline $\mathrm{mg}$ & Milligrams \\
\hline MOF & Major osteoporotic fracture \\
\hline MORE & Multiple Outcomes of Raloxifene Evaluation \\
\hline $\mathrm{N}$ & Number \\
\hline NA & Not applicable \\
\hline NIA & National Institute on Aging \\
\hline NIAMS & National Institute of Arthritis and Musculoskeletal and Skin Diseases \\
\hline NIH & National Institute of Health \\
\hline NR & Not reported \\
\hline NS & Not significant \\
\hline NSAIDS & Nonsteroidal anti-inflammatory drugs \\
\hline NTX & N-terminal telopeptide of type 1 collagen \\
\hline ODP & Office of Disease Prevention \\
\hline ONJ & Osteonecrosis of the jaw \\
\hline OR & Odds ratio \\
\hline P2P & Pathways to Prevention \\
\hline P1NP & Procollagen type 1 amino-terminal propeptide \\
\hline $\mathrm{PE}$ & Pulmonary embolism \\
\hline PICOTS & Population, Interventions, Comparators, Outcomes, Timing, and Settings \\
\hline PM & Postmenopausal \\
\hline PRISMA & Preferred Reporting Items for Systematic Reviews and Meta-Analyses \\
\hline PTH & Parathyroid hormone \\
\hline HRpQCT & High resolution peripheral quantitative computed tomography \\
\hline QD & Per day \\
\hline RCT & Randomized controlled trial \\
\hline RD & Risk difference \\
\hline $\mathrm{RH}$ & Relative hazard \\
\hline ROB & Risk of bias \\
\hline $\mathrm{RR}$ & Risk ratio \\
\hline RVFx & Radiographic vertebral fracture \\
\hline SAE & Serious adverse event \\
\hline SC & Subcutaneous \\
\hline SD & Standard deviation \\
\hline SE & Standard error \\
\hline SEADS & Supplemental Evidence and Data for Systematic Reviews \\
\hline SERM & Selective estrogen receptor modulator \\
\hline SOE & Strength of Evidence \\
\hline SSRI & Selective Serotonin Reuptake Inhibitors \\
\hline TEP & Technical Expert Panel \\
\hline TH & Total hip \\
\hline TH-BMD & Total hip bone mineral density \\
\hline VFx & Vertebral fracture \\
\hline VTE & Venous thromboembolism \\
\hline
\end{tabular}


WHO World Health Organization

yr

Year 


\section{Appendix A. Search Strategy}

\section{Database: Ovid MEDLINE(R) Epub Ahead of Print, In-Process \& Other Non-Indexed Citations, Ovid MEDLINE(R) Daily and Ovid MEDLINE(R) Search Strategy}

1 exp Osteoporosis/ (56601)

2 osteoporosis.ti. (24986)

3 Bone Density/ (53169)

4 exp Fractures, Bone/ (178905)

5 or/1-4 (249251)

6 Bone Density Conservation Agents/ (13618)

7 exp Diphosphonates/ (26164)

8 bisphosphonate*.ti. (6667)

9 alendronate.ti. (2190)

10 ibandronate.ti. (497)

11 risedronate.ti. (732)

12 zoledronic acid.ti. (1876)

13 or/7-12 (27852)

14 denosumab.ti. (998)

15 exp Anabolic Agents/ (15267)

16 teriparatide.ti. (835)

17 abaloparatide.ti. (22)

18 or/15-17 (16105)

19 exp Selective Estrogen Receptor Modulators/ (28675)

20 raloxifene.ti. (1433)

21 or/19-20 (28784)

22 Hormone Replacement Therapy/ (9611)

23 Estrogen Replacement Therapy/ (15549)

24 Estrogens, Conjugated/ (3783)

25 (conjugated adj2 estrogens).ti. (442)

26 (conjugated adj2 oestrogens).ti. (52)

27 bazedoxifene.ti. (203)

28 parathyroid.ti. (21206)

29 pth.ti. (2443)

3024 or 25 or 26 or 27 or 28 or 29 (27047)

31 Romosozumab.ti. (37)

326 or 13 or 14 or 18 or 21 or 22 or 23 or 30 or 31 (124263)

335 and 32 (19473)

34 meta analysis as topic/ (17374)

35 meta-analy\$.tw. (132466)

36 metaanaly\$.tw. (1964) 
meta-analysis/ (94826)

38 (systematic adj (review\$1 or overview\$1)).tw. (121503)

39 exp Review Literature as Topic/ (10321)

40 or/34-39 (237928)

41 cochrane.ab. (61695)

42 embase.ab. (65904)

43 (psychlit or psyclit).ab. (957)

44 (psychinfor or psycinfo).ab. (18590)

45 or/41-44 (100777)

46 reference list\$.ab. (15891)

47 bibliograph\$.ab. (16312)

48 hand search.ab. (1444)

49 relevant journals.ab. (1105)

50 manual search\$.ab. (3843)

51 or/46-50 (36058)

52 selection criteria.ab. (28294)

53 data extraction.ab. (16712)

5452 or $53(42825)$

55 review/ (2480829)

$56 \quad 54$ and 55 (28679)

57 comment/ (735829)

58 letter/ (1035382)

59 editorial/ (470175)

$60 \mathrm{animal} /(6598636)$

61 human/ (18057963)

6260 not (61 and 60) (4708384)

63 or/57-59,62 (6336940)

6440 or 45 or 51 or 56 (281525)

$65 \quad 64$ not 63 (266871)

66 randomized controlled trials as topic/ (123612)

67 randomized controlled trial/ (505454)

68 random allocation/ (101086)

69 double blind method/ (159463)

70 single blind method/ (27137)

71 clinical trial/ (553719)

72 clinical trial, phase i.pt. (20676)

73 clinical trial, phase ii.pt. (33325)

74 clinical trial, phase iii.pt. (15646)

75 clinical trial, phase iv.pt. (1673)

76 controlled clinical trial.pt. (100423)

77 randomized controlled trial.pt. (505454)

78 multicenter study.pt. (254739)

79 clinical trial.pt. (553719)

80 exp Clinical trials as topic/ (335805)

81 or/66-80 (1338507)

82 (clinical adj trial\$).tw. (327584) 
83 ((singl\$ or doubl\$ or treb\$ or tripl\$) adj (blind\$3 or mask\$3)).tw. (169807)

84 placebos/ (36670)

85 placebo\$.tw. (211894)

86 randomly allocated.tw. (25617)

87 (allocated adj2 random\$).tw. (28819)

$88 \quad 82$ or 83 or 84 or 85 or 86 or 87 (592975)

8981 or $88(1569425)$

90 case report.tw. (279795)

91 case report.tw. (279795)

92 letter/ (1035382)

93 historical article/ (358172)

9490 or 91 or 92 or 93 (1658574)

9589 not $94(1533984)$

96 exp cohort studies/ (1866525)

97 cohort\$.tw. (483043)

98 controlled clinical trial.pt. (100423)

99 epidemiologic methods/ (32559)

100 limit 99 to $\mathrm{yr}=1971-1983$ (5524)

10196 or 97 or 98 or 100 (2167906)

102 exp case-control study/ (974951)

103 (case\$ and control\$).tw. (459124)

104102 or $103(1309725)$

10565 or 95 or 101 or 104 (3860160)

$106 \quad 33$ and 65 (723)

$107 \quad 33$ and 95 (6177)

10833 and 101 (3725)

10933 and 104 (1519)

110106 or 107 or 108 or 109 (8692)

111 limit 110 to animals (636)

112 limit 111 to humans (415)

113110 not 111 (8056)

114113 or 112 (8471)

115 limit 114 to "all child (0 to 18 years)" (541)

116 limit 115 to "all adult (19 plus years)" (276)

117114 not 115 (7930)

118117 or $116(8206)$

119 limit 118 to (addresses or autobiography or bibliography or biography or case reports or comment or dataset or dictionary or directory or editorial or interactive tutorial or interview or legal cases or legislation or letter or news or newspaper article or patient education handout or periodical index or personal narratives or portraits or validation studies or video-audio media or webcasts) (389)

120118 not 119 (7817)

121 limit 120 to ("young adult (19 to 24 years)" or "adult (19 to 44 years)") (1684)

122 limit 121 to ("middle age (45 to 64 years)" or "middle aged (45 plus years)" or "all aged (65 and over)" or "aged (80 and over)") (1411)

123120 not $121(6133)$ 
124123 or $122(7544)$

125 limit 124 to english language (6901)

126 limit 125 to yr="1995 -Current" (6527)

\section{Database: Embase Classic+Embase Search Strategy}

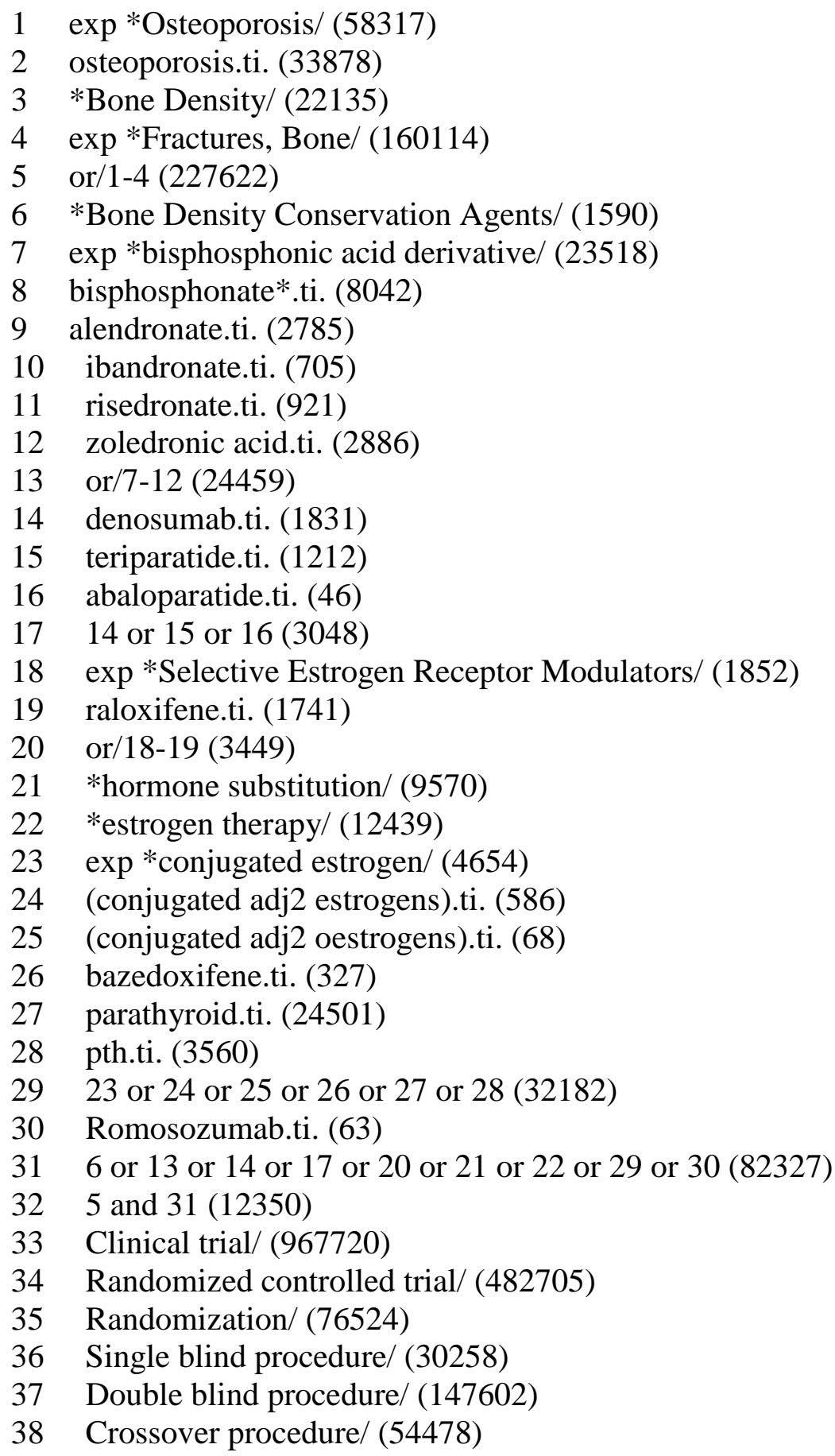


39 Placebo/ (321912)

40 Randomi?ed controlled trial\$.tw. (171939)

41 Rct.tw. (26582)

42 Random allocation.tw. (1803)

43 Randomly allocated.tw. (29202)

44 Allocated randomly.tw. (2326)

45 (allocated adj2 random).tw. (951)

4633 or 34 or 35 or 36 or 37 or 38 or 39 or 40 or 41 or 42 or 43 or 44 or 45 (1492328)

47 Case study/ (60326)

48 Case report.tw. (370340)

49 Abstract report/ or letter/ (1042296)

5047 or 48 or $49(1464628)$

5146 not 50 (1452601)

52 Clinical study/ (169001)

53 exp case control study/ (139905)

54 family study/ (27182)

55 longitudinal study/ (108891)

56 retrospective study/ (601258)

57 prospective study/ (419321)

58 cohort analysis/ (336075)

59 (cohort adj stud*).mp. (217474)

60 (observational adj stud*).mp. (172157)

61 (case control adj stud*).mp. (177221)

62 (follow up adj stud*).mp. (64043)

63 (epidemiologic* adj stud*).mp. (100966)

64 (cross sectional adj stud*).mp. (282797)

65 or/52-64 (2122395)

6651 or 65 (3372681)

$67 \quad 32$ and 66 (5210)

68 limit 67 to (embryo < first trimester $>$ or infant $<$ to one year $>$ or child $<$ unspecified age $>$ or preschool child $<1$ to 6 years $>$ or school child $<7$ to 12 years $>$ or adolescent $<13$ to 17 years $>$ ) (112)

69 limit 68 to (adult $<18$ to 64 years $>$ or aged $<65+$ years $>$ ) (55)

$70 \quad 67$ not $68(5098)$

7170 or $69(5153)$

72 limit 71 to (amphibia or ape or bird or cat or cattle or chicken or dog or "ducks and geese" or fish or "frogs and toads" or goat or guinea pig or "hamsters and gerbils" or horse or monkey or mouse or "pigeons and doves" or "rabbits and hares" or rat or reptile or sheep or swine) (104)

7371 not 72 (5049)

74 limit 73 to (abstract report or books or "book review" or chapter or conference abstract or "conference review" or editorial or letter or note or patent or short survey or tombstone) (1313)

7573 not $74(3736)$

76 limit 75 to yr="1995 -Current" (3564)

77 limit 76 to English language (3174) 


\section{Appendix B. Risk of Bias Assessment Decision Aid}

\section{Selection Bias}

Systematic differences between baseline characteristics of the groups that arise from selfselection of treatments, physician-directed selection of treatments, or association of treatment assignments with demographic, clinical, or social characteristics.

Good randomization produces study groups that are likely comparable for known and unknown risk factors, removes investigator bias in allocation, and allow the most valid statistical inference in comparing outcomes between groups. In randomized studies, whether there is bias in allocation of study participants to treatment groups is a function both of whether the methods of randomization are good AND whether randomization successfully achieved a balance between treatment groups in risk factors or prognostic covariates.

- Randomized: The study reports that it was randomized

o Good Methodology of Randomization: The study used a randomization method such as random numbers table, computer-generated random number producing algorithm, blocked randomization, stratified randomization, adaptive randomization (e.g., minimization).

o Unclear Methodology of Randomization: Study reports that allocation/assignment was randomized, but gives no further detail.

- Not Randomized:

o Systematic allocation of treatment by investigator: Systematic and predictable investigator allocation of treatment assignment (e.g., alternation, based on day of week, based on the month of birthday)

o Observational study: Treatment allocation not assigned by investigator (i.e. noninterventional study)

- Balance in prognostic variables. A study is assumed to have achieved a balance between known/measured risk factors or prognostic variables when participant characteristics are balanced between treatment groups. Balance between unmeasured risk factors or prognostic variables cannot be determined. 
Figure B1. Selection bias decision aid

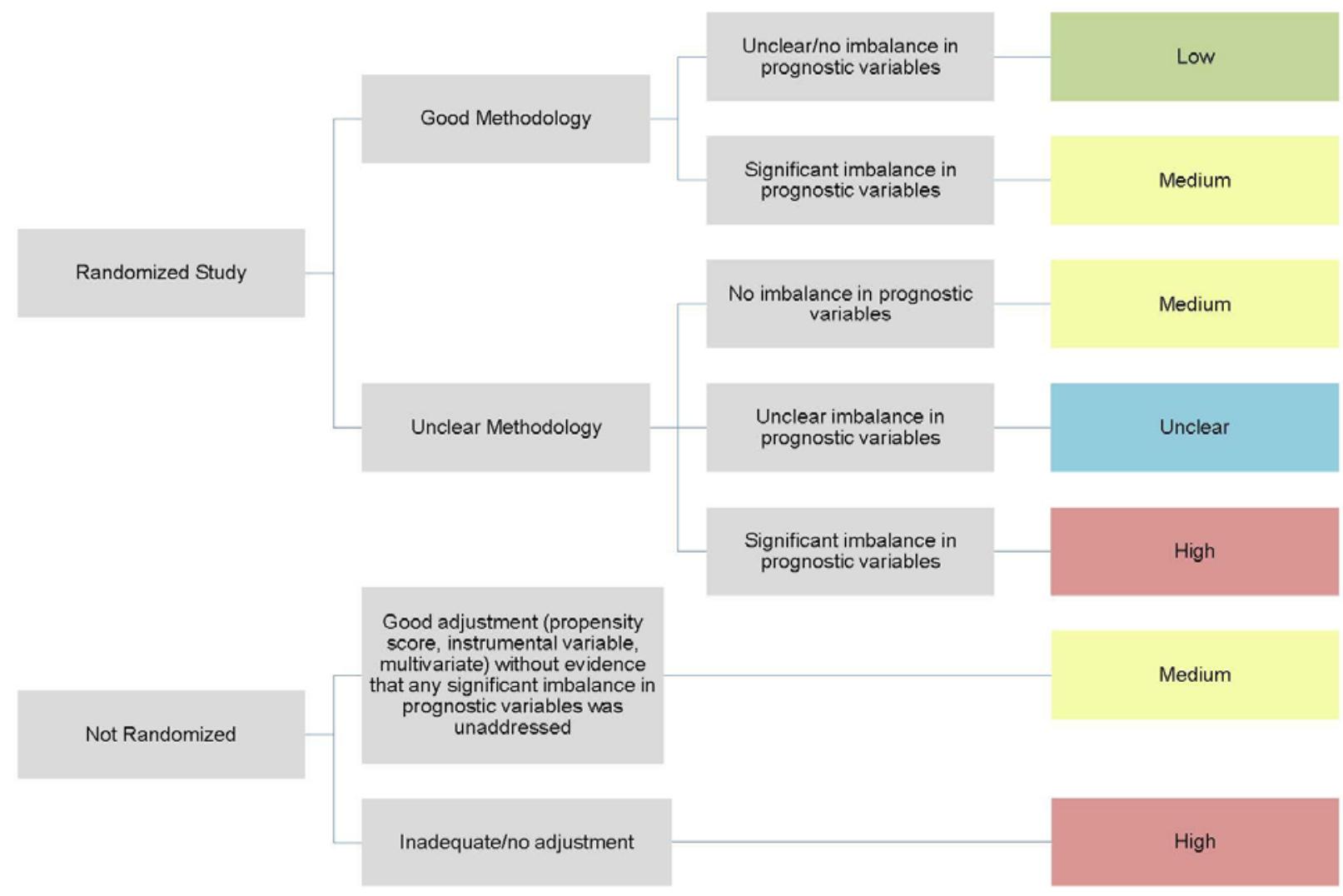

B-2 


\section{Performance Bias}

Systematic differences in the care provided to participants and protocol deviation. Examples include contamination of the control group with the exposure or intervention, unbalanced provision of additional interventions or co-interventions, difference in co-interventions, and inadequate blinding of providers and participants.

- Intention-to-Treat Principle (ITT) is when the study counts events in all randomized participants according to their treatment assignment, regardless of whether they received assigned treatment. It does not exclude participants from analysis for nonadherence, protocol deviations, withdrawal, or anything else that happens after randomization. To exclude such participants undercuts the benefit of randomization in minimizing selection bias.

- Modified ITT (mITT) is where analyses exclude randomized participants who did not receive any of their assigned treatment. This is not strictly ITT, but is accepted as such by the FDA in evaluating drug trials for approval.

- Adjustment for known potential confounders.

o Adequate adjustment includes adjustment for at least the following three categories: [1] age, [2] fracture risk (e.g., history of past fracture, BMD, FRAX or other risk calculator) and [3] some estimate of comorbidity.

o Partially adequate adjustment adjusts for 1 or 2 of these potential confounder categories.

o Inadequate adjustment does not adjust for any of these potential confounder categories.

Table B1. Performance bias decision aid

\begin{tabular}{|c|c|c|c|}
\hline Domain & Options & & \\
\hline 1a. & Yes & & \\
\hline & No & & \\
\hline & Unclearl & eported & \\
\hline & N/A (if $n$ & & \\
\hline 1b. & Adequat & & \\
\hline for CCTs, observational studies, and RCTs & Partially & uate & \\
\hline variables) & Inadequ & & \\
\hline & N/A (if $R$ & th balanced baselin & variables) \\
\hline $2 . \quad 2.425$ & Yes & & \\
\hline CCTs & No & & \\
\hline & Unclear & & \\
\hline & N/A (obs & onal) & \\
\hline $\begin{array}{l}\text { Overall Performance } \\
\text { Rating }\end{array}$ & ategories & $\begin{array}{l}\text { Medium }= \\
\text { Low/Medium mix }\end{array}$ & $\begin{array}{l}\text { High = } \\
\text { High in a }\end{array}$ \\
\hline
\end{tabular}




\section{Reporting Bias}

Systematic differences between reported and unreported findings (e.g., differential reporting of outcomes or harms, incomplete reporting of study findings).

- Were all outcomes reported in the methods section reported in the result section and vice versa?

Table B2. Reporting bias decision aid

\begin{tabular}{|l|l|l|}
\hline Domain & Options & Rating \\
\hline \multirow{3}{*}{ All outcomes reported } & Yes & Low \\
\cline { 2 - 3 } & No & Medium \\
\cline { 2 - 3 } & Unclear & Unclear \\
\hline
\end{tabular}

Notes to Reviewers for Using Decision Aid: Reviewers should first rate reporting bias based on the information in the article. In the ideal world, reviewers will have the protocol available for all the studies and will go back to look for whether all major outcomes (those for which we are assessing ROB and SOE) reported in the protocol methods were reported in the paper results. This may allow us to find reporting bias that wasn't apparent from the paper, which could result in a downgrading of the overall ROB rating.

To save work, we may decide that if a study already has an overall high ROB without checking the protocol, there is no good reason to check the protocol. If the study has an overall low or medium ROB, we can check the protocol for evidence of reporting bias. 


\section{Attrition}

Loss of participants from the study, potential systematic differences in that loss to follow-up, and how losses were accounted for in the results (e.g., incomplete follow-up, differential attrition). Those who drop out of the study or who are lost to follow-up may be systematically different from those who remain in the study. Attrition bias can potentially change the collective (group) characteristics of the relevant groups and their observed outcomes in ways that affect study results by confounding and spurious associations.

- Are reasons for incomplete/missing data adequately explained?

- Do the authors attempt to address attrition in the analysis?

- Analysis should be done with appropriate method (i.e. sensitivity analysis with various scenarios), noting that this may help explain the size and direction of the potential bias, but they don't eliminate the bias.

- Overall attrition refers to attrition in all treatment groups combined for a given outcome comparison and timepoint.

- Differential attrition refers to the absolute difference between treatment groups in attrition for a given outcome comparison and timepoint.

Figure B2. Attrition bias decision aid

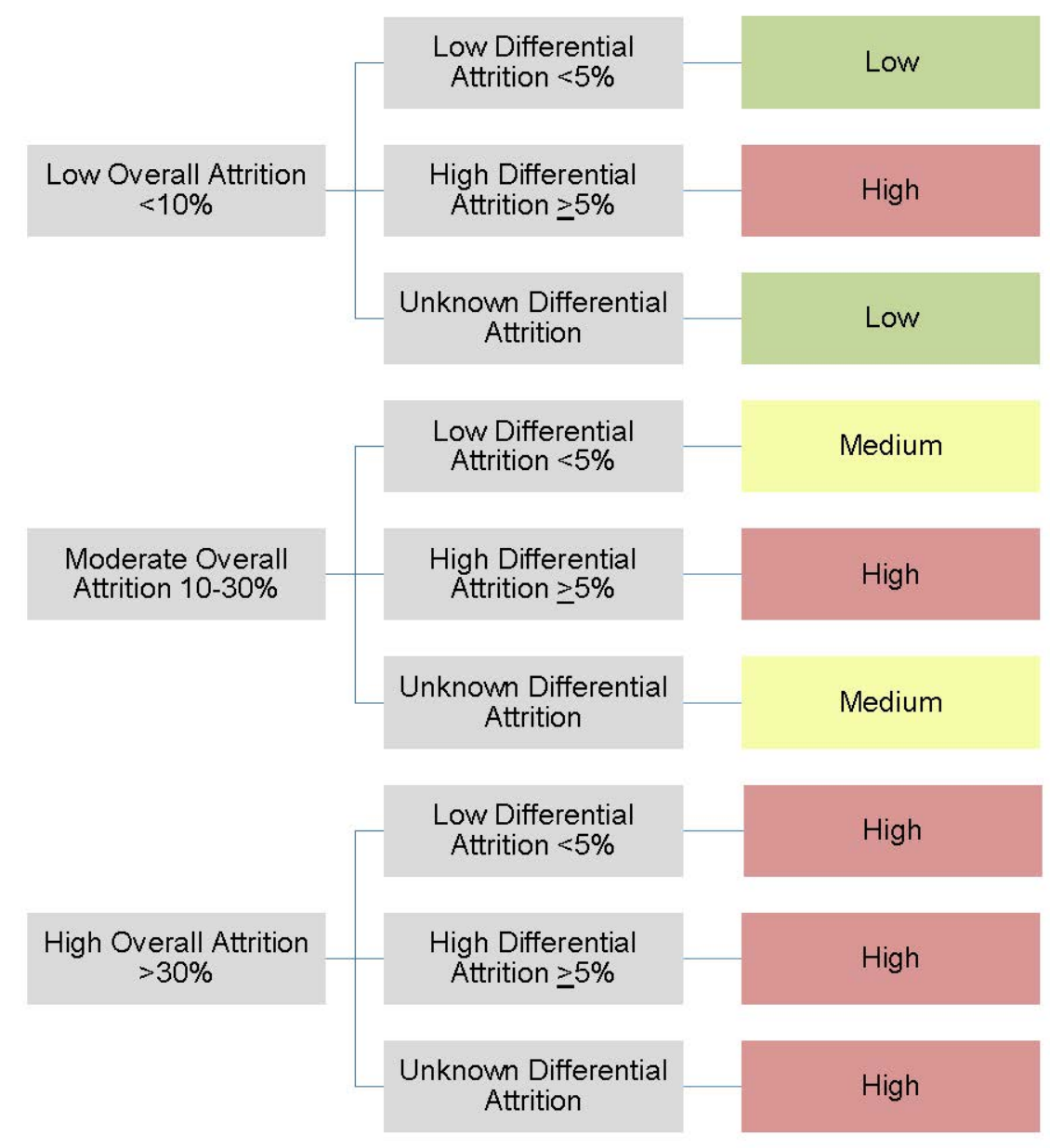




\section{Detection Bias}

Systematic differences in outcomes assessment among groups being compared, including systematic misclassification of the exposure or intervention, covariates, or outcomes because of variable definitions and timings, diagnostic thresholds, recall from memory, inadequate assessor blinding, and faulty measurement techniques. Erroneous statistical analysis might also affect the validity of effect estimates.

- Were the outcome assessors blinded to the treatment assignment?

- Were outcome measures validated, reliable, and were the groups assessed using comparable outcome measures? Were outcomes confirmed/adjudicated, either by direct measurement in clinic, review of study records (e.g. study x-rays) or review of clinical records?

Table B3. Detection bias decision aid

\begin{tabular}{|l|l|}
\hline Domain & Options \\
\hline Outcome assessor blinded to treatment & Yes \\
assignment & \\
\cline { 2 - 3 } & No \\
\cline { 2 - 2 } & Unclear \\
\hline \multirow{3}{*}{$\begin{array}{l}\text { High quality Instrument/measurement or } \\
\text { outcome confirmed/adjudicated }\end{array}$} & Yes \\
\cline { 2 - 2 } & No (unconfirmed self-report outcomes) \\
\cline { 2 - 2 } & Intermediate (outcomes based on administrative data) \\
\cline { 2 - 2 } & Unclear \\
\hline
\end{tabular}

\begin{tabular}{|l|l|l|l|}
\hline $\begin{array}{l}\text { Overall } \\
\text { Performance } \\
\text { Rating }\end{array}$ & $\begin{array}{l}\text { Low }=2 \text { Yes OR } 1 \text { Yes, } 1 \\
\text { Unclear }\end{array}$ & $\begin{array}{l}\text { Medium = all unclear or } \\
\text { intermediate }\end{array}$ & High = at least 1 No \\
\hline
\end{tabular}




\section{Overall Risk of Bias Guide}

ASSESSMENT GUIDANCE: Overall risk of bias is determined by reviewer, or team, consensus. Figure B3 provides a guide for how to rate overall risk of bias, based on the assessment of each individual domain. Reviewers should use this guide when making judgements about overall risk of bias. However, there may be cases where deviation from this guide is necessary and appropriate. For clarification and transparency, reviewers should provide a brief written justification for these deviations. 


\section{Figure B3. Overall risk of bias decision aid}

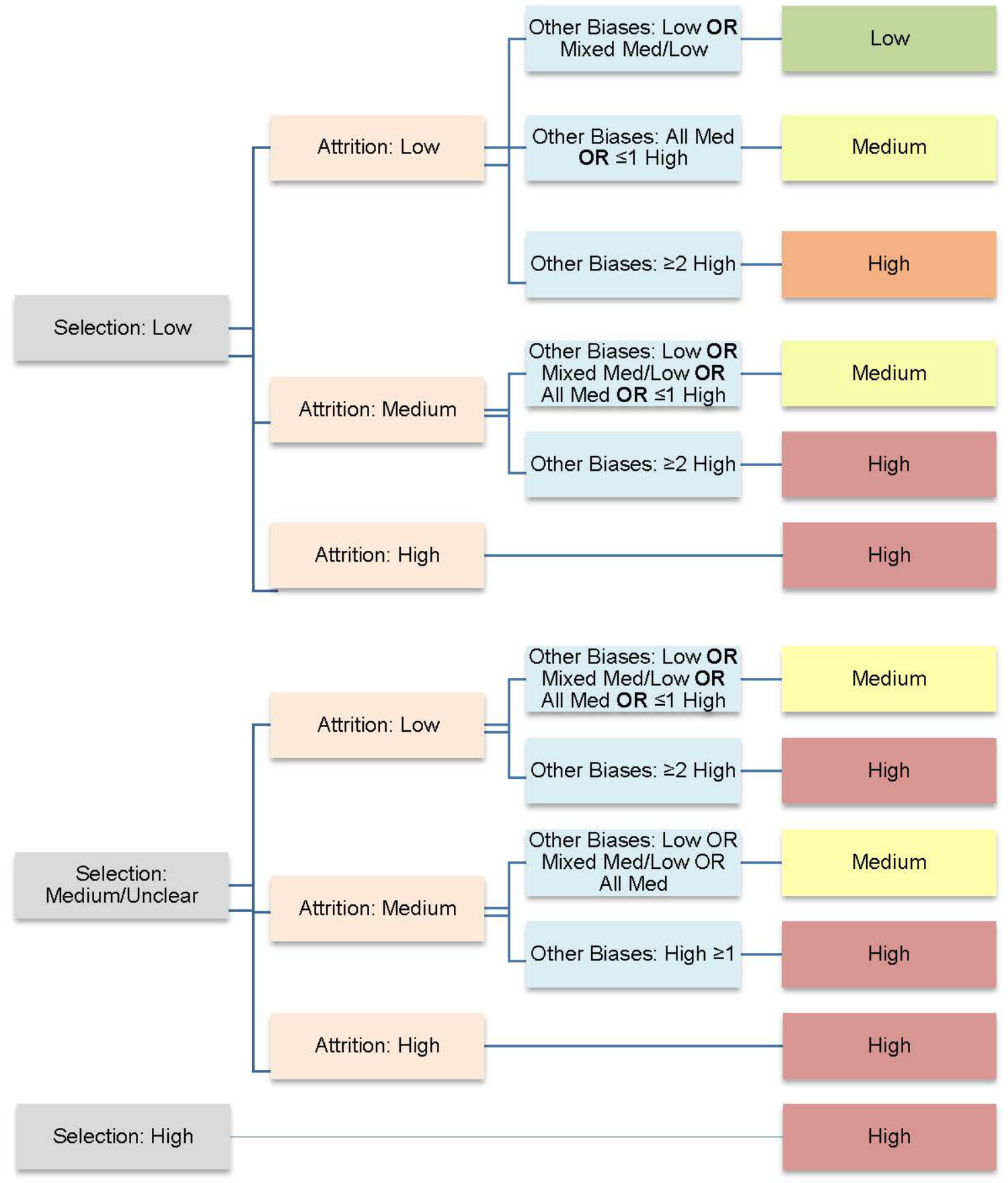




\section{Appendix C. Included References Risk of Bias Assessment}

Table C1. Included studies with summary risk of bias assessments

\begin{tabular}{|c|c|c|c|c|c|c|c|c|}
\hline Intervention & Study & $\begin{array}{l}\text { Selection } \\
\text { Bias }\end{array}$ & Attrition & $\begin{array}{l}\text { Performance } \\
\text { Bias }\end{array}$ & $\begin{array}{l}\text { Detection } \\
\text { Bias }\end{array}$ & $\begin{array}{l}\text { Reporting } \\
\text { Bias }\end{array}$ & $\begin{array}{l}\text { Overall } \\
\text { Rating }\end{array}$ & $\begin{array}{l}\text { Justification (if deviated } \\
\text { from decision aid) }\end{array}$ \\
\hline \multirow{16}{*}{$\begin{array}{l}\text { Bisphosphonate } \\
\text { Alendronate }\end{array}$} & Abrahamsen 2009 & Medium & Low & Low & Medium & Low & Medium & $\mathrm{NA}$ \\
\hline & Abrahamsen 2010 & Medium & High & Medium & High & Low & High & NA \\
\hline & Abrahamsen 2016 & Medium & High & Low & High & Low & High & NA \\
\hline & Bauer 2000 & Low & High & Low & Low & Low & Medium & $\begin{array}{l}\text { Pooled data from long-term } \\
\text { RCT and extension(s) with } \\
\text { differing attrition }\end{array}$ \\
\hline & Bauer 2006 & Medium & High & Medium & Low & Low & Medium & $\begin{array}{l}\text { Pooled data from long-term } \\
\text { RCT and extension(s) with } \\
\text { differing attrition }\end{array}$ \\
\hline & Black 2006 & Low & Low & Low & Low & Low & Low & NA \\
\hline & Black 2010 & Low & Medium & Medium & Low & Low & Medium & NA \\
\hline & Bone 2004 & Low & High & Low & Low & Low & Medium & $\begin{array}{l}\text { Low overall attrition, high } \\
\text { differential attrition }\end{array}$ \\
\hline & Chapurlat 2005 & Medium & High & High & Medium & Low & High & NA \\
\hline & Chiu 2014 & Medium & Medium & Medium & Medium & Low & Medium & NA \\
\hline & Chiu 2018 & High & Low & High & Medium & Low & High & $\mathrm{NA}$ \\
\hline & Cummings 1998 & Low & Low & Low & Low & Low & Low & NA \\
\hline & Donaldson 2012 & Low & Medium & Low & Low & Low & Medium & $\mathrm{NA}$ \\
\hline & Ensrud 2004 & Low & Low & Low & Low & Low & Low & NA \\
\hline & Jamal 2007 & Medium & Low & Medium & Low & Low & Medium & NA \\
\hline & Lin 2014 & Medium & Medium & Low & Medium & Low & Medium & $\mathrm{NA}$ \\
\hline
\end{tabular}




\begin{tabular}{|c|c|c|c|c|c|c|c|c|}
\hline Intervention & Study & $\begin{array}{l}\text { Selection } \\
\text { Bias }\end{array}$ & Attrition & $\begin{array}{l}\text { Performance } \\
\text { Bias }\end{array}$ & $\begin{array}{l}\text { Detection } \\
\text { Bias }\end{array}$ & $\begin{array}{l}\text { Reporting } \\
\text { Bias }\end{array}$ & $\begin{array}{l}\text { Overall } \\
\text { Rating }\end{array}$ & $\begin{array}{l}\text { Justification (if deviated } \\
\text { from decision aid) }\end{array}$ \\
\hline & Nevitt 1999 & Medium & High & Medium & Low & Low & High & NA \\
\hline & Quandt 2005 & Medium & Medium & Low & Low & Low & Medium & NA \\
\hline & Ryder 2008 & Medium & Medium & Low & Low & Medium & Medium & NA \\
\hline & Schwartz 2010 & Low & Medium & Low & Low & Medium & Medium & NA \\
\hline & Tonino 2000 & Low & High & High & Low & Low & Medium & $\begin{array}{l}\text { High attrition overall, } \\
\text { unable to determine } \\
\text { attrition for extension study } \\
\text { only }\end{array}$ \\
\hline & Wang, 2016 & High & Low & Low & High & Low & High & NA \\
\hline \multirow{5}{*}{$\begin{array}{l}\text { Bisphosphonate } \\
\text { Zoledronic acid }\end{array}$} & Black 2012 & Low & Medium & Low & Low & Low & Low & NA \\
\hline & Black 2015 & Low & Medium & Low & Low & Low & Low & NA \\
\hline & Cosman 2014 & Medium & Medium & High & Low & Low & High & NA \\
\hline & McClung 2009 & Low & Medium & Low & Low & Low & Low & NA \\
\hline & Reid 2018 & Low & Low & Low & Low & Low & Low & NA \\
\hline \multirow{2}{*}{$\begin{array}{l}\text { Bisphosphonate } \\
\text { Risedronate }\end{array}$} & Sorensen 2003 & Low & High & Medium & Low & Low & High & NA \\
\hline & Ste 2004 & Medium & High & Low & Low & Low & High & NA \\
\hline $\begin{array}{l}\text { Bisphosphonate } \\
\text { Ibandronate }\end{array}$ & Miller 2012 & Medium & Medium & Medium & Low & Low & High & $\begin{array}{l}\text { Partial randomization not } \\
\text { described clearly, missing } \\
\text { data not clearly defined }\end{array}$ \\
\hline \multirow{3}{*}{$\begin{array}{l}\text { Bisphosphonate } \\
\text { Pooled }\end{array}$} & Adams 2018 & Medium & Low & Low & Medium & Low & Medium & NA \\
\hline & Erviti 2013 & Medium & Low & Low & Medium & Low & Medium & NA \\
\hline & Kim 2011 & Medium & Medium & Low & Medium & Low & Medium & NA \\
\hline
\end{tabular}




\begin{tabular}{|c|c|c|c|c|c|c|c|c|}
\hline Intervention & Study & $\begin{array}{l}\text { Selection } \\
\text { Bias }\end{array}$ & Attrition & $\begin{array}{l}\text { Performance } \\
\text { Bias }\end{array}$ & $\begin{array}{l}\text { Detection } \\
\text { Bias }\end{array}$ & $\begin{array}{l}\text { Reporting } \\
\text { Bias }\end{array}$ & $\begin{array}{l}\text { Overall } \\
\text { Rating }\end{array}$ & $\begin{array}{l}\text { Justification (if deviated } \\
\text { from decision aid) }\end{array}$ \\
\hline & Koh 2017 & Medium & Low & Medium & Low & Low & Medium & NA \\
\hline & Lee 2018 & High & Medium & Medium & Low & Low & High & NA \\
\hline & Lim 2018 & Medium & Medium & Low & Low & Low & Medium & NA \\
\hline & Meier 2012 & High & Low & Medium & Low & Low & High & NA \\
\hline & Park-Wylie 2011 & Medium & Medium & Low & Medium & Low & Medium & NA \\
\hline & Schilcher 2014 & Medium & Low & Medium & Low & Low & Medium & NA \\
\hline \multirow[t]{2}{*}{ Denosumab } & Leder 2015 & Low & High & High & Low & Low & High & NA \\
\hline & Miller 2008 & Low & High & Low & Medium & Low & Medium & NA \\
\hline \multirow[t]{8}{*}{ Raloxifene } & Barrett 2002 & Low & Medium & Low & Low & Low & Low & $\begin{array}{l}\text { Data from long-term RCT } \\
\text { with attrition well explained } \\
\text { and addressed in analysis }\end{array}$ \\
\hline & Barrett 2004 & Low & Medium & Low & Low & Low & Low & $\begin{array}{l}\text { Data from long-term RCT } \\
\text { with attrition well explained } \\
\text { and addressed in analysis }\end{array}$ \\
\hline & Barrett 2009 & Low & Medium & Low & Low & Low & Low & $\begin{array}{l}\text { Data from long-term RCT } \\
\text { with attrition addressed in } \\
\text { analysis }\end{array}$ \\
\hline & Cauley 2001 & Low & Medium & Low & Low & Low & Low & $\begin{array}{l}\text { Data from long-term RCT } \\
\text { with attrition addressed in } \\
\text { analysis }\end{array}$ \\
\hline & Delmas 2002 & Medium & Medium & Low & Low & Low & Low & $\begin{array}{l}\text { Data from long-term RCT } \\
\text { with attrition well explained } \\
\text { and addressed in analysis }\end{array}$ \\
\hline & Duvernoy 2005 & Low & Medium & Low & Low & Low & Low & $\begin{array}{l}\text { Data from long-term RCT } \\
\text { with attrition addressed in } \\
\text { analysis }\end{array}$ \\
\hline & Ensrud 2006 & Low & Medium & Low & Low & Low & Medium & NA \\
\hline & Grady 2004 & Low & Medium & Low & Low & Low & Low & $\begin{array}{l}\text { Data from long-term RCT } \\
\text { with attrition addressed in } \\
\text { analysis }\end{array}$ \\
\hline
\end{tabular}




\begin{tabular}{|c|c|c|c|c|c|c|c|c|}
\hline Intervention & Study & $\begin{array}{l}\text { Selection } \\
\text { Bias }\end{array}$ & Attrition & $\begin{array}{l}\text { Performance } \\
\text { Bias }\end{array}$ & $\begin{array}{l}\text { Detection } \\
\text { Bias }\end{array}$ & $\begin{array}{l}\text { Reporting } \\
\text { Bias }\end{array}$ & $\begin{array}{l}\text { Overall } \\
\text { Rating }\end{array}$ & $\begin{array}{l}\text { Justification (if deviated } \\
\text { from decision aid) }\end{array}$ \\
\hline & Grady 2010 & Low & High & Low & Low & Low & Medium & $\begin{array}{l}\text { Data from long-term RCT } \\
\text { and extension(s) with } \\
\text { attrition addressed in } \\
\text { analysis }\end{array}$ \\
\hline & Johnell 2004 & Low & Medium & Low & Low & Low & Medium & $\begin{array}{l}\text { Data from long-term RCT } \\
\text { with attrition addressed in } \\
\text { analysis }\end{array}$ \\
\hline & Martino 2004 & Low & Medium & Low & Low & Low & Medium & NA \\
\hline & Martino 2005 & Low & Medium & Low & Low & Low & Medium & NA \\
\hline & Siris 2005 & Medium & Medium & Medium & Low & Low & Medium & NA \\
\hline & Sontag 2010 & Low & Medium & Low & Low & Low & Low & $\begin{array}{l}\text { Data from long-term RCT } \\
\text { with attrition addressed in } \\
\text { analysis }\end{array}$ \\
\hline \multirow[t]{3}{*}{$\begin{array}{l}\text { Hormone } \\
\text { therapy }\end{array}$} & $\begin{array}{l}\text { Wimalawansa } \\
\text { (Estrogen/Progestin) } \\
1998\end{array}$ & Low & Medium & High & Low & Low & Medium & NA \\
\hline & $\begin{array}{l}\text { Cauley } \\
\text { (Estrogen/Progestin) } \\
2003\end{array}$ & Low & Low & Low & Low & Low & Low & NA \\
\hline & $\begin{array}{l}\text { Jackson } \\
\text { (Estrogen) } \\
2006\end{array}$ & Low & Low & Low & Low & Low & Low & NA \\
\hline \multirow{3}{*}{$\begin{array}{l}\text { Osteoporosis } \\
\text { drugs }\end{array}$} & Vestergaard 2010 & Medium & Medium & Medium & Medium & Low & Medium & NA \\
\hline & Vestergaard 2011 & Medium & Medium & Low & Medium & Low & Medium & NA \\
\hline & Vestergaard 2012 & Medium & Medium & Medium & Medium & Low & Medium & NA \\
\hline
\end{tabular}




\section{Appendix D. Evidence Tables}

Table D1. Characteristics of observational studies with low or medium risk of bias

\begin{tabular}{|c|c|c|c|c|c|c|}
\hline $\begin{array}{l}\text { Study, Year } \\
\text { Design } \\
\text { Location } \\
\text { Funder } \\
\text { Risk of Bias }\end{array}$ & $\begin{array}{l}\text { Intervention } \\
\text { Comparison(s) } \\
\text { Dosage } \\
\text { Delivery route } \\
\text { Duration }\end{array}$ & $\begin{array}{l}\text { Overall sample } \\
\mathrm{N}= \\
\text { Inclusion criteria } \\
\text { Mean age } \\
\% \text { Female } \\
\text { Comorbidities } \\
\text { (\% with DM, CKD, } \\
\text { CVD) }\end{array}$ & $\begin{array}{l}\text { Intervention sample } \\
\mathrm{n}= \\
\text { Mean age } \\
\% \text { Female } \\
\text { Comorbidities } \\
\text { Baseline BMD } \\
\text { Fracture history } \\
\text { Fracture risk }\end{array}$ & $\begin{array}{l}\text { Comparison sample } \\
\mathrm{n}= \\
\text { Mean age } \\
\% \text { Female } \\
\text { Comorbidities } \\
\text { Baseline BMD } \\
\text { Fracture history } \\
\text { Fracture risk }\end{array}$ & $\begin{array}{l}\text { Followup } \\
\text { Duration }\end{array}$ & $\begin{array}{l}\text { Outcomes } \\
\text { Reported }\end{array}$ \\
\hline $\begin{array}{l}\text { Abrahamsen, } 2009 \\
\text { Database cohort } \\
\text { Denmark } \\
\text { Foundation } \\
\text { Medium }\end{array}$ & $\begin{array}{l}\text { Alendronate } \\
\text { Dose NR } \\
\text { Delivery route NR } \\
\text { Duration }>6 \mathrm{yr}\end{array}$ & $\begin{array}{l}\mathrm{N}=15561 \\
\text { Born in } 1945 \text { or earlier, } \\
\text { sustained hospital } \\
\text { treated Fx except hip } \\
\text { Fx between } 1997 \text { and } \\
2005 \\
\text { Age } 73 \\
\text { Female } 90 \% \\
\text { Comorbidities NR }\end{array}$ & $\begin{array}{l}\text { Alendronate } \\
\mathrm{n}=5187 \\
\text { Age } 73 \\
\text { Female 90\% } \\
\text { Comorbidities NR } \\
\text { Baseline BMD NR } \\
\text { Baseline fracture } \\
\text { Spine 8\% } \\
\text { Humerus 12\% } \\
\text { Forearm 35\% } \\
\text { Other 45\% } \\
\text { Fx risk NR }\end{array}$ & $\begin{array}{l}\text { No treatment } \\
\mathrm{n}=10374 \\
\\
\text { Age } 73 \\
\text { Female 90\% } \\
\text { Comorbidities NR } \\
\text { Baseline BMD NR } \\
\text { Baseline fracture } \\
\text { Spine 8\% } \\
\text { Humerus 12\% } \\
\text { Forearm 35\% } \\
\text { Other 45\% } \\
\text { Fx risk NR }\end{array}$ & $\begin{array}{l}0-9 \text { yr } \\
\text { (longterm } \\
\text { outcomes } \\
\text { reported for } \\
6-9 \text { years) }\end{array}$ & $\begin{array}{l}\text { Fracture: NR } \\
\text { Predictors: NR } \\
\text { Harms: AFF } \\
\text { Rare harms: AFF }\end{array}$ \\
\hline $\begin{array}{l}\text { Adams, } 2018 \\
\text { Database cohort } \\
\text { US } \\
\text { Industry } \\
\text { Medium }\end{array}$ & \begin{tabular}{|l} 
Bisphosphonates \\
(mostly alendronate \\
$(99 \%)$, risedronate, \\
ibandronate, etidronate, \\
pamidronate, \\
zolendronic acid) $\geq 3 \mathrm{yr}$ \\
BP drug holiday (no use \\
$\geq 1 \mathrm{yr})$ \\
Dose NR \\
Oral or injectable
\end{tabular} & $\begin{array}{l}\mathrm{N}=39502 \\
\text { Women aged } \geq 45 \text { who } \\
\text { initiated } \\
\text { bisphosphonate use } \\
1998-2008 \text { and } \\
\text { continued use } \geq 3 \mathrm{yr} \\
\text { with } \geq 50 \% \text { adherence } \\
\text { Age } 70 \\
\text { Female } 100 \% \\
\text { Comorbidities NR }\end{array}$ & $\begin{array}{l}\text { BP use } \geq 3 \text { yr } \\
\mathrm{n}=17123 \\
\text { Age } 71 \\
\text { Female } 100 \% \\
\text { Quan-Charlson } \\
\text { Comorbidity Index } 1.3 \\
\text { FN T-score -2.3 } \\
\text { FRAX (any osteoporotic } \\
\text { fx) } 14.9 \\
\text { Prior fx } 12.9 \% \\
\text { Vetebral T-score: }-1.8 \\
\text { T-score } \leq-2.5: 48 \%\end{array}$ & $\begin{array}{l}\text { BP drug holiday } \geq 1 \mathrm{yr} \\
\mathrm{n}=11497 \\
\text { Age } 69 \\
\text { Female } 100 \% \\
\text { Quan-Charlson } \\
\text { Comorbidity Index } 1.1 \\
\text { FN T-score -1.6 } \\
\text { FRAX (any } \\
\text { osteoporotic fx) } 13.4 \\
\text { Prior fx 11.2\% } \\
\text { Vetebral T-score: }-1.6 \\
\text { T-score } \leq-2.5: 37 \%\end{array}$ & $\begin{array}{l}\text { Mean } \\
\text { followup } 4 \text { yr } \\
\text { (4.9 yr for } \\
\text { drug holiday } \\
\text { group; } 3.5 \text { for } \\
\text { comparison } \\
\text { group) }\end{array}$ & $\begin{array}{l}\text { Fracture: All } \\
\text { osteoporosis related } \\
\text { fx, hip/femur fx, VFx } \\
\text { Predictors: FRAX } \\
\text { Harms: NR } \\
\text { Rare harms: AFF }\end{array}$ \\
\hline
\end{tabular}




\begin{tabular}{|c|c|c|c|c|c|c|}
\hline $\begin{array}{l}\text { Study, Year } \\
\text { Design } \\
\text { Location } \\
\text { Funder } \\
\text { Risk of Bias }\end{array}$ & $\begin{array}{l}\text { Intervention } \\
\text { Comparison(s) } \\
\text { Dosage } \\
\text { Delivery route } \\
\text { Duration }\end{array}$ & $\begin{array}{l}\text { Overall sample } \\
\mathrm{N}= \\
\text { Inclusion criteria } \\
\text { Mean age } \\
\% \text { Female } \\
\text { Comorbidities } \\
\text { (\% with DM, CKD, } \\
\text { CVD) }\end{array}$ & $\begin{array}{l}\text { Intervention sample } \\
n= \\
\text { Mean age } \\
\% \text { Female } \\
\text { Comorbidities } \\
\text { Baseline BMD } \\
\text { Fracture history } \\
\text { Fracture risk }\end{array}$ & $\begin{array}{l}\text { Comparison sample } \\
\text { n= } \\
\text { Mean age } \\
\text { \% Female } \\
\text { Comorbidities } \\
\text { Baseline BMD } \\
\text { Fracture history } \\
\text { Fracture risk }\end{array}$ & $\begin{array}{l}\text { Followup } \\
\text { Duration }\end{array}$ & \begin{tabular}{|l|} 
Outcomes \\
Reported
\end{tabular} \\
\hline $\begin{array}{l}\text { Chiu, } 2014 \\
\text { Retrospective cohort } \\
\text { Taiwan } \\
\text { Funding NR } \\
\text { Medium }\end{array}$ & \begin{tabular}{|l|} 
Alendronate \\
Dose NR \\
Delivery route NR \\
Mean duration 4 yr (0-11 \\
yr)
\end{tabular} & $\begin{array}{l}\mathrm{N}=7332 \text { alendronate } \\
\text { users } \\
\text { Women } \geq 50 \text { and men } \\
\geq 60 \text { yr old prescribed } \\
\text { alendronate via hospital } \\
\text { pharmacy. Excluded } \\
\text { adults with head or } \\
\text { neck cancer } \\
\text { before/during treatment. } \\
\text { Age } 72 \\
\text { Female } 88 \% \\
\text { DM } 16 \% \\
\text { CKD } 2 \% \\
\text { Hypertension } 44 \%\end{array}$ & $\begin{array}{l}\text { ONJ } \\
n=40 \\
\text { Age } 75 \\
\text { Female } 98 \% \\
\text { DM } 35 \% \\
\text { CKD 3\% } \\
\text { BMD NR } \\
\text { Fx history or risk NR }\end{array}$ & \begin{tabular}{|l} 
Did not have ONJ \\
$\mathrm{n}=7292$ \\
Age 72 \\
Female $88 \%$ \\
DM $19 \%$ \\
CKD $2 \%$ \\
BMD NR \\
Fx history of risk NR
\end{tabular} & $\begin{array}{l}\text { Mean drug } \\
\text { duration } 4 \mathrm{yr} \\
(0-11 \mathrm{yr})\end{array}$ & $\begin{array}{l}\text { Fracture: NR } \\
\text { Predictors: NR } \\
\text { Harms: NR } \\
\text { Rare harms: ONJ }\end{array}$ \\
\hline $\begin{array}{l}\text { Lin, } 2014 \\
\text { Case control } \\
\text { Taiwan } \\
\text { Government } \\
\text { Medium }\end{array}$ & \begin{tabular}{|l|} 
Alendronate \\
Nonbisphoshonate \\
osteoporosis \\
medications (raloxifene \\
or calcitonin) \\
Dose NR \\
Delivery route NR \\
Duration 6 yr (range NR) \\
\end{tabular} & $\begin{array}{l}\mathrm{N}=32006 \\
\text { Women and men aged } \\
\geq 50 \text { with newly } \\
\text { diagnosed hip or VFx } \\
\text { new to alendronate } \\
\text { within } 30 \text { days of Fx } \\
\text { compared with } \\
\text { propensity-matched } \\
\text { raloxifene or calcitonin } \\
\text { users. } \\
\text { Age } 74 \\
\text { Female } 84 \% \\
\text { DM } 24 \% \\
\text { Renal disease } 3 \%\end{array}$ & $\begin{array}{l}\text { Alendronate } \\
\mathrm{n}=16003 \\
\text { Age } 74 \\
\text { Female 84\% } \\
\text { DM 24\% } \\
\text { Renal disease 3\% } \\
\text { BMD NR } \\
\text { Index hip Fx 22\% } \\
\text { Index VFx 78\% } \\
\text { Fx risk NR }\end{array}$ & $\begin{array}{l}\text { Raloxifene or } \\
\text { calcitonin } \\
\mathrm{n}=16003 \\
\text { Age } 74 \\
\text { Female 84\% } \\
\text { DM } 24 \% \\
\text { Renal disease 3\% } \\
\text { BMD NR } \\
\text { Index hip Fx 22\% } \\
\text { Index VFx 78\% } \\
\text { Fx risk NR }\end{array}$ & $6 \mathrm{yr}$ & $\begin{array}{l}\text { Fracture: NR } \\
\text { Predictors: NR } \\
\text { Harms: NR } \\
\text { Rare harms: ONJ }\end{array}$ \\
\hline
\end{tabular}




\begin{tabular}{|c|c|c|c|c|c|c|}
\hline $\begin{array}{l}\text { Study, Year } \\
\text { Design } \\
\text { Location } \\
\text { Funder } \\
\text { Risk of Bias }\end{array}$ & $\begin{array}{l}\text { Intervention } \\
\text { Comparison(s) } \\
\text { Dosage } \\
\text { Delivery route } \\
\text { Duration }\end{array}$ & $\begin{array}{l}\text { Overall sample } \\
\mathrm{N}= \\
\text { Inclusion criteria } \\
\text { Mean age } \\
\% \text { Female } \\
\text { Comorbidities } \\
\text { (\% with DM, CKD, } \\
\text { CVD) }\end{array}$ & $\begin{array}{l}\text { Intervention sample } \\
\mathrm{n}= \\
\text { Mean age } \\
\% \text { Female } \\
\text { Comorbidities } \\
\text { Baseline BMD } \\
\text { Fracture history } \\
\text { Fracture risk }\end{array}$ & $\begin{array}{l}\text { Comparison sample } \\
\mathrm{n}= \\
\text { Mean age } \\
\text { \% Female } \\
\text { Comorbidities } \\
\text { Baseline BMD } \\
\text { Fracture history } \\
\text { Fracture risk }\end{array}$ & $\begin{array}{l}\text { Followup } \\
\text { Duration }\end{array}$ & $\begin{array}{l}\text { Outcomes } \\
\text { Reported }\end{array}$ \\
\hline $\begin{array}{l}\text { Erviti, } 2013 \\
\text { Case control } \\
\text { Spain } \\
\text { Spanish Ministry of } \\
\text { Health } \\
\text { Medium }\end{array}$ & $\begin{array}{l}\text { Bisphosphonates } \\
\text { Dose NR } \\
\text { Delivery route NR } \\
>3 \mathrm{yr}\end{array}$ & $\begin{array}{l}\mathrm{N}=264 \\
\text { Women aged }>65 \\
\text { Age } 82 \\
\text { Female } 100 \% \\
\text { Comorbidities } \\
\text { DM } 21 \% \\
\text { CKD } 6 \% \\
\text { CVD NR }\end{array}$ & $\begin{array}{l}\text { Cases } \\
\mathrm{n}=44 \\
\text { Age } 82 \\
\text { Female } 100 \% \\
\text { DM } 18 \% \\
\text { CKD 5\% } \\
\text { CVD NR }\end{array}$ & $\begin{array}{l}\text { Controls } \\
\mathrm{n}=220 \\
\text { Age } 82 \\
\text { Female } 100 \% \\
\text { DM } 21 \% \\
\text { CKD 5\% } \\
\text { CVD NR }\end{array}$ & $>3 \mathrm{yr}$ & $\begin{array}{l}\text { Fracture: NR } \\
\text { Predictors: NR } \\
\text { Harms: NR } \\
\text { Rare harms: AFF }\end{array}$ \\
\hline $\begin{array}{l}\text { Kim, } 2011 \\
\text { Database cohort } \\
\text { US } \\
\text { No funding } \\
\text { Medium }\end{array}$ & $\begin{array}{l}\text { Bisphosphonates } \\
\text { (alendronate, } \\
\text { risedronate, etidronate) } \\
\text { Raloxifene or calcitonin } \\
\text { nasal spray } \\
\\
\text { Dose NR } \\
\text { Oral (except calcitonin) } \\
\text { >90 days }\end{array}$ & $\begin{array}{l}\mathrm{N}=33815 \\
\text { Medicare beneficiaries } \\
\text { with at least one } \\
\text { osteoporosis treatment } \\
\text { prescription and one } \\
\text { medical claim in } \\
\text { previous } 3 \text { consecutive } \\
6 \text { month periods; Jan } \\
1996-D e c \text { 2006 } \\
\text { Age } 80 \\
\text { Female 97\% } \\
\text { White 95\% } \\
\text { DM 26\% } \\
\text { CKD 3\% } \\
\text { Hypertension 67\% } \\
\text { CHF 22\% }\end{array}$ & $\begin{array}{l}\text { Bisphosphonates } \\
n=17028 \\
\text { Age } 80 \\
\text { Female 97\% } \\
\text { White 95\% } \\
\text { DM 26\% } \\
\text { CKD 3\% } \\
\text { Hypertension 66\% } \\
\text { CHF 22\% } \\
\text { BMD NR } \\
\text { Hip Fx 4\% } \\
\text { VFx 11\% } \\
\text { Fx risk NR }\end{array}$ & $\begin{array}{l}\text { Raloxifene or } \\
\text { calcitonin } \\
\mathrm{n}=16787 \\
\text { Age } 80 \\
\text { Female 97\% } \\
\text { White } 95 \% \\
\text { DM 26\% } \\
\text { CKD 3\% } \\
\text { Hypertension 67\% } \\
\text { CHF 22\% } \\
\text { BMD NR } \\
\text { Hip Fx 4\% } \\
\text { VFx 11\% } \\
\text { Fx NR }\end{array}$ & \begin{tabular}{|l} 
Up to $9.5 \mathrm{yr}$ \\
(est) (mean 2 \\
$\mathrm{yr})$
\end{tabular} & $\begin{array}{l}\text { Fracture: NR } \\
\text { Predictors: none } \\
\text { Harms: NR } \\
\text { Rare harms: } \\
\text { subtrochanteric and } \\
\text { diaphyseal femur fx } \\
\text { (proxy for AFF) }\end{array}$ \\
\hline
\end{tabular}




\begin{tabular}{|c|c|c|c|c|c|c|}
\hline $\begin{array}{l}\text { Study, Year } \\
\text { Design } \\
\text { Location } \\
\text { Funder } \\
\text { Risk of Bias }\end{array}$ & $\begin{array}{l}\text { Intervention } \\
\text { Comparison(s) } \\
\text { Dosage } \\
\text { Delivery route } \\
\text { Duration }\end{array}$ & $\begin{array}{l}\text { Overall sample } \\
\mathrm{N}= \\
\text { Inclusion criteria } \\
\text { Mean age } \\
\% \text { Female } \\
\text { Comorbidities } \\
\text { (\% with DM, CKD, } \\
\text { CVD) }\end{array}$ & $\begin{array}{l}\text { Intervention sample } \\
\mathrm{n}= \\
\text { Mean age } \\
\% \text { Female } \\
\text { Comorbidities } \\
\text { Baseline BMD } \\
\text { Fracture history } \\
\text { Fracture risk }\end{array}$ & $\begin{array}{l}\text { Comparison sample } \\
\mathrm{n}= \\
\text { Mean age } \\
\text { \% Female } \\
\text { Comorbidities } \\
\text { Baseline BMD } \\
\text { Fracture history } \\
\text { Fracture risk }\end{array}$ & $\begin{array}{l}\text { Followup } \\
\text { Duration }\end{array}$ & $\begin{array}{l}\text { Outcomes } \\
\text { Reported }\end{array}$ \\
\hline $\begin{array}{l}\text { Koh, } 2017 \\
\text { Case control } \\
\text { South Korea } \\
\text { Funding NR } \\
\text { Medium }\end{array}$ & $\begin{array}{l}\text { Bisphosphonates } \\
\text { (alendronate, } \\
\text { risedronate, } \\
\text { ibandronate, or } \\
\text { zolendronate) } \\
\\
\text { Dose NR } \\
\text { Delivery route NR } \\
\text { Duration: varied (See } \\
\text { Cases and Controls) }\end{array}$ & $\begin{array}{l}\mathrm{N}=172 \text { out of } 35,104 \\
\text { patients prescribed } \\
\text { bisphosphonates } \geq 1 \mathrm{yr}, \\
\text { Cancer history, } \\
\text { hyperparathyroidism, } \\
\text { renal osteodystrophy, } \\
\text { and osteomalacia } \\
\text { excluded. Cases } \\
\text { experienced AFF. } \\
\text { Controls received } \\
\text { bisphosphonates } \\
\text { without AFF matched } \\
\text { 1:3 from EHR. } \\
\text { Age } 68 \\
\text { Female } 100 \% \\
\text { Race NR } \\
\text { DM } 13 \%\end{array}$ & $\begin{array}{l}\text { Cases: AFF } \\
\text { n=43 } \\
\text { Mean drug duration: } 7.3 \mathrm{yr} \\
\text { Age } 68 \text { (NR) } \\
\text { Female 100\% } \\
\text { Race NR } \\
\text { DM 19\% } \\
\text { BMD NR } \\
\text { Previous osteoporotic Fx } \\
19 \% \\
\text { Fx risk NR } \\
\text { Had drug holiday } 16 \% \\
\text { Glucocorticoid } \geq 1 \mathrm{yr} 37 \% \\
\end{array}$ & $\begin{array}{l}\text { Controls: No AFF } \\
\mathrm{n}=129 \\
\text { Mean drug duration: } \\
5.2 \mathrm{yr} \\
\\
\text { Age } 68 \text { (NR) } \\
\text { Female 100\% } \\
\text { Race NR } \\
\text { DM } 11 \% \\
\text { BMD NR } \\
\text { Previous osteoporotic } \\
\text { Fx } 12 \% \\
\text { Fx risk NR } \\
\text { Discontinued drug } \\
36 \% \\
\text { Glucocorticoid } \geq 1 \mathrm{yr} \\
9 \%\end{array}$ & $\begin{array}{l}\text { Mean } 5.7 \mathrm{yr} \\
\text { (range NR) }\end{array}$ & $\begin{array}{l}\text { Fracture: NR } \\
\text { Predictors: NR } \\
\text { Harms: NR } \\
\text { Rare harms: AFF }\end{array}$ \\
\hline $\begin{array}{l}\text { Lim, } 2018 \\
\text { Case control } \\
\text { Korea } \\
\text { Industry } \\
\text { Medium }\end{array}$ & $\begin{array}{l}\text { Bisphosphonates } \\
\text { (alendronate, } \\
\text { risedronate, } \\
\text { ibandronate, } \\
\text { pamidronate or } \\
\text { zoledronate) } \\
\\
\text { Dose NR } \\
\text { Delivery route NR } \\
\text { Duration mean } 5.2 \mathrm{yr} \\
\text { (range 1-17yr) for cases }\end{array}$ & $\begin{array}{l}\mathrm{N}=290 \text { from a cohort of } \\
6644 \text { patients with hip } \\
\text { and femoral fractures } \\
\text { identified via medical } \\
\text { record diagnosis codes } \\
\text { Age } 72 \\
\text { Female } 100 \% \\
\text { Comorbidities } \\
\text { DM } 20 \% \\
\text { CKD NR } \\
\text { CVD } 10 \%\end{array}$ & $\begin{array}{l}\text { Cases: AFF } \\
\mathrm{n}=196 \\
\text { Age } 72 \\
\text { Female 100\% } \\
\text { Comorbidities } \\
\text { DM 16\% } \\
\text { CKD NR } \\
\text { CVD 13\% } \\
\text { T-score <-2.0 85\% }\end{array}$ & $\begin{array}{l}\text { Controls: Typical } \\
\text { femoral fracture } \\
\mathrm{n}=94 \\
\text { Age } 71 \\
\text { Female 100\% } \\
\text { Comorbidities } \\
\text { DM 28\% } \\
\text { CKD NR } \\
\text { CVD 5\% } \\
\text { T-score <-2.0 18\% }\end{array}$ & $\begin{array}{l}\text { Cases: } \\
\text { mean 5.2 yr } \\
\text { (range 1-17 } \\
\text { yr) }\end{array}$ & $\begin{array}{l}\text { Fracture: NR } \\
\text { Predictors: NR } \\
\text { Harms: NR } \\
\text { Rare harms: AFF }\end{array}$ \\
\hline
\end{tabular}




\begin{tabular}{|c|c|c|c|c|c|c|}
\hline $\begin{array}{l}\text { Study, Year } \\
\text { Design } \\
\text { Location } \\
\text { Funder } \\
\text { Risk of Bias }\end{array}$ & $\begin{array}{l}\text { Intervention } \\
\text { Comparison(s) } \\
\text { Dosage } \\
\text { Delivery route } \\
\text { Duration }\end{array}$ & $\begin{array}{l}\text { Overall sample } \\
\mathrm{N}= \\
\text { Inclusion criteria } \\
\text { Mean age } \\
\% \text { Female } \\
\text { Comorbidities } \\
\text { (\% with DM, CKD, } \\
\text { CVD) }\end{array}$ & $\begin{array}{l}\text { Intervention sample } \\
\mathrm{n}= \\
\text { Mean age } \\
\% \text { Female } \\
\text { Comorbidities } \\
\text { Baseline BMD } \\
\text { Fracture history } \\
\text { Fracture risk }\end{array}$ & $\begin{array}{l}\text { Comparison sample } \\
\mathrm{n}= \\
\text { Mean age } \\
\% \text { Female } \\
\text { Comorbidities } \\
\text { Baseline BMD } \\
\text { Fracture history } \\
\text { Fracture risk }\end{array}$ & $\begin{array}{l}\text { Followup } \\
\text { Duration }\end{array}$ & $\begin{array}{l}\text { Outcomes } \\
\text { Reported }\end{array}$ \\
\hline $\begin{array}{l}\text { Park-Wyllie, } 2011 \\
\text { Case control } \\
\text { Canada } \\
\text { Government } \\
\text { Medium }\end{array}$ & \begin{tabular}{|l} 
Bisphosphonates \\
(alendronate, \\
risedronate or \\
etidronate) \\
Dose NR \\
$<100$ days (reference) to \\
$<5 \mathrm{yr}$
\end{tabular} & $\begin{array}{l}\mathrm{N}=4296 \\
\text { Postmenopausal } \\
\text { women aged } \geq 50 \text {. } \\
\text { Excluded women with } \\
10 \text { yr history of cancer, } \\
\text { conditions with altered } \\
\text { bone integrity, gastric } \\
\text { bypass, used other } \\
\text { osteoporosis drugs, } \\
\text { high energy trauma, } \\
\text { and Fx treated with hip } \\
\text { replacement. Cases } \\
\text { matched with up to } 5 \\
\text { controls. } \\
\\
\text { Age } 83 \text { (median) } \\
\text { Female 100\% } \\
\text { Race NR } \\
\text { CHF 6\% } \\
\text { DM 7\% }\end{array}$ & $\begin{array}{l}\text { Cases: Bisphosphonates } \\
\geq 5 \text { yr } \\
n=716 \\
\text { Age } 83 \text { (median) } \\
\text { Female } 100 \% \\
\text { Race NR } \\
\text { CHF } 13 \% \\
\text { DM } 12 \% \\
\text { BMD NR } \\
\text { Osteoporotic Fx in prior } 5 \\
\text { yr } 70 \% \\
\text { Falls prior } 5 \text { yr: } 67 \%\end{array}$ & $\begin{array}{l}\text { Controls: } \\
\text { Bisphosphonates 3-5 } \\
\text { yr } \\
\mathrm{n}=3580 \\
\text { Age } 83 \text { (median) } \\
\text { Female } 100 \% \\
\text { Race NR } \\
\text { CHF } 5 \% \\
\text { DM } 6 \% \\
\text { BMD NR } \\
\text { Osteoporotic Fx in } \\
\text { prior } 5 \text { yr } 24 \% \\
\text { Falls prior } 5 \text { yr: } 6 \%\end{array}$ & $\begin{array}{l}\text { Cases: } \\
\text { median } 4 \text { yr } \\
\text { (range 2.6- } \\
5.4 \text { yr) }\end{array}$ & $\begin{array}{l}\text { Fracture: NR } \\
\text { Predictors: drug } \\
\text { duration } \\
\text { Harms: NR } \\
\text { Rare harms: AFF }\end{array}$ \\
\hline $\begin{array}{l}\text { Schilcher, } 2015 \\
\text { Cohort/Case control } \\
\text { Sweden } \\
\text { Swedish Research } \\
\text { Council } \\
\text { Medium }\end{array}$ & $\begin{array}{l}\text { Bisphosphonates } \\
\text { Dose NR } \\
\text { Delivery route NR } \\
4-5 \mathrm{yr}\end{array}$ & $\begin{array}{l}\text { Cohort of } 2.9 \text { million } \\
\text { women and men aged } \\
\geq 55 \text { yr over the study } \\
\text { period of } 2008-2010, \\
97,900 \text { with a history of } \\
\text { bisphosphonate use } \\
\text { (87\% women) }\end{array}$ & $\begin{array}{l}\text { Cases: AFF } \\
n=172 \\
\text { Age } 76 \\
\text { Female } 93 \% \\
\text { Comorbidities } \\
\text { DM } 4 \% \\
\text { CKD } 30 \% \\
\text { CVD } 46 \%\end{array}$ & $\begin{array}{l}\text { Controls: Ordinary } \\
\text { shaft fx } \\
\mathrm{n}=952 \\
\text { Age } 82 \\
\text { Female } 81 \% \\
\text { Comorbidities } \\
\text { DM } 16 \% \\
\text { CKD 40\% } \\
\text { CVD 60\% }\end{array}$ & $4-5 \mathrm{yr}$ & $\begin{array}{l}\text { Fracture: NR } \\
\text { Predictors: age } \\
\text { Harms: NR } \\
\text { Rare harms: AFF }\end{array}$ \\
\hline
\end{tabular}




\begin{tabular}{|c|c|c|c|c|c|c|}
\hline $\begin{array}{l}\text { Study, Year } \\
\text { Design } \\
\text { Location } \\
\text { Funder } \\
\text { Risk of Bias }\end{array}$ & $\begin{array}{l}\text { Intervention } \\
\text { Comparison(s) } \\
\text { Dosage } \\
\text { Delivery route } \\
\text { Duration }\end{array}$ & $\begin{array}{l}\text { Overall sample } \\
\mathrm{N}= \\
\text { Inclusion criteria } \\
\text { Mean age } \\
\% \text { Female } \\
\text { Comorbidities } \\
\text { (\% with DM, CKD, } \\
\text { CVD) }\end{array}$ & \begin{tabular}{|l} 
Intervention sample \\
$\mathrm{n}=$ \\
Mean age \\
$\%$ Female \\
Comorbidities \\
Baseline BMD \\
Fracture history \\
Fracture risk
\end{tabular} & $\begin{array}{l}\text { Comparison sample } \\
\mathrm{n}= \\
\text { Mean age } \\
\% \text { Female } \\
\text { Comorbidities } \\
\text { Baseline BMD } \\
\text { Fracture history } \\
\text { Fracture risk }\end{array}$ & $\begin{array}{l}\text { Followup } \\
\text { Duration }\end{array}$ & \begin{tabular}{|l|} 
Outcomes \\
Reported
\end{tabular} \\
\hline $\begin{array}{l}\text { Vestergaard Series } \\
\text { Vestergaard 2010, } \\
\text { Vestergaard 2011, } \\
\text { Vestergaard } 2012 \\
\text { Database cohort } \\
\text { Denmark } \\
\text { Industry, foundation } \\
\text { Medium }\end{array}$ & \begin{tabular}{|l} 
Osteoporosis drugs \\
(bisphosphonates \\
including alendronate, \\
risedronate, zoledronate, \\
ibandronate), raloxifene, \\
teriparatide \\
Dose varied \\
Delivery route varied \\
3.8 yr observation \\
(mean)
\end{tabular} & $\begin{array}{l}\text { N= varied } \\
\text { Danish people who } \\
\text { filled a prescription for a } \\
\text { drug used to treat or } \\
\text { prevent osteoporosis } \\
\text { Jan 1, 1996-Dec 31, } \\
\text { 2006; matched (1:3) by } \\
\text { age and gender to } \\
\text { Danish people who had } \\
\text { not filled such a } \\
\text { prescription } \\
\text { Age } 71 \\
\text { Female } 85 \% \\
\text { White NR } \\
\text { DM } 2 \% \\
\text { CKD NR } \\
\text { Hypertension } 42 \% \\
\text { CHF } 2 \%\end{array}$ & $\begin{array}{l}\text { n= varied } \\
\text { Age } 71 \\
\text { Female 85\% } \\
\text { White NR } \\
\text { DM 2\% } \\
\text { CKD NR } \\
\text { Hypertension 37\% } \\
\text { CHF 3\% }\end{array}$ & $\begin{array}{l}\mathrm{n}=\text { varied } \\
\\
\text { Age } 71 \\
\text { Female } 85 \% \\
\text { White NR } \\
\text { DM 2\% } \\
\text { CKD NR } \\
\text { Hypertension 34\% } \\
\text { CHF 2\% }\end{array}$ & \begin{tabular}{|l|}
$3.8 \mathrm{yr}$ (mean, \\
after date of \\
exposure)
\end{tabular} & $\begin{array}{l}\text { Fracture: NR } \\
\text { Predictors: } \\
\text { Cumulative dose } \\
\text { Harms: NR } \\
\text { Rare Harms: } \\
\text { Inflammatory jaw } \\
\text { events (proxy for } \\
\text { ONJ) (Vestergaard } \\
\text { 2012), } \\
\text { Femoral shaft and } \\
\text { subtrochanteric } \\
\text { fractures (proxy for } \\
\text { AFF) (Vestergaard } \\
\text { 2011), } \\
\text { AFib or atrial flutter } \\
\text { (Vestergaard 2010) }\end{array}$ \\
\hline
\end{tabular}

Abbreviations: Afib=atrial fibrillation; AFF=atypical femoral fracture; ALP=alkaline phosphatase; BMD=bone mineral density; BP=bisphosphonates; BSALP=bone specific alkaline phosphatase; $\mathrm{CFx}=$ clinical fracture; $\mathrm{CHF}=$ congestive heart failure; $\mathrm{CKD}=$ chronic kidney disease; $\mathrm{CVD}=$ cardiovascular disease; $\mathrm{DM}=$ diabetes mellitus; $\mathrm{EHR}=$ electronic health record; FIT=Fracture Intervention Trial; FN=femoral neck; FRAX=World Health Organization (WHO) Fracture Risk Assessment Tool; Fx=fracture; g/cm²=grams per centimeter squared; LS=lumbar spine; mg=milligrams; N=number; NR=not reported; NTX=N-terminal telopeptide of type 1 collagen; ONJ=osteonecrosis of the jaw; QD=per day; RCT=randomized controlled trial; TH=total hip; UGI=upper Gastrointestinal; VFx=vertebral fracture; vs=versus; $y r=y e a r s$ 
Table D2. Characteristics of RCTs and CCTs with low or medium risk of bias

\begin{tabular}{|c|c|c|c|c|c|c|}
\hline $\begin{array}{l}\text { Study, Year } \\
\text { Design } \\
\text { Location } \\
\text { Funder } \\
\text { Risk of Bias }\end{array}$ & $\begin{array}{l}\text { Intervention } \\
\text { Dosage } \\
\text { Delivery Route } \\
\text { Duration }\end{array}$ & $\begin{array}{l}\text { Comparison } \\
\text { Dosage } \\
\text { Delivery Route } \\
\text { Duration }\end{array}$ & \begin{tabular}{|l} 
Sample \\
\# Randomized \\
Mean age \\
$\%$ Female \\
$\%$ White Race \\
Comorbidities \\
(\% with DM, CKD, \\
CVD) \\
Fracture History \\
Fracture Risk \\
\end{tabular} & $\begin{array}{l}\text { Inclusion criteria } \\
\text { Baseline BMD }\end{array}$ & $\begin{array}{l}\text { Followup } \\
\text { Duration }\end{array}$ & $\begin{array}{l}\text { Outcomes } \\
\text { Reported }\end{array}$ \\
\hline $\begin{array}{l}\text { Bauer, } 2000 \\
\text { Pooled FIT-I and FIT-II } \\
\text { RCTs } \\
\text { US } \\
\text { Industry } \\
\text { Medium }\end{array}$ & $\begin{array}{l}\text { Alendronate } \\
5 \mathrm{mg} \text { QD } \times 2 \mathrm{yr} \text { then } 10 \\
\text { mg QD thereafter } \\
\text { Oral } \\
\text { FIT-I: mean } 2.9 \mathrm{yr} \\
\text { FIT-II: mean } 4.3 \mathrm{yr}\end{array}$ & $\begin{array}{l}\text { Placebo } \\
\text { QD } \\
\text { Oral } \\
\text { FIT-I: mean } 2.9 \mathrm{yr} \\
\text { FIT-II: mean } 4.3 \mathrm{yr}\end{array}$ & $\begin{array}{l}\mathrm{N}=6459 \\
\text { Age } 69 \\
\text { Female } 100 \% \\
\text { Race NR } \\
\text { Comorbidities NR } \\
\text { Prior Fx NR }\end{array}$ & $\begin{array}{l}\text { Postmenopausal women } \\
\text { with or without baseline } \\
\text { VFx. } \\
\text { FN BMD } \leq 0.68 \mathrm{~g} / \mathrm{cm}^{2} \\
\text { (T score } \leq-1.6 \text { ) }\end{array}$ & $\begin{array}{l}\text { Mean } 3.8 \mathrm{yr} ; \\
\text { (median NR); } \\
\text { pooled study } \\
\text { arms with } \\
\text { mean } 2.9 \mathrm{yr} \\
\text { and } 4.3 \mathrm{yr} \\
\text { followup) }\end{array}$ & $\begin{array}{l}\text { Fracture: NR } \\
\text { Predictors: age, UGI } \\
\text { disease, NSAID use. } \\
\text { Harms: UGI only (any, } \\
\text { gastric/ duodenal, } \\
\text { esophageal) } \\
\text { Rare harms: NR }\end{array}$ \\
\hline $\begin{array}{l}\text { Bauer, } 2006 \\
\text { Pooled FIT-I and FIT-II } \\
\text { RCTs } \\
\text { USA, } 11 \text { centers } \\
\text { Industry } \\
\text { Medium }\end{array}$ & $\begin{array}{l}\text { Alendronate } \\
5 \text { mg QD } x 2 \text { yr then } 10 \\
\text { mg QD thereafter } \\
\text { Oral } \\
\text { FIT-I: } 3 \mathrm{yr} \\
\text { FIT-II: } 4 \text { yr } \\
\text { Calcium and Vitamin D } \\
\text { supplements }\end{array}$ & $\begin{array}{l}\text { Placebo } \\
\text { QD } \\
\text { Oral } \\
\text { FIT-I: } 3 \mathrm{yr} \\
\text { FIT-II: } 4 \mathrm{yr} \\
\text { Calcium and Vitamin D } \\
\text { supplements }\end{array}$ & $\begin{array}{l}\mathrm{N}=6186 \text { (completed } \\
\text { trial, complete data) } \\
\text { Age } 69 \mathrm{yr} \\
\text { Female } 100 \% \\
\text { Race NR } \\
\text { Comorbidities NR } \\
\text { Prior fracture: NR } \\
\text { Baseline VFx: } 31 \%\end{array}$ & $\begin{array}{l}\text { Postmenopausal women } \\
\text { with or without baseline } \\
\text { VFx and } \\
\text { FN-BMD } \leq 0.68 \mathrm{~g} / \mathrm{cm}^{2} \\
\text { (T score } \leq-1.6 \text { ) }\end{array}$ & $\begin{array}{l}\text { "Mean } 3.2 \mathrm{yr} " \\
\text { (median NR) }\end{array}$ & $\begin{array}{l}\text { Fracture: clinical } \\
\text { (pathologic or severe } \\
\text { trauma excluded), } \\
\text { radiographic vertebral } \\
\text { Predictors: } \\
\text { pretreatment bone } \\
\text { turnover; osteoporosis } \\
\text { Harms: NR } \\
\text { Rare harms: NR }\end{array}$ \\
\hline $\begin{array}{l}\text { Black, } 2006 \\
\text { Ensrud, } 2004 \\
\text { FLEX } \\
\text { RCT extension with } \\
\text { (rerandomization and } \\
\text { discontinuation } \\
\text { US } \\
\text { Industry } \\
\text { Low }\end{array}$ & $\begin{array}{l}\text { Alendronate } \\
5 \mathrm{mg} \text { QD } \\
\text { Oral } \\
5 \mathrm{yr} \text { (5-10 yr total) } \\
\text { Alendronate } 10 \mathrm{mg} \\
\text { Daily } \\
\text { Oral } \\
5 \mathrm{yr} \text { (5-10 yr total) } \\
1.6 \% \text { on raloxifene or } \\
\text { HRT. Calcium 500mg } \\
\text { and } 250 \text { IU Vitamin D } \\
\text { offered. }\end{array}$ & $\begin{array}{l}\text { Placebo (discontinue } \\
\text { alendronate after } 5 \mathrm{yr} \text { ) } \\
\text { QD } \\
\text { Oral } \\
5 \mathrm{yr} \\
2.7 \% \text { on raloxifene or } \\
\text { HRT. Calcium 500mg } \\
\text { and } 250 \text { IU Vitamin D } \\
\text { offered. }\end{array}$ & $\begin{array}{l}\mathrm{N}=1099 \text { (39\% of } 2852 \\
\text { ALN users from FIT) } \\
\text { with mean of } 5 \mathrm{yr} \text {. prior } \\
\text { ALN use) } \\
\text { Age } 73 \\
\text { Female } 100 \% \\
\text { White } 98 \% \\
\text { Comorbidities NR } \\
\text { Prior Fx } \geq 45 \text { yr } 60 \% \\
\text { Prevalent VFx } 34 \%\end{array}$ & $\begin{array}{l}\text { Prior enrollment in ALN } \\
\text { arm of FIT (I or II), took } \\
\geq 3 \text { yr of ALN, with TH } \\
\text { BMD >-3.5 at FLEX } \\
\text { baseline }\end{array}$ & $\begin{array}{l}5 \mathrm{yr} \\
\text { (5-10 yr FIT } \\
\text { extension) }\end{array}$ & $\begin{array}{l}\text { Fractures: } \\
\text { CVFx, RVFx, all } \\
\text { nonVFx, forearm fx, } \\
\text { hip fx; pathologic, } \\
\text { skull, or excess } \\
\text { trauma fxs excluded. } \\
\text { Other outcomes: } \\
\text { Change in TH BMD, } \\
\text { new RVFx } \\
\text { Predictors: FN BMD, } \\
\text { prevalent VFx } \\
\text { Harms: UGI, death } \\
\text { Rare harms: ONJ }\end{array}$ \\
\hline
\end{tabular}




\begin{tabular}{|c|c|c|c|c|c|c|}
\hline $\begin{array}{l}\text { Study, Year } \\
\text { Design } \\
\text { Location } \\
\text { Funder } \\
\text { Risk of Bias }\end{array}$ & \begin{tabular}{|l} 
Intervention \\
Dosage \\
Delivery Route \\
Duration
\end{tabular} & \begin{tabular}{|l|} 
Comparison \\
Dosage \\
Delivery Route \\
Duration
\end{tabular} & $\begin{array}{l}\text { Sample } \\
\text { \# Randomized } \\
\text { Mean age } \\
\text { \% Female } \\
\% \text { White Race } \\
\text { Comorbidities } \\
\text { (\% with DM, CKD, } \\
\text { CVD) } \\
\text { Fracture History } \\
\text { Fracture Risk }\end{array}$ & \begin{tabular}{|l|} 
Inclusion criteria \\
Baseline BMD
\end{tabular} & $\begin{array}{l}\text { Followup } \\
\text { Duration }\end{array}$ & $\begin{array}{l}\text { Outcomes } \\
\text { Reported }\end{array}$ \\
\hline $\begin{array}{l}\text { Black, } 2010 \\
\text { RCTs (pooled Fit-I and } \\
\text { Fit-II, FLEX) } \\
\text { USA } \\
\text { Industry } \\
\text { Low }\end{array}$ & $\begin{array}{l}\text { Alendronate } \\
\text { Pooled FIT-I and FIT-II: } \\
\text { Alendronate } \\
5 \text { mg QD x } 2 \text { yr then } 10 \\
\text { mg QD thereafter } \\
\text { Oral } \\
\text { FIT-I: mean } 2.9 \mathrm{yr} \\
\text { FIT-II: mean } 4.3 \mathrm{yr} \\
\text { Calcium and Vitamin D: } \\
\text { NR } \\
\text { FLEX: } \\
\text { Alendronate } \\
5 \text { mg QD } \\
\text { Oral } \\
5 \text { yr (5-10 yr total) } \\
\text { Alendronate } 10 \text { mg } \\
\text { Daily } \\
\text { Oral } \\
5 \text { yr (5-10 yr total) }\end{array}$ & $\begin{array}{l}\text { Pooled FIT-I and FIT-II: } \\
\text { Placebo } \\
\text { QD } \\
\text { Oral } \\
\text { FIT-I: mean } 2.9 \mathrm{yr} \\
\text { FIT-II: mean } 4.3 \mathrm{yr} \\
\text { Calcium and Vitamin D: } \\
\text { NR } \\
\text { FLEX: } \\
\text { Placebo (discontinued } \\
\text { alendronate after } 5 \mathrm{yr} \text { ) } \\
\text { QD } \\
\text { Oral } \\
5 \mathrm{yr}\end{array}$ & $\begin{array}{l}\text { Pooled FIT-I and FIT-II: } \\
\text { N=6459 } \\
\text { For baseline } \\
\text { characteristics, see } \\
\text { Donaldson, } 2012 \\
\\
\text { FLEX: } \\
\mathrm{N}=1099 \\
\text { For baseline } \\
\text { characteristics, see } \\
\text { Schwartz, } 2010\end{array}$ & $\begin{array}{l}\text { Pooled FIT-I and FIT-II: } \\
\text { see Donaldson, } 2012 \\
\text { FLEX: } \\
\text { see Schwartz, } 2010\end{array}$ & $\begin{array}{l}\text { Pooled FIT-I } \\
\text { and FIT-II: } \\
\text { "Median } \\
1457 \text { days" } \\
\text { (4 yr) } \\
\text { [31.4\% } \\
\text { median } 3 \text { yr.; } \\
68.6 \% \\
\text { median } 4 \text { yr.] } \\
\text { FLEX: } \\
5 \text { yr } \\
\text { (5-10 yr FIT } \\
\text { extension) }\end{array}$ & $\begin{array}{l}\text { Fracture: NR } \\
\text { Predictors: } \\
\text { NR } \\
\text { Harms: NR } \\
\text { Rare harms: AFF }\end{array}$ \\
\hline $\begin{array}{l}\text { Bone, } 2004 \\
\text { Prospective CCT (2 } \\
\text { pooled partial RCT } \\
\text { extension with } \\
\text { discontinuation; subset } \\
\text { of Tonino, 2000) } \\
\text { Multicenter } \\
\text { Industry } \\
\text { Medium }\end{array}$ & $\begin{array}{l}\text { Alendronate } \\
\text { Continue ALN } 5 \mathrm{mg} \\
\text { QD } \\
\text { oral } \\
\text { Continue ALN } 10 \mathrm{mg} \\
\text { QD } \\
\text { oral } \\
500 \mathrm{mg} \text { calcium, no } \\
\text { vitamin D }\end{array}$ & $\begin{array}{l}\text { placebo/discontinue QD } \\
\text { Oral } \\
\text { 500mg calcium, no } \\
\text { vitamin D }\end{array}$ & \begin{tabular}{|l}
$\mathrm{N}=247$ \\
Age NR \\
Female 100\% \\
Comorbidities NR \\
Fx history or risk NR
\end{tabular} & See Tonino, 2000 & $8-10 \mathrm{yr}$ & $\begin{array}{l}\text { Fracture: RVFx, (6-10 } \\
\text { yr) CFx, nonVFx } \\
\text { (traumatic fxs } \\
\text { included) } \\
\text { Other: change in LS } \\
\text { BMD, FN BMD, } \\
\text { trochanteric BMD, TH } \\
\text { BMD, total body BMD, } \\
\text { forearm BMD; NTX, } \\
\text { BSALP, ALP. } \\
\text { Predictors: NR } \\
\text { Harms: death, UGI } \\
\text { Rare harms: NR }\end{array}$ \\
\hline
\end{tabular}




\begin{tabular}{|c|c|c|c|c|c|c|}
\hline $\begin{array}{l}\text { Study, Year } \\
\text { Design } \\
\text { Location } \\
\text { Funder } \\
\text { Risk of Bias }\end{array}$ & \begin{tabular}{|l} 
Intervention \\
Dosage \\
Delivery Route \\
Duration
\end{tabular} & \begin{tabular}{|l|} 
Comparison \\
Dosage \\
Delivery Route \\
Duration
\end{tabular} & $\begin{array}{l}\text { Sample } \\
\text { \# Randomized } \\
\text { Mean age } \\
\text { \% Female } \\
\% \text { White Race } \\
\text { Comorbidities } \\
\text { (\% with DM, CKD, } \\
\text { CVD) } \\
\text { Fracture History } \\
\text { Fracture Risk } \\
\end{array}$ & \begin{tabular}{|l|} 
Inclusion criteria \\
Baseline BMD
\end{tabular} & $\begin{array}{l}\text { Followup } \\
\text { Duration }\end{array}$ & $\begin{array}{l}\text { Outcomes } \\
\text { Reported }\end{array}$ \\
\hline $\begin{array}{l}\text { Cummings, } 1998 \\
\text { FIT-II (CFx arm) } \\
\text { RCT } \\
\text { US } \\
\text { Industry } \\
\text { Low }\end{array}$ & $\begin{array}{l}\text { Alendronate } \\
5 \text { mg QD } \times 2 \text { yr then } 10 \\
\text { mg QD x } 2 \text { yr } \\
\text { Oral } \\
4 \text { yr } \\
\text { Calcium and Vitamin D } \\
\text { supplements }\end{array}$ & $\begin{array}{l}\text { Placebo } \\
\text { QD } \\
\text { Oral } \\
4 \text { yr } \\
\text { Calcium and Vitamin D } \\
\text { supplements }\end{array}$ & $\begin{array}{l}\mathrm{N}=4432 \\
\text { Age } 68 \\
\text { Female } 100 \% \\
\text { White } 97 \% \\
\text { Comorbidities NR } \\
\text { Prior Fx } \geq 45 \text { yr } 36 \% \\
\text { Baseline VFx 0\% }\end{array}$ & $\begin{array}{l}\text { Postmenopausal women } \\
\text { without baseline VFx and } \\
\text { FN BMD } \leq 0.68 \mathrm{~g} / \mathrm{cm}^{2} \\
\text { (T score } \leq-1.6)\end{array}$ & $\begin{array}{l}4 \mathrm{yr} \\
\text { (mean } 4.2 \mathrm{yr} \text { ) }\end{array}$ & $\begin{array}{l}\text { Fracture } \\
\text { 10: CFx (nonspine, } \\
\text { hip, wrist, CVFx, } \\
\text { other) } \\
\text { 2:: RVFx } \\
\text { Predictors: FN BMD } \\
\text { Harms: any, death, GI } \\
\text { Rare harms: NR }\end{array}$ \\
\hline $\begin{array}{l}\text { Donaldson, } 2012 \\
\text { Pooled FIT-I and FIT-II } \\
\text { RCTs } \\
\text { USA, } 11 \text { centers } \\
\text { Industry } \\
\text { Medium }\end{array}$ & $\begin{array}{l}\text { Alendronate } \\
5 \text { mg QD x } 2 \text { yr then } 10 \\
\text { mg QD thereafter } \\
\text { Oral } \\
\text { FIT-I: mean } 2.9 \mathrm{yr} \\
\text { FIT-II: mean } 4.3 \mathrm{yr} \\
\text { Calcium and Vitamin D: } \\
\text { NR }\end{array}$ & $\begin{array}{l}\text { Placebo } \\
\text { QD } \\
\text { Oral } \\
\text { FIT-I: mean } 2.9 \mathrm{yr} \\
\text { FIT-II: mean } 4.3 \mathrm{yr} \\
\text { Calcium and Vitamin D: } \\
\text { NR }\end{array}$ & $\begin{array}{l}\text { N=6459 (FIT-I 2027, } \\
\text { FIT-II 4432) } \\
\\
\text { Age 68 yr } \\
\text { Female 100\% } \\
\text { Race NR } \\
\text { Comorbidities NR } \\
\text { Prior Fx: 44\% } \\
\text { Baseline VFx: 31\% }\end{array}$ & $\begin{array}{l}\text { Postmenopausal } 2+\mathrm{yr} ., \\
\text { with or without baseline } \\
\text { VFx. } \\
\text { FN-BMD } \leq 0.68 \mathrm{~g} / \mathrm{cm}^{2} \\
\text { (T score } \leq-1.6 \text { ) }\end{array}$ & $\begin{array}{l}\text { Median } 1457 \\
\text { days }\left(4 \mathrm{yr}^{\star}\right) \\
{\left[31.4 \% \%^{\star}\right.} \\
\text { median } 3 \mathrm{yr} . ; \\
68.6 \%^{\star} \\
\text { median } 4 \mathrm{yr} .]\end{array}$ & $\begin{array}{l}\text { Fracture: NonVFx; } \\
\text { assessed CFx, MOF } \\
\text { and RadVFx but } \\
\text { results NR } \\
\text { (interactions NS) } \\
\text { Predictors: FRAX } \\
\text { score (with/without } \\
\text { FN-BMD). Baseline } \\
\text { FRAX excluded } \\
\text { baseline RadVFx); } \\
\text { FN-BMD } \\
\text { Harms: NR } \\
\text { Rare harms: NR } \\
\end{array}$ \\
\hline $\begin{array}{l}\text { Jamal, } 2007 \\
\text { Pooled FIT-I and FIT-II } \\
\text { RCTs } \\
\text { USA } \\
\text { Industry } \\
\text { Medium } \\
\text { [Cummings 1998, } \\
\text { Black 1996] }\end{array}$ & $\begin{array}{l}\text { Alendronate } \\
5 \text { mg QD x } 2 \text { yr then } 10 \\
\text { mg QD thereafter } \\
\text { Oral } \\
\text { FIT-I: } 3 \mathrm{yr} \\
\text { FIT-II: } 4 \mathrm{yr} \\
\text { Calcium and Vitamin D } \\
\text { supplements }\end{array}$ & $\begin{array}{l}\text { Placebo } \\
\text { QD } \\
\text { Oral } \\
\text { FIT-I: } 3 \mathrm{yr} \\
\text { FIT-II: } 4 \mathrm{yr} \\
\text { Calcium and Vitamin D } \\
\text { supplements }\end{array}$ & $\begin{array}{l}\mathrm{N}=6458 \\
\text { Age } 69 \mathrm{yr} \\
\text { Female: } 100 \% \\
\text { White race: } 97 \% \\
\text { CKD (eGFR<45 } \\
\text { ml/min): } 9 \% * ; \text { DM NR; } \\
\text { CVD NR } \\
\text { Prior fracture: } 42 \% \\
\text { Baseline VFx: } 31 \%\end{array}$ & $\begin{array}{l}\text { Postmenopausal women } \\
\text { with or without baseline } \\
\text { VFx and } \\
\text { FN-BMD } \leq 0.68 \mathrm{~g} / \mathrm{cm}^{2} \\
\text { (T score } \leq-1.6 \text { ) }\end{array}$ & $\begin{array}{l}\text { Average of } 4 \\
\text { yrs (3 yr FIT- } \\
\text { I, } 4 \text { yr FIT-II; } \\
\text { pooled } \\
\text { median NR) }\end{array}$ & \begin{tabular}{|l|} 
Fracture: clinical, \\
radiographic vertebral. \\
Predictors: renal \\
function (eGFR $<45$ \\
ml/min vs $\geq 45 \mathrm{ml} / \mathrm{min})$ \\
Harms: by renal \\
function \\
Rare harms: NR
\end{tabular} \\
\hline
\end{tabular}




\begin{tabular}{|c|c|c|c|c|c|c|}
\hline $\begin{array}{l}\text { Study, Year } \\
\text { Design } \\
\text { Location } \\
\text { Funder } \\
\text { Risk of Bias }\end{array}$ & \begin{tabular}{|l} 
Intervention \\
Dosage \\
Delivery Route \\
Duration
\end{tabular} & \begin{tabular}{|l} 
Comparison \\
Dosage \\
Delivery Route \\
Duration
\end{tabular} & $\begin{array}{l}\text { Sample } \\
\text { \# Randomized } \\
\text { Mean age } \\
\text { \% Female } \\
\% \text { White Race } \\
\text { Comorbidities } \\
\text { (\% with DM, CKD, } \\
\text { CVD) } \\
\text { Fracture History } \\
\text { Fracture Risk }\end{array}$ & \begin{tabular}{|l} 
Inclusion criteria \\
Baseline BMD
\end{tabular} & $\begin{array}{l}\text { Followup } \\
\text { Duration }\end{array}$ & $\begin{array}{l}\text { Outcomes } \\
\text { Reported }\end{array}$ \\
\hline $\begin{array}{l}\text { Quandt, } 2005 \\
\text { Subgroup of pooled } \\
\text { FIT I \& FIT-II } \\
\text { Industry } \\
\text { Medium }\end{array}$ & $\begin{array}{l}\text { Alendronate } \\
5 \mathrm{mg} \text { QD } \times 2 \text { yr then } 10 \\
\text { mg QD thereafter } \\
\text { Oral } \\
\text { FIT-I: } 3 \mathrm{yr} \\
\text { FIT-II: } 4 \mathrm{yr} \\
\text { Calcium and Vitamin D } \\
\text { supplements }\end{array}$ & $\begin{array}{l}\text { Placebo } \\
\text { QD } \\
\text { Oral } \\
\text { FIT-I: } 3 \mathrm{yr} \\
\text { FIT-II: } 4 \mathrm{yr} \\
\text { Calcium and Vitamin D } \\
\text { supplements }\end{array}$ & $\begin{array}{l}\mathrm{N}=2037 \\
\text { Age } 68 \\
\text { Female } 100 \% \\
\text { Race NR } \\
\text { Comorbidities NR } \\
\text { Fracture history and } \\
\text { risk NR }\end{array}$ & \begin{tabular}{|l|} 
Postmenopausal $2+$ yr., \\
with or without baseline \\
VFx., FN-BMD $\leq$ \\
$0.68 \mathrm{~g} / \mathrm{cm}^{2}($ T-score $\leq-1.6)$ \\
BMD NR
\end{tabular} & 4 years & $\begin{array}{l}\text { Fracture: NonVFX } \\
\text { Predictors: FRAX } \\
\text { score (with/without } \\
\text { FN-BMD) } \\
\text { Harms: NR } \\
\text { Rare harms: NR }\end{array}$ \\
\hline $\begin{array}{l}\text { Ryder, } 2008 \\
\text { Post hoc subgroup of } \\
\text { FIT-II RCT } \\
\text { Industry } \\
\text { Medium }\end{array}$ & $\begin{array}{l}\text { Alendronate } \\
5 \mathrm{mg} \text { QD } \times 2 \text { yr then } 10 \\
\text { mg QD thereafter } \\
\text { Oral } \\
\text { FIT-I: } 3 \mathrm{yr} \\
\text { FIT-II: } 4 \mathrm{yr} \\
\text { Calcium and Vitamin D } \\
\text { supplements }\end{array}$ & $\begin{array}{l}\text { Placebo } \\
\text { QD } \\
\text { Oral } \\
\text { FIT-I: } 3 \mathrm{yr} \\
\text { FIT-II: } 4 \mathrm{yr} \\
\text { Calcium and Vitamin D } \\
\text { supplements }\end{array}$ & $\begin{array}{l}\mathrm{N}=2785 \\
\text { Age } 68 \\
\text { Female } 100 \% \\
\text { Race NR } \\
\text { Comorbidities NR } \\
\text { Fracture history and } \\
\text { risk NR }\end{array}$ & $\begin{array}{l}\text { Postmenopausal } 2+y r ., \\
\text { with or without baseline } \\
\text { VFx., FN-BMD } \leq \\
0.68 \mathrm{~g} / \mathrm{cm}^{2}(\mathrm{~T} \text {-score } \leq-1.6) \\
\text { BMD NR }\end{array}$ & 4 years & $\begin{array}{l}\text { Fracture: NonVFX } \\
\text { Predictors: FRAX } \\
\text { score (with/without } \\
\text { FN-BMD) } \\
\text { Harms: NR } \\
\text { Rare harms: NR }\end{array}$ \\
\hline $\begin{array}{l}\text { Schwartz, } 2010 \\
\text { FLEX (Post hoc RCT } \\
\text { subgroup*subgroup } \\
\text { analysis) } \\
\text { US } \\
\text { Industry } \\
\text { Medium }\end{array}$ & $\begin{array}{l}\text { Alendronate } \\
5 \mathrm{mg} \text { QD } \\
\text { Oral } \\
5 \mathrm{yr} \text { (5-10 yr total) } \\
\text { Alendronate } 10 \mathrm{mg} \\
\text { Daily } \\
\text { Oral } \\
5 \mathrm{yr} \text { (5-10 yr total) } \\
\text { (groups pooled for } \\
\text { analyses) }\end{array}$ & $\begin{array}{l}\text { Placebo (discontinued } \\
\text { alendronate after } 5 \mathrm{yr} \text { ) } \\
\text { QD } \\
\text { Oral } \\
5 \mathrm{yr}\end{array}$ & $\begin{array}{l}\mathrm{N}=1099(39 \% \text { of } 2852 \\
\text { ALN users from } \mathrm{FIT}) \\
\text { with mean of } 5 \text { yr prior } \\
\text { ALN use } \\
\text { Age } 73 \\
\text { Female } 100 \% \\
\text { White } 98 \% \\
\text { Comorbidities NR } \\
\text { Prior Fx } \geq 45 \text { yr } 60 \% \\
\text { Prevalent VFx } 34 \%\end{array}$ & $\begin{array}{l}\text { Prior enrollment in ALN } \\
\text { arm of FIT (I or II), took } \\
\geq 3 \text { yr ALN } \\
\text { TH BMD >-3.5 at FLEX } \\
\text { baseline }\end{array}$ & $\begin{array}{l}5 \mathrm{yr} \\
\text { (5-10 yr FIT } \\
\text { extension) }\end{array}$ & $\begin{array}{l}\text { Fractures: First } \\
\text { NonVFx, CVFx, RVFx. } \\
\text { (Pathologic, skull, or } \\
\text { excess trauma fxs } \\
\text { excluded) } \\
\text { Predictors: baseline } \\
\text { VFx, FN BMD, change } \\
\text { in BMD prior to FLEX } \\
\text { Harms: NR } \\
\text { Rare harms: NR }\end{array}$ \\
\hline
\end{tabular}




\begin{tabular}{|c|c|c|c|c|c|c|}
\hline $\begin{array}{l}\text { Study, Year } \\
\text { Design } \\
\text { Location } \\
\text { Funder } \\
\text { Risk of Bias }\end{array}$ & \begin{tabular}{|l} 
Intervention \\
Dosage \\
Delivery Route \\
Duration
\end{tabular} & \begin{tabular}{|l|} 
Comparison \\
Dosage \\
Delivery Route \\
Duration
\end{tabular} & $\begin{array}{l}\text { Sample } \\
\text { \# Randomized } \\
\text { Mean age } \\
\text { \% Female } \\
\% \text { White Race } \\
\text { Comorbidities } \\
\text { (\% with DM, CKD, } \\
\text { CVD) } \\
\text { Fracture History } \\
\text { Fracture Risk }\end{array}$ & \begin{tabular}{|l|} 
Inclusion criteria \\
Baseline BMD
\end{tabular} & $\begin{array}{l}\text { Followup } \\
\text { Duration }\end{array}$ & $\begin{array}{l}\text { Outcomes } \\
\text { Reported }\end{array}$ \\
\hline $\begin{array}{l}\text { Tonino, } 2000 \\
\text { Prospective CCT (2 } \\
\text { pooled partial RCT } \\
\text { extension with } \\
\text { discontinuation) } \\
\text { Multicenter } \\
\text { Industry } \\
\text { Medium }\end{array}$ & $\begin{array}{l}\text { Alendronate } \\
\text { Continue ALN } 5 \mathrm{mg} \\
\text { QD } \\
\text { oral } \\
\text { Continue ALN } 10 \mathrm{mg} \\
\text { QD } \\
\text { oral } \\
\text { placebo/discontinue QD } \\
\text { Oral } \\
500 m g \text { calcium, no } \\
\text { vitamin D }\end{array}$ & $\begin{array}{l}\text { placebo/discontinue } \\
\text { QD } \\
\text { Oral } \\
\text { 500mg calcium, no } \\
\text { vitamin D }\end{array}$ & \begin{tabular}{|l}
$\mathrm{N}=350$ \\
Age NR \\
Female $100 \%$ \\
Comorbidities NR \\
Fx history or risk NR
\end{tabular} & $\begin{array}{l}\text { Postmenopausal women } \\
\text { with osteoporosis per } \\
\text { LS BMD } \leq 0.80 \mathrm{~g} / \mathrm{cm}^{2} \\
(\leq-2.5 \text { SD) } \\
\text { BMD NR }\end{array}$ & $6-7 \mathrm{yr}$ & $\begin{array}{l}\text { Fracture: symptomatic } \\
\text { VFx, CFx (traumatic } \\
\text { fxs included) } \\
\text { Other outcomes: } \\
\text { change in LS BMD, } \\
\text { change in FN, } \\
\text { trochanteric, TH, total } \\
\text { body, \& forearm BMD; } \\
\text { NTX, BSALP } \\
\text { Predictors: NR } \\
\text { Harms: UGI, serious, } \\
\text { any } \\
\text { Rare harms: NR }\end{array}$ \\
\hline $\begin{array}{l}\text { Black, } 2012 \\
\text { HORIZON } \\
\text { RCT } \\
\text { Multinational } \\
\text { Industry } \\
\text { Low }\end{array}$ & $\begin{array}{l}\text { Zoledronic acid } \\
5 \mathrm{mg} \text { once per year } \\
\text { IV } \\
6 \mathrm{yr} \\
\text { with calcium 1000-1500 } \\
\text { mg QD and vitamin D } \\
400-1200 \text { IU QD }\end{array}$ & $\begin{array}{l}\text { Placebo } \\
\text { with calcium 1000-1500 } \\
\text { mg QD and vitamin D } \\
\text { 400-1200 IU QD }\end{array}$ & $\begin{array}{l}\mathrm{N}=1233 \\
\text { Age } 76 \\
\text { Female } 100 \% \\
\text { Race NR } \\
\text { Comorbidities NR } \\
\text { Fx history or risk NR }\end{array}$ & $\begin{array}{l}\text { Osteoporotic women } \\
\text { BMD g/cm² } \\
\text { FN BMD } 0.57 \\
\text { TH BMD } 0.69 \\
\text { LS BMD } 0.82 \\
\text { FN T-score }-2.57\end{array}$ & $\begin{array}{l}6 \mathrm{yr} \\
\text { (3 yr } \\
\text { extension of } \\
\text { a } 3 \mathrm{yr} \text { trial) }\end{array}$ & $\begin{array}{l}\text { Fracture: CFx and } \\
\text { RVFx } \\
\text { Predictors: } \\
\text { BMD (percentage } \\
\text { change) } \\
\text { Rare harms: ONJ, } \\
\text { AFF, Afib }\end{array}$ \\
\hline $\begin{array}{l}\text { Black, } 2015 \\
\text { HORIZON } \\
\text { RCT } \\
\text { Multinational } \\
\text { Industry } \\
\text { Low }\end{array}$ & $\begin{array}{l}\text { Zoledronic acid } \\
5 \mathrm{mg} \text { once per year } \\
\text { IV } \\
9 \mathrm{yr} \\
\text { with calcium } 1000-1500 \\
\text { mg QD and vitamin D } \\
400-1200 \text { IU QD }\end{array}$ & $\begin{array}{l}\text { Placebo } \\
\text { with calcium 1000-1500 } \\
\text { mg QD and vitamin D } \\
\text { 400-1200 IU QD }\end{array}$ & $\begin{array}{l}\text { N=190 } \\
\text { Age } 78 \\
\text { Female } 100 \% \\
\text { Race NR } \\
\text { Comorbidities NR } \\
\text { Fx history or risk NR }\end{array}$ & $\begin{array}{l}\text { Osteoporotic women } \\
\text { BMD } \mathrm{g} / \mathrm{cm}^{2} \\
\text { FN BMD } 0.58 \\
\text { TH BMD } 0.70 \\
\text { FN T-score }-2.44\end{array}$ & $\begin{array}{l}9 \mathrm{yr} \\
\text { (second } 3 \mathrm{yr} \\
\text { extension of } \\
\text { a } 3 \text { yr trial) }\end{array}$ & $\begin{array}{l}\text { Fracture: CFx and } \\
\text { RVFx } \\
\text { Predictors: } \\
\text { TH BMD (percentage } \\
\text { change) } \\
\text { Rare harms: } \\
\text { ONJ, AFF, Afib }\end{array}$ \\
\hline
\end{tabular}




\begin{tabular}{|c|c|c|c|c|c|c|}
\hline $\begin{array}{l}\text { Study, Year } \\
\text { Design } \\
\text { Location } \\
\text { Funder } \\
\text { Risk of Bias }\end{array}$ & \begin{tabular}{|l} 
Intervention \\
Dosage \\
Delivery Route \\
Duration
\end{tabular} & \begin{tabular}{|l|} 
Comparison \\
Dosage \\
Delivery Route \\
Duration
\end{tabular} & $\begin{array}{l}\text { Sample } \\
\text { \# Randomized } \\
\text { Mean age } \\
\text { \% Female } \\
\% \text { White Race } \\
\text { Comorbidities } \\
\text { (\% with DM, CKD, } \\
\text { CVD) } \\
\text { Fracture History } \\
\text { Fracture Risk } \\
\end{array}$ & \begin{tabular}{|l|} 
Inclusion criteria \\
Baseline BMD
\end{tabular} & $\begin{array}{l}\text { Followup } \\
\text { Duration }\end{array}$ & $\begin{array}{l}\text { Outcomes } \\
\text { Reported }\end{array}$ \\
\hline $\begin{array}{l}\text { McClung, } 2009 \\
\text { RCT } \\
\text { US } \\
\text { Industry } \\
\text { Low }\end{array}$ & \begin{tabular}{|l|} 
Zoledronic acid \\
$5 \mathrm{mg} / \mathrm{yr}$ for $1 \mathrm{yr}$ then \\
placebo for $1 \mathrm{yr}$ or $5 \mathrm{mg} / \mathrm{yr}$ \\
for $2 \mathrm{yr}$ \\
IV \\
$2 \mathrm{yr}$ \\
with calcium $500-1200$ \\
mg QD and vitamin D \\
$400-800$ IU QD
\end{tabular} & $\begin{array}{l}\text { Placebo } \\
\text { with calcium 500-1200 } \\
\text { mg QD and vitamin D } \\
400-800 \text { IU QD }\end{array}$ & $\begin{array}{l}\mathrm{N}=581 \\
\text { Age } 60 \\
\text { Female } 100 \% \\
\text { White } 93 \% \\
\text { Comorbidities NR } \\
\text { Fx history or risk NR }\end{array}$ & $\begin{array}{l}\text { Postmenopausal women } \\
>45 \text { yr with T-score <-1.0 } \\
\text { and >-2.5 at LS and T- } \\
\text { score }>-2.5 \text { at FN } \\
\text { BMD g/cm } 2 \\
\text { FN BMD } 0.69 \\
\text { TH BMD } 0.83 \\
\text { LS BMD } 0.86\end{array}$ & $2 \mathrm{yr}$ & $\begin{array}{l}\text { Fracture: CFx } \\
\text { Predictors: none } \\
\text { Harms: Serious } \\
\text { adverse events } \\
\text { Rare harms: Afib, } \\
\text { ONJ }\end{array}$ \\
\hline $\begin{array}{l}\text { Reid, } 2018 \\
\text { RCT } \\
\text { New Zealand } \\
\text { Government } \\
\text { Industry (Novartis, } \\
\text { medication only) } \\
\text { Low }\end{array}$ & \begin{tabular}{|l|} 
Zoledronic acid \\
5 mg at $18-$ month \\
intervals \\
IV \\
6 years \\
With vitamin D $1.25 \mathrm{mg}$ \\
per month + advised \\
calcium $1 \mathrm{~g}$ per day
\end{tabular} & Placebo (normal saline) & \begin{tabular}{|l|}
$\mathrm{N}=2000$ \\
Age 71 \\
Female $100 \%$ \\
White $95 \%$ \\
Comorbidities NR \\
Hx of NVF after age 45 \\
$24 \%$ \\
Prevalent VFx $13 \%$ \\
Median $10-y$ risk of \\
osteoporotic Fx $12 \%$ \\
Median $10-y$ r risk of hip \\
Fx $2.4 \%$
\end{tabular} & \begin{tabular}{|l|} 
Postmenopausal women \\
$\geq 65$ yr with T-score of -1.0 \\
to -2.5 at either TH or FN \\
on either side \\
BMD g/cm 2 \\
LS BMD 1.08 \\
TH BMD 0.85 \\
FN BMD 0.81 \\
Total body DMB 1.06 \\
T-score \\
LS T-score -0.89 \\
TH T-score -1.26 \\
FN T-score -1.64 \\
Total body T-score -0.81
\end{tabular} & $6 \mathrm{yr}$ & 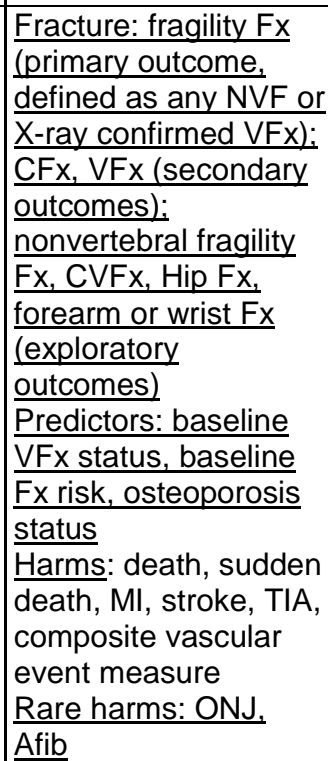 \\
\hline
\end{tabular}




\begin{tabular}{|c|c|c|c|c|c|c|}
\hline $\begin{array}{l}\text { Study, Year } \\
\text { Design } \\
\text { Location } \\
\text { Funder } \\
\text { Risk of Bias }\end{array}$ & \begin{tabular}{|l} 
Intervention \\
Dosage \\
Delivery Route \\
Duration
\end{tabular} & \begin{tabular}{|l|} 
Comparison \\
Dosage \\
Delivery Route \\
Duration
\end{tabular} & \begin{tabular}{|l} 
Sample \\
\# Randomized \\
Mean age \\
\% Female \\
\% White Race \\
Comorbidities \\
(\% with DM, CKD, \\
CVD) \\
Fracture History \\
Fracture Risk \\
\end{tabular} & \begin{tabular}{|l|} 
Inclusion criteria \\
Baseline BMD
\end{tabular} & $\begin{array}{l}\text { Followup } \\
\text { Duration }\end{array}$ & $\begin{array}{l}\text { Outcomes } \\
\text { Reported }\end{array}$ \\
\hline $\begin{array}{l}\text { Miller, } 2008 \\
\text { RCT } \\
\text { US } \\
\text { Industry } \\
\text { Medium }\end{array}$ & $\begin{array}{l}\text { Denosumab } \\
\text { various doses (6 mg or } \\
14 \text { mg every } 3 \text { months; } \\
\text { or } 14 \text { mg, } 60 \text { mg, or } 100 \\
\text { mg every } 6 \text { months) for } 2 \\
\text { yr; then } 60 \text { mg every } 6 \\
\text { months subcutaneously } \\
\text { for } 2 \text { yr } \\
\text { with calcium } 1000 \text { mg } \\
\text { QD and vitamin D } 400 \text { IU } \\
\text { QD }\end{array}$ & $\begin{array}{l}\text { Placebo } \\
\text { QD } \\
4 \mathrm{yr} \\
\text { with calcium } 1000 \mathrm{mg} \\
\text { QD and vitamin D } 400 \mathrm{IU} \\
\text { QD }\end{array}$ & $\begin{array}{l}\mathrm{N}=365 \\
\text { Age } 63 \\
\text { Female } 100 \% \\
\text { White } 85 \% \\
\text { Comorbidities NR } \\
\text { Yr since menopause } 15 \\
\text { Fx history or risk NR } \\
\text { (from McClung 2006) }\end{array}$ & $\begin{array}{l}\text { Postmenopausal women } \\
<80 \text { yr old with T-score at } \\
\text { LS }-1.8 \text { to }-4.0 \text { or T-score } \\
\text { at FN or TH }-1.8 \text { to }-3.5\end{array}$ & $4 \mathrm{yr}$ & $\begin{array}{l}\text { Fracture: CFx, } \\
\text { osteoporotic fx, BMD } \\
\text { Predictors: NR } \\
\text { Harms: Arthralgia, } \\
\text { infections, malignant } \\
\text { neoplasms } \\
\text { Rare harms: NR }\end{array}$ \\
\hline $\begin{array}{l}\text { Barrett-Connor, } 2002 \\
\text { Barrett-Connor, } 2004 \\
\text { Barrett-Connor, } 2009 \\
\text { Cauley, } 2001 \\
\text { Delmas, } 2002 \\
\text { Duvernoy, } 2005 \\
\text { Grady, } 2004 \\
\text { Johnell, } 2004 \\
\text { Sontag, } 2010 \\
\text { MORE } \\
\text { RCT } \\
\text { Multinational } \\
\text { Industry (Eli Lilly) } \\
\text { Low (all reports from } \\
\text { MORE trial have low } \\
\text { RoB except Johnell } \\
2004, \text { which has } \\
\text { medium) }\end{array}$ & $\begin{array}{l}\text { Raloxifene } \\
60 \text { mg QD (1 placebo } \\
\text { and one } 60 \mathrm{mg} \text { tablet) } \\
120 \mathrm{mg} \text { QD (two } 60 \mathrm{mg} \\
\text { tablets) } \\
\text { Oral } \\
\text { with calcium } 500 \mathrm{mg} \text { QD } \\
\text { and cholecalciferol } 400 \\
\text { to } 600 \text { mg QD }\end{array}$ & $\begin{array}{l}\text { Placebo } \\
2 \text { tablets QD } \\
\text { Oral } \\
4 \mathrm{yr} \\
\text { with calcium } 500 \mathrm{mg} \text { QD } \\
\text { and cholecalciferol } 400 \\
\text { to } 600 \mathrm{mg} \text { QD }\end{array}$ & $\begin{array}{l}\mathrm{N}=7705 \\
\text { Age } 66 \\
\text { Female } 100 \% \\
\text { White } 96 \% \\
\text { Hypertension NR } \\
\text { CVD 3\% } \\
\text { Stroke 5\% } \\
\text { DM 5\% } \\
\text { Family history of breast } \\
\text { cancer 12\% } \\
\text { History of VFx (n=6828 } \\
\text { with followup } \\
\text { radiograph) 37\% } \\
\text { Yr post menopause } 19 \\
\text { Fx history or risk NR } \\
\\
\text { (Cauley 2001, Barrett- } \\
\text { Connor 2002, Delmas } \\
\text { 2002, Barrett-Connor } \\
\text { 2009) }\end{array}$ & $\begin{array}{l}\text { Postmenopausal for } \geq 2 \mathrm{yr} \\
\text { Osteoporosis (prior VFx } \\
\text { or T-score <-2.5) } \\
\text { Baseline BMD ( } \mathrm{n}=6828 \\
\text { with followup vertebral } \\
\text { Xray) } \\
\text { FN BMD } 0.62 \mathrm{~g} / \mathrm{cm}^{2} \\
\text { LS BMD } 0.81 \mathrm{~g} / \mathrm{cm}^{2} \\
\text { Baseline T-score }(\mathrm{n}=6828 \\
\text { with followup vertebral } \\
\text { Xray) } \\
\text { FN T-score }-2.33 \\
\text { LS T-score }-2.57\end{array}$ & $\begin{array}{l}4 \text { yr (mean } \\
3.3 \mathrm{yr})\end{array}$ & $\begin{array}{l}\text { Fracture: NR } \\
\text { Predictors: } \\
\text { cardiovascular risk, } \\
\text { prevalent CHD, } \\
\text { baseline VFx status, } \\
\text { drug dose } \\
\text { Harms: hot flashes, } \\
\text { stroke, PE, DVT, } \\
\text { mortality } \\
\text { Rare harms: NR }\end{array}$ \\
\hline
\end{tabular}




\begin{tabular}{|c|c|c|c|c|c|c|}
\hline $\begin{array}{l}\text { Study, Year } \\
\text { Design } \\
\text { Location } \\
\text { Funder } \\
\text { Risk of Bias }\end{array}$ & \begin{tabular}{|l|} 
Intervention \\
Dosage \\
Delivery Route \\
Duration
\end{tabular} & \begin{tabular}{|l|} 
Comparison \\
Dosage \\
Delivery Route \\
Duration
\end{tabular} & $\begin{array}{l}\text { Sample } \\
\text { \# Randomized } \\
\text { Mean age } \\
\text { \% Female } \\
\text { \% White Race } \\
\text { Comorbidities } \\
\text { (\% with DM, CKD, } \\
\text { CVD) } \\
\text { Fracture History } \\
\text { Fracture Risk }\end{array}$ & \begin{tabular}{|l|} 
Inclusion criteria \\
Baseline BMD
\end{tabular} & $\begin{array}{l}\text { Followup } \\
\text { Duration }\end{array}$ & \begin{tabular}{|l|} 
Outcomes \\
Reported
\end{tabular} \\
\hline $\begin{array}{l}\text { Grady } 2010 \\
\text { MORE/CORE } \\
\text { RCT } \\
\text { Multinational } \\
\text { Industry (Eli Lilly) } \\
\text { Medium }\end{array}$ & $\begin{array}{l}\text { Raloxifene } \\
60 \mathrm{mg} \text { QD } \\
\text { Oral } \\
8 \mathrm{yr}\end{array}$ & $\begin{array}{l}\text { Placebo } \\
\text { QD } \\
\text { Oral } \\
8 \mathrm{yr}\end{array}$ & $\begin{array}{l}\text { From beginning of } \\
\text { MORE trial, raloxifene } \\
60 \text { mg and placebo } \\
\text { groups: } \\
\mathrm{N}=5133 \\
\text { Age } 67 \\
\text { Female } 100 \% \\
\text { White } 96 \% \\
\text { Hypertension } 30 \% \\
\text { CVD } 2 \% \\
\text { Stroke } 6 \% \\
\text { DM 3\% } \\
\text { Yr post menopause } 19 \\
\text { Fx history or risk NR } \\
\\
\text { Delmas } 2002 \text { Barrett- } \\
\text { Connor } 2002\end{array}$ & $\begin{array}{l}\text { Postmenopausal for } \geq 2 \mathrm{yr} \\
\text { Osteoporosis (prior VFx } \\
\text { or T-score <-2.5) } \\
\text { Was enrolled in MORE }\end{array}$ & $8 \mathrm{yr}$ & $\begin{array}{l}\text { Fracture: NR } \\
\text { Predictors: NR } \\
\text { Harms: Mortality } \\
\text { Rare harms: NR }\end{array}$ \\
\hline
\end{tabular}




\begin{tabular}{|c|c|c|c|c|c|c|}
\hline $\begin{array}{l}\text { Study, Year } \\
\text { Design } \\
\text { Location } \\
\text { Funder } \\
\text { Risk of Bias }\end{array}$ & \begin{tabular}{|l} 
Intervention \\
Dosage \\
Delivery Route \\
Duration
\end{tabular} & $\begin{array}{l}\text { Comparison } \\
\text { Dosage } \\
\text { Delivery Route } \\
\text { Duration }\end{array}$ & \begin{tabular}{|l|} 
Sample \\
\# Randomized \\
Mean age \\
\% Female \\
$\%$ White Race \\
Comorbidities \\
(\% with DM, CKD, \\
CVD) \\
Fracture History \\
Fracture Risk \\
\end{tabular} & $\begin{array}{l}\text { Inclusion criteria } \\
\text { Baseline BMD }\end{array}$ & $\begin{array}{l}\text { Followup } \\
\text { Duration }\end{array}$ & $\begin{array}{l}\text { Outcomes } \\
\text { Reported }\end{array}$ \\
\hline $\begin{array}{l}\text { Ensrud, } 2006 \\
\text { Martino, } 2004 \\
\text { Martino, } 2005 \\
\text { Siris, 2005 } \\
\text { MORE/CORE } \\
\text { RCT } \\
\text { Multinational } \\
\text { Industry (Eli Lilly) } \\
\text { Medium }\end{array}$ & $\begin{array}{l}\text { Raloxifene } \\
60 \mathrm{mg} \text { QD } \\
\text { Oral } \\
8 \mathrm{yr}\end{array}$ & $\begin{array}{l}\text { Placebo } \\
\text { QD } \\
\text { Oral } \\
8 \mathrm{yr}\end{array}$ & $\begin{array}{l}\text { From beginning of } \\
\text { MORE trial, placebo } \\
\text { and raloxifene } 60 \mathrm{mg} \\
\text { groups: } \\
\mathrm{N}=4011 \\
\text { Age } 66 \\
\text { Female } 100 \% \\
\text { White } 96 \% \\
\text { Hypertension } 28 \% \\
\text { CVD NR } \\
\text { Stroke } 0.3 \% \\
\text { DM } 3 \% \\
\text { BMD NR } \\
\text { History of VFx NR } \\
\text { Yr post menopause } 18 \\
\text { Fx history or risk NR }\end{array}$ & $\begin{array}{l}\text { Postmenopausal for } \geq 2 \mathrm{yr} \\
\text { Osteoporosis (prior VFx } \\
\text { or T-score }<-2.5 \text { ) } \\
\text { Was enrolled in MORE }\end{array}$ & $8 \mathrm{yr}$ & $\begin{array}{l}\text { Fracture: CFx } \\
\text { (nonvertebral), } \\
\text { fracture at } 6 \text { sites } \\
\text { Predictors: age, BMD, } \\
\text { prevalent vertebral Fx } \\
\text { Harms: Stroke, PE, } \\
\text { DVT, hot flashes, } \\
\text { mortality, serious } \\
\text { adverse events } \\
\text { Rare harms: none } \\
\end{array}$ \\
\hline $\begin{array}{l}\text { Cauley, } 2003 \\
\text { WHI } \\
\text { RCT } \\
\text { USA, } 40 \text { centers } \\
\text { Government } \\
\text { Low }\end{array}$ & $\begin{array}{l}\text { Conjugated equine } \\
\text { estrogen } 0.625 \mathrm{mg} \text { QD + } \\
\text { medroxyprogesterone } \\
\text { acetate } 2.5 \mathrm{mg} \text { QD } \\
\text { Oral } \\
6 \mathrm{yr}\end{array}$ & $\begin{array}{l}\text { Placebo } \\
\text { QD } \\
\text { Oral } \\
6 \mathrm{yr}\end{array}$ & $\begin{array}{l}\mathrm{N}=16608 \\
\mathrm{~N} \text { for } \mathrm{BMD} \\
\text { subset=1024 } \\
\text { Age } 63 \\
\text { Female } 100 \% \\
\text { White } 84 \% \\
\text { Comorbidities NR } \\
\text { Prior Fx } 39 \% \\
\text { High fracture risk } 23 \%\end{array}$ & $\begin{array}{l}\text { Postmenopausal women } \\
\text { with intact uterus, } 50-79 \\
\text { years at baseline } \\
\text { No BMD requirement for } \\
\text { inclusion } \\
\text { TH BMD } 0.83 \text { (T score } \\
-0.93 \text { ) } \\
\text { LS BMD } 0.94 \text { (T score } \\
-1.28 \text { ) }\end{array}$ & Mean $5.6 \mathrm{yr}$ & $\begin{array}{l}\text { Fracture } \\
\text { CFx, hip } \\
\text { Predictors: History of } \\
\text { fracture, baseline } \\
\text { BMD, fracture risk } \\
\text { Harms: global index } \\
\text { Rare harms: none }\end{array}$ \\
\hline
\end{tabular}




\begin{tabular}{|c|c|c|c|c|c|c|}
\hline $\begin{array}{l}\text { Study, Year } \\
\text { Design } \\
\text { Location } \\
\text { Funder } \\
\text { Risk of Bias }\end{array}$ & \begin{tabular}{|l} 
Intervention \\
Dosage \\
Delivery Route \\
Duration
\end{tabular} & \begin{tabular}{|l|} 
Comparison \\
Dosage \\
Delivery Route \\
Duration
\end{tabular} & $\begin{array}{l}\text { Sample } \\
\text { \# Randomized } \\
\text { Mean age } \\
\% \text { Female } \\
\% \text { White Race } \\
\text { Comorbidities } \\
\text { (\% with DM, CKD, } \\
\text { CVD) } \\
\text { Fracture History } \\
\text { Fracture Risk } \\
\end{array}$ & $\begin{array}{l}\text { Inclusion criteria } \\
\text { Baseline BMD }\end{array}$ & $\begin{array}{l}\text { Followup } \\
\text { Duration }\end{array}$ & $\begin{array}{l}\text { Outcomes } \\
\text { Reported }\end{array}$ \\
\hline $\begin{array}{l}\text { Jackson, } 2006 \\
\text { WHI } \\
\text { RCT } \\
\text { USA, } 40 \text { centers } \\
\text { Government } \\
\text { Low }\end{array}$ & $\begin{array}{l}\text { Conjugated equine } \\
\text { estrogen } 0.625 \mathrm{mg} \text { QD } \\
\text { Oral } \\
7 \mathrm{yr}\end{array}$ & $\begin{array}{l}\text { Placebo } \\
\text { QD } \\
\text { Oral } \\
7 \mathrm{yr}\end{array}$ & $\begin{array}{l}\mathrm{N}=10739 \\
\mathrm{~N} \text { for BMD subset=938 } \\
\text { Age } 64 \\
\text { Female } 100 \% \\
\text { White } 75 \% \\
\text { Comorbidities NR } \\
\text { Prior Fx } 39 \% \\
\text { High fracture risk } 28 \%\end{array}$ & $\begin{array}{l}\text { Postmenopausal women } \\
\text { with prior hysterectomy, } \\
50-79 \text { years at baseline } \\
\text { No BMD requirement for } \\
\text { inclusion } \\
\text { TH BMD T score } \\
-0.81 \\
\text { LS BMD T score } \\
-1.16 \\
\end{array}$ & Mean $7.1 \mathrm{yr}$ & $\begin{array}{l}\text { Fracture } \\
\text { Cfx, hip } \\
\text { Predictors: History of } \\
\text { fracture, baseline } \\
\text { BMD, fracture risk } \\
\text { Harms: global index } \\
\text { Rare harms: none }\end{array}$ \\
\hline $\begin{array}{l}\text { Wimalawansa } 1998 \\
\text { RCT } \\
\text { Unclear, possibly the } \\
\text { UK } \\
\text { Funder NR } \\
\text { Medium }\end{array}$ & \begin{tabular}{|l|} 
HRT, prempack C \\
(premarin $0.625 \mathrm{mg}$ daily \\
and norgestrel $150 \mathrm{mg}$ \\
12 days each month) \\
Oral \\
with $1 \mathrm{~g}$ elemental \\
calcium (Sandocal 1,000 \\
effervescent) and 400 \\
units of vitamin D \\
(ergocaliciferol 10 \\
mg) \\
QD \\
Oral \\
$4 \mathrm{yr}$
\end{tabular} & $\begin{array}{l}\text { Control, } 1 \text { g elemental } \\
\text { calcium (Sandocal 1,000 } \\
\text { effervescent) } \\
\text { and } 400 \text { units of vitamin } \\
\text { D (ergocaliciferol } 10 \\
\text { mg) } \\
\text { QD } \\
\text { Oral } \\
4 \mathrm{yr}\end{array}$ & $\begin{array}{l}\mathrm{N}=36 \\
\text { Age } 65 \\
\text { Female } 100 \% \\
\text { White } 100 \% \\
\text { Comorbidities NR } \\
\text { Prior RVFx } 100 \%\end{array}$ & $\begin{array}{l}\text { Evidence of osteoporosis } \\
\text { as determined by at least } \\
1 \text { (but not more than } 4 \text { ) } \\
\text { radiographically } \\
\text { demonstrable atraumatic } \\
\text { thoracic vertebral crush } \\
\text { fractures, and spine BMD } \\
2.0 \text { standard deviations } \\
\text { below the reference range } \\
\text { using DXA. } \\
\text { Mean LS BMD } 0.82 \mathrm{~g} / \mathrm{cm}^{2} \\
\text { Mean FN BMD } 0.67 \mathrm{~g} / \mathrm{cm}^{2}\end{array}$ & $4 \mathrm{yr}$ & $\begin{array}{l}\text { Fractures: } \\
\text { NonVFx, RVFx } \\
\text { Predictors: NR } \\
\text { Harms: withdrawals } \\
\text { due to AEs or other } \\
\text { medical problems } \\
\text { Rare harms: NR } \\
\text { Other outcomes: } \\
\text { Change in TH BMD }\end{array}$ \\
\hline
\end{tabular}

Abbreviations: Afib=atrial fibrillation; AFF=atypical femoral fracture; $\mathrm{ALN}=$ alendronate; $\mathrm{BMD}=\mathrm{bone}$ mineral density; $\mathrm{BSALP}=\mathrm{bone}$ specific alkaline phosphatase; $\mathrm{CFx}=\mathrm{clinical}$ fracture; $\mathrm{CHD}=$ coronary heart disease; $\mathrm{CKD}=$ acute kidney disease; $\mathrm{CORE}=$ Continuing Outcomes Relevant to Evista; $\mathrm{CVD}=$ cardiovascular disease; $\mathrm{DM}=$ diabetes mellitus; DVT=deep vein thrombosis; FIT=Fracture Intervention Trial; FLEX=Fracture Intervention Trial Long Term Extension; FN=femoral neck; Fx=fracture; g/cm2=grams per centimeter squared; GI=gastrointestinal; HORIZON=Health Outcomes and Reduced Incidence with Zoledronic Acid Once Yearly trial; IU=international units; IV=Intravenous infusion; LS=lumbar spine; MORE=Multiple Outcomes of Raloxifene Evaluation; mg=milligram; N=number; NSAID=nonsteroidal anti-inflammatory drug; NR=not reported; ONJ=osteonecrosis of the jaw; $\mathrm{PE}=$ pulmonary embolism; RCT=randomized controlled trial; RVFx=Radiographic vertebral fracture; $\mathrm{SD}=$ standard deviation; TH=total hip; UGI=upper gastrointestinal; VFx=vertebral fracture; yr=year

* Includes pooled RCTs and RCT subgroup analyses 
Table D3. Key Questions 1 and 2 evidence overview

\begin{tabular}{|c|c|c|c|c|}
\hline $\begin{array}{l}\text { Drug } \\
\text { Comparison }\end{array}$ & $\begin{array}{l}\text { Study } \\
\text { (Trial) } \\
\text { Followup } \\
\text { Risk of Bias }\end{array}$ & $\begin{array}{l}\text { Final Outcomes } \\
\text { Incident Clinical Fracture }\end{array}$ & $\begin{array}{l}\text { Intermediate Outcomes } \\
\text { Incident Radiographic } \\
\text { Vertebral Fracture } \\
\text { DXA BMD Change }\end{array}$ & $\begin{array}{l}\text { Predictors } \\
\text { (Patient, Bone, Drug) }\end{array}$ \\
\hline $\begin{array}{l}\text { Alendronate vs } \\
\text { placebo }\end{array}$ & $\begin{array}{l}\text { Bauer, } 2006 \\
\text { (Pooled FIT-I \& } \\
\text { FIT-II RCTs } \\
3 \text { yr FIT-I; } \\
4 \text { yr FIT-II) } \\
\text { Medium }\end{array}$ & NR & $\mathrm{NR}$ & 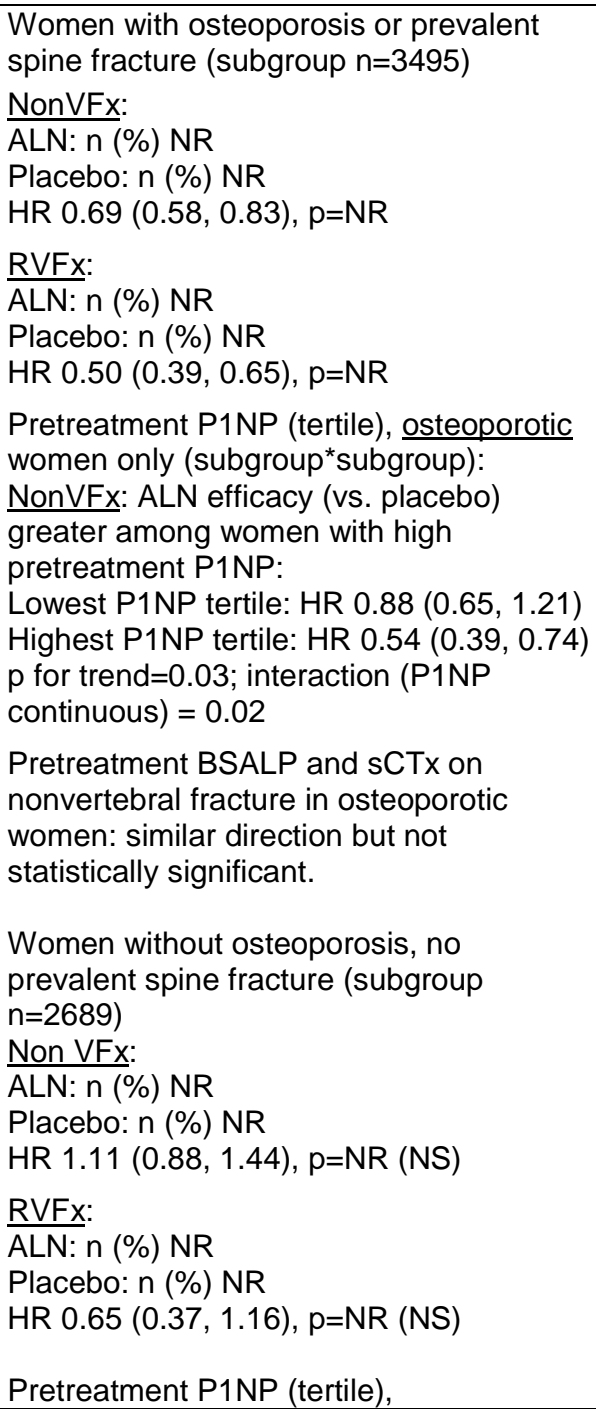 \\
\hline
\end{tabular}




\begin{tabular}{|c|c|c|c|c|}
\hline $\begin{array}{l}\text { Drug } \\
\text { Comparison }\end{array}$ & $\begin{array}{l}\text { Study } \\
\text { (Trial) } \\
\text { Followup } \\
\text { Risk of Bias }\end{array}$ & $\begin{array}{l}\text { Final Outcomes } \\
\text { Incident Clinical Fracture }\end{array}$ & $\begin{array}{l}\text { Intermediate Outcomes } \\
\text { Incident Radiographic } \\
\text { Vertebral Fracture } \\
\text { DXA BMD Change }\end{array}$ & $\begin{array}{l}\text { Predictors } \\
\text { (Patient, Bone, Drug) }\end{array}$ \\
\hline & & & & $\begin{array}{l}\text { nonosteoporotic women only: } \\
\text { NonVFx: } \\
\text { ALN vs. placebo } \\
\text { Low P1NP tertile: HR } 1.46(0.98,2.17) \\
\text { High P1NP tertile: HR } 0.77(0.51,1.17) \\
\text { p for trend=0.03; interaction (P1NP } \\
\text { continuous) = } 0.08 \\
\text { No evidence of a relationship between } \\
\text { preatment BSALP and sCTx on } \\
\text { nonvertebral fracture in nonosteoporotic } \\
\text { women. } \\
\text { RVFx: } \\
\text { Higher pretreatment BSALP was } \\
\text { associated with greater alendronate } \\
\text { efficacy on vertebral fracture } \\
\text { Low BSLAP tertile: OR } 1.22 \text { (0.45, 3.33) } \\
\text { High BSALP tertile: OR 0.25 (0.07, 0.88) } \\
\text { p for trend }=0.05 ; \text { interaction p value } \\
\text { (BSALP continuous) = 0.08 } \\
\text { Results did not change when authors } \\
\text { adjusted for baseline BMD (data NR) }\end{array}$ \\
\hline & $\begin{array}{l}\text { Cummings } \\
1998 \\
\text { (FIT-II) } \\
4 \mathrm{yr} \\
\\
\text { Low }\end{array}$ & $\begin{array}{l}\text { Up to } 4 \text { yr Multiple fxs in respective } \\
\text { categories: } \\
\text { Any CFx, baseline N/group: } \\
\text { ALN: } 272 / 2214(12.3 \%) \\
\text { Placebo: } 312 / 2218(14.1 \%) \\
\text { HR=0.86 }(95 \% \mathrm{Cl} 0.73,1.01) p=0.07 \\
\text { Any nonVFx: } \\
\text { ALN: } 261 / 2214(11.8 \%) \\
\text { Placebo:294/2218 }(13.3 \%) \\
\text { RH=0.88 }(95 \% \mathrm{Cl} 0.74,1.04) p=0.13 \\
\text { Hip: } \\
\text { ALN: } 19 / 2214(0.9 \%) \\
\text { Placebo: } 24 / 2218(1.1 \%)\end{array}$ & 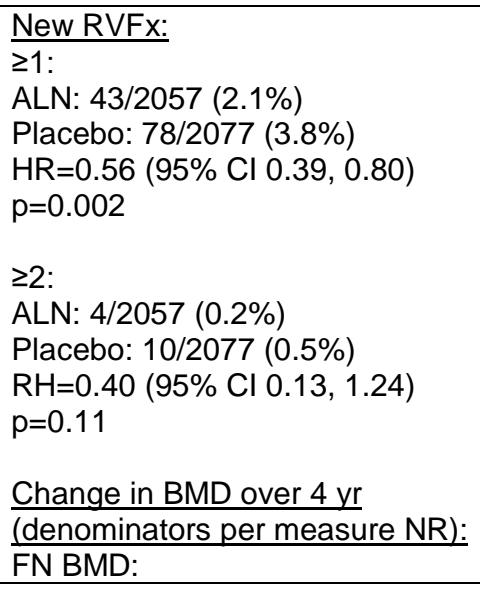 & $\begin{array}{l}\text { CFx by baseline FN BMD T-score. } \\
\text { Interaction } p \text { value (BMD } \\
\text { continuous)=0.01: } \\
\text { <-2.5: } \\
\text { ALN: } 107 / N R(13.1 \%) \\
\text { Placebo: } 159 / N R(19.6 \%) \\
\text { HR=0.64 }(95 \% \mathrm{Cl} 0.50,0.82) \\
-2.5 \text { to }-2.0: \\
\text { ALN: } 92 / N R(12.7 \%) \\
\text { Placebo: } 87 / N R(12.3 \%) \\
\text { HR=1.03 (95\% Cl 0.77, } 1.39) \\
-2.0 \text { to }-1.6: \\
\text { ALN: } 73 / N R(10.9 \%) \\
\text { Placebo: } 66 / N R(9.5 \%)\end{array}$ \\
\hline
\end{tabular}




\begin{tabular}{|c|c|c|c|c|}
\hline $\begin{array}{l}\text { Drug } \\
\text { Comparison }\end{array}$ & $\begin{array}{l}\text { Study } \\
\text { (Trial) } \\
\text { Followup } \\
\text { Risk of Bias }\end{array}$ & $\begin{array}{l}\text { Final Outcomes } \\
\text { Incident Clinical Fracture }\end{array}$ & $\begin{array}{l}\text { Intermediate Outcomes } \\
\text { Incident Radiographic } \\
\text { Vertebral Fracture } \\
\text { DXA BMD Change }\end{array}$ & $\begin{array}{l}\text { Predictors } \\
\text { (Patient, Bone, Drug) }\end{array}$ \\
\hline & & $\begin{array}{l}\text { HR=0.79 }(95 \% \mathrm{Cl} 0.43,1.44) \mathrm{p}=0.44 \\
\text { Wrist: } \\
\text { ALN: } 83 / 2214(3.7 \%) \\
\text { Placebo: } 70 / 2218(3.2 \%) \\
\text { HR=1.19 }(95 \% \mathrm{Cl} 0.87,1.64) \mathrm{p}=0.28 \\
\text { Other than hip, wrist or spine: } \\
\text { ALN: } 182 / 2214(8.2 \%) \\
\text { Placebo: } 227 / 2218(10.2 \%) \\
\text { HR=0.79 }(95 \% \mathrm{Cl} 0.65,0.96) \mathrm{p}=0.02\end{array}$ & $\begin{array}{l}\text { ALN: }+3.8 \% \\
\text { Placebo: }-0.8 \% \\
\text { Difference: } 4.6 \%(p<0.001) \\
\text { TH BMD: } \\
\text { ALN: }+3.4 \% \\
\text { Placebo: }-1.6 \% \\
\text { Difference: } 5.0 \%(p<0.001) \\
\text { LS BMD: } \\
\text { ALN: }+8.3 \% \\
\text { Placebo: }+1.5 \% \\
\text { Difference: } 6.6 \%(p<0.001)\end{array}$ & $\begin{array}{l}\text { RH=1.14 }(95 \% \mathrm{Cl} 0.82,1.60) \\
\text { Post hoc incident hip fracture by } \\
\text { dichotomized baseline FN-BMD T-score: } \\
\text { <-2.5: } \\
\text { ALN: } 8 / \mathrm{NR}(1.0 \%) \\
\text { Placebo: } 18 / \mathrm{NR}(2.2 \%) \text {; } \\
\text { HR }(95 \% \mathrm{CI}): 0.44(0.18,0.97), \mathrm{p}=\mathrm{NR} \\
\text { >2.5: } \\
\text { ALN: } 11 / \mathrm{NR}(0.8 \%) \\
\text { Placebo: } 6 / \mathrm{NR}(0.4 \%) \\
\text { HR }(95 \% \mathrm{Cl}): 1.84(0.70,5.36), \mathrm{p}=\mathrm{NR} \\
\text { RVFx by FN BMD T score: } \\
\text { <-2.5: } \\
\text { ALN: } 22 / \mathrm{NR}(2.9 \%) \\
\text { Placebo: } 44 / \mathrm{NR}(5.8 \%) \\
\text { HR=0.50 }(95 \% \mathrm{Cl} 0.31,0.82) \\
-2.5 \text { to }-2.0: \\
\text { ALN: } 13 / \mathrm{NR}(1.9 \%) \\
\text { Placebo: } 24 / \mathrm{NR}(3.6 \%) \\
\text { HR=0.54 }(95 \% \mathrm{Cl} 0.28,1.04) \\
-2.0 \text { to }-1.6: \\
\text { ALN: } 8 / \mathrm{NR}(1.3 \%) \\
\text { Placebo: } 10 / \mathrm{NR}(1.5 \%) \\
\text { HR=0.82 }(95 \% \mathrm{Cl} 0.33,2.07)\end{array}$ \\
\hline & $\begin{array}{l}\text { Donaldson } \\
2012 \\
\text { (Pooled FIT I \& } \\
\text { II RCTs) } \\
3 \text { yr FIT-I; } \\
4 \text { yr FIT-II } \\
\text { Medium }\end{array}$ & $\begin{array}{l}\text { Overall: First NonVFx: } \\
\text { ALN: NR/3236 (\% NR) } \\
\text { Placebo: NR/3223 (\% NR) } \\
\text { IRR=0.86 (95\% Cl 0.75, 0.99) p=NR }\end{array}$ & $\begin{array}{l}\text { RVFX: } \\
\text { Data not reported/shown. } \\
\text { Interaction between treatment } \\
\text { (ALN/placebo) and (FRAX with } \\
\text { BMD): } p=0.88\end{array}$ & $\begin{array}{l}\text { First NonVFx by FRAX (with FN BMD); by } \\
\text { FRAX tertiles, per } 100 \text { person years: } \\
\text { FRAX 4.75-22.06: } \\
\text { ALN rate: } 2.21 \\
\text { Placebo rate: } 2.55 \\
\text { IRR }=0.87(95 \% \mathrm{Cl} 0.65,1.15) \\
\text { RD }=-0.34(95 \% \mathrm{Cl}-1.0,0.33) \\
\\
\text { FRAX } 22.07-34.19: \\
\text { ALN rate: } 3.32 \\
\text { Placebo rate: } 3.73 \\
\text { IRR }=0.89(95 \% \mathrm{Cl} 0.70,1.13)\end{array}$ \\
\hline
\end{tabular}




\begin{tabular}{|c|c|c|c|c|}
\hline $\begin{array}{l}\text { Drug } \\
\text { Comparison }\end{array}$ & $\begin{array}{l}\text { Study } \\
\text { (Trial) } \\
\text { Followup } \\
\text { Risk of Bias }\end{array}$ & $\begin{array}{l}\text { Final Outcomes } \\
\text { Incident Clinical Fracture }\end{array}$ & $\begin{array}{l}\text { Intermediate Outcomes } \\
\text { Incident Radiographic } \\
\text { Vertebral Fracture } \\
\text { DXA BMD Change }\end{array}$ & $\begin{array}{l}\text { Predictors } \\
\text { (Patient, Bone, Drug) }\end{array}$ \\
\hline & & & & $\begin{array}{l}\text { RD=-0.41 }(95 \% \mathrm{Cl}-1.3,0.43) \\
\text { FRAX 34.2-85.36: } \\
\text { ALN rate: } 4.75 \\
\text { Placebo rate: } 5.66 \\
\text { IRR=0.84 }(95 \% \mathrm{Cl} 0.68,1.03) \\
\text { RD=-0.91 (95\% Cl -2.0, 0.17) } \\
\text { First NonVFx by baseline FN BMD: } \\
\text { Interaction between treatment and FN } \\
\text { BMD: } p=0.014 . \\
\text { Within FN T-score } \leq-2.5 \text { stratum: } \\
\text { ALN: NR/stratum NR (\% NR) } \\
\text { Placebo: NR/stratum NR (\% NR) } \\
\text { IRR=0.76 ( } 95 \% \mathrm{Cl} 0.62,0.93) \mathrm{p}=\mathrm{NR} \\
\text { "No evidence of benefit" of ALN in women } \\
\text { with FN T-score >-2.5; data not shown: } \\
\text { IRR=0.96 (95\% Cl 0.80, 1.16) } \\
\text { Also assessed CFx, MOF and RVFx but } \\
\text { results NR (interactions NS) }\end{array}$ \\
\hline & $\begin{array}{l}\text { Jamal } 2007 \\
\text { (Pooled FIT-I \& } \\
\text { FIT-II RCTs } \\
3 \text { yr FIT-I; } \\
4 \text { yr FIT-II) } \\
\text { Medium }\end{array}$ & $\begin{array}{l}\frac{\mathrm{CFX}:}{\text { "ALN (vs. placebo) reduced the risk of clinical }} \text { fractures regardless of renal function": } \\
\text { OR }(95 \% \mathrm{Cl}): 0.8(0.70,0.9) ; \mathrm{n}, \% \text {, and } \mathrm{p} \\
\text { value NR }\end{array}$ & $\begin{array}{l}\text { RVFX: } \\
\text { "Overall, alendronate (vs. } \\
\text { placebo) reduced the risk of } \\
\text { spine fractures" } \\
\text { OR }(95 \% \mathrm{Cl}): 0.54(0.37,0.78) \\
\mathrm{n}, \% \text {, and } \mathrm{p} \text { value NR }\end{array}$ & $\begin{array}{l}\text { All women, by renal function (eGFR }<45 \\
\mathrm{ml} / \mathrm{min} \text { vs } \geq 45 \mathrm{ml} / \mathrm{min}) . \mathrm{n}=6458 \\
\mathrm{CFx}: \\
\mathrm{P} \text { value for interaction= }=0.89: \\
\text { With reduced eGFR: } \\
\text { Alendronate: } \mathrm{n}(\%)=\mathrm{NR} \\
\text { Placebo: } \mathrm{n}(\%)=\mathrm{NR} \\
\text { OR }(95 \% \mathrm{Cl}): 0.78(0.51,1.21) \\
\text { Without reduced eGFR: } \\
\text { Alendronate: } \mathrm{n}(\%)=\mathrm{NR} \\
\text { Placebo: } \mathrm{n}(\%)=\mathrm{NR} \\
\text { OR }(95 \% \mathrm{Cl}): 0.80(0.70,0.93) \\
\text { RVFx: } \\
\text { P value for interaction=0.44: } \\
\text { With reduced eGFR: } \\
\text { Alendronate: } \mathrm{n}(\%)=\mathrm{NR}\end{array}$ \\
\hline
\end{tabular}




\begin{tabular}{|c|c|c|c|c|}
\hline $\begin{array}{l}\text { Drug } \\
\text { Comparison }\end{array}$ & $\begin{array}{l}\text { Study } \\
\text { (Trial) } \\
\text { Followup } \\
\text { Risk of Bias }\end{array}$ & $\begin{array}{l}\text { Final Outcomes } \\
\text { Incident Clinical Fracture }\end{array}$ & $\begin{array}{l}\text { Intermediate Outcomes } \\
\text { Incident Radiographic } \\
\text { Vertebral Fracture } \\
\text { DXA BMD Change }\end{array}$ & $\begin{array}{l}\text { Predictors } \\
\text { (Patient, Bone, Drug) }\end{array}$ \\
\hline & & & & $\begin{array}{l}\text { Placebo: } \mathrm{n}(\%)=\mathrm{NR} \\
\text { OR (95\% Cl): } 0.72(0.31,1.7) \\
\text { Without reduced eGFR: } \\
\text { Alendronate: } \mathrm{n}(\%)=\mathrm{NR} \\
\text { Placebo: } \mathrm{n}(\%)=\mathrm{NR}+ \\
\text { OR }(95 \% \mathrm{Cl}): 0.50(0.32,0.76) \\
\text { Subgroup with osteoporosis (FN BMD T } \\
\text { score <-2.5, } \mathrm{n}=3214) \\
\mathrm{CFx} \\
\text { P value for interaction=0.72: } \\
\text { With reduced eGFR: } \\
\text { Alendronate: } \mathrm{n}(\%)=\mathrm{NR} \\
\text { Placebo: } \mathrm{n}(\%)=\mathrm{NR} \\
\text { OR (95\% Cl): } 0.84(0.45,1.54) \\
\text { Without reduced eGFR: } \\
\text { Alendronate: } \mathrm{n}(\%)=\mathrm{NR} \\
\text { Placebo: } \mathrm{n}(\%)=\mathrm{NR} \\
\text { OR (95\% Cl): } 0.74(0.61,0.91) \\
\text { RVFx: } \\
\text { P value for interaction=0.49: } \\
\text { With reduced eGFR: } \\
\text { Alendronate: } \mathrm{n}(\%)=\mathrm{NR} \\
\text { Placebo: } \mathrm{n}(\%)=\mathrm{NR} \\
\text { OR }(95 \% \mathrm{Cl}): 1.01(0.29,3.6) \\
\text { Without reduced eGFR: } \\
\text { Alendronate: } \mathrm{n}(\%)=\mathrm{NR} \\
\text { Placebo: } \mathrm{n}(\%)=\mathrm{NR}+ \\
\text { OR (95\% Cl): } 0.62(0.36,1.10)\end{array}$ \\
\hline & $\begin{array}{l}\text { Ryder } 2008 \\
\text { (FIT-II RCT } \\
\text { subgroup) } \\
4.2 \text { yr } \\
\text { Medium }\end{array}$ & $\begin{array}{l}\text { NonVFX, in FIT-II women with low BMD: } \\
\text { ALN 158/1389 (11.4\%) } \\
\text { Placebo: } 143 / 1396(10.2 \%) \\
\text { HR=1.12 }(95 \% \mathrm{Cl} 0.89,1.40)\end{array}$ & NR & $\begin{array}{l}\text { NonVFX, in FIT-II women with low BMD, } \\
\text { stratified by prior fx after age } 45: \\
\text { interaction p value }=0.37 \\
\text { No prior fx since age } 45 \text { : } \\
\text { ALN: } 88 / 951(9.3 \%)^{\star} \\
\text { Placebo: } 86 / 954(9.0 \%)^{\star} \\
\text { HR=1.02 }(95 \% \mathrm{Cl} 0.76,1.38) \\
\text { Yes, prior fx since age } 45\end{array}$ \\
\hline
\end{tabular}




\begin{tabular}{|c|c|c|c|c|}
\hline $\begin{array}{l}\text { Drug } \\
\text { Comparison }\end{array}$ & $\begin{array}{l}\text { Study } \\
\text { (Trial) } \\
\text { Followup } \\
\text { Risk of Bias }\end{array}$ & $\begin{array}{l}\text { Final Outcomes } \\
\text { Incident Clinical Fracture }\end{array}$ & $\begin{array}{l}\text { Intermediate Outcomes } \\
\text { Incident Radiographic } \\
\text { Vertebral Fracture } \\
\text { DXA BMD Change }\end{array}$ & $\begin{array}{l}\text { Predictors } \\
\text { (Patient, Bone, Drug) }\end{array}$ \\
\hline & & & & $\begin{array}{l}\text { ALN: } 70 / 438(16.0 \%)^{\star} \\
\text { Placebo: } 57 / 442(12.9 \%)^{\star} \\
\text { HR=1.26 }(95 \% \mathrm{Cl} 0.89,1.79)\end{array}$ \\
\hline & $\begin{array}{l}\text { Quandt } 2005 \\
\text { (FIT-I and FIT- } \\
\text { II subgroup) } \\
3-4.5 \mathrm{yr} \\
\text { Medium }\end{array}$ & $\begin{array}{l}\text { FIT-II subgroup with }>3 \text { yr followup: } \\
\text { CVFx (women without baseline VFx): } \\
\text { ALN: } 6 / 1394(0.43 \%)^{\star} \\
\text { Placebo: } 13 / 1403(0.93 \%)^{\star} \\
\text { (denominators/group from Table 1) } \\
\text { RR }=0.46(95 \% \mathrm{Cl} 0.16,1.17)\end{array}$ & $\begin{array}{l}\text { FIT-II subgroup (>3 yr followup): } \\
\text { RVFx (>4 yr), women without } \\
\text { baseline VFx: } \\
\text { ALN: } 21 / \text { ITD } \\
\text { Placebo: } 33 / U T D \\
\text { RR=0.64 (95\% CI 0.38, 1.10) } \\
\text { Denominators NR for FIT-II } \\
\text { subgroup, this outcome. Not all } \\
\text { women had } x \text {-rays (total N for } \\
\text { RVFx = 3532; total N for this } \\
\text { study }=3737)\end{array}$ & NR for outcomes $>3 \mathrm{yr}$ \\
\hline $\begin{array}{l}\text { Zoledronic acid } \\
\text { vs placebo }\end{array}$ & $\begin{array}{l}\text { Black 2012 } \\
\text { (HORIZON) } \\
6 \text { yr } \\
\text { Low }\end{array}$ & $\begin{array}{l}\text { Incident CFx, all } \\
6 \text { yr } \\
\text { HR=1.04 (95\% Cl 0.71, 1.54) } \\
\text { Incident CVFx } \\
\text { HR=1.81 (95\% Cl 0.53, 6.2) } \\
\text { Incident nonCVFx } \\
\text { Zoledronic acid: } 45 / 616\left(7.3 \%^{\star}\right) \\
\text { Placebo: } 47 / 617\left(7.6 \% \%^{\star}\right) \\
\text { HR=0.99 (95\% Cl 0.7, 1.5) } \\
\text { Incident CFX, hip } \\
\text { Zoledronic acid: } 7 / 616(1.1 \%) \\
\text { Placebo: } 8 / 617(1.3 \%) \\
\text { HR=0.90 }(95 \% \mathrm{Cl} 0.33,2.49)\end{array}$ & 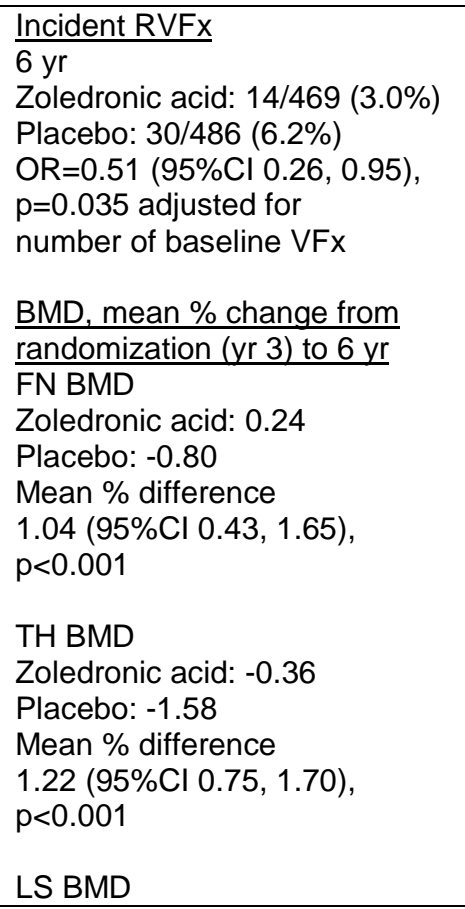 & NR \\
\hline
\end{tabular}




\begin{tabular}{|c|c|c|c|c|}
\hline $\begin{array}{l}\text { Drug } \\
\text { Comparison }\end{array}$ & $\begin{array}{l}\text { Study } \\
\text { (Trial) } \\
\text { Followup } \\
\text { Risk of Bias }\end{array}$ & $\begin{array}{l}\text { Final Outcomes } \\
\text { Incident Clinical Fracture }\end{array}$ & $\begin{array}{l}\text { Intermediate Outcomes } \\
\text { Incident Radiographic } \\
\text { Vertebral Fracture } \\
\text { DXA BMD Change }\end{array}$ & $\begin{array}{l}\text { Predictors } \\
\text { (Patient, Bone, Drug) }\end{array}$ \\
\hline & & & $\begin{array}{l}\text { Zoledronic acid: } 3.2 \\
\text { Placebo: } 1.18 \\
\text { Mean \% difference } \\
2.03(95 \% \mathrm{Cl} 0.76,3.29), p=- \\
0.002\end{array}$ & \\
\hline & $\begin{array}{l}\text { Black 2015 } \\
\text { (HORIZON) } \\
9 \mathrm{yr} \\
\text { Low }\end{array}$ & $\begin{array}{l}\text { Incident CFX, all } \\
9 \mathrm{yr} \\
\text { Zoledronic acid: } 10 / 95(12.2 \%)^{\star} \\
\text { Placebo: } 9 / 95(9.5 \%)^{\star} \\
\text { HR=1.11 }(95 \% \mathrm{Cl} 0.45,2.73)\end{array}$ & $\begin{array}{l}\frac{\text { Incident RVFx }}{9 \text { yr }} \\
\text { Zoledronic acid: } 3 / 68(4.4 \%) \\
\text { Placebo: } 5 / 69(7.2 \%) \\
\text { OR=0.61 (95\% Cl 0.14, 2.77) } \\
\text { BMD, mean \% change from } \\
\text { randomization (yr 6) to } 9 \mathrm{yr} \\
\text { FN BMD } \\
\text { Zoledronic acid: }-1.11 \\
\text { Placebo: }-1.17 \\
\text { Mean \% difference=0.06 (95\% } \\
\text { Cl -1.41, 1.53) } p=0.935 \\
\text { TH BMD } \\
\text { Zoledronic acid: }-0.54 \\
\text { Placebo: }-1.31 \\
\text { Mean \% difference=0.78 }(95 \% \\
\text { Cl -0.37, 1.93) } p=0.183\end{array}$ & NR \\
\hline & $\begin{array}{l}\text { Reid, } 2018 \\
6 \mathrm{yr} \\
\text { Low }\end{array}$ & 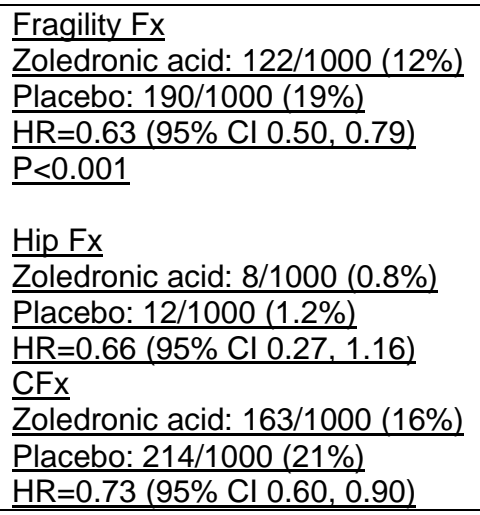 & $\begin{array}{l}\text { Percent change in LS BMD } \\
\text { (estimated from Figure S1) } \\
\text { Baseline to Year } 3 \\
\text { Zoledronic acid: } 5.5 \% \\
\text { Placebo: }-1 \% \\
\text { Baseline to Year 6: } \\
\text { Zoledronic acid: } 7 \% \\
\text { Placebo: }-1 \% \\
\text { Percent change in TH BMD } \\
\text { (estimated from Figure S1) } \\
\text { Baseline to Year } 3 \\
\text { Zoledronic acid: } 3.5 \% \\
\end{array}$ & $\begin{array}{l}\text { Predictor: Baseline VFx status } \\
\text { Outcome: Fragility Fx } \\
\text { p for interaction }=0.27 \\
\text { HR among participants without baseline } \\
\text { VFx }=0.65 \text { (95\% Cl 0.50, 0.83) } \\
\text { Predictor: Osteoporosis status } \\
\text { Outcome: Fragility Fx } \\
\text { HR excluding osteoporotic } \\
\text { participants=0.63 (95\% Cl } 0.49,0.80) \\
\text { Predictor: Baseline Fx risk } \\
\text { Outcome: Fragility Fx }\end{array}$ \\
\hline
\end{tabular}




\begin{tabular}{|c|c|c|c|c|}
\hline $\begin{array}{l}\text { Drug } \\
\text { Comparison }\end{array}$ & $\begin{array}{l}\text { Study } \\
\text { (Trial) } \\
\text { Followup } \\
\text { Risk of Bias }\end{array}$ & $\begin{array}{l}\text { Final Outcomes } \\
\text { Incident Clinical Fracture }\end{array}$ & $\begin{array}{l}\text { Intermediate Outcomes } \\
\text { Incident Radiographic } \\
\text { Vertebral Fracture } \\
\text { DXA BMD Change }\end{array}$ & $\begin{array}{l}\text { Predictors } \\
\text { (Patient, Bone, Drug) }\end{array}$ \\
\hline & & $\begin{array}{l}\text { Total VFx } \\
\text { Zoledronic acid: } 23 / 1000(2.3 \%) \\
\text { Placebo: } 49 / 1000(4.9 \%) \\
\text { OR=0.45 (95\% Cl } 0.27,0.73) \\
\text { CVFx } \\
\text { Zoledronic acid: } 14 / 1000(1.4 \%) \\
\text { Placebo: } 34 / 1000(3.4 \%) \\
\text { HR=0.41 }(95 \% \mathrm{Cl} 0.22,0.75) \\
\text { NV Fragility Fx } \\
\text { Zoledronic acid: } 101 / 1000(10 \%) \\
\text { Placebo: } 148 / 1000(15 \%) \\
\text { HR=0.66 } 95 \% \mathrm{Cl} 0.27,1.16) \\
\text { Forearm or wrist Fx } \\
\text { Zoledronic acid: } 36 / 1000(3.6 \%) \\
\text { Placebo: } 63 / 1000(6.3 \%) \\
\text { HR=0.56 (95\% Cl } 0.37,0.85) \\
\text { All counts represent number of participants } \\
\text { with event, not total number of events }\end{array}$ & $\begin{array}{l}\text { Placebo: }-2 \% \\
\text { Baseline to Year 6: } \\
\text { Zoledronic acid: } 3.5 \% \\
\text { Placebo: }-3.8 \% \\
\text { Percent change in Total Body } \\
\text { BMD (estimated from Figure S1) } \\
\text { Baseline to Year } 3 \\
\text { Zoledronic acid: } 1.3 \% \\
\text { Placebo: }-1.6 \% \\
\text { Baseline to Year 6: } \\
\text { Zoledronic acid: } 1.8 \% \\
\text { Placebo: }-2 \%\end{array}$ & $\begin{array}{l}\text { HR excluding participants with hip Fx risk } \\
>3 \% \text { or osteoporotic Fx risk }>20 \%=0.60 \\
\text { (95\% Cl 0.44, 0.81) } \\
\text { HR excluding participants with hip Fx } \\
\text { risk }>3 \% \text { or osteoporotic Fx risk }>20 \% \text { or } \\
\text { hx of NVFx after age } 45=0.60(95 \% \mathrm{Cl} \\
0.39,0.91) \\
\text { Predictor: Baseline Fx risk } \\
\text { Outcome: NVFx } \\
\text { HR excluding participants with hip Fx } \\
\text { risk }>3 \% \text { or osteoporotic } \mathrm{Fx} \text { risk }>20 \% \text { or } \\
\text { hx of NVFx after age } 45=0.57(95 \% \mathrm{Cl} \\
0.37,0.86)\end{array}$ \\
\hline $\begin{array}{l}\text { Denosumab vs } \\
\text { placebo }\end{array}$ & $\begin{array}{l}\text { Miller } 2008 \\
4 \mathrm{yr} \\
\text { Medium }\end{array}$ & $\begin{array}{l}\frac{C F x}{\text { Denosumab } 33 / 314(11 \%)} \\
\text { Placebo } 5 / 46(11 \%) \\
\text { RR=0.97 }(95 \% \mathrm{Cl} 0.40,2.35)^{\star} \\
\text { p>0.99 } \\
\text { Osteoporotic fx } \\
\text { Denosumab: } 22 / 314(7 \%) \\
\text { Placebo: } 4 / 46(9 \%) \\
\text { RR=0.81 }(95 \% \mathrm{Cl} 0.29,2.23)^{\star}\end{array}$ & $\begin{array}{l}\text { BMD, \% change from baseline } \\
\underline{\text { LS }} \\
\text { Continued denosumab: }(n=153) \\
\text { Placebo: } 11.8 \\
p<0.001 \\
\text { Discontinued denosumab }(n=31) \\
\text { at } 36 \text { months: }-6.6 \\
\text { Discontinued/retreatment } \\
\text { denosumab ( } n=30): 9.0 \\
p<0.05 \text { for both groups vs } \\
\text { placebo } \\
\frac{\text { TH }}{\text { Continued denosumab: } 4.0 \text { to }} \\
6.1\end{array}$ & $\mathrm{NR}$ \\
\hline
\end{tabular}




\begin{tabular}{|c|c|c|c|c|}
\hline $\begin{array}{l}\text { Drug } \\
\text { Comparison }\end{array}$ & $\begin{array}{l}\text { Study } \\
\text { (Trial) } \\
\text { Followup } \\
\text { Risk of Bias }\end{array}$ & $\begin{array}{l}\text { Final Outcomes } \\
\text { Incident Clinical Fracture }\end{array}$ & $\begin{array}{l}\text { Intermediate Outcomes } \\
\text { Incident Radiographic } \\
\text { Vertebral Fracture } \\
\text { DXA BMD Change }\end{array}$ & $\begin{array}{l}\text { Predictors } \\
\text { (Patient, Bone, Drug) }\end{array}$ \\
\hline & & & $\begin{array}{l}\text { Placebo: }-3.5 \\
p<0.001 \text { for continued vs } \\
\text { placebo } \\
\text { Discontinued denosumab }(n=31) \\
\text { at } 36 \text { months: }-5.3 \\
\text { Discontinued/retreatment } \\
\text { denosumab }(n=30): 3.9 \\
p<0.05 \text { for both groups vs. } \\
\text { placebo }\end{array}$ & \\
\hline $\begin{array}{l}\text { Raloxifene vs } \\
\text { placebo }\end{array}$ & $\begin{array}{l}\text { Sontag } 2010 \\
\text { Johnell } 2004 \\
\text { (Raloxifene } 60 \\
\text { mg and } \\
\text { placebo } \\
\text { groups only) } \\
\text { (MORE) } \\
\text { 3.3 yr (mean) } \\
\text { Low (Sontag) } \\
\text { Medium } \\
\text { (Johnell) }\end{array}$ & $\begin{array}{l}\text { Incident CVFx } \\
4 \text { yr } \\
\text { Raloxifene } 60 \mathrm{mg}: 62 / 2549(2 \%) \\
\text { Placebo: } 107 / 2565(4 \%)^{\star} p=0.003 \\
\text { (Data from Sontag } 2010 ; \text { also reported in } \\
\text { Johnell 2004) }\end{array}$ & $\begin{array}{l}\text { Incident RVFx } \\
\text { Raloxifene: } 60 \text { mg 181/2259 } \\
\text { (8\%) } \\
\text { Placebo: 288/2292 (13\%)* } \\
\text { (Data from Sontag 2010; also } \\
\text { reported in Johnell 2004) }\end{array}$ & $\begin{array}{l}\text { Predictor: Baseline VFx status } \\
\text { Outcome: CVFx } \\
\text { No baseline VFx } \\
\text { Raloxifene } 60 \mathrm{mg}: 8 / 1574(0.5 \%) \\
\text { Placebo: } 26 / 1629(2 \%) \\
\text { With baseline VFx } \\
\text { Raloxifene } 60 \mathrm{mg}: 54 / 975(6 \%) \\
\text { Placebo: } 81 / 936(9 \%) \\
\text { p for interaction=0.127 } \\
\text { Outcome: RVFx } \\
\text { No baseline VFx } \\
\text { Raloxifene } 60 \mathrm{mg}: 41 / 1574(3 \%) \\
\text { Placebo: } 84 / 1629(5 \%) \\
\text { With baseline VFx } \\
\text { Raloxifene } 60 \mathrm{mg}: 140 / 975 \text { (14\%) } \\
\text { Placebo: } 204 / 936(22 \%) \\
\text { p for interaction=0.402 } \\
\text { (Data from Sontag 2010; also reported in } \\
\text { Johnell 2004) }\end{array}$ \\
\hline $\begin{array}{l}\text { Raloxifene vs } \\
\text { placebo }\end{array}$ & $\begin{array}{l}\text { Delmas } 2002 \\
\text { (subjects with } \\
\text { a baseline and } \\
\geq 1 \text { post- } \\
\text { baseline } \\
\text { vertebral Xray, }\end{array}$ & $\begin{array}{l}\text { Incident Hip fx } \\
\text { Raloxifene } 60 \mathrm{mg}+120 \mathrm{mg}: 56 / 4536(1.1 \%) \\
\text { Placebo: } 29 / 2292(1.1 \%) \\
\text { RR=0.97 (95\% Cl } 0.62 \text { to } 1.52) \\
\text { (Data from Delmas } 2002 ; \text { also reported in }\end{array}$ & $\begin{array}{l}\text { Incident RVFx, Kaplan-Meier } \\
\text { log-rank test } \\
\text { Raloxifene } 60 \mathrm{mg}: \mathrm{n} / \mathrm{N}=\mathrm{NR} \\
\text { Raloxifene } 120 \mathrm{mg}: \mathrm{n} / \mathrm{N}=\mathrm{NR} \\
\text { Placebo: } \mathrm{n} / \mathrm{N}=\mathrm{NR} \\
\text { Raloxifene } 60 \mathrm{mg} \text { vs placebo }\end{array}$ & $\begin{array}{l}\text { Predictor: Baseline VFx } \\
\text { Outcome: RVFx } \\
\text { No baseline VFx } \\
\text { Raloxifene } 60 \mathrm{mg} \text { vs placebo } \\
\text { RR }=0.51(95 \% \mathrm{Cl} 0.35,0.73) \\
\text { Raloxifene } 120 \mathrm{mg} \text { vs placebo }\end{array}$ \\
\hline
\end{tabular}




\begin{tabular}{|c|c|c|c|c|}
\hline $\begin{array}{l}\text { Drug } \\
\text { Comparison }\end{array}$ & $\begin{array}{l}\text { Study } \\
\text { (Trial) } \\
\text { Followup } \\
\text { Risk of Bias }\end{array}$ & $\begin{array}{l}\text { Final Outcomes } \\
\text { Incident Clinical Fracture }\end{array}$ & $\begin{array}{l}\text { Intermediate Outcomes } \\
\text { Incident Radiographic } \\
\text { Vertebral Fracture } \\
\text { DXA BMD Change }\end{array}$ & $\begin{array}{l}\text { Predictors } \\
\text { (Patient, Bone, Drug) }\end{array}$ \\
\hline & $\begin{array}{l}\mathrm{n}=6828 \text { ) } \\
\text { Barrett-Connor } \\
2004 \\
\text { (MORE) } \\
3.3 \text { yr (mean) } \\
\text { Low }\end{array}$ & Barrett-Connor 2004) & $\begin{array}{l}\text { RR=0.64 }(95 \% \mathrm{Cl} 0.53,0.76) \\
\text { Raloxifene } 120 \mathrm{mg} \text { vs placebo } \\
\text { RR=0.57 ( } 95 \% \mathrm{Cl} 0.48,0.69) \\
\text { p<0.001 for difference between } \\
\text { raloxifene groups and placebo } \\
\text { Change in LS BMD vs placebo, } \\
\text { ANOVA } \\
\text { Raloxifene } 60 \mathrm{mg}: 2.6 \% \\
\text { Raloxifene } 120 \mathrm{mg}: 2.5 \% \\
\text { p<0.001 } \\
\text { Change in FN BMD vs placebo, } \\
\text { ANOVA } \\
\text { Raloxifene } 60 \mathrm{mg}: 2.1 \% \\
\text { Raloxifene } 120 \mathrm{mg}: 2.3 \% \\
\text { p<0.001 } \\
\text { (Data from Delmas } 2002)\end{array}$ & $\begin{array}{l}\text { RR=0.62 (95\% Cl 0.44, 0.87) } \\
\text { With baseline VFx } \\
\text { Raloxifene } 60 \mathrm{mg} \text { vs placebo } \\
\mathrm{RR}=0.66(95 \% \mathrm{Cl} 0.55,0.81) \\
\text { Raloxifene } 120 \mathrm{mg} \text { vs placebo } \\
\mathrm{RR}=0.54(95 \% \mathrm{Cl} 0.44,0.66) \\
\text { (Data from Delmas } 2002)\end{array}$ \\
\hline $\begin{array}{l}\text { Raloxifene vs } \\
\text { placebo }\end{array}$ & $\begin{array}{l}\text { Siris } 2005 \\
\text { (MORE/CORE) } \\
8 \mathrm{yr} \\
\text { Medium }\end{array}$ & $\begin{array}{l}\text { Incident clinical nonvertebral fracture } \\
\text { Raloxifene } 60 \mathrm{mg} \mathrm{n} / \mathrm{N}=22.8 \% \\
\text { Placebo } \mathrm{n} / \mathrm{N}=22.9 \% \\
\text { Raloxifene } 60 \mathrm{mg} \text { vs placebo } \\
\text { HR=1.00 }(0.82,1.21) \\
\frac{\text { Incident clinical nonvertebral-6 fracture }}{\text { (clavicle, humerus, wrist, pelvis, hip, lower }} \\
\frac{\text { leg) }}{\text { Raloxifene } 60 \mathrm{mg} \mathrm{n} / \mathrm{N}=17.5 \%} \\
\text { Placebo } \mathrm{n} / \mathrm{N}=17.5 \% \\
\text { Raloxifene } 60 \mathrm{mg} \text { vs placebo } \\
\mathrm{HR}=1.01(0.81,1.26)\end{array}$ & $\begin{array}{l}\text { BMD } \\
\text { Reported in Siris } 2005 \text { (high } \\
\text { ROB) }\end{array}$ & $\begin{array}{l}\frac{\text { Baseline age }}{\text { Treatment-by-subgroup interaction NS }} \\
\frac{\text { Baseline BMD }}{\text { Treatment-by-subgroup interaction NS }} \\
\frac{\text { Prevalent vertebral fracture }}{\text { Treatment-by-subgroup interaction }} \\
p<0.10 \text { (significant) } \\
\begin{array}{l}\text { No significant reduction in nonvertebral of } \\
\text { nonvertebral-6 fracture in either subgroup } \\
\text { (with or without vertebral fracture) }\end{array}\end{array}$ \\
\hline $\begin{array}{l}\text { Hormone } \\
\text { therapy vs } \\
\text { control }\end{array}$ & $\begin{array}{l}\text { Wimalawansa } \\
4 \mathrm{yr} \\
\text { Medium }\end{array}$ & $\begin{array}{l}\text { Incident nonCVFx } \\
4 \text { yrHormone therapy: } 1 / 15(6.7 \%) \\
\text { Control: } 1 / 14(7.1 \%) P=1.0 \text { (Fisher's exact } \\
\text { test) * }\end{array}$ & $\begin{array}{l}\text { Incident RVFx } \\
4 \text { yrHormone therapy: } 2 / 15 \\
(13.3 \%) \\
\text { Control: } 5 / 14(35.7 \%) \\
\end{array}$ & NR \\
\hline
\end{tabular}




\begin{tabular}{|c|c|c|c|c|}
\hline $\begin{array}{l}\text { Drug } \\
\text { Comparison }\end{array}$ & $\begin{array}{l}\text { Study } \\
\text { (Trial) } \\
\text { Followup } \\
\text { Risk of Bias }\end{array}$ & $\begin{array}{l}\text { Final Outcomes } \\
\text { Incident Clinical Fracture }\end{array}$ & $\begin{array}{l}\text { Intermediate Outcomes } \\
\text { Incident Radiographic } \\
\text { Vertebral Fracture } \\
\text { DXA BMD Change }\end{array}$ & $\begin{array}{l}\text { Predictors } \\
\text { (Patient, Bone, Drug) }\end{array}$ \\
\hline & & & 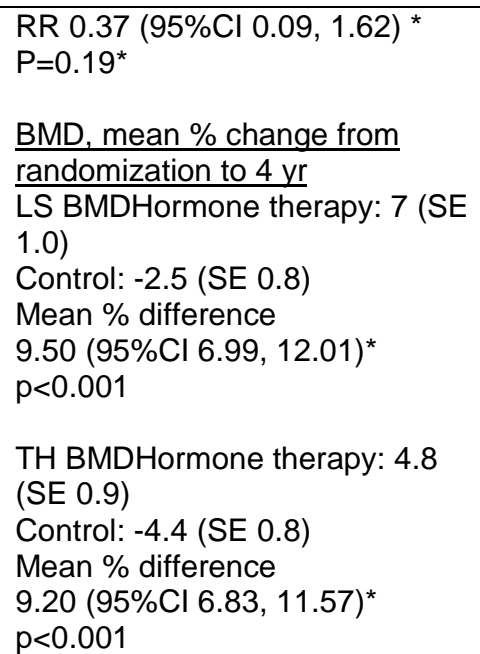 & \\
\hline
\end{tabular}




\begin{tabular}{|c|c|c|c|c|}
\hline $\begin{array}{l}\text { Drug } \\
\text { Comparison }\end{array}$ & $\begin{array}{l}\text { Study } \\
\text { (Trial) } \\
\text { Followup } \\
\text { Risk of Bias }\end{array}$ & $\begin{array}{l}\text { Final Outcomes } \\
\text { Incident Clinical Fracture }\end{array}$ & $\begin{array}{l}\text { Intermediate Outcomes } \\
\text { Incident Radiographic } \\
\text { Vertebral Fracture } \\
\text { DXA BMD Change }\end{array}$ & $\begin{array}{l}\text { Predictors } \\
\text { (Patient, Bone, Drug) }\end{array}$ \\
\hline & & $\begin{array}{l}\text { HR } 0.65(0.46,0.92) \\
\text { Note these outcomes are for the total study } \\
\text { population, which includes women who are } \\
\text { neither osteoporotic nor osteopenic. }\end{array}$ & & $\begin{array}{l}\text { Placebo: 22/NR (annualized percentage } \\
3.2 \% \text { ) } \\
\text { HR 0.53 (95\% Cl 0.25, 1.10) } \\
\text { T score } \leq 2.5: \\
\text { Hormone therapy: 44/NR (annualized } \\
\text { percentage } 1.7 \% \text { ) } \\
\text { Placebo: } 43 / \mathrm{NR} \text { (annualized percentage } \\
1.9 \%) \\
\text { HR 0.87 (95\% Cl } 0.57,1.34) \\
\text { p for interaction=0.15 } \\
\\
\text { Predictor: History of fracture } \\
\text { Outcome: Incident Hip Fx } \\
\text { With history of fracture: } \\
\text { Hormone therapy: } 31 / 2950(1.1 \%, \\
\text { annualized percentage } 0.19 \%) \\
\text { Placebo: } 41 / 2947(1.4 \%, \text { annualized } \\
\text { percentage } 0.26 \%) \\
\text { HR 0.77 (95\% Cl 0.48, } 1.22) \\
\text { Without history of fracture: } \\
\text { Hormone therapy: } 15 / 5556(0.27 \%, \\
\text { annualized percentage } 0.06 \%) \\
\text { Placebo: } 28 / 5155(0.54 \%, \text { annualized } \\
\text { percentage } 0.11 \%) \\
\text { HR 0.52 (95\% Cl } 0.28,0.98) \\
\text { p for interaction }=0.34\end{array}$ \\
\hline $\begin{array}{l}\frac{\text { Hormone }}{\text { therapy }} \\
\text { (estrogen) vs } \\
\text { placebo }\end{array}$ & $\begin{array}{l}\text { Jackson } 2006 \\
7 \text { yr } \\
\text { Low }\end{array}$ & $\begin{array}{l}\frac{\text { Incident CFX, all }}{7.1 \text { yr }} \\
\text { Hormone therapy: } 540 / 5310(10.2 \%, \\
\text { annualized percentage } 1.4 \%) \\
\text { Placebo: } 761 / 5429(14.0 \%, \text { annualized } \\
\text { percentage } 2.0 \%) \\
\text { HR } 0.71(95 \% \mathrm{Cl} 0.64,0.80) \\
\\
\text { Incident Hip Fx } \\
7.1 \text { yr } \\
\text { Hormone therapy: } 46 / 5310(0.87 \%, \\
\text { annualized percentage } 0.12 \%) \\
\text { Placebo: } 73 / 5429(1.3 \%, \text { annualized } \\
\text { percentage } 0.19 \%)\end{array}$ & NR for subgroups of interest & $\begin{array}{l}\text { Predictor: History of fracture } \\
\text { Outcome: Incident CFx } \\
\text { With history of fracture: } \\
\text { Hormone therapy: } 267 / 1916(13.9 \%, \\
\text { annualized percentage } 2.0 \%) \\
\text { Placebo: } 354 / 1900(18.6 \%, \text { annualized } \\
\text { percentage } 2.7 \%) \\
\text { HR } 0.73(95 \% \mathrm{Cl} 0.62,0.86) \\
\text { Without history of fracture: } \\
\text { Hormone therapy: } 220 / 3394(6.5 \%, \\
\text { annualized percentage } 1.1 \%) \\
\text { Placebo: } 321 / 3529(9.1 \%, \text { annualized } \\
\text { percentage } 1.6 \%) \\
\text { HR } 0.68(95 \% \mathrm{Cl} 0.57,0.81)\end{array}$ \\
\hline
\end{tabular}




\begin{tabular}{|c|c|c|c|c|}
\hline $\begin{array}{l}\text { Drug } \\
\text { Comparison }\end{array}$ & $\begin{array}{l}\text { Study } \\
\text { (Trial) } \\
\text { Followup } \\
\text { Risk of Bias }\end{array}$ & $\begin{array}{l}\text { Final Outcomes } \\
\text { Incident Clinical Fracture }\end{array}$ & $\begin{array}{l}\text { Intermediate Outcomes } \\
\text { Incident Radiographic } \\
\text { Vertebral Fracture } \\
\text { DXA BMD Change }\end{array}$ & $\begin{array}{l}\text { Predictors } \\
\text { (Patient, Bone, Drug) }\end{array}$ \\
\hline & & 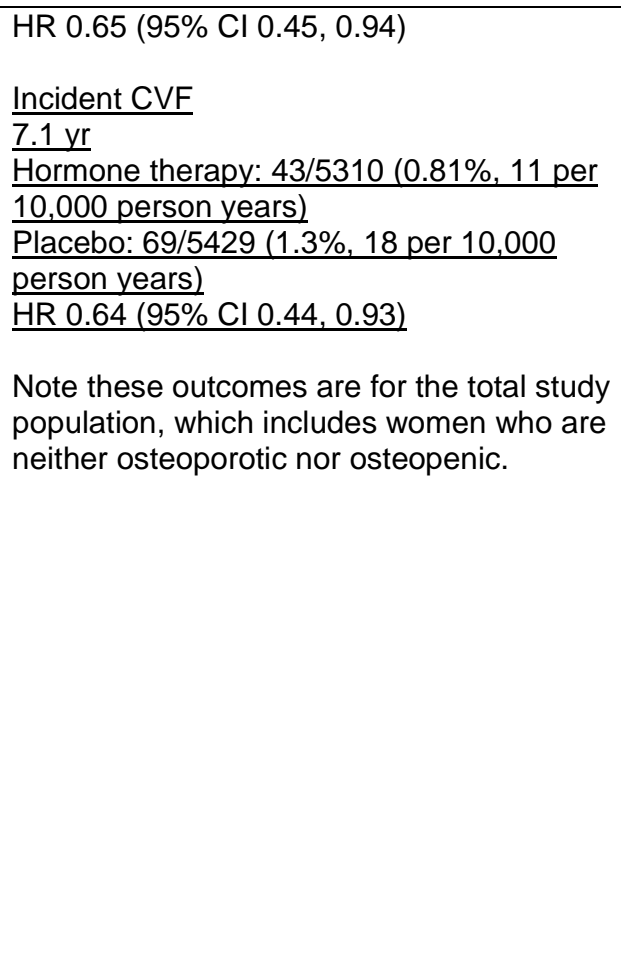 & & 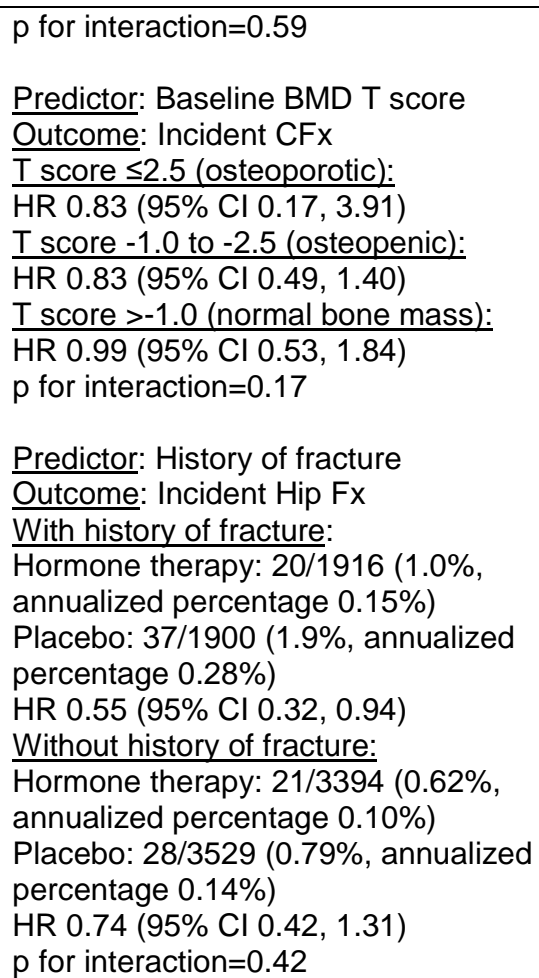 \\
\hline
\end{tabular}

Abbreviations: ALN=alendronate; BMD=bone mineral density; BMI=body mass index; $\mathrm{CFx}=$ clinical fracture; CORE=Continuing Outcomes Relevant to Evista; $\overline{\mathrm{DXA}=\mathrm{Dual}-}$ (WHO) Fracture Risk Assessment Tool; Fx=fracture; HORIZON=Health Outcomes and Reduced Incidence with Zoledronic Acid Once Yearly trial; HR=hazard ratio;

IRR=incidence rate ratio; LS=lumbar spine; $\mathrm{mg}=$ milligrams; MOF=major osteoporotic fracture; MORE=Multiple Outcomes of Raloxifene Evaluation; N=number; NR=not reported; NS=not significant; $\mathrm{RD}=$ risk difference; $\mathrm{RH}=$ relative hazard; $\mathrm{RVFx}=$ radiographic vertebral fracture; $\mathrm{RR}=$ risk ratio; $\mathrm{TH}=$ total hip; $\mathrm{VFx}=\mathrm{vertebral}$ fracture; vs=versus; yr=years

*Calculated by EPC 
Table D4. Key Questions 3 and 4 evidence overview

\begin{tabular}{|c|c|c|c|c|}
\hline $\begin{array}{l}\text { Drug } \\
\text { Comparison }\end{array}$ & $\begin{array}{l}\text { Study } \\
\text { (Trial) } \\
\text { Followup } \\
\text { Risk of Bias }\end{array}$ & $\begin{array}{l}\text { Harms } \\
\text { Drug class-specific harms }\end{array}$ & $\begin{array}{l}\text { Rare Harms } \\
\text { AFF, ONJ, Afib }\end{array}$ & $\begin{array}{l}\text { Predictors } \\
\text { (Patient, Bone, Drug) }\end{array}$ \\
\hline \multirow[t]{2}{*}{$\begin{array}{l}\text { Alendronate vs } \\
\text { placebo }\end{array}$} & $\begin{array}{l}\text { Bauer } 2000 \\
\text { (Pooled FIT I \& II) } \\
\text { Mean 3.8 yr } \\
\text { Medium }\end{array}$ & $\begin{array}{l}\text { Any upper GI AE } \\
\text { ALN: } 1536 / 3236(47.5 \%) \\
\text { Placebo: } 1490 / 3223(46.2 \%) \\
\text { RR } 1.02(95 \% \mathrm{Cl} 0.95,1.10) \\
\text { Any gastric or duodenal AE } \\
\text { ALN: } 130 / 3236(4.0 \%) \\
\text { Placebo: } 129 / 3223(4.0 \%) \\
\text { RR: NR } \\
\text { Gastroduodenal } \\
\text { perforation/ulcer/bleed } \\
\text { ALN: } 53 / 3236(1.6 \%) \\
\text { Placebo: } 61 / 3223(1.9 \%) \\
\text { RR: NR } \\
\text { Any esophageal AE } \\
\text { ALN: } 322 / 3236(10.0 \%) \\
\text { Placebo: } 309 / 3223(9.4 \%) \\
\text { RR: NR }\end{array}$ & NR & $\begin{array}{l}\text { Age (Fig 3): "perforation/ulcer/bleed } \\
\text { increased with age but no evidence of } \\
\text { disproportionate \# of events with ALN" } \\
\text { (pg 522) } \\
\text { History of UGI disease } \\
\text { Gastroduodenal } \\
\text { perforation/ulcer/bleed, esophageal } \\
\text { events more common with history of } \\
\text { UGI disease; no evidence of } \\
\text { disproportionate events on ALN. } \\
\text { NSAIDs } \\
\text { Events more common on NSAIDs (both } \\
\text { groups) }\end{array}$ \\
\hline & $\begin{array}{l}\text { Black } 2010 \\
\text { (pooled Fit-I and } \\
\text { Fit-II) } \\
\text { Mean } 3.8 \mathrm{yr} \\
\text { Low }\end{array}$ & NR & $\begin{array}{l}\text { Subtrochanteric or diaphyseal } \\
\text { femur fracture } \\
\text { ALN: } 1 / 3236(0.031 \%) \\
\text { Placebo: } 1 / 3223(0.031 \%) \\
\mathrm{RH}=1.03(95 \% \mathrm{Cl} 0.06,16.46) \\
\mathrm{p}=0.98\end{array}$ & NR \\
\hline
\end{tabular}




\begin{tabular}{|c|c|c|c|c|}
\hline $\begin{array}{l}\text { Drug } \\
\text { Comparison }\end{array}$ & $\begin{array}{l}\text { Study } \\
\text { (Trial) } \\
\text { Followup } \\
\text { Risk of Bias }\end{array}$ & $\begin{array}{l}\text { Harms } \\
\text { Drug class-specific harms }\end{array}$ & $\begin{array}{l}\text { Rare Harms } \\
\text { AFF, ONJ, Afib }\end{array}$ & $\begin{array}{l}\text { Predictors } \\
\text { (Patient, Bone, Drug) }\end{array}$ \\
\hline & $\begin{array}{l}\text { Cummings } 1998 \\
\text { (FIT-II) } \\
4 \mathrm{yr} \\
\text { Low }\end{array}$ & $\begin{array}{l}\text { Any upper GI event } \\
\text { ALN: } 1052 / 2214(47.5 \%) \\
\text { Placebo: } 1047 / 2218(47.2 \%) \\
\text { RH=1.00 (95\% Cl 0.92, 1.09) } \\
\text { Serious AE category: NR } \\
\text { Any AE resulting in hospitalization: } \\
\text { ALN: } 644 / 2214(29.1 \%) \\
\text { Placebo: } 596 / 2218(26.9 \%) \\
\text { HR (95\% Cl): } 1.09(0.98,1.22) \\
\text { p=NR } \\
\text { Death } \\
\text { ALN: } 37 / 2214(1.7 \%) \\
\text { Placebo: } 40 / 2218(1.8 \%) \\
\text { HR=0.92 }(95 \% \mathrm{Cl} 0.59,1.45)\end{array}$ & NR & NR \\
\hline
\end{tabular}




\begin{tabular}{|c|c|c|c|c|}
\hline $\begin{array}{l}\text { Drug } \\
\text { Comparison }\end{array}$ & $\begin{array}{l}\text { Study } \\
\text { (Trial) } \\
\text { Followup } \\
\text { Risk of Bias }\end{array}$ & $\begin{array}{l}\text { Harms } \\
\text { Drug class-specific harms }\end{array}$ & $\begin{array}{l}\text { Rare Harms } \\
A F F, \text { ONJ, Afib }\end{array}$ & $\begin{array}{l}\text { Predictors } \\
\text { (Patient, Bone, Drug) }\end{array}$ \\
\hline & $\begin{array}{l}\text { Jamal } 2007 \\
\text { Pooled (FIT-I + } \\
\text { FIT-II) } \\
\text { Mean follow-up } 3 \\
\text { yr FIT-I, } 4 \text { yr FIT- } \\
\text { II } \\
\text { Medium }\end{array}$ & NR & NR & $\begin{array}{l}\text { Reduced renal function (eGFR }<45 \\
\text { ml/min, } \mathrm{n}=581 \text { ) vs without reduced } \\
\text { renal function (eGFR } \geq 45 \mathrm{ml} / \mathrm{min}, \\
\mathrm{n}=5877 \text { ). } \\
\text { Gl events } \\
\text { With reduced eGFR: } 4.5 \% \\
\text { Without reduced eGFR: } 5.2 \% \mathrm{p}=0.5 \\
\text { Arrhythmias } \\
\text { With reduced eGFR: } 2.4 \% \\
\text { Without reduced eGFR: } 2.1 \% \mathrm{p}=0.7 \\
\text { Cardiovascular events } \\
\text { With reduced eGFR: } 2.6 \% \\
\text { Without reduced eGFR: } 3.2 \% \mathrm{p}=0.4 \\
\text { Renal AEs } \\
\text { With reduced eGFR: } 12 / 581(2.1 \%) \\
\text { Without reduced eGFR: } 137 / 5877 \\
\text { (2.3\%), p=0.68 } \\
\text { Cancer (any) } \\
\text { With reduced eGFR: } 4.3 \% \\
\text { Without reduced eGFR: } 5.0 \% \mathrm{p}=0.4 \\
\text { Death } \\
\text { With reduced eGFR: } 1.6 \% \\
\text { Without reduced eGFR: } 1.9 \% \mathrm{p}=0.5\end{array}$ \\
\hline
\end{tabular}




\begin{tabular}{|c|c|c|c|c|}
\hline $\begin{array}{l}\text { Drug } \\
\text { Comparison }\end{array}$ & $\begin{array}{l}\text { Study } \\
\text { (Trial) } \\
\text { Followup } \\
\text { Risk of Bias }\end{array}$ & $\begin{array}{l}\text { Harms } \\
\text { Drug class-specific harms }\end{array}$ & $\begin{array}{l}\text { Rare Harms } \\
A F F, \text { ONJ, Afib }\end{array}$ & $\begin{array}{l}\text { Predictors } \\
\text { (Patient, Bone, Drug) }\end{array}$ \\
\hline $\begin{array}{l}\text { Alendronate vs active } \\
\text { comparator }\end{array}$ & $\begin{array}{l}\text { Chiu } 2014 \\
4 \mathrm{yr} \\
\text { Medium }\end{array}$ & NR & $\begin{array}{l}\text { ONJ: defined by } 3 \text { criteria } \\
\text { [Principal hospital diagnosis of } \\
\text { inflammatory dental condition } \\
\text { (526.3X, } 526.4 X, 730.1 X \\
733.4 X ; \text { Claim for } \geq 1 \text { dentistry } \\
\text { visit at hospital; Review } x \text {-ray, } \\
\text { chart, surgical and pathology } \\
\text { reports for ONJ confirmation] } \\
\text { Crude incidence ONJ: } 40 / 7332 \\
\text { ( } 0.05 \%) \\
\text { Incidence per } 100,000 \text { persons } \\
\text { per year: } \\
\text { Alendronate: } 318 / 100,000 \\
\text { Raloxifene: } 35 / 100,000 \\
\text { Attributable risk of ONJ } \\
\text { associated with alendronate: } \\
283 / 100,000 \text { person years } \\
\text { aHR } 7.42 \text { ( } 95 \% \text { Cl } 1.02,54.09 \text { ) } \\
\text { p=0.48 }\end{array}$ & 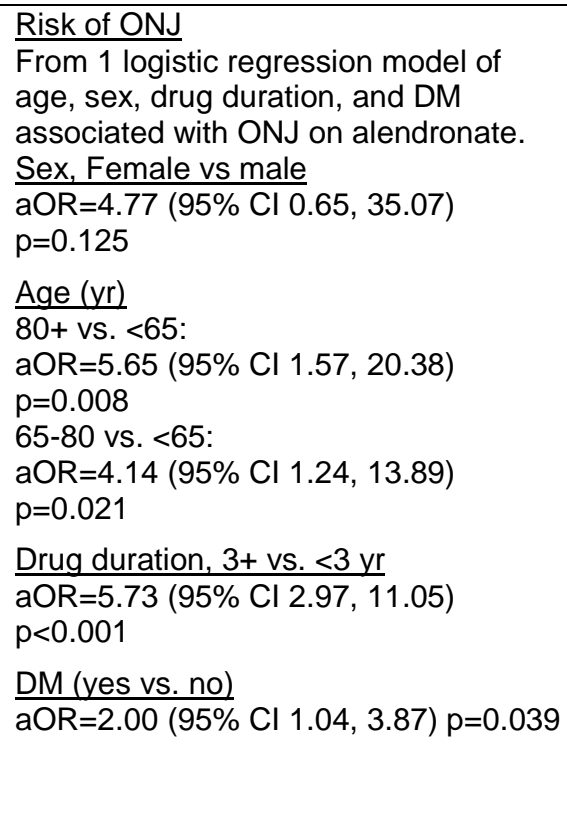 \\
\hline
\end{tabular}




\begin{tabular}{|c|c|c|c|c|}
\hline $\begin{array}{l}\text { Drug } \\
\text { Comparison }\end{array}$ & $\begin{array}{l}\text { Study } \\
\text { (Trial) } \\
\text { Followup } \\
\text { Risk of Bias }\end{array}$ & $\begin{array}{l}\text { Harms } \\
\text { Drug class-specific harms }\end{array}$ & $\begin{array}{l}\text { Rare Harms } \\
\text { AFF, ONJ, Afib }\end{array}$ & $\begin{array}{l}\text { Predictors } \\
\text { (Patient, Bone, Drug) }\end{array}$ \\
\hline $\begin{array}{l}\text { Alendronate vs } \\
\text { raloxifene/calcitonin }\end{array}$ & $\begin{array}{l}\text { Lin } 2014 \\
6 \text { yr } \\
\text { Medium }\end{array}$ & NR & $\begin{array}{l}\text { ONJ: diagnostic codes (ICD-9: } \\
73008,73000,73340,73349, \\
73018,73010,73020,73345, \\
73399,52689,7339,5264, \\
5289,5259, \text { and 5269). } \\
\text { Confirmed the diagnosis } \\
\text { persisted for } 8 \text { weeks or longer } \\
\text { while allowing a 30-day gap } \\
\text { between records. } \\
\text { Incidence } \\
\text { ALN: } 24 / 16003(0.15 \%) \\
\text { Raloxifene or calcitonin: } \\
\text { 13/16003 (0.08\%) } \\
\text { Incidence per } 10,000 \text { person-yr } \\
\text { ALN: } 8.2 \\
\text { Raloxifene or calcitonin: } 6.9 \\
\text { aHR=0.86 (95\% Cl } 0.44,1.69) \\
\text { p=0.659 }\end{array}$ & NR \\
\hline $\begin{array}{l}\text { Alendronate vs no } \\
\text { treatment }\end{array}$ & $\begin{array}{l}\text { Abrahamsen } \\
2009 \\
0-9 \mathrm{yr}\end{array}$ & NR & 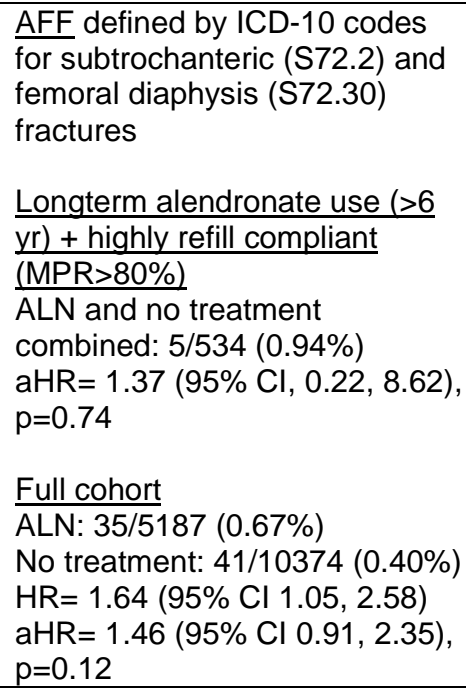 & NR \\
\hline
\end{tabular}




\begin{tabular}{|c|c|c|c|c|}
\hline $\begin{array}{l}\text { Drug } \\
\text { Comparison }\end{array}$ & $\begin{array}{l}\text { Study } \\
\text { (Trial) } \\
\text { Followup } \\
\text { Risk of Bias }\end{array}$ & $\begin{array}{l}\text { Harms } \\
\text { Drug class-specific harms }\end{array}$ & $\begin{array}{l}\text { Rare Harms } \\
A F F, \text { ONJ, Afib }\end{array}$ & $\begin{array}{l}\text { Predictors } \\
\text { (Patient, Bone, Drug) }\end{array}$ \\
\hline \multirow[t]{2}{*}{$\begin{array}{l}\text { Zoledronic acid vs } \\
\text { placebo }\end{array}$} & $\begin{array}{l}\text { Black 2012 } \\
\text { (HORIZON } \\
\text { extension) } \\
6 \text { yr } \\
\text { Low }\end{array}$ & $\begin{array}{l}\text { Mortality } \\
\text { Zoledronic acid: } 26 / 613(4.2 \%) \\
\text { Placebo: } 18 / 616(2.9 \%) \\
p=0.22 \\
\text { Myocardial infarction, any } \\
\text { Zoledronic acid: } 6 / 613(1.0 \%) \\
\text { Placebo: } 4 / 616(0.6 \%) \\
\text { Myocardial infarction, severe } \\
\text { Zoledronic acid: } 5 / 13(0.8 \%) \\
\text { Placebo: } 4 / 616(0.6 \%)\end{array}$ & 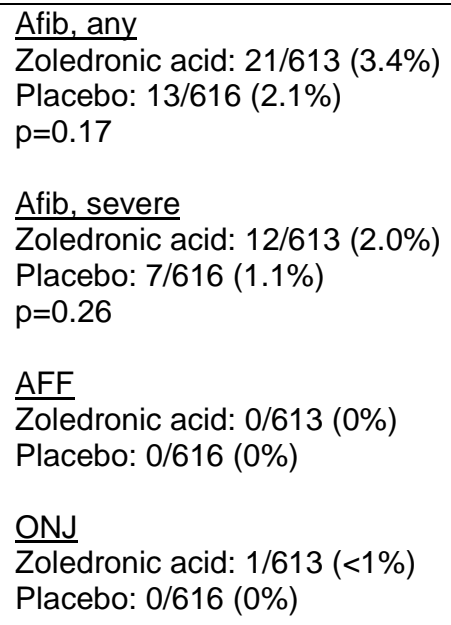 & \\
\hline & $\begin{array}{l}\text { Black 2015 } \\
\text { (HORIZON } \\
\text { extension) } \\
9 \text { yr } \\
\text { Low }\end{array}$ & $\begin{array}{l}\text { Mortality } \\
\text { Zoledronic acid: } 1 / 92(1.1 \%) \\
\text { Placebo: } 5 / 95 \text { (5.3\%) } \\
p=0.21\end{array}$ & $\begin{array}{l}\text { Afib, any } \\
\text { Zoledronic acid: } 5 / 92 \text { (5.4\%) } \\
\text { Placebo: } 1 / 95(1.1 \%) \\
\text { p=0.11 } \\
\text { Afib, severe } \\
\text { Zoledronic acid: } 1 / 92 \text { (1.1\%) } \\
\text { Placebo: } 1 / 95(1.1 \%) \\
\text { p=1.0 } \\
\text { AFF } \\
\text { Zoledronic acid: 0/92 (0\%) } \\
\text { Placebo: 0/95 (0\%) } \\
\text { ONJ } \\
\text { Zoledronic acid: } 0 / 92(0 \%) \\
\text { Placebo: 0/95 (0\%) }\end{array}$ & NR \\
\hline
\end{tabular}




\begin{tabular}{|c|c|c|c|c|}
\hline & $\begin{array}{l}\text { Reid, } 2018 \\
6 \text { yr } \\
\text { Low }\end{array}$ & 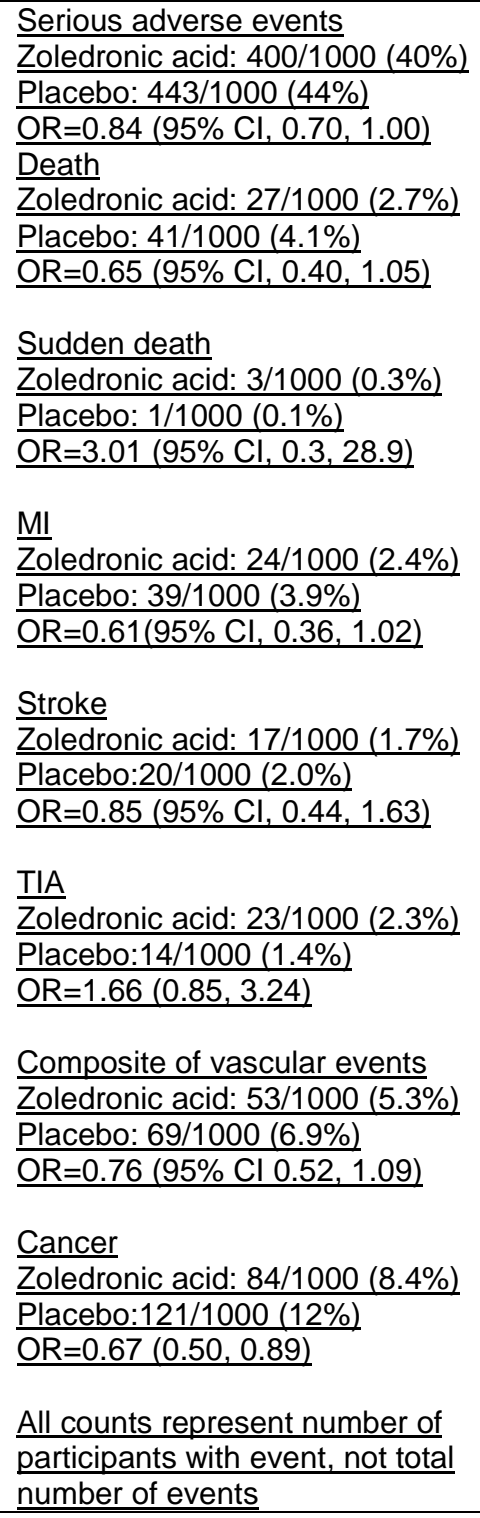 & 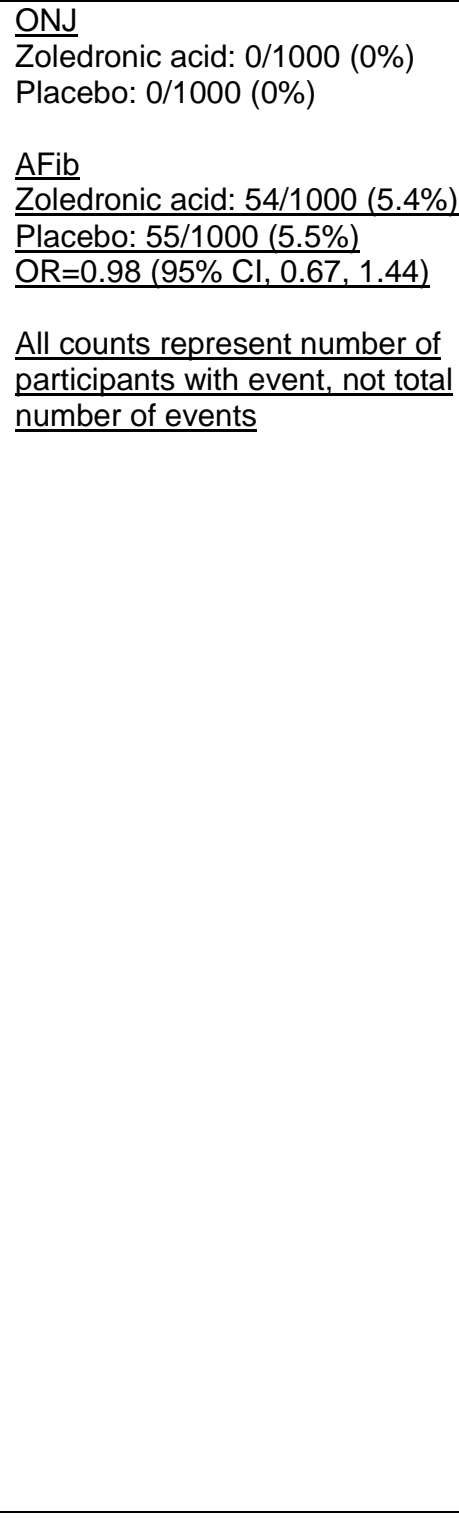 & $\mathrm{NR}$ \\
\hline $\begin{array}{l}\text { Bisphosphonates } \\
\text { vs active comparator }\end{array}$ & $\begin{array}{l}\text { Erviti } 2013 \\
>3 \mathrm{yr} \\
\text { Medium }\end{array}$ & $\overline{N R}$ & $\begin{array}{l}\text { AFF defined by ICD-10 codes } \\
\text { for subtrochanteric or shaft } \\
\text { (S722, S723) fracture and }\end{array}$ & NR \\
\hline
\end{tabular}




\begin{tabular}{|c|c|c|}
\hline . & 列 & $\begin{array}{l}\text { reviewed by team blinded to } \\
\text { exposure status } \\
\text { Any bisphosphonate } \\
\text { Time since first bisphosphonate } \\
\text { prescription, }>3 \mathrm{yr} \\
\text { Case: } 6 / 44(14 \%) \\
\text { Control: } 8 / 220(4 \%) \\
\text { Duration of use, >3 yr } \\
\text { Case: } 5 / 44(11 \%) \\
\text { Control: } 3 / 220(1 \%) \\
\text { Time since first bisphosphonate } \\
\text { prescription } \\
\text { Model } 1 \\
\text { aOR=4.71 (95\% Cl 1.52, 14.6) } \\
\text { Model } 2 \\
\text { aOR=9.46 (95\% Cl 2.17, 41.3) } \\
\text { Duration } \\
\text { Model } 1 \\
\text { aOR=9.18 (95\% Cl 2.12, 38.9) } \\
\text { Model } 2 \\
\text { aOR=31.9 (95\% Cl 4.05, 251) } \\
\text { Model 1: Adjusted for matching } \\
\text { variables } \\
\text { Model 2: Adjusted for matching } \\
\text { variables, smoking, alcoholism, } \\
\text { BMl, previous fracture, kidney } \\
\text { disease, malabsorption, stroke, } \\
\text { dementia, rheumatoid arthritis, } \\
\text { diabetes, epilepsy, Parkinson } \\
\text { disease, thyroid disease, PPI, } \\
\text { anxiolytics, sedatives, } \\
\text { antidepressants, } \\
\text { antihypertensives, } \\
\text { corticosteroids, raloxifene, } \\
\text { hormone therapy and } \\
\text { thiazolidinediones. } \\
\text { p=0.0007 for trend over duration } \\
\text { of use }\end{array}$ \\
\hline
\end{tabular}




\begin{tabular}{|c|c|c|c|c|}
\hline $\begin{array}{l}\text { Drug } \\
\text { Comparison }\end{array}$ & $\begin{array}{l}\text { Study } \\
\text { (Trial) } \\
\text { Followup } \\
\text { Risk of Bias }\end{array}$ & $\begin{array}{l}\text { Harms } \\
\text { Drug class-specific harms }\end{array}$ & $\begin{array}{l}\text { Rare Harms } \\
\text { AFF, ONJ, Afib }\end{array}$ & $\begin{array}{l}\text { Predictors } \\
\text { (Patient, Bone, Drug) }\end{array}$ \\
\hline & $\begin{array}{l}\text { Koh } 2017 \\
5.7 \mathrm{yr} \\
\text { Medium }\end{array}$ & NR & $\begin{array}{l}\text { AFF defined by subtrochanteric } \\
\text { or diaphyseal femoral fracture } \\
\text { (ICD-10 S722, S723, S724, or } \\
\text { S728), plus x-ray confirmation } \\
\text { per 2014 ASBMR AFF criteria } \\
\text { Bisphosphonate users from } \\
\text { which cases \& controls were } \\
\text { selected: } 43 / 35104 \text { (0.12\%) } \\
\text { Mean bisphosphonate use: } \\
\text { Cases: } 7.3 \mathrm{yr} \\
\text { Controls: } 5.2 \mathrm{yr} \\
\text { Cox Model 1: factors associated } \\
\text { with AFF } \\
\text { Bisphosphonate without drug } \\
\text { holiday } \\
\text { aHR=5.17 (95\% Cl 2.0, 13.36) } \\
\text { Glucocorticoid } \geq 1 \mathrm{yr} \\
\text { aHR=3.04 ( } 95 \% \mathrm{Cl} 1.40,6.57 \text { ) } \\
\text { BMI } \\
\text { aHR=1.24 (95\% Cl 1.1, 1.39) }\end{array}$ & $\begin{array}{l}\text { Risk of AFF } \\
\text { Bisphosphonate without drug holiday } \\
\text { aHR=5.17 }(95 \% \mathrm{Cl} 2.0,13.36) \\
\text { Glucocorticoid } \geq 1 \mathrm{yr} \\
\text { aHR=3.04 }(95 \% \mathrm{Cl} 1.40,6.57) \\
\text { BMl } \\
\text { aHR=1.24 }(95 \% \mathrm{Cl} 1.1,1.39)\end{array}$ \\
\hline & $\begin{array}{l}\text { Lim } 2018 \\
5.2 \text { yr (mean) } \\
\text { Medium }\end{array}$ & NR & $\begin{array}{l}\text { AFF defined by subtrochanteric } \\
\text { or diaphyseal femoral fracture } \\
\text { (ICD-10 S72.0-S72.9) plus x-ray } \\
\text { confirmation per } 2013 \text { ASBMR } \\
\text { AFF criteria } \\
\text { Bisphosphonate use } \\
\text { In AFF cases: } 140 / 196 \\
\text { In TFF cases: } 10 / 94 \\
\text { aOR=25.65 ( } 95 \% \mathrm{Cl} 10.74, \\
61.28)\end{array}$ & \\
\hline
\end{tabular}




\begin{tabular}{|c|c|c|c|c|}
\hline $\begin{array}{l}\text { Drug } \\
\text { Comparison }\end{array}$ & $\begin{array}{l}\text { Study } \\
\text { (Trial) } \\
\text { Followup } \\
\text { Risk of Bias }\end{array}$ & $\begin{array}{l}\text { Harms } \\
\text { Drug class-specific harms }\end{array}$ & $\begin{array}{l}\text { Rare Harms } \\
\text { AFF, ONJ, Afib }\end{array}$ & $\begin{array}{l}\text { Predictors } \\
\text { (Patient, Bone, Drug) }\end{array}$ \\
\hline & $\begin{array}{l}\text { Park-Wyllie } 2011 \\
4.0 \mathrm{yr} \\
\text { Medium }\end{array}$ & NR & $\begin{array}{l}\text { AFF, subtrochanteric or femoral } \\
\text { shaft fracture in bisphosphonate } \\
\text { users } \\
\text { Overall incidence, } \\
\text { bisphosphonate users }>7 \mathrm{yr}: \\
716 / 205466(0.35 \%) \\
\text { Absolute risk, bisphosphonate } \\
\text { use }>5 \text { yr: } \\
6 \text { th yr: } 71 / 52595(0.13 \%) \\
7 \text { th yr: } 117 / 52595(0.22 \%) \\
\text { No x-ray confirmation. Only first } \\
\text { fractures counted. } \\
\text { Reference=bisphosphonate use } \\
<100 \text { days. In women on } \\
\text { bisphosphonates }>5 y r, 64 \% \text { of } \\
\text { subtrochanteric/femoral shaft } \\
\text { fractures were attributable to } \\
\text { bisphosphonate use. }\end{array}$ & $\begin{array}{l}\text { Risk of subtrochanteric or femoral shaft } \\
\text { fracture, by drug duration. } \\
\text { 3-5 yr bisphosphonate use } \\
\text { Cases: } 204 / 716(28.5 \%) \\
\text { Controls: } 1070 / 3580(29.9 \%) \\
\text { aOR=1.59 }(95 \% \mathrm{Cl} 0.80,3.15) \\
\\
\text { 5+ yr bisphosphonate use } \\
\text { Cases: } 121 / 716(16.9 \%) \\
\text { Controls: } 460 / 3580(12.9 \%) \\
\text { aOR=2.74 }(95 \% \mathrm{Cl} 1.25,6.02) \\
\text { Adjusted for socioeconomic status, } \\
\text { comedications, drug count, } \\
\text { comorbidities, recent medical visits, } \\
\text { prior fall, prior osteoporotic fracture, } \\
\text { BMD test past } 5 \text { yr. }\end{array}$ \\
\hline
\end{tabular}




\begin{tabular}{|c|c|c|c|c|}
\hline $\begin{array}{l}\text { Drug } \\
\text { Comparison }\end{array}$ & $\begin{array}{l}\text { Study } \\
\text { (Trial) } \\
\text { Followup } \\
\text { Risk of Bias }\end{array}$ & $\begin{array}{l}\text { Harms } \\
\text { Drug class-specific harms }\end{array}$ & $\begin{array}{l}\text { Rare Harms } \\
A F F, \text { ONJ, Afib }\end{array}$ & $\begin{array}{l}\text { Predictors } \\
\text { (Patient, Bone, Drug) }\end{array}$ \\
\hline & $\begin{array}{l}\text { Schilcher } 2014 \\
4-5 \mathrm{yr} \\
\text { Medium }\end{array}$ & $\mathrm{NR}$ & $\begin{array}{l}\text { Cohort study } \\
\text { age-adjusted RR by duration of } \\
\text { use vs. no use } \\
\geq 4 \text { yr } \\
\text { RR=126 (95\% CI 55, 288) } \\
\text { Absolute risk=11 (95\% Cl 7, 14) } \\
\text { fractures per 10,000 patient-yr } \\
\text { Case/control study } \\
172 \text { cases with AFF vs. } 952 \\
\text { controls with ordinary femoral } \\
\text { shaft Fx } \\
\text { AFF defined by ICPC-2 codes } \\
\text { for subtrochanteric or diaphyseal } \\
\text { fracture and manually reviewed } \\
\text { by team blinded to exposure } \\
\text { status } \\
\text { Any bisphosphonate } \\
4-5 \text { yr } \\
\text { Case: } 13 / 172 \text { (8\%) } \\
\text { Control (ordinary shaft fx): } 3 / 952 \\
(0.3 \%) \\
\text { aOR=116 (95\%Cl 58, 234) } \\
5+\text { yr } \\
\text { Case: } 11 / 172(6 \%) \\
\text { Control (ordinary shaft fx): 5/952 } \\
\text { (0.5\%) } \\
\text { aOR=93 (95\% Cl 66, 132) } \\
\text { *Adjusted by age, sex, cortisone } \\
\text { use, and Charlson's comorbidity } \\
\text { Index }\end{array}$ & $\begin{array}{l}\text { Risk of AFF } \\
\text { Age } \leq 80 \mathrm{yr} \\
\mathrm{RR}=100(95 \% \mathrm{Cl} 40,253) \\
\text { Absolute risk }=10(95 \% \mathrm{Cl} 5,15) \\
\text { fractures per } 10,000 \text { patient-yr } \\
\text { Age }>80 \mathrm{yr} \\
\mathrm{RR}=163(95 \% \mathrm{Cl} 39,687) \\
\text { Absolute risk }=11(95 \% \mathrm{Cl} 7,16) \\
\text { fractures per } 10,000 \text { patient-yr }\end{array}$ \\
\hline
\end{tabular}




\begin{tabular}{|c|c|c|c|c|}
\hline $\begin{array}{l}\text { Drug } \\
\text { Comparison }\end{array}$ & $\begin{array}{l}\text { Study } \\
\text { (Trial) } \\
\text { Followup } \\
\text { Risk of Bias }\end{array}$ & $\begin{array}{l}\text { Harms } \\
\text { Drug class-specific harms }\end{array}$ & $\begin{array}{l}\text { Rare Harms } \\
A F F, \text { ONJ, Afib }\end{array}$ & $\begin{array}{l}\text { Predictors } \\
\text { (Patient, Bone, Drug) }\end{array}$ \\
\hline $\begin{array}{l}\text { Biphosphonates vs } \\
\text { raloxifene/calcitonin }\end{array}$ & $\begin{array}{l}\text { Kim } 2011 \\
<9.5 \text { yr (mean } 2 \\
\text { yr) } \\
\text { Medium }\end{array}$ & NR & $\begin{array}{l}\text { Subtrochanteric or diaphyseal } \\
\text { femur fracture defined by ICD-9 } \\
\text { codes } 820.22 \text { and } 821.0 x: \\
\text { 3-5 yr treatment } \\
\text { Biphosphonates: } 15 / 2591(0.6 \%) \\
\text { Raloxifene/calcitonin: } 11 / 2309 \\
(0.5 \%) \\
\text { HR }=1.20 \text { ( } 95 \% \mathrm{Cl} 0.55,2.61) \\
\geq 5 \text { yr treatment } \\
\text { Biphosphonates: } 6 / 2371(0.3 \%) \\
\text { Raloxifene/calcitonin: } 2 / 1726 \\
(0.1 \%) \\
\text { HR=2.02 (95\% Cl 0.41, 10.00) } \\
\text { Cox proportional hazards } \\
\text { Propensity score matched on } \\
\text { demographics, health care } \\
\text { utilization, comorbidities, other } \\
\text { medications }\end{array}$ & NR \\
\hline $\begin{array}{l}\text { Denosumab vs } \\
\text { placebo }\end{array}$ & $\begin{array}{l}\text { Miller } 2008 \\
4 \mathrm{yr} \\
\text { Medium }\end{array}$ & 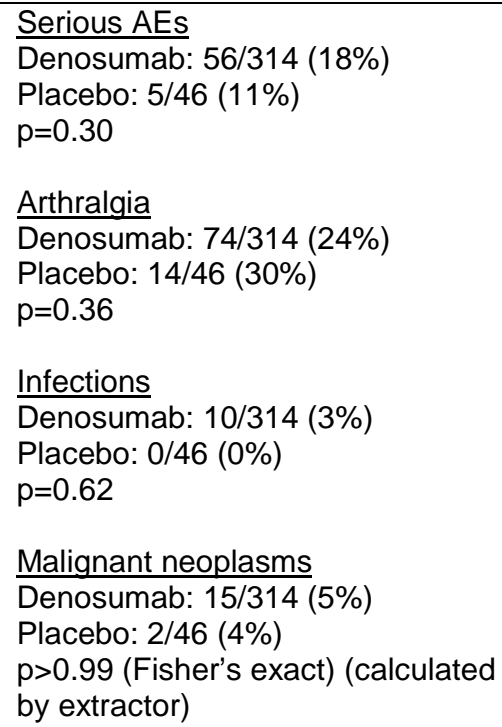 & $\mathrm{NR}$ & NR \\
\hline
\end{tabular}




\begin{tabular}{|c|c|c|c|c|}
\hline $\begin{array}{l}\text { Raloxifene vs } \\
\text { placebo }\end{array}$ & $\begin{array}{l}\text { Sontag } 2010 \\
\text { (Raloxifene } 60 \\
\text { mg and placebo } \\
\text { groups only) } \\
\text { Barrett-Connor } \\
2004 \text { Barrett- } \\
\text { Connor } 2009 \\
\text { (combined } \\
\text { raloxifene } 60 \text { mg } \\
\text { and raloxifene } \\
120 \text { mg groups) } \\
\text { (MORE) } \\
3.3 \text { yr (mean) } \\
\text { Low }\end{array}$ & $\begin{array}{l}\text { Stroke } \\
\text { Raloxifene } 60 \mathrm{mg}+120 \mathrm{mg}: \\
44 / 5129(0.25 \%) \\
\text { Placebo: } 32 / 2576(0.37 \%) \\
\text { HR=0.68 }(95 \% \mathrm{Cl} 0.43,1.07) \\
\text { PE } \\
\text { Raloxifene } 60 \mathrm{mg}+120 \mathrm{mg}: \\
\text { 16/5129 }(0.09 \%) \\
\text { Placebo: } 2 / 2576(0.02 \%) \\
\text { HR=3.97 }(95 \% \mathrm{Cl} 0.91,17.3) \\
\text { Mortality } \\
\text { Raloxifene } 60 \mathrm{mg}+120 \mathrm{mg}: \\
62 / 5129(0.35 \%) \\
\text { Placebo: } 36 / 2576(0.41 \%) \\
\text { HR=0.85 (95\% Cl 0.56, } 1.28) \\
\text { Cox proportional hazards } \\
\text { (Data from Barrett-Connor 2004. } \\
\text { Also reported in Barrett-Connor } \\
\text { 2009) }\end{array}$ & NR & $\begin{array}{l}\text { Stroke } \\
\text { No baseline vertebral fracture } \\
\text { Raloxifene: } 60 \mathrm{mg} 23 / 1574(1.5 \%) \\
\text { Placebo: } 27 / 1629(1.7 \%) \\
\text { With baseline vertebral fracture } \\
\text { Raloxifene } 60 \mathrm{mg}: 20 / 975(2.05 \%) \\
\text { Placebo: } 29 / 936(3.1 \%) \\
\text { p for interaction=0.47 } \\
\text { PE } \\
\text { No baseline vertebral fracture } \\
\text { Raloxifene } 60 \mathrm{mg}: 6 / 1574(2.05 \%) \\
\text { Placebo: } 3 / 1629(0.18 \%) \\
\text { With baseline vertebral fracture } \\
\text { Raloxifene } 60 \mathrm{mg}: 5 / 975(0.51 \%) \\
\text { Placebo: } 1 / 936(0.11 \%) \\
\text { p for interaction=0.52 } \\
\text { DVT } \\
\text { No baseline vertebral fracture } \\
\text { Raloxifene } 60 \mathrm{mg}: 12 / 1574(0.76 \%) \\
\text { Placebo: } 6 / 1629(0.37 \%) \\
\text { With baseline vertebral fracture } \\
\text { Raloxifene } 60 \mathrm{mg}: 8 / 975(0.82 \%) \\
\text { Placebo: } 2 / 936(0.21 \%) \\
\text { p for interaction=0.51 } \\
\text { Hot flashes } \\
\text { No baseline vertebral fracture } \\
\text { Raloxifene } 60 \mathrm{mg}: 158 / 1574(10.0 \%) \\
\text { Placebo: } 103 / 1629(6.3 \%) \\
\text { With baseline vertebral fracture } \\
\text { Raloxifene } 60 \mathrm{mg}: 79 / 975(8.1 \%) \\
\text { Placebo: } 47 / 936(5.02 \%) \\
\text { p for interaction=0.99 } \\
\text { Mortality } \\
\text { No baseline vertebral fracture } \\
\text { Raloxifene } 60 \mathrm{mg}: 10 / 1574(0.64 \%) \\
\text { Placebo: } 13 / 1629(0.80 \%)\end{array}$ \\
\hline
\end{tabular}




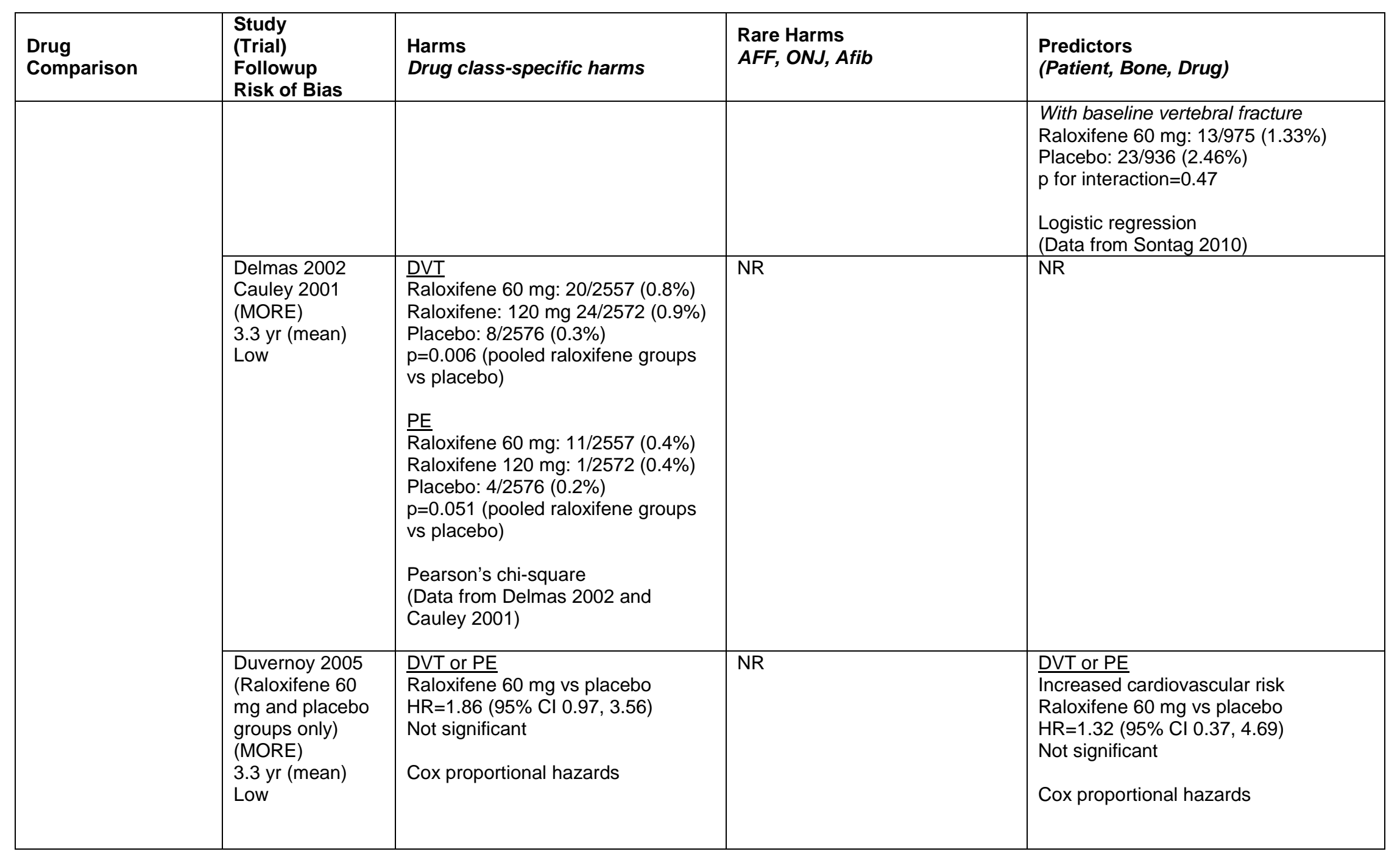




\begin{tabular}{|c|c|c|c|c|}
\hline $\begin{array}{l}\text { Drug } \\
\text { Comparison }\end{array}$ & $\begin{array}{l}\text { Study } \\
\text { (Trial) } \\
\text { Followup } \\
\text { Risk of Bias }\end{array}$ & $\begin{array}{l}\text { Harms } \\
\text { Drug class-specific harms }\end{array}$ & $\begin{array}{l}\text { Rare Harms } \\
A F F, \text { ONJ, Afib }\end{array}$ & $\begin{array}{l}\text { Predictors } \\
\text { (Patient, Bone, Drug) }\end{array}$ \\
\hline & $\begin{array}{l}\text { Grady } 2004 \\
\text { (MORE) } \\
3.3 \text { yr (mean) } \\
\text { Low }\end{array}$ & $\begin{array}{l}\text { DVT } \\
\text { Raloxifene } 60 \mathrm{mg}+120 \mathrm{mg}: \\
\text { 43/5129 }(0.8 \%) \\
\text { Placebo: } 7 / 2576(0.3 \%) \\
\mathrm{RR}=3.1(95 \% \mathrm{Cl} 1.4,6.9) \\
\mathrm{p}<0.01 \\
\text { PE } \\
\text { Raloxifene } 60 \mathrm{mg}+120 \mathrm{mg}: \\
\text { 18/5129 }(0.4 \%) \\
\text { Placebo: } 2 / 2576(0.08 \%) \\
\text { RR=4.5 }(95 \% \mathrm{Cl} 1.1,19.5) \\
\mathrm{p}=0.05 \\
\text { Logistic regression }\end{array}$ & $\mathrm{NR}$ & $\begin{array}{l}\text { DVT or PE } \\
\text { Drug dose } \\
\text { Raloxifene } 60 \mathrm{mg} \text { vs placebo: } \\
\text { RR }=1.9(95 \% \mathrm{Cl} 1.0,3.6) \\
\text { Raloxifene } 120 \mathrm{mg} \text { vs placebo: } \\
\text { RR=2.4 }(95 \% \mathrm{Cl} 1.3,4.4) \\
\mathrm{P} \text { for difference between } \mathrm{RRs}=0.4 \\
\text { Treatment duration } \\
\text { DVT } \\
\mathrm{p} \text { for time } x \text { treatment interaction }=0.01 \\
\mathrm{PE} \\
\mathrm{p} \text { for time } x \text { treatment interaction }=0.8\end{array}$ \\
\hline & $\begin{array}{l}\text { Barrett-Connor } \\
2002 \\
\text { (MORE) } \\
3.3 \text { yr (mean) } \\
\text { Low }\end{array}$ & $\begin{array}{l}\text { Stroke } \\
\text { Raloxifene } 60 \mathrm{mg}: 22 / 2557(1.2 \%) \\
\text { Raloxifene } 120 \mathrm{mg}: 26 / 2572(0.9 \%) \\
\text { Placebo: } 32 / 2576(1.0 \%) \\
\text { Raloxifene } 60 \mathrm{mg} \text { vs placebo } \\
\text { RR=0.69 ( } 95 \% \mathrm{Cl} 0.40,1.81) \\
\text { Raloxifene } 120 \mathrm{mg} \text { vs placebo } \\
\text { RR=0.81 }(95 \% \mathrm{Cl} 0.49,1.36)\end{array}$ & NR & $\begin{array}{l}\text { Stroke } \\
\text { Increased cardiovascular risk } \\
\text { Raloxifene } 60 \mathrm{mg}: 6 / 359(1.7 \%) \\
\text { Raloxifene } 120 \mathrm{mg}: 6 / 359(1.7 \%) \\
\text { Placebo: } 14 / 317(4.4 \%) \\
\text { Raloxifene } 60 \mathrm{mg} \text { vs placebo: } \mathrm{RR}=0.38 \\
(0.15,0.94) \\
\text { Raloxifene } 120 \mathrm{mg} \text { vs placebo: } \\
\text { RR=0.38 }(0.15,0.94) \\
\text { Prevalent } \mathrm{CHD} \\
\text { Raloxifene } 60 \mathrm{mg}: 1 / 56(1.7 \%) \\
\text { Raloxifene } 120 \mathrm{mg}: 2 / 80(2.5 \%) \\
\text { Placebo: } 6 / 66(9 \%) \\
\text { Raloxifene } 60 \mathrm{mg} \text { vs placebo: } \mathrm{RR}=0.20 \\
\text { (95\% Cl } 0.03,1.25) \\
\text { Raloxifene } 120 \mathrm{mg} \text { vs placebo: } \\
\text { RR=0.28 (95\% Cl } 0.06,1.18)\end{array}$ \\
\hline
\end{tabular}




\begin{tabular}{|c|c|c|c|c|}
\hline $\begin{array}{l}\text { Drug } \\
\text { Comparison }\end{array}$ & $\begin{array}{l}\text { Study } \\
\text { (Trial) } \\
\text { Followup } \\
\text { Risk of Bias }\end{array}$ & $\begin{array}{l}\text { Harms } \\
\text { Drug class-specific harms }\end{array}$ & $\begin{array}{l}\text { Rare Harms } \\
A F F, \text { ONJ, Afib }\end{array}$ & $\begin{array}{l}\text { Predictors } \\
\text { (Patient, Bone, Drug) }\end{array}$ \\
\hline & $\begin{array}{l}\text { Grady } 2010 \\
\text { (MORE/CORE) } \\
8 \mathrm{yr} \\
\text { Medium }\end{array}$ & $\begin{array}{l}\text { Mortality } \\
\text { Raloxifene: } 45 / 2557(1.8 \%) \\
\text { Placebo: } 65 / 2576(2.5 \%) \\
\text { HR=0.68 (95\% Cl 0.46, 0.99) } \\
p=0.04\end{array}$ & NR & NR \\
\hline & $\begin{array}{l}\text { Martino } 2004 \\
\text { Martino } 2005 \\
\text { Ensrud } 2006 \\
\text { Grady 2010 } \\
\text { (MORE/CORE) } \\
8 \text { yr } \\
\text { Medium }\end{array}$ & $\begin{array}{l}\text { Stroke } \\
\text { Raloxifene: } 49 / 2725(1.8 \%) \\
\text { Placebo: } 19 / 1286(1.5 \%) \\
\text { HR=1.22 (95\% Cl } 0.72,2.08) \\
p=0.45 \\
\text { PE } \\
\text { Raloxifene: } 17 / 2725(0.6 \%) \\
\text { Placebo: } 2 / 1286(0.2 \%) \\
p=0.048 \\
\text { DVT } \\
\text { Raloxifene: } 31 / 2725(1.1 \%) \\
\text { Placebo: } 10 / 1286(0.8 \%) \\
p=0.32 \\
\text { Hot flashes } \\
\text { Raloxifene: } 342 / 2725(12.6 \%) \\
\text { Placebo: } 89 / 1286(6.9 \%) \\
p<0.001 \\
\text { Mortality } \\
\text { Raloxifene: } 47 / 2725(1.7 \%) \\
\text { Placebo: } 29 / 1286(2.3 \%) \\
p=0.27\end{array}$ & NR & NR \\
\hline
\end{tabular}




\begin{tabular}{|c|c|c|c|c|}
\hline $\begin{array}{l}\text { Drug } \\
\text { Comparison }\end{array}$ & $\begin{array}{l}\text { Study } \\
\text { (Trial) } \\
\text { Followup } \\
\text { Risk of Bias }\end{array}$ & $\begin{array}{l}\text { Harms } \\
\text { Drug class-specific harms }\end{array}$ & $\begin{array}{l}\text { Rare Harms } \\
A F F, \text { ONJ, Afib }\end{array}$ & $\begin{array}{l}\text { Predictors } \\
\text { (Patient, Bone, Drug) }\end{array}$ \\
\hline $\begin{array}{l}\text { Hormone therapy vs } \\
\text { control }\end{array}$ & $\begin{array}{l}\text { Wimalawansa } \\
4 \mathrm{yr} \\
\text { Medium }\end{array}$ & $\begin{array}{l}\text { Withdrawals due to estrogen-related } \\
\text { AEs } \\
\text { HRT: } 3 / 18(17 \%) \\
\text { Control: } 0 / 18 \\
\text { Withdrawals due to inability to } \\
\text { tolerate medications } \\
\text { HRT: } 0 / 18 \\
\text { Control: } 1 / 18(6 \%) \\
\text { Withdrawals due to other medical } \\
\text { problems } \\
\text { HRT: } 0 / 18 \\
\text { Control: } 2 / 18(11 \%)\end{array}$ & NR & NR \\
\hline $\begin{array}{l}\text { Hormone therapy } \\
\text { (estrogen }+ \\
\text { progestin) vs placebo }\end{array}$ & $\begin{array}{l}\text { Cauley } 2003 \\
6 \text { yr } \\
\text { Low }\end{array}$ & $\begin{array}{l}\text { Global index } \\
15 \% \text { increase in hormone therapy } \\
\text { group vs placebo group indicating } \\
\text { more harm than benefit } \\
\text { Note this outcome is for the total } \\
\text { study population, which includes } \\
\text { women who are neither osteoporotic } \\
\text { nor osteopenic }\end{array}$ & NR & $\begin{array}{l}\text { Predictor: Fracture risk } \\
\text { Outcome: Global index } \\
\text { Lowest tertile fracture risk: } \\
\text { HR } 1.20(95 \% \mathrm{Cl} 0.93,1.58) \\
\text { Medium tertile fracture risk: } \\
\text { HR } 1.23(95 \% \mathrm{Cl} 1.04,1.46) \\
\text { Highest tertile fracture risk: } \\
\text { HR } 1.03(95 \% \mathrm{Cl} 0.88,1.24) \\
\text { p for interaction= } 0.54 \\
\\
\text { Note this outcome is for the total study } \\
\text { population, which includes women who } \\
\text { are neither osteoporotic nor osteopenic }\end{array}$ \\
\hline $\begin{array}{l}\text { Hormone therapy } \\
\text { (estrogen) vs placebo }\end{array}$ & $\begin{array}{l}\text { Jackson } 2006 \\
7 \text { yr } \\
\text { Low }\end{array}$ & NR & NR & $\begin{array}{l}\text { Predictor: Fracture risk } \\
\text { Outcome: Global index } \\
\text { Lowest tertile fracture risk: } \\
\text { HR } 0.81(95 \% \mathrm{Cl} 0.62,1.05) \\
\text { Medium tertile fracture risk: } \\
\text { HR } 1.09(95 \% \mathrm{Cl} 0.92,1.30) \\
\text { Highest tertile fracture risk: } \\
\text { HR } 1.04(95 \% \mathrm{Cl} 0.88,1.23) \\
\text { p for interaction= } 0.42 \\
\\
\text { Note this outcome is for the total study } \\
\text { population, which includes women who } \\
\text { are neither osteoporotic nor osteopenic }\end{array}$ \\
\hline
\end{tabular}




\begin{tabular}{|c|c|c|c|c|}
\hline $\begin{array}{l}\text { Drug } \\
\text { Comparison }\end{array}$ & $\begin{array}{l}\text { Study } \\
\text { (Trial) } \\
\text { Followup } \\
\text { Risk of Bias }\end{array}$ & $\begin{array}{l}\text { Harms } \\
\text { Drug class-specific harms }\end{array}$ & $\begin{array}{l}\text { Rare Harms } \\
A F F, \text { ONJ, Afib }\end{array}$ & $\begin{array}{l}\text { Predictors } \\
\text { (Patient, Bone, Drug) }\end{array}$ \\
\hline $\begin{array}{l}\text { Osteoporosis drugs } \\
\text { vs active comparator }\end{array}$ & $\begin{array}{l}\text { Vestergaard } \\
2012 \\
3.8 \mathrm{yr} \\
\text { Medium }\end{array}$ & NR & $\begin{array}{l}\text { Inflammatory jaw event (proxy } \\
\text { for ONJ) } \\
\text { Any osteoporosis drug: } \\
33 / 103,562(0.03 \%) \\
\text { No osteoporosis drug: } \\
37 / 310,683(0.01 \%) \\
\text { RR=2.68 }(95 \% \mathrm{Cl} 1.70,4.20) \\
\text { p<0.01 } \\
\text { Alendronate: } 15 / 55,090(0.03 \%) \\
\text { No osteoporosis drug: } \\
\text { 13/310,683 (0.004\%) } \\
\text { RR=3.46 (95\% Cl 1.72, 6.95) } \\
\text { p<0.01 } \\
\text { HR=3.15 (95\% Cl 1.44, 6.87) } \\
\text { Cox proportional hazards } \\
\text { Adjusted for DMs, Sjögren's } \\
\text { syndrome, chemotherapy, } \\
\text { irradiation, use of systemic } \\
\text { corticosteroids, alcoholism, and } \\
\text { jaw events before start of drug } \\
\text { Raloxifene: } 0 / 4,831(0 \%) \\
\text { No osteoporosis drug: } \\
\text { 2/310,683 (0.0006\%) } \\
\text { RR=0 (unadjusted) } \\
\text { p=0.41 }\end{array}$ & NR \\
\hline
\end{tabular}




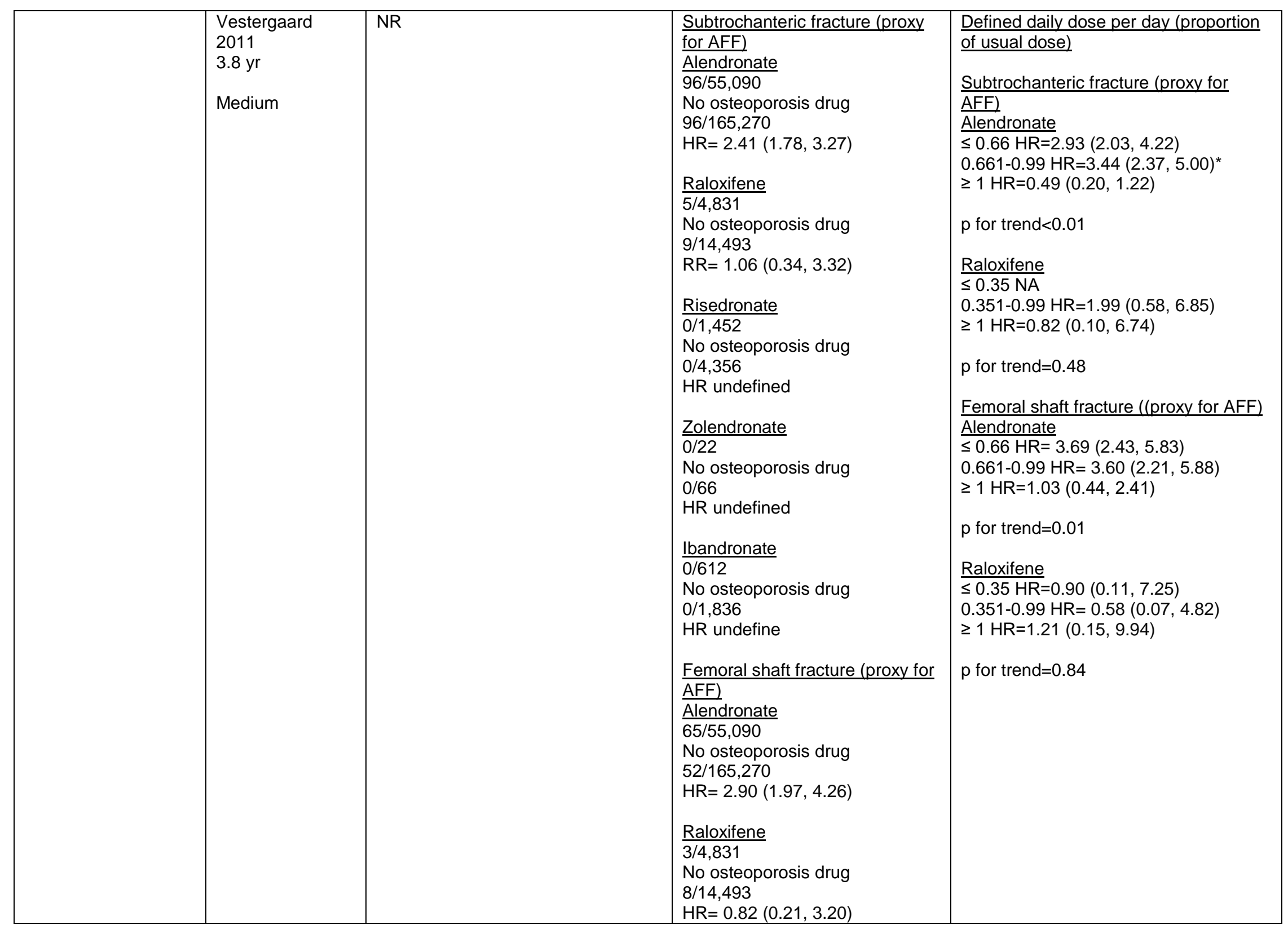




\begin{tabular}{|l|l|l|l|l|}
\hline $\begin{array}{l}\text { Drug } \\
\text { Comparison }\end{array}$ & $\begin{array}{l}\text { Study } \\
\text { (Trial) } \\
\text { Followup } \\
\text { Risk of Bias }\end{array}$ & $\begin{array}{l}\text { Harms } \\
\text { Drug class-specific harms }\end{array}$ & $\begin{array}{l}\text { Rare Harms } \\
\text { AFF, ONJ, Afib }\end{array}$ & $\begin{array}{l}\text { Predictors } \\
\text { (Patient, Bone, Drug) }\end{array}$ \\
\hline & & & $\begin{array}{l}\text { Risedronate } \\
0 / 1,452 \\
\text { No osteoporosis drug } \\
0 / 4,356 \\
\text { HR undefined }\end{array}$ & \\
& & & & \\
& & & \\
\hline
\end{tabular}




\begin{tabular}{|c|c|c|c|c|}
\hline $\begin{array}{l}\text { Drug } \\
\text { Comparison }\end{array}$ & $\begin{array}{l}\text { Study } \\
\text { (Trial) } \\
\text { Followup } \\
\text { Risk of Bias }\end{array}$ & $\begin{array}{l}\text { Harms } \\
\text { Drug class-specific harms }\end{array}$ & $\begin{array}{l}\text { Rare Harms } \\
A F F, \text { ONJ, Afib }\end{array}$ & $\begin{array}{l}\text { Predictors } \\
\text { (Patient, Bone, Drug) }\end{array}$ \\
\hline & $\begin{array}{l}\text { Vestergaard } \\
2010 \\
3.8 \mathrm{yr} \\
\text { Medium }\end{array}$ & NR & $\begin{array}{l}\text { AFib or atrial flutter } \\
\text { Alendronate: } \\
\text { 729/55,090 (1.3\%) } \\
\text { No osteoporosis drug: } \\
1,635 / 310,683(0.53 \%) \\
\text { HR=1.05 (95\% Cl 0.96, 1.15) } \\
\text { Raloxifene: } 55 / 4,831(1.1 \%) \\
\text { No osteoporosis drug: } \\
\text { 168/310,683 (0.05\%) } \\
\text { HR=0.82 (95\% Cl 0.60, 1.13) } \\
\text { Risedronate: 0/1,452 (0\%) } \\
\text { No osteoporosis drug: 0/310,683 } \\
\text { (0\%) } \\
\text { HR undefined } \\
\text { Zolendronate: 0/22 (0\%) } \\
\text { No osteoporosis drug: 0/310,683 } \\
\text { (0\%) } \\
\text { HR undefined } \\
\text { Ibandronate: } 0 / 612 \text { (0\%) } \\
\text { No osteoporosis drug: 0/310,683 } \\
\text { (0\%) } \\
\text { HR undefined } \\
\text { Teriparatide: 0/303 (0\%) } \\
\text { No osteoporosis drug: 0/310,683 } \\
\text { (0\%) } \\
\text { HR undefined } \\
\text { Cox proportional hazards } \\
\text { HRs adjusted for AFib before } \\
\text { starting drug, heart valve } \\
\text { disease, CHF, hyperthyroidism, } \\
\text { diuretics, cardiovaascular drugs, } \\
\text { COPD, durgs treating COPD, } \\
\text { alcoholism }\end{array}$ & NR \\
\hline
\end{tabular}

mass index; $\mathrm{CHF}=$ congestive heart failure; $\mathrm{CI}=$ confidence interval; $\mathrm{COPD}=$ chronic obstructive pulmonary disease; $\mathrm{CORE}=\mathrm{Continuing}$ Outcomes Relevant to Evista trial; 
DM=diabetes mellitus; DVT=deep vein thrombosis; FIT=Fracture Intervention Trial; GI=gastrointestinal; HORIZON=Health Outcomes and Reduced Incidence with Zoledronic Acid Once Yearly trial; HR=hazard ratio; mg=milligrams; MORE =Multiple Outcomes of Raloxifene Evaluation; N=number; NSAID=nonsteroidal anti-inflammatory drug; $\mathrm{NR}=$ not reported; $\mathrm{ONJ}=$ osteonecrosis of the jaw; $\mathrm{OR}=$ odds ratio; $\mathrm{PE}=$ =pulmonary embolism; $\mathrm{RCT}=$ randomized controlled trial; $\mathrm{RH}=$ relative hazard ; $\mathrm{RR}=$ risk ratio; $\mathrm{UGI}=$ upper gastrointestinal; yr=year 
Table D5. Key Questions 5 and 6 evidence overview

\begin{tabular}{|c|c|c|c|c|}
\hline $\begin{array}{l}\text { Drug } \\
\text { Comparison }\end{array}$ & $\begin{array}{l}\text { Study } \\
\text { (Trial) } \\
\text { Followup } \\
\text { Risk of Bias }\end{array}$ & $\begin{array}{l}\text { Final Outcomes } \\
\text { Incident Clinical Fracture }\end{array}$ & $\begin{array}{l}\text { Intermediate Outcomes } \\
\text { Incident Radiographic Vertebral } \\
\text { Fracture } \\
\text { DXA BMD Change }\end{array}$ & $\begin{array}{l}\text { Predictors } \\
\text { (Patient, Bone, Drug) }\end{array}$ \\
\hline $\begin{array}{l}\text { Alendronate vs } \\
\text { Discontinue } \\
\text { Alendronate } \\
\text { (Placebo) }\end{array}$ & $\begin{array}{l}\text { Black } 2006 \\
\text { (FLEX: RCT } \\
\text { extension with } \\
\text { rerandomization } \\
\text { and } \\
\text { discontinuation) } \\
5-10 \text { yr } \\
\text { Low }\end{array}$ & 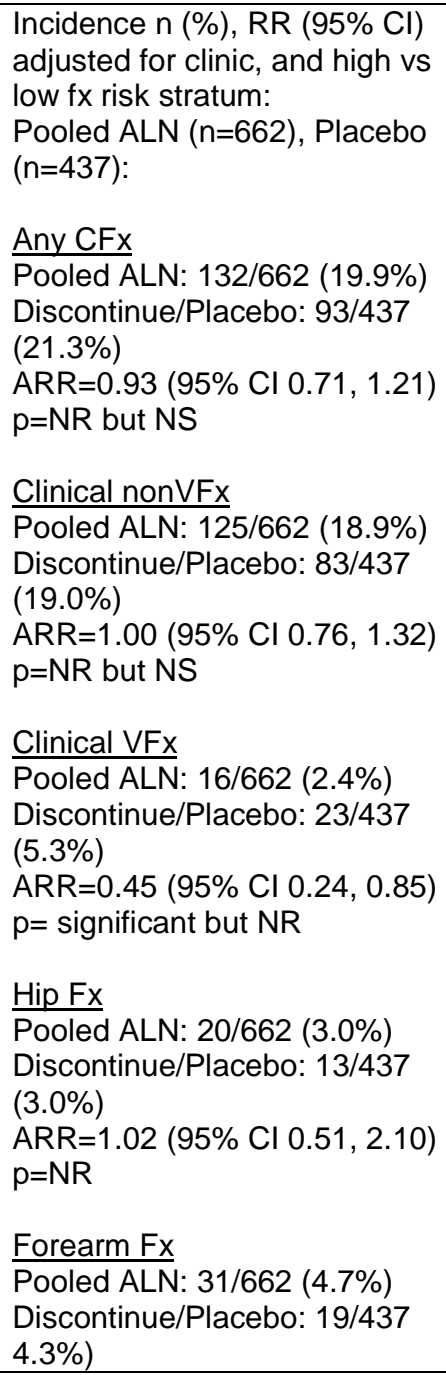 & 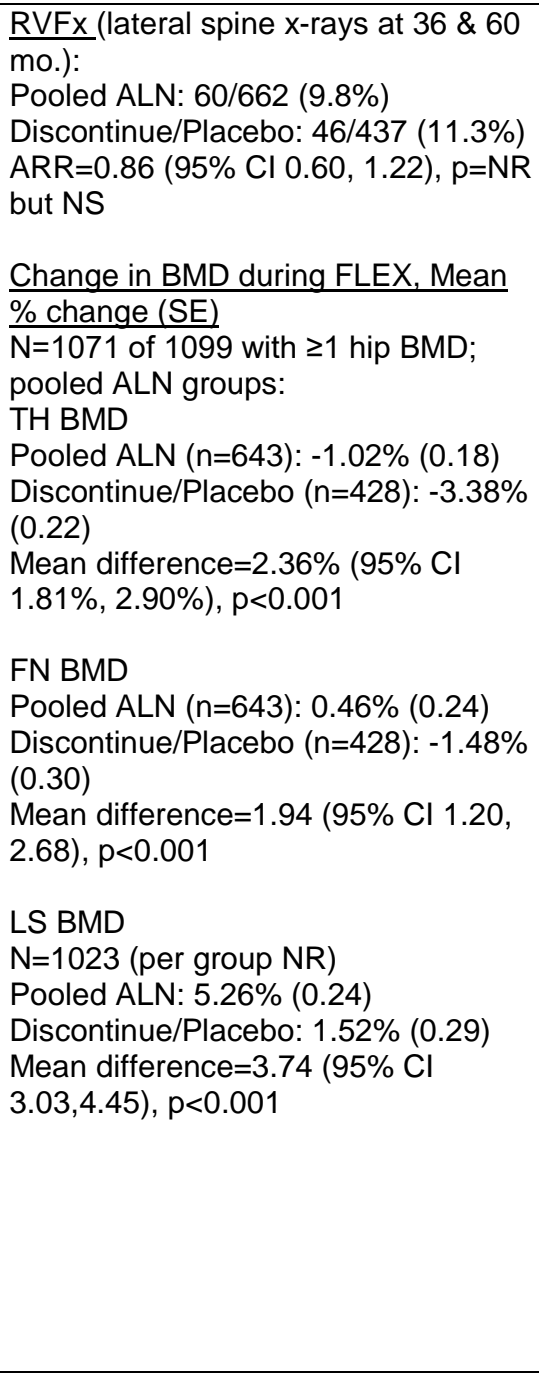 & 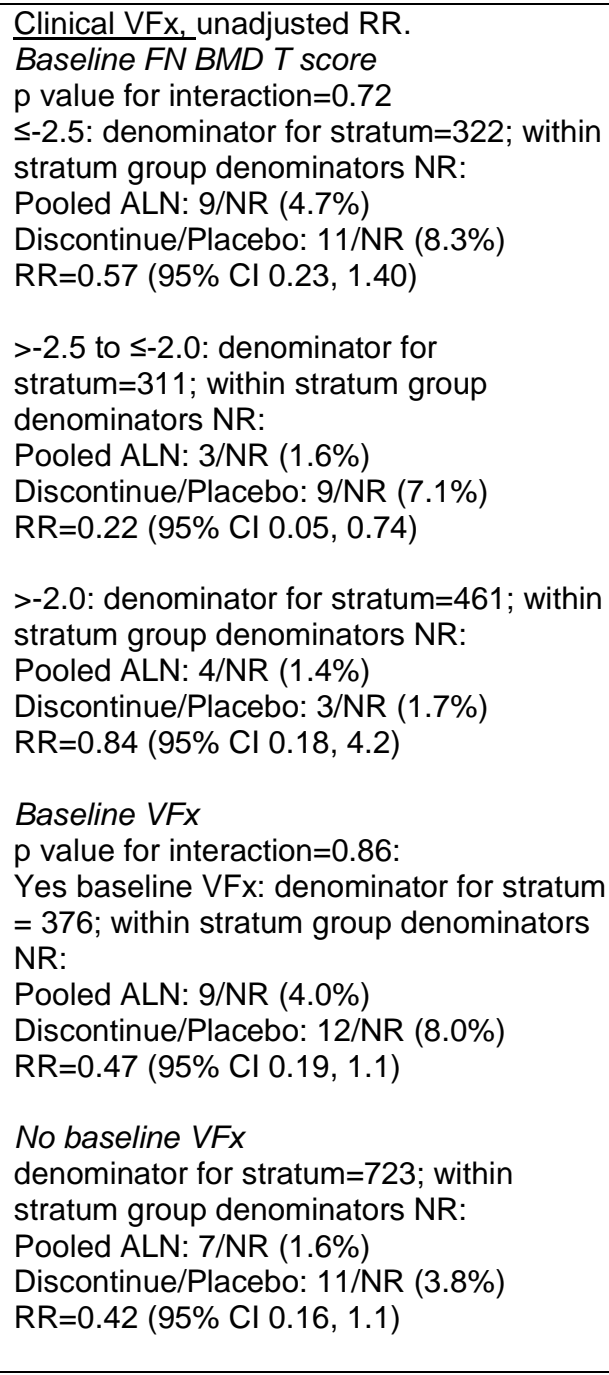 \\
\hline
\end{tabular}




\begin{tabular}{|c|c|c|c|c|}
\hline $\begin{array}{l}\text { Drug } \\
\text { Comparison }\end{array}$ & $\begin{array}{l}\text { Study } \\
\text { (Trial) } \\
\text { Followup } \\
\text { Risk of Bias }\end{array}$ & $\begin{array}{l}\text { Final Outcomes } \\
\text { Incident Clinical Fracture }\end{array}$ & $\begin{array}{l}\text { Intermediate Outcomes } \\
\text { Incident Radiographic Vertebral } \\
\text { Fracture } \\
\text { DXA BMD Change }\end{array}$ & $\begin{array}{l}\text { Predictors } \\
\text { (Patient, Bone, Drug) }\end{array}$ \\
\hline & & $\begin{array}{l}\text { ARR=1.09 }(95 \% \mathrm{Cl} 0.62,1.96) \\
p=N R\end{array}$ & & 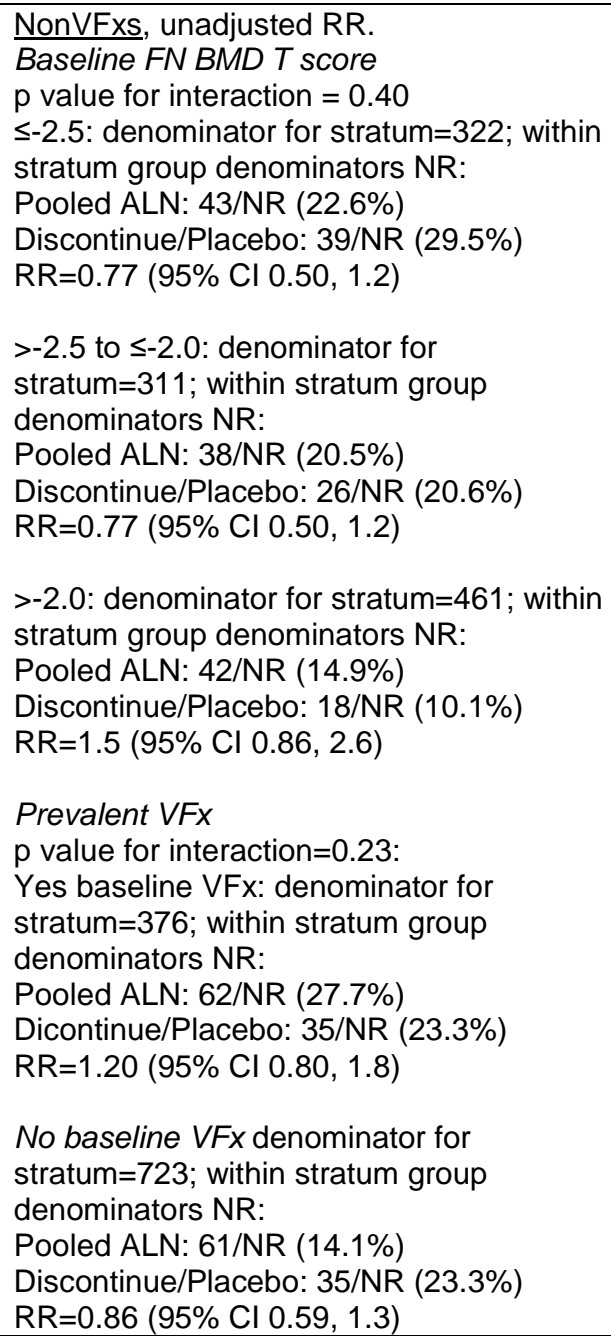 \\
\hline & $\begin{array}{l}\text { Schwartz } 2010 \\
\text { Post hoc FLEX } \\
\text { analysis) }\end{array}$ & NR & NR & $\begin{array}{l}\text { Risk of first nonVFx in women without } \\
\text { baseline VFx }\left(n=720 \text { of } 1094^{\star}\right) \text {, by FLEX } \\
\text { baseline T score. } P \text { value for interaction= }\end{array}$ \\
\hline
\end{tabular}




\begin{tabular}{|c|c|c|c|c|}
\hline $\begin{array}{l}\text { Drug } \\
\text { Comparison }\end{array}$ & $\begin{array}{l}\text { Study } \\
\text { (Trial) } \\
\text { Followup } \\
\text { Risk of Bias }\end{array}$ & $\begin{array}{l}\text { Final Outcomes } \\
\text { Incident Clinical Fracture }\end{array}$ & $\begin{array}{l}\text { Intermediate Outcomes } \\
\text { Incident Radiographic Vertebral } \\
\text { Fracture } \\
\text { DXA BMD Change }\end{array}$ & $\begin{array}{l}\text { Predictors } \\
\text { (Patient, Bone, Drug) }\end{array}$ \\
\hline & $\begin{array}{l}5-10 \mathrm{yr} \\
\text { Medium }\end{array}$ & & & 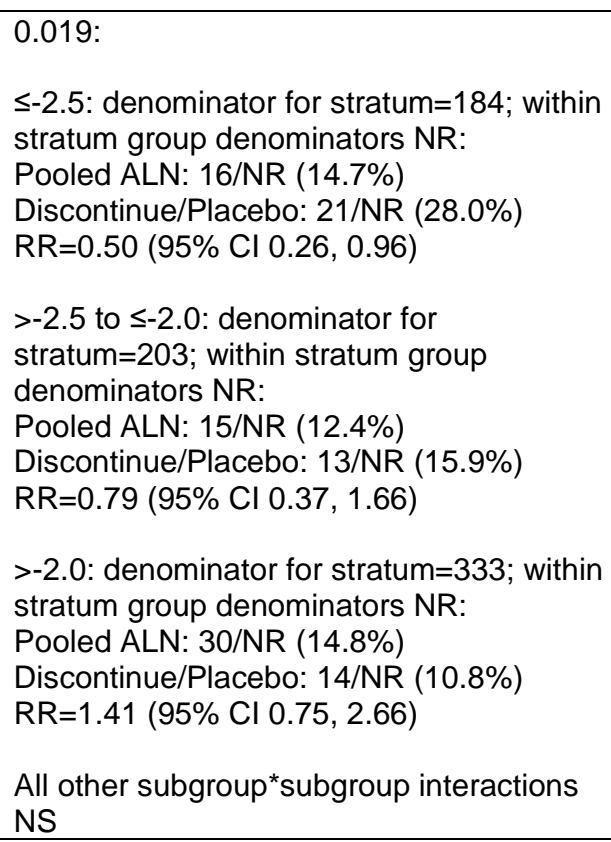 \\
\hline & $\begin{array}{l}\text { Bone } 2004 \\
\text { Prospective } \\
\text { CCT 8-10 yr } \\
\text { with } \\
\text { discontinuation } \\
\text { Medium }\end{array}$ & $\begin{array}{l}\text { CFx: collected, NR } \\
\text { First NonVFx } \\
\text { 8-10 yr. N=247 } \\
\text { ALN } 10 \mathrm{mg}: 8.1 \% \\
\text { ALN } 5 \mathrm{mg}: 11.5 \% \\
\text { ALN 20/ALN } 5 / \text { placebo: } 12.0 \% \\
\text { p=NR but NS }\end{array}$ & $\begin{array}{l}\frac{R V F x}{6-10 \text { yr }(\mathrm{n}=228)} \\
\text { ALN } 10 \mathrm{mg}: 5.0 \% \\
\text { ALN } 5 \mathrm{mg}: 13.9 \% \\
\text { ALN 20/ALN 5/placebo: } 6.6 \% \\
\text { p=NR but NS } \\
\text { Mean \% change in BMD } \\
\text { 8-10 yr. Reports denominator range } \\
\text { for } 3 \text { groups together, each } \\
\text { comparison: } \\
\text { LS BMD } \\
\text { n=71-81/group } \\
\text { ALN } 10 \mathrm{mg}: 2.3 \%(95 \% \mathrm{Cl} 1.4,3.1) \\
\text { p<0.001 } \\
\text { ALN } 5 \mathrm{mg}: 1.2 \%(95 \% \mathrm{Cl} 0.2,2.1)\end{array}$ & $\mathrm{NR}$ \\
\hline
\end{tabular}




\begin{tabular}{|c|c|c|c|c|}
\hline $\begin{array}{l}\text { Drug } \\
\text { Comparison }\end{array}$ & $\begin{array}{l}\text { Study } \\
\text { (Trial) } \\
\text { Followup } \\
\text { Risk of Bias }\end{array}$ & $\begin{array}{l}\text { Final Outcomes } \\
\text { Incident Clinical Fracture }\end{array}$ & $\begin{array}{l}\text { Intermediate Outcomes } \\
\text { Incident Radiographic Vertebral } \\
\text { Fracture } \\
\text { DXA BMD Change }\end{array}$ & $\begin{array}{l}\text { Predictors } \\
\text { (Patient, Bone, Drug) }\end{array}$ \\
\hline & & & $\begin{array}{l}\text { p<0.05 } \\
\text { Placebo /discontinue: } 0.2 \%(9 \% \mathrm{Cl}- \\
0.7,1.1) \text { NS } \\
\text { FN BMD } \\
\text { n=71-76/group } \\
\text { ALN } 10 \mathrm{mg}: 1.0 \%(95 \% \mathrm{Cl}-0.3,2.4) \\
\text { NS } \\
\text { ALN } 5 \mathrm{mg}: 0.3 \%(95 \% \mathrm{Cl}-1.2,1.7) \\
\text { NS } \\
\text { Placebo /discontinue: }-1.7 \%(95 \% \mathrm{Cl} \\
-3.0,-0.3) \text { p<0.05 } \\
\text { TH BMD } \\
\text { n=46-50/group } \\
\text { ALN } 10 \mathrm{mg}: 0.1 \%(95 \% \mathrm{Cl}-1.1,1.3) \\
\text { NS } \\
\text { ALN } 5 \mathrm{mg}:-0.2 \%(-1.4,1.0) \mathrm{NS} \\
\text { Placebo/discontinue: }-1.6(95 \% \mathrm{Cl}- \\
\text { 2.8, -0.4) p<0.05 }\end{array}$ & \\
\hline & $\begin{array}{l}\text { Tonino } 2000 \\
6-7 \text { yr with } \\
\text { discontinuation } \\
\text { Medium }\end{array}$ & $\begin{array}{l}\frac{\text { NonVFx }}{6-7 y r(p=n o t ~ c a l c u l a t e d ~ b y ~} \\
\text { authors) } \\
\text { ALN } 10 \mathrm{mg}: 8 / 122(6.6 \%) \\
\text { ALN } 5 \mathrm{mg}: 8 / 113(7.1 \%) \\
\text { ALN 20/ALN 5/placebo: } 9 / 115 \\
(7.8 \%) \\
\text { Symptomatic VFx } \\
\text { 6-7 yr ( } \mathrm{p}=\text { not calculated by } \\
\text { authors) } \\
\text { ALN } 10 \mathrm{mg}: 8 / 122(6.6 \%) \\
\text { ALN } 5 \mathrm{mg}: 7 / 113(6.2 \%) \\
\text { ALN 20/ALN 5/placebo: } 8 / 115 \\
(7.0 \%)\end{array}$ & $\begin{array}{l}\text { Denominators: ALN } 10 \mathrm{mg}(\mathrm{n}=122) \\
\text { ALN } 5 \mathrm{mg}(\mathrm{n}=113), \text { ALN 20/ALN } \\
\text { 5/placebo }(\mathrm{n}=115) \\
\text { LS BMD } \\
6-7 \mathrm{yr}: \mathrm{p}=\mathrm{NR} \text { (all 3) } \\
\text { ALN } 10 \mathrm{mg}:+1.6 \% \\
\text { ALN } 5 \mathrm{mg}:+1.5 \% \\
\text { ALN 20/ALN 5/placebo: }+0.2 \% \\
\text { FN BMD } \\
6-7 \text { yr: } \\
\text { ALN } 10 \mathrm{mg}:+0.5 \%, \mathrm{p}=\mathrm{NS} \\
\text { ALN } 5 \mathrm{mg:}+0.3 \%, \mathrm{p}=\mathrm{NS} \\
\text { ALN } 20 / \mathrm{ALN} 5 / \mathrm{placebo}: \\
-0.5 \%\end{array}$ & NR \\
\hline
\end{tabular}




\begin{tabular}{|c|c|c|c|c|}
\hline $\begin{array}{l}\text { Drug } \\
\text { Comparison }\end{array}$ & $\begin{array}{l}\text { Study } \\
\text { (Trial) } \\
\text { Followup } \\
\text { Risk of Bias }\end{array}$ & $\begin{array}{l}\text { Final Outcomes } \\
\text { Incident Clinical Fracture }\end{array}$ & $\begin{array}{l}\text { Intermediate Outcomes } \\
\text { Incident Radiographic Vertebral } \\
\text { Fracture } \\
\text { DXA BMD Change }\end{array}$ & $\begin{array}{l}\text { Predictors } \\
\text { (Patient, Bone, Drug) }\end{array}$ \\
\hline $\begin{array}{l}\text { Zoledronic Acid } \\
\text { vs Zoledronic } \\
\text { Acid then } \\
\text { placebo }\end{array}$ & $\begin{array}{l}\text { McClung, } 2009 \\
2 \text { yr } \\
\text { Low }\end{array}$ & $\begin{array}{l}\text { CFx: } \\
\text { ZOL 5mg/yr x1 year } \\
\text { (discontinuation): } 4 / 181(2 \%) \\
\text { ZOL } 5 \mathrm{mg} / \mathrm{yr} \times 2 \text { year: } 6 / 198(3 \%) \\
\text { RR } 0.73(0.21,2.54) * \\
{ }^{*} \text { Calculated }\end{array}$ & 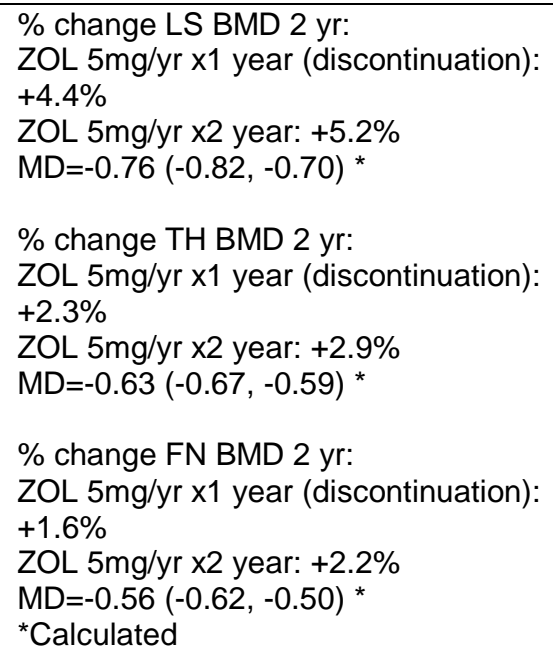 & NR \\
\hline
\end{tabular}

Abbreviations: ALN=alendronate; ARR=absolute risk reduction; BMD=bone mineral density; BP=bisphosphonates; CI=confidence interval; DXA= Dual-energy x-ray absorptiometry; FIT=Fracture Intervention Trial; FLEX=Fracture Intervention Trial Long Term Extension; FN=femoral neck; Fx=fracture; LS=lumbar spine; mg=milligrams; $\mathrm{N}=$ number; NR=not reported; NS=not significant; RCT=randomized controlled trial; RR=risk ratio; SE=standard error; TH=total hip; VFx=vertebral fracture; $y r=y e a r$ 
Table D6. Key Questions 7 and 8 evidence overview

\begin{tabular}{|c|c|c|c|c|}
\hline $\begin{array}{l}\text { Drug } \\
\text { Comparison }\end{array}$ & $\begin{array}{l}\text { Study } \\
\text { (Trial) } \\
\text { Followup } \\
\text { Risk of Bias }\end{array}$ & $\begin{array}{l}\text { Harms } \\
\text { Drug class specific harms }\end{array}$ & $\begin{array}{l}\text { Rare Harms } \\
\text { AFF, ONJ, Afib }\end{array}$ & $\begin{array}{l}\text { Predictors } \\
\text { (Patient, Bone, Drug) }\end{array}$ \\
\hline \multirow[t]{4}{*}{$\begin{array}{l}\text { Alendronate vs } \\
\text { Discontinue } \\
\text { Alendronate } \\
\text { (Placebo) }\end{array}$} & $\begin{array}{l}\text { Black } 2006 \\
\text { (FLEX) } \\
\text { Continue vs } \\
\text { stop ALN } \\
5 \text { yr: } 5-10 \text { year } \\
\text { FIT extension } \\
\text { Low }\end{array}$ & $\begin{array}{l}\frac{\text { Serious AEs, discontinuations due to AES, }}{\text { rates of death, UGI or serious UGI AEs }} \\
\text { Data not shown. Pooled ALN vS. } \\
\text { discontinue/placebo } p=\text { not significant }\end{array}$ & None & NR \\
\hline & $\begin{array}{l}\text { Black } 2010 \\
\text { (FLEX) } \\
\text { Continue vs } \\
\text { stop ALN } \\
5 \text { yr: } 5-10 \text { year } \\
\text { FIT extension } \\
\text { Low }\end{array}$ & NR & $\begin{array}{l}\text { Subtrochanteric or diaphyseal } \\
\text { femur fracture determined by } \\
\text { review of radiology reports and } \\
\text { medical records; high-energy } \\
\text { trauma and pathologic fractures } \\
\text { excluded } \\
\text { ALN: } 2 / 662(0.030 \%) \\
\text { Placebo: } 1 / 437(0.023 \%) \\
\text { RH= } 1.33(95 \% \mathrm{Cl} 0.12,14.67) \\
p=0.82\end{array}$ & NR \\
\hline & $\begin{array}{l}\text { Bone } 2004 \\
\text { 8-10 yr CCT } \\
\text { extension with } \\
\text { discontinuation } \\
\text { Medium } \\
\text { (subset of } \\
\text { Tonino 2000) }\end{array}$ & $\begin{array}{l}\text { Any serious clinical event: } \\
\text { ALN } 10 \mathrm{mg}: 18 / 86(20.9 \%) \\
\text { ALN } 5 \mathrm{mg}: 25 / 78(32.1 \%) \\
\text { ALN 20/ALN 5/placebo: } 18 / 83(21.7 \%) \\
\text { p=NR } \\
\text { Any upper GI event }(\geq 1): \\
\text { ALN } 10 \mathrm{mg}: 24 / 86(27.9 \%) \\
\text { ALN } 5 \mathrm{mg}: 11 / 78(14.1 \%) \\
\text { ALN 20/ALN 5/placebo : } 20 / 83(24.1 \%) \\
\text { p=NR }\end{array}$ & $\begin{array}{l}\text { Reported "no insufficiency } \\
\text { fractures" }\end{array}$ & $\begin{array}{l}8-10 \text { yr: } 30-36 \% \text { of women in } \\
\text { each group used aspirin and } \\
41-53 \% \text { used nonsteroidal or } \\
\text { glucocorticoid drugs. No } \\
\text { apparent adverse interaction } \\
\text { between these drugs and } \\
\text { alendronate. }\end{array}$ \\
\hline & $\begin{array}{l}\text { Ensrud } 2004 \\
\text { (FLEX } 3 \text { year } \\
\text { interim } \\
\text { analysis) } \\
\text { Black 2006 } \\
3 \text { yr } \\
\text { Low }\end{array}$ & $\begin{array}{l}\text { Any UGI event(s) } \\
\text { Pooled ALN: } 197 / 662(29.8 \%) \\
\text { Discontinue/placebo: } 156 / 437(35.7 \%) \\
p=0.04 \\
\text { Death } \\
\text { Pooled ALN: } 16 / 662(2.4 \%) \\
\text { Discontinue/placebo: } 8 / 437(1.8 \%) \\
p=0.67\end{array}$ & NR & NR \\
\hline
\end{tabular}




\begin{tabular}{|c|c|c|c|c|}
\hline $\begin{array}{l}\text { Drug } \\
\text { Comparison }\end{array}$ & $\begin{array}{l}\text { Study } \\
\text { (Trial) } \\
\text { Followup } \\
\text { Risk of Bias }\end{array}$ & $\begin{array}{l}\text { Harms } \\
\text { Drug class specific harms }\end{array}$ & $\begin{array}{l}\text { Rare Harms } \\
A F F, \text { ONJ, Afib }\end{array}$ & $\begin{array}{l}\text { Predictors } \\
\text { (Patient, Bone, Drug) }\end{array}$ \\
\hline & $\begin{array}{l}\text { Tonino } 2000 \\
6-7 \text { yr CCT } \\
\text { extension } \\
\text { with } \\
\text { discontinuation } \\
\text { Medium }\end{array}$ & 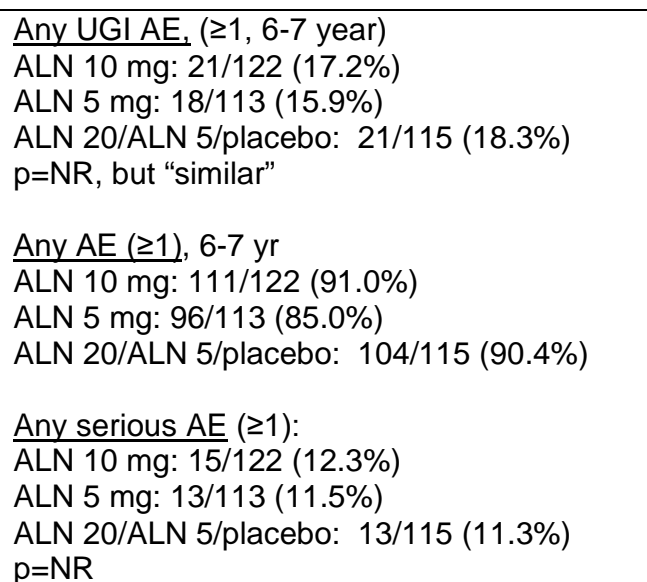 & NR & NR \\
\hline $\begin{array}{l}\text { Bisphosphonate drug } \\
\text { holiday ( } \geq 1 \text { yr without } \\
\text { use) vs } \\
\text { Bisphosphonate use } \\
\geq 3 \mathrm{yr}\end{array}$ & $\begin{array}{l}\text { Adams } 2018 \\
\text { Mean } 4 \text { yr } \\
\text { Medium }\end{array}$ & NR & $\begin{array}{l}\text { AFF } \\
\text { BP drug holiday group } 3 / 11497 \\
(0.03 \%) \\
\text { Persistent and non-persistent } \\
\text { groups pooled } 44 / 28005(0.15 \%)\end{array}$ & NR \\
\hline $\begin{array}{l}\text { Zoledronic Acid vs } \\
\text { Zoledronic Acid then } \\
\text { placebo }\end{array}$ & $\begin{array}{l}\text { McClung, } 2009 \\
2 \text { yr } \\
\text { Low }\end{array}$ & $\begin{array}{l}\text { Serious AEs } \\
\text { ZOL } 5 \mathrm{mg} / \mathrm{yr} \times 1 \text { year (discontinuation): } 17 / 181 \\
(9 \%) \\
\text { ZOL } 5 \mathrm{mg} / \mathrm{yr} \times 2 \text { year: } 21 / 198(11 \%) \\
\text { RR } 0.89(0.48,1.62){ }^{*} \\
\text { Death } \\
\text { ZOL } 5 \mathrm{mg} / \mathrm{yr} \times 1 \text { year (discontinuation): 0/181 } \\
\text { ZOL } 5 \mathrm{mg} / \mathrm{yr} \times 2 \text { year: } 1 / 198(<1 \%) \\
{ }^{*} \text { Calculated }\end{array}$ & $\begin{array}{l}\text { Afib } 0 \\
\underline{\text { ONJ } 0}\end{array}$ & NR \\
\hline
\end{tabular}

Abbreviations: Afib=atrial fibrillation; $\mathrm{AFF}=$ atypical femoral fracture; $\mathrm{AE}=$ adverse event; $\mathrm{ALN}=$ alendronate; $\mathrm{mg}=$ =milligram; $\mathrm{BP}=$ bisphosphonates; $\mathrm{NR}=$ not reported;

$\mathrm{ONJ}=$ osteonecrosis of the jaw; RCT=randomized controlled trial; UGI=upper gastrointestinal 
Table D7. Characteristics of eligible studies with high risk of bias

\begin{tabular}{|c|c|c|c|c|c|}
\hline Intervention & $\begin{array}{l}\text { Study, Year } \\
\text { Design (Trial) } \\
\text { Location } \\
\text { Funding }\end{array}$ & $\begin{array}{l}\text { Study Demographics } \\
\text { Comorbidities }\end{array}$ & \begin{tabular}{|l} 
Duration of \\
Treatment \\
Drug Holiday?
\end{tabular} & Outcomes Reported & $\begin{array}{l}\text { Patient, Bone, } \\
\text { Drug } \\
\text { Predictors } \\
\text { Reported? }\end{array}$ \\
\hline \multirow[t]{3}{*}{ Alendronate } & $\begin{array}{l}\text { Abrahamsen, } 2010 \\
\text { Retrospective cohort, } \\
\text { administrative data } \\
\text { Denmark } \\
\text { Industry }\end{array}$ & $\begin{array}{l}\text { Male and female incident alendronate } \\
\text { users and untreated matched controls } \\
\text { DM 4\% } \\
\text { CVD NR } \\
\text { Previous MI } 2 \% \\
\text { CKD NR } \\
\text { Renal failure } 0.4 \% \\
\text { BMD NR } \\
\text { Spine fracture } 1 \% \\
\text { Hip fracture } 0 \% \text { (excluded) } \\
\text { Fracture risk NR }\end{array}$ & $\begin{array}{l}\text { NR } \\
2 \text { months } 25 \% \\
1.1 \text { yr } 25 \% \\
3.7 \text { yr } 25 \% \\
8.7 \text { yr } 25 \% \\
3.4 \text { yr (mean } \\
\text { followup time) } \\
\text { No }\end{array}$ & $\begin{array}{l}\text { Incident hip fractures } \\
\text { Adverse events: incident fractures of the } \\
\text { subtrochanteric femur or femoral shaft }\end{array}$ & $\begin{array}{l}\text { Sex, treatment } \\
\text { duration }\end{array}$ \\
\hline & $\begin{array}{l}\text { Abrahamsen, } 2016 \\
\text { Case control, } \\
\text { administrative data } \\
\text { Denmark } \\
\text { Industry }\end{array}$ & $\begin{array}{l}\text { Male and female incident alendronate } \\
\text { users and untreated matched controls } \\
\text { aged } 50-94 \\
\text { DM } 5 \% \\
\text { CVD NR } \\
\text { Previous MI } 6 \% \\
\text { CKD } 1 \% \\
\text { BMD NR } \\
\text { MOF } 31 \% \\
\text { Fracture risk NR }\end{array}$ & $\begin{array}{l}\text { NR } \\
<5 \text { yr } 76 \% \\
5-10 \text { yr } 21 \% \\
\geq 10 \text { yr } 3 \% \\
6.9 \text { yr (median } \\
\text { ascertainment) } \\
\text { No }\end{array}$ & $\begin{array}{l}\text { Incident hip fractures } \\
\text { Adverse events: incident } \\
\text { subtrochanteric femur or femoral shaft } \\
\text { fractures }\end{array}$ & $\begin{array}{l}\text { Treatment } \\
\text { duration }\end{array}$ \\
\hline & $\begin{array}{l}\text { Chiu, } 2018 \\
\text { Retrospective cohort, } \\
\text { administrative data } \\
\text { Taiwan } \\
\text { Government }\end{array}$ & $\begin{array}{l}\text { Women aged } \geq 50 \text { or men aged } \geq 60 \\
\text { using alendronate } \geq 30 \text { days and } \\
\text { unmatched controls taking raloxifene } \\
\geq 30 \text { days } \\
\text { DM } 25 \% \\
\text { CKD } 4 \% \\
\text { CVD NR } \\
\text { Hypertension } 52 \% \\
\text { BMD NR } \\
\text { Fracture risk NR }\end{array}$ & $\begin{array}{l}1-8 \mathrm{yr} \\
\text { No }\end{array}$ & Adverse events: ONJ & $\begin{array}{l}\text { Treatment } \\
\text { duration }\end{array}$ \\
\hline
\end{tabular}




\begin{tabular}{|c|c|c|c|c|c|}
\hline \multirow[t]{4}{*}{ Intervention } & $\begin{array}{l}\text { Study, Year } \\
\text { Design (Trial) } \\
\text { Location } \\
\text { Funding }\end{array}$ & $\begin{array}{l}\text { Study Demographics } \\
\text { Comorbidities }\end{array}$ & \begin{tabular}{|l} 
Duration of \\
Treatment \\
Drug Holiday?
\end{tabular} & Outcomes Reported & $\begin{array}{l}\text { Patient, Bone, } \\
\text { Drug } \\
\text { Predictors } \\
\text { Reported? }\end{array}$ \\
\hline & $\begin{array}{l}\text { Chapurlat, } 2005 \\
\text { RCT (FIT) } \\
\text { US } \\
\text { Industry }\end{array}$ & $\begin{array}{l}\text { Postmenopausal women aged } 55 \text { to } 80 \\
\text { with femoral neck T-score } \leq-1.6 \text { who } \\
\text { adhered to treatment but lost BMD } \\
\text { during first yr of treatment } \\
\text { DM NR } \\
\text { CVD NR } \\
\text { CKD NR } \\
\text { BMD, mean, g/cm }{ }^{2} \\
\text { Hip } 0.700 \\
\text { LS } 0.826 \\
\text { Fracture since age } 45: 41 \% \\
\text { Fracture risk NR }\end{array}$ & $\begin{array}{l}3.2 \mathrm{yr} \text { (mean) } \\
\text { No }\end{array}$ & Radiographic vertebral fractures & $\begin{array}{l}\text { BMD change } \\
\text { during } 1 \text { or } 2 \text { yr } \\
\text { of treatment }\end{array}$ \\
\hline & $\begin{array}{l}\text { Nevitt, } 1999 \\
\text { RCT (FIT) } \\
\text { US } \\
\text { Industry }\end{array}$ & $\begin{array}{l}\text { Postmenopausal (at least } 2 \mathrm{yr} \text { ) women } \\
\text { aged } 55-80 \text { with FN BMD } \leq 0.68 \mathrm{~g} / \mathrm{cm}^{2} \\
\text { (T-score } \leq-1.6) \text { who had lateral spine } \\
\text { radiographs and baseline and one } \\
\text { followup visit } \\
\text { DM NR } \\
\text { CVD NR } \\
\text { CKD NR } \\
\text { BMD, g/cm² } \\
\text { LS } 0.83 \\
\text { FN } 0.59 \\
\text { TH } 0.69 \\
\text { Vertebral fracture NR } \\
\text { Fracture risk NR }\end{array}$ & $\begin{array}{l}3.8 \\
\text { No }\end{array}$ & $\begin{array}{l}\text { Incident radiographic vertebral fractures } \\
\text { Bone density as a predictor of fractures }\end{array}$ & $\begin{array}{l}\text { Fracture } \\
\text { history } \\
\text { BMD (LS, FN, } \\
\text { TH) }\end{array}$ \\
\hline & $\begin{array}{l}\text { Wang, } 2016 \\
\text { Retrospective cohort } \\
\text { Taiwan } \\
\text { University }\end{array}$ & $\begin{array}{l}\text { Women aged } \geq 50 \text { with osteoporosis } \\
\text { Comorbidities NR } \\
\text { BMD NR } \\
\text { Fracture during } 1 \text { yr prior to study } 20 \%\end{array}$ & $\begin{array}{l}5 \mathrm{yr} \\
\text { No }\end{array}$ & Adverse events: AFF & $\begin{array}{l}\text { Treatment } \\
\text { duration }\end{array}$ \\
\hline Zoledronic Acid & $\begin{array}{l}\text { Cosman, } 2014 \\
\text { RCT (HORIZON } \\
\text { extension) }\end{array}$ & $\begin{array}{l}\text { Postmenopausal women aged } 65 \text { to } 89 \\
\text { yr with FN T-score } \leq-2.5 \text {, or FN T- } \\
\text { score } \leq-1.5 \text {, with } \geq 2 \text { mild radiologic }\end{array}$ & $\begin{array}{l}3 \mathrm{yr} \\
\text { Yes }\end{array}$ & $\begin{array}{l}\text { Clinical nonvertebral fractures, } \\
\text { radiographic vertebral fractures }\end{array}$ & $\begin{array}{l}\text { Incident } \\
\text { radiographic } \\
\text { vertebral } \\
\end{array}$ \\
\hline
\end{tabular}




\begin{tabular}{|c|c|c|c|c|c|}
\hline Intervention & $\begin{array}{l}\text { Study, Year } \\
\text { Design (Trial) } \\
\text { Location } \\
\text { Funding }\end{array}$ & $\begin{array}{l}\text { Study Demographics } \\
\text { Comorbidities }\end{array}$ & $\begin{array}{l}\text { Duration of } \\
\text { Treatment } \\
\text { Drug Holiday? }\end{array}$ & Outcomes Reported & $\begin{array}{l}\text { Patient, Bone, } \\
\text { Drug } \\
\text { Predictors } \\
\text { Reported? }\end{array}$ \\
\hline & $\begin{array}{l}\text { US } \\
\text { Industry }\end{array}$ & $\begin{array}{l}\text { vertebral fractures or one moderate } \\
\text { vertebral fracture; who had received } \\
\text { zoledronate for } 3 \text { yr in the core study } \\
\text { Comorbidities at start of extension } \\
\text { study } \\
\text { DM NR } \\
\text { CVD NR } \\
\text { CKD NR } \\
\text { BMD } \\
\text { FN T-score } \leq-2.5: 55 \% \\
\text { TH T-score } \leq-2.5: 26 \% \\
\text { Spine fracture } 61 \% \\
\text { Hip fracture NR } \\
\text { Fracture risk NR }\end{array}$ & & & $\begin{array}{l}\text { fractures } \\
\text { during initial } \\
\text { study, } \\
\text { TH BMD, FN } \\
\text { BMD }\end{array}$ \\
\hline \multirow[t]{2}{*}{ Risedronate } & $\begin{array}{l}\text { Sorensen, } 2002 \\
\text { RCT (VERT) } \\
\text { Multinational } \\
\text { Industry }\end{array}$ & $\begin{array}{l}\text { Postmenopausal women aged } \leq 85 \mathrm{yr} \\
\text { with } \geq 2 \text { vertebral fractures; compliant in } \\
\text { core study, no new medications known } \\
\text { to affect bone metabolism } \\
\text { DM NR } \\
\text { CVD NR } \\
\text { CKD NR } \\
\text { BMD, g/cm² } \\
\text { LS } 0.80 \\
\text { FN } 0.64 \\
\text { Number of vertebral fractures } 4.4 \\
\text { Fracture risk NR }\end{array}$ & $\begin{array}{l}5 \mathrm{yr} \\
\text { No }\end{array}$ & $\begin{array}{l}\text { Vertebral fractures, nonvertebral } \\
\text { fractures, hip fractures, BMD changes } \\
\text { Adverse events: serious adverse } \\
\text { events, GI intolerance }\end{array}$ & None \\
\hline & $\begin{array}{l}\text { Ste-Marie, } 2004 \\
\text { RCT (VERT) } \\
\text { Multinational } \\
\text { Industry }\end{array}$ & $\begin{array}{l}\text { Postmenopausal women with } 2 \\
\text { prevalent vertebral fractures or } 1 \\
\text { prevalent vertebral fracture and LS T- } \\
\text { score } \leq-2 \text {; completed core study and } \\
\text { underwent } 3 \text { yr iliac crest biopsy } \\
\text { DM NR } \\
\text { CVD NR } \\
\text { CKD NR }\end{array}$ & $\begin{array}{l}5 \mathrm{yr} \\
\text { No }\end{array}$ & $\begin{array}{l}\text { Adverse events: vertebral fractures, } \\
\text { nonvertebral fractures, BMD change }\end{array}$ & None \\
\hline
\end{tabular}




\begin{tabular}{|c|c|c|c|c|c|}
\hline \multirow[t]{2}{*}{ Intervention } & $\begin{array}{l}\text { Study, Year } \\
\text { Design (Trial) } \\
\text { Location } \\
\text { Funding }\end{array}$ & $\begin{array}{l}\text { Study Demographics } \\
\text { Comorbidities }\end{array}$ & \begin{tabular}{|l} 
Duration of \\
Treatment \\
Drug Holiday?
\end{tabular} & Outcomes Reported & $\begin{array}{l}\text { Patient, Bone, } \\
\text { Drug } \\
\text { Predictors } \\
\text { Reported? }\end{array}$ \\
\hline & & $\begin{array}{l}\text { BMD, g/cm² NR } \\
\text { LS T-score }-1.8 \\
\text { Number of vertebral fractures } 1.8 \\
\text { Fracture risk NR }\end{array}$ & & & \\
\hline Ibandronate & $\begin{array}{l}\text { Miller, } 2011 \\
\text { RCT (MOBILE long- } \\
\text { term extension) } \\
\text { Multinational } \\
\text { Industry }\end{array}$ & $\begin{array}{l}\text { Postmenopausal women aged } 55 \text { to } 80 \\
\text { yr with LS T-score }<-2.5 \text { and } \geq-5.0, \\
\geq 75 \% \text { compliant in core study } \\
\text { DM NR } \\
\text { CVD NR } \\
\text { CKD NR } \\
\text { BMD, } / \mathrm{cm}^{2} \\
\text { LS } 0.79 \\
\text { TH } 0.78 \\
\text { Vertebral fracture NR } \\
\text { Fracture risk NR }\end{array}$ & $\begin{array}{l}5 \mathrm{yr} \\
\text { No }\end{array}$ & $\begin{array}{l}\text { Adverse events: upper GI intolerance, } \\
\text { musculoskeletal pain, serious adverse } \\
\text { events }\end{array}$ & Dose \\
\hline $\begin{array}{l}\text { Bisphosphonates } \\
\text { (Alendronate } \\
\text { Ibandronate } \\
\text { Risedronate } \\
\text { Zoledronic Acid) }\end{array}$ & $\begin{array}{l}\text { Meier, } 2012 \\
\text { Case control, } \\
\text { administrative data } \\
\text { US } \\
\text { Funding NR }\end{array}$ & $\begin{array}{l}\text { Women and men aged } \geq 50 \mathrm{yr} \text { admitted } \\
\text { to a trauma center with a } \\
\text { subtrochanteric } \\
\text { or femoral shaft fracture } \\
\text { DM NR } \\
\text { CVD NR } \\
\text { CKD NR } \\
\text { BMD NR } \\
\text { History of fracture NR } \\
\text { Fracture risk NR }\end{array}$ & $\begin{array}{l}\text { NR } \\
\text { None } 87 \% \\
<2 \text { yr } 3 \% \\
2-5 \text { yr } 4 \% \\
5-9 \text { yr } 4 \% \\
\geq 9 \text { yr } 2 \% \\
12 \text { yr } \\
\text { ascertainment } \\
\text { No }\end{array}$ & Adverse events: AFF & $\begin{array}{l}\text { Treatment } \\
\text { duration }\end{array}$ \\
\hline $\begin{array}{l}\text { Bisphosphonates } \\
\text { (any) }\end{array}$ & $\begin{array}{l}\text { Lee, } 2018 \\
\text { Retrospective cohort, } \\
\text { administrative data } \\
\text { Korea } \\
\text { Foundation }\end{array}$ & $\begin{array}{l}\text { Women and men } \geq 45 \text { yr without history } \\
\text { of femoral surgery who took } \\
\text { bisphosphonate at study hospital } \\
\text { DM NR } \\
\text { CVD NR } \\
\text { BMD NR } \\
\text { History of fracture NR } \\
\text { Fracture risk NR }\end{array}$ & $\begin{array}{l}4.5 \mathrm{yr} \text { (mean in } \\
\text { AFF patients) } \\
10 \mathrm{yr} \\
\text { ascertainment } \\
\text { No }\end{array}$ & Adverse events: AFF & $\begin{array}{l}\text { Treatment } \\
\text { duration }\end{array}$ \\
\hline
\end{tabular}




\begin{tabular}{|c|c|c|c|c|c|}
\hline Intervention & $\begin{array}{l}\text { Study, Year } \\
\text { Design (Trial) } \\
\text { Location } \\
\text { Funding }\end{array}$ & $\begin{array}{l}\text { Study Demographics } \\
\text { Comorbidities }\end{array}$ & \begin{tabular}{|l} 
Duration of \\
Treatment \\
Drug Holiday?
\end{tabular} & Outcomes Reported & $\begin{array}{l}\text { Patient, Bone, } \\
\text { Drug } \\
\text { Predictors } \\
\text { Reported? }\end{array}$ \\
\hline $\begin{array}{l}\text { Denosumab } \\
\text { Teriparatide }\end{array}$ & \begin{tabular}{|l} 
Leder, 2015 \\
RCT (DATA-Switch) \\
US \\
Industry
\end{tabular} & $\begin{array}{l}\text { Postmenopausal women aged } \geq 45 \text { with } \\
\text { T-score } \leq-2.5 \text { at the spine, hip, or FN; } \\
\text { T-score } \leq-2.0 \text { with } \geq 1 \text { BMD- } \\
\text { independent risk factor; or T-score } \leq \text { - } \\
1.0 \text { with history of fragility fracture } \\
\text { DM NR } \\
\text { CVD NR } \\
\text { Previous MI NR } \\
\text { CKD NR } \\
\text { BMD, g/cm }{ }^{2} \\
\text { Posterior-anterior spine } 0.84 \\
\text { FN } 0.64 \\
\text { TH } 0.75 \\
\text { Clinical fracture age }>4542 \%\end{array}$ & $\begin{array}{l}4 \mathrm{yr} \\
\text { No }\end{array}$ & $\begin{array}{l}\text { Adverse events: hypercalcemia, serious } \\
\text { adverse events }\end{array}$ & None \\
\hline
\end{tabular}

Abbreviations: $\mathrm{AFF}=$ atypical femoral fracture; $\mathrm{BMD}=$ =bone mineral density; $\mathrm{CKD}=$ chronic kidney disease; $\mathrm{CVD}=$ cardiovascular disease; DM=diabetes mellitus; FIT=Fracture Intervention Trial; FN=femoral neck; HORIZON=Health Outcomes and Reduced Incidence with Zoledronic Acid Once Yearly; LS=lumbar spine; MOF=major osteoporotic fracture; NR=not reported; ONJ=osteonecrosis of the jaw; RCT=randomized controlled trial; TH=total hip; yr=years 


\section{Strength of Evidence Tables}

- High: Very confident that estimate of effect lies close to true effect. Few or no deficiencies in body of evidence, findings believed to be stable.

- Moderate: Moderately confident that estimate of effect lies close to true effect. Some deficiencies in body of evidence; findings likely to be stable, but some doubt.

- Low: Limited confidence that estimate of effect lies close to true effect; major or numerous deficiencies in body of evidence. Additional evidence necessary before concluding that findings are stable or that estimate of effect is close to true effect.

- Insufficient: No evidence, unable to estimate an effect, or no confidence in estimate of effect. No evidence is available or the body of evidence precludes judgment.

\section{Alendronate}

Table D8. Strength of evidence assessments: Long-term treatment with alendronate versus placebo from RCTs and CCTs

\begin{tabular}{|c|c|c|c|c|c|c|c|c|c|}
\hline $\begin{array}{l}\text { Outcome } \\
\text { Time frame }\end{array}$ & $\begin{array}{l}\text { \# Studies } \\
\text { (n/N } \\
\text { events/participants } \\
\text { evaluated) } \\
\text { Population }\end{array}$ & $\begin{array}{l}\text { Summary } \\
\text { statistics } \\
{[95 \% \mathrm{Cl}]}\end{array}$ & $\begin{array}{l}\text { Study } \\
\text { limitations }\end{array}$ & Directness & Precision & Consistency & $\begin{array}{l}\text { Reporting } \\
\text { Bias }\end{array}$ & $\begin{array}{l}\text { Optional } \\
\text { Components }\end{array}$ & SOE \\
\hline \multirow[t]{3}{*}{$\begin{array}{l}\text { Incident } \\
\text { clinical } \\
\text { fracture } \\
0-4 \text { yrs }\end{array}$} & $\begin{array}{l}1(584 / 4432) \\
\text { PM women with FN } \\
\text { BMD T-score } \leq-1.6 \\
\text { and no baseline } \\
\text { RVF }\end{array}$ & $\begin{array}{l}\text { Cummings } \\
1998 \\
\text { NS difference } \\
\text { in risk with } \\
\text { ALN vs. PBO: } \\
\text { ALN } 12.3 \% \\
\text { PBO } 14.1 \% \text {; } \\
\text { HR }=0.86 \\
{[0.73,1.01]}\end{array}$ & Low & Direct & Imprecise & $\begin{array}{l}\text { Unknown but } \\
\text { multisite trial }\end{array}$ & Undetected & NA & Low \\
\hline & $\begin{array}{l}1\left(266 / 1631^{*}\right) \\
\text { PM women with FN } \\
\text { BMD T-score } \leq-2.5 \\
\text { and no baseline } \\
\text { RVF }\end{array}$ & $\begin{array}{l}\text { Cummings } \\
1998 \\
\text { Lower risk with } \\
\text { ALN vs. PBO: } \\
\text { ALN 13.1\%, } \\
\text { PBO } 19.6 \% \text {; } \\
\text { HR = 0.64 } \\
{[0.50,0.82]}\end{array}$ & $\begin{array}{l}\text { Medium } \\
\text { (subgroup) }\end{array}$ & Direct & Precise & $\begin{array}{l}\text { Unknown but } \\
\text { multisite trial }\end{array}$ & Undetected & $\begin{array}{l}\text { A priori } \\
\text { subgroup } \\
\text { analysis. } \\
\text { Interaction } \\
\text { p value } 0.01 .\end{array}$ & Moderate \\
\hline & $\begin{array}{l}1(179 / 1436) \\
\text { PM women with FN } \\
\text { BMD T-score }-2.5 \text { to }\end{array}$ & $\begin{array}{l}\text { Cummings } \\
1998\end{array}$ & $\begin{array}{l}\text { Medium } \\
\text { (subgroup) }\end{array}$ & Direct & Imprecise & $\begin{array}{l}\text { Unknown but } \\
\text { multisite trial }\end{array}$ & Undetected & $\begin{array}{l}\text { A priori } \\
\text { subgroup } \\
\text { analysis. }\end{array}$ & Low \\
\hline
\end{tabular}




\begin{tabular}{|c|c|c|c|c|c|c|c|c|c|}
\hline $\begin{array}{l}\text { Outcome } \\
\text { Time frame }\end{array}$ & $\begin{array}{l}\text { \# Studies } \\
\text { (n/N } \\
\text { events/participants } \\
\text { evaluated) } \\
\text { Population } \\
\end{array}$ & $\begin{array}{l}\text { Summary } \\
\text { statistics } \\
{[95 \% \mathrm{CI}]}\end{array}$ & $\begin{array}{l}\text { Study } \\
\text { limitations }\end{array}$ & Directness & Precision & Consistency & $\begin{array}{l}\text { Reporting } \\
\text { Bias }\end{array}$ & $\begin{array}{l}\text { Optional } \\
\text { Components }\end{array}$ & SOE \\
\hline & $\begin{array}{l}-2 \text { and no baseline } \\
\text { RVF }\end{array}$ & $\begin{array}{l}\text { NS difference } \\
\text { in risk with } \\
\text { ALN vs. PBO: } \\
\text { ALN } 12.7 \% \text {, } \\
\text { PBO } 12.3 \% \\
\text { HR = } 1.03 \\
{[0.77,1.39]}\end{array}$ & & & & & & $\begin{array}{l}\text { Interaction } p \\
\text { value } 0.01\end{array}$ & \\
\hline & $\begin{array}{l}1(139 / 1315) \\
\text { PM women with FN } \\
\text { BMD T-score }-2 \text { to - } \\
1.6 \text { and no baseline } \\
\text { RVF }\end{array}$ & $\begin{array}{l}\text { Cummings } \\
1998 \\
\text { NS difference } \\
\text { in risk with } \\
\text { ALN vs. PBO: } \\
\text { ALN } 10.9 \% \text {, } \\
\text { PBO } 9.5 \% \text {; } \\
\text { HR = } 1.14 \\
{[0.82,1.60]}\end{array}$ & $\begin{array}{l}\text { Medium } \\
\text { (subgroup) }\end{array}$ & Direct & Imprecise & $\begin{array}{l}\text { Unknown but } \\
\text { multisite trial }\end{array}$ & Undetected & $\begin{array}{l}\text { A priori } \\
\text { subgroup } \\
\text { analysis. } \\
\text { Interaction p } \\
\text { value } 0.01 \text {. }\end{array}$ & Low \\
\hline $\begin{array}{l}\text { Incident } \\
\text { nonvertebral } \\
\text { fracture } \\
0-4 \text { yrs }\end{array}$ & $\begin{array}{l}1(555 / 4432) \\
\text { PM women with FN } \\
\text { BMD T-score } \leq-1.6 \\
\text { and no baseline } \\
\text { RVF }\end{array}$ & $\begin{array}{l}\text { Cummings } \\
1998 \\
\text { NS difference } \\
\text { in risk with } \\
\text { ALN vs PBO: } \\
\text { ALN } 11.8 \% \\
\text { PBO } 13.3 \% \text {; } \\
\text { HR = } 0.88 \\
{[0.74,1.04]}\end{array}$ & Low & Direct & Imprecise & $\begin{array}{l}\text { Unknown but } \\
\text { multisite trial }\end{array}$ & Undetected & NA & Low \\
\hline $\begin{array}{l}\text { Incident hip } \\
\text { fracture } \\
0-4 \text { yrs }\end{array}$ & $\begin{array}{l}1(43 / 4432) \\
\text { PM women with FN } \\
\text { BMD T-score } \leq-1.6 \\
\text { and no baseline } \\
\text { RVF }\end{array}$ & $\begin{array}{l}\text { Cummings } \\
1998 \\
\text { NS difference } \\
\text { in risk with } \\
\text { ALN vs. PBO: } \\
\text { ALN 0.9\%, } \\
\text { PBO } 1.1 \% ; \\
\text { HR = } 0.79 \\
{[0.43,1.44]} \\
\end{array}$ & Low & Direct & $\begin{array}{l}\text { Highly } \\
\text { Imprecise }\end{array}$ & $\begin{array}{l}\text { Unknown but } \\
\text { multisite trial }\end{array}$ & Undetected & NA & Low \\
\hline $\begin{array}{l}\text { Incident } \\
\text { nonhip } \\
\text { nonvertebral } \\
\text { fracture }\end{array}$ & 0 & NR & NA & NA & NA & NA & NA & NA & Insufficient \\
\hline
\end{tabular}




\begin{tabular}{|c|c|c|c|c|c|c|c|c|c|}
\hline $\begin{array}{l}\text { Outcome } \\
\text { Time frame }\end{array}$ & $\begin{array}{l}\text { \# Studies } \\
\text { (n/N } \\
\text { events/participants } \\
\text { evaluated) } \\
\text { Population } \\
\end{array}$ & $\begin{array}{l}\text { Summary } \\
\text { statistics } \\
{[95 \% \mathrm{Cl}]}\end{array}$ & $\begin{array}{l}\text { Study } \\
\text { limitations }\end{array}$ & Directness & Precision & Consistency & $\begin{array}{l}\text { Reporting } \\
\text { Bias }\end{array}$ & $\begin{array}{l}\text { Optional } \\
\text { Components }\end{array}$ & SOE \\
\hline $\begin{array}{l}\text { Incident major } \\
\text { osteoporotic } \\
\text { fracture }\end{array}$ & 0 & NR & NA & NA & NA & NA & NA & NA & Insufficient \\
\hline $\begin{array}{l}\text { Incident } \\
\text { clinical } \\
\text { vertebral } \\
\text { fracture }\end{array}$ & 0 & NR & NA & NA & NA & NA & NA & NA & Insufficient \\
\hline \multirow[t]{4}{*}{$\begin{array}{l}\text { Incident } \\
\text { radiographic } \\
\text { vertebral } \\
\text { fracture } † \\
0-4 \text { yrs }\end{array}$} & $\begin{array}{l}1(121 / 4134) \\
\text { PM women with FN } \\
\text { BMD T-score } \leq-1.6 \\
\text { and no baseline } \\
\text { RVF }\end{array}$ & $\begin{array}{l}\text { Cummings } \\
1998 \\
\text { Lower risk with } \\
\text { ALN vs. PBO: } \\
\text { ALN 2.1\%, } \\
\text { PBO 3.8\%; } \\
\text { HR = 0.56 } \\
{[0.39,0.80]}\end{array}$ & Low & Direct & Precise & $\begin{array}{l}\text { Unknown but } \\
\text { multisite trial }\end{array}$ & Undetected & NA & High \\
\hline & $\begin{array}{l}1(66 /<1631) \\
\text { PM women with FN } \\
\text { BMD T-score } \leq-2.5 \\
\text { and no baseline } \\
\text { RVF }\end{array}$ & $\begin{array}{l}\text { Cummings } \\
1998 \\
\text { Lower risk with } \\
\text { ALN vs. PBO: } \\
\text { ALN } 2.9 \% \text {, } \\
\text { PBO 5.8\%; } \\
\text { HR = 0.50 } \\
{[0.31,0.82]}\end{array}$ & $\begin{array}{l}\text { Medium } \\
\text { (subgroup) }\end{array}$ & Direct & Precise & $\begin{array}{l}\text { Unknown but } \\
\text { multisite trial }\end{array}$ & Undetected & $\begin{array}{l}\text { A priori } \\
\text { subgroup } \\
\text { analysis. } \\
\text { Interaction p } \\
\text { value NR. }\end{array}$ & Moderate \\
\hline & $\begin{array}{l}1(37 /<1436) \\
\text { PM women with FN } \\
\text { BMD T-score }-2.5 \text { to } \\
-2 \text { and no baseline } \\
\text { RVF }\end{array}$ & $\begin{array}{l}\text { Cummings } \\
1998 \\
\text { Lower risk with } \\
\text { ALN vs. PBO: } \\
\text { ALN } 1.9 \% \text {, } \\
\text { PBO } 3.6 \% ; \\
\text { HR = 0.54 } \\
{[0.28,1.04]}\end{array}$ & $\begin{array}{l}\text { Medium } \\
\text { (subgroup) }\end{array}$ & Direct & Imprecise & $\begin{array}{l}\text { Unknown but } \\
\text { multisite trial }\end{array}$ & Undetected & $\begin{array}{l}\text { A priori } \\
\text { subgroup } \\
\text { analysis. } \\
\text { Interaction p } \\
\text { value NR. }\end{array}$ & Low \\
\hline & $\begin{array}{l}1(18 /<1315) \\
\text { PM women with FN } \\
\text { BMD T-score }-2 \text { to - } \\
1.6 \text { and no baseline } \\
\text { RVF }\end{array}$ & $\begin{array}{l}\text { Cummings } \\
1998 \\
\text { Lower risk with } \\
\text { ALN vs. PBO: } \\
\text { ALN 1.3\%, } \\
\text { PBO 1.5\%; }\end{array}$ & $\begin{array}{l}\text { Medium } \\
\text { (subgroup) }\end{array}$ & Direct & Imprecise & $\begin{array}{l}\text { Unknown but } \\
\text { multisite trial }\end{array}$ & Undetected & $\begin{array}{l}\text { A priori } \\
\text { subgroup } \\
\text { analysis. } \\
\text { Interaction p } \\
\text { value NR. }\end{array}$ & Insufficient \\
\hline
\end{tabular}




\begin{tabular}{|c|c|c|c|c|c|c|c|c|c|}
\hline $\begin{array}{l}\text { Outcome } \\
\text { Time frame }\end{array}$ & $\begin{array}{l}\text { \# Studies } \\
\text { (n/N } \\
\text { events/participants } \\
\text { evaluated) } \\
\text { Population }\end{array}$ & $\begin{array}{l}\text { Summary } \\
\text { statistics } \\
{[95 \% \mathrm{Cl}]}\end{array}$ & $\begin{array}{l}\text { Study } \\
\text { limitations }\end{array}$ & Directness & Precision & Consistency & $\begin{array}{l}\text { Reporting } \\
\text { Bias }\end{array}$ & $\begin{array}{l}\text { Optional } \\
\text { Components }\end{array}$ & SOE \\
\hline & & $\begin{array}{l}\mathrm{HR}=0.82 \\
{[0.33,2.07]}\end{array}$ & & & & & & & \\
\hline \multirow[t]{2}{*}{$\begin{array}{l}\text { Serious } \\
\text { adverse } \\
\text { events } \\
0-4 \text { yrs }\end{array}$} & $\begin{array}{l}1(4432) \\
\text { PM women with FN- } \\
\text { BMD T-score } \leq-1.6 \\
\text { and no baseline } \\
\text { RVF }\end{array}$ & $\begin{array}{l}\text { Cummings } \\
1998 \\
\text { AE causing } \\
\text { hospitalization } \\
\text { NS difference } \\
\text { with ALN vs. } \\
\text { PBO: } \\
\text { ALN } 29.1 \% \text {, } \\
\text { PBO } 26.9 \% \text {; } \\
\text { HR }=1.09 \\
{[0.98,1.22]}\end{array}$ & Low & Indirect & Precise & $\begin{array}{l}\text { Unknown but } \\
\text { multisite trial }\end{array}$ & $\begin{array}{l}\text { Possible } \\
\text { (serious AE } \\
\text { outcome } \\
\text { NR) }\end{array}$ & NA & Low \\
\hline & $\begin{array}{l}1(4432) \\
\text { PM women with FN- } \\
\text { BMD T-score } \leq-1.6 \\
\text { and no baseline } \\
\text { RVF }\end{array}$ & $\begin{array}{l}\text { Cummings } \\
1998 \\
\text { Mortality } \\
\text { NS difference } \\
\text { with ALN vs. } \\
\text { PBO: } \\
\text { ALN } 1.7 \% \text {, } \\
\text { PBO } 1.8 \% \\
\text { HR }=0.92 \\
{[0.59,1.45]}\end{array}$ & Low & Indirect & Imprecise & $\begin{array}{l}\text { Unknown but } \\
\text { multisite trial }\end{array}$ & Undetected & NA & Low \\
\hline $\begin{array}{l}\text { ST/FS } \\
\text { fracture (rare } \\
\text { x-ray review } \\
\text { for } \\
\text { confirmation } \\
\text { of AFF } \\
\text { features) } \\
3-4.5 \text { yrs }\end{array}$ & $\begin{array}{l}1 \text { (6459) } \\
\text { PM women with FN- } \\
\text { BMD T-score } \leq-1.6\end{array}$ & $\begin{array}{l}\text { Black } 2010 \\
\text { NS difference } \\
\text { with ALN vs. } \\
\text { PBO: } \\
\text { ALN } 0.031 \% \\
(n=1), P B O \\
0.031 \%(n=1) \\
\text { HR= } 1.03 \\
{[0.06,16.46]}\end{array}$ & Low & Direct & $\begin{array}{l}\text { Highly } \\
\text { Imprecise }\end{array}$ & $\begin{array}{l}\text { Unknown but } \\
\text { multisite trial }\end{array}$ & Undetected & NA & Insufficient \\
\hline $\begin{array}{l}\text { Osteonecrosi } \\
\text { s of the jaw }\end{array}$ & 0 & NR & NA & NA & NA & NA & NA & NA & Insufficient \\
\hline
\end{tabular}

Abbreviations: AE=adverse events; $\mathrm{ALN}=$ alendronate; $\mathrm{BMD}=$ bone mineral density; $\mathrm{CI}=$ confidence interval; FIT=Fracture Intervention Trial; FN=femoral neck; HR=hazard ratio; LS=lumbar spine; $\mathrm{NA}=$ not applicable; $\mathrm{NR}=$ not reported; $\mathrm{PBO}=$ placebo; $\mathrm{PM}=$ =postmenopausal; $\mathrm{RCT}=$ randomized controlled trial; $\mathrm{SOE}=$ strength of evidence;

$\mathrm{ST} / \mathrm{FS}=$ subtrochanteric/femoral shaft; $\mathrm{TH}=$ total hip; $\mathrm{yr}=$ years 
*Calculated by EPC

$\lceil 95 \%$ of survivors to the year 4 visit completed followup vertebral x-rays at that visit, but the percentage of completers is not reported by BMD subgroup, so the number in each BMD population subgroup is some unknown number likely slightly smaller than that listed.

\begin{tabular}{|c|c|c|c|c|c|c|c|c|}
\hline Outcome & $\begin{array}{l}\text { \# Studies } \\
\text { (n/N } \\
\text { events/participants } \\
\text { evaluated) } \\
\text { Population } \\
\end{array}$ & $\begin{array}{l}\text { Summary statistics } \\
{[95 \% \mathrm{Cl}]}\end{array}$ & $\begin{array}{l}\text { Study } \\
\text { limitations }\end{array}$ & Directness & Precision & Consistency & $\begin{array}{l}\text { Reporting } \\
\text { Bias }\end{array}$ & SOE \\
\hline $\begin{array}{l}\text { Incident } \\
\text { clinical } \\
\text { fracture }\end{array}$ & $\begin{array}{l}1(225 / 1099) \\
\text { PM women } \\
\text { previously received } \\
5 \text { yrs ALN for FN } \\
\text { BMD T-score } \leq-1.6\end{array}$ & $\begin{array}{l}\text { Black } 2006 \\
\text { NS difference in risk with } 10 \\
\text { yrs ALN (ALN10) vs. } 5 \text { yrs } \\
\text { ALN + } 5 \text { yrs PBO } \\
\text { (ALN5/PBO5): } \\
\text { ALN10 19.9\%, ALN5/PBO5 } \\
21.3 \% ; \text { RR }=0.93[0.71,1.21]\end{array}$ & Low & Direct & Imprecise & $\begin{array}{l}\text { Unknown but } \\
\text { multisite trial }\end{array}$ & Undetected & Moderate \\
\hline \multirow[t]{3}{*}{$\begin{array}{l}\text { Incident } \\
\text { nonvertebral } \\
\text { fracture }\end{array}$} & $\begin{array}{l}1 \text { (208/1099) } \\
\text { PM women } \\
\text { previously received } \\
5 \text { yrs ALN for FN } \\
\text { BMD T-score } \leq-1.6\end{array}$ & $\begin{array}{l}\text { Black } 2006 \\
\text { NS difference in risk with } 10 \\
\text { yrs ALN (ALN10) vs. } 5 \text { yrs } \\
\text { ALN + } 5 \text { yrs PBO } \\
\text { (ALN5/PBO5): } \\
\text { ALN10 18.9\%, ALN5/PBO5 } \\
19.0 \% ; \text { RR }=1.00[0.76,1.32]\end{array}$ & Low & Direct & Imprecise & $\begin{array}{l}\text { Unknown but } \\
\text { multisite trial }\end{array}$ & Undetected & Moderate \\
\hline & $\begin{array}{l}1(25 / 350) \\
\text { PM women } \\
\text { previously received } \\
5 \text { yrs ALN for LS } \\
\text { BMD T-score } \leq-2.5\end{array}$ & $\begin{array}{l}\text { Tonino } 2000 \\
\text { No apparent difference in risk } \\
\text { with } 7 \text { yrs ALN (ALN7) vs. } 5 \\
\text { yrs ALN + } 2 \text { yrs PBO } \\
\text { (ALN5/PBO2): } \\
\text { ALN7 } 5 \mathrm{mg} / \mathrm{d} 7.1 \%, \text { ALN7 } 10 \\
\text { mg/d } 6.6 \%, \text { ALN5/PBO2 } 7.8 \% \\
\text { ALN7 vs. ALN5/PBO } \\
\text { RR }=0.87[0.40,1.91]\end{array}$ & Low & Direct & $\begin{array}{l}\text { Highly } \\
\text { imprecise }\end{array}$ & $\begin{array}{l}\text { Unknown but } \\
\text { multisite trial }\end{array}$ & Undetected & Insufficient \\
\hline & $\begin{array}{l}1(26 / 247) \\
\text { PM women } \\
\text { previously received } \\
5 \text { yrs ALN for LS } \\
\text { BMD T-score } \leq-2.5\end{array}$ & $\begin{array}{l}\text { Bone } 2004 \\
\text { No apparent difference in risk } \\
\text { with } 10 \text { yrs ALN (ALN10) vs. } 7 \\
\text { yrs ALN }+3 \text { yrs PBO } \\
\text { (ALN7/PBO3): } \\
\text { ALN10 } 5 \mathrm{mg} / \mathrm{d} 11.5 \% \text {, ALN10 } \\
10 \mathrm{mg} / \mathrm{d} 8.1 \%, \text { ALN7/PBO3 } \\
12.0 \%\end{array}$ & Medium & Direct & $\begin{array}{l}\text { Highly } \\
\text { imprecise }\end{array}$ & $\begin{array}{l}\text { Unknown but } \\
\text { multisite trial }\end{array}$ & Undetected & Insufficient \\
\hline
\end{tabular}




\begin{tabular}{|c|c|c|c|c|c|c|c|c|}
\hline Outcome & $\begin{array}{l}\text { \# Studies } \\
\text { (n/N } \\
\text { events/participants } \\
\text { evaluated) } \\
\text { Population }\end{array}$ & $\begin{array}{l}\text { Summary statistics } \\
{[95 \% \mathrm{Cl}]}\end{array}$ & $\begin{array}{l}\text { Study } \\
\text { limitations }\end{array}$ & Directness & Precision & Consistency & $\begin{array}{l}\text { Reporting } \\
\text { Bias }\end{array}$ & SOE \\
\hline & & $\begin{array}{l}\text { ALN10 vs. ALN7/PBO } \\
\text { RR }=0.81[0.38,1.71]\end{array}$ & & & & & & \\
\hline $\begin{array}{l}\text { Incident hip } \\
\text { fracture }\end{array}$ & $\begin{array}{l}1(33 / 1099) \\
\text { PM women } \\
\text { previously received } \\
5 \text { yrs ALN for FN } \\
\text { BMD T-score } \leq-1.6 \\
\text { and no baseline } \\
\text { RVF }\end{array}$ & $\begin{array}{l}\text { Black } 2006 \\
\text { NS difference in risk with } 10 \\
\text { yrs ALN (ALN10) vs. } 5 \text { yrs } \\
\text { ALN + } 5 \text { yrs PBO } \\
\text { (ALN5/PBO5): } \\
\text { ALN10 3.0\%, ALN5/PBO5 } \\
3.0 \% \text {; RR = } 1.02[0.51,2.10]\end{array}$ & Low & Direct & $\begin{array}{l}\text { Highly } \\
\text { imprecise }\end{array}$ & $\begin{array}{l}\text { Unknown but } \\
\text { multisite trial }\end{array}$ & Undetected & Insufficient \\
\hline $\begin{array}{l}\text { Incident } \\
\text { nonhip } \\
\text { nonvertebral } \\
\text { fracture }\end{array}$ & 0 & NR & $\mathrm{NA}$ & NA & $\mathrm{NA}$ & NA & NA & Insufficient \\
\hline $\begin{array}{l}\text { Incident } \\
\text { major } \\
\text { osteoporotic } \\
\text { fracture }\end{array}$ & 0 & NR & NA & NA & NA & NA & NA & Insufficient \\
\hline \multirow[t]{2}{*}{$\begin{array}{l}\text { Incident } \\
\text { clinical } \\
\text { vertebral } \\
\text { fracture }\end{array}$} & $\begin{array}{l}1(39 / 1099) \\
\text { PM women } \\
\text { previously received } \\
5 \text { yrs ALN for FN } \\
\text { BMD T-score } \leq-1.6\end{array}$ & $\begin{array}{l}\text { Black } 2006 \\
\text { Lower risk with } 10 \text { yrs ALN } \\
\text { (ALN10) vs. } 5 \text { yrs ALN + } 5 \text { yrs } \\
\text { PBO (ALN5/PBO5): } \\
\text { ALN10 2.4\%, ALN5/PBO5 } \\
5.3 \% ; \\
\text { RR = } 0.45[0.24,0.85]\end{array}$ & Low & Direct & Imprecise & $\begin{array}{l}\text { Unknown but } \\
\text { multisite trial }\end{array}$ & Undetected & Moderate \\
\hline & $\begin{array}{l}1(23 / 350) \\
\text { PM women } \\
\text { previously received } \\
5 \text { yrs ALN for LS } \\
\text { BMD T-score } \leq-2.5\end{array}$ & $\begin{array}{l}\text { Tonino } 2000 \\
\text { No apparent difference in risk } \\
\text { with } 7 \text { yrs ALN (ALN7) vs. } 5 \\
\text { yrs ALN + } 2 \text { yrs PBO } \\
\text { (ALN5/PBO2): } \\
\text { ALN7 } 5 \mathrm{mg} / \mathrm{d} 6.2 \%, \text { ALN7 } 10 \\
\text { mg/d } 6.6 \%, \text { ALN5/PBO2 } 7.0 \% \\
\text { ALN7 vs ALN5/PBO } \\
\text { RR }=0.92[0.40,2.10]\end{array}$ & Low & Direct & $\begin{array}{l}\text { Highly } \\
\text { imprecise }\end{array}$ & $\begin{array}{l}\text { Unknown but } \\
\text { multisite trial }\end{array}$ & Undetected & Insufficient \\
\hline $\begin{array}{l}\text { Incident } \\
\text { radiographic } \\
\text { vertebral }\end{array}$ & $\begin{array}{l}1(106 / 1099) \\
\text { PM women } \\
\text { previously received }\end{array}$ & $\begin{array}{l}\text { Black } 2006 \\
\text { NS difference in risk with } 10 \\
\text { yrs ALN (ALN10) vs. } 5 \text { yrs }\end{array}$ & Low & Direct & Imprecise & $\begin{array}{l}\text { Unknown but } \\
\text { multisite trial }\end{array}$ & Undetected & Moderate \\
\hline
\end{tabular}




\begin{tabular}{|c|c|c|c|c|c|c|c|c|}
\hline Outcome & $\begin{array}{l}\text { \# Studies } \\
\text { (n/N } \\
\text { events/participants } \\
\text { evaluated) } \\
\text { Population }\end{array}$ & $\begin{array}{l}\text { Summary statistics } \\
{[95 \% \mathrm{Cl}]}\end{array}$ & $\begin{array}{l}\text { Study } \\
\text { limitations }\end{array}$ & Directness & Precision & Consistency & $\begin{array}{l}\text { Reporting } \\
\text { Bias }\end{array}$ & SOE \\
\hline \multirow[t]{2}{*}{ fracture } & $\begin{array}{l}5 \text { yrs ALN for FN } \\
\text { BMD T-score } \leq-1.6\end{array}$ & $\begin{array}{l}\text { ALN + } 5 \text { yrs PBO } \\
\text { (ALN5/PBO5): } \\
\text { ALN10 9.8\%, ALN5/PBO5 } \\
\text { 11.3\%; } \\
\text { RR = } 0.86[0.60,1.22]\end{array}$ & & & & & & \\
\hline & $\begin{array}{l}1(\mathrm{NR} / 228) \\
\text { PM women } \\
\text { previously received } \\
5 \text { yrs ALN for LS } \\
\text { BMD T-score } \leq-2.5\end{array}$ & $\begin{array}{l}\text { Bone } 2004 \\
\text { No apparent difference in risk } \\
\text { with } 10 \text { yrs ALN (ALN10) vs. } 5 \\
\text { yrs ALN }+5 \text { yrs PBO } \\
\text { (ALN5/PBO2): } \\
\text { ALN7 } 5 \text { mg/d } 5 \%, \text { ALN7 } 10 \\
\text { mg/d } 6.6 \%, \text { ALN5/PBO2 } \\
13.9 \% ; \\
\text { For ALN10 vs. ALN5/P5: RR } \\
1.40[0.52,3.74]\end{array}$ & Medium & Direct & $\begin{array}{l}\text { Highly } \\
\text { imprecise }\end{array}$ & $\begin{array}{l}\text { Unknown but } \\
\text { multisite trial }\end{array}$ & Undetected & Insufficient \\
\hline \multirow[t]{3}{*}{$\begin{array}{l}\text { Serious } \\
\text { adverse } \\
\text { events }\end{array}$} & $\begin{array}{l}1 \text { (NR/1099) } \\
\text { PM women } \\
\text { previously received } \\
5 \text { yrs ALN for FN- } \\
\text { BMD T-score } \leq-1.6\end{array}$ & $\begin{array}{l}\text { Black } 2006 \\
\text { NS difference in risk of } \\
\text { serious AEs, mortality, UGI } \\
\text { AEs, or serious UGI AEs } \\
\text { between ALN10 and } \\
\text { ALN5/PBO5 (no numerical } \\
\text { data provided) }\end{array}$ & Low & Direct & $\begin{array}{l}\text { Unknown } \\
\text { (no data) }\end{array}$ & $\begin{array}{l}\text { Unknown but } \\
\text { multisite trial }\end{array}$ & Detected & Insufficient \\
\hline & $\begin{array}{l}1(41 / 350) \\
\text { PM women } \\
\text { previously received } \\
5 \text { yrs ALN for LS } \\
\text { BMD T-score } \leq-2.5\end{array}$ & $\begin{array}{l}\text { Tonino } 2000 \\
\text { NS difference in risk with } 7 \text { yrs } \\
\text { ALN (ALN7) vs. } 5 \text { yrs ALN + } 2 \\
\text { yrs PBO (ALN5/PBO2): } \\
\text { ALN7 } 5 \text { mg/d 11.5\%, ALN7 } 10 \\
\text { mg/d } 12.3 \%, \text { ALN5/PBO2 } \\
11.3 \% \\
\text { ALN7 vs ALN5/PBO } \\
\text { RR = } 1.05[0.57,1.96]\end{array}$ & Low & Direct & $\begin{array}{l}\text { Highly } \\
\text { imprecise }\end{array}$ & $\begin{array}{l}\text { Unknown but } \\
\text { multisite trial }\end{array}$ & Detected & Insufficient \\
\hline & $\begin{array}{l}1(61 / 247) \\
\text { PM women } \\
\text { previously received } \\
5 \text { yrs ALN for LS } \\
\text { BMD T-score } \leq-2.5\end{array}$ & $\begin{array}{l}\text { Bone } 2004 \\
\text { NS difference in risk with } 10 \\
\text { yrs ALN (ALN10) vs. } 7 \text { yrs } \\
\text { ALN + } 3 \text { yrs PBO } \\
\text { (ALN7/PBO3): } \\
\text { ALN10 } 5 \text { mg/d } 32.1 \%, \text { ALN10 } \\
10 \mathrm{mg} / \mathrm{d} 20.9 \%, \text { ALN7/PBO3 }\end{array}$ & Medium & Direct & Imprecise & $\begin{array}{l}\text { Unknown but } \\
\text { multisite trial }\end{array}$ & Detected & Insufficient \\
\hline
\end{tabular}




\begin{tabular}{|c|c|c|c|c|c|c|c|c|}
\hline Outcome & $\begin{array}{l}\text { \# Studies } \\
\text { (n/N } \\
\text { events/participants } \\
\text { evaluated) } \\
\text { Population } \\
\end{array}$ & $\begin{array}{l}\text { Summary statistics } \\
{[95 \% \mathrm{Cl}]}\end{array}$ & $\begin{array}{l}\text { Study } \\
\text { limitations }\end{array}$ & Directness & Precision & Consistency & $\begin{array}{l}\text { Reporting } \\
\text { Bias }\end{array}$ & SOE \\
\hline & & $\begin{array}{l}21.7 \% \\
\text { ALN10 vs. ALN7/PBO3 } \\
\text { RR }=1.21[0.75,1.96]\end{array}$ & & & & & & \\
\hline $\begin{array}{l}\text { ST/FS } \\
\text { fracture (rare } \\
\text { x-ray review } \\
\text { for } \\
\text { confirmation } \\
\text { of AFF } \\
\text { features) }\end{array}$ & $\begin{array}{l}1(3 / 1099) \\
\text { PM women } \\
\text { previously received } \\
5 \text { yrs ALN for FN- } \\
\text { BMD } \leq-1.6\end{array}$ & $\begin{array}{l}\text { Black } 2006 \\
\text { NS difference in risk with } 10 \\
\text { yrs ALN (ALN10) vs. } 5 \text { yrs } \\
\text { ALN + } 5 \text { yrs PBO } \\
\text { (ALN5/PBO5): } \\
\text { ALN10 0.030\%, ALN5/PBO5 } \\
0.023 \% \\
\text { HR }=1.33[0.12,14.67]\end{array}$ & Low & Direct & $\begin{array}{l}\text { Highly } \\
\text { Imprecise }\end{array}$ & $\begin{array}{l}\text { Unknown but } \\
\text { multisite trial }\end{array}$ & Undetected & Insufficient \\
\hline $\begin{array}{l}\text { ST/FS } \\
\text { fracture } \\
\text { without x-ray } \\
\text { review for } \\
\text { confirmation } \\
\text { of AFF } \\
\text { features }\end{array}$ & $\begin{array}{l}1(47 / 39502) \\
\text { Women aged } \geq 45 \mathrm{yr} \\
\text { with } \geq 3 \text { yr prior } \\
\geq 50 \% \text { adherent } \\
\text { bisphosphonate use } \\
\text { ( } 99 \% \text { alendronate) }\end{array}$ & $\begin{array}{l}\text { Adams } 2018 \\
\text { Increased risk with } \\
\text { bisphosphonate (alendronate) } \\
\text { continuation vs. } \\
\text { discontinuation } \\
\text { Pooled persistent and } \\
\text { nonpersistent continuation } \\
\text { groups } 0.15 \%(44 / 28005) \text { vs. } \\
\text { discontinuation } 0.03 \% \\
(3 / 11497) \\
\text { OR=6.03 }[1.87,19.42]\end{array}$ & Medium & Direct & Imprecise & Unknown & Undetected & Low \\
\hline $\begin{array}{l}\text { Osteonecrosi } \\
\text { s of the jaw }\end{array}$ & $\begin{array}{l}1(0 / 1099) \\
\text { PM women } \\
\text { previously received } \\
5 \text { yrs ALN for FN } \\
\text { BMD T-score } \leq-1.6\end{array}$ & $\begin{array}{l}\text { Black } 2006 \\
\text { "No cases of ONJ were } \\
\text { observed." }\end{array}$ & Low & Direct & $\begin{array}{l}\text { Highly } \\
\text { Imprecise }\end{array}$ & $\begin{array}{l}\text { Unknown but } \\
\text { multisite trial }\end{array}$ & Undetected & Insufficient \\
\hline
\end{tabular}

Abbreviations: $\mathrm{AE}=$ adverse events; $\mathrm{ALN}=$ alendronate; $\mathrm{BMD}=$ bone mineral density; $\mathrm{CF}=$ clinical fracture; $\mathrm{CI}=$ confidence interval; FIT=Fracture Intervention Trial; FN=femoral

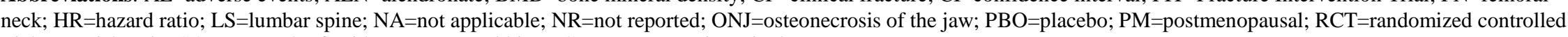
trial; RR=risk ratio; $\mathrm{SOE}=$ =strength of evidence; $\mathrm{TH}=$ total hip; $\mathrm{UGI}=$ upper gastrointestinal; yr=years 
Table D10. Strength of evidence assessments: Long-term alendronate treatment versus no treatment

\begin{tabular}{|c|c|c|c|c|c|c|c|c|c|}
\hline Outcome & $\begin{array}{l}\text { \# Studies } \\
\text { (n/N } \\
\text { events/participants } \\
\text { evaluated) } \\
\text { Study description }\end{array}$ & $\begin{array}{l}\text { Summary } \\
\text { statistics } \\
{[95 \% \mathrm{Cl}]}\end{array}$ & $\begin{array}{l}\text { Study } \\
\text { Limitations }\end{array}$ & Directness & Precision & Consistency & $\begin{array}{l}\text { Reporting } \\
\text { Bias }\end{array}$ & $\begin{array}{l}\text { Optional } \\
\text { Components }\end{array}$ & SOE \\
\hline $\begin{array}{l}\text { Incident } \\
\text { clinical } \\
\text { fracture }\end{array}$ & 0 & NR & NA & NA & NA & NA & NA & NA & Insufficient \\
\hline $\begin{array}{l}\text { Incident } \\
\text { nonvertebral } \\
\text { fracture }\end{array}$ & 0 & NR & NA & NA & NA & NA & NA & NA & Insufficient \\
\hline $\begin{array}{l}\text { Incident hip } \\
\text { fracture }\end{array}$ & 0 & NR & NA & NA & NA & NA & NA & NA & Insufficient \\
\hline $\begin{array}{l}\text { Incident } \\
\text { nonhip } \\
\text { nonvertebral } \\
\text { fracture }\end{array}$ & 0 & NR & NA & NA & NA & NA & NA & NA & Insufficient \\
\hline $\begin{array}{l}\text { Incident major } \\
\text { osteoporotic } \\
\text { fracture }\end{array}$ & 0 & NR & NA & NA & NA & NA & NA & NA & Insufficient \\
\hline $\begin{array}{l}\text { Incident } \\
\text { clinical } \\
\text { vertebral } \\
\text { fracture }\end{array}$ & 0 & & NA & NA & NA & NA & NA & NA & Insufficient \\
\hline $\begin{array}{l}\text { Incident } \\
\text { radiographic } \\
\text { vertebral } \\
\text { fracture }\end{array}$ & 0 & NR & NA & NA & NA & NA & NA & NA & Insufficient \\
\hline $\begin{array}{l}\text { Serious } \\
\text { adverse } \\
\text { events }\end{array}$ & 0 & NR & NA & NA & NA & NA & NA & NA & Insufficient \\
\hline
\end{tabular}




\begin{tabular}{|c|c|c|c|c|c|c|c|c|c|}
\hline Outcome & $\begin{array}{l}\text { \# Studies } \\
\text { (n/N } \\
\text { events/participants } \\
\text { evaluated) } \\
\text { Study description }\end{array}$ & $\begin{array}{l}\text { Summary } \\
\text { statistics } \\
{[95 \% \mathrm{Cl}]}\end{array}$ & $\begin{array}{l}\text { Study } \\
\text { Limitations }\end{array}$ & Directness & Precision & Consistency & $\begin{array}{l}\text { Reporting } \\
\text { Bias }\end{array}$ & $\begin{array}{l}\text { Optional } \\
\text { Components }\end{array}$ & SOE \\
\hline \multirow[t]{2}{*}{$\begin{array}{l}\text { ST/FS } \\
\text { fracture } \\
\text { without x-ray } \\
\text { confirmation } \\
\text { of AFF } \\
\text { features }\end{array}$} & $\begin{array}{l}1(309 / 220,360) \\
\text { Retrospective } \\
\text { cohort. AFF defined } \\
\text { by diagnosis codes } \\
\text { only. ALN treatment } \\
\text { duration range } 0-11 \\
\text { yrs (mean } \sim 3.8)\end{array}$ & $\begin{array}{l}\text { Vestergaard } \\
2012 \\
\text { Higher risk with } \\
\text { ALN vs. no } \\
\text { osteoporosis } \\
\text { drug treatment } \\
\text { Subtrochanteric } \\
\text { fracture: } \\
\text { ALN 0.017\%, } \\
\text { No treatment } \\
0.006 \% \text {; } \\
\text { HR=2.41 [1.78, } \\
3.27] \\
\text { Femoral shaft } \\
\text { fracture: } \\
\text { ALN } 0.012 \%, \\
\text { No treatment } \\
0.003 \% \text {; } \\
\text { HR=2.90 [1.97, } \\
4.26]\end{array}$ & Medium & Direct & Imprecise & Unknown & Undetected & $\begin{array}{l}\text { Large } \\
\text { magnitude of } \\
\text { effect }\end{array}$ & Low \\
\hline & $\begin{array}{l}1(5 / 534) \\
\text { Retrospective } \\
\text { cohort, AFF defined } \\
\text { by diagnosis codes } \\
\text { only. Subgroup with } \\
\text { ALN treatment } \\
\text { duration }>6 \text { years }\end{array}$ & $\begin{array}{l}\text { Abrahamsen } \\
2009 \\
\text { No difference in } \\
\text { risk with ALN } \\
\text { vs. no } \\
\text { bisphosphonate } \\
\text { treatment } \\
\text { ALN \% NR, No } \\
\text { bisphosphonate } \\
\% \text { NR; } \\
\text { HR=1.37 [0.22, } \\
8.62]\end{array}$ & Medium & Direct & $\begin{array}{l}\text { Highly } \\
\text { Imprecise }\end{array}$ & Unknown & Undetected & NA & Insufficient \\
\hline
\end{tabular}




\begin{tabular}{|c|c|c|c|c|c|c|c|c|c|}
\hline Outcome & $\begin{array}{l}\text { \# Studies } \\
\text { (n/N } \\
\text { events/participants } \\
\text { evaluated) } \\
\text { Study description }\end{array}$ & $\begin{array}{l}\text { Summary } \\
\text { statistics } \\
{[95 \% \mathrm{Cl}]}\end{array}$ & $\begin{array}{l}\text { Study } \\
\text { Limitations }\end{array}$ & Directness & Precision & Consistency & $\begin{array}{l}\text { Reporting } \\
\text { Bias }\end{array}$ & $\begin{array}{l}\text { Optional } \\
\text { Components }\end{array}$ & SOE \\
\hline $\begin{array}{l}\text { Osteonecrosi } \\
\mathrm{s} \text { of the jaw }\end{array}$ & $\begin{array}{l}1(28 / 220,360) \\
\text { Retrospective } \\
\text { cohort. ONJ defined } \\
\text { by diagnosis codes } \\
\text { only. ALN treatment } \\
\text { duration range } 0-11 \\
\text { yrs (mean } \sim 3.8)\end{array}$ & $\begin{array}{l}\text { Vestergaard } \\
2012 \\
\text { Higher risk with } \\
\text { ALN vs no } \\
\text { treatment } \\
\text { Any jaw event: } \\
\text { ALN } 0.03 \% \text { vs } \\
\text { No treatment } \\
0.01 \% \text {; } \\
\text { HR=3.15 [1.44, } \\
6.87]\end{array}$ & Medium & Direct & Imprecise & Unknown & Undetected & $\begin{array}{l}\text { Large } \\
\text { magnitude of } \\
\text { effect }\end{array}$ & Low \\
\hline
\end{tabular}

Abbreviations: AFF=atypical femoral fracture; AE=adverse events; ALN=alendronate; $\mathrm{CI}=$ confidence interval; HR=hazard ratio; ICF=incident clinical fracture; LS=lumbar spine; MOF=major osteoporotic fracture; NA=not applicable; NR=not reported; ONJ=osteonecrosis of the jaw; SOE=strength of evidence; ST/FS=subtrochanteric/femoral shaft; yr=years

Table D11. Strength of evidence assessments: Long-term alendronate treatment versus raloxifene or calcitonin or versus raloxifene alone

\begin{tabular}{|c|c|c|c|c|c|c|c|c|c|}
\hline Outcome & $\begin{array}{l}\text { \# Studies } \\
\text { (n/N } \\
\text { events/participants } \\
\text { evaluated) } \\
\text { Study description } \\
\end{array}$ & $\begin{array}{l}\text { Summary } \\
\text { statistics } \\
{[95 \% \mathrm{Cl}]}\end{array}$ & $\begin{array}{l}\text { Study } \\
\text { Limitations }\end{array}$ & Directness & Precision & Consistency & $\begin{array}{l}\text { Reporting } \\
\text { Bias }\end{array}$ & $\begin{array}{l}\text { Optional } \\
\text { Components }\end{array}$ & SOE \\
\hline $\begin{array}{l}\text { Incident } \\
\text { clinical } \\
\text { fracture }\end{array}$ & 0 & $\mathrm{NR}$ & NA & NA & NA & NA & NA & NA & Insufficient \\
\hline $\begin{array}{l}\text { Incident } \\
\text { nonvertebral } \\
\text { fracture }\end{array}$ & 0 & $\mathrm{NR}$ & NA & NA & NA & NA & NA & NA & Insufficient \\
\hline $\begin{array}{l}\text { Incident hip } \\
\text { fracture }\end{array}$ & 0 & NR & NA & NA & NA & NA & NA & NA & Insufficient \\
\hline $\begin{array}{l}\text { Incident } \\
\text { nonhip } \\
\text { nonvertebral } \\
\text { fracture } \\
\end{array}$ & 0 & NR & NA & NA & NA & NA & NA & NA & Insufficient \\
\hline $\begin{array}{l}\text { Incident } \\
\text { major } \\
\text { osteoporotic } \\
\text { fracture } \\
\end{array}$ & 0 & NR & NA & NA & NA & NA & NA & NA & Insufficient \\
\hline
\end{tabular}




\begin{tabular}{|c|c|c|c|c|c|c|c|c|c|}
\hline Outcome & $\begin{array}{l}\text { \# Studies } \\
\text { (n/N } \\
\text { events/participants } \\
\text { evaluated) } \\
\text { Study description }\end{array}$ & $\begin{array}{l}\text { Summary } \\
\text { statistics } \\
{[95 \% \mathrm{Cl}]}\end{array}$ & $\begin{array}{l}\text { Study } \\
\text { Limitations }\end{array}$ & Directness & Precision & Consistency & $\begin{array}{l}\text { Reporting } \\
\text { Bias }\end{array}$ & $\begin{array}{l}\text { Optional } \\
\text { Components }\end{array}$ & SOE \\
\hline $\begin{array}{l}\text { Incident } \\
\text { clinical } \\
\text { vertebral } \\
\text { fracture }\end{array}$ & 0 & NR & NA & NA & NA & NA & NA & NA & Insufficient \\
\hline $\begin{array}{l}\text { Incident } \\
\text { radiographic } \\
\text { vertebral } \\
\text { fracture }\end{array}$ & 0 & NR & NA & NA & NA & NA & NA & NA & Insufficient \\
\hline $\begin{array}{l}\text { Serious } \\
\text { adverse } \\
\text { events }\end{array}$ & 0 & NR & NA & NA & NA & NA & NA & NA & Insufficient \\
\hline $\begin{array}{l}\text { Atypical } \\
\text { femoral } \\
\text { fracture }\end{array}$ & 0 & NR & NA & NA & NA & NA & NA & NA & Insufficient \\
\hline \multirow[t]{2}{*}{$\begin{array}{l}\text { Osteonecrosi } \\
\text { s of the jaw }\end{array}$} & $\begin{array}{l}1(353 / 8,354) \\
\text { ONJ defined by } \\
\text { diagnosis codes, } \\
\text { radiographic and } \\
\text { pathological } \\
\text { confirmation. ALN } \\
\text { duration range } 0-11 \\
\text { yrs (mean } 4 \text { yr in } \\
\text { ONJ group). }\end{array}$ & $\begin{array}{l}\text { Chiu } 2014 \\
\text { Higher risk } \\
\text { with ALN } \\
\text { vs. } \\
\text { raloxifene: } \\
\text { HR=7.42 } \\
{[1.02,} \\
54.09]\end{array}$ & Medium & Direct & Imprecise & Unknown & Undetected & $\begin{array}{l}\text { Large } \\
\text { magnitude of } \\
\text { effect }\end{array}$ & Low \\
\hline & $\begin{array}{l}1(37 / 43,645) \\
\text { ONJ defined only by } \\
\text { diagnosis codes. } \\
\text { ALN duration up to } \\
6 \text { yrs. }\end{array}$ & $\begin{array}{l}\text { Lin } 2014 \\
\text { No } \\
\text { difference in } \\
\text { risk with } \\
\text { ALN vs. } \\
\text { raloxifene or } \\
\text { calcitonin: } \\
\text { ALN } 0.15 \% \\
\text { vs. } \\
\text { raloxifene/c } \\
\text { alcitonin } \\
0.08 \% ; \\
\text { HR }=0.86 \\
{[0.44,1.69]}\end{array}$ & Medium & Direct & $\begin{array}{l}\text { Highly } \\
\text { Imprecise }\end{array}$ & Unknown & Undetected & NA & Insufficient \\
\hline
\end{tabular}


Abbreviations: $\mathrm{AE}=$ adverse events; $\mathrm{ALN}=$ alendronate; $\mathrm{CI}=$ confidence interval; $\mathrm{HR}=$ hazard ratio; $\mathrm{MOF}=$ major osteoporotic fracture; $\mathrm{NA}=$ not applicable; $\mathrm{NR}=$ not reported; $\mathrm{ONJ}=$ osteonecrosis of the jaw; $\mathrm{SOE}=$ strength of evidence; $y \mathrm{r}=$ years 


\section{Zoledronic Acid}

Table D12. Strength of evidence assessments: Long-term zoledronic acid treatment (6 yr) versus placebo

\begin{tabular}{|c|c|c|c|c|c|c|c|c|}
\hline Outcome & $\begin{array}{l}\text { \# Studies } \\
\text { (n/N } \\
\text { events/participants } \\
\text { evaluated) }\end{array}$ & $\begin{array}{l}\text { Summary statistics } \\
{[95 \% \mathrm{Cl}]}\end{array}$ & $\begin{array}{l}\text { Study } \\
\text { Limitations }\end{array}$ & Directness & Precision & Consistency & $\begin{array}{l}\text { Reporting } \\
\text { Bias }\end{array}$ & SOE \\
\hline $\begin{array}{l}\text { Incident clinical } \\
\text { fracture }\end{array}$ & $1(377 / 2000)$ & $\begin{array}{l}\text { Reid } 2018 \\
\text { Lower risk with zoledronic } \\
\text { acid vs. placebo: } \\
\text { ZOL } 16 \% \text { vs. placebo } \\
21 \% \\
\text { HR }=0.73[0.60,0.90]\end{array}$ & Low & Direct & Imprecise & Unknown & Undetected & Moderate \\
\hline $\begin{array}{l}\text { Incident } \\
\text { nonvertebral } \\
\text { fracture }\end{array}$ & $1(249 / 2000)$ & $\begin{array}{l}\text { Reid } 2018 \\
\text { Lower risk with zoledronic } \\
\text { acid vs. placebo } \\
\text { ZOL } 10 \% \text { vs placebo } 15 \% \\
\text { HR }=0.66[0.51,0.85]\end{array}$ & Low & Direct & Precise & Unknown & Undetected & High \\
\hline $\begin{array}{l}\text { Incident hip } \\
\text { fracture }\end{array}$ & $1(20 / 2000)$ & $\begin{array}{l}\text { Reid } 2018 \\
\text { ND in risk with zoledronic } \\
\text { acid vs. placebo } \\
\text { ZOL } 0.8 \% \text { vs placebo } \\
1.2 \% \\
\text { HR }=0.66[0.27,1.16]\end{array}$ & Low & Direct & $\begin{array}{l}\text { Highly } \\
\text { Imprecise }\end{array}$ & Unknown & Undetected & Low \\
\hline $\begin{array}{l}\text { Incident nonhip } \\
\text { nonvertebral } \\
\text { fracture }\end{array}$ & 0 & NR & NA & NA & NA & NA & NA & Insufficient \\
\hline $\begin{array}{l}\text { Incident major } \\
\text { osteoporotic } \\
\text { fracture }\end{array}$ & 0 & NR & NA & NA & NA & NA & NA & Insufficient \\
\hline $\begin{array}{l}\text { Incident clinical } \\
\text { vertebral fracture }\end{array}$ & $1(48 / 2000)$ & $\begin{array}{l}\text { Reid } 2018 \\
\text { Lower risk with zoledronic } \\
\text { acid vs. placebo: } \\
\text { ZOL } 1.4 \% \text { vs placebo } \\
3.4 \% \\
\text { HR }=0.41[0.22,0.75]\end{array}$ & Low & Direct & Imprecise & Unknown & Undetected & Moderate \\
\hline $\begin{array}{l}\text { Incident } \\
\text { radiographic } \\
\text { vertebral fracture }\end{array}$ & NR & NR & NA & NA & NA & NA & NA & Insufficient \\
\hline
\end{tabular}




\begin{tabular}{|c|c|c|c|c|c|c|c|c|}
\hline Outcome & $\begin{array}{l}\text { \# Studies } \\
\text { (n/N } \\
\text { events/participants } \\
\text { evaluated) }\end{array}$ & $\begin{array}{l}\text { Summary statistics } \\
{[95 \% \mathrm{Cl}]}\end{array}$ & $\begin{array}{l}\text { Study } \\
\text { Limitations }\end{array}$ & Directness & Precision & Consistency & $\begin{array}{l}\text { Reporting } \\
\text { Bias }\end{array}$ & SOE \\
\hline $\begin{array}{l}\text { Serious adverse } \\
\text { events }\end{array}$ & $1(843 / 2000)$ & $\begin{array}{l}\text { Reid } 2018 \\
\text { ND in risk with zoledronic } \\
\text { acid vs. placebo: } \\
\text { ZOL } 40 \% \text { vs placebo } 44 \% \\
\text { OR }=0.84[0.70,1.00]\end{array}$ & Low & Direct & Imprecise & Unknown & Undetected & Low \\
\hline $\begin{array}{l}\text { Atypical femoral } \\
\text { fracture }\end{array}$ & 0 & NR & NA & NA & NA & NA & NA & Insufficient \\
\hline $\begin{array}{l}\text { Osteonecrosis of } \\
\text { the jaw }\end{array}$ & $1(0 / 2000)$ & $\begin{array}{l}\text { Reid } 2018 \\
\text { No cases occured }\end{array}$ & Low & Direct & NA & Unknown, & Undetected & Insufficient \\
\hline
\end{tabular}

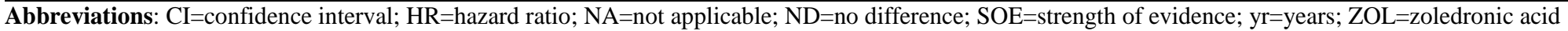

Table D13. Strength of evidence assessments: zoledronic acid continuation (2 years zoledronic acid) versus discontinuation (1 year zoledronic acid followed by 1 year placebo)

\begin{tabular}{|c|c|c|c|c|c|c|c|c|}
\hline Outcome & $\begin{array}{l}\text { \# Studies } \\
\text { (n/N } \\
\text { events/participants } \\
\text { evaluated) }\end{array}$ & $\begin{array}{l}\text { Summary statistics } \\
{[95 \% \mathrm{Cl}]}\end{array}$ & $\begin{array}{l}\text { Study } \\
\text { Limitations }\end{array}$ & Directness & Precision & Consistency & $\begin{array}{l}\text { Reporting } \\
\text { Bias }\end{array}$ & SOE \\
\hline $\begin{array}{l}\text { Incident clinical } \\
\text { fracture }\end{array}$ & $1(10 / 379)$ & $\begin{array}{l}\text { McClung } 2009 \\
\text { NS difference in risk with } \\
\text { zoledronic acid } 2 \text { yrs (Z2) } \\
\text { vs. zoledronic acid } 1 \mathrm{yr} \\
\text { followed by placebo } 1 \mathrm{yr} \\
\text { (Z1/P1): } \\
\text { Z2 } 3.0 \% \text { vs. Z1/P1 } 2.2 \% \text {; } \\
\text { RR=1.37 [0.39, } 4.78]\end{array}$ & Low & Direct & $\begin{array}{l}\text { Highly } \\
\text { imprecise }\end{array}$ & $\begin{array}{l}\text { Unknown, } \\
\text { but multisite } \\
\text { trial }\end{array}$ & Undetected & Insufficient \\
\hline $\begin{array}{l}\text { Incident } \\
\text { nonvertebral } \\
\text { fracture }\end{array}$ & 0 & NR & NA & NA & NA & NA & NA & Insufficient \\
\hline $\begin{array}{l}\text { Incident hip } \\
\text { fracture }\end{array}$ & 0 & NR & NA & NA & NA & NA & NA & Insufficient \\
\hline $\begin{array}{l}\text { Incident nonhip } \\
\text { nonvertebral } \\
\text { fracture }\end{array}$ & 0 & NR & NA & NA & NA & NA & NA & Insufficient \\
\hline $\begin{array}{l}\text { Incident major } \\
\text { osteoporotic } \\
\text { fracture }\end{array}$ & 0 & NR & NA & NA & NA & NA & NA & Insufficient \\
\hline $\begin{array}{l}\text { Incident clinical } \\
\text { vertebral fracture }\end{array}$ & 0 & NR & NA & NA & NA & NA & NA & Insufficient \\
\hline
\end{tabular}




\begin{tabular}{|c|c|c|c|c|c|c|c|c|}
\hline Outcome & $\begin{array}{l}\text { \# Studies } \\
\text { (n/N } \\
\text { events/participants } \\
\text { evaluated) }\end{array}$ & $\begin{array}{l}\text { Summary statistics } \\
{[95 \% \mathrm{Cl}]}\end{array}$ & $\begin{array}{l}\text { Study } \\
\text { Limitations }\end{array}$ & Directness & Precision & Consistency & $\begin{array}{l}\text { Reporting } \\
\text { Bias }\end{array}$ & SOE \\
\hline $\begin{array}{l}\text { Incident } \\
\text { radiographic } \\
\text { vertebral fracture }\end{array}$ & 0 & NR & NA & NA & NA & NA & NA & Insufficient \\
\hline $\begin{array}{l}\text { Serious adverse } \\
\text { events }\end{array}$ & $1(38 / 379)$ & $\begin{array}{l}\text { McClung } 2009 \\
\text { NS difference in risk with } \\
\text { Z2 vs. Z1/P1: } \\
\text { Z2 } 9.4 \% \text { vs. Z1/P1 10.6\%; } \\
\text { RR=0.91 }[0.50,1.67]\end{array}$ & Low & Direct & $\begin{array}{l}\text { Highly } \\
\text { imprecise }\end{array}$ & $\begin{array}{l}\text { Unknown, } \\
\text { but multisite } \\
\text { trial }\end{array}$ & Undetected & Low \\
\hline $\begin{array}{l}\text { Atypical femoral } \\
\text { fracture }\end{array}$ & 0 & NR & NA & NA & NA & NA & NA & Insufficient \\
\hline $\begin{array}{l}\text { Osteonecrosis of } \\
\text { the jaw }\end{array}$ & $1(0 / 379)$ & $\begin{array}{l}\text { McClung } 2009 \\
\text { No cases occurred }\end{array}$ & Low & Direct & $\begin{array}{l}\text { Highly } \\
\text { Imprecise }\end{array}$ & $\begin{array}{l}\text { Unknown, } \\
\text { but multisite } \\
\text { trial }\end{array}$ & Undetected & Insufficient \\
\hline
\end{tabular}

Abbreviations: $\mathrm{AE}=$ adverse events; $\mathrm{CI}=$ confidence interval; $\mathrm{NA}=$ not applicable; $\mathrm{NR}=$ not reported; $\mathrm{P}=$ placebo; $\mathrm{RR}=$ risk ratio; $\mathrm{SOE}=$ strength of evidence; $\mathrm{yr}=\mathrm{years}$; $\mathrm{Z}=\mathrm{zoledronic}$ acid

Table D14. Strength of evidence assessments: zoledronic acid continuation (6 years zoledronic acid) versus discontinuation ( 3 years zoledronic acid followed by 3 years placebo)

\begin{tabular}{|c|c|c|c|c|c|c|c|c|}
\hline Outcome & $\begin{array}{l}\text { \# Studies } \\
\text { (n/N } \\
\text { events/participants } \\
\text { evaluated) }\end{array}$ & $\begin{array}{l}\text { Summary statistics } \\
{[95 \% \mathrm{Cl}]}\end{array}$ & $\begin{array}{l}\text { Study } \\
\text { Limitations }\end{array}$ & Directness & Precision & Consistency & $\begin{array}{l}\text { Reporting } \\
\text { Bias }\end{array}$ & SOE \\
\hline $\begin{array}{l}\text { Incident } \\
\text { clinical fracture }\end{array}$ & $1(\mathrm{NR} / 1233)$ & $\begin{array}{l}\text { Black } 2012 \\
\text { NS difference in risk with } \\
\text { zoledronic acid } 6 \text { yrs (Z6) vs. } \\
\text { zoledronic acid } 3 \text { yrs followed } \\
\text { by placebo } 3 \text { yrs (Z3/P3): } \\
\text { Incidence NR; } \\
\text { HR=1.04 [0.71, 1.54] }\end{array}$ & Low & Direct & Imprecise & $\begin{array}{l}\text { Unknown, } \\
\text { but multisite } \\
\text { trial }\end{array}$ & $\begin{array}{l}\text { Number of } \\
\text { events not } \\
\text { reported }\end{array}$ & Moderate \\
\hline $\begin{array}{l}\text { Incident } \\
\text { nonvertebral } \\
\text { fracture }\end{array}$ & $1(92 / 1233)$ & $\begin{array}{l}\text { Black } 2012 \\
\text { NS difference in risk with Z6 vs. } \\
\text { Z3/P3: } \\
\text { Z6 } 7.6 \% \text { vs. Z3/P3 8.2\%; } \\
\text { HR=0.99 }[0.7,1.5]\end{array}$ & Low & Direct & Imprecise & $\begin{array}{l}\text { Unknown, } \\
\text { but multisite } \\
\text { trial }\end{array}$ & Undetected & Moderate \\
\hline $\begin{array}{l}\text { Incident hip } \\
\text { fracture }\end{array}$ & $1(15 / 1233)$ & $\begin{array}{l}\text { Black } 2012 \\
\text { NS difference in risk with Z6 vs. } \\
\text { Z3/P3: } \\
\text { Z6 1.3\% vs. Z3/P3 1.4\%; } \\
\text { HR }=0.90[0.33,2.49]\end{array}$ & Low & Direct & $\begin{array}{l}\text { Highly } \\
\text { imprecise }\end{array}$ & $\begin{array}{l}\text { Unknown, } \\
\text { but multisite } \\
\text { trial }\end{array}$ & Undetected & Insufficient \\
\hline
\end{tabular}




\begin{tabular}{|c|c|c|c|c|c|c|c|c|}
\hline Outcome & $\begin{array}{l}\text { \# Studies } \\
\text { (n/N } \\
\text { events/participants } \\
\text { evaluated) }\end{array}$ & $\begin{array}{l}\text { Summary statistics } \\
{[95 \% \mathrm{Cl}]}\end{array}$ & $\begin{array}{l}\text { Study } \\
\text { Limitations }\end{array}$ & Directness & Precision & Consistency & $\begin{array}{l}\text { Reporting } \\
\text { Bias }\end{array}$ & SOE \\
\hline $\begin{array}{l}\text { Incident } \\
\text { nonhip } \\
\text { nonvertebral } \\
\text { fracture }\end{array}$ & 0 & NR & NA & NA & NA & NA & NA & Insufficient \\
\hline $\begin{array}{l}\text { Incident major } \\
\text { osteoporotic } \\
\text { fracture }\end{array}$ & 0 & NR & NA & NA & NA & NA & NA & Insufficient \\
\hline $\begin{array}{l}\text { Incident } \\
\text { clinical } \\
\text { vertebral } \\
\text { fracture }\end{array}$ & $1(\mathrm{NR} / 1233)$ & $\begin{array}{l}\text { Black } 2012 \\
\text { NS difference in risk with } Z 6 \text { vs. } \\
\text { Z3/P3: } \\
\text { HR=1.81 }[0.53,6.2]\end{array}$ & Low & Direct & $\begin{array}{l}\text { Highly } \\
\text { imprecise }\end{array}$ & $\begin{array}{l}\text { Unknown, } \\
\text { but multisite } \\
\text { trial }\end{array}$ & $\begin{array}{l}\text { Number of } \\
\text { events not } \\
\text { reported }\end{array}$ & Insufficient \\
\hline $\begin{array}{l}\text { Incident } \\
\text { radiographic } \\
\text { vertebral } \\
\text { fracture }\end{array}$ & $1(44 / 1233)$ & $\begin{array}{l}\text { Black } 2012 \\
\text { Lower risk with Z6 vs. Z3/P3: } \\
\text { Z6 } 3.0 \% \text { vs. Z3/P3 6.2\%; } \\
\text { OR=0.51 [0.26, } 0.95]\end{array}$ & Low & Direct & $\begin{array}{l}\text { Highly } \\
\text { Imprecise }\end{array}$ & $\begin{array}{l}\text { Unknown, } \\
\text { but multisite } \\
\text { trial }\end{array}$ & Undetected & Low \\
\hline $\begin{array}{l}\text { Serious } \\
\text { adverse } \\
\text { events }\end{array}$ & $1(359 / 1233)$ & $\begin{array}{l}\text { Black } 2012 \\
\text { NS difference in risk with Z6 vs. } \\
\text { Z3/P3: } \\
\text { Z6 31\% vs. Z3/P3 27\%; } \\
\text { RR=1.14 }[0.96,1.36]\end{array}$ & Low & Direct & Imprecise & $\begin{array}{l}\text { Unknown, } \\
\text { but multisite } \\
\text { trial }\end{array}$ & Undetected & Low \\
\hline $\begin{array}{l}\text { "Atypical } \\
\text { femoral } \\
\text { fracture" (not } \\
\text { defined) }\end{array}$ & $1(0 / 1233)$ & $\begin{array}{l}\text { Black } 2012 \\
\text { No cases occurred }\end{array}$ & Low & Direct & $\begin{array}{l}\text { Highly } \\
\text { imprecise }\end{array}$ & $\begin{array}{l}\text { Unknown, } \\
\text { but multisite } \\
\text { trial }\end{array}$ & Undetected & Insufficient \\
\hline $\begin{array}{l}\text { Osteonecrosis } \\
\text { of the jaw }\end{array}$ & $1(1 / 1233)$ & $\begin{array}{l}\text { Black } 2012 \\
\text { One case of ONJ in Z6 group, } \\
\text { none in Z3/P3 group }\end{array}$ & Low & Direct & $\begin{array}{l}\text { Highly } \\
\text { imprecise }\end{array}$ & $\begin{array}{l}\text { Unknown, } \\
\text { but multisite } \\
\text { trial }\end{array}$ & Undetected & Insufficient \\
\hline
\end{tabular}

Abbreviations: $\mathrm{AE}=$ adverse events; $\mathrm{CI}=$ confidence interval; $\mathrm{HR}=$ hazard ratio; $\mathrm{NA}=$ not applicable; $\mathrm{NR}=$ not reported; $\mathrm{ONJ}=\mathrm{osteonecrosis}$ of the jaw; $\mathrm{P}=\mathrm{placebo}$; $\mathrm{RR}=$ risk ratio; $\mathrm{SOE}=$ strength of evidence; $\mathrm{yr}=$ years; $\mathrm{Z}=$ zoledronic acid 
Table D15. Strength of evidence assessments: zoledronic acid continuation (9 years zoledronic acid) versus discontinuation ( 6 years zoledronic acid followed by 3 years placebo)

\begin{tabular}{|c|c|c|c|c|c|c|c|c|}
\hline Outcome & $\begin{array}{l}\text { \# Studies } \\
\text { (n/N } \\
\text { events/participants } \\
\text { evaluated) }\end{array}$ & $\begin{array}{l}\text { Evidence Summary } \\
\text { Summary statistics } \\
{[95 \% \mathrm{Cl}]}\end{array}$ & $\begin{array}{l}\text { Study } \\
\text { Limitations }\end{array}$ & Directness & Precision & Consistency & $\begin{array}{l}\text { Reporting } \\
\text { Bias }\end{array}$ & SOE \\
\hline $\begin{array}{l}\text { Incident } \\
\text { clinical fracture }\end{array}$ & $1(19 / 190)$ & $\begin{array}{l}\text { Black } 2015 \\
\text { NS difference in risk with } \\
\text { zoledronic acid } 9 \text { yrs (Z9) vs. } \\
\text { zoledronic acid } 6 \text { yrs followed } \\
\text { by placebo } 3 \text { yrs (Z6/P3): } \\
\text { Z9 } 11 \% \text { vs Z6/P3 9\%; HR=1.11 } \\
{[0.45,2.73]}\end{array}$ & Low & Direct & $\begin{array}{l}\text { Highly } \\
\text { imprecise }\end{array}$ & $\begin{array}{l}\text { Unknown, } \\
\text { but multisite } \\
\text { trial }\end{array}$ & Undetected & Insufficient \\
\hline $\begin{array}{l}\text { Incident } \\
\text { nonvertebral } \\
\text { fracture }\end{array}$ & 0 & NR & NA & NA & NA & NA & NA & Insufficient \\
\hline $\begin{array}{l}\text { Incident hip } \\
\text { fracture }\end{array}$ & 0 & NR & NA & NA & NA & NA & NA & Insufficient \\
\hline $\begin{array}{l}\text { Incident } \\
\text { nonhip } \\
\text { nonvertebral } \\
\text { fracture } \\
\end{array}$ & 0 & NR & NA & NA & NA & NA & NA & Insufficient \\
\hline $\begin{array}{l}\text { Incident major } \\
\text { osteoporotic } \\
\text { fracture }\end{array}$ & 0 & NR & NA & NA & NA & NA & NA & Insufficient \\
\hline $\begin{array}{l}\text { Incident } \\
\text { clinical } \\
\text { vertebral } \\
\text { fracture }\end{array}$ & 0 & NR & NA & NA & NA & NA & NA & Insufficient \\
\hline $\begin{array}{l}\text { Incident } \\
\text { radiographic } \\
\text { vertebral } \\
\text { fracture }\end{array}$ & $1(8 / 190)$ & $\begin{array}{l}\text { Black } 2015 \\
\text { NS difference in risk with Z9 vs. } \\
\text { Z6/P3: } \\
\text { Z9 3.2\% vs Z6/P3 5.3\%; } \\
\text { OR=0.58 }[0.13,2.55]\end{array}$ & Low & Direct & $\begin{array}{l}\text { Highly } \\
\text { imprecise }\end{array}$ & $\begin{array}{l}\text { Unknown, } \\
\text { but multisite } \\
\text { trial }\end{array}$ & Undetected & Insufficient \\
\hline $\begin{array}{l}\text { Serious } \\
\text { adverse } \\
\text { events }\end{array}$ & $1(52 / 190)$ & $\begin{array}{l}\text { Black } 2015 \\
\text { NS difference in risk with Z9 vs. } \\
\text { Z6/P3: } \\
\text { Z9 26\% vs Z6/P3 30\%; } \\
\text { RR=0.86 [0.54, 1.36] }\end{array}$ & Low & Direct & Imprecise & $\begin{array}{l}\text { Unknown, } \\
\text { but multisite } \\
\text { trial }\end{array}$ & Undetected & Low \\
\hline
\end{tabular}




\begin{tabular}{|c|c|c|c|c|c|c|c|c|}
\hline Outcome & $\begin{array}{l}\text { \# Studies } \\
\text { (n/N } \\
\text { events/participants } \\
\text { evaluated) }\end{array}$ & $\begin{array}{l}\text { Evidence Summary } \\
\text { Summary statistics } \\
{[95 \% \mathrm{Cl}]}\end{array}$ & $\begin{array}{l}\text { Study } \\
\text { Limitations }\end{array}$ & Directness & Precision & Consistency & $\begin{array}{l}\text { Reporting } \\
\text { Bias }\end{array}$ & SOE \\
\hline $\begin{array}{l}\text { Atypical } \\
\text { femoral } \\
\text { fracture with } \\
\text { confirmed } \\
\text { radiologic } \\
\text { features }\end{array}$ & $1(0 / 190)$ & $\begin{array}{l}\text { Black } 2015 \\
\text { No cases occurred }\end{array}$ & Low & Direct & $\begin{array}{l}\text { Highly } \\
\text { imprecise }\end{array}$ & Unknown & Undetected & Insufficient \\
\hline $\begin{array}{l}\text { Osteonecrosis } \\
\text { of the jaw }\end{array}$ & $1(0 / 190)$ & $\begin{array}{l}\text { Black } 2015 \\
\text { No cases occurred }\end{array}$ & Low & Direct & $\begin{array}{l}\text { Highly } \\
\text { imprecise }\end{array}$ & Unknown & Undetected & Insufficient \\
\hline
\end{tabular}

Abbreviations: $\mathrm{CI}=$ confidence interval; $\mathrm{HR}=$ hazard ratio; NA=not applicable; NR=not reported; $\mathrm{P}=$ placebo; RR=risk ratio; $\mathrm{SOE}=$ strength of evidence; yr=years; $\mathrm{Z}=$ =zoledronic acid 


\section{Any Bisphosphonate}

Table D16. Strength of evidence assessments: any bisphosphonate use $>\mathbf{3}$ years versus no bisphosphonate

\begin{tabular}{|c|c|c|c|c|c|c|c|c|c|}
\hline Outcome & $\begin{array}{l}\text { \# Studies } \\
\text { (n/N events/ } \\
\text { participants } \\
\text { evaluated) }\end{array}$ & $\begin{array}{l}\text { Summary statistics } \\
{[95 \% \mathrm{Cl}]}\end{array}$ & $\begin{array}{l}\text { Study } \\
\text { Limitations }\end{array}$ & Directness & Precision & Consistency & $\begin{array}{l}\text { Reporting } \\
\text { Bias }\end{array}$ & $\begin{array}{l}\text { Optional } \\
\text { Components }\end{array}$ & SOE \\
\hline $\begin{array}{l}\text { Incident } \\
\text { clinical } \\
\text { fracture }\end{array}$ & 0 & NR & NA & NA & $\mathrm{NA}$ & NA & NA & NA & Insufficient \\
\hline $\begin{array}{l}\text { Incident } \\
\text { nonvertebral } \\
\text { fracture }\end{array}$ & 0 & NR & NA & NA & NA & NA & $\mathrm{NA}$ & NA & Insufficient \\
\hline $\begin{array}{l}\text { Incident hip } \\
\text { fracture }\end{array}$ & 0 & NR & NA & NA & $\mathrm{NA}$ & NA & $\mathrm{NA}$ & $\mathrm{NA}$ & Insufficient \\
\hline $\begin{array}{l}\text { Incident } \\
\text { nonhip } \\
\text { nonvertebral } \\
\text { fracture }\end{array}$ & 0 & NR & $\mathrm{NA}$ & NA & $\mathrm{NA}$ & $\mathrm{NA}$ & $\mathrm{NA}$ & $\mathrm{NA}$ & Insufficient \\
\hline $\begin{array}{l}\text { Incident } \\
\text { major } \\
\text { osteoporotic } \\
\text { fracture }\end{array}$ & 0 & NR & NA & NA & $\mathrm{NA}$ & $\mathrm{NA}$ & $\mathrm{NA}$ & $\mathrm{NA}$ & Insufficient \\
\hline $\begin{array}{l}\text { Incident } \\
\text { clinical } \\
\text { vertebral } \\
\text { fracture }\end{array}$ & 0 & NR & NA & NA & $\mathrm{NA}$ & $\mathrm{NA}$ & $\mathrm{NA}$ & $\mathrm{NA}$ & Insufficient \\
\hline $\begin{array}{l}\text { Incident } \\
\text { radiographic } \\
\text { vertebral } \\
\text { fracture }\end{array}$ & 0 & NR & NA & NA & NA & NA & NA & NA & Insufficient \\
\hline $\begin{array}{l}\text { Serious } \\
\text { adverse } \\
\text { events }\end{array}$ & 0 & NR & NA & NA & $\mathrm{NA}$ & NA & $\mathrm{NA}$ & $\mathrm{NA}$ & Insufficient \\
\hline
\end{tabular}




\begin{tabular}{|c|c|c|c|c|c|c|c|c|c|}
\hline Outcome & $\begin{array}{l}\text { \# Studies } \\
\text { (n/N events/ } \\
\text { participants } \\
\text { evaluated) }\end{array}$ & $\begin{array}{l}\text { Summary statistics } \\
{[95 \% \mathrm{Cl}]}\end{array}$ & $\begin{array}{l}\text { Study } \\
\text { Limitations }\end{array}$ & Directness & Precision & Consistency & $\begin{array}{l}\text { Reporting } \\
\text { Bias }\end{array}$ & $\begin{array}{l}\text { Optional } \\
\text { Components }\end{array}$ & SOE \\
\hline \multirow[t]{2}{*}{$\begin{array}{l}\text { Atypical } \\
\text { femoral } \\
\text { fracture }\end{array}$} & $\begin{array}{l}2 \\
(216 / \sim 2.8 \\
\text { million for } \\
\text { cohort; } 244 \\
\text { BP } \\
\text { users/1124 } \\
\text { participants } \\
\text { for case- } \\
\text { control) }\end{array}$ & $\begin{array}{l}\text { Schilcher } 2015 \\
\text { Higher risk of AFF } \\
\text { Retrospective cohort } \\
\text { analysis: } \\
\geq 4 \text { yr BP use: } \\
\text { RR=126 [55, 288] } \\
\text { Case-control } \\
\text { analysis: } \\
3-4 \text { yr BP use: } \\
\text { OR=40 [17, 91] } \\
4-5 \text { yr BP use: } \\
\text { OR=116 [58, 234] } \\
>5 \text { yr BP use: OR=93 } \\
\text { [66, 132] }\end{array}$ & Medium & Direct & Precise & Consistent & Undetected & $\begin{array}{l}\text { Large } \\
\text { magnitude of } \\
\text { effect }\end{array}$ & Low \\
\hline & $\begin{array}{l}1 \\
\text { (158 BP } \\
\text { users/290 } \\
\text { participants) }\end{array}$ & $\begin{array}{l}\text { Lim } 2018 \\
\text { Case control } \\
\text { Higher risk of AFF } \\
\text { with BP use (mean } \\
5.2 \text { yr) } \\
\text { OR=25.65 [10.74, } \\
61.28]\end{array}$ & Medium & Direct & Precise & Consistent & Undetected & $\begin{array}{l}\text { Large } \\
\text { magnitude of } \\
\text { effect }\end{array}$ & Low \\
\hline $\begin{array}{l}\text { ST/FS } \\
\text { fracture } \\
\text { without } \\
\text { radiologic } \\
\text { confirmation } \\
\text { of AFF } \\
\text { features }\end{array}$ & $\begin{array}{l}1 \\
\text { (228 BP } \\
\text { users/264 } \\
\text { participants) }\end{array}$ & $\begin{array}{l}\text { Erviti } 2013 \\
\text { Case control } \\
\text { Higher risk of } \\
\text { subtrochanteric or } \\
\text { femoral shaft fracture } \\
>3 \text { yr BP use: } \\
\text { OR=9.46 [2.17, 41.3] }\end{array}$ & Medium & Direct & Precise & Unknown & Undetected & $\begin{array}{l}\text { Large } \\
\text { magnitude of } \\
\text { effect }\end{array}$ & Low \\
\hline $\begin{array}{l}\text { Osteonecros } \\
\text { is of the jaw }\end{array}$ & 0 & NR & NA & NA & NA & NA & NA & & Insufficient \\
\hline
\end{tabular}

Abbreviations: $\mathrm{AFF}=$ atypical femoral fracture; $\mathrm{BP}=$ bisphosphonate; $\mathrm{NA}=$ not applicable; $\mathrm{NR}=$ not reported; OR=adjusted odds ration; RR=risk ratio; $\mathrm{SOE}=$ strength of evidence; yr=years

*calculated by EPC 
Table D17. Strength of evidence assessments: Any bisphosphonate use $>\mathbf{3}$ years versus past bisphosphonate use

\begin{tabular}{|c|c|c|c|c|c|c|c|c|c|}
\hline Outcome & $\begin{array}{l}\text { \# Studies } \\
\text { (n/N eventsl } \\
\text { participants } \\
\text { evaluated) }\end{array}$ & $\begin{array}{l}\text { Summary statistics } \\
{[95 \% \mathrm{Cl}]}\end{array}$ & \begin{tabular}{|l} 
Study \\
Limitations
\end{tabular} & Directness & Precision & Consistency & \begin{tabular}{|l} 
Reporting \\
Bias
\end{tabular} & $\begin{array}{l}\text { Optional } \\
\text { Factors }\end{array}$ & SOE \\
\hline $\begin{array}{l}\text { Incident clinical } \\
\text { fracture }\end{array}$ & 0 & NR & NA & NA & NA & NA & NA & NA & Insufficient \\
\hline $\begin{array}{l}\text { Incident } \\
\text { nonvertebral } \\
\text { fracture }\end{array}$ & 0 & NR & NA & NA & NA & NA & NA & NA & Insufficient \\
\hline $\begin{array}{l}\text { Incident hip } \\
\text { fracture }\end{array}$ & 0 & NR & NA & NA & NA & NA & NA & NA & Insufficient \\
\hline $\begin{array}{l}\text { Incident nonhip } \\
\text { nonvertebral } \\
\text { fracture }\end{array}$ & 0 & NR & NA & NA & NA & NA & NA & NA & Insufficient \\
\hline $\begin{array}{l}\text { Incident major } \\
\text { osteoporotic } \\
\text { fracture } \\
\end{array}$ & 0 & NR & NA & NA & NA & NA & NA & NA & Insufficient \\
\hline $\begin{array}{l}\text { Incident clinical } \\
\text { vertebral } \\
\text { fracture }\end{array}$ & 0 & NR & NA & NA & NA & NA & NA & NA & Insufficient \\
\hline $\begin{array}{l}\text { Incident } \\
\text { radiographic } \\
\text { vertebral } \\
\text { fracture }\end{array}$ & 0 & NR & NA & NA & NA & NA & NA & NA & Insufficient \\
\hline $\begin{array}{l}\text { Serious adverse } \\
\text { events }\end{array}$ & 0 & NR & NA & NA & NA & NA & NA & NA & Insufficient \\
\hline $\begin{array}{l}\text { Atypical femoral } \\
\text { fracture with } \\
\text { confirmed AFF } \\
\text { radiologic } \\
\text { features }\end{array}$ & $\begin{array}{l}1 \\
(43 / 172)\end{array}$ & $\begin{array}{l}\text { Koh } 2017 \\
\text { Higher risk AFF with } \\
\geq 5 \text { yr BP use vs. past } \\
\text { BP use lasted } \geq 1 \mathrm{yr} \\
\text { but stopped } \geq 6 \text { mo } \\
\text { ago: } \\
\text { HR=5.17 [2.0, 13.36] } \\
\text { HR=2.37 [1.68, } \\
11.41] \\
\text { HR=3.36 [1.77, } \\
11.91]\end{array}$ & Medium & Direct & Precise & Unknown & Undetected & $\begin{array}{l}\text { Large } \\
\text { magnitude } \\
\text { of effect }\end{array}$ & Low \\
\hline
\end{tabular}




\begin{tabular}{|c|c|c|c|c|c|c|c|c|c|}
\hline Outcome & $\begin{array}{l}\text { \# Studies } \\
\text { (n/N events/ } \\
\text { participants } \\
\text { evaluated) }\end{array}$ & $\begin{array}{l}\text { Summary statistics } \\
{[95 \% \mathrm{Cl}]}\end{array}$ & $\begin{array}{l}\text { Study } \\
\text { Limitations }\end{array}$ & Directness & Precision & Consistency & $\begin{array}{l}\text { Reporting } \\
\text { Bias }\end{array}$ & $\begin{array}{l}\text { Optional } \\
\text { Factors }\end{array}$ & SOE \\
\hline $\begin{array}{l}\text { ST/FS fracture } \\
\text { without } \\
\text { confirmed } \\
\text { radiologic AFF } \\
\text { features }\end{array}$ & $\begin{array}{l}1 \\
(325 / 1855)\end{array}$ & $\begin{array}{l}\text { Park-Wyllie } 2011 \\
\text { Higher risk } \\
\text { subtrochanteric or } \\
\text { femoral shaft fracture } \\
\text { with longterm BP use } \\
\text { vs. past BP use } \\
\text { lasted <100 days: } \\
3-5 \text { yr BP use: } \\
\text { OR=1.59 [0.80, 3.15] } \\
\geq 5 \text { yr BP use: } \\
\text { OR=2.74 [1.25, 6.02] }\end{array}$ & Medium & Direct & Imprecise & Unknown & Undetected & & Low \\
\hline $\begin{array}{l}\text { Osteonecrosis } \\
\text { of the jaw }\end{array}$ & 0 & NR & NA & NA & NA & NA & NA & & Insufficient \\
\hline
\end{tabular}

Abbreviations: $\mathrm{AFF}=$ atypical femoral fracture; $\mathrm{BP}=$ bisphosphonate; $\mathrm{HR}=$ hazard ratio; $\mathrm{NR}=$ not reported; $\mathrm{NA}=$ not applicable; OR=adjusted odds ration; RR=risk ratio;

$\mathrm{SOE}=$ strength of evidence; ST/FS=subtrochanteric/femoral shaft; yr=years

*calculated by EPC

Table D18. Strength of evidence assessments: Any bisphosphonate use $>3$ years versus different osteoporosis drug treatment

\begin{tabular}{|c|c|c|c|c|c|c|c|c|c|}
\hline Outcome & $\begin{array}{l}\text { \# Studies } \\
\text { (n/N eventsI } \\
\text { participants } \\
\text { evaluated) }\end{array}$ & $\begin{array}{l}\text { Summary statistics } \\
{[95 \% \mathrm{Cl}]}\end{array}$ & $\begin{array}{l}\text { Study } \\
\text { Limitations }\end{array}$ & Directness & Precision & Consistency & $\begin{array}{l}\text { Reporting } \\
\text { Bias }\end{array}$ & $\begin{array}{l}\text { Optional } \\
\text { Factors }\end{array}$ & SOE \\
\hline $\begin{array}{l}\text { Incident clinical } \\
\text { fracture }\end{array}$ & 0 & NR & NA & NA & NA & NA & NA & NA & Insufficient \\
\hline $\begin{array}{l}\text { Incident } \\
\text { nonvertebral } \\
\text { fracture }\end{array}$ & 0 & NR & NA & NA & NA & NA & NA & NA & Insufficient \\
\hline $\begin{array}{l}\text { Incident hip } \\
\text { fracture }\end{array}$ & 0 & NR & NA & NA & NA & NA & NA & NA & Insufficient \\
\hline $\begin{array}{l}\text { Incident nonhip } \\
\text { nonvertebral } \\
\text { fracture }\end{array}$ & 0 & NR & NA & NA & NA & NA & NA & NA & Insufficient \\
\hline $\begin{array}{l}\text { Incident major } \\
\text { osteoporotic } \\
\text { fracture }\end{array}$ & 0 & NR & NA & NA & NA & NA & NA & NA & Insufficient \\
\hline $\begin{array}{l}\text { Incident clinical } \\
\text { vertebral } \\
\text { fracture }\end{array}$ & 0 & NR & NA & NA & NA & NA & NA & NA & Insufficient \\
\hline
\end{tabular}




\begin{tabular}{|c|c|c|c|c|c|c|c|c|c|}
\hline Outcome & $\begin{array}{l}\text { \# Studies } \\
\text { (n/N events/ } \\
\text { participants } \\
\text { evaluated) } \\
\end{array}$ & $\begin{array}{l}\text { Summary statistics } \\
{[95 \% \mathrm{Cl}]}\end{array}$ & \begin{tabular}{|l|} 
Study \\
Limitations
\end{tabular} & Directness & Precision & Consistency & \begin{tabular}{|l|} 
Reporting \\
Bias
\end{tabular} & $\begin{array}{l}\text { Optional } \\
\text { Factors }\end{array}$ & SOE \\
\hline $\begin{array}{l}\text { Incident } \\
\text { radiographic } \\
\text { vertebral } \\
\text { fracture }\end{array}$ & 0 & NR & NA & NA & NA & NA & NA & NA & Insufficient \\
\hline $\begin{array}{l}\text { Serious adverse } \\
\text { events }\end{array}$ & 0 & NR & NA & NA & NA & NA & NA & NA & Insufficient \\
\hline $\begin{array}{l}\text { Subtrochanteric } \\
\text { or femoral shaft } \\
\text { fracture without } \\
\text { confirmed } \\
\text { radiologic AFF } \\
\text { features }\end{array}$ & $\begin{array}{l}1 \\
3-5 \text { yr use } \\
(26 / 4900) \\
>5 \text { yr use } \\
(8 / 4097)\end{array}$ & $\begin{array}{l}\text { Kim } 2011 \\
\text { NS difference in risk } \\
\text { of subtrochanteric or } \\
\text { femoral shaft fracture } \\
\text { with longterm use of } \\
\text { any BP vs. longterm } \\
\text { raloxifene or } \\
\text { calcitonin: } \\
3-5 \text { yr BP use: } \\
\text { HR=1.20 [0.55, 2.61] } \\
>5 \text { yr BP use: } \\
\text { HR=2.02 [0.41,10.0] }\end{array}$ & Medium & Direct & $\begin{array}{l}\text { Highly } \\
\text { Imprecise }\end{array}$ & Unknown & Undetected & None & Insufficient \\
\hline $\begin{array}{l}\text { Osteonecrosis } \\
\text { of the jaw }\end{array}$ & 0 & NR & NA & NA & NA & NA & NA & & Insufficient \\
\hline
\end{tabular}

Abbreviations: $\mathrm{AFF}=$ atypical femoral fracture; $\mathrm{BP}=$ bisphosphonate; $\mathrm{HR}=$ hazard ratio; NA=not applicable; NR=not reported; NS=not significant; OR=adjusted odds ration;

$\mathrm{RR}=$ risk ratio; $\mathrm{SOE}=$ strength of evidence; $\mathrm{yr}=$ years

*calculated by EPC 


\section{Denosumab}

Table D19. Strength of evidence assessments: long-term treatment with denosumab versus placebo

\begin{tabular}{|c|c|c|c|c|c|c|c|c|}
\hline $\begin{array}{l}\text { Outcome } \\
\text { Time frame }\end{array}$ & $\begin{array}{l}\text { \# Studies } \\
\text { (n/N=events/ participants) } \\
\text { Population }\end{array}$ & $\begin{array}{l}\text { Summary Statistics } \\
{[95 \% \mathrm{Cl}]}\end{array}$ & $\begin{array}{l}\text { Study } \\
\text { Limitations }\end{array}$ & Directness & Precision & Consistency & $\begin{array}{l}\text { Reporting } \\
\text { Bias }\end{array}$ & SOE \\
\hline $\begin{array}{l}\text { Incident clinical } \\
\text { fracture } \\
0-4 \text { yrs }\end{array}$ & $\begin{array}{l}1(38 / 360) \\
\text { PM women with LS-BMD } \\
\text { T-score }-1.8 \text { to }-4 \text { or hip T- } \\
\text { score }-1.8 \text { to }-3.5\end{array}$ & $\begin{array}{l}\text { Miller } 2008 \\
\text { NS difference in risk } \\
\text { with denosumab vs. } \\
\text { placebo: } \\
10.5 \% \text { vs } 10.9 \% ; \\
\text { RR }=0.97[0.40,2.35]\end{array}$ & Medium & Indirect & $\begin{array}{l}\text { Highly } \\
\text { imprecise }\end{array}$ & $\begin{array}{l}\text { Unknown but } \\
\text { multisite trial }\end{array}$ & Undetected & Insufficient \\
\hline $\begin{array}{l}\text { Incident } \\
\text { nonvertebral } \\
\text { fracture }\end{array}$ & 0 & NR & NA & NA & NA & NA & NA & Insufficient \\
\hline $\begin{array}{l}\text { Incident hip } \\
\text { fracture }\end{array}$ & 0 & NR & NA & NA & NA & NA & NA & Insufficient \\
\hline $\begin{array}{l}\text { Incident nonhip } \\
\text { nonvertebral } \\
\text { fracture }\end{array}$ & 0 & NR & NA & NA & NA & NA & NA & Insufficient \\
\hline $\begin{array}{l}\text { Incident major } \\
\text { osteoporotic } \\
\text { fracture }\end{array}$ & 0 & NR & NA & NA & NA & NA & NA & Insufficient \\
\hline $\begin{array}{l}\text { Incident clinical } \\
\text { vertebral fracture }\end{array}$ & 0 & NR & NA & NA & NA & NA & NA & Insufficient \\
\hline $\begin{array}{l}\text { Incident } \\
\text { radiographic } \\
\text { vertebral fracture }\end{array}$ & 0 & NR & NA & NA & NA & NA & NA & Insufficient \\
\hline $\begin{array}{l}\text { Serious adverse } \\
\text { events } \\
0-4 \text { yrs }\end{array}$ & $\begin{array}{l}1(61 / 360) \\
\text { PM women with LS-BMD } \\
\text { T-score }-1.8 \text { to }-4 \text { or hip T- } \\
\text { score }-1.8 \text { to }-3.5\end{array}$ & $\begin{array}{l}\text { Miller } 2008 \\
\text { NS difference in risk } \\
\text { with denosumab vs. } \\
\text { placebo: } 17.8 \% \text { vs } \\
10.9 \% ; \\
\text { RR=1.64 }[0.69,3.88]\end{array}$ & Medium & Indirect & $\begin{array}{l}\text { Highly } \\
\text { imprecise }\end{array}$ & $\begin{array}{l}\text { Unknown but } \\
\text { multisite trial }\end{array}$ & Undetected & Insufficient \\
\hline $\begin{array}{l}\text { Atypical femoral } \\
\text { fracture }\end{array}$ & 0 & NR & NA & NA & NA & NA & NA & Insufficient \\
\hline $\begin{array}{l}\text { Osteonecrosis of } \\
\text { the jaw }\end{array}$ & 0 & NR & NA & NA & NA & NA & NA & Insufficient \\
\hline
\end{tabular}

Abbreviations: AE=adverse events; BMD=bone mineral density; LS=lumbar spine; NA=not applicable; NR=not reported; PM=postmenopausal; RR=risk ratio; SOE=strength of evidence; yr=years 
Table D20. Strength of evidence assessments: denosumab continuation ( 4 years denosumab) versus discontinuation ( 2 years denosumab followed by 2 years placebo or by 1 year placebo and 1 year of denosumab)

\begin{tabular}{|c|c|c|c|c|c|c|c|c|}
\hline $\begin{array}{l}\text { Outcome } \\
\text { Time frame }\end{array}$ & $\begin{array}{l}\text { \# Studies } \\
\text { (n/N=events/ participants) } \\
\text { Population }\end{array}$ & $\begin{array}{l}\text { Summary Statistics } \\
{[95 \% \mathrm{Cl}]}\end{array}$ & $\begin{array}{l}\text { Study } \\
\text { Limitations }\end{array}$ & Directness & Precision & Consistency & $\begin{array}{l}\text { Reporting } \\
\text { Bias }\end{array}$ & SOE \\
\hline $\begin{array}{l}\text { Incident clinical } \\
\text { fracture } \\
0-4 \text { yrs }\end{array}$ & $\begin{array}{l}1(33 / 314) \\
\text { PM women with LS-BMD } \\
\text { T-score }-1.8 \text { to }-4 \text { or hip T- } \\
\text { score }-1.8 \text { to }-3.5\end{array}$ & $\begin{array}{l}\text { Miller } 2008 \\
\text { Among women } \\
\text { assigned denosumab, } \\
\text { "no increase in } \\
\text { fracture incidence } \\
\text { among small number } \\
\text { of patients who } \\
\text { discontinued } \\
\text { denosumab," but no } \\
\text { numerical data } \\
\text { provided. }\end{array}$ & NA & NA & NA & NA & NA & Insufficient \\
\hline $\begin{array}{l}\text { Incident } \\
\text { nonvertebral } \\
\text { fracture }\end{array}$ & 0 & NR & NA & NA & NA & NA & NA & Insufficient \\
\hline $\begin{array}{l}\text { Incident hip } \\
\text { fracture }\end{array}$ & 0 & NR & NA & NA & $\mathrm{NA}$ & $\mathrm{NA}$ & NA & Insufficient \\
\hline $\begin{array}{l}\text { Incident nonhip } \\
\text { nonvertebral } \\
\text { fracture }\end{array}$ & 0 & NR & NA & NA & NA & NA & NA & Insufficient \\
\hline $\begin{array}{l}\text { Incident major } \\
\text { osteoporotic } \\
\text { fracture }\end{array}$ & 0 & NR & NA & NA & NA & NA & NA & Insufficient \\
\hline $\begin{array}{l}\text { Incident clinical } \\
\text { vertebral fracture }\end{array}$ & 0 & NR & NA & NA & NA & $\mathrm{NA}$ & NA & Insufficient \\
\hline $\begin{array}{l}\text { Incident } \\
\text { radiographic } \\
\text { vertebral fracture }\end{array}$ & 0 & NR & NA & NA & NA & NA & NA & Insufficient \\
\hline $\begin{array}{l}\text { Serious adverse } \\
\text { events } \\
0-4 \text { yrs }\end{array}$ & $\begin{array}{l}1(56 / 314) \\
\text { PM women with LS-BMD } \\
\text { T-score }-1.8 \text { to }-4 \text { or hip T- } \\
\text { score }-1.8 \text { to }-3.5\end{array}$ & $\begin{array}{l}\text { Miller } 2008 \\
\text { Authors only reported } \\
\text { results for denosumab } \\
\text { continuation and } \\
\text { discontinuation } \\
\text { groups pooled } \\
\text { together. }\end{array}$ & NA & NA & NA & NA & NA & Insufficient \\
\hline $\begin{array}{l}\text { Atypical femoral } \\
\text { fracture }\end{array}$ & 0 & NR & NA & NA & NA & NA & NA & Insufficient \\
\hline $\begin{array}{l}\text { Osteonecrosis of } \\
\text { the jaw }\end{array}$ & 0 & NR & NA & NA & $\mathrm{NA}$ & $\mathrm{NA}$ & NA & Insufficient \\
\hline
\end{tabular}


Abbreviations: $\mathrm{AE}=$ adverse events; $\mathrm{BMD}=$ bone mineral density; $\mathrm{LS}=$ =lumbar spine; $\mathrm{NA}=$ not applicable; $\mathrm{NR}=$ not reported; $\mathrm{PM=postmenopausal;} \mathrm{RR=risk} \mathrm{ratio;} \mathrm{SOE}=$ strength of evidence; yr=years 


\section{Raloxifene}

Table D21. Strength of evidence assessments: long-term treatment with raloxifene versus placebo

\begin{tabular}{|c|c|c|c|c|c|c|c|c|}
\hline $\begin{array}{l}\text { Outcome } \\
\text { Time frame }\end{array}$ & $\begin{array}{l}\text { \# Studies } \\
\text { (n/N=events/participant } \\
\text { S evaluated) } \\
\text { Population }\end{array}$ & $\begin{array}{l}\text { Summary statistics } \\
{[95 \% \mathrm{Cl}]}\end{array}$ & $\begin{array}{l}\text { Study } \\
\text { Limitations }\end{array}$ & Directness & Precision & Consistency & $\begin{array}{l}\text { Reporting } \\
\text { Bias }\end{array}$ & SOE \\
\hline $\begin{array}{l}\text { Incident } \\
\text { clinical fracture }\end{array}$ & 0 & NR & NA & NA & NA & NA & NA & Insufficient \\
\hline $\begin{array}{l}\text { Incident } \\
\text { nonvertebral } \\
\text { fracture } \\
0-4 \text { yrs }\end{array}$ & $\begin{array}{l}1 \text { ( } 844 / 6828) \\
\text { PM women with T-score } \\
\text { - } 2.5 \text { and/or baseline } \\
\text { RVF }\end{array}$ & $\begin{array}{l}\text { Barrett-Connor } 2004 \\
\text { NS difference in risk } \\
\text { with raloxifene (pooled } \\
60 \mathrm{mg} / \mathrm{d}+120 \mathrm{mg} / \mathrm{d} \text { ) } \\
\text { vs. placebo: } \\
11 \% \text { vs. } 12 \% ; \\
\text { RR=0.93 [0.81, 1.06] }\end{array}$ & Low & Direct & Precise & $\begin{array}{l}\text { Unknown, but } \\
\text { multisite trial }\end{array}$ & Undetected & High \\
\hline $\begin{array}{l}\text { Incident } \\
\text { nonvertebral } \\
\text { fracture } \\
5-8 \text { yrs }\end{array}$ & $\begin{array}{l}1\left(915^{\star} / 4011\right) \\
\text { PM women previously } \\
\text { received raloxifene vs. } \\
\text { placebo for T-score } \leq-2.5 \\
\text { and/or baseline RVF }\end{array}$ & $\begin{array}{l}\text { Siris } 2005 \\
\text { NS difference in risk } \\
\text { with raloxifene } 60 \mathrm{mg} / \mathrm{d} \\
\text { vs. placebo: } \\
23 \% \text { vs. } 23 \% \text {; } \\
\mathrm{HR}=1.00[0.82,1.21]\end{array}$ & Medium & Direct & Precise & $\begin{array}{l}\text { Unknown, but } \\
\text { multisite trial }\end{array}$ & Undetected & Moderate \\
\hline $\begin{array}{l}\text { Incident hip } \\
\text { fracture } \\
0-4 \text { yrs }\end{array}$ & $\begin{array}{l}1 \text { ( } 85 / 6828) \\
\text { PM women with T-score } \\
\leq-2.5 \text { and/or baseline } \\
\text { RVF }\end{array}$ & $\begin{array}{l}\text { Delmas } 2002 \\
\text { NS difference in risk } \\
\text { with raloxifene (pooled } \\
60 \mathrm{mg} / \mathrm{d}+120 \mathrm{mg} / \mathrm{d}) \\
\text { vs. placebo: } \\
1.1 \% \text { vs. } 1.1 \% ; \\
\text { RR=0.97 [0.62, 1.52] }\end{array}$ & Low & Direct & Imprecise & $\begin{array}{l}\text { Unknown, but } \\
\text { multisite trial }\end{array}$ & Undetected & Moderate \\
\hline $\begin{array}{l}\text { Incident hip } \\
\text { fracture } \\
5-8 \text { yrs }\end{array}$ & 0 & NR & NA & NA & NA & NA & NA & Insufficient \\
\hline $\begin{array}{l}\text { Incident } \\
\text { nonvertebral } \\
\text { nonhip fracture }\end{array}$ & 0 & NR & NA & NA & NA & NA & NA & Insufficient \\
\hline $\begin{array}{l}\text { Incident major } \\
\text { osteoporotic } \\
\text { fracture }\end{array}$ & 0 & NR & NA & NA & NA & NA & NA & Insufficient \\
\hline $\begin{array}{l}\text { Incident } \\
\text { clinical }\end{array}$ & $\begin{array}{l}1 \text { (169/5114) } \\
\text { PM women with T-score }\end{array}$ & Sontag 2010 & Low & Direct & Precise & $\begin{array}{l}\text { Unknown, but } \\
\text { multisite trial }\end{array}$ & Undetected & High \\
\hline
\end{tabular}




\begin{tabular}{|c|c|c|c|c|c|c|c|c|}
\hline $\begin{array}{l}\text { Outcome } \\
\text { Time frame }\end{array}$ & $\begin{array}{l}\text { \# Studies } \\
\text { (n/N=events/participant } \\
\text { S evaluated) } \\
\text { Population }\end{array}$ & $\begin{array}{l}\text { Summary statistics } \\
{[95 \% \mathrm{Cl}]}\end{array}$ & $\begin{array}{l}\text { Study } \\
\text { Limitations }\end{array}$ & Directness & Precision & Consistency & $\begin{array}{l}\text { Reporting } \\
\text { Bias }\end{array}$ & SOE \\
\hline $\begin{array}{l}\text { vertebral } \\
\text { fracture } \\
0-4 \text { yrs }\end{array}$ & $\begin{array}{l}\leq-2.5 \text { and/or baseline } \\
\text { RVF }\end{array}$ & $\begin{array}{l}\text { Lower risk with } \\
\text { raloxifene } 60 \mathrm{mg} / \mathrm{d} \text { vs } \\
\text { placebo: } \\
2 \% \text { vs. } 4 \% ; \\
\mathrm{RR}=0.58[0.43,0.79]\end{array}$ & & & & & & \\
\hline $\begin{array}{l}\text { Incident } \\
\text { clinical } \\
\text { vertebral } \\
\text { fracture } \\
5-8 \text { yrs }\end{array}$ & 0 & $\mathrm{NR}$ & NA & NA & NA & NA & NA & Insufficient \\
\hline $\begin{array}{l}\text { Incident } \\
\text { radiographic } \\
\text { vertebral } \\
\text { fracture } \\
0-4 \text { yrs }\end{array}$ & $\begin{array}{l}1\left(615^{\star} / 6828\right) \\
\text { PM women with T-score } \\
\leq-2.5 \text { and/or baseline } \\
\text { RVF }\end{array}$ & $\begin{array}{l}\text { Delmas } 2002 \\
\text { Lower risk with } \\
\text { raloxifene vs. placebo: } \\
60 \mathrm{mg} / \mathrm{d} \text { vs. placebo } \\
8 \% \text { vs. } 12 \% ; \mathrm{RR}= \\
0.64[0.53,0.76] \\
120 \mathrm{mg} / \mathrm{d} \text { vs placebo: } \\
7 \% \text { vs. } 12 \% ; \\
\mathrm{RR}=0.57[0.48 \text { to } 0.69]\end{array}$ & Low & Direct & Precise & $\begin{array}{l}\text { Unknown, but } \\
\text { multisite trial }\end{array}$ & Undetected & High \\
\hline $\begin{array}{l}\text { Incident } \\
\text { radiographic } \\
\text { vertebral } \\
\text { fracture } \\
5-8 \text { yrs }\end{array}$ & 0 & NR & NA & NA & $\mathrm{NA}$ & NA & NA & Insufficient \\
\hline $\begin{array}{l}\text { Serious } \\
\text { adverse } \\
\text { events } \\
0-4 \text { yrs }\end{array}$ & 0 & NR & NA & NA & $\mathrm{NA}$ & NA & NA & Insufficient \\
\hline $\begin{array}{l}\text { Serious } \\
\text { adverse } \\
\text { events } \\
5-8 \text { yrs }\end{array}$ & $\begin{array}{l}1(1739 / 4011) \\
\text { PM women previously } \\
\text { received raloxifene vs. } \\
\text { placebo for T-score } \leq-2.5 \\
\text { and/or baseline RVF }\end{array}$ & $\begin{array}{l}\text { Martino } 2004 \\
\text { NS difference in risk } \\
\text { with raloxifene } 60 \mathrm{mg} / \mathrm{d} \\
\text { vs. placebo: } \\
42 \% \text { vs. } 46 \% ; \\
\text { RR=0.93 }[0.86,1.00]^{\star}\end{array}$ & Medium & Direct & Imprecise & $\begin{array}{l}\text { Unknown, but } \\
\text { multisite trial }\end{array}$ & Undetected & Low \\
\hline $\begin{array}{l}\text { Atypical } \\
\text { femoral } \\
\text { fractures }\end{array}$ & 0 & NR & NA & NA & NA & NA & NA & Insufficient \\
\hline
\end{tabular}




\begin{tabular}{|l|l|l|l|l|l|l|l|}
\hline $\begin{array}{l}\text { Outcome } \\
\text { Time frame }\end{array}$ & $\begin{array}{l}\text { \# Studies } \\
\text { (n/N=events/participant } \\
\text { s evaluated) } \\
\text { Population }\end{array}$ & $\begin{array}{l}\text { Summary statistics } \\
{[95 \% \mathrm{Cl}]}\end{array}$ & $\begin{array}{l}\text { Study } \\
\text { Limitations }\end{array}$ & Directness & Precision & Consistency & $\begin{array}{l}\text { Reporting } \\
\text { Bias }\end{array}$ \\
\hline $\begin{array}{l}\text { Osteonecrosis } \\
\text { of the jaw }\end{array}$ & 0 & NR & NA & NA & NA & NA & Insufficient \\
\hline
\end{tabular}

Abbreviations: BMD=bone mineral density; $\mathrm{d}=$ day; $\mathrm{HR}=$ hazard ratio; $\mathrm{LS}=$ =lumbar spine; $\mathrm{MG}=$ milligrams; $\mathrm{NA}=$ not applicable; NR=not reported; NS=not significant;

$\mathrm{PM}=$ postmenopausal; $\mathrm{RR}=$ risk ratio; $\mathrm{RVF}=$ radiographic vertebral fracture; $\mathrm{SOE}=$ strength of evidence; yr=years

*calculated by EPC

Table D22. Strength of evidence assessments: long-term treatment with raloxifene versus no osteoporosis drug treatment

\begin{tabular}{|c|c|c|c|c|c|c|c|c|}
\hline Outcome & $\begin{array}{l}\text { \# Studies } \\
\text { (n/N=events/participants } \\
\text { evaluated) } \\
\text { Study description }\end{array}$ & $\begin{array}{l}\text { Summary statistics } \\
{[95 \% \mathrm{Cl}]}\end{array}$ & $\begin{array}{l}\text { Study } \\
\text { Limitations }\end{array}$ & Directness & Precision & Consistency & $\begin{array}{l}\text { Reporting } \\
\text { Bias }\end{array}$ & SOE \\
\hline $\begin{array}{l}\text { Incident } \\
\text { clinical fracture }\end{array}$ & NR & NA & NA & NA & NA & NA & NA & Insufficient \\
\hline $\begin{array}{l}\text { Incident } \\
\text { nonvertebral } \\
\text { fracture }\end{array}$ & NR & NA & NA & NA & NA & NA & NA & Insufficient \\
\hline $\begin{array}{l}\text { Incident hip } \\
\text { fracture }\end{array}$ & NR & NA & NA & NA & NA & NA & NA & Insufficient \\
\hline $\begin{array}{l}\text { Incident } \\
\text { nonhip } \\
\text { nonvertebral } \\
\text { fracture }\end{array}$ & NR & NA & NA & NA & NA & NA & NA & Insufficient \\
\hline $\begin{array}{l}\text { Incident major } \\
\text { osteoporotic } \\
\text { fracture }\end{array}$ & NR & NA & NA & NA & NA & NA & NA & Insufficient \\
\hline $\begin{array}{l}\text { Incident } \\
\text { clinical } \\
\text { vertebral } \\
\text { fracture }\end{array}$ & NR & NA & NA & NA & NA & NA & NA & Insufficient \\
\hline $\begin{array}{l}\text { Incident } \\
\text { radiographic } \\
\text { vertebral } \\
\end{array}$ & NR & NA & NA & NA & NA & NA & NA & Insufficient \\
\hline $\begin{array}{l}\text { Serious } \\
\text { adverse } \\
\text { events } \\
\end{array}$ & NR & NA & NA & NA & NA & NA & NA & Insufficient \\
\hline
\end{tabular}




\begin{tabular}{|c|c|c|c|c|c|c|c|c|}
\hline Outcome & $\begin{array}{l}\text { \# Studies } \\
\text { (n/N=events/participants } \\
\text { evaluated) } \\
\text { Study description }\end{array}$ & $\begin{array}{l}\text { Summary statistics } \\
{[95 \% \mathrm{Cl}]}\end{array}$ & $\begin{array}{l}\text { Study } \\
\text { Limitations }\end{array}$ & Directness & Precision & Consistency & $\begin{array}{l}\text { Reporting } \\
\text { Bias }\end{array}$ & SOE \\
\hline $\begin{array}{l}\text { ST/FS fracture } \\
\text { without } \\
\text { radiologic } \\
\text { confirmation of } \\
\text { AFF features }\end{array}$ & $\begin{array}{l}1 \text { (25/19324) } \\
\text { Retrospective cohort. AFF } \\
\text { defined by diagnosis } \\
\text { codes only. Duration 0-11 } \\
\text { yr (mean } \sim 3.8) .\end{array}$ & $\begin{array}{l}\text { Vestergaard } 2011 \\
\text { NS difference in risk } \\
\text { with raloxifene vs. no } \\
\text { osteoporosis drug } \\
\text { treatment: } \\
\text { Subtrochanteric } \\
\text { fracture: HR=1.06 } \\
{[0.34,3.32]} \\
\text { Femoral shaft fracture: } \\
\text { HR }=0.82[0.21,3.20]\end{array}$ & Medium & Direct & $\begin{array}{l}\text { Highly } \\
\text { Imprecise }\end{array}$ & Unknown & Undetected & Insufficient \\
\hline $\begin{array}{l}\text { Osteonecrosis } \\
\text { of the jaw }\end{array}$ & $\begin{array}{l}1(2 / 19324) \\
\text { Retrospective cohort. ONJ } \\
\text { defined by diagnosis } \\
\text { codes only. Duration 0-11 } \\
\text { yrs (mean } \sim 3.8) .\end{array}$ & $\begin{array}{l}\text { Vestergaard } 2012 \\
2 \text { cases of } \\
\text { inflammatory jaw } \\
\text { events in no treatment } \\
\text { group }\end{array}$ & Medium & Direct & $\begin{array}{l}\text { Highly } \\
\text { Imprecise }\end{array}$ & Unknown & Undetected & Insufficient \\
\hline
\end{tabular}

Abbreviations: AFF=atypical femoral fracture; $\mathrm{CI}=$ confidence interval; IRR=incident risk ratio; NA=not applicable; NR=not reported; NS=not significant ONJ=osteonecrosis of the jaw; SOE=strength of evidence; $y r=y e a r s$ 


\section{Hormone Therapy}

Table D23. Strength of evidence assessments: long-term treatment with hormone therapy (estrogen/progestin) versus control

\begin{tabular}{|c|c|c|c|c|c|c|c|c|}
\hline Outcome & $\begin{array}{l}\text { \# Studies } \\
\text { (n/N=events/participants } \\
\text { evaluated) }\end{array}$ & $\begin{array}{l}\text { Summary Statistics } \\
{[95 \% \mathrm{Cl}]}\end{array}$ & $\begin{array}{l}\text { Study } \\
\text { Limitations }\end{array}$ & Directness & Precision & Consistency & $\begin{array}{l}\text { Reporting } \\
\text { Bias }\end{array}$ & SOE \\
\hline \multirow[t]{3}{*}{$\begin{array}{l}\text { Incident } \\
\text { clinical } \\
\text { fracture }\end{array}$} & $\begin{array}{l}1 \text { (WHI trial, PM women } \\
\text { with an intact uterus at } \\
\text { baseline) } \\
\text { (1629/16608) } \\
\text { Full study population }\end{array}$ & $\begin{array}{l}\text { Cauley } 2003 \\
\text { Lower risk with } \\
\text { estrogen/progestin vs. } \\
\text { placebo: } \\
8.6 \% \text { vs. } 11.1 \% \text {; HR } 0.76 \\
(95 \% \mathrm{Cl} 0.69,0.83)\end{array}$ & Low & Direct & Precise & $\begin{array}{l}\text { Unknown, } \\
\text { but large } \\
\text { multisite trial }\end{array}$ & Undetected & High \\
\hline & $\begin{array}{l}1 \\
\text { (747/5897) } \\
\text { History of fracture }\end{array}$ & $\begin{array}{l}\text { Cauley } 2003 \\
\text { Lower risk with } \\
\text { estrogen/progestin vs. } \\
\text { placebo: } 11.2 \% \text { vs. } \\
14.1 \% ; \mathrm{HR} 0.78(95 \% \mathrm{Cl} \\
0.68,0.91)\end{array}$ & $\begin{array}{l}\text { Medium } \\
\text { (low for the } \\
\text { WHI trial but } \\
\text { this } \\
\text { subgroup } \\
\text { analysis } \\
\text { was not } \\
\text { specified a } \\
\text { priori) }\end{array}$ & Direct & Imprecise & $\begin{array}{l}\text { Unknown, } \\
\text { but large } \\
\text { multisite trial }\end{array}$ & Undetected & Low \\
\hline & $\begin{array}{l}1 \\
(33 / \mathrm{NR}) \\
\text { T score } \leq-2.5\end{array}$ & $\begin{array}{l}\text { Cauley } 2003 \\
\text { No difference in risk with } \\
\text { estrogen/progestin vs. } \\
\text { placebo: } \\
\text { Annualized percentage } \\
1.4 \% \text { vs } 3.2 \% \\
\text { HR } 0.53(0.25,1.10)\end{array}$ & $\begin{array}{l}\text { Medium } \\
\text { (see above) }\end{array}$ & Direct & $\begin{array}{l}\text { Highly } \\
\text { Imprecise }\end{array}$ & $\begin{array}{l}\text { Unknown, } \\
\text { and only } \\
\text { included } 3 / 40 \\
\text { study sites }\end{array}$ & Undetected & Insufficient \\
\hline $\begin{array}{l}\text { Incident } \\
\text { nonvertebral } \\
\text { fracture }\end{array}$ & $\begin{array}{l}1 \\
(2 / 29)\end{array}$ & $\begin{array}{l}\text { Wimalawansa } 1998 \\
\text { NS difference in risk with } \\
\text { estrogen/progestin vs. } \\
\text { control: } \\
7 \% \text { vs. } 7 \% \text {; } \\
\text { RR=0.93 }[0.06,13.5]\end{array}$ & Medium & Direct & $\begin{array}{l}\text { Highly } \\
\text { Imprecise }\end{array}$ & Unknown & Undetected & Insufficient \\
\hline $\begin{array}{l}\text { Incident hip } \\
\text { fracture }\end{array}$ & $\begin{array}{l}1 \\
(125 / 16608) \\
\text { Full study population }\end{array}$ & $\begin{array}{l}\text { Cauley } 2003 \\
\text { Lower risk with } \\
\text { estrogen/progestin vs. } \\
\text { placebo: } \\
0.61 \% \text { vs. } 0.90 \% ; \mathrm{HR} \\
0.67(95 \% \mathrm{Cl} 0.47,0.96)\end{array}$ & Low & Direct & Imprecise & $\begin{array}{l}\text { Unknown, } \\
\text { but large } \\
\text { multisite trial }\end{array}$ & Undetected & Moderate \\
\hline
\end{tabular}




\begin{tabular}{|c|c|c|c|c|c|c|c|c|}
\hline Outcome & $\begin{array}{l}\text { \# Studies } \\
\text { (n/N=events/participants } \\
\text { evaluated) }\end{array}$ & $\begin{array}{l}\text { Summary Statistics } \\
{[95 \% \mathrm{Cl}]}\end{array}$ & $\begin{array}{l}\text { Study } \\
\text { Limitations }\end{array}$ & Directness & Precision & Consistency & $\begin{array}{l}\text { Reporting } \\
\text { Bias }\end{array}$ & SOE \\
\hline & $\begin{array}{l}1 \\
(72 / 5897) \\
\text { History of fracture }\end{array}$ & $\begin{array}{l}\text { Cauley } 2003 \\
\text { No difference in risk with } \\
\text { estrogen/progestin vs. } \\
\text { placebo: } 1.1 \% \text { vs } 1.4 \% \text {; } \\
\text { HR } 0.77(95 \% \text { Cl } 0.48 \text {, } \\
1.22)\end{array}$ & $\begin{array}{l}\text { Medium } \\
\text { (see above) }\end{array}$ & Direct & Imprecise & $\begin{array}{l}\text { Unknown, } \\
\text { but large } \\
\text { multisite trial }\end{array}$ & Undetected & Low \\
\hline $\begin{array}{l}\text { Incident } \\
\text { nonhip } \\
\text { nonvertebral } \\
\text { fracture }\end{array}$ & 0 & $\mathrm{NR}$ & NA & NA & NA & NA & NA & Insufficient \\
\hline $\begin{array}{l}\text { Incident major } \\
\text { osteoporotic } \\
\text { fracture }\end{array}$ & 0 & NR & NA & NA & NA & NA & NA & Insufficient \\
\hline $\begin{array}{l}\text { Incident } \\
\text { clinical } \\
\text { vertebral } \\
\text { fracture }\end{array}$ & $\begin{array}{l}1 \\
(101 / 16608) \\
\text { Full study population }\end{array}$ & $\begin{array}{l}\text { Cauley } 2003 \\
\text { Lower risk with } \\
\text { estrogen/progestin vs. } \\
\text { placebo: } \\
0.48 \% \text { vs. } 0.74 \% \text {; } \\
\text { HR } 0.65(0.46,0.92)\end{array}$ & Low & Direct & Imprecise & $\begin{array}{l}\text { Unknown, } \\
\text { but large } \\
\text { multisite trial }\end{array}$ & Undetected & Moderate \\
\hline $\begin{array}{l}\text { Incident } \\
\text { radiographic } \\
\text { vertebral } \\
\text { fracture }\end{array}$ & $\begin{array}{l}1 \\
(7 / 29)\end{array}$ & $\begin{array}{l}\text { Wimalawansa } 1998 \\
\text { NS difference in risk with } \\
\text { estrogen/progestin vs. } \\
\text { control: } \\
13 \% \text { vs. } 36 \% ; \\
\text { RR }=0.37[0.09,1.62]\end{array}$ & Medium & Direct & $\begin{array}{l}\text { Highly } \\
\text { Imprecise }\end{array}$ & Unknown & Undetected & Insufficient \\
\hline $\begin{array}{l}\text { Serious } \\
\text { adverse } \\
\text { events }\end{array}$ & 0 & NR & NA & NA & NA & NA & NA & Insufficient \\
\hline $\begin{array}{l}\text { Atypical } \\
\text { femoral } \\
\text { fracture }\end{array}$ & 0 & NR & NA & NA & NA & NA & NA & Insufficient \\
\hline $\begin{array}{l}\text { Osteonecrosis } \\
\text { of the jaw }\end{array}$ & 0 & NR & NA & NA & NA & NA & NA & Insufficient \\
\hline
\end{tabular}

Abbreviations: NA=not applicable; NR=not reported; PM=postmenopausal; RR=risk ratio; $\mathrm{SOE}=$ strength of evidence; yr=years 
Table D24. Strength of evidence assessments: long-term treatment with hormone therapy (estrogen) versus placebo

\begin{tabular}{|c|c|c|c|c|c|c|c|c|}
\hline Outcome & $\begin{array}{l}\text { \# Studies } \\
\text { (n/N=events/participants } \\
\text { evaluated) } \\
\text { Population }\end{array}$ & $\begin{array}{l}\text { Summary Statistics } \\
{[95 \% \mathrm{Cl}]}\end{array}$ & $\begin{array}{l}\text { Study } \\
\text { Limitations }\end{array}$ & Directness & Precision & Consistency & $\begin{array}{l}\text { Reporting } \\
\text { Bias }\end{array}$ & SOE \\
\hline \multirow[t]{4}{*}{$\begin{array}{l}\text { Incident clinical } \\
\text { fracture }\end{array}$} & $\begin{array}{l}1 \text { (WHI trial, PM women } \\
\text { with hysterectomy) } \\
\text { (1301/10739) } \\
\text { Full study population }\end{array}$ & $\begin{array}{l}\text { Jackson } 2006 \\
\text { Lower risk with estrogen } \\
\text { vs. placebo: } \\
10 \% \text { vs. } 14 \% \text {; HR } 0.71 \\
(95 \% \text { Cl } 0.64,0.80)\end{array}$ & Low & Direct & Precise & $\begin{array}{l}\text { Unknown, } \\
\text { but large } \\
\text { multisite trial }\end{array}$ & Undetected & High \\
\hline & $\begin{array}{l}1 \\
(621 / 3816) \\
\text { History of fracture }\end{array}$ & $\begin{array}{l}\text { Jackson } 2006 \\
\text { Lower risk with estrogen } \\
\text { vs. placebo: } \\
\text { 14\% vs. } 19 \% \text {; HR } 0.73 \\
(95 \% \text { Cl } 0.62,0.86)\end{array}$ & $\begin{array}{l}\text { Medium (low } \\
\text { for the WHI } \\
\text { trial but this } \\
\text { subgroup } \\
\text { analysis was } \\
\text { not specified } \\
\text { a priori) }\end{array}$ & Direct & Imprecise & $\begin{array}{l}\text { Unknown, } \\
\text { but large } \\
\text { multisite trial }\end{array}$ & Undetected & Low \\
\hline & $\begin{array}{l}1 \\
\left(\mathrm{NR} / 363^{\star}\right) \\
\text { T score between }-1.0 \text { and } \\
-2.5\end{array}$ & $\begin{array}{l}\text { Jackson } 2006 \\
\text { No difference in risk with } \\
\text { estrogen vs. placebo: } \\
\text { HR } 0.83(95 \% \mathrm{Cl} 0.49 \text {, } \\
1.40)\end{array}$ & $\begin{array}{l}\text { Medium } \\
\text { (see above) }\end{array}$ & Direct & $\begin{array}{l}\text { Highly } \\
\text { Imprecise }\end{array}$ & $\begin{array}{l}\text { Unknown, } \\
\text { and only } \\
\text { included } 3 / 40 \\
\text { study sites }\end{array}$ & Undetected & Insufficient \\
\hline & $\begin{array}{l}1 \\
\left(\mathrm{NR} / 53^{\star}\right) \\
\text { T score } \leq-2.5\end{array}$ & $\begin{array}{l}\text { Jackson } 2006 \\
\text { No difference in risk with } \\
\text { estrogen vs. placebo: } \\
\text { HR } 0.83(95 \% \mathrm{Cl} 0.17 \text {, } \\
3.91)\end{array}$ & $\begin{array}{l}\text { Medium } \\
\text { (see above) }\end{array}$ & Direct & $\begin{array}{l}\text { Highly } \\
\text { Imprecise }\end{array}$ & $\begin{array}{l}\text { Unknown, } \\
\text { and only } \\
\text { included } 3 / 40 \\
\text { study sites }\end{array}$ & Undetected & Insufficient \\
\hline $\begin{array}{l}\text { Incident } \\
\text { nonvertebral } \\
\text { fracture }\end{array}$ & 0 & $\mathrm{NR}$ & NA & NA & NA & NA & NA & Insufficient \\
\hline \multirow[t]{2}{*}{$\begin{array}{l}\text { Incident hip } \\
\text { fracture }\end{array}$} & $\begin{array}{l}1 \\
(119 / 10739) \\
\text { Full study population }\end{array}$ & $\begin{array}{l}\text { Jackson } 2006 \\
\text { Lower risk with estrogen } \\
\text { vs. placebo: } \\
0.87 \% \text { vs } 1.3 \% \text {; HR } 0.65 \\
(95 \% \text { Cl } 0.45,0.94)\end{array}$ & Low & Direct & Imprecise & $\begin{array}{l}\text { Unknown, } \\
\text { but large } \\
\text { multisite trial }\end{array}$ & Undetected & Moderate \\
\hline & $\begin{array}{l}1 \\
(57 / 3816) \\
\text { History of fracture }\end{array}$ & $\begin{array}{l}\text { Jackson } 2006 \\
\text { Lower risk with hormone } \\
\text { therapy vs. placebo: } \\
1.0 \% \text { vs } 1.9 \% \text {; HR } 0.55 \\
(95 \% \text { Cl } 0.32,0.94)\end{array}$ & $\begin{array}{l}\text { Medium } \\
\text { (see above) }\end{array}$ & Direct & Imprecise & $\begin{array}{l}\text { Unknown, } \\
\text { but large } \\
\text { multisite trial }\end{array}$ & Undetected & Low \\
\hline $\begin{array}{l}\text { Incident nonhip } \\
\text { nonvertebral } \\
\text { fracture }\end{array}$ & 0 & NR & NA & NA & NA & NA & NA & Insufficient \\
\hline
\end{tabular}




\begin{tabular}{|c|c|c|c|c|c|c|c|c|}
\hline Outcome & $\begin{array}{l}\text { \# Studies } \\
\text { (n/N=events/participants } \\
\text { evaluated) } \\
\text { Population }\end{array}$ & $\begin{array}{l}\text { Summary Statistics } \\
{[95 \% \mathrm{Cl}]}\end{array}$ & $\begin{array}{l}\text { Study } \\
\text { Limitations }\end{array}$ & Directness & Precision & Consistency & $\begin{array}{l}\text { Reporting } \\
\text { Bias }\end{array}$ & SOE \\
\hline $\begin{array}{l}\text { Incident major } \\
\text { osteoporotic } \\
\text { fracture }\end{array}$ & 0 & NR & NA & NA & NA & NA & NA & Insufficient \\
\hline $\begin{array}{l}\text { Incident clinical } \\
\text { vertebral } \\
\text { fracture }\end{array}$ & $\begin{array}{l}1 \\
(112 / 10739) \\
\text { Full study population }\end{array}$ & $\begin{array}{l}\text { Jackson } 2006 \\
\text { Lower risk with estrogen } \\
\text { vs. placebo: } \\
0.81 \% \text { vs } 1.3 \% \text {; HR } 0.64 \\
(95 \% \mathrm{Cl} 0.44,0.93)\end{array}$ & Low & Direct & Imprecise & $\begin{array}{l}\text { Unknown, } \\
\text { but large } \\
\text { multisite trial }\end{array}$ & Undetected & Moderate \\
\hline $\begin{array}{l}\text { Incident } \\
\text { radiographic } \\
\text { vertebral } \\
\text { fracture }\end{array}$ & 0 & NR & NA & NA & NA & NA & NA & Insufficient \\
\hline $\begin{array}{l}\text { Serious adverse } \\
\text { events }\end{array}$ & 0 & NR & NA & NA & NA & NA & NA & Insufficient \\
\hline $\begin{array}{l}\text { Atypical femoral } \\
\text { fracture }\end{array}$ & 0 & NR & NA & NA & NA & NA & NA & Insufficient \\
\hline $\begin{array}{l}\text { Osteonecrosis } \\
\text { of the jaw }\end{array}$ & 0 & NR & NA & NA & NA & NA & NA & Insufficient \\
\hline
\end{tabular}

Abbreviations: NA=not applicable; NR=not reported; $\mathrm{PM=postmenopausal;} \mathrm{HR=hazard} \mathrm{ratio;} \mathrm{SOE=strength} \mathrm{of} \mathrm{evidence}$

*Calculated by EPC 


\section{Appendix E. Excluded References}

1. Abdelazim I, A. Abdelrazak, K, M. Al, Kadi M, Yehia, A, H. Nusair, B, M. Faza, M, A. Effect of raloxifene hydrochloride on bone mineral density and bone turnover in Kuwaiti postmenopausal women with osteoporosis. Archives of Osteoporosis. 2014;9:189. PMID: 25037077. Ineligible study design.

2. Abelson A, Ringe, J, D. Gold, D, T. Lange, J, L. Thomas, T,. Longitudinal change in clinical fracture incidence after initiation of bisphosphonates. Osteoporosis International. 2010 Jun;21(6):1021-9. PMID: 19722103. Ineligible study design.

3. Abrahamsen B, Stilgren, L, S. Hermann, A, P. Tofteng, C, L. Barenholdt, O, Vestergaard, P, Brot, C, Nielsen, S, P. Discordance between changes in bone mineral density measured at different skeletal sites in perimenopausal women-implications for assessment of bone loss and response to therapy: The Danish Osteoporosis Prevention Study. Journal of Bone \& Mineral Research. 2001 Jul;16(7):1212-9. PMID: 11450696. Ineligible population.

4. Abrahamsen B, Eiken, P, Brixen, K, Atrial fibrillation in fracture patients treated with oral bisphosphonates. Journal of Internal Medicine. 2009 May;265(5):58192. PMID: 19141097. Ineligible treatment duration.

5. Abrahamsen B. Adverse effects of bisphosphonates. Calcified Tissue International. 2010 Jun;86(6):421-35. PMID: 20407762. Ineligible study design.

6. Abrahamsen B, Eiken, P, Eastell, R,. Proton pump inhibitor use and the antifracture efficacy of alendronate. Archives of Internal Medicine. 2011 Jun 13;171(11):998-1004. PMID: 21321287. Ineligible treatment duration.

7. Abrahamsen B, Pazianas M, Eiken P, et al. Esophageal and gastric cancer incidence and mortality in alendronate users. J Bone Miner
Res. 2012 Mar;27(3):679-86. doi: 10.1002/jbmr.1481. PMID: 22113985. Ineligible population.

8. Adachi J, D. Sargeant, E, J. Sagle, M, A. Lamont, D, Fawcett, P, D. Bensen, W, G. McQueen, M, Nazir, D, J. Goldsmith, C, H. A double-blind randomised controlled trial of the effects of medroxyprogesterone acetate on bone density of women taking oestrogen replacement therapy. British Journal of Obstetrics \& Gynaecology. 1997 Jan;104(1):64-70. PMID: 8988699. Ineligible population.

9. Adachi J, D. Adami, S, Kulkarni, P, M. Wong, M, Stock, J, L. Similar proportions of women lose bone mineral density with raloxifene or alendronate treatment. Journal of Clinical Densitometry. 2005 Fall;8(3):273-7. PMID: 16055956. No outcomes of interest.

10. Adachi J, D. Lyles, K, Boonen, S, Colon, Emeric C, Hyldstrup, L, Nordsletten, L, Pieper, C, Recknor, C, Su, G, Bucci, Rechtweg C, Magaziner, J,. Subtrochanteric fractures in bisphosphonatenaive patients: results from the HORIZONrecurrent fracture trial. Calcified Tissue International. 2011 Dec;89(6):427-33. PMID: 22038744. No outcomes of interest.

11. Adachi J, D. Bone, H, G. Daizadeh, N, S. Dakin, P, Papapoulos, S, Hadji, P, Recknor, C, Bolognese, M, A. Wang, A, Lin, C. J, F. Wagman, R, B. Ferrari, S,. Influence of subject discontinuation on longterm nonvertebral fracture rate in the denosumab FREEDOM Extension study. BMC Musculoskeletal Disorders. 201727 Apr;18 (1) (no pagination)(174) PMID: 615687631. Ineligible study design.

12. Adami S, Passeri, M, Ortolani, S, Broggini, M, Carratelli, L, Caruso, I, Gandolini, G, Gnessi, L, Laurenzi, M, Lombardi, A, et al.,. Effects of oral alendronate and intranasal salmon calcitonin on bone mass and biochemical markers of bone turnover in postmenopausal women with osteoporosis. Bone. 1995 
Oct;17(4):383-90. PMID: 8573412.

Ineligible treatment duration.

13. Adami S, Felsenberg, D, Christiansen, C, Robinson, J, Lorenc, R, S. Mahoney, P, Coutant, K, Schimmer, R, C. Delmas, P, D. Efficacy and safety of ibandronate given by intravenous injection once every 3 months. Bone. 2004 May;34(5):881-9. PMID: 15121020. Ineligible treatment duration.

14. Adami S, San, Martin J, Munoz, Torres M, Econs, M, J. Xie, L, Dalsky, G, P. McClung, M, Felsenberg, D, Brown, J, P. Brandi, M, L. Sipos, A,. Effect of raloxifene after recombinant teriparatide [hPTH(1-34)] treatment in postmenopausal women with osteoporosis. Osteoporosis International. 2008 Jan;19(1):87-94. PMID: 17938984. No outcomes of interest.

15. Adami S, Giannini, S, Bianchi, G, Sinigaglia, L, Di, Munno O, Fiore, C, E. Minisola, S, Rossini, M,. Vitamin D status and response to treatment in postmenopausal osteoporosis. Osteoporosis International. 2009 Feb;20(2):239-44.

PMID: 18551242. No outcomes of interest.

16. Adami S, Libanati, C, Boonen, S, Cummings, S, R. Ho, P, R. Wang, A, Siris, E, Lane, J, Freedom FractureHealing Writing, Group Adachi, J, D. Bhandari, M, de, Gregorio L, Gilchrist, N, Lyritis, G, Moller, G, Palacios, S, Pavelka, K, Heinrich, R, Roux, C, Uebelhart, D,. Denosumab treatment in postmenopausal women with osteoporosis does not interfere with fracture-healing: results from the FREEDOM trial. Journal of Bone \& Joint Surgery - American Volume. 2012 Dec 05;94(23):2113-9. PMID: 23097066. No outcomes of interest.

17. Adami S, Palacios, S, Rizzoli, R, Levine, A, B. Sutradhar, S, Chines, A, A. The efficacy and safety of bazedoxifene in postmenopausal women by baseline kidney function status. Climacteric. 2014 Jun;17(3):273-84. PMID: 23937421. Ineligible treatment duration.

18. Adler R, A. Osteoporosis in men: what has changed? Current Osteoporosis Reports.
2011 Mar;9(1):31-5. PMID: 21113693. Ineligible study design.

19. Adler R, A. El-Hajj, Fuleihan G, Bauer, D, C. Camacho, P, M. Clarke, B, L. Clines, G, A. Compston, J, E. Drake, M, T. Edwards, B, J. Favus, M, J. Greenspan, S, L. McKinney, R, Jr, Pignolo, R, J. Sellmeyer, D, E. Managing Osteoporosis in Patients on Long-Term Bisphosphonate Treatment: Report of a Task Force of the American Society for Bone and Mineral Research. Journal of Bone \& Mineral Research. 2016 Jan;31(1):16-35. PMID: 26350171. Ineligible study design.

20. Adler RA. Update on osteoporosis in men. Best Practice and Research: Clinical Endocrinology and Metabolism. 2018 PMID: 2000853205. Ineligible study design.

21. Agnusdei D, Gennari, C, Bufalino, L,. Prevention of early postmenopausal bone loss using low doses of conjugated estrogens and the non-hormonal, bone-active drug ipriflavone. Osteoporosis International. 1995;5(6):462-6. PMID: 8695969. No outcomes of interest.

22. Aki S, Gulbaba, R, G. Eskiyurt, N,. Effect of alendronate on bone density and bone markers in postmenopausal osteoporosis. Journal of Back and Musculoskeletal Rehabilitation. 2004;17(1):27-31. PMID: 38578531. No outcomes of interest.

23. Al BM, M. Alkhorayef, M, A. Bystrom, J, Akanle, O, A. Al, Adhoubi N, K. Jawad, A, S. Mageed, R, A. Favorable therapeutic response of osteoporosis patients to treatment with intravenous zoledronate compared with oral alendronate. Saudi Medical Journal. 2015 Nov;36(11):1305-11. PMID: 26593163. Ineligible study design.

24. Alexandersen B, Christiansen,. Piperazine oestrone sulphate and interrupted norethisterone in postmenopausal women: effects on bone mass, lipoprotein metabolism, climacteric symptoms, and adverse effects. Bjog. 2000;107(3):356-64. PMID: CN-00276681. Ineligible treatment duration. 
25. Alexandersen P, Riis, B, J. Christiansen, C,. Monofluorophosphate combined with hormone replacement therapy induces a synergistic effect on bone mass by dissociating bone formation and resorption in postmenopausal women: a randomized study. Journal of Clinical Endocrinology \& Metabolism. 1999 Sep;84(9):3013-20. PMID: 10487657. Ineligible treatment duration.

26. Aloia J, F. Vaswani, A, Yeh, J, K. Russo, L,. Differential effects of dietary calcium augmentation and hormone replacement therapy on bone turnover and serum levels of calcitrophic hormones. Osteoporosis International. 1996;6(1):55-62. PMID: 8845601. Ineligible treatment duration.

27. Alonso CP, Garcia, Franco A, L. Guyatt, G, Moynihan, R,. Drugs for preosteoporosis: prevention or disease mongering? BMJ. 2008 Jan 19;336(7636):126-9. PMID: 18202066. Ineligible study design.

28. Ambrus C, Almasi, C, Berta, K, Deak, G, Marton, A, Molnar, M, Z. Nemeth, Z, Horvath, C, Lakatos, P, Szathmari, M, Mucsi, I,. Bone mineral density and parathyroid function in patients on maintenance hemodialysis. International Urology \& Nephrology. 2011 Mar;43(1):191-201. PMID: 20091221. Ineligible study design.

29. Anagnostis P, Paschou, S, A. Mintziori, G, Ceausu, I, Depypere, H, Lambrinoudaki, I, Mueck, A, Perez, Lopez F, R. Rees, M, Senturk, L, M. Simoncini, T, Stevenson, J, C. Stute, P, Tremollieres, F, A. Goulis, D, G. Drug holidays from bisphosphonates and denosumab in postmenopausal osteoporosis: EMAS position statement. Maturitas. 2017 Jul;101:23-30. PMID: 28539165. Ineligible study design.

30. Anastasilakis A, D. Goulis, D, G. Polyzos, S, A. Gerou, S, Koukoulis, G, N. Efstathiadou, Z, Kita, M, Avramidis, A,. Head-to-head comparison of risedronate vs. teriparatide on bone turnover markers in women with postmenopausal osteoporosis: a randomised trial. International Journal of
Clinical Practice. 2008 Jun;62(6):919-24. PMID: 18422590. No outcomes of interest.

31. Anastasilakis A, D. Toulis, K, A. Polyzos, S, A. Anastasilakis, C, D. Makras, P,. Long-term treatment of osteoporosis: safety and efficacy appraisal of denosumab. Therapeutics \& Clinical Risk Management. 2012;8:295-306. PMID: 22767993. Ineligible study design.

32. Anastasilakis A, D. Polyzos, S, A. Gkiomisi, A, Bisbinas, I, Gerou, S, Makras, P,. Comparative effect of zoledronic acid versus denosumab on serum sclerostin and dickkopf-1 levels of naive postmenopausal women with low bone mass: a randomized, head-to-head clinical trial. Journal of Clinical Endocrinology \& Metabolism. 2013 Aug;98(8):3206-12. PMID: 23788684. Ineligible treatment duration.

33. Anastasilakis A, D. Polyzos, S, A. Gkiomisi, A, Saridakis, Z, G. Digkas, D, Bisbinas, I, Sakellariou, G, T. Papatheodorou, A, Kokkoris, P, Makras, $\mathrm{P}$,. Denosumab versus zoledronic acid in patients previously treated with zoledronic acid. Osteoporosis International. 2015 Oct;26(10):2521-7. PMID: 25990355. No outcomes of interest.

34. Anderson G, L. Limacher, M, Assaf, A, R. Bassford, T, Beresford, S, A. Black, $\mathrm{H}$, Bonds, D, Brunner, R, Brzyski, R, Caan, B, Chlebowski, R, Curb, D, Gass, M, Hays, J, Heiss, G, Hendrix, S, Howard, B, V. Hsia, J, Hubbell, A, Jackson, R, Johnson, K, C. Judd, H, Kotchen, J, M. Kuller, L, LaCroix, A, Z. Lane, D, Langer, R, D. Lasser, N, Lewis, C, E. Manson, J, Margolis, K, Ockene, J, O'Sullivan, M, J. Phillips, L, Prentice, R, L. Ritenbaugh, C, Robbins, J, Rossouw, J, E. Sarto, G, Stefanick, M, L. Van, Horn L, Wactawski, Wende J, Wallace, R, Wassertheil, Smoller S, Women's Health Initiative, Steering Committee,. Effects of conjugated equine estrogen in postmenopausal women with hysterectomy: the Women's Health Initiative randomized controlled trial. JAMA. 2004 Apr 14;291(14):1701-12. PMID: 15082697. Ineligible population. 
35. Ando H, Otoda, T, Ookami, H, Nagai, Y, Inano, A, Takamura, T, Ushijima, K, Hosohata, K, Matsushita, E, Saito, T, Kaneko, S, Fujimura, A,. Dosing timedependent effect of raloxifene on plasma plasminogen activator inhibitor-1 concentrations in post-menopausal women with osteoporosis. Clinical \& Experimental Pharmacology \& Physiology. 2013 Mar;40(3):227-32. PMID: 23323567. No outcomes of interest.

36. Angsanuntsukh C, Wajanavisit, W, Woratanarat, P,. Efficacy of Aldren 70 compared with Fosamax in terms of bone resorption marker in postmenopausal osteoporosis. Journal of the Medical Association of Thailand. 2017 May;100(5):488-95. PMID: 616837309. Ineligible treatment duration.

37. Anonymous. Effects of hormone therapy on bone mineral density: results from the postmenopausal estrogen/progestin interventions (PEPI) trial. The Writing Group for the PEPI. JAMA. 1996 Nov 06;276(17):1389-96. PMID: 8892713. Ineligible treatment duration.

38. Anonymous. Summaries for patients. Bone loss after stopping estrogen or alendronate therapy. Annals of internal medicine. 20023 Dec;137(11):I31. PMID: 35509505. Ineligible study design.

39. Anonymous. Study confirms long-term renal safety of annual zoledronic acid infusions. Nature Clinical Practice Nephrology. 2008 October;4(10):522. PMID: 352441234. Ineligible study design.

40. Anonymous. Reduction in vertebral fracture risk persists after discontinuation of risedronate. Nature Clinical Practice Endocrinology and Metabolism. 200811 Jun;4(6):307. PMID: 351733471. Ineligible study design.

41. Anonymous. Twice-monthly risedronate is as effective as daily dosing for osteoporosis therapy. Nature Clinical Practice Endocrinology and Metabolism. 2008
October;4(10):535. PMID: 352413990. Ineligible study design.

42. Anonymous. Effect of raloxifene on bone mineral density is enhanced in women with stage 3 CKD. Nature Clinical Practice Nephrology. 2008 November;4(11):584. PMID: 352577917. Ineligible study design.

43. Anonymous. Denosumab. Limited efficacy in fracture prevention, too many adverse effects. Prescrire International. 2011 Jun;20(117):145-8. PMID: 21678700. Ineligible study design.

44. Anonymous. In brief: delayed-release risedronate (Atelvia). Medical Letter on Drugs \& Therapeutics. 2011 Mar 21;53(1360):24. PMID: 21412209. Ineligible study design.

45. Anonymous. Zoledronic acid prevents fractures in men with osteoporosis. BMJ (Online). 201210 Nov;345 (7882) (no pagination)(e7471) PMID: 366023558. Ineligible study design.

46. Antoniucci D, M. Vittinghoff, E, Blackwell, T, Black, D, M. Sellmeyer, D, E. Vitamin D insufficiency does not affect bone mineral density response to raloxifene. Journal of Clinical Endocrinology \& Metabolism. 2005 Aug;90(8):4566-72. PMID: 15899959. Ineligible treatment duration.

47. Antoniucci D, M. Vittinghoff, E, Palermo, L, Black, D, M. Sellmeyer, D, E. Vitamin $\mathrm{D}$ insufficiency does not affect response of bone mineral density to alendronate. Osteoporosis International. 2009 Jul;20(7):1259-66. PMID: 19043656. No outcomes of interest.

48. Arabi A, Garnero, P, Porcher, R, Pelissier, C, Benhamou, C, L. Roux, C,. Changes in body composition during post-menopausal hormone therapy: a 2 year prospective study. Human Reproduction. 2003 Aug;18(8):1747-52. PMID: 12871894. Ineligible treatment duration.

49. Archer D, F. Pinkerton, J, V. Utian, W, H. Menegoci, J, C. de, Villiers T, J. Yuen, 
C, K. Levine, A, B. Chines, A, A. Constantine, G, D. Bazedoxifene, a selective estrogen receptor modulator: effects on the endometrium, ovaries, and breast from a randomized controlled trial in osteoporotic postmenopausal women. Menopause. 2009 Nov-Dec;16(6):1109-15. PMID: 19543129. No outcomes of interest.

50. Archer D, F. Lewis, V, Carr, B, R. Olivier, S, Pickar, J, H. Bazedoxifene/conjugated estrogens (BZA/CE): incidence of uterine bleeding in postmenopausal women. Fertility and Sterility. 2009 September;92(3):1039-44. PMID: 50591767. Ineligible treatment duration.

51. Arrenbrecht C, Garnero, Felsenberg,. The effect of continuous oestradiol with intermittent norgestimate on bone mineral density and bone turnover in postmenopausal women. Maturitas. 2004;48(3):197-207. PMID: CN-00502235. Ineligible treatment duration.

52. Arrenbrecht S, Boermans, A, J. Effects of transdermal estradiol delivered by a matrix patch on bone density in hysterectomized, postmenopausal women: a 2-year placebocontrolled trial. Osteoporosis International. 2002;13(2):176-83. PMID: 11908492. Ineligible treatment duration.

53. Asaoka D, Nagahara, A, Hojo, M, Ueyama, H, Matsumoto, K, Izumi, K, Takeda, T, Komori, H, Akazawa, Y, Shimada, Y, Osada, T, Watanabe, S,. Efficacy of alfacalcidol and alendronate on lumbar bone mineral density in osteoporotic patients using proton pump inhibitors. Biomedical Reports. 2016 August;5(2):16570. PMID: 611150914. No interventions of interest.

54. Ascott EB, H. Guanabens, N, Kivinen, S, Stuckey, B, G. Magaril, C, H. Vandormael, K, Stych, B, Melton, M, E. Alendronate prevents loss of bone density associated with discontinuation of hormone replacement therapy: a randomized controlled trial. Archives of Internal Medicine. 2003 Apr 14;163(7):789-94. PMID: 12695269. No outcomes of interest.
55. Atmaca A, Gedik, O,. Effects of alendronate and risedronate on bone mineral density and bone turnover markers in late postmenopausal women with osteoporosis. Advances in Therapy. 2006 NovDec;23(6):842-53. PMID: 17276952. No outcomes of interest.

56. Aubertin LM, Melancon, M, O. Chaput, J, P. Dionne, I, J. Past-users of HRT are osteopenic four months after discontinuation:an observational and crosssectional study. Journal of Women \& Aging. 2006;18(4):19-29. PMID: 17200061. Ineligible treatment duration.

57. Austin M, Yang, Y, C. Vittinghoff, E, Adami, S, Boonen, S, Bauer, D, C. Bianchi, G, Bolognese, M, A. Christiansen, C, Eastell, R, Grauer, A, Hawkins, F, Kendler, D, L. Oliveri, B, McClung, M, R. Reid, I, R. Siris, E, S. Zanchetta, J, Zerbini, C, A. Libanati, C, Cummings, S, R. Freedom, Trial.

Relationship between bone mineral density changes with denosumab treatment and risk reduction for vertebral and nonvertebral fractures. Journal of Bone \& Mineral Research. 2012 Mar;27(3):687-93. PMID: 22095631. Ineligible treatment duration.

58. Axelsson KF, Wallander M, Johansson H, et al. Hip fracture risk and safety with alendronate treatment in the oldest-old. Journal of Internal Medicine. 2017 Dec;282(6):546-59. PMID: 28857352. No outcomes of interest.

59. Bagger Y, Z. Tanko, L, B. Alexandersen, P, Ravn, P, Christiansen, C,. Alendronate has a residual effect on bone mass in postmenopausal Danish women up to 7 years after treatment withdrawal. Bone. 2003 Sep;33(3):301-7. PMID: 13678770. Ineligible population.

60. Bagger Y, Tankó, Lb, Alexandersen, Ravn, Christiansen,. Alendronate has a residual effect on bone mass in postmenopausal Danish women up to 7 years after treatment withdrawal. Bone. 2003;33(3):301-7. PMID: CN-00469953. Ineligible population. 
61. Bagger Y, Tankó, Lb, Alexandersen, Hansen, Hb, Møllgaard, Ravn, Qvist, Kanis, Ja, Christiansen,. Two to three years of hormone replacement treatment in healthy women have long-term preventive effects on bone mass and osteoporotic fractures: the PERF study. Bone. 2004;34(4):728-35. PMID: CN-00489218. Ineligible study design.

62. Bai H, Jing, D, Guo, A, Yin, S, Randomized controlled trial of zoledronic acid for treatment of osteoporosis in women. Journal of International Medical Research. 2013 Jun;41(3):697-704. PMID: 23669294. No outcomes of interest.

63. Baillargeon J, Kuo, Y, F. Lin, Y, L. Wilkinson, G, S. Goodwin, J, S. Osteonecrosis of the jaw in older osteoporosis patients treated with intravenous bisphosphonates. Annals of Pharmacotherapy. 2011 Oct;45(10):1199206. PMID: 21954448. No outcomes of interest.

64. Bakhireva L, N. Shainline, M, R. Carter, S, Robinson, S, Beaton, S, J. Nawarskas, J, J. Gunter, M, J. Synergistic effect of statins and postmenopausal hormone therapy in the prevention of skeletal fractures in elderly women. Pharmacotherapy:The Journal of Human Pharmacology \& Drug Therapy. 2010 Sep;30(9):879-87. PMID: 20795843. No outcomes of interest.

65. Bala Y, Chapurlat, R, Cheung, A, M. Felsenberg, D, LaRoche, M, Morris, E, Reeve, J, Thomas, T, Zanchetta, J, Bock, O, Ghasem, Zadeh A, Djoumessi, R, M. Seeman, E, Rizzoli, R,. Risedronate slows or partly reverses cortical and trabecular microarchitectural deterioration in postmenopausal women. Journal of Bone \& Mineral Research. 2014 Feb;29(2):380-8. PMID: 24115129. No outcomes of interest.

66. Bang U, C. Hyldstrup, L, Jensen, J, E. The impact of recombinant parathyroid hormone on malignancies and mortality: 7 years of experience based on nationwide Danish registers. Osteoporosis International. 2014 Feb;25(2):639-44. PMID: 23943162. Ineligible study design.
67. Banks. Raloxifene and breast cancer risk. Breast cancer research. 1999;1(1) PMID: $\mathrm{CN}-01166839$. Ineligible study design.

68. Banks E, Beral, V, Reeves, G, Balkwill, A, Barnes, I,. Fracture Incidence in Relation to the Pattern of Use of Hormone Therapy in Postmenopausal Women. Journal of the American Medical Association. 2004 12 May;291(18):2212-20. PMID: 38608170. Ineligible treatment duration.

69. Barasch A, Cunha, Cruz J, Curro, F, A. Hujoel, P, Sung, A, H. Vena, D, Voinea, Griffin A, E. Condor Collaborative, Group Beadnell, S, Craig, R, G. DeRouen, T, Desaranayake, A, Gilbert, A, Gilbert, G, H. Goldberg, K, Hauley, R, Hashimoto, M, Holmes, J, Latzke, B, Leroux, B, Lindblad, A, Richman, J, Safford, M, Ship, J, Thompson, V, P. Williams, O, D. Yin, W,. Risk factors for osteonecrosis of the jaws: a case-control study from the CONDOR dental PBRN.[Reprint in Tex Dent J. 2013 Apr 130(4):299-307 PMID: 23767159]. Journal of Dental Research. 2011 Apr;90(4):439-44. PMID: 21317246. Ineligible population.

70. Barnard K, Lakey, W, C. Batch, B, C. Chiswell, K, Tasneem, A, Green, J, B. Recent Clinical Trials in Osteoporosis: A Firm Foundation or Falling Short? PLoS ONE [Electronic Resource]. 2016;11(5):e0156068. PMID: 27191848. Ineligible study design.

71. Barreira J, C. Messina, O, D. Maldonado, Cocco J, A. Roldan, E, J. Site-dependent bone mineral density response to oral pamidronate and calcium in postmenopausal osteoporosis: a preliminary report. Clinical Rheumatology. 1997 Jun;16(4):346-52. PMID: 9259247. No interventions of interest.

72. Barrett CE, Young, R, Notelovitz, M, Sullivan, J, Wiita, B, Yang, H, M. Nolan, J,. A two-year, double-blind comparison of estrogen-androgen and conjugated estrogens in surgically menopausal women. Effects on bone mineral density, symptoms and lipid profiles. Journal of Reproductive Medicine. 
1999 Dec;44(12):1012-20. PMID: 10649811. Ineligible population.

73. Barrett CE, Ke, Harper, Mason, Tm, Sashegyi, Krueger, Ka, Anderson, Pw, Post hoc analysis of data from the Multiple Outcomes of Raloxifene Evaluation (MORE) trial on the effects of three years of raloxifene treatment on glycemic control and cardiovascular disease risk factors in women with and without type 2 diabetes. Clinical therapeutics. 2003;25(3):919-30. PMID: CN-00439164. No outcomes of interest.

74. Barrett CE, Mosca, L, Collins, P, Geiger, M, J. Grady, D, Kornitzer, M, McNabb, M, A. Wenger, N, K. Raloxifene Use for The Heart, Trial Investigators,. Effects of raloxifene on cardiovascular events and breast cancer in postmenopausal women. New England Journal of Medicine. $2006 \mathrm{Jul}$ 13;355(2):125-37. PMID: 16837676. Ineligible population.

75. Bauer D, C. Black, D, M. Garnero, P, Hochberg, M, Ott, S, Orloff, J, Thompson, D, E. Ewing, S, K. Delmas, P, D. Fracture Intervention Trial, Study Group,. Change in bone turnover and hip, non-spine, and vertebral fracture in alendronate-treated women: the fracture intervention trial. Journal of Bone \& Mineral Research. 2004 Aug;19(8):1250-8. PMID: 15231011. Ineligible treatment duration.

76. Bauer D, C. Garnero, P, Bilezikian, J, P. Greenspan, S, L. Ensrud, K, E. Rosen, C, J. Palermo, L, Black, D, M. Short-term changes in bone turnover markers and bone mineral density response to parathyroid hormone in postmenopausal women with osteoporosis. Journal of Clinical Endocrinology \& Metabolism. 2006 Apr;91(4):1370-5. PMID: 16449339. No interventions of interest.

77. Bauer D, C. Schwartz, A, Palermo, L, Cauley, J, Hochberg, M, Santora, A, Cummings, S, R. Black, D, M. Fracture prediction after discontinuation of 4 to 5 years of alendronate therapy: the FLEX study. JAMA Internal Medicine. 2014
Jul;174(7):1126-34. PMID: 24798675. Ineligible study design.

78. Bauer DC, Black DM, Bouxsein ML, et al. Treatment-Related Changes in Bone Turnover and Fracture Risk Reduction in Clinical Trials of Anti-Resorptive Drugs: A Meta-Regression. Journal of Bone and Mineral Research. 2018 April;33(4):634-42. PMID: 620161276. Ineligible study design.

79. Bawa H, S. Weick, J, Dirschl, D, R. AntiOsteoporotic Therapy After Fragility Fracture Lowers Rate of Subsequent Fracture: Analysis of a Large Population Sample. Journal of Bone \& Joint Surgery American Volume. 2015 Oct 07;97(19):1555-62. PMID: 26446962. Ineligible study design.

80. Bea J, W. Zhao, Q, Cauley, J, A. Lacroix, A, Z. Bassford, T, Lewis, C, E. Jackson, R, D. Tylavsky, F, A. Chen, Z,. Effect of hormone therapy on lean body mass, falls, and fractures: 6-year results from the Women's Health Initiative hormone trials. Menopause. 2011 January;18(1):44-52. PMID: 51022026. Ineligible population.

81. Beck T, J. Stone, K, L. Oreskovic, T, L. Hochberg, M, C. Nevitt, M, C. Genant, H, K. Cummings, S, R. Effects of current and discontinued estrogen replacement therapy on hip structural geometry: The study of osteoporotic fractures. Journal of Bone and Mineral Research. 2001;16(11):2103-10. PMID: 32995402. Ineligible treatment duration.

82. Beekman K, M. Veldhuis, Vlug A, G. den, Heijer M, Maas, M, Oleksik, A, M. Tanck, M, W. Ott, S, M. van 't, Hof R, J. Lips, P, Bisschop, P, H. Bravenboer, N,. The effect of raloxifene on bone marrow adipose tissue and bone turnover in postmenopausal women with osteoporosis. Bone. 2017 Oct 11;11:11. PMID: 29032175. Ineligible study design.

83. Bejhed R, S. Kharazmi, M, Hallberg, P,. Identification of Risk Factors for Bisphosphonate-Associated Atypical Femoral Fractures and Osteonecrosis of the Jaw in a Pharmacovigilance Database. Annals of Pharmacotherapy. 2016 
Aug;50(8):616-24. PMID: 27179251.

Ineligible treatment duration.

84. Bell K, J. Hayen, A, Glasziou, P, Irwig, L, Eastell, R, Harrison, S, L. Black, D, M. Bauer, D, C. Potential Usefulness of BMD and Bone Turnover Monitoring of Zoledronic Acid Therapy Among Women With Osteoporosis: Secondary Analysis of Randomized Controlled Trial Data. Journal of Bone \& Mineral Research. 2016

Sep;31(9):1767-73. PMID: 27027655. No interventions of interest.

85. Bell N, H. Bilezikian, J, P. Bone, H, G. rd, Kaur, A, Maragoto, A, Santora, A, C. M. K. Study Group. Alendronate increases bone mass and reduces bone markers in postmenopausal African-American women. Journal of Clinical Endocrinology \& Metabolism. 2002 Jun;87(6):2792-7. PMID: 12050252. No outcomes of interest.

86. Benhamou C, L. Effects of osteoporosis medications on bone quality. Joint, Bone, Spine: Revue du Rhumatisme. 2007 Jan;74(1):39-47. PMID: 17196423. Ineligible study design.

87. Benjamin B, Benjamin, M, A. Swe, M, Sugathan, S,. Review on the comparison of effectiveness between denosumab and bisphosphonates in post-menopausal osteoporosis. Osteoporosis and Sarcopenia. 201601 Jun;2(2):77-81. PMID: 610939976. Ineligible study design.

88. Beral V, Banks, E, Reeves, G,. Evidence from randomised trials on the long-term effects of hormone replacement therapy. Lancet. 2002 Sep 21;360(9337):942-4. PMID: 12354487. Ineligible study design.

89. Beral V, Million Women Study C. Breast cancer and hormone-replacement therapy in the Million Women Study. Lancet. 2003 Aug 9;362(9382):419-27. PMID: 12927427. Ineligible population.

90. Bergström F, Landgren, Bm,. Physical training and hormone replacement therapy reduce the decrease in bone mineral density in perimenopausal women: a pilot study. Osteoporosis international. 2005;16(7):823-

\section{PMID: $\mathrm{CN}-00527685$. Ineligible population.}

91. Bergstrom I, Freyschuss, B, Landgren, B, M. Physical training and hormone replacement therapy reduce the decrease in bone mineral density in perimenopausal women: a pilot study. Osteoporosis International. 2005 Jul;16(7):823-8. PMID: 15536539. Ineligible study design.

92. Beth TN, H. Mayer, Benjamin, Hussein, Heba, Zolk, Oliver,. Interventions for managing medication-related osteonecrosis of the jaw. Cochrane Database of Systematic Reviews. 2017(10) PMID: CD012432. Ineligible study design.

93. Bhandari M, Jin, L, See, K, Burge, R, Gilchrist, N, Witvrouw, R, Krohn, K, D. Warner, M, R. Ahmad, Q, I. Mitlak, B,. Does Teriparatide Improve Femoral Neck Fracture Healing: Results From A Randomized Placebo-controlled Trial. Clinical Orthopaedics \& Related Research. 2016 May;474(5):1234-44. PMID: 26932738. Ineligible treatment duration.

94. Bilbao J, Pugh, A, Aspy, C, B. In postmenopausal women with osteoporosis, is zoledronic acid (Reclast) superior in improving T-scores and decreasing fracture rates when compared with placebo? Journal - Oklahoma State Medical Association. 2010 Aug;103(8):374-5. PMID: 21049709. Ineligible study design.

95. Bilezikian J, P. Kurland, E, S. Therapy of male osteoporosis with parathyroid hormone. Calcified Tissue International. 2001 Oct;69(4):248-51. PMID: 11730261. Ineligible study design.

96. Bindon B, Adams W, Balasubramanian N, et al. Osteoporotic Fractures during Bisphosphonate Drug Holiday. Endocrine Practice. 2018 Feb;24(2):163-9. PMID: 29144808. Ineligible treatment duration.

97. Bjarnason N, H. Christiansen, C,. The influence of thinness and smoking on bone loss and response to hormone replacement therapy in early postmenopausal women. Journal of Clinical Endocrinology \& 
Metabolism. 2000 Feb;85(2):590-6. PMID: 10690860. Ineligible treatment duration.

98. Bjarnason N, H. Sarkar, S, Duong, T, Mitlak, B, Delmas, P, D. Christiansen, $\mathrm{C}$. Six and twelve month changes in bone turnover are related to reduction in vertebral fracture risk during 3 years of raloxifene treatment in postmenopausal osteoporosis. Osteoporosis International. 2001;12(11):922-30. PMID: 11808544. Ineligible treatment duration.

99. Bjarnason N, H. Alexandersen, P, Christiansen, C,. Number of years since menopause: spontaneous bone loss is dependent but response to hormone replacement therapy is independent. Bone. 2002 Apr;30(4):637-42. PMID: 11934658. Ineligible treatment duration.

100. Bjarnason N, Nielsen, Tf, Jørgensen, Hl, Christiansen,. The influence of smoking on bone loss and response to nasal estradiol. Climacteric. 2009;12(1):59-65. PMID: CN00685182. Ineligible treatment duration.

101. Black D, M. Cummings, S, R. Karpf, D, B. Cauley, J, A. Thompson, D, E. Nevitt, M, C. Bauer, D, C. Genant, H, K. Haskell, W, L. Marcus, R, Ott, S, M. Torner, J, C. Quandt, S, A. Reiss, T, F. Ensrud, K, E. Randomised trial of effect of alendronate on risk of fracture in women with existing vertebral fractures. Fracture Intervention Trial Research Group. Lancet. 1996 Dec 07;348(9041):1535-41. PMID: 8950879. No outcomes of interest.

102. Black D, M. Thompson, D, E. The effect of alendronate therapy on osteoporotic fracture in the vertebral fracture arm of the Fracture Intervention Trial. International Journal of Clinical Practice. Supplement. 1999 Apr;101:46-50. PMID: 12669740. No outcomes of interest.

103. Black D, M. Thompson, D, E. Bauer, D, C. Ensrud, K, Musliner, T, Hochberg, M, C. Nevitt, M, C. Suryawanshi, S, Cummings, S, R. Fracture, Intervention Trial,. Fracture risk reduction with alendronate in women with osteoporosis: the Fracture Intervention Trial. FIT Research Group.[Erratum appears in J Clin
Endocrinol Metab 2001 Feb 86(2):938]. Journal of Clinical Endocrinology \& Metabolism. 2000 Nov;85(11):4118-24. PMID: 11095442. Ineligible treatment duration.

104. Black D, M. Greenspan, S, L. Ensrud, K, E. Palermo, L, McGowan, J, A. Lang, T, F. Garnero, P, Bouxsein, M, L. Bilezikian, J, P. Rosen, C, J. Pa, T. H. Study Investigators. The effects of parathyroid hormone and alendronate alone or in combination in postmenopausal osteoporosis. New England Journal of Medicine. 2003 Sep 25;349(13):1207-15. PMID: 14500804. No outcomes of interest.

105. Black D, M. Bilezikian, J, P. Ensrud, K, E. Greenspan, S, L. Palermo, L, Hue, T, Lang, T, F. McGowan, J, A. Rosen, C, J. $\mathrm{Pa}, \mathrm{T}$. H. Study Investigators. One year of alendronate after one year of parathyroid hormone (1-84) for osteoporosis. New England Journal of Medicine. 2005 Aug 11;353(6):555-65. PMID: 16093464. No outcomes of interest.

106. Black D, M. Delmas, P, D. Eastell, R, Reid, I, R. Boonen, S, Cauley, J, A. Cosman, F, Lakatos, P, Leung, P, C. Man, Z, Mautalen, C, Mesenbrink, P, Hu, H, Caminis, J, Tong, K, Rosario, Jansen T, Krasnow, J, Hue, T, F. Sellmeyer, D, Eriksen, E, F. Cummings, S, R. Horizon Pivotal Fracture, Trial. Onceyearly zoledronic acid for treatment of postmenopausal osteoporosis. New England Journal of Medicine. 2007 May 03;356(18):1809-22. PMID: 17476007. No outcomes of interest.

107. Black D, M. Bouxsein, M, L. Palermo, L, McGowan, J, A. Newitt, D, C. Rosen, E, Majumdar, S, Rosen, C, J. P. T. H. OnceWeekly Research Group. Randomized trial of once-weekly parathyroid hormone (1-84) on bone mineral density and remodeling. Journal of Clinical Endocrinology \& Metabolism. 2008 Jun;93(6):2166-72. PMID: 18349061. Ineligible treatment duration.

108. Blumentals W, A. Harris, S, T. Cole, R, E. Huang, L, Silverman, S, L. Risk of severe gastrointestinal events in women 
treated with monthly ibandronate or weekly alendronate and risedronate. Annals of Pharmacotherapy. 2009 Apr;43(4):577-85. PMID: 19318598. Ineligible treatment duration.

109. Bock O, Borst, H, Beller, G, Armbrecht, G, Degner, C, Martus, P, Roth, H, J. Felsenberg, D,. Impact of oral ibandronate $150 \mathrm{mg}$ once monthly on bone structure and density in post-menopausal osteoporosis or osteopenia derived from in vivo muCT.

Bone. 2012 Jan;50(1):317-24. PMID: 22067902. No outcomes of interest.

110. Body J, J. Gaich, G, A. Scheele, W, H. Kulkarni, P, M. Miller, P, D. Peretz, A, Dore, R, K. Correa, Rotter R, Papaioannou, A, Cumming, D, C. Hodsman, A, B. A randomized doubleblind trial to compare the efficacy of teriparatide [recombinant human parathyroid hormone (1-34)] with alendronate in postmenopausal women with osteoporosis. Journal of Clinical Endocrinology \& Metabolism. 2002 Oct;87(10):4528-35. PMID: 12364430. No outcomes of interest.

111. Body J, J. How to manage postmenopausal osteoporosis? Acta Clinica Belgica. 2011 Nov-Dec;66(6):443-7. PMID: 22338309. Ineligible study design.

112. Body J, J. Bergmann, P, Boonen, S, Devogelaer, J, P. Gielen, E, Goemaere, S, Kaufman, J, M. Rozenberg, S, Reginster, J, Y. Extraskeletal benefits and risks of calcium, vitamin D and anti-osteoporosis medications. Osteoporosis International. 2012 February;23(SUPPL. 1):S1-S23. PMID: 364305818. Ineligible study design.

113. Bogado C, E. Massari, F, E. Zanchetta, J, R. Parathyroid hormone (1-84) and teriparatide in the treatment of postmenopausal osteoporosis. Women's health. 2006 May;2(3):447-57. PMID: 19803916. Ineligible study design.

114. Bogado C, E. Boailchuk, J, A. Zanchetta, M, B. Massari, F, E. Zanchetta, J, R. Denosumab: an update. Drugs of Today. 2011 Aug;47(8):605-13. PMID: 21850283. Ineligible study design.
115. Bogado C, E. Zanchetta, M, B. Boailchuk, J, A. Massari, F, E. Zanchetta, J, R. Denosumab: What's new? Current Osteoporosis Reports. $2011 \mathrm{Mar} ; 9(1): 12-9$. PMID: 21132405. Ineligible study design.

116. Bolanos R, Francia, J,. Isoflavones versus hormone therapy for reduction of vertebral fracture risk: indirect comparison. Menopause. 2010 Nov-Dec;17(6):1201-5. PMID: 20613672. Ineligible study design.

117. Bolognese M, Teglbjærg, Cs, Zanchetta, Jr, Lippuner, McClung, Mr, Brandi, Ml, Høiseth, Lakatos, Moffett, Ah, Lorenc, Rs, Wang, Libanati,. Denosumab significantly increases DXA BMD at both trabecular and cortical sites: results from the FREEDOM study. Journal of clinical densitometry. 2013;16(2):147-53. PMID: CN-00969747. No outcomes of interest.

118. Bone H, G. Downs, R, W. Jr, Tucci, J, R. Harris, S, T. Weinstein, R, S. Licata, A, A. McClung, M, R. Kimmel, D, B. Gertz, B, J. Hale, E, Polvino, W, J. Doseresponse relationships for alendronate treatment in osteoporotic elderly women. Alendronate Elderly Osteoporosis Study Centers. Journal of Clinical Endocrinology \& Metabolism. 1997 Jan;82(1):265-74. PMID: 8989272. Ineligible treatment duration.

119. Bone H, G. Greenspan, S, L. McKeever, C, Bell, N, Davidson, M, Downs, R, W. Emkey, R, Meunier, P, J. Miller, S, S. Mulloy, A, L. Recker, R, R. Weiss, S, R. Heyden, N, Musliner, T, Suryawanshi, S, Yates, A, J. Lombardi, A,. Alendronate and estrogen effects in postmenopausal women with low bone mineral density. Alendronate/Estrogen Study Group. Journal of Clinical Endocrinology \& Metabolism. 2000 Feb;85(2):720-6. PMID: 10690882. No outcomes of interest.

120. Bone H, G. Bolognese, M, A. Yuen, C, K. Kendler, D, L. Wang, H, Liu, Y, San, Martin J,. Effects of denosumab on bone mineral density and bone turnover in postmenopausal women. Journal of Clinical Endocrinology \& Metabolism. 2008 
Jun;93(6):2149-57. PMID: 18381571.

Ineligible treatment duration.

121. Bone H, G. Bolognese, M, A. Yuen, C, K. Kendler, D, L. Miller, P, D. Yang, Y, C. Grazette, L, San, Martin J, Gallagher, J, C. Effects of denosumab treatment and discontinuation on bone mineral density and bone turnover markers in postmenopausal women with low bone mass. Journal of Clinical Endocrinology \& Metabolism. 2011 Apr;96(4):972-80. PMID: 21289258. Ineligible study design.

122. Bone H, G. Chapurlat, R, Brandi, M, L. Brown, J, P. Czerwinski, E, Krieg, M, A. Mellstrom, D, Radominski, S, C.

Reginster, J, Y. Resch, H, Ivorra, J, A. Roux, C, Vittinghoff, E, Daizadeh, N, S. Wang, A, Bradley, M, N. Franchimont, N, Geller, M, L. Wagman, R, B. Cummings, S, R. Papapoulos, S,. The effect of three or six years of denosumab exposure in women with postmenopausal osteoporosis: results from the FREEDOM extension. Journal of Clinical Endocrinology \& Metabolism. 2013 Nov;98(11):4483-92. PMID: 23979955. Ineligible study design.

123. Bone H, G. Wagman, R, B. Brandi, M, L. Brown, J, P. Chapurlat, R, Cummings, S, R. Czerwinski, E, Fahrleitner, Pammer A, Kendler, D, L. Lippuner, K, Reginster, J, Y. Roux, C, Malouf, J, Bradley, M, N. Daizadeh, N, S. Wang, A, Dakin, P, Pannacciulli, N, Dempster, D, W. Papapoulos, S,. 10 years of denosumab treatment in postmenopausal women with osteoporosis: results from the phase 3 randomised FREEDOM trial and open-label extension. The Lancet Diabetes \& Endocrinology. 2017 Jul;5(7):513-23. PMID: 28546097. Ineligible study design.

124. Bone HG, Cosman F, Miller PD, et al. ACTIVExtend: 24 Months of Alendronate after 18 Months of Abaloparatide or Placebo for Postmenopausal Osteoporosis. Journal of Clinical Endocrinology \& Metabolism. 2018 May 24;24:24. PMID: 29800372. Ineligible study design.

125. Bonnick S, Saag, K, G. Kiel, D, P. McClung, M, Hochberg, M, Burnett, S, M. Sebba, A, Kagan, R, Chen, E,
Thompson, D, E. de, Papp A, E. Comparison of weekly treatment of postmenopausal osteoporosis with alendronate versus risedronate over two years.[Erratum appears in J Clin Endocrinol Metab. 2007 Aug 92(8):3032]. Journal of Clinical Endocrinology \& Metabolism. 2006 Jul;91(7):2631-7. PMID: 16636120. No outcomes of interest.

126. Bonnick S, Broy, S, Kaiser, F, Teutsch, C, Rosenberg, E, DeLucca, P, Melton, $\mathrm{M}$,. Treatment with alendronate plus calcium, alendronate alone, or calcium alone for postmenopausal low bone mineral density. Current Medical Research \& Opinion. 2007 Jun;23(6):1341-9. PMID: 17594775. No outcomes of interest.

127. Boonen S, McClung, M, R. Eastell, R, ElHajj, Fuleihan G, Barton, I, P. Delmas, $\mathrm{P}$,. Safety and efficacy of risedronate in reducing fracture risk in osteoporotic women aged 80 and older: implications for the use of antiresorptive agents in the old and oldest old. Journal of the American Geriatrics Society. 2004 Nov;52(11):1832-9. PMID: 15507059. Ineligible treatment duration.

128. Boonen S, Marin, F, Mellstrom, D, Xie, L, Desaiah, D, Krege, J, H. Rosen, C, J. Safety and efficacy of teriparatide in elderly women with established osteoporosis: bone anabolic therapy from a geriatric perspective. Journal of the American Geriatrics Society. 2006 May;54(5):782-9. PMID: 16696744. Ineligible treatment duration.

129. Boonen S, Sellmeyer, D, E. Lippuner, K, Orlov, Morozov A, Abrams, K, Mesenbrink, P, Eriksen, E, F. Miller, P, D. Renal safety of annual zoledronic acid infusions in osteoporotic postmenopausal women. Kidney International. 2008 Sep;74(5):641-8. PMID: 18509324. Ineligible treatment duration.

130. Boonen S, Orwoll, E, S. Wenderoth, D, Stoner, K, J. Eusebio, R, Delmas, P, D. Once-weekly risedronate in men with osteoporosis: results of a 2-year, placebocontrolled, double-blind, multicenter study. Journal of Bone \& Mineral Research. 2009 
Apr;24(4):719-25. PMID: 19049326. No outcomes of interest.

131. Boonen S, Klemes, A, B. Zhou, X, Lindsay, R,. Assessment of the relationship between age and the effect of risedronate treatment in women with postmenopausal osteoporosis: a pooled analysis of four studies. Journal of the American Geriatrics Society. 2010 Apr;58(4):658-63. PMID: 20345865. Ineligible treatment duration.

132. Boonen S, Black, D, M. Colon, Emeric C, S. Eastell, R, Magaziner, J, S. Eriksen, E, F. Mesenbrink, P, Haentjens, P, Lyles, $\mathrm{K}, \mathrm{W}$. Efficacy and safety of a once-yearly intravenous zoledronic acid $5 \mathrm{mg}$ for fracture prevention in elderly postmenopausal women with osteoporosis aged 75 and older. Journal of the American Geriatrics Society. 2010 Feb;58(2):292-9. PMID: 20070415. Ineligible treatment duration.

133. Boonen S, Orwoll, E, Magaziner, J, Colon, Emeric C, S. Adachi, J, D. Bucci, Rechtweg C, Haentjens, P, Kaufman, J, M. Rizzoli, R, Vanderschueren, D, Claessens, F, Sermon, A, Witvrouw, R, Milisen, K, Su, G, Lyles, K, W. Horizon Recurrent Fracture, Trial. Once-yearly zoledronic acid in older men compared with women with recent hip fracture. Journal of the American Geriatrics Society. 2011 Nov;59(11):2084-90. PMID: 22091563. Ineligible treatment duration.

134. Boonen S, Adachi, J, D. Man, Z, Cummings, S, R. Lippuner, K, Torring, O, Gallagher, J, C. Farrerons, J, Wang, A, Franchimont, N, San, Martin J, Grauer, A, McClung, M,. Treatment with denosumab reduces the incidence of new vertebral and hip fractures in postmenopausal women at high risk. Journal of Clinical Endocrinology \& Metabolism. 2011 Jun;96(6):1727-36. PMID: 21411557. Ineligible treatment duration.

135. Boonen S, Reginster, J, Y. Kaufman, J, M. Lippuner, K, Zanchetta, J, Langdahl, B, Rizzoli, R, Lipschitz, S, Dimai, H, P. Witvrouw, R, Eriksen, E, Brixen, K, Russo, L, Claessens, F, Papanastasiou, P, Antunez, O, Su, G, Bucci, Rechtweg C,
Hruska, J, Incera, E, Vanderschueren, D, Orwoll, E,. Fracture risk and zoledronic acid therapy in men with osteoporosis. New England Journal of Medicine. 201211 01;367(18):1714-23. PMID: 23113482. Ineligible treatment duration.

136. Boonen S, Ferrari, S, Miller, P, D. Eriksen, E, F. Sambrook, P, N. Compston, J, Reid, I, R. Vanderschueren, D, Cosman, F,. Postmenopausal osteoporosis treatment with antiresorptives: effects of discontinuation or long-term continuation on bone turnover and fracture risk--a perspective. Journal of Bone \& Mineral Research. 2012 May;27(5):963-74. PMID: 22467094. Ineligible study design.

137. Boonen S, Eastell, R, Su, G, Mesenbrink, P, Cosman, F, Cauley, J, A. Reid, I, R. Claessens, F, Vanderschueren, D, Lyles, K, W. Black, D, M. Time to onset of antifracture efficacy and year-by-year persistence of effect of zoledronic acid in women with osteoporosis. Journal of Bone \& Mineral Research. 2012 Jul;27(7):148793. PMID: 22431413. No outcomes of interest.

138. Borromeo G, L. Brand, C, Clement, J, G. McCullough, M, Thomson, W, Flitzanis, E, Wark, J, D. Is bisphosphonate therapy for benign bone disease associated with impaired dental healing? A case-controlled study. BMC Musculoskeletal Disorders. 2011 Apr 10;12:71. PMID: 21477374. Ineligible study design.

139. Bourke S, Bolland, M, J. Grey, A, Horne, A, M. Wattie, D, J. Wong, S, Gamble, G, D. Reid, I, R. The impact of dietary calcium intake and vitamin D status on the effects of zoledronate. Osteoporosis International. 2013 Jan;24(1):349-54. PMID: 22893357. No outcomes of interest.

140. Bouxsein M, L. Parker, R, A. Greenspan, $\mathrm{S}$, L. Forearm bone mineral densitometry cannot be used to monitor response to alendronate therapy in postmenopausal women. Osteoporosis International. 1999;10(6):505-9. PMID: 10663352. No outcomes of interest. 
141. Bouxsein M, L. Chen, P, Glass, E, V. Kallmes, D, F. Delmas, P, D. Mitlak, B, $\mathrm{H}$. Teriparatide and raloxifene reduce the risk of new adjacent vertebral fractures in postmenopausal women with osteoporosis. Results from two randomized controlled trials. Journal of Bone \& Joint Surgery American Volume. 2009 Jun;91(6):1329-38. PMID: 19487509. Ineligible treatment duration.

142. Boytsov N, Zhang, X, Sugihara, T, Taylor, K, Swindle, R,. Osteoporotic fractures and associated hospitalizations among patients treated with teriparatide compared to a matched cohort of patients not treated with teriparatide. Current Medical Research \& Opinion. 2015;31(9):1665-75. PMID: 26121328. Ineligible study design.

143. Braga V, Gatti, D, Colapietro, F, Battaglia, E, Righetti, D, Prizzi, R, Rossini, M, Adami, S,. Intravenous intermittent neridronate in the treatment of postmenopausal osteoporosis. Bone. 2003 Sep;33(3):342-5. PMID: 13678775. No interventions of interest.

144. Brandi M, L. Raloxifene reduces vertebral fracture risk in postmenopausal women with osteoporosis. Clinical \& Experimental Rheumatology. 2000 May-Jun;18(3):309-10. PMID: 10895366. Ineligible study design.

145. Breart G, Cooper, C, Meyer, O, Speirs, C, Deltour, N, Reginster, J, Y. Osteoporosis and venous thromboembolism: a retrospective cohort study in the UK General Practice Research Database. Osteoporosis International. 2010 Jul;21(7):1181-7. PMID: 19806285. No outcomes of interest.

146. Bridgeman M, B. Pathak, R,. Denosumab for the reduction of bone loss in postmenopausal osteoporosis: a review. Clinical Therapeutics. 2011 Nov;33(11):1547-59. PMID: 22108301. Ineligible study design.

147. Briot K, Benhamou, C, L. Roux, C,. Hip cortical thickness assessment in postmenopausal women with osteoporosis and strontium ranelate effect on hip geometry. Journal of Clinical Densitometry. 2012 Apr-Jun;15(2):176-85. PMID:

22321661. No interventions of interest.

148. Brown J, P. Kendler, D, L. McClung, M, R. Emkey, R, D. Adachi, J, D. Bolognese, M, A. Li, Z, Balske, A, Lindsay, R,. The efficacy and tolerability of risedronate once a week for the treatment of postmenopausal osteoporosis. Calcified Tissue International. 2002 Aug;71(2):10311. PMID: 12085156. Ineligible treatment duration.

149. Brown J, P. Prince, R, L. Deal, C, Recker, R, R. Kiel, D, P. de, Gregorio L, H. Hadji, P, Hofbauer, L, C. Alvaro, Gracia J, M. Wang, H, Austin, M, Wagman, R, B. Newmark, R, Libanati, C, San, Martin J, Bone, H, G. Comparison of the effect of denosumab and alendronate on BMD and biochemical markers of bone turnover in postmenopausal women with low bone mass: a randomized, blinded, phase 3 trial. Journal of Bone \& Mineral Research. 2009 Jan;24(1):153-61. PMID: 18767928. No outcomes of interest.

150. Brown J, P. Adachi, J, D. Kendler, D, L. Rigal, R, Deutsch, G, Leclerc, J, M. A community-based clinical trial of IntraVenous zOledRonic acid once Yearly in comparison to oral bisphosphonates in postmenopausal women with osteoporosis: the IVORY trial Methodological considerations. Contemporary Clinical Trials. 2011 Sep;32(5):741-6. PMID: 21628001. Ineligible study design.

151. Brown J, P. Roux, C, Torring, O, Ho, P, R. Beck, Jensen J, E. Gilchrist, N, Recknor, C, Austin, M, Wang, A, Grauer, A, Wagman, R, B.

Discontinuation of denosumab and associated fracture incidence: analysis from the Fracture Reduction Evaluation of Denosumab in Osteoporosis Every 6 Months (FREEDOM) trial. Journal of Bone \& Mineral Research. 2013 Apr;28(4):746-52. PMID: 23109251. Ineligible study design.

152. Brown J, Roux, Törring, Ho, Pr, Beck, Jensen, Je Gilchrist, Recknor, Austin, Wang, Grauer, Wagman, Rb,. Discontinuation of denosumab and 
associated fracture incidence: analysis from the Fracture Reduction Evaluation of Denosumab in Osteoporosis Every 6 Months (FREEDOM) trial. Journal of bone and mineral research. 2013;28(4):746-52. PMID: CN-01261357. Ineligible treatment duration.

153. Brown J, P. Morin, S, Leslie, W, Papaioannou, A, Cheung, A, M. Davison, K, S. Goltzman, D, Hanley, D, A. Hodsman, A, Josse, R, Jovaisas, A, Juby, A, Kaiser, S, Karaplis, A, Kendler, D, Khan, A, Ngui, D, Olszynski, W, Ste, Marie L, G. Adachi, J,. Bisphosphonates for treatment of osteoporosis: expected benefits, potential harms, and drug holidays. Canadian Family Physician. 2014 Apr;60(4):324-33. PMID: 24733321. Ineligible study design.

154. Brown J, P. Roux, C, Ho, P, R. Bolognese, M, A. Hall, J, Bone, H, G. Bonnick, S, van den, Bergh J, P. Ferreira, I, Dakin, P, Wagman, R, B. Recknor, C,. Denosumab significantly increases bone mineral density and reduces bone turnover compared with monthly oral ibandronate and risedronate in postmenopausal women who remained at higher risk for fracture despite previous suboptimal treatment with an oral bisphosphonate. Osteoporosis International. 2014 Jul;25(7):1953-61. PMID: 24676847. No outcomes of interest.

155. Brown J, Reid, Ir, Wagman, Rb, Kendler, Miller, Pd, Jensen, Je, Bolognese, Ma, Daizadeh, Valter, Zerbini, Ca, Dempster, Dw,. Effects of up to 5 years of denosumab treatment on bone histology and histomorphometry: the FREEDOM study extension. Journal of bone and mineral research. 2014;29(9):2051-6. PMID: CN01117418. Ineligible study design.

156. Brozek W, Reichardt, B, Zwerina, J, Dimai, H, P. Klaushofer, K, Zwettler, E,. Antiresorptive therapy and risk of mortality and refracture in osteoporosis-related hip fracture: a nationwide study. Osteoporosis International. 2016 Jan;27(1):387-96. PMID: 26576544. Ineligible study design.

157. Bruyere O, Collette, J, Rizzoli, R, Decock, C, Ortolani, S, Cormier, C,
Detilleux, J, Reginster, J, Y. Relationship between 3-month changes in biochemical markers of bone remodelling and changes in bone mineral density and fracture incidence in patients treated with strontium ranelate for 3 years. Osteoporosis International. 2010 Jun;21(6):1031-6. PMID: 19813043. No interventions of interest.

158. Bruyere O, Detilleux, J, Chines, A, Reginster, J, Y. Relationships between changes in bone mineral density or bone turnover markers and vertebral fracture incidence in patients treated with bazedoxifene. Calcified Tissue International. 2012 Oct;91(4):244-9. PMID: 22945761. No interventions of interest.

159. Bueno JA, H. Arias, L, Yu, C, R. Williams, R, Komm, B, S. Efficacy and safety of bazedoxifene in postmenopausal Latino women with osteoporosis. Menopause. 2017 Sep;24(9):1033-9. PMID: 28837504. No outcomes of interest.

160. Buist D, S. LaCroix, A, Z. Black, D, M. Harris, F, Blank, J, Ensrud, K, Edgerton, D, Rubin, S, Fox, K, M. Inclusion of older women in randomized clinical trials: factors associated with taking study medication in the fracture intervention trial. Journal of the American Geriatrics Society. 2000 Sep;48(9):1126-31. PMID: 10983914. No outcomes of interest.

161. Burkard D, Beckett T, Kourtjian E, et al. Effects of bone remodeling agents following teriparatide treatment. Osteoporosis International. 201814 Mar:1-7. PMID: 621252474. Ineligible study design.

162. Burshell A, L. Song, J, Dowsett, S, A. Mershon, J, L. Delmas, P, D. Secrest, R, J. Cauley, J, A. Relationship between bone mass, invasive breast cancer incidence and raloxifene therapy in postmenopausal women with low bone mass or osteoporosis. Current Medical Research \& Opinion. 2008 Mar;24(3):807-13. PMID: 18254988. No outcomes of interest.

163. Cadarette S, M. Katz, J, N. Brookhart, M, A. Sturmer, T, Stedman, M, R. Solomon, $D, H$. Relative effectiveness of osteoporosis drugs for preventing nonvertebral 
fracture.[Summary for patients in Ann Intern Med. 2008 May 6 148(9):I28 PMID: 18458273]. Annals of Internal Medicine. 2008 May 06;148(9):637-46. PMID: 18458276. Ineligible study design.

164. Caffarelli C, Montagnani A, Nuti R, et al. Bisphosphonates, atherosclerosis and vascular calcification: update and systematic review of clinical studies. Clinical Interventions In Aging. 2017;12:1819-28. PMID: 29133976. Ineligible study design.

165. Canderelli R, Leccesse, L, A. Miller, N, L. Unruh, Davidson J,. Benefits of hormone replacement therapy in postmenopausal women. Journal of the American Academy of Nurse Practitioners. 2007 Dec;19(12):635-41. PMID: 18042129. Ineligible study design.

166. Cantatore F, P. Cicinelli, E, Galantino, P, Pepe, V, Popolizio, A, Savino, F, Balzano, G, Epifani, S,. Evaluation of bone turnover and bone mass during tapering estrogen therapy: a preliminary report. Arthritis \& Rheumatism. 1995 Feb;38(2):292-4. PMID: 7848323. Ineligible population.

167. Caplan L, Pittman, C, B. Zeringue, A, L. Scherrer, J, F. Wehmeier, K, R. Cunningham, F, E. Eisen, S, A. McDonald, J, R. An observational study of musculoskeletal pain among patients receiving bisphosphonate therapy. Mayo Clinic Proceedings. 2010 Apr;85(4):341-8. PMID: 20231335. No outcomes of interest.

168. Capozzi A, Lello, S, Pontecorvi, A,. The inhibition of RANK-ligand in the management of postmenopausal osteoporosis and related fractures: the role of denosumab. Gynecological Endocrinology. 2014 Jun;30(6):403-8. PMID: 24592987. Ineligible study design.

169. Cardwell C, R. Abnet, C, C. Cantwell, M, M. Murray, L, J. Exposure to oral bisphosphonates and risk of esophageal cancer. JAMA. 2010 Aug 11;304(6):657-63. PMID: 20699457. Ineligible treatment duration.
170. Cartsos V, M. Zhu, S, Zavras, A, I. Bisphosphonate use and the risk of adverse jaw outcomes: a medical claims study of 714,217 people. Journal of the American Dental Association. 2008 Jan;139(1):23-30. PMID: 18167381. Ineligible treatment duration.

171. Casadei K, Becker, C,. Once-monthly risedronate for postmenopausal osteoporosis. International Journal of Women's Health. 2010 Aug 09;1:1-9. PMID: 21072270. Ineligible study design.

172. Castelo BC, Figueras, F, Sanjuan, A, Vicente, J, J. Martinez de, Osaba M, J. Pons, F, Balasch, J, Vanrell, J, A. Longterm compliance with estrogen replacement therapy in surgical postmenopausal women: benefits to bone and analysis of factors associated with discontinuation. Menopause. 1999 Winter;6(4):307-11. PMID: 10614677. Ineligible population.

173. Castelo BC, Vicente, J, J. Figueras, F, Sanjuan, A, Martinez de, Osaba M, J. Casals, E, Pons, F, Balasch, J, Vanrell, $\mathrm{J}$, A. Comparative effects of estrogens plus androgens and tibolone on bone, lipid pattern and sexuality in postmenopausal women. Maturitas. 2000 Feb 15;34(2):1618. PMID: 10714911. Ineligible treatment duration.

174. Cauley C, Secrest, Disch, Mershon,. Riskbenefit profile of raloxifene: influence of breast cancer risk factors. 2003 PMID: CN00989612. Ineligible study design.

175. Cauley J, A. Seeley, D, G. Browner, W, S. Ensrud, K, Kuller, L, H. Lipschutz, R, C. Hulley, S, B. Estrogen replacement therapy and mortality among older women. The study of osteoporotic fractures. Archives of Internal Medicine. 1997 Oct 27;157(19):2181-7. PMID: 9342994. Ineligible population.

176. Cauley J, A. Zmuda, J, M. Ensrud, K, E. Bauer, D, C. Ettinger, B, Study of Osteoporotic Fractures, Research Group,. Timing of estrogen replacement therapy for optimal osteoporosis prevention. Journal of Clinical Endocrinology \& Metabolism. 2001 
Dec;86(12):5700-5. PMID: 11739424.

Ineligible treatment duration.

177. Cauley J, A. Black, D, Boonen, S, Cummings, S, R. Mesenbrink, P, Palermo, L, Man, Z, Hadji, P, Reid, I, R. Horizon Pivotal Fracture, Group. Onceyearly zoledronic acid and days of disability, bed rest, and back pain: randomized, controlled HORIZON Pivotal Fracture Trial. Journal of Bone \& Mineral Research. 2011 May;26(5):984-92. PMID: 21542001. No outcomes of interest.

178. Cavalli L, Brandi, M, L. Targeted approaches in the treatment of osteoporosis: differential mechanism of action of denosumab and clinical utility. Therapeutics \& Clinical Risk Management. 2012;8:25366. PMID: 22745560. Ineligible study design.

179. Cecilia J, Fernández, Resines, Hawkins,. Effect of alendronate in elderly patients after low trauma hip fracture repair. Osteoporosis international. 2009;20(6):903-10. PMID: CN-00742972. No outcomes of interest.

180. Celi L, Ragno, Minisola, D'Erasmo, Mazzuoli, Gf,. Effects of the combined raloxifene-sodium fluoride therapy on bone mass and bone turnover in women with postmenopausal osteoporosis. Minerva medica. 2002;93(6):471-8. PMID: CN00615540. Not available in English.

181. Celikkanat H, Moroy, P, Senoz, S, Cettindag, I, Gokmen, O,. The effects of various hormone replacement therapy regimens on bone mineral density after 2 years of treatment. Marmara Medical Journal. 1996;9(4):165-8. PMID: 26377366. Ineligible population.

182. Center J, R. Bliuc, D, Nguyen, N, D. Nguyen, T, V. Eisman, J, A. Osteoporosis medication and reduced mortality risk in elderly women and men. Journal of Clinical Endocrinology \& Metabolism. 2011

Apr;96(4):1006-14. PMID: 21289270. No outcomes of interest.

183. Cetinkaya M, B. Kokcu, A, Yanik, F, F. Basoglu, T, Malatyalioglu, E, Alper, T,.
Comparison of the effects of transdermal estrogen, oral estrogen, and oral estrogenprogestogen therapy on bone mineral density in postmenopausal women. Journal of Bone \& Mineral Metabolism. 2002;20(1):44-8. PMID: 11810416. Ineligible population.

184. Chailurkit L, Ongphiphadhanakul, Piaseu, Saetung, Rajatanavin,. Biochemical markers of bone turnover and response of bone mineral density to intervention in early postmenopausal women: an experience in a clinical laboratory. Clinical chemistry. 2001;47(6):1083-8. PMID: CN-00442242. Ineligible treatment duration.

185. Chailurkit L, O. Jongjaroenprasert, W, Rungbunnapun, S, Ongphiphadhanakul, B, Sae, tung S, Rajatanavin, R,. Effect of alendronate on bone mineral density and bone turnover in Thai postmenopausal osteoporosis. Journal of Bone \& Mineral Metabolism. 2003;21(6):421-7. PMID: 14586800. No outcomes of interest.

186. Chan C, K. Mason, A, Cooper, C, Dennison, E,. Novel advances in the treatment of osteoporosis. British Medical Bulletin. 2016 Sep;119(1):129-42. PMID: 27558130. Ineligible study design.

187. Chan D, C. Yang, R, S. Ho, C, H. Tsai, Y, S. Wang, J, J. Tsai, K, T. The use of alendronate is associated with a decreased incidence of type 2 diabetes mellitus--a population-based cohort study in Taiwan. PLoS ONE [Electronic Resource]. 2015;10(4):e0123279. PMID: 25874962. No outcomes of interest.

188. Chao M, Hua, Q, Yingfeng, Z, Guang, W, Shufeng, S, Yuzhen, D, Wei, W, Haifeng, T,. Study on the role of zoledronic acid in treatment of postmenopausal osteoporosis women. Pakistan Journal of Medical Sciences. 2013 Nov;29(6):1381-4. PMID: 24550958. No outcomes of interest.

189. Chapurlat R, D. Ewing, S, K. Bauer, D, C. Cummings, S, R. Influence of smoking on the antiosteoporotic efficacy of raloxifene. Journal of Clinical Endocrinology \& Metabolism. 2001 Sep;86(9):4178-82. PMID: 11549646. No outcomes of interest. 
190. Chapurlat R, D. Treatment of osteoporosis with annual iv zoledronic acid: effects on hip fracture. Therapeutics \& Clinical Risk Management. 2009 Apr;5(2):169-75. PMID: 19536314. Ineligible study design.

191. Chapurlat R, D. Laroche, M, Thomas, T, Rouanet, S, Delmas, P, D. de, Vernejoul $\mathrm{M}, \mathrm{C}$. Effect of oral monthly ibandronate on bone microarchitecture in women with osteopenia-a randomized placebo-controlled trial. Osteoporosis International. 2013 Jan;24(1):311-20. PMID: 22402673. Ineligible treatment duration.

192. Checa M, A. Garrido, A, Prat, M, Conangla, M, Rueda, C, Carreras, R,. A comparison of raloxifene and calcium plus vitamin $\mathrm{D}$ on vaginal atrophy after discontinuation of long-standing postmenopausal hormone therapy in osteoporotic women. A randomized, masked-evaluator, one-year, prospective study. Maturitas. 2005 Sep 16;52(1):70-7. PMID: 16143228. No outcomes of interest.

193. Chen C, K. Chang, H, T. Chou, H, P. Lee, M, H. Chen, Y, C. Huang, Y, C. Chen, T, J. Chang, H, L. Shih, C, C. Alendronate and risk of lower limb ischemic vascular events: a population-based cohort study. Osteoporosis International. 2014 Feb;25(2):673-80. PMID: 23943167. Ineligible treatment duration.

194. Chen L, X. Ning, G, Z. Zhou, Z, R. Li, Y, L. Zhang, D, Wu, Q, L. Zhang, T, S. Cheng, L, Feng, S, Q. The carcinogenicity of alendronate in patients with osteoporosis: evidence from cohort studies. PLoS ONE [Electronic Resource].

2015;10(4):e0123080. PMID: 25881304. Ineligible study design.

195. Chen P, Satterwhite, J, H. Licata, A, A. Lewiecki, E, M. Sipos, A, A. Misurski, D, M. Wagman, R, B. Early changes in biochemical markers of bone formation predict BMD response to teriparatide in postmenopausal women with osteoporosis. Journal of Bone \& Mineral Research. 2005 Jun;20(6):962-70. PMID: 15883636. Ineligible treatment duration.
196. Chen P, Miller, P, D. Delmas, P, D. Misurski, D, A. Krege, J, H. Change in lumbar spine BMD and vertebral fracture risk reduction in teriparatide-treated postmenopausal women with osteoporosis. Journal of Bone \& Mineral Research. 2006 Nov;21(11):1785-90. PMID: 17002571. Ineligible treatment duration.

197. Chen Y, C. Lin, W, C. Can antiosteoporotic therapy reduce adjacent fracture in magnetic resonance imagingproven acute osteoporotic vertebral fractures? BMC Musculoskeletal Disorders. 2016 Apr 06;17:151. PMID: 27052323. Ineligible study design.

198. Cheng B, C. Chen, Y, C. Can lumbar spine bone mineral density predict readmission in denosumab-Treated patients with chronic kidney disease? Journal of Investigative Medicine. 2017 January;65(1):53-6. PMID: 616353872. Ineligible study design.

199. Cheng M, H. Chen, J, F. Fuh, J, L. Lee, W, L. Wang, P, H. Osteoporosis treatment in postmenopausal women with pre-existing fracture. Taiwanese Journal of Obstetrics \& Gynecology. 2012 Jun;51(2):153-66. PMID: 22795089. Ineligible study design.

200. Cheng S, Sipila, S, Taaffe, D, R. Puolakka, J, Suominen, H,. Change in bone mass distribution induced by hormone replacement therapy and high-impact physical exercise in post-menopausal women. Bone. 2002 Jul;31(1):126-35. PMID: 12110425. No outcomes of interest.

201. Chesnut C, H. rd, McClung, M, R. Ensrud, K, E. Bell, N, H. Genant, H, K. Harris, S, T. Singer, F, R. Stock, J, L. Yood, R, A. Delmas, P, D. et al.,. Alendronate treatment of the postmenopausal osteoporotic woman: effect of multiple dosages on bone mass and bone remodeling. American Journal of Medicine. 1995 Aug;99(2):144-52. PMID: 7625419. Ineligible treatment duration.

202. Chesnut C, H. rd, Bell, N, H. Clark, G, S. Drinkwater, B, L. English, S, C. Johnson, C, C. Jr, Notelovitz, M, Rosen, C, Cain, 
D, F. Flessland, K, A. Mallinak, N, J. Hormone replacement therapy in postmenopausal women: urinary $\mathrm{N}$ telopeptide of type I collagen monitors therapeutic effect and predicts response of bone mineral density. American Journal of Medicine. 1997 Jan;102(1):29-37. PMID: 9209198. Ineligible treatment duration.

203. Chesnut C, H. rd, Skag, A, Christiansen, C, Recker, R, Stakkestad, J, A. Hoiseth, A, Felsenberg, D, Huss, H, Gilbride, J, Schimmer, R, C. Delmas, P, D. Oral Ibandronate Osteoporosis Vertebral Fracture Trial in, North America, Europe,. Effects of oral ibandronate administered daily or intermittently on fracture risk in postmenopausal osteoporosis. Journal of Bone \& Mineral Research. 2004 Aug;19(8):1241-9. PMID: 15231010. Ineligible treatment duration.

204. Chesnut C, H. Ettinger, M, P. Miller, P, D. Baylink, D, J. Emkey, R, Harris, S, T. Wasnich, R, D. Watts, N, B. Schimmer, R, C. Recker, R, R. Ibandronate produces significant, similar antifracture efficacy in North American and European women: new clinical findings from BONE. Current Medical Research \& Opinion. 2005 Mar;21(3):391-401. PMID: 15811208. Ineligible treatment duration.

205. Cheung A, M. Frame, H, Ho, M, Mackinnon, E, S. Brown, J, P. Bone strength and management of postmenopausal fracture risk with antiresorptive therapies: Considerations for women's health practice. International Journal of Women's Health. 201628 Sep;8:537-47. PMID: 612819539. Ineligible study design.

206. Chiang C, H. Huang, C, C. Chan, W, L. Huang, P, H. Chen, T, J. Chung, C, M. Lin, S, J. Chen, J, W. Leu, H, B. Oral alendronate use and risk of cancer in postmenopausal women with osteoporosis: A nationwide study. Journal of Bone \& Mineral Research. 2012 Sep;27(9):1951-8. PMID: 22532232. No outcomes of interest.

207. Chilibeck P, D. Davison, K, S. Whiting, S, J. Suzuki, Y, Janzen, C, L. Peloso, P,. The effect of strength training combined with bisphosphonate (etidronate) therapy on bone mineral, lean tissue, and fat mass in postmenopausal women. Canadian Journal of Physiology \& Pharmacology. 2002 Oct;80(10):941-50. PMID: 12450060. Ineligible treatment duration.

208. Chitre M, Shechter, D, Grauer, A,. Denosumab for treatment of postmenopausal osteoporosis. American Journal of HealthSystem Pharmacy. 2011 Aug 01;68(15):1409-18. PMID: 21785030. Ineligible study design.

209. Chlebowski R, Chen, Cauley, Ja, Anderson, Rodabough, Rj, McTiernan, Lane, Ds, Manson, Je, Snetselaar, Yasmeen, O'Sullivan, Mj, Safford, Hendrix, Sl, Wallace, Rb,. Oral bisphosphonate use and breast cancer incidence in postmenopausal women. Journal of clinical oncology. 2010;28(22):3582-90. PMID: CN00889145. Ineligible treatment duration.

210. Chlebowski R, Barrington, Aragaki, Ak, Manson, Jae, Sarto, O'Sullivan, Mj, Wu, Cauley, Ja, Qi, Wallace, Rl, Prentice, $\mathrm{Rl}$,. Estrogen alone and health outcomes in black women by African ancestry: a secondary analyses of a randomized controlled trial. Menopause (new york, N.Y.). 2017;24(2):133-41. PMID: CN01328303. Ineligible population.

211. Choi H, Kim, Mj, Kim, Yh, Noh, Yh, Lee, Jw, Lee, Tw, Kim, Mg, Bae, Ks,. Pharmacokinetic Characteristics of Ibandronate and Tolerability of DP-R206 (150 mg Ibandronate/24,000 IU Vitamin D3) Compared to the Ibandronate (150 mg) Monotherapy in Healthy Adults. Transl clin pharmacol. 2014;22(1):22-9. PMID: CN01305762. Ineligible treatment duration.

212. Choi N, K. Solomon, D, H. Tsacogianis, T, N. Landon, J, E. Song, H, J. Kim, S, C. Comparative Safety and Effectiveness of Denosumab Versus Zoledronic Acid in Patients With Osteoporosis: A Cohort Study. Journal of Bone \& Mineral Research. 2017 Mar;32(3):611-7. PMID: 27736041. Ineligible treatment duration. 
213. Chrischilles E, A. Dasbach, E, J. Rubenstein, L, M. Cook, J, R. Tabor, H, K. Black, D, M. Fracture Intervention Trial, Research Group,. The effect of alendronate on fracture-related healthcare utilization and costs: the fracture intervention trial. Osteoporosis International. 2001;12(8):654-60. PMID: 11580079. No outcomes of interest.

214. Christiansen C, Chesnut, C, H. rd, Adachi, J, D. Brown, J, P. Fernandes, C, E. Kung, A, W. Palacios, S, Levine, A, B. Chines, A, A. Constantine, G, D. Safety of bazedoxifene in a randomized, double-blind, placebo- and active-controlled Phase 3 study of postmenopausal women with osteoporosis. BMC Musculoskeletal Disorders. 2010 Jun 22;11:130. PMID: 20569451. No outcomes of interest.

215. Christopher GJ, Palacios, S, Ryan, K, A. Yu, C, R. Pan, K, Kendler, D, L. Mirkin, S, Komm, B, S. Effect of conjugated estrogens/bazedoxifene on postmenopausal bone loss: Pooled analysis of two randomized trials. Menopause. 201601 Oct;23(10):1083-91. PMID: 611215758. Ineligible treatment duration.

216. Chuengsamarn S, Suwanwalaikorn, S,. Comparing the effect of short term post meals and bedtime calcium supplementation on the C-terminal telopeptide crosslinks and PTH levels in postmenopausal osteopenic women. Journal of the Medical Association of Thailand. 2005 Jun;88 Suppl 1:S12-20. PMID: 16858933. Ineligible treatment duration.

217. Cicinelli E, Cantatore, F, P. Galantino, P, Rubini, G, Cerundolo, M, Balzano, G, D'Aquino, T, M. Effects of continuous percutaneous estradiol administration on skeletal turnover in postmenopausal women: a 1-year prospective controlled study. European Journal of Obstetrics, Gynecology, \& Reproductive Biology. 1996 Nov;69(2):109-13. PMID: 8902442. No outcomes of interest.

218. Cipriani C, Irani, D, Bilezikian, J, P. Safety of osteoanabolic therapy: a decade of experience.[Erratum appears in J Bone Miner Res. 2013 Feb 28(2):431 Note:
Capriani, Cristiana [corrected to Cipriani, Cristiana]]. Journal of Bone \& Mineral Research. 2012 Dec;27(12):2419-28. PMID: 23165426. Ineligible study design.

219. Civitelli R, Pilgram, T, K. Dotson, M, Muckerman, J, Lewandowski, N, Armamento, Villareal R, Yokoyama, Crothers N, Kardaris, E, E. Hauser, J, Cohen, S, Hildebolt, C, F. Alveolar and postcranial bone density in postmenopausal women receiving hormone/estrogen replacement therapy: a randomized, doubleblind, placebo-controlled trial.[Erratum appears in Arch Intern Med. 2004 Jan 12 164(1):96]. Archives of Internal Medicine. 2002 Jun 24;162(12):1409-15. PMID: 12076241. Ineligible treatment duration.

220. Clark E, M. Durup, D,. Inflammatory eye reactions with bisphosphonates and other osteoporosis medications: what are the risks? Therapeutic Advances in Musculoskeletal Disease. 2015 Feb;7(1):116. PMID: 25650170. Ineligible study design.

221. Clemmesen B, Ravn, P, Zegels, B, Taquet, A, N. Christiansen, C, Reginster, J, Y. A 2-year phase II study with 1-year of follow-up of risedronate (NE-58095) in postmenopausal osteoporosis. Osteoporosis International. 1997;7(5):488-95. PMID: 9425508. Ineligible treatment duration.

222. Cobin R, H. Goodman, N, F. Aace Reproductive Endocrinology Scientific, Committee. American Association of Clinical Endocrinologists and American College of Endocrinology Position Statement on Menopause-2017 Update. Endocrine Practice. 2017 Jul;23(7):869-80. PMID: 28703650. Ineligible study design.

223. Cohran V, Cassedy, A, Hawkins, A, Bean, J, Heubi, J,. Oral risedronate sodium improves bone mineral density in nonambulatory patients: A randomized, doubleblind, placebo controlled trial. Journal of Pediatric Rehabilitation Medicine. 2013;6(2):85-93. PMID: 369389391. Ineligible population.

224. Cole Z, Dennison, E, Cooper, C,. Update on the treatment of post-menopausal 
osteoporosis. British Medical Bulletin. 2008;86:129-43. PMID: 18477578. Ineligible study design.

225. Collinge C, Favela, J,. Use of teriparatide in osteoporotic fracture patients. Injury. 2016 Jan;47 Suppl 1:S36-8. PMID: 26768289. Ineligible study design.

226. Coloma P, M. de, Ridder M, Bezemer, I, Herings, R, M. Gini, R, Pecchioli, S, Scotti, L, Rijnbeek, P, Mosseveld, M, van der, Lei J, Trifiro, G, Sturkenboom, M, Eu-Adr, Consortium. Risk of cardiac valvulopathy with use of bisphosphonates: a population-based, multi-country casecontrol study. Osteoporosis International. 2016 May;27(5):1857-67. PMID: 26694594. Ineligible study design.

227. Colon EC, S. Mesenbrink, P, Lyles, K, W. Pieper, C, F. Boonen, S, Delmas, P, Eriksen, E, F. Magaziner, J,. Potential mediators of the mortality reduction with zoledronic acid after hip fracture. Journal of Bone \& Mineral Research. 2010 Jan;25(1):91-7. PMID: 19580467. Ineligible treatment duration.

228. Colon EC, S. Lyles, K, W. Su, G, Pieper, C, F. Magaziner, J, S. Adachi, J, D. Bucci, Rechtweg C, M. Haentjens, P, Boonen, S, Horizon Recurrent Fracture, Trial. Clinical risk factors for recurrent fracture after hip fracture: a prospective study. Calcified Tissue International. 2011 May;88(5):425-31. PMID: 21331567. Ineligible treatment duration.

229. Compston J. The use of combination therapy in the treatment of postmenopausal osteoporosis. Endocrine. 2012 February;41(1):11-8. PMID: 51691000. Ineligible study design.

230. Compston J, Cooper, A, Cooper, C, Gittoes, N, Gregson, C, Harvey, N, Hope, S, Kanis, J, A. McCloskey, E, V. Poole, K. E, S. Reid, D, M. Selby, P, Thompson, F, Thurston, A, Vine, N, National Osteoporosis, Guideline Group,. UK clinical guideline for the prevention and treatment of osteoporosis. Archives of Osteoporosis. 2017 Dec;12(1):43. PMID: 28425085. Ineligible study design.
231. Cooke A, L. Metge, C, Lix, L, Prior, H, J. Leslie, W, D. Tamoxifen use and osteoporotic fracture risk: a populationbased analysis. Journal of Clinical Oncology. 2008 Nov 10;26(32):5227-32. PMID: 18838712. Ineligible study design.

232. Cooper C, Emkey, R, D. McDonald, R, H. Hawker, G, Bianchi, G, Wilson, K, Schimmer, R, C. Efficacy and safety of oral weekly ibandronate in the treatment of postmenopausal osteoporosis. Journal of Clinical Endocrinology \& Metabolism. 2003 Oct;88(10):4609-15. PMID: 14557430. Ineligible treatment duration.

233. Cooper C, Reginster, J, Y. Cortet, B, Diaz, Curiel M, Lorenc, R, S. Kanis, J, A. Rizzoli, R,. Long-term treatment of osteoporosis in postmenopausal women: a review from the European Society for Clinical and Economic Aspects of Osteoporosis and Osteoarthritis (ESCEO) and the International Osteoporosis Foundation (IOF). Current Medical Research \& Opinion. 2012 Mar;28(3):47591. PMID: 22356102. Ineligible study design.

234. Cornell C. CORR Insights $<$ sup $></$ sup $>$ : does Teriparatide Improve Femoral Neck Fracture Healing: results From A Randomized Placebo-controlled Trial. Clinical orthopaedics and related research. 2016;474(5):1245-6. PMID: CN-01265136. Ineligible study design.

235. Corrao G, Zambon, A, Nicotra, F, Conti, V, Nappi, R, E. Merlino, L,. Issues concerning the use of hormone replacement therapy and risk of fracture: a populationbased, nested case-control study. British Journal of Clinical Pharmacology. 2008 Jan;65(1):123-9. PMID: 17953723. Ineligible study design.

236. Cortet B, Bera, Louville A, Gauthier, P, Gauthier, A, Marchandise, X, Delcambre, B,. Comparative efficacy and safety study of etidronate and alendronate in postmenopausal osteoporosis. effect of adding hormone replacement therapy. Joint, Bone, Spine: Revue du Rhumatisme. 2001 
Oct;68(5):410-5. PMID: 11707007.

Ineligible study design.

237. Cosman F, Nieves, J, Woelfert, L, Formica, C, Gordon, S, Shen, V, Lindsay, R,. Parathyroid hormone added to established hormone therapy: effects on vertebral fracture and maintenance of bone mass after parathyroid hormone withdrawal. Journal of Bone \& Mineral Research. 2001 May;16(5):925-31. PMID: 11341338. No outcomes of interest.

238. Cosman F, Nieves, J, Zion, M, Woelfert, L, Luckey, M, Lindsay, R,. Daily and cyclic parathyroid hormone in women receiving alendronate. New England Journal of Medicine. 2005 Aug 11;353(6):566-75. PMID: 16093465. No outcomes of interest.

239. Cosman F, Borges, J, L. Curiel, M, D. Clinical evaluation of novel bisphosphonate dosing regimens in osteoporosis: the role of comparative studies and implications for future studies. Clinical Therapeutics. 2007 Jun;29(6):1116-27. PMID: 17692726. Ineligible study design.

240. Cosman F, Nieves, J, W. Zion, M, Barbuto, N, Lindsay, R,. Effect of prior and ongoing raloxifene therapy on response to PTH and maintenance of BMD after PTH therapy. Osteoporosis International. 2008 Apr;19(4):529-35. PMID: 17929072. No outcomes of interest.

241. Cosman F, Wermers, R, A. Recknor, C, Mauck, K, F. Xie, L, Glass, E, V. Krege, $\mathrm{J}, \mathrm{H}$. Effects of teriparatide in postmenopausal women with osteoporosis on prior alendronate or raloxifene: differences between stopping and continuing the antiresorptive agent. Journal of Clinical Endocrinology \& Metabolism. 2009 Oct;94(10):3772-80. PMID: 19584192. No outcomes of interest.

242. Cosman F, Nieves, J, W. Zion, M, Barbuto, N, Lindsay, R,. Retreatment with teriparatide one year after the first teriparatide course in patients on continued long-term alendronate. Journal of Bone \& Mineral Research. 2009 Jun;24(6):1110-5. PMID: 19113926. No outcomes of interest.
243. Cosman F, Eriksen, E, F. Recknor, C, Miller, P, D. Guanabens, N, Kasperk, C, Papanastasiou, P, Readie, A, Rao, H, Gasser, J, A. Bucci, Rechtweg C, Boonen, $\mathrm{S}$,. Effects of intravenous zoledronic acid plus subcutaneous teriparatide [rhPTH(134)] in postmenopausal osteoporosis. Journal of Bone \& Mineral Research. 2011 Mar;26(3):503-11. PMID: 20814967. Ineligible treatment duration.

244. Cosman F, Keaveny, T, M. Kopperdahl, D, Wermers, R, A. Wan, X, Krohn, K, D. Krege, J, H. Hip and spine strength effects of adding versus switching to teriparatide in postmenopausal women with osteoporosis treated with prior alendronate or raloxifene. Journal of Bone \& Mineral Research. 2013 Jun;28(6):1328-36. PMID: 23281041. No outcomes of interest.

245. Cosman F. Anabolic and antiresorptive therapy for osteoporosis: combination and sequential approaches. Current Osteoporosis Reports. 2014 Dec;12(4):385-95. PMID: 25341476. Ineligible study design.

246. Cosman F, Nieves, J, W. Zion, M, Garrett, P, Neubort, S, Dempster, D, Lindsay, R,. Daily or Cyclical Teriparatide Treatment in Women With Osteoporosis on no Prior Therapy and Women on Alendronate. Journal of Clinical Endocrinology \& Metabolism. 2015 Jul;100(7):2769-76. PMID: 25961136. Ineligible treatment duration.

247. Cosman F, Crittenden, D, B. Adachi, J, D. Binkley, N, Czerwinski, E, Ferrari, S, Hofbauer, L, C. Lau, E, Lewiecki, E, M. Miyauchi, A, Zerbini, C, A. Milmont, C, E. Chen, L, Maddox, J, Meisner, P, D. Libanati, C, Grauer, A,. Romosozumab Treatment in Postmenopausal Women with Osteoporosis. New England Journal of Medicine. 2016 Oct 20;375(16):1532-43. PMID: 27641143. No outcomes of interest.

248. Cosman F, Hattersley, G, Hu, M, Y. Williams, G, C. Fitzpatrick, L, A. Black, D, M. Effects of Abaloparatide-SC on Fractures and Bone Mineral Density in Subgroups of Postmenopausal Women With Osteoporosis and Varying Baseline Risk 
Factors. Journal of Bone \& Mineral Research. 2017 Jan;32(1):17-23. PMID: 27612281. Ineligible treatment duration.

249. Costa A, G. Bilezikian, J, P. How Long to Treat with Denosumab. Current Osteoporosis Reports. 2015 Dec;13(6):41520. PMID: 26474564. Ineligible study design.

250. Cranney A, Adachi, J, D. Guyatt, G, Papaioannou, A, Robinson, V, A. Shea, B, J. Tugwell, P, Waldegger, L, M. Weaver, B, Wells, G, Zytaruk, N,. WITHDRAWN: Risedronate for the prevention and treatment of postmenopausal osteoporosis. Cochrane Database of Systematic Reviews. 2007 Jul 18(1):CD004523. PMID: 17636764. Ineligible study design.

251. Cryer B, Miller, P, Petruschke, R, A. Chen, E, Geba, G, P. Papp, A, E. Upper gastrointestinal tolerability of once weekly alendronate $70 \mathrm{mg}$ with concomitant nonsteroidal anti-inflammatory drug use. Alimentary Pharmacology \& Therapeutics. 2005 Mar 01;21(5):599-607. PMID: 15740544. Ineligible treatment duration.

252. Cummings S, R. Eckert, S, Krueger, K, A. Grady, D, Powles, T, J. Cauley, J, A. Norton, L, Nickelsen, T, Bjarnason, N, H. Morrow, M, Lippman, M, E. Black, D, Glusman, J, E. Costa, A, Jordan, V, C. The effect of raloxifene on risk of breast cancer in postmenopausal women: results from the MORE randomized trial. Multiple Outcomes of Raloxifene

Evaluation.[Erratum appears in JAMA 1999

Dec 8 282(22):2124]. JAMA. 1999 Jun 16;281(23):2189-97. PMID: 10376571. No outcomes of interest.

253. Cummings S, R. Palermo, L, Browner, W, Marcus, R, Wallace, R, Pearson, J, Blackwell, T, Eckert, S, Black, D,. Monitoring osteoporosis therapy with bone densitometry: misleading changes and regression to the mean. Fracture Intervention Trial Research Group. JAMA. 2000 Mar 08;283(10):1318-21. PMID: 10714731. No outcomes of interest.
254. Cummings S, R. Duong, T, Kenyon, E, Cauley, J, A. Whitehead, M, Krueger, K, A. Multiple Outcomes of Raloxifene, Evaluation Trial,. Serum estradiol level and risk of breast cancer during treatment with raloxifene. JAMA. 2002 Jan 09;287(2):21620. PMID: 11779264. No outcomes of interest.

255. Cummings S, R. San, Martin J, McClung, M, R. Siris, E, S. Eastell, R, Reid, I, R. Delmas, P, Zoog, H, B. Austin, M, Wang, A, Kutilek, S, Adami, S, Zanchetta, J, Libanati, C, Siddhanti, S, Christiansen, C, Freedom, Trial.

Denosumab for prevention of fractures in postmenopausal women with osteoporosis.[Erratum appears in N Engl J Med. 2009 Nov 5 361(19):1914]. New England Journal of Medicine. 2009 Aug 20;361(8):756-65. PMID: 19671655. Ineligible treatment duration.

256. Cummings S, R. Ensrud, K, Delmas, P, D. LaCroix, A, Z. Vukicevic, S, Reid, D, M. Goldstein, S, Sriram, U, Lee, A, Thompson, J, Armstrong, R, A. Thompson, D, D. Powles, T, Zanchetta, J, Kendler, D, Neven, P, Eastell, R, Pearl Study, Investigators. Lasofoxifene in postmenopausal women with osteoporosis.[Erratum appears in N Engl J Med. 2011 Jan 20 364(3):290 Note: Kucu kdeveci A [corrected to Kucukdeveci, A]]. New England Journal of Medicine. 2010 Feb 25;362(8):686-96. PMID: 20181970. No interventions of interest.

257. Cummings SR, Ferrari S, Eastell R, et al. Vertebral Fractures After Discontinuation of Denosumab: A Post Hoc Analysis of the Randomized Placebo-Controlled FREEDOM Trial and Its Extension. Journal of Bone and Mineral Research. 2018 Februaryy;33(2):190-8. PMID: 619391692. Ineligible study design.

258. Curtis J, R. Westfall, A, O. Cheng, H, Delzell, E, Saag, K, G. Risk of hip fracture after bisphosphonate discontinuation: implications for a drug holiday. Osteoporosis International. 2008 Nov;19(11):1613-20. PMID: 18483689. Ineligible study design. 
259. Curtis J, R. Westfall, A, O. Cheng, H, Saag, K, G. Delzell, E,. RisedronatE and ALendronate Intervention over Three Years (REALITY): minimal differences in fracture risk reduction. Osteoporosis International. 2009 Jun;20(6):973-8. PMID: 18946630. Ineligible study design.

260. Curtis J, R. Delzell, E, Chen, L, Black, D, Ensrud, K, Judd, S, Safford, M, M. Schwartz, A, V. Bauer, D, C. The relationship between bisphosphonate adherence and fracture: is it the behavior or the medication? Results from the placebo arm of the fracture intervention trial. Journal of Bone \& Mineral Research. 2011 Apr;26(4):683-8. PMID: 20939064. Ineligible study design.

261. Curtis J, R. Yun, H, Lange, J, L. Matthews, R, Sharma, P, Saag, K, G. Delzell, E,. Does medication adherence itself confer fracture protection? An investigation of the healthy adherer effect in observational data. Arthritis care \& research. 2012 Dec;64(12):1855-63. PMID: 22730364. Ineligible study design.

262. Cusano N, E. Bilezikian, J, P. Combination Anabolic and Antiresorptive Therapy for Osteoporosis. Endocrinology and Metabolism Clinics of North America. 2012 September;41(3):643-54. PMID: 365405578. Ineligible study design.

263. Dagdelen S, Sener, D, Bayraktar, M,. Influence of type 2 diabetes mellitus on bone mineral density response to bisphosphonates in late postmenopausal osteoporosis. Advances in Therapy. 2007 Nov-Dec;24(6):1314-20. PMID: 18165214. Ineligible study design.

264. Daly E, Vessey, M, P. Hawkins, M, M. Carson, J, L. Gough, P, Marsh, S,. Risk of venous thromboembolism in users of hormone replacement therapy. Lancet. 1996 Oct 12;348(9033):977-80. PMID: 8855852. Ineligible study design.

265. D'Amelio P, Muratore, M, Tinelli, F, Tamone, C, Cosentino, L, Quarta, E, Calcagnile, F, Carlo, Isaia G,. Effect of raloxifene and clodronate on bone density in postmenopausal osteoporotic women.
International Journal of Tissue Reactions. 2003;25(2):73-8. PMID: 14518596. Ineligible treatment duration.

266. Dando T, M. Noble, S,. Once-monthly ibandronate. Treatments in Endocrinology. 2005;4(6):381-7 discussion 9-90. PMID: 16318406. Ineligible study design.

267. Dane C, Dane, B, Cetin, A, Erginbas, M, Comparison of the effects of raloxifene and low-dose hormone replacement therapy on bone mineral density and bone turnover in the treatment of postmenopausal osteoporosis. Gynecological Endocrinology. 2007 Jul;23(7):398-403. PMID: 17701771. No outcomes of interest.

268. Davas I, Altintas, A, Yoldemir, T, Varolan, A, Yazgan, A, Baksu, B,. Effect of daily hormone therapy and alendronate use on bone mineral density in postmenopausal women. Fertility \& Sterility. 2003 Sep;80(3):536-40. PMID: 12969694. Ineligible study design.

269. Dave M, Criswell, D, F. Question: In postmenopausal women with osteoporosis is once monthly oral ibandronate (150 mg) therapy preferred to once weekly oral bisphosphonate therapy. Journal - Oklahoma State Medical Association. 2012 Mar;105(3):89-90. PMID: 22685920. Ineligible study design.

270. Davey D, A. Osteoporosis, osteopenia and fracture risk: widening the therapeutic horizons. South African Medical Journal. Suid-Afrikaanse Tydskrif Vir Geneeskunde. 2012 Mar 08;102(5):285-8. PMID: 22554331. Ineligible study design.

271. Davis J, W. Ross, P, D. Johnson, N, E. Wasnich, R, D. Estrogen and calcium supplement use among Japanese-American women: effects upon bone loss when used singly and in combination. Bone. 1995 Oct;17(4):369-73. PMID: 8573410. Ineligible study design.

272. Davis J, W. Ross, P, D. Wasnich, R, D. A longitudinal study of estrogen and calcium supplement use among Japanese women living in Hawaii. Preventive Medicine. 1995 
Mar;24(2):159-65. PMID: 7597018.

Ineligible study design.

273. Dawson HB, Chen, P, Krege, J, H. Response to teriparatide in patients with baseline 25-hydroxyvitamin D insufficiency or sufficiency. Journal of Clinical

Endocrinology \& Metabolism. 2007

Dec;92(12):4630-6. PMID: 17911178.

Ineligible treatment duration.

274. de AD, Gambacciani, M, Altieri, P, Ciaponi, M, Ventura, V, Mura, M, Genazzani, A, R. Bottiglioni, F,. Bone density changes in postmenopausal women with the administration of ipriflavone alone or in association with low-dose ERT. Gynecological Endocrinology. 1997 Aug;11(4):289-93. PMID: 9272427. Ineligible treatment duration.

275. de VF, Cooper, A, L. Cockle, S, M. van, Staa T, P. Cooper, C,. Fracture risk in patients receiving acid-suppressant medication alone and in combination with bisphosphonates. Osteoporosis International. 2009 Dec;20(12):1989-98. PMID: 19333676. No outcomes of interest.

276. de VT, J. Bazedoxifene: a novel selective estrogen receptor modulator for postmenopausal osteoporosis. Climacteric. 2010 Jun;13(3):210-8. PMID: 20184423. Ineligible study design.

277. de VT, J. Chines, A, A. Palacios, S, Lips, P, Sawicki, A, Z. Levine, A, B. Codreanu, C, Kelepouris, N, Brown, J, P. Safety and tolerability of bazedoxifene in postmenopausal women with osteoporosis: results of a 5-year, randomized, placebocontrolled phase 3 trial. Osteoporosis International. 2011 Feb;22(2):567-76. PMID: 20535606. No interventions of interest.

278. Deal C, L. Draper, M, W. Raloxifene: a selective estrogen-receptor modulator for postmenopausal osteoporosis - a clinical update on efficacy and safety. Women's health. 2006 Mar;2(2):199-210. PMID: 19803890. Ineligible study design.
279. Deeks ED. Denosumab: A Review in Postmenopausal Osteoporosis.[Erratum appears in Drugs Aging. 2018 Mar 9;:; PMID: 29524105]. Drugs \& Aging. 2018 02;35(2):163-73. PMID: 29435849. Ineligible study design.

280. Dell R, M. Adams, A, L. Greene, D, F. Funahashi, T, T. Silverman, S, L. Eisemon, E, O. Zhou, H, Burchette, R, J. Ott, S, M. Incidence of atypical nontraumatic diaphyseal fractures of the femur. Journal of Bone \& Mineral Research. 2012 Dec;27(12):2544-50. PMID: 22836783. Ineligible study design.

281. Delmas P, D. Bjarnason, N, H. Mitlak, B, H. Ravoux, A, C. Shah, A, S. Huster, W, J. Draper, M, Christiansen, C,. Effects of raloxifene on bone mineral density, serum cholesterol concentrations, and uterine endometrium in postmenopausal women. New England Journal of Medicine. 1997 Dec 04;337(23):1641-7. PMID: 9385122. Ineligible treatment duration.

282. Delmas P, D. Hardy, P, Garnero, P, Dain, $\mathrm{M}$,. Monitoring individual response to hormone replacement therapy with bone markers. Bone. 2000 Jun;26(6):553-60. PMID: 10831925. Ineligible treatment duration.

283. Delmas P, D. Confavreux, E, Garnero, P, Fardellone, P, de, Vernejoul M, C. Cormier, C, Arce, J, C. A combination of low doses of 17 beta-estradiol and norethisterone acetate prevents bone loss and normalizes bone turnover in postmenopausal women. Osteoporosis International. 2000;11(2):177-87. PMID: 10793878. Ineligible treatment duration.

284. Delmas P, D. Genant, H, K. Crans, G, G. Stock, J, L. Wong, M, Siris, E, Adachi, J, D. Severity of prevalent vertebral fractures and the risk of subsequent vertebral and nonvertebral fractures: results from the MORE trial. Bone. 2003 Oct;33(4):522-32. PMID: 14555255. Ineligible treatment duration.

285. Delmas P, D. Recker, R, R. Chesnut, C, H. rd, Skag, A, Stakkestad, J, A. Emkey, R, Gilbride, J, Schimmer, R, C. 
Christiansen, C,. Daily and intermittent oral ibandronate normalize bone turnover and provide significant reduction in vertebral fracture risk: results from the BONE study. Osteoporosis International. 2004

Oct;15(10):792-8. PMID: 15071723.

Ineligible treatment duration.

286. Delmas P, Marianowski, Perez, Ade, C. Ribot, Pornel, Palacios, Lopes,. Prevention of postmenopausal bone loss by pulsed estrogen therapy: comparison with transdermal route. Maturitas. 2004;48(2):8596. PMID: CN-00489674. Ineligible treatment duration.

287. Delmas P, D. Adami, S, Strugala, C, Stakkestad, J, A. Reginster, J, Y. Felsenberg, D, Christiansen, C, Civitelli, R, Drezner, M, K. Recker, R, R. Bolognese, M, Hughes, C, Masanauskaite, D, Ward, P, Sambrook, P, Reid, D, M. Intravenous ibandronate injections in postmenopausal women with osteoporosis: one-year results from the dosing intravenous administration study. Arthritis \& Rheumatism. 2006 Jun;54(6):1838-46. PMID: 16729277. Ineligible treatment duration.

288. Delmas P, D. Licata, A, A. Reginster, J, Y. Crans, G, G. Chen, P, Misurski, D, A. Wagman, R, B. Mitlak, B, H. Fracture risk reduction during treatment with teriparatide is independent of pretreatment bone turnover. Bone. 2006 Aug;39(2):237-43. PMID: 16563890. Ineligible treatment duration.

289. Delmas P, D. Benhamou, C, L. Man, Z, Tlustochowicz, W, Matzkin, E, Eusebio, R, Zanchetta, J, Olszynski, W, P. Recker, R, R. McClung, M, R. Monthly dosing of $75 \mathrm{mg}$ risedronate on 2 consecutive days a month: efficacy and safety results. Osteoporosis International. 2008 Jul;19(7):1039-45. PMID: 18087660. Ineligible treatment duration.

290. Delmas P, D. McClung, M, R. Zanchetta, J, R. Racewicz, A, Roux, C, Benhamou, C, L. Man, Z, Eusebio, R, A. Beary, J, F. Burgio, D, E. Matzkin, E, Boonen, S,. Efficacy and safety of risedronate $150 \mathrm{mg}$ once a month in the treatment of postmenopausal osteoporosis. Bone. 2008 Jan;42(1):36-42. PMID: 17920005. Ineligible treatment duration.

291. Delmas P, D. Munoz, F, Black, D, M. Cosman, F, Boonen, S, Watts, N, B. Kendler, D, Eriksen, E, F. Mesenbrink, P, G. Eastell, R,. Effects of yearly zoledronic acid $5 \mathrm{mg}$ on bone turnover markers and relation of PINP with fracture reduction in postmenopausal women with osteoporosis. Journal of Bone and Mineral Research. 2009;24(9):1544-51. PMID: 355840767. Ineligible treatment duration.

292. Dempster D, W. Lambing, C, L. Kostenuik, P, J. Grauer, A,. Role of RANK ligand and denosumab, a targeted RANK ligand inhibitor, in bone health and osteoporosis: a review of preclinical and clinical data. Clinical Therapeutics. 2012 Mar;34(3):521-36. PMID: 22440513. Ineligible study design.

293. Dempster DW, Brown JP, FahrleitnerPammer A, et al. Effects of Long-term Denosumab on Bone Histomorphometry and Mineralization in Women With Postmenopausal Osteoporosis. Journal of Clinical Endocrinology \& Metabolism. 2018 Apr 16;16:16. PMID: 29672714. No outcomes of interest.

294. Devine D, Im, Dhaliwal, Ss, Naheed, Beilby, Prince, Rl,. Prediction of incident osteoporotic fractures in elderly women using the free estradiol index. Osteoporosis international. 2005;16(2):216-21. PMID: CN-00511425. No interventions of interest.

295. Devogelaer J, P. Broll, H, Correa, Rotter R, Cumming, D, C. De, Deuxchaisnes C, N. Geusens, P, Hosking, D, Jaeger, P, Kaufman, J, M. Leite, M, Leon, J, Liberman, U, Menkes, C, J. Meunier, P, J. Reid, I, Rodriguez, J, Romanowicz, A, Seeman, E, Vermeulen, A, Hirsch, L, J. Lombardi, A, Plezia, K, Santora, A, C. Yates, A, J. Yuan, W,. Oral alendronate induces progressive increases in bone mass of the spine, hip, and total body over 3 years in postmenopausal women with osteoporosis.[Erratum appears in Bone 1996 Jul 19(1):78]. Bone. 1996 Feb;18(2):141-50. 
PMID: 8833208. Ineligible treatment duration.

296. Devogelaer J, P. Lecart, C, Dupret, P, De, Nayer P, Nagant De, Deuxchaisnes C, Long-term effects of percutaneous estradiol on bone loss and bone metabolism in postmenopausal hysterectomized women. Maturitas. 1998 Jan 12;28(3):243-9. PMID: 9571600. Ineligible treatment duration.

297. Devogelaer J, P. Brown, J, P. Burckhardt, $\mathrm{P}$, Meunier, P, J. Goemaere, S, Lippuner, K, Body, J, J. Samsioe, G, Felsenberg, D, Fashola, T, Sanna, L, Ortmann, C, E. Trechsel, U, Krasnow, J, Eriksen, E, F. Garnero, P,. Zoledronic acid efficacy and safety over five years in postmenopausal osteoporosis. Osteoporosis International. 2007 Sep;18(9):1211-8. PMID: 17516022. Ineligible study design.

298. Dhillon S. Zoledronic Acid

(Reclast $<$ sup $></$ sup $>$,

Aclasta $<$ sup $></$ sup $>$ ): A Review in

Osteoporosis. Drugs. 2016

Nov;76(17):1683-97. PMID: 27864686.

Ineligible study design.

299. Diab D, L. Watts, N, B. Bisphosphonates in the Treatment of Osteoporosis. Endocrinology and Metabolism Clinics of North America. 2012 September;41(3):487506. PMID: 365405570. Ineligible study design.

300. Diez PA. Bisphosphonates. Maturitas. 2002 30 Aug;43(SUPPL.):S19-S26. PMID: 35279477. Ineligible study design.

301. Diez PA, Adachi, J, D. Adami, S, Anderson, F, A. Jr, Boonen, S, Chapurlat, R, Compston, J, E. Cooper, C, Gehlbach, S, H. Greenspan, S, L. Hooven, F, H. LaCroix, A, Z. Nieves, J, W. Netelenbos, J, C. Pfeilschifter, J, Rossini, M, Roux, C, Saag, K, G. Silverman, S, Siris, E, S. Wyman, A, Rushton, Smith S, K. Watts, N, B. Global Longitudinal Study of Osteoporosis in, Women Investigators,. Risk factors for treatment failure with antiosteoporosis medication: the global longitudinal study of osteoporosis in women (GLOW). Journal of Bone \& Mineral
Research. 2014 Jan;29(1):260-7. PMID: 23794198. Ineligible study design.

302. Dobnig H, Sipos, A, Jiang, Y, Fahrleitner, Pammer A, Ste, Marie L, G. Gallagher, J, C. Pavo, I, Wang, J, Eriksen, E, F. Early changes in biochemical markers of bone formation correlate with improvements in bone structure during teriparatide therapy. Journal of Clinical Endocrinology \& Metabolism. 2005 Jul;90(7):3970-7. PMID: 15840739. No outcomes of interest.

303. Dobnig H, Hofbauer, L, C. Viereck, V, Obermayer, Pietsch B, Fahrleitner, Pammer A,. Changes in the RANK ligand/osteoprotegerin system are correlated to changes in bone mineral density in bisphosphonate-treated osteoporotic patients. Osteoporosis International. 2006;17(5):693-703. PMID: 16435076. No outcomes of interest.

304. Donggrell S, A. New horizons for zoledronic acid: Results of the HORIZON trials in postmenopausal women with osteoporosis and after hip fracture. Expert Opinion on Pharmacotherapy. 2008 March;9(4):663-8. PMID: 351425393. Ineligible study design.

305. Doren M, Reuther, G, Minne, H, W. Schneider, H, P. Superior compliance and efficacy of continuous combined oral estrogen-progestogen replacement therapy in postmenopausal women. American Journal of Obstetrics \& Gynecology. 1995 Nov;173(5):1446-51. PMID: 7503183. Ineligible treatment duration.

306. Downs R, W. Jr, Bone, H, G. McIlwain, H, Baker, M, Z. Yates, A, J. Lombardi, A, Krupa, D, Harning, R,. An open-label extension study of alendronate treatment in elderly women with osteoporosis. Calcified Tissue International. 1999 Jun;64(6):463-9. PMID: 10341016. Ineligible study design.

307. Downs R, W. Jr, Bell, N, H. Ettinger, M, P. Walsh, B, W. Favus, M, J. Mako, B, Wang, L, Smith, M, E. Gormley, G, J. Melton, M, E. Comparison of alendronate and intranasal calcitonin for treatment of osteoporosis in postmenopausal women. Journal of Clinical Endocrinology \& 
Metabolism. 2000 May;85(5):1783-8. PMID: 10843152. No outcomes of interest.

308. Drake M, Srinivasan, Mödder, Ui, Peterson, Jm, McCready, Lk, Riggs, Bl, Dwyer, Stolina, Kostenuik, Khosla,. Effects of parathyroid hormone treatment on circulating sclerostin levels in postmenopausal women. Journal of clinical endocrinology and metabolism. 2010;95(11):5056-62. PMID: CN00768559. Ineligible treatment duration.

309. Drake W, M. Brown, J, P. Banville, C, Kendler, D, L. Use of phalangeal bone mineral density and multi-site speed of sound conduction to monitor therapy with alendronate in postmenopausal women. Osteoporosis International. 2002 Mar;13(3):249-56. PMID: 11991446. No outcomes of interest.

310. Drake W, M. Kendler, D, L. Rosen, C, J. Orwoll, E, S. An investigation of the predictors of bone mineral density and response to therapy with alendronate in osteoporotic men. Journal of Clinical Endocrinology \& Metabolism. 2003 Dec;88(12):5759-65. PMID: 14671165. No outcomes of interest.

311. Dresner PR, Mayer, M, Hochner, Celiniker $\mathrm{D}$,. The decrease in serum bone-specific alkaline phosphatase predicts bone mineral density response to hormone replacement therapy in early postmenopausal women. Calcified Tissue International. 2000 Feb;66(2):104-7. PMID: 10652956. Ineligible population.

312. Duan D, H. Different Dose HormoneReplacement Therapy in Treatment of Postmenopausal Osteoporosis. Fudan university journal of medical sciences. 2003;30(5):477-9. PMID: CN-00464203. Not available in English.

313. Duggan S, T. McKeage, K,. Bazedoxifene: a review of its use in the treatment of postmenopausal osteoporosis. Drugs. 2011 Nov 12;71(16):2193-212. PMID: 22035517. Ineligible study design.
314. Dulipsingh L, De, Souza M, J. Ikram, Z, Fall, P, Willard, A, Fang, Z, Prestwood, $\mathrm{K}$, Raisz, L,. Clinical utility of urinary markers of bone turnover in response to antiresorptive therapies. Endocrinologist. 2003 June;13(3):293-9. PMID: 36750104. Ineligible study design.

315. Dundar U, Kavuncu, V, Ciftci, I, H. Evcik, D, Solak, O, Cakir, T,. The effect of risedronate treatment on serum cytokines in postmenopausal osteoporosis: a 6-month randomized and controlled study. Journal of Bone \& Mineral Metabolism. 2009;27(4):464-70. PMID: 19301089. Ineligible treatment duration.

316. Durchschlag E, Paschalis, E, P. Zoehrer, R, Roschger, P, Fratzl, P, Recker, R, Phipps, R, Klaushofer, K,. Bone material properties in trabecular bone from human iliac crest biopsies after 3- and 5-year treatment with risedronate. Journal of Bone \& Mineral Research. 2006 Oct;21(10):158190. PMID: 16995813. No outcomes of interest.

317. Dursun N, Dursun, E, Yalcin, S,. Comparison of alendronate, calcitonin and calcium treatments in postmenopausal osteoporosis. International Journal of Clinical Practice. 2001 Oct;55(8):505-9. PMID: 11695068. No outcomes of interest.

318. Duvernoy C, Yeo, Aa, Wong, Cox, Da, Kim, Hm,. Antiplatelet therapy use and the risk of venous thromboembolic events in the Raloxifene Use for the Heart (RUTH) trial. Journal of women's health (2002). 2010;19(8):1459-65. PMID: CN-00813285. Ineligible population.

319. Eastell R, Barton, I, Hannon, R, A. Chines, A, Garnero, P, Delmas, P, D. Relationship of early changes in bone resorption to the reduction in fracture risk with risedronate. Journal of Bone \& Mineral Research. 2003 Jun;18(6):1051-6. PMID: 12817758. Ineligible treatment duration.

320. Eastell R, Black, D, M. Boonen, S, Adami, S, Felsenberg, D, Lippuner, K, Cummings, S, R. Delmas, P, D. Palermo, L, Mesenbrink, P, Cauley, J, A. Horizon Pivotal Fracture, Trial. Effect of once-yearly 
zoledronic acid five milligrams on fracture risk and change in femoral neck bone mineral density. Journal of Clinical Endocrinology \& Metabolism. 2009 Sep;94(9):3215-25. PMID: 19567517. Ineligible treatment duration.

321. Eastell R, Nickelsen, T, Marin, F, Barker, C, Hadji, P, Farrerons, J, Audran, M, Boonen, S, Brixen, K, Gomes, J, M. Obermayer, Pietsch B, Avramidis, A, Sigurdsson, G, Gluer, C, C. Sequential treatment of severe postmenopausal osteoporosis after teriparatide: final results of the randomized, controlled European Study of Forsteo (EUROFORS). Journal of Bone \& Mineral Research. 2009 Apr;24(4):726-36. PMID: 19049337. No outcomes of interest.

322. Eastell R, Lang, T, Boonen, S, Cummings, S, Delmas, P, D. Cauley, J, A. Horowitz, Z, Kerzberg, E, Bianchi, G, Kendler, D, Leung, P, Man, Z, Mesenbrink, P, Eriksen, E, F. Black, D, M. Horizon Pivotal Fracture, Trial. Effect of once-yearly zoledronic acid on the spine and hip as measured by quantitative computed tomography: results of the HORIZON Pivotal Fracture Trial. Osteoporosis International. 2010 Jul;21(7):1277-85. PMID: 19802508. No outcomes of interest.

323. Eastell R, Hannon, R, A. Wenderoth, D, Rodriguez, Moreno J, Sawicki, A,. Effect of stopping risedronate after long-term treatment on bone turnover. Journal of Clinical Endocrinology \& Metabolism. 2011 Nov;96(11):3367-73. PMID: 21865359. Ineligible study design.

324. Eastell R, Vrijens, B, Cahall, D, L. Ringe, J, D. Garnero, P, Watts, N, B. Bone turnover markers and bone mineral density response with risedronate therapy: relationship with fracture risk and patient adherence. Journal of Bone \& Mineral Research. 2011 Jul;26(7):1662-9. PMID: 21312265. Ineligible study design.

325. Eastell R, Christiansen, C, Grauer, A, Kutilek, S, Libanati, C, McClung, M, R. Reid, I, R. Resch, H, Siris, E, Uebelhart, D, Wang, A, Weryha, G, Cummings, S,
R. Effects of denosumab on bone turnover markers in postmenopausal osteoporosis. Journal of Bone \& Mineral Research. 2011 Mar;26(3):530-7. PMID: 20839290. No outcomes of interest.

326. Ebata S, Takahashi, J, Hasegawa, T, Mukaiyama, K, Isogai, Y, Ohba, T, Shibata, Y, Ojima, T, Yamagata, Z, Matsuyama, Y, Haro, H,. Role of Weekly Teriparatide Administration in Osseous Union Enhancement within Six Months After Posterior or Transforaminal Lumbar Interbody Fusion for OsteoporosisAssociated Lumbar Degenerative Disorders: A Multicenter, Prospective Randomized Study. Journal of Bone \& Joint Surgery American Volume. 2017 Mar 01;99(5):36572. PMID: 28244906. Ineligible study design.

327. Ebeling P, R. Osteoporosis in men. Current Opinion in Rheumatology. 2013 Jul;25(4):542-52. PMID: 23719364. Ineligible study design.

328. Edwards B, J. Migliorati, C, A. Osteoporosis and its implications for dental patients. Journal of the American Dental Association. 2008 May;139(5):545-52 quiz 625-6. PMID: 18451370. Ineligible study design.

329. Eekman D, A. Vis, M, Bultink, I, E. Derikx, H, J. Dijkmans, B, A. Lems, W, F. Treatment with intravenous pamidronate is a good alternative in case of gastrointestinal side effects or contraindications for oral bisphosphonates. BMC Musculoskeletal Disorders. 2009 Jul 15;10:86. PMID: 19604377. Ineligible study design.

330. Eiken P, Kolthoff, N, Nielsen, S, P. Effect of 10 years' hormone replacement therapy on bone mineral content in postmenopausal women. Bone. 1996 Nov;19(5 Suppl):191S3S. PMID: 8922661. Ineligible population.

331. Eiken P, Nielsen, S, P. Kolthoff, N,. Effects on bone mass after eight years of hormonal replacement therapy. British Journal of Obstetrics \& Gynaecology. 1997 Jun;104(6):702-7. PMID: 9197874. Ineligible population. 
332. Eiken P, Vestergaard, P,. Treatment of osteoporosis after alendronate or risedronate. Osteoporosis International. 201601 Jan;27(1):1-12. PMID: 606536788. Ineligible study design.

333. Eisenstein N, Kasavkar, G, Bhavsar, D, Khan, F, S. Paskins, Z,. Incidence and medical management of bisphosphonateassociated atypical femoral fractures in a major trauma centre: a retrospective observational study. BMC Musculoskeletal Disorders. 201723 Jan;18(1):1-6. PMID: 614196065. Ineligible study design.

334. Eisman J, A. Civitelli, R, Adami, S, Czerwinski, E, Recknor, C, Prince, R, Reginster, J, Y. Zaidi, M, Felsenberg, D, Hughes, C, Mairon, N, Masanauskaite, D, Reid, D, M. Delmas, P, D. Recker, R, R. Efficacy and tolerability of intravenous ibandronate injections in postmenopausal osteoporosis: 2-year results from the DIVA study. Journal of Rheumatology. 2008 Mar;35(3):488-97. PMID: 18260172. Ineligible treatment duration.

335. El HM, H. Eltoukhy, H, G. Hasanein, M, M. A pilot study to assess the efficacy and side effects of silymarin alone and in combination with alendronate in treatment of postmenopausal osteoporosis. International Journal of Pharmaceutical Sciences and Research. 2016;7(3):1012-20. PMID: 608858209. No outcomes of interest.

336. Elraiyah T, Ahmed, A, H. Wang, Z, Farr, J, N. Murad, M, H. Drake, M, T.

Predictors of teriparatide treatment failure in patients with low bone mass. Bone Reports. 2016 June 01;4:17-22. PMID: 607058962. Ineligible study design.

337. Emkey R, Delmas, P, D. Bolognese, M, Borges, J, L. Cosman, F, Ragi, Eis S, Recknor, C, Zerbini, C, A. Neate, C, Sedarati, F, Epstein, S,. Efficacy and tolerability of once-monthly oral ibandronate (150 mg) and once-weekly oral alendronate (70 mg): additional results from the Monthly Oral Therapy With Ibandronate For Osteoporosis Intervention (MOTION) study. Clinical Therapeutics. 2009
Apr;31(4):751-61. PMID: 19446148. No outcomes of interest.

338. Engel P, Fabre, A, Fournier, A, Mesrine, S, Boutron, Ruault M, C. Clavel, Chapelon F,. Risk of osteoporotic fractures after discontinuation of menopausal hormone therapy: results from the E3N cohort. American Journal of Epidemiology. 2011 Jul 01;174(1):12-21. PMID: 21555715. Ineligible population.

339. Engelke K, Fuerst, T, Dasic, G, Davies, R, Y. Genant, H, K. Regional distribution of spine and hip QCT BMD responses after one year of once-monthly ibandronate in postmenopausal osteoporosis. Bone. 2010 Jun;46(6):1626-32. PMID: 20226286. No outcomes of interest.

340. Ensrud K, E. Black, D, M. Palermo, L, Bauer, D, C. Barrett, Connor E, Quandt, S, A. Thompson, D, E. Karpf, D, B. Treatment with alendronate prevents fractures in women at highest risk: results from the Fracture Intervention Trial. Archives of Internal Medicine. 1997 Dec 822;157(22):2617-24. PMID: 9531231. Ineligible treatment duration.

341. Ensrud K, E. Black, D, M. Palermo, L,. Alendronate reduced new fractures in postmenopausal women who had low bonemineral density and existing vertebral fractures. Evidence-Based Medicine. 1998 July/August;3(4):119. PMID: 28389832. Ineligible study design.

342. Ensrud K, E. Stock, J, L. Barrett, Connor E, Grady, D, Mosca, L, Khaw, K, T. Zhao, Q, Agnusdei, D, Cauley, J, A. Effects of raloxifene on fracture risk in postmenopausal women: the Raloxifene Use for the Heart Trial. Journal of Bone \& Mineral Research. 2008 Jan;23(1):112-20. PMID: 17892376. Ineligible population.

343. Erenus M, Gurler, A, Binoz, S,. Effect of adding alendronate to hormone replacement therapy on bone mineral density in established postmenopausal osteoporosis. Marmara Medical Journal. 2000;13(4):196200. PMID: 32002928. No outcomes of interest. 
344. Eriguchi R, Umakoshi, J, Miura, S, Sato, $\mathrm{Y},$. Raloxifene ameliorates progressive bone loss in postmenopausal dialysis patients with controlled parathyroid hormone levels. Clinical Nephrology. 2009 Dec;72(6):423-9. PMID: 19954718. Ineligible study design.

345. Erviti J, Alonso, A, Gorricho, J, Lopez, A,. Oral bisphosphonates may not decrease hip fracture risk in elderly Spanish women: A nested case-control study. BMJ Open. 2013;3 (2) (no pagination)(e002084) PMID: 368520524. Ineligible study design.

346. Ettinger B, Black, D, M. Mitlak, B, H. Knickerbocker, R, K. Nickelsen, T, Genant, H, K. Christiansen, C, Delmas, P, D. Zanchetta, J, R. Stakkestad, J, Gluer, C, C. Krueger, K, Cohen, F, J. Eckert, S, Ensrud, K, E. Avioli, L, V. Lips, P, Cummings, S, R. Reduction of vertebral fracture risk in postmenopausal women with osteoporosis treated with raloxifene: results from a 3-year randomized clinical trial. Multiple Outcomes of Raloxifene Evaluation (MORE) Investigators.[Erratum appears in JAMA 1999 Dec 8 282(22):2124]. JAMA. 1999 Aug 18;282(7):637-45. PMID: 10517716. Ineligible treatment duration.

347. Ettinger B, San, Martin J, Crans, G, Pavo, I,. Differential effects of teriparatide on BMD after treatment with raloxifene or alendronate. Journal of Bone \& Mineral Research. 2004 May;19(5):745-51. PMID: 15068497. Ineligible study design.

348. Ettinger M, P. Felsenberg, D, Harris, S, T. Wasnich, R, Skag, A, Hiltbrunner, V, Wilson, K, Schimmer, R, C. Miller, P, D. Safety and tolerability of oral daily and intermittent ibandronate are not influenced by age. Journal of Rheumatology. 2005 Oct;32(10):1968-74. PMID: 16206354. Ineligible treatment duration.

349. Evans S, F. Davie, M, W. Low and conventional dose transdermal oestradiol are equally effective at preventing bone loss in spine and femur at all post-menopausal ages. Clinical Endocrinology. 1996 Jan;44(1):7984. PMID: 8706298. No outcomes of interest.
350. Evio S, Tiitinen, A, Laitinen, K, Ylikorkala, O, Valimaki, M, J. Effects of alendronate and hormone replacement therapy, alone and in combination, on bone mass and markers of bone turnover in elderly women with osteoporosis. Journal of Clinical Endocrinology \& Metabolism. 2004 Feb;89(2):626-31. PMID: 14764773. No outcomes of interest.

351. Evio S, Tarkkila, L, Sorsa, T, Furuholm, J, Valimaki, M, J. Ylikorkala, O, Tiitinen, A, Meurman, J, H. Effects of alendronate and hormone replacement therapy, alone and in combination, on saliva, periodontal conditions and gingival crevicular fluid matrix metalloproteinase-8 levels in women with osteoporosis. Oral Diseases. 2006 Mar;12(2):187-93. PMID: 16476042. No outcomes of interest.

352. Fadda V, Maratea, D, Trippoli, S, Messori, A,. Gastrointestinal and renal side effects of bisphosphonates: differentiating between no proof of difference and proof of no difference. Journal of Endocrinological Investigation. 2015 Feb;38(2):189-92. PMID: 25412945. Ineligible study design.

353. Feldstein A, C. Weycker, D, Nichols, G, A. Oster, G, Rosales, G, Boardman, D, L. Perrin, N,. Effectiveness of bisphosphonate therapy in a community setting. Bone. 2009 Jan;44(1):153-9. PMID: 18926939. Ineligible study design.

354. Feldstein A, C. Black, D, Perrin, N, Rosales, A, G. Friess, D, Boardman, D, Dell, R, Santora, A, Chandler, J, M. Rix, M, M. Orwoll, E,. Incidence and demography of femur fractures with and without atypical features. Journal of Bone \& Mineral Research. 2012 May;27(5):977-86. PMID: 22275107. No outcomes of interest.

355. Fellows J, L. Rindal, D, B. Barasch, A, Gullion, C, M. Rush, W, Pihlstrom, D, J. Richman, J, Dpbrn Collaborative, Group. ONJ in two dental practice-based research network regions.[Reprint in Tex Dent J. 2013 Apr 130(4):311-8 PMID: 23767160]. Journal of Dental Research. 2011 Apr;90(4):433-8. PMID: 21317245. Ineligible population. 
356. Felsenberg D, Alenfeld, F, Bock, O, Hammermeister, C, Gowan, W,. Placebocontrolled multicenter study of oral alendronate in postmenopausal osteoporotic women. FOSIT-Study-Group. Fosamax International Trial. Maturitas. $1998 \mathrm{Nov}$ 30;31(1):35-44. PMID: 10091203. No outcomes of interest.

357. Felsenberg D, Miller, P, Armbrecht, G, Wilson, K, Schimmer, R, C. Papapoulos, $S$, E. Oral ibandronate significantly reduces the risk of vertebral fractures of greater severity after 1,2 , and 3 years in postmenopausal women with osteoporosis. Bone. 2005 Nov;37(5):651-4. PMID: 16126016. No outcomes of interest.

358. Felsenberg D, Bock, O, Borst, H, Armbrecht, G, Beller, G, Degner, C, Stephan, Oelkers M, Schacht, E, Mazor, Z, Hashimoto, J, Roth, H, J. Martus, P, Runge, M,. Additive impact of alfacalcidol on bone mineral density and bone strength in alendronate treated postmenopausal women with reduced bone mass. Journal of Musculoskeletal Neuronal Interactions. 2011 Mar;11(1):34-45. PMID: 21364273. No outcomes of interest.

359. Ferguson S, Feudjo, Tepie M, Taylor, A, Roddam, A, Critchlow, C, Iqbal, M, Spangler, L, Bayly, J,. The impact of persistence with bisphosphonates on health resource utilization and fracture risk in the UK: a study of patient records from the UK Clinical Practice Research Datalink. Journal of Evaluation in Clinical Practice. 2016 Feb;22(1):31-9. PMID: 26279458. Ineligible study design.

360. Ferrari LS, Ferrari, S,. Do RANKL inhibitors (denosumab) affect inflammation and immunity? Osteoporosis International. 2011 Feb;22(2):435-46. PMID: 20571772. Ineligible study design.

361. Ferrari S, Nakamura, T, Hagino, H, Fujiwara, S, Lange, J, L. Watts, N, B. Longitudinal change in hip fracture incidence after starting risedronate or raloxifene: an observational study. Journal of Bone \& Mineral Metabolism. 2011
Sep;29(5):561-70. PMID: 21225297. Ineligible study design.

362. Ferrari S, Adachi, J, D. Lippuner, K, Zapalowski, C, Miller, P, D. Reginster, J, Y. Torring, O, Kendler, D, L. Daizadeh, N, S. Wang, A, O'Malley, C, D.

Wagman, R, B. Libanati, C, Lewiecki, E, $\mathrm{M}$. Further reductions in nonvertebral fracture rate with long-term denosumab treatment in the FREEDOM open-label extension and influence of hip bone mineral density after 3 years. Osteoporosis International. 2015 Dec;26(12):2763-71. PMID: 26068295. Ineligible study design.

363. Figueras F, Castelo, Branco C, Pons, F, Sanjuan, A, Vanrell, J, A. Effect of continuous and sequential oral estrogenprogestogen replacement regimens on postmenopausal bone loss: a 2-year prospective study. European Journal of Obstetrics, Gynecology, \& Reproductive Biology. 2001 Dec 01;99(2):261-5. PMID: 11788184. Ineligible treatment duration.

364. Fink H, A. Ensrud, K, E. Nelson, D, B. Kerani, R, P. Schreiner, P, J. Zhao, Y, Cummings, S, R. Nevitt, M, C. Disability after clinical fracture in postmenopausal women with low bone density: the fracture intervention trial (FIT). Osteoporosis International. 2003 Jan;14(1):69-76. PMID: 12577187. Ineligible study design.

365. Finkelstein J, S. Hayes, A, Hunzelman, J, L. Wyland, J, J. Lee, H, Neer, R, M. The effects of parathyroid hormone, alendronate, or both in men with osteoporosis. New England Journal of Medicine. 2003 Sep 25;349(13):1216-26. PMID: 14500805. No outcomes of interest.

366. Finkelstein J, S. Leder, B, Z. Burnett, S, M. Wyland, J, J. Lee, H, de la, Paz A, V. Gibson, K, Neer, R, M. Effects of teriparatide, alendronate, or both on bone turnover in osteoporotic men. Journal of Clinical Endocrinology \& Metabolism. 2006 Aug;91(8):2882-7. PMID: 16684825. No outcomes of interest.

367. Finkelstein J, S. Wyland, J, J. Leder, B, Z. Burnett, Bowie S, M. Lee, H, Juppner, H, Neer, R, M. Effects of teriparatide 
retreatment in osteoporotic men and women. Journal of Clinical Endocrinology \& Metabolism. 2009 Jul;94(7):2495-501. PMID: 19401368. Ineligible study design.

368. Finkelstein J, S. Wyland, J, J. Lee, H, Neer, R, M. Effects of teriparatide, alendronate, or both in women with postmenopausal osteoporosis. Journal of Clinical Endocrinology \& Metabolism. 2010 Apr;95(4):1838-45. PMID: 20164296. No outcomes of interest.

369. Flodin L, Saaf, M, Cederholm, T, Al, Ani A, N. Ackermann, P, W. Samnegard, E, Dalen, N, Hedstrom, M,. Additive effects of nutritional supplementation, together with bisphosphonates, on bone mineral density after hip fracture: a 12-month randomized controlled study. Clinical Interventions In Aging. 2014;9:1043-50. PMID: 25045257. No outcomes of interest.

370. Flodin S, Cederholm, Al, Ani An, Ackermann, Pw, Samnegård, Dalen, Hedström,. Additive effects of nutritional supplementation, together with bisphosphonates, on bone mineral density after hip fracture: a 12-month randomized controlled study. Clinical interventions in aging. 2014;9:1043-50. PMID: CN00994603. Ineligible treatment duration.

371. Fogelman I, Ribot, C, Smith, R, Ethgen, D, Sod, E, Reginster, J, Y. Risedronate reverses bone loss in postmenopausal women with low bone mass: results from a multinational, double-blind, placebocontrolled trial. BMD-MN Study Group. Journal of Clinical Endocrinology \& Metabolism. 2000 May;85(5):1895-900. PMID: 10843171. Ineligible treatment duration.

372. Fogelman I, Fordham, J, N. Fraser, W, D. Spector, T, D. Christiansen, C, Morris, S, A. Fox, J,. Parathyroid hormone(1-84) treatment of postmenopausal women with low bone mass receiving hormone replacement therapy. Calcified Tissue International. 2008 Aug;83(2):85-92. PMID: 18626566. No interventions of interest.
373. Folkesson J, Goldenstein, J, Carballido, Gamio J, Kazakia, G, Burghardt, A, J. Rodriguez, A, Krug, R, de, Papp A, E. Link, T, M. Majumdar, S,. Longitudinal evaluation of the effects of alendronate on MRI bone microarchitecture in postmenopausal osteopenic women. Bone. 2011 Mar 01;48(3):611-21. PMID: 21059422. Ineligible study design.

374. Folsom A, R. Mink, P, J. Sellers, T, A. Hong, C, P. Zheng, W, Potter, J, D. Hormonal replacement therapy and morbidity and mortality in a prospective study of postmenopausal women. American Journal of Public Health. 1995 Aug;85(8 Pt 1):1128-32. PMID: 7625511. Ineligible study design.

375. Foster S, A. Shi, N, Curkendall, S, Stock, J, Chu, B, C. Burge, R, Diakun, D, R. Krege, J, H. Fractures in women treated with raloxifene or alendronate: a retrospective database analysis. BMC Women's Health. 2013 Mar 23;13:15. PMID: 23521803. Ineligible population.

376. Fowler J, R. Craig, M, R. Association of low-energy femoral shaft fractures and bisphosphonate use. Orthopedics. 2012 Jan 16;35(1):e38-40. PMID: 22229611. Ineligible study design.

377. Frampton J, E. Risedronate on two consecutive days per month. Drugs \& Aging. 2009;26(4):355-62. PMID: 19476402. Ineligible study design.

378. Franek E. Denosumab--a new option in the treatment of osteoporosis. Endokrynologia Polska. 2011 Jan-Feb;62(1):79-83. PMID: 21365584. Not available in English.

379. Frankel B, Krishna, V, Vandergrift, A, Bauer, D, C. Nicholas, J,. Natural history and risk factors for adjacent vertebral fractures in the fracture intervention trial. Spine. 2013 Dec 01;38(25):2201-7. PMID: 24285276. No outcomes of interest.

380. Fraser L, A. Papaioannou, A, Adachi, J, D. Ma, J, Thabane, L, CaMos, Research Group,. Fractures are increased and bisphosphonate use decreased in individuals 
with insulin-dependent diabetes: a 10 year

cohort study. BMC Musculoskeletal

Disorders. 2014 Jun 11;15:201. PMID:

24919660. Ineligible treatment duration.

381. Fratzl P, Roschger, P, Fratzl, Zelman N, Paschalis, E, P. Phipps, R, Klaushofer, $\mathrm{K}$,. Evidence that treatment with risedronate in women with postmenopausal osteoporosis affects bone mineralization and bone volume. Calcified Tissue International. 2007 Aug;81(2):73-80. PMID: 17612779. No outcomes of interest.

382. Frediani B, Allegri, A, Bisogno, S, Marcolongo, R,. Effects of combined treatment with calcitriol plus alendronate on bone mass and bone turnover in postmenopausal osteoporosis: Two years of continuous treatment. Clinical Drug Investigation. 1998;15(3):235-44. PMID: 28163750. No outcomes of interest.

383. Frediani B. Effects of two administration schemes of intramuscular clodronic acid on bone mineral density: a randomized, openlabel, parallel-group study. Clinical Drug Investigation. 2011;31(1):43-50. PMID: 20932069. Ineligible study design.

384. Freedman M, San, Martin J, O'Gorman, J, Eckert, S, Lippman, M, E. Lo, S, C. Walls, E, L. Zeng, J,. Digitized mammography: a clinical trial of postmenopausal women randomly assigned to receive raloxifene, estrogen, or placebo. Journal of the National Cancer Institute. 2001 Jan 03;93(1):51-6. PMID: 11136842. Ineligible treatment duration.

385. Frost M, L. Siddique, M, Blake, G, M. Moore, A, E. Marsden, P, K. Schleyer, P, J. Eastell, R, Fogelman, I,. Regional bone metabolism at the lumbar spine and hip following discontinuation of alendronate and risedronate treatment in postmenopausal women. Osteoporosis International. 2012 Aug;23(8):2107-16. PMID: 21983795. Ineligible study design.

386. Fu S, H. Wang, C, Y. Yang, R, S. Wu, F, L. Hsiao, F, Y. Bisphosphonate Use and the Risk of Undergoing Total Knee Arthroplasty in Osteoporotic Patients with Osteoarthritis: A Nationwide Cohort Study in Taiwan. Journal of Bone \& Joint Surgery - American Volume. 2017 Jun 07;99(11):938-46. PMID: 28590379. Ineligible treatment duration.

387. Fujita T, Fukunaga, M, Itabashi, A, Tsutani, K, Nakamura, T,. Once-Weekly Injection of Low-Dose Teriparatide (28.2 mug) Reduced the Risk of Vertebral Fracture in Patients with Primary Osteoporosis. Calcified Tissue International. 2014 Feb;94(2):170-5. PMID: 23963633. No outcomes of interest.

388. Furness S, Roberts, Helen, Marjoribanks, Jane, Lethaby, Anne,. Hormone therapy in postmenopausal women and risk of endometrial hyperplasia. Cochrane Database of Systematic Reviews. 2012(8) PMID: CD000402. Ineligible study design.

389. Gallagher J, C. Rapuri, P, B. Haynatzki, G, Detter, J, R. Effect of discontinuation of estrogen, calcitriol, and the combination of both on bone density and bone markers. Journal of Clinical Endocrinology \& Metabolism. 2002 Nov;87(11):4914-23. PMID: 12414850. Ineligible population.

390. Gallagher J, C. Genant, H, K. Crans, G, G. Vargas, S, J. Krege, J, H. Teriparatide reduces the fracture risk associated with increasing number and severity of osteoporotic fractures. Journal of Clinical Endocrinology \& Metabolism. 2005 Mar;90(3):1583-7. PMID: 15613428. Ineligible treatment duration.

391. Gallagher J, C. Rosen, C, J. Chen, P, Misurski, D, A. Marcus, R,. Response rate of bone mineral density to teriparatide in postmenopausal women with osteoporosis. Bone. 2006 Dec;39(6):1268-75. PMID: 16884968. No outcomes of interest.

392. Gambacciani M, Spinetti, A, Orlandi, R, Piaggesi, L, Cappagli, B, Weiss, C, Ciaponi, M, Genazzani, A, R. Effects of a new estrogen/progestin combination in the treatment of postmenopausal syndrome. Maturitas. 1995;22(2):115-20. PMID: 25289405. Ineligible treatment duration. 
393. Gambacciani M, Ciaponi, M, Cappagli, B, Piaggesi, L, Genazzani, A, R. Effects of combined low dose of the isoflavone derivative ipriflavone and estrogen replacement on bone mineral density and metabolism in postmenopausal women. Maturitas. 1997 Sep;28(1):75-81. PMID: 9391998. Ineligible treatment duration.

394. Gambacciani M, Ciaponi, M, Cappagli, B, Genazzani, A, R. Effects of low-dose continuous combined conjugated estrogens and medroxyprogesterone acetate on menopausal symptoms, body weight, bone density, and metabolism in postmenopausal women. American Journal of Obstetrics \& Gynecology. 2001 Nov;185(5):1180-5. PMID: 11717654. Ineligible treatment duration.

395. Gambacciani M, Ciaponi, M, Cappagli, B, Monteleone, P, Benussi, C, Bevilacqua, G, Genazzani, A, R. Postmenopausal femur bone loss: effects of a low dose hormone replacement therapy. Maturitas. $2003 \mathrm{Jul}$ 25;45(3):175-83. PMID: 12818462. Ineligible treatment duration.

396. Gambacciani M, Levancini, M, Management of postmenopausal osteoporosis and the prevention of fractures. Panminerva Medica. 2014 Jun;56(2):115-31. PMID: 24942322. Ineligible study design.

397. Gambacciani M, Levancini, M,. Hormone replacement therapy and the prevention of postmenopausal osteoporosis. Przeglad Menopauzalny. 2014;13(4):213-20. PMID: 602972042. Ineligible study design.

398. García PM, Moreno, Mercer Tarín, Jj, Cano,. Similar efficacy of low and standard doses of transdermal estradiol in controlling bone turnover in postmenopausal women. Gynecological endocrinology. 2006;22(4):179-84. PMID: CN-00565546. Ineligible study design.

399. Garnero P, Bauer, D, C. Mareau, E, Bilezikian, J, P. Greenspan, S, L. Rosen, C, Black, D,. Effects of PTH and alendronate on type I collagen isomerization in postmenopausal women with osteoporosis: the PaTH study. Journal of Bone \& Mineral Research. 2008
Sep;23(9):1442-8. PMID: 18442311. No outcomes of interest.

400. Gass M, Liu, J, Rebar, R, W. The effect of low-dose conjugated equine estrogens and cyclic MPA on bone density. Maturitas. 200226 Feb;41(2):143-7. PMID: 34142503. Ineligible treatment duration.

401. Gates B, J. Das, S,. Risedronate's role in reducing hip fracture in postmenopausal women with established osteoporosis. Clinical Medicine Insights: Arthritis and Musculoskeletal Disorders. 201205 Jan;5:114. PMID: 364236090. Ineligible study design.

402. Gatti D, Viapiana, O, Idolazzi, L, Fracassi, E, Rossini, M, Adami, S,. The waning of teriparatide effect on bone formation markers in postmenopausal osteoporosis is associated with increasing serum levels of DKK1. Journal of Clinical Endocrinology \& Metabolism. 2011 May;96(5):1555-9. PMID: 21367927. No outcomes of interest.

403. Gatti D, Viapiana, O, Fracassi, E, Idolazzi, L, Dartizio, C, Povino, M, R. Adami, S, Rossini, M,. Sclerostin and DKK1 in postmenopausal osteoporosis treated with denosumab. Journal of Bone \& Mineral Research. 2012 Nov;27(11):225963. PMID: 22692843. Ineligible study design.

404. Genant H, K. Lucas, J, Weiss, S, Akin, M, Emkey, R, McNaney, Flint $\mathrm{H}$, Downs, R, Mortola, J, Watts, N, Yang, H, M. Banav, N, Brennan, J, J. Nolan, J, C. Low-dose esterified estrogen therapy: effects on bone, plasma estradiol concentrations, endometrium, and lipid levels. Estratab/Osteoporosis Study Group. Archives of Internal Medicine. 1997 Dec 822;157(22):2609-15. PMID: 9531230. Ineligible treatment duration.

405. Genant H, K. Lang, T, Fuerst, T, Pinette, K, V. Zhou, C, Thiebaud, D, Diez, Perez A,. Treatment with raloxifene for 2 years increases vertebral bone mineral density as measured by volumetric quantitative computed tomography. Bone. 2004 
Nov;35(5):1164-8. PMID: 15542042. No outcomes of interest.

406. Genant H, K. Halse, J, Briney, W, G. Xie, L, Glass, E, V. Krege, J, H. The effects of teriparatide on the incidence of back pain in postmenopausal women with osteoporosis. Current Medical Research \& Opinion. 2005 Jul;21(7):1027-34. PMID: 16004669. No outcomes of interest.

407. Genant H, K. Siris, E, Crans, G, G. Desaiah, D, Krege, J, H. Reduction in vertebral fracture risk in teriparatide-treated postmenopausal women as assessed by spinal deformity index. Bone. 2005 Aug;37(2):170-4. PMID: 15961357. No outcomes of interest.

408. Genant H, K. Engelke, K, Hanley, D, A. Brown, J, P. Omizo, M, Bone, H, G. Kivitz, A, J. Fuerst, T, Wang, H, Austin, M, Libanati, C,. Denosumab improves density and strength parameters as measured by QCT of the radius in postmenopausal women with low bone mineral density. Bone. 2010 Jul;47(1):131-9. PMID: 20399288. Ineligible treatment duration.

409. Genant H, K. Bazedoxifene: a new selective estrogen receptor modulator for postmenopausal osteoporosis. Menopause International. 2011 Jun;17(2):44-9. PMID: 21693498. Ineligible study design.

410. Genant H, K. Lewiecki, E, M. Fuerst, T, Fries, M,. Effect of monthly ibandronate on hip structural geometry in men with low bone density. Osteoporosis International. 2012 Jan;23(1):257-65. PMID: 21811866. Ineligible study design.

411. Genant H, K. Libanati, C, Engelke, K, Zanchetta, J, R. Hoiseth, A, Yuen, C, K. Stonkus, S, Bolognese, M, A. Franek, E, Fuerst, T, Radcliffe, H, S. McClung, M, $\mathrm{R}$. Improvements in hip trabecular, subcortical, and cortical density and mass in postmenopausal women with osteoporosis treated with denosumab. Bone. 2013 Oct;56(2):482-8. PMID: 23871852. No outcomes of interest.
412. Genant H, K. Engelke, K, Bolognese, M, A. Mautalen, C, Brown, J, P. Recknor, C, Goemaere, S, Fuerst, T, Yang, Y, C. Grauer, A, Libanati, C,. Effects of Romosozumab Compared With Teriparatide on Bone Density and Mass at the Spine and Hip in Postmenopausal Women With Low Bone Mass. Journal of Bone and Mineral Research. 201701 Jan;32(1):181-7. PMID: 612961570. No outcomes of interest.

413. Gennari L, Merlotti, D, Nuti, R,. Selective estrogen receptor modulator (SERM) for the treatment of osteoporosis in postmenopausal women: focus on lasofoxifene. Clinical Interventions In Aging. 2010 Feb 02;5:1929. PMID: 20169039. Ineligible study design.

414. Gennari L, Merlotti, D, Stolakis, K, Nuti, R,. Pharmacokinetic evaluation of toremifene and its clinical implications for the treatment of osteoporosis. Expert Opinion On Drug Metabolism \& Toxicology. 2012 Apr;8(4):505-13. PMID: 22356442. Ineligible study design.

415. Ghidini G, Manfredi M, Giovannacci I, et al. Medication-related osteonecrosis of the jaw: risk factors in patients under biphosphonate versus patients under antiresorptiveantiangiogenic drugs. Minerva Stomatologica. 2017 Aug;66(4):135-40. PMID: 28497661. Ineligible study design.

416. Ghirardi A, Scotti, L, Zambon, A, Della, Vedova G, Cavalieri, D'oro L, Lapi, F, Cipriani, F, Caputi, A, P. Vaccheri, A, Gregori, D, Gesuita, R, Vestri, A, Staniscia, T, Mazzaglia, G, Corrao, G, Best, Investigators. Risk of severe upper gastrointestinal complications among oral bisphosphonate users. PLoS ONE

[Electronic Resource]. 2013;8(12):e73159. PMID: 24348985. Ineligible study design.

417. Ghirardi A, Di, Bari M, Zambon, A, Scotti, L, Della, Vedova G, Lapi, F, Cipriani, F, Caputi, A, P. Vaccheri, A, Gregori, D, Gesuita, R, Vestri, A, Staniscia, T, Mazzaglia, G, Corrao, G, Best, investigators. Effectiveness of oral bisphosphonates for primary prevention of osteoporotic fractures: evidence from the AIFA-BEST observational study. European 
Journal of Clinical Pharmacology. 2014 Sep;70(9):1129-37. PMID: 24951915. Ineligible treatment duration.

418. Ghirardi A, Scotti, L, Vedova, G, D. D'Oro, L, C. Lapi, F, Cipriani, F, Caputi, A, P. Vaccheri, A, Gregori, D, Gesuita, R, Vestri, A, Staniscia, T, Mazzaglia, G, Corrao, G, Aifa-Best, Investigators. Oral bisphosphonates do not increase the risk of severe upper gastrointestinal complications: a nested case-control study. BMC

Gastroenterology. 2014 Jan 07;14:5. PMID: 24397769. Ineligible study design.

419. Giske L, Hall, Rud, Landgren, Bm,. The effect of 17beta-estradiol at doses of $0.5,1$ and $2 \mathrm{mg}$ compared with placebo on early postmenopausal bone loss in hysterectomized women. Osteoporosis international. 2002;13(4):309-16. PMID: CN-00380253. Ineligible population.

420. Giusti A, Hamdy, N, A. Dekkers, O, M. Ramautar, S, R. Dijkstra, S, Papapoulos, S, E. Atypical fractures and bisphosphonate therapy: a cohort study of patients with femoral fracture with radiographic adjudication of fracture site and features. Bone. 2011 May 01;48(5):966-71. PMID: 21195812. No outcomes of interest.

421. Giusti A, Bianchi, G,. Treatment of primary osteoporosis in men. Clinical Interventions In Aging. 2015;10:105-15. PMID: 25565793. Ineligible study design.

422. Gonnelli S, Cepollaro, C, Pondrelli, C, Martini, S, Monaco, R, Gennari, C,. The usefulness of bone turnover in predicting the response to transdermal estrogen therapy in postmenopausal osteoporosis. Journal of Bone \& Mineral Research. 1997 Apr;12(4):624-31. PMID: 9101374. No outcomes of interest.

423. Gonnelli S, Cepollaro, C, Pondrelli, C, Martini, S, Montagnani, A, Monaco, R, Gennari, C,. Bone turnover and the response to alendronate treatment in postmenopausal osteoporosis. Calcified Tissue International. 1999 Nov;65(5):35964. PMID: 10541760. No outcomes of interest.
424. Gonnelli S, Martini, G, Caffarelli, C, Salvadori, S, Cadirni, A, Montagnani, A, Nuti, R,. Teriparatide's effects on quantitative ultrasound parameters and bone density in women with established osteoporosis. Osteoporosis International. 2006 Oct;17(10):1524-31. PMID: 16767526. No outcomes of interest.

425. Gorai I, Chaki, O, Taguchi, Y, Nakayama, M, Osada, H, Suzuki, N, Katagiri, N, Misu, Y, Minaguchi, H,. Early postmenopausal bone loss is prevented by estrogen and partially by 1 alpha-OH-vitamin D3: therapeutic effects of estrogen and/or 1alpha-OH-vitamin D3. Calcified Tissue International. 1999 Jul;65(1):16-22. PMID: 10369728. Ineligible treatment duration.

426. Gorai I, Hattori, S, Tanaka, Y, Iwaoki, Y,. Alfacalcidol-supplemented raloxifene therapy has greater bone-sparing effect than raloxifene-alone therapy in postmenopausal Japanese women with osteoporosis or osteopenia. Journal of Bone \& Mineral Metabolism. 2012 May;30(3):349-58. PMID: 22130786. No outcomes of interest.

427. Grady D, Wenger, N, K. Herrington, D, Khan, S, Furberg, C, Hunninghake, D, Vittinghoff, E, Hulley, S,. Postmenopausal hormone therapy increases risk for venous thromboembolic disease. The Heart and Estrogen/progestin Replacement Study. Annals of Internal Medicine. 2000 May 02;132(9):689-96. PMID: 10787361. Ineligible population.

428. Grady D, Cauley, J, A. Geiger, M, J. Kornitzer, M, Mosca, L, Collins, P, Wenger, N, K. Song, J, Mershon, J, Barrett, Connor E, Raloxifene Use for The Heart, Trial Investigators,. Reduced incidence of invasive breast cancer with raloxifene among women at increased coronary risk. Journal of the National Cancer Institute. 2008 Jun 18;100(12):85461. PMID: 18544744. Ineligible population.

429. Graham J, Irgit, K, Smith, W, R. Bowen, T, R. Diaphyseal femur fractures associated with bisphosphonate use. Acta Orthopaedica et Traumatologica Turcica. 2013;47(4):255- 
60. PMID: 23999513. Ineligible study design.

430. Graves L, L. Bukata, S, V. Aghazadehsanai, N, Chang, T, I. Garrett, N, R. Friedlander, A, H. Patients Receiving Parenteral Bisphosphonates for Malignant Disease and Having Developed an Atypical Femoral Fracture Are at Risk of Concomitant Osteonecrosis of the Jaw: An Evidence-Based Review. Journal of Oral \& Maxillofacial Surgery. 2016

Dec;74(12):2403-8. PMID: 27376180. Ineligible study design.

431. Grbic J, T. Landesberg, R, Lin, S, Q. Mesenbrink, P, Reid, I, R. Leung, P, C. Casas, N, Recknor, C, P. Hua, Y, Delmas, P, D. Eriksen, E, F. Incidence of osteonecrosis of the jaw in women with postmenopausal osteoporosis in the health outcomes and reduced incidence with zoledronic acid once yearly pivotal fracture trial. Journal of the American Dental Association. 2008 January;139(1):32-40. PMID: 351205770. Ineligible treatment duration.

432. Grbic J, T. Black, D, M. Lyles, K, W. Reid, D, M. Orwoll, E, McClung, M, Bucci, Rechtweg C, Su, G,. The incidence of osteonecrosis of the jaw in patients receiving 5 milligrams of zoledronic acid: data from the health outcomes and reduced incidence with zoledronic acid once yearly clinical trials program. Journal of the American Dental Association. 2010 Nov;141(11):1365-70. PMID: 21037195. Ineligible treatment duration.

433. Green J, Czanner, G, Reeves, G, Watson, J, Wise, L, Beral, V,. Oral bisphosphonates and risk of cancer of oesophagus, stomach, and colorectum: casecontrol analysis within a UK primary care cohort. BMJ. 2010 Sep 01;341:c4444. PMID: 20813820. Ineligible study design.

434. Greendale G, A. Espeland, M, Slone, S, Marcus, R, Barrett, Connor E, Pepi Safety Follow-Up Study, Investigators. Bone mass response to discontinuation of long-term hormone replacement therapy: results from the Postmenopausal Estrogen/Progestin Interventions (PEPI) Safety Follow-up
Study. Archives of Internal Medicine. 2002 Mar 25;162(6):665-72. PMID: 11911720. Ineligible treatment duration.

435. Greenspan S, L. Holland, S, Maitland, Ramsey L, Poku, M, Freeman, A, Yuan, W, Kher, U, Gertz, B,. Alendronate stimulation of nocturnal parathyroid hormone secretion: a mechanism to explain the continued improvement in bone mineral density accompanying alendronate therapy.[Erratum appears in Proc Assoc Am Physicians 1996 Jul 108(4):followi].

Proceedings of the Association of American Physicians. 1996 May;108(3):230-8. PMID: 8774056. Ineligible treatment duration.

436. Greenspan S, L. Parker, R, A. Ferguson, L, Rosen, H, N. Maitland, Ramsey L, Karpf, D, B. Early changes in biochemical markers of bone turnover predict the longterm response to alendronate therapy in representative elderly women: a randomized clinical trial. Journal of Bone \& Mineral Research. 1998 Sep;13(9):1431-8. PMID: 9738515. No outcomes of interest.

437. Greenspan S, L. Rosen, H, N. Parker, R, A. Early changes in serum N-telopeptide and C-telopeptide cross-linked collagen type 1 predict long-term response to alendronate therapy in elderly women. Journal of Clinical Endocrinology \& Metabolism. 2000 Oct;85(10):3537-40. PMID: 11061497. No outcomes of interest.

438. Greenspan S, L. Emkey, R, D. Bone, H, G. Weiss, S, R. Bell, N, H. Downs, R, W. McKeever, C, Miller, S, S. Davidson, M, Bolognese, M, A. Mulloy, A, L. Heyden, N, Wu, M, Kaur, A, Lombardi, A,. Significant differential effects of alendronate, estrogen, or combination therapy on the rate of bone loss after discontinuation of treatment of postmenopausal osteoporosis. A randomized, double-blind, placebocontrolled trial.[Summary for patients in Ann Intern Med. 2002 Dec 3 137(11):I31 PMID: 12459003]. Annals of Internal Medicine. 2002 Dec 03;137(11):875-83. PMID: 12458987. No outcomes of interest.

439. Greenspan S, L. Schneider, D, L. McClung, M, R. Miller, P, D. Schnitzer, 
T, J. Bonin, R, Smith, M, E. DeLucca, P, Gormley, G, J. Melton, M, E. Alendronate improves bone mineral density in elderly women with osteoporosis residing in longterm care facilities. A randomized, doubleblind, placebo-controlled trial.[Summary for patients in Ann Intern Med. 2002 May 21 136(10):I54 PMID: 12020160]. Annals of Internal Medicine. 2002 May 21;136(10):742-6. PMID: 12020142. No outcomes of interest.

440. Greenspan S, L. Resnick, N, M. Parker, $\mathrm{R}, \mathrm{A}$. Combination therapy with hormone replacement and alendronate for prevention of bone loss in elderly women: a randomized controlled trial. JAMA. 2003 May 21;289(19):2525-33. PMID: 12759324. Ineligible treatment duration.

441. Greenspan S, L. Beck, T, J. Resnick, N, M. Bhattacharya, R, Parker, R, A. Effect of hormone replacement, alendronate, or combination therapy on hip structural geometry: a 3-year, double-blind, placebocontrolled clinical trial. Journal of Bone \& Mineral Research. 2005 Sep;20(9):1525-32. PMID: 16059624. Ineligible treatment duration.

442. Greenspan S, L. Resnick, N, M. Parker, $\mathrm{R}, \mathrm{A}$. Early changes in biochemical markers of bone turnover are associated with longterm changes in bone mineral density in elderly women on alendronate, hormone replacement therapy, or combination therapy: a three-year, double-blind, placebocontrolled, randomized clinical trial. Journal of Clinical Endocrinology \& Metabolism. 2005 May;90(5):2762-7. PMID: 15713726. No outcomes of interest.

443. Greenspan S, L. Bone, H, G. Ettinger, M, P. Hanley, D, A. Lindsay, R, Zanchetta, J, R. Blosch, C, M. Mathisen, A, L. Morris, S, A. Marriott, T, B. Treatment of Osteoporosis with Parathyroid Hormone, Study Group,. Effect of recombinant human parathyroid hormone (1-84) on vertebral fracture and bone mineral density in postmenopausal women with osteoporosis: a randomized trial.[Summary for patients in Ann Intern Med. 2007 Mar 6 146(5):I20 PMID: 17339614]. Annals of Internal Medicine. 2007 Mar 06;146(5):326-39.
PMID: 17339618. No interventions of interest.

444. Greenspan S, L. Perera, S, Ferchak, M, A. Nace, D, A. Resnick, N, M. Efficacy and safety of single-dose zoledronic acid for osteoporosis in frail elderly women: a randomized clinical trial. JAMA Internal Medicine. 2015 Jun;175(6):913-21. PMID: 25867538. No outcomes of interest.

445. Greenspan S, L. Vujevich, K, Britton, C, Herradura, A, Gruen, G, Tarkin, I, Siska, P, Hamlin, B, Perera, S,. Teriparatide for treatment of patients with bisphosphonateassociated atypical fracture of the femur. Osteoporosis International. 201730 Oct:1-6. PMID: 619065536. Ineligible treatment duration.

446. Greenwald M, Gluck, Os, Lang, Rakov,. Oral hormone therapy with 17beta-estradiol and 17beta-estradiol in combination with norethindrone acetate in the prevention of bone loss in early postmenopausal women: dose-dependent effects. Menopause (new york, N.Y.). 2005;12(6):741-8. PMID: CN00570019. Ineligible treatment duration.

447. Grey A, Reid, I, R. Differences between the bisphosphonates for the prevention and treatment of osteoporosis. Therapeutics \& Clinical Risk Management. 2006 Mar;2(1):77-86. PMID: 18360583. Ineligible study design.

448. Grey A, Bolland, M, J. Wattie, D, Horne, A, Gamble, G, Reid, I, R. The antiresorptive effects of a single dose of zoledronate persist for two years: a randomized, placebo-controlled trial in osteopenic postmenopausal women. Journal of Clinical Endocrinology \& Metabolism. 2009 Feb;94(2):538-44. PMID: 19050050. No outcomes of interest.

449. Grey A, Bolland, M, Wattie, D, Horne, A, Gamble, G, Reid, I, R. Prolonged antiresorptive activity of zoledronate: a randomized, controlled trial. Journal of Bone \& Mineral Research. 2010 Oct;25(10):2251-5. PMID: 20499349. No outcomes of interest. 
450. Grey A, Bolland, M, J. Horne, A, Wattie, D, House, M, Gamble, G, Reid, I, R. Five years of anti-resorptive activity after a single dose of zoledronate--results from a randomized double-blind placebo-controlled trial. Bone. 2012 Jun;50(6):1389-93. PMID: 22465268. No outcomes of interest.

451. Grey A, Bolland, M, Wong, S, Horne, A, Gamble, G, Reid, I, R. Low-dose zoledronate in osteopenic postmenopausal women: a randomized controlled trial. Journal of Clinical Endocrinology \& Metabolism. 2012 Jan;97(1):286-92. PMID: 22072741. Ineligible treatment duration.

452. Grey A, Bolland, M, Mihov, B, Wong, S, Horne, A, Gamble, G, Reid, I, R. Duration of antiresorptive effects of lowdose zoledronate in osteopenic postmenopausal women: a randomized, placebo-controlled trial. Journal of Bone \& Mineral Research. 2014 Jan;29(1):166-72. PMID: 23761303. Ineligible treatment duration.

453. Grodstein F, Stampfer, M, J. Falkeborn, M, Naessen, T, Persson, I,. Postmenopausal hormone therapy and risk of cardiovascular disease and hip fracture in a cohort of Swedish women. Epidemiology. 1999 Sep;10(5):476-80. PMID: 10468418. Ineligible population.

454. Gu J, M. Wang, L, Lin, H, Chen, D, C. Tang, H, Jin, X, L. Xia, W, B. Hu, Y, Q. Fu, W, Z. He, J, W. Zhang, H, Wang, C, Yue, H, Hu, W, W. Liu, Y, J. Zhang, Z, L. The efficacy and safety of weekly 35-mg risedronate dosing regimen for Chinese postmenopausal women with osteoporosis or osteopenia: 1-year data. Zhongguo Yao Li Xue Bao/Acta Pharmacologica Sinica. 2015 Jul;36(7):841-6. PMID: 26051110. Ineligible treatment duration.

455. Gur A, Colpan, L, Cevik, R, Nas, K, Jale, Sarac A,. Comparison of zinc excretion and biochemical markers of bone remodelling in the assessment of the effects of alendronate and calcitonin on bone in postmenopausal osteoporosis. Clinical Biochemistry. 2005 Jan;38(1):66-72. PMID: 15607319. Ineligible treatment duration.
456. Gutteridge D, H. Stewart, G, O. Prince, R, L. Price, R, I. Retallack, R, W. Dhaliwal, S, S. Stuckey, B, G. Drury, P, Jones, C, E. Faulkner, D, L. Kent, G, N. Bhagat, C, I. Nicholson, G, C. Jamrozik, K,. A randomized trial of sodium fluoride $(60 \mathrm{mg})$ +/- estrogen in postmenopausal osteoporotic vertebral fractures: increased vertebral fractures and peripheral bone loss with sodium fluoride concurrent estrogen prevents peripheral loss, but not vertebral fractures. Osteoporosis International. 2002;13(2):158-70. PMID: 11908491. No interventions of interest.

457. Guven Z, Karadag, Saygi E, Unlu, Ozkan F, Akyuz, G,. The effects of daily alendronate, daily calcitonin and alendronate every other day on bone mineral density in osteoporotic men. Aging Male. 2007 Dec;10(4):197-201. PMID: 18033629. No outcomes of interest.

458. Hadji P. Managing bone health with zoledronic acid: a review of randomized clinical study results. Climacteric. 2011 Jun;14(3):321-32. PMID: 21182431. Ineligible study design.

459. Hadji P, Gamerdinger, D, Spieler, W, Kann, P, H. Loeffler, H, Articus, K, Moricke, R, Ziller, V,. Rapid Onset and Sustained Efficacy (ROSE) study: results of a randomised, multicentre trial comparing the effect of zoledronic acid or alendronate on bone metabolism in postmenopausal women with low bone mass. Osteoporosis International. 2012 Feb;23(2):625-33. PMID: 21442459. No outcomes of interest.

460. Hadji P. The evolution of selective estrogen receptor modulators in osteoporosis therapy. Climacteric. 2012 Dec;15(6):513-23. PMID: 22853318. Ineligible study design.

461. Hadji P, Felsenberg, D, Amling, M, Hofbauer, L, C. Kandenwein, J, A. Kurth, A,. The non-interventional BonViva Intravenous Versus Alendronate (VIVA) study: real-world adherence and persistence to medication, efficacy, and safety, in patients with postmenopausal osteoporosis. Osteoporosis International. 2014 
Jan;25(1):339-47. PMID: 24091594. No outcomes of interest.

462. Hadji P, Ryan, K, A. Yu, C, R. Mirkin, S, Komm, B, S. CE/BZA effects on bone and quality of life in European postmenopausal women: a pooled analysis. Climacteric. 2016 Oct;19(5):482-7. PMID: 27631562. Ineligible treatment duration.

463. Hadji Z, Kreiss, Articus,. Comparison of the effect of $5 \mathrm{mg}$ zoledronic acid i.v. and 70mg alendronate p.o. on bone metabolism in the treatment of patients with postmenopausal osteoporosis over one year. Medizinische klinik. 2007;102(4):17. PMID: CN-00741369. Not available in English.

464. Hadji Z, Loffler, Kolter, Articus,. Comparison of speed to take effect and the long-lasting effect of $5 \mathrm{mg}$ zoledronic acid i.v. and 70mg alendronate p.o. in the treatment of patients with post-menopausal osteoporosis. Medizinische klinik. 2008;103(3):17. PMID: CN-00741367. Not available in English.

465. Hagino H, Nishizawa, Y, Sone, T, Morii, H, Taketani, Y, Nakamura, T, Itabashi, A, Mizunuma, H, Ohashi, Y, Shiraki, M, Minamide, T, Matsumoto, T,. A doubleblinded head-to-head trial of minodronate and alendronate in women with postmenopausal osteoporosis. Bone. 2009 Jun;44(6):1078-84. PMID: 19264155. No outcomes of interest.

466. Hagino H, Shiraki, M, Fukunaga, M, Nakano, T, Takaoka, K, Ohashi, Y, Nakamura, T, Matsumoto, T,. Number and severity of prevalent vertebral fractures and the risk of subsequent vertebral fractures in Japanese women with osteoporosis: results from the minodronate trial. Journal of Bone \& Mineral Metabolism. 2013

Sep;31(5):544-50. PMID: 23529800. No interventions of interest.

467. Hagino H, Yoshida, S, Hashimoto, J, Matsunaga, M, Tobinai, M, Nakamura, $\mathrm{T}$,. Increased bone mineral density with monthly intravenous ibandronate contributes to fracture risk reduction in patients with primary osteoporosis: three-year analysis of the MOVER study. Calcified Tissue International. 2014 Dec;95(6):557-63. PMID: 25377907. Ineligible treatment duration.

468. Hagino H, Kishimoto, H, Ohishi, H, Horii, S, Nakamura, T,. Efficacy, tolerability and safety of once-monthly administration of $75 \mathrm{mg}$ risedronate in Japanese patients with involutional osteoporosis: a comparison with a $2.5 \mathrm{mg}$ once-daily dosage regimen. Bone. 2014 Feb;59:44-52. PMID: 24184313. Ineligible treatment duration.

469. Hagino I, Hashimoto, Yamamoto, Endo, Katsumata, Asao, Matsumoto, Nakano, Mizunuma, Nakamura,. Monthly oral ibandronate $100 \mathrm{mg}$ is as effective as monthly intravenous ibandronate $1 \mathrm{mg}$ in patients with various pathologies in the MOVEST study. Journal of bone and mineral metabolism. 2017:1-8. PMID: CN01366511. No outcomes of interest.

470. Haines C, J. Yim, S, F. Chung, T, K. Lam, C, W. Lau, E, W. Ng, M, H. Chin, $\mathrm{R}$, Lee, D, T. A prospective, randomized, placebo-controlled study of the dose effect of oral estradiol on bone mineral density in postmenopausal Chinese women. Maturitas. 2003 Jul 25;45(3):169-73. PMID: 12818461. Ineligible treatment duration.

471. Hajcsar E, E. Hawker, G, Bogoch, E, R. Investigation and treatment of osteoporosis in patients with fragility fractures. CMAJ Canadian Medical Association Journal. 2000 Oct 03;163(7):819-22. PMID: 11033708. Ineligible study design.

472. Han L, W. Ma, D, D. Xu, X, J. Lu, F, Liu, Y, Xia, W, B. Jiang, Y, Wang, O, Xing, X, P. Li, M,. Association Between Geranylgeranyl Pyrophosphate Synthase Gene Polymorphisms and Bone Phenotypes and Response to Alendronate Treatment in Chinese Osteoporotic Women. Chinese Medical Sciences Journal. 201601 Mar;31(1):8-16. PMID: 611250298. Ineligible treatment duration.

473. Han R, K. Pimlott, N, Heisey, R,. Does raloxifene reduce postmenopausal women's risk of breast cancer? Canadian Family 
Physician. 2000 Jan;46:77-80. PMID: 10660790. Ineligible study design.

474. Handler R, P. Prior bisphosphonate therapy of osteoporosis attenuates and blocks response to subsequent parathyroid hormone. JCR: Journal of Clinical Rheumatology. 2008 Apr;14(2):122-4. PMID: 18391687. Ineligible study design.

475. Hansen L, Petersen, K, D. Eriksen, S, A. Gerstoft, F, Vestergaard, P,. Subjects with osteoporosis to remain at high risk for fracture despite benefit of prior bisphosphonate treatment-a Danish casecontrol study. Osteoporosis International. 2017 Jan;28(1):321-8. PMID: 27475930. Ineligible study design.

476. Harrington J, T. Ste, Marie L, G. Brandi, M, L. Civitelli, R, Fardellone, P, Grauer, A, Barton, I, Boonen, S,. Risedronate rapidly reduces the risk for nonvertebral fractures in women with postmenopausal osteoporosis. Calcified Tissue International. 2004 Feb;74(2):129-35. PMID: 14648009. Ineligible treatment duration.

477. Harris K, B. Nealy, K, L. Jackson, D, J. Thornton, P, L. The clinical use of denosumab for the management of low bone mineral density in postmenopausal women. Journal of Pharmacy Practice. 2012 Jun;25(3):310-8. PMID: 22550161. Ineligible study design.

478. Harris S, T. Watts, N, B. Genant, H, K. McKeever, C, D. Hangartner, T, Keller, M, Chesnut, C, H. rd, Brown, J, Eriksen, E, F. Hoseyni, M, S. Axelrod, D, W. Miller, P, D. Effects of risedronate treatment on vertebral and nonvertebral fractures in women with postmenopausal osteoporosis: a randomized controlled trial. Vertebral Efficacy With Risedronate Therapy (VERT) Study Group. JAMA. 1999 Oct 13;282(14):1344-52. PMID: 10527181. No outcomes of interest.

479. Harris S, T. Eriksen, E, F. Davidson, M, Ettinger, M, P. Moffett, Jr. A, H. Jr, Baylink, D, J. Crusan, C, E. Chines, A, A. Effect of combined risedronate and hormone replacement therapies on bone mineral density in postmenopausal women.
Journal of Clinical Endocrinology \& Metabolism. 2001 May;86(5):1890-7. PMID: 11344179. Ineligible treatment duration.

480. Harris S, T. Watts, N, B. Li, Z, Chines, A, A. Hanley, D, A. Brown, J, P. Twoyear efficacy and tolerability of risedronate once a week for the treatment of women with postmenopausal osteoporosis.[Erratum appears in Curr Med Res Opin. 2004 Oct 20(10):1690]. Current Medical Research \& Opinion. 2004 May;20(5):757-64. PMID: 15140343. Ineligible treatment duration.

481. Harris S, T. Reginster, J, Y. Harley, C, Blumentals, W, A. Poston, S, A. Barr, C, E. Silverman, S, L. Risk of fracture in women treated with monthly oral ibandronate or weekly bisphosphonates: the eValuation of IBandronate Efficacy (VIBE) database fracture study. Bone. 2009 May;44(5):758-65. PMID: 19168160. Ineligible treatment duration.

482. Hartle JE, Tang X, Kirchner HL, et al. Bisphosphonate therapy, death, and cardiovascular events among female patients with CKD: a retrospective cohort study. American Journal of Kidney Diseases. 2012;59(5):636-44. Ineligible population.

483. Harvey N, C. Kanis, J, A. Oden, A, Burge, R, T. Mitlak, B, H. Johansson, H, McCloskey, E, V. FRAX and the effect of teriparatide on vertebral and non-vertebral fracture. Osteoporosis International. 2015 Nov;26(11):2677-84. PMID: 26092063. Ineligible treatment duration.

484. Harvey N, C. Kanis, J, A. Oden, A, Nakamura, T, Shiraki, M, Sugimoto, T, Kuroda, T, Johansson, H, McCloskey, E, V. Efficacy of weekly teriparatide does not vary by baseline fracture probability calculated using FRAX. Osteoporosis International. 2015 Sep;26(9):2347-53. PMID: 26092062. Ineligible treatment duration.

485. Hashimoto M, Akishita, Hosoi, Toba, Kozaki, Yoshizumi, Ouchi,. Effects of long-term and reduced-dose hormone replacement therapy on endothelial function and intima-media thickness in 
postmenopausal women. Menopause (new york, N.Y.). 2002;9(1):58-64. PMID: CN00377065. Ineligible study design.

486. Hassler N, Gamsjaeger, S, Hofstetter, B, Brozek, W, Klaushofer, K, Paschalis, E, P. Effects of long-term alendronate treatment on postmenopausal osteoporosis bone material properties. Osteoporosis International. 2015 Jan;26(1):339-52. PMID: 25315260. Ineligible study design.

487. Hathirat S, Evans, M, F. Does raloxifene reduce risk of vertebral fractures? Is this another, brighter way to treat osteoporosis? Canadian Family Physician. 2001 Oct;47:1982-4. PMID: 11723592. Ineligible study design.

488. Hayashi T, Ina, K, Maeda, M, Nomura, $H$,. The effects of selective estrogen receptor modulator treatment following hormone replacement therapy on elderly postmenopausal women with osteoporosis. Nitric Oxide. 2011 May 31;24(4):199-203. PMID: 21513812. No outcomes of interest.

489. Heaney R, P. Zizic, T, M. Fogelman, I, Olszynski, W, P. Geusens, P, Kasibhatla, C, Alsayed, N, Isaia, G, Davie, M, W. Chesnut, C, H. rd,. Risedronate reduces the risk of first vertebral fracture in osteoporotic women. Osteoporosis International. 2002;13(6):501-5. PMID: 12107665. Ineligible treatment duration.

490. Heckbert S, R. Li, G, Cummings, S, R. Smith, N, L. Psaty, B, M. Use of alendronate and risk of incident atrial fibrillation in women. Archives of Internal Medicine. 2008 Apr 28;168(8):826-31. PMID: 18443257. Ineligible treatment duration.

491. Heijckmann A, C. Juttmann, J, R. Wolffenbuttel, B, H. Intravenous pamidronate compared with oral alendronate for the treatment of postmenopausal osteoporosis. Netherlands Journal of Medicine. 2002 Sep;60(8):315-9. PMID: 12481878. Ineligible study design.

492. Heikkinen A, M. Parviainen, M, Niskanen, L, Komulainen, M, Tuppurainen, M, T.
Kroger, H, Saarikoski, S,. Biochemical bone markers and bone mineral density during postmenopausal hormone replacement therapy with and without vitamin D3: a prospective, controlled, randomized study. Journal of Clinical Endocrinology \& Metabolism. 1997 Aug;82(8):2476-82. PMID: 9253321. Ineligible treatment duration.

493. Heikkinen A, Kröger, Niskanen, Komulainen, Mh, Ryynänen, Parviainen, Mt, Tuppurainen, Mt, Honkanen, Saarikoski,. Does apolipoprotein E genotype relate to BMD and bone markers in postmenopausal women? Maturitas. 2000;34(1):33-41. PMID: CN-00275357. Ineligible population.

494. Heikkinen J, E. Selander, K, S. Laitinen, K, Arnala, I, Vaananen, H, K. Short-term intravenous bisphosphonates in prevention of postmenopausal bone loss. Journal of Bone \& Mineral Research. 1997 Jan;12(1):103-10. PMID: 9240732. Ineligible treatment duration.

495. Heikkinen J, Vaheri, R, Kainulainen, P, Timonen, U,. Long-term continuous combined hormone replacement therapy in the prevention of postmenopausal bone loss: a comparison of high- and low-dose estrogen-progestin regimens. Osteoporosis International. 2000;11(11):929-37. PMID: 11193245. No outcomes of interest.

496. Heikkinen J, Vaheri, R, Haapalahti, J, Timonen, U,. A 10-year follow-up of the effect of continuous-combined hormone replacement therapy and its discontinuation on bone in postmenopausal women. Menopause International. 2008 Jun;14(2):70-7. PMID: 18519268. Ineligible population.

497. Heikkinen K, Kurttila, Matero Wilén, Rosenqvist Lankinen, Ks, Rita, Väänänen, Hk,. HRT and exercise: effects on bone density, muscle strength and lipid metabolism. A placebo controlled 2-year prospective trial on two estrogen-progestin regimens in healthy postmenopausal women. Maturitas. 1997;26(2):139-49. PMID: CN-00138266. Ineligible treatment duration. 
498. Heikkinen V, Timonen,. A 10-year followup of postmenopausal women on long-term continuous combined hormone replacement therapy: update of safety and quality-of-life findings. Journal of the British Menopause Society. 2006;12(3):115-25. PMID: CN00567838. Ineligible population.

499. Hennekens C, H. Barice, E, J. Calcium supplements and risk of myocardial infarction: a hypothesis formulated but not yet adequately tested. American Journal of Medicine. 2011 Dec;124(12):1097-8. PMID: 21798509. Ineligible study design.

500. Hernandez E, Valera, R, Alonzo, E, Bajares, Lilue M, Carlini, R, Capriles, F, Martinis, R, Bellorin, Font E, Weisinger, J, R. Effects of raloxifene on bone metabolism and serum lipids in postmenopausal women on chronic hemodialysis. Kidney International. 2003 Jun;63(6):2269-74. PMID: 12753317. No outcomes of interest.

501. Hirao M, Hashimoto, J, Ando, W, Ono, $\mathrm{T}$, Yoshikawa, H,. Response of serum carboxylated and undercarboxylated osteocalcin to alendronate monotherapy and combined therapy with vitamin K2 in postmenopausal women. Journal of Bone \& Mineral Metabolism. 2008;26(3):260-4. PMID: 18470667. No interventions of interest.

502. Hirvonen E, Cacciatore, B, Wahlstrom, T, Rita, H, Wilen, Rosenqvist G,. Effects of transdermal oestrogen therapy in postmenopausal women: a comparative study of an oestradiol gel and an oestradiol delivering patch. British Journal of Obstetrics \& Gynaecology. 1997 Nov;104 Suppl 16:26-31. PMID: 9389780. Ineligible treatment duration.

503. Hirvonen E, Lamberg, Allardt C, Lankinen, K, S. Geurts, P, Wilen, Rosenqvist G,. Transdermal oestradiol gel in the treatment of the climacterium: a comparison with oral therapy. British Journal of Obstetrics \& Gynaecology. 1997 Nov;104 Suppl 16:19-25. PMID: 9389779. Ineligible treatment duration.
504. Ho A, Y. Kung, A, W. Efficacy and tolerability of alendronate once weekly in Asian postmenopausal osteoporotic women. Annals of Pharmacotherapy. 2005 Sep;39(9):1428-33. PMID: 16076919. No outcomes of interest.

505. Ho Y, V. Frauman, A, G. Thomson, W, Seeman, E,. Effects of alendronate on bone density in men with primary and secondary osteoporosis. Osteoporosis International. 2000;11(2):98-101. PMID: 10793867. Ineligible study design.

506. Hochberg M, C. Ross, P, D. Black, D, Cummings, S, R. Genant, H, K. Nevitt, M, C. Barrett, Connor E, Musliner, T, Thompson, D,. Larger increases in bone mineral density during alendronate therapy are associated with a lower risk of new vertebral fractures in women with postmenopausal osteoporosis. Fracture Intervention Trial Research Group. Arthritis \& Rheumatism. 1999 Jun;42(6):1246-54. PMID: 10366118. Ineligible study design.

507. Hochberg M, C. Thompson, D, E. Black, D, M. Quandt, S, A. Cauley, J, Geusens, P, Ross, P, D. Baran, D, F. I. T. Research Group. Effect of alendronate on the agespecific incidence of symptomatic osteoporotic fractures. Journal of Bone \& Mineral Research. 2005 Jun;20(6):971-6. PMID: 15883637. Ineligible treatment duration.

508. Hochberg M, C. Silverman, S, L. Barr, C, E. Miller, P, D. The utility of changes in serum levels of C-terminal telopeptide of type I collagen in predicting patient response to oral monthly ibandronate therapy. Journal of Clinical Densitometry. 2010 AprJun;13(2):181-9. PMID: 20347369. No outcomes of interest.

509. Hodsman A, B. Fraher, L, J. Watson, P, H. Ostbye, T, Stitt, L, W. Adachi, J, D. Taves, D, H. Drost, D,. A randomized controlled trial to compare the efficacy of cyclical parathyroid hormone versus cyclical parathyroid hormone and sequential calcitonin to improve bone mass in postmenopausal women with osteoporosis. Journal of Clinical Endocrinology \& 
Metabolism. 1997 Feb;82(2):620-8. PMID: 9024265. No interventions of interest.

510. Hodsman A, Kisiel, Adachi, Jd, Fraher, $\mathrm{Lj}$, Watson, Ph,. Histomorphometric evidence for increased bone turnover without change in cortical thickness or porosity after 2 years of cyclical hPTH(1$34)$ therapy in women with severe osteoporosis. Bone. 2000;27(2):311-8. PMID: CN-00298588. No outcomes of interest.

511. Hodsman A, B. Hanley, D, A. Ettinger, M, P. Bolognese, M, A. Fox, J, Metcalfe, A, J. Lindsay, R,. Efficacy and safety of human parathyroid hormone-(1-84) in increasing bone mineral density in postmenopausal osteoporosis. Journal of Clinical Endocrinology \& Metabolism. 2003 Nov;88(11):5212-20. PMID: 14602752. No interventions of interest.

512. Holland E, F. Leather, A, T. Studd, J, W. Increase in bone mass of older postmenopausal women with low mineral bone density after one year of percutaneous oestradiol implants. British Journal of Obstetrics \& Gynaecology. 1995 Mar;102(3):238-42. PMID: 7794850. Ineligible study design.

513. Honig S, Rajapakse, C, S. Chang, G,. Current treatment approaches to osteoporosis - 2013. Bulletin of the Hospital for Joint Disease (2013). 2013;71(3):184-8. PMID: 24151942. Ineligible study design.

514. Hooper M, J. Ebeling, P, R. Roberts, A, P. Graham, J, J. Nicholson, G, C. D'Emden, M, Ernst, T, F. Wenderoth, D,. Risedronate prevents bone loss in early postmenopausal women: a prospective randomized, placebo-controlled trial. Climacteric. 2005 Sep;8(3):251-62. PMID: 16390757. Ineligible treatment duration.

515. Hopkins R, B. Goeree, R, Pullenayegum, E, Adachi, J, D. Papaioannou, A, Xie, F, Thabane, L,. The relative efficacy of nine osteoporosis medications for reducing the rate of fractures in post-menopausal women. BMC Musculoskeletal Disorders. 2011 Sep 26;12:209. PMID: 21943363. Ineligible study design.
516. Hosking D, Chilvers, C, E. Christiansen, C, Ravn, P, Wasnich, R, Ross, P, McClung, M, Balske, A, Thompson, D, Daley, M, Yates, A, J. Prevention of bone loss with alendronate in postmenopausal women under 60 years of age. Early Postmenopausal Intervention Cohort Study Group. New England Journal of Medicine. 1998 Feb 19;338(8):485-92. PMID: 9443925. Ineligible treatment duration.

517. Hosking D, J. Favus, M, Yates, A, J. Alendronate in the treatment of postmenopausal osteoporosis. International Journal of Clinical Practice. Supplement. 1999 Apr;101:27-35. PMID: 12669738. Ineligible treatment duration.

518. Hosking D, Adami, S, Felsenberg, D, Andia, J, C. Valimaki, M, Benhamou, L, Reginster, J, Y. Yacik, C, Rybak, Feglin A, Petruschke, R, A. Zaru, L, Santora, A, C. Comparison of change in bone resorption and bone mineral density with once-weekly alendronate and daily risedronate: a randomised, placebocontrolled study. Current Medical Research \& Opinion. 2003;19(5):383-94. PMID: 13678475. No outcomes of interest.

519. Hsiao F, Y. Huang, W, F. Chen, Y, M. Wen, Y, W. Kao, Y, H. Chen, L, K. Tsai, Y, W. Hip and subtrochanteric or diaphyseal femoral fractures in alendronate users: a 10-year, nationwide retrospective cohort study in Taiwanese women. Clinical Therapeutics. 2011 Nov;33(11):1659-67. PMID: 22018450. No outcomes of interest.

520. Hsiao F, Y. Hsu, W, W. Comparative risks for cancer associated with use of calcitonin, bisphosphonates or selective estrogen receptor modulators among osteoporosis patients: a population-based cohort study. Japanese Journal of Clinical Oncology. 2017 Oct 01;47(10):935-41. PMID: 28981741. No outcomes of interest.

521. Huang A, Ettinger, Vittinghoff, Ensrud, Ke, Johnson, Kc, Cummings, Sr,. Endogenous estrogen levels and the effects of ultra-low-dose transdermal estradiol therapy on bone turnover and BMD in postmenopausal women. Journal of bone 
and mineral research. 2007;22(11):1791-7. PMID: CN-00700670. No outcomes of interest.

522. Huang W, F. Tsai, Y, W. Wen, Y, W. Hsiao, F, Y. Kuo, K, N. Tsai, C, R. Osteoporosis treatment and atrial fibrillation: alendronate versus raloxifene. Menopause. 2010 Jan-Feb;17(1):57-63. PMID: 19680161. Ineligible treatment duration.

523. Huang W, F. Chang, L, C. Kao, Y, H. Wen, Y, W. Hsiao, F, Y. Peng, L, N. Tsai, Y, W. Chen, L, K. Prolonged risk of subtrochanteric and diaphyseal femur fractures after discontinuing alendronate treatment: A nationwide nested case-control study in Taiwan. Journal of Clinical Gerontology and Geriatrics. 201501 Jun;6(2):54-8. PMID: 604812155. Ineligible treatment duration.

524. Huang Y, F. Chang, C, T. Muo, C, H. Tsai, C, H. Shen, Y, F. Wu, C, Z. Impact of bisphosphonate-related osteonecrosis of the jaw on osteoporotic patients after dental extraction: A population-based cohort study. PLoS ONE. 201516 Apr;10 (4) (no pagination)(e0120756) PMID: 603863824. Ineligible treatment duration.

525. Hulley S, Furberg, C, Barrett, Connor E, Cauley, J, Grady, D, Haskell, W, Knopp, R, Lowery, M, Satterfield, S, Schrott, H, Vittinghoff, E, Hunninghake, D, Hers Research, Group. Noncardiovascular disease outcomes during 6.8 years of hormone therapy: Heart and Estrogen/progestin Replacement Study follow-up (HERS II). JAMA. 2002 Jul 03;288(1):58-66. PMID: 12090863. Ineligible population.

526. Hwang J, S. Chin, L, S. Chen, J, F. Yang, T, S. Chen, P, Q. Tsai, K, S. Leung, P, C. The effects of intravenous zoledronic acid in Chinese women with postmenopausal osteoporosis. Journal of Bone \& Mineral Metabolism. 2011 May;29(3):328-33. PMID: 20922438. No outcomes of interest.

527. Iannitti T, Rosini, S, Lodi, D, Frediani, B, Rottigni, V, Palmieri, B,. Bisphosphonates: focus on inflammation and bone loss. American Journal of Therapeutics. 2012 May;19(3):228-46. PMID: 22549638. Ineligible study design.

528. Idolazzi L, Fassio, A, Gatti, D, Tamanini, S, Viapiana, O, Rossini, M, Adami, S,. Duration of treatment for osteoporosis. Reumatismo. 2013 Mar 28;65(1):22-35. PMID: 23550257. Ineligible study design.

529. Iizuka T, Matsukawa, M.. Potential excessive suppression of bone turnover with long-term oral bisphosphonate therapy in postmenopausal osteoporotic patients. Climacteric. 2008 Aug;11(4):287-95. PMID: 18645694. No outcomes of interest.

530. Ilter E, Karalok, H, Tufekci, E, C. Batur, $\mathrm{O}$,. Efficacy and acceptability of risedronate $5 \mathrm{mg}$ daily compared with $35 \mathrm{mg}$ once weekly for the treatment of postmenopausal osteoporosis. Climacteric. 2006 Apr;9(2):129-34. PMID: 16698659. Ineligible treatment duration.

531. Inderjeeth C, A. Glendenning, P, Ratnagobal, S, Inderjeeth, D, C. Ondhia, C,. Long-term efficacy, safety, and patient acceptability of ibandronate in the treatment of postmenopausal osteoporosis. International Journal of Women's Health. 2015;7:7-17. PMID: 25565901. Ineligible study design.

532. Inoue D, Muraoka, R, Okazaki, R, Nishizawa, Y, Sugimoto, T,. Efficacy and Safety of Risedronate in Osteoporosis Subjects with Comorbid Diabetes, Hypertension, and/or Dyslipidemia: A Post Hoc Analysis of Phase III Trials Conducted in Japan. Calcified Tissue International. 2016 Feb;98(2):114-22. PMID: 26466937. Ineligible treatment duration.

533. Iqbal J, Sun, L, Zaidi, M,. Denosumab for the treatment of osteoporosis. Current Osteoporosis Reports. 2010 Dec;8(4):163-7. PMID: 20857349. Ineligible study design.

534. Ishani A, Blackwell, T, Jamal, S, A. Cummings, S, R. Ensrud, K, E. More, Investigators. The effect of raloxifene treatment in postmenopausal women with CKD. Journal of the American Society of 
Nephrology. 2008 Jul;19(7):1430-8. PMID: 18400939. Ineligible treatment duration.

535. Ishibashi H, Crittenden, D, B. Miyauchi, A, Libanati, C, Maddox, J, Fan, M, Chen, L, Grauer, A,. Romosozumab increases bone mineral density in postmenopausal Japanese women with osteoporosis: A phase 2 study. Bone. 2017 Oct;103:209-15. PMID: 28687496. No outcomes of interest.

536. Ishida Y, Kawai, S,. Comparative efficacy of hormone replacement therapy, etidronate, calcitonin, alfacalcidol, and vitamin $\mathrm{K}$ in postmenopausal women with osteoporosis: The Yamaguchi Osteoporosis Prevention Study. American Journal of Medicine. 2004 Oct 15;117(8):549-55. PMID: 15465502. No interventions of interest.

537. Ishida Y, Mine, T, Taguchi, T,. Effect of progestins with different glucocorticoid activity on bone metabolism. Clinical Endocrinology. 2008 Mar;68(3):423-8. PMID: 17973947. No outcomes of interest.

538. Ishtiaq S, Fogelman, I, Hampson, G, Treatment of post-menopausal osteoporosis: beyond bisphosphonates. Journal of Endocrinological Investigation. 2015 Jan;38(1):13-29. PMID: 25194424. Ineligible study design.

539. Ismail A, Bekhet AH, Abushouk AI, et al. Denosumab and atypical femoral fractures: A scoping literature review. Trauma Monthly. 2018 January;23 (1) (no pagination)(e42869) PMID: 621923122. Ineligible study design.

540. Itabashi A, Yoh, K, Chines, A, A. Miki, T, Takada, M, Sato, H, Gorai, I, Sugimoto, T, Mizunuma, H, Ochi, H, Constantine, G, D. Ohta, H,. Effects of bazedoxifene on bone mineral density, bone turnover, and safety in postmenopausal Japanese women with osteoporosis. Journal of Bone \& Mineral Research. 2011 Mar;26(3):519-29. PMID: 20839291. No interventions of interest.

541. Itabashi A, Yoh, K, Chines, A, A. Miki, T, Takada, M, Sato, H, Gorai, I,
Sugimoto, T, Mizunuma, H, Ochi, H, Constantine, G, D. Ohta, H,. Bridging analysis of the efficacy and safety of bazedoxifene in Japanese and global populations of postmenopausal women with osteoporosis. Journal of Bone \& Mineral Metabolism. 2015 Jan;33(1):61-72. PMID: 24714934. No interventions of interest.

542. Ito M, Oishi, R, Fukunaga, M, Sone, T, Sugimoto, T, Shiraki, M, Nishizawa, Y, Nakamura, T,. The effects of once-weekly teriparatide on hip structure and biomechanical properties assessed by CT. Osteoporosis International. 2014 Mar;25(3):1163-72. PMID: 24345886. No outcomes of interest.

543. Ito M, Tobinai, M, Yoshida, S, Hashimoto, J, Nakamura, T,. Effect of monthly intravenous ibandronate injections on vertebral or non-vertebral fracture risk in Japanese patients with high-risk osteoporosis in the MOVER study. Journal of Bone \& Mineral Metabolism. 2017 Jan;35(1):58-64. PMID: 26614597. Ineligible treatment duration.

544. Ito M, Sone, T, Shiraki, M, Tanaka, S, Irie, C, Ota, Y, Nakamura, T,. The effect of once-yearly zoledronic acid on hip structural and biomechanical properties derived using computed tomography (CT) in Japanese women with osteoporosis. Bone. 2018 Jan;106:179-86. PMID: 29033381. No outcomes of interest.

545. Itoi H, Minakami, H, Sato, I,. Comparison of the long-term effects of oral estriol with the effects of conjugated estrogen, 1-alphahydroxyvitamin D3 and calcium lactate on vertebral bone loss in early menopausal women. Maturitas. 1997 Sep;28(1):11-7. PMID: 9391990. Ineligible treatment duration.

546. Ivaska K, K. Pettersson, K, Nenonen, A, Uusi, Rasi K, Heinonen, A, Kannus, P, Vaananen, H, K. Urinary osteocalcin is a useful marker for monitoring the effect of alendronate therapy. Clinical Chemistry. 2005 Dec;51(12):2362-5. PMID: 16306097. Ineligible treatment duration. 
547. Iwamoto I, Kosha, S, Noguchi, S, Murakami, M, Fujino, T, Douchi, T, Nagata, Y,. A longitudinal study of the effect of vitamin K2 on bone mineral density in postmenopausal women a comparative study with vitamin D3 and estrogen-progestin therapy. Maturitas. 1999 Jan 04;31(2):161-4. PMID: 10227010. Ineligible treatment duration.

548. Iwamoto J, Takeda, T, Ichimura, S,. Effect of menatetrenone on bone mineral density and incidence of vertebral fractures in postmenopausal women with osteoporosis: a comparison with the effect of etidronate. Journal of Orthopaedic Science. 2001;6(6):487-92. PMID: 11793169. No interventions of interest.

549. Iwamoto J, Takeda, T, Ichimura, S, Uzawa, M,. Early response to alendronate after treatment with etidronate in postmenopausal women with osteoporosis. Keio Journal of Medicine. 2003 Jun;52(2):113-9. PMID: 12862363. No outcomes of interest.

550. Iwamoto J, Takeda, T, Sato, Y, Uzawa, M,. Effects of alendronate on metacarpal and lumbar bone mineral density, bone resorption, and chronic back pain in postmenopausal women with osteoporosis. Clinical Rheumatology. 2004 Oct;23(5):383-9. PMID: 15278749. No outcomes of interest.

551. Iwamoto J, Takeda, T, Sato, Y, Uzawa, M,. Comparison of effect of treatment with etidronate and alendronate on lumbar bone mineral density in elderly women with osteoporosis. Yonsei Medical Journal. 2005 Dec 31;46(6):750-8. PMID: 16385649. No outcomes of interest.

552. Iwamoto J, Sato, Y, Uzawa, M, Takeda, T, Matsumoto, H,. Comparison of effects of alendronate and raloxifene on lumbar bone mineral density, bone turnover, and lipid metabolism in elderly women with osteoporosis. Yonsei Medical Journal. 2008 Feb 29;49(1):119-28. PMID: 18306478. No outcomes of interest.

553. Iwamoto J, Sato, Y, Uzawa, M, Takeda, $\mathrm{T}$, Matsumoto, H,. Comparison of the effects of alendronate and alfacalcidol on hip bone mineral density and bone turnover in Japanese men having osteoporosis or osteopenia with clinical risk factors for fractures. Yonsei Medical Journal. 2009 Aug 31;50(4):474-81. PMID: 19718394. No outcomes of interest.

554. Iwamoto J, Sato, Y, Takeda, T, Matsumoto, H,. Efficacy of antiresorptive agents for preventing fractures in Japanese patients with an increased fracture risk: review of the literature. Drugs \& Aging. 2012 Mar 01;29(3):191-203. PMID: 22372723. Ineligible study design.

555. Iwamoto J, Sato, Y, Uzawa, M, Takeda, $\mathrm{T}$, Matsumoto, H,. Three-year experience with alendronate treatment in postmenopausal osteoporotic Japanese women with or without renal dysfunction: a retrospective study. Drugs \& Aging. 2012 Feb 01;29(2):133-42. PMID: 22233456. Ineligible study design.

556. Iwamoto J, Okano, H, Furuya, T, Urano, T, Hasegawa, M, Hirabayashi, H, Kumakubo, T, Makita, K,. Patient preference for monthly bisphosphonate versus weekly bisphosphonate in a clusterrandomized, open-label, crossover trial: Minodroate Alendronate/Risedronate Trial in Osteoporosis (MARTO). Journal of Bone \& Mineral Metabolism. 2016

Mar;34(2):201-8. PMID: 25794468. Ineligible treatment duration.

557. Jacobsen C, Metzler, P, Obwegeser, J, A. Zemann, W, Graetz, K, W. Osteopathology of the jaw associated with bone resorption inhibitors: what have we learned in the last 8 years? Swiss Medical Weekly. 2012 Jun 26;142:w13605. PMID: 22736052. Ineligible study design.

558. Jacobsen D, E. Melis, R, J. Verhaar, H, J. Olde, Rikkert M, G. Raloxifene and tibolone in elderly women: a randomized, double-blind, double-dummy, placebocontrolled trial. Journal of the American Medical Directors Association. 2012 Feb;13(2):189.e1-7. PMID: 21741883. Ineligible treatment duration. 
559. Jacques R, M. Boonen, S, Cosman, F, Reid, I, R. Bauer, D, C. Black, D, M. Eastell, R,. Relationship of changes in total hip bone mineral density to vertebral and nonvertebral fracture risk in women with postmenopausal osteoporosis treated with once-yearly zoledronic acid $5 \mathrm{mg}$ : the HORIZON-Pivotal Fracture Trial (PFT). Journal of Bone \& Mineral Research. 2012 Aug;27(8):1627-34. PMID: 22532515. Ineligible treatment duration.

560. Jamal S, A. Ljunggren, O, Stehman, Breen C, Cummings, S, R. McClung, M, R. Goemaere, S, Ebeling, P, R. Franek, E, Yang, Y, C. Egbuna, O, I. Boonen, S, Miller, P, D. Effects of denosumab on fracture and bone mineral density by level of kidney function. Journal of Bone \& Mineral Research. 2011 Aug;26(8):1829-35. PMID: 21491487. Ineligible treatment duration.

561. Jawad A. Atypical femoral fractures and biphosphonates. Saudi Medical Journal. 2017 Aug;38(8):872. PMID: 28762443. Ineligible study design.

562. Jeffcoat M. Safety of oral bisphosphonates: controlled studies on alveolar bone. International journal of oral \& maxillofacial implants. 2006;21(3):349-53. PMID: CN00557102. Ineligible population.

563. Jensen L, B. Vestergaard, P, Hermann, A, P. Gram, J, Eiken, P, Abrahamsen, B, Brot, C, Kolthoff, N, Sorensen, O, H. Beck, Nielsen H, Nielsen, S, P. Charles, P, Mosekilde, L,. Hormone replacement therapy dissociates fat mass and bone mass, and tends to reduce weight gain in early postmenopausal women: a randomized controlled 5-year clinical trial of the Danish Osteoporosis Prevention Study. Journal of Bone \& Mineral Research. 2003 Feb;18(2):333-42. PMID: 12568411. Ineligible population.

564. Jirapinyo M, Theppisai, U, Manonai, J, Suchartwatnachai, C, Jorgensen, L, N. Effect of combined oral estrogen/progestogen preparation (Kliogest) on bone mineral density, plasma lipids and postmenopausal symptoms in HRT-naive Thai women. Acta Obstetricia et Gynecologica Scandinavica. 2003
Sep;82(9):857-66. PMID: 12911449. Ineligible treatment duration.

565. Jodar GE. Full length parathyroid hormone (1-84) in the treatment of osteoporosis in postmenopausal women. Clinical Interventions In Aging. 2007;2(1):163-74. PMID: 18044089. Ineligible study design.

566. Johnell O, Scheele, W, H. Lu, Y, Reginster, J, Y. Need, A, G. Seeman, E,. Additive effects of raloxifene and alendronate on bone density and biochemical markers of bone remodeling in postmenopausal women with osteoporosis. Journal of Clinical Endocrinology \& Metabolism. 2002 Mar;87(3):985-92. PMID: 11889149. No outcomes of interest.

567. Johnell O, Jonsson, B, Jonsson, L, Black, $\mathrm{D}$,. Cost effectiveness of alendronate (fosamax) for the treatment of osteoporosis and prevention of fractures.

Pharmacoeconomics. 2003;21(5):305-14. PMID: 12627984. Ineligible study design.

568. Johnell O, Kanis, J, A. Black, D, M. Balogh, A, Poor, G, Sarkar, S, Zhou, C, Pavo, I,. Associations between baseline risk factors and vertebral fracture risk in the Multiple Outcomes of Raloxifene Evaluation (MORE) Study. Journal of Bone \& Mineral Research. 2004 May;19(5):76472. PMID: 15068500. Ineligible treatment duration.

569. Johnston C, C. Jr, Bjarnason, N, H. Cohen, F, J. Shah, A, Lindsay, R, Mitlak, B, H. Huster, W, Draper, M, W. Harper, K, D. Heath, H, rd, Gennari, C, Christiansen, C, Arnaud, C, D. Delmas, $\mathrm{P}, \mathrm{D}$. Long-term effects of raloxifene on bone mineral density, bone turnover, and serum lipid levels in early postmenopausal women: three-year data from 2 double-blind, randomized, placebo-controlled trials. Archives of Internal Medicine. 2000 Dec 11-25;160(22):3444-50. PMID: 11112238. Ineligible treatment duration.

570. Jolly E, E. Bjarnason, N, H. Neven, P, Plouffe, L, Jr, Johnston, C, C. Jr, Watts, S, D. Arnaud, C, D. Mason, T, M. Crans, G, Akers, R, Draper, M, W. Prevention of osteoporosis and uterine effects in 
postmenopausal women taking raloxifene for 5 years. Menopause. 2003 JulAug;10(4):337-44. PMID: 12851517. Ineligible population.

571. Jorgensen L, Vik, A, Emaus, N, Brox, J, Hansen, J, B. Mathiesen, E, Vestergaard, $\mathrm{P}$, . Bone loss in relation to serum levels of osteoprotegerin and nuclear factor-kappaB ligand: the Tromso Study. Osteoporosis International. 2010 Jun;21(6):931-8. PMID: 19701599. Ineligible treatment duration.

572. Jung S, Y. Sohn, H, S. Park, E, J. Suh, H, S. Park, J, W. Kwon, J, W. Oral Bisphosphonates and Upper Gastrointestinal Cancer Risks in Asians with Osteoporosis: A Nested Case-Control Study Using National Retrospective Cohort Sample Data from Korea. PLoS ONE [Electronic Resource]. 2016;11(3):e0150531. PMID: 26937968. Ineligible study design.

573. Kagan R. The tissue selective estrogen complex: a novel approach to the treatment of menopausal symptoms. Journal of Women's Health. 2012 Sep;21(9):975-81. PMID: 22759213. Ineligible study design.

574. Kalder K, Hars, Kauka, Hadji,. Comparison of combined low-dose hormone therapy vs. tibolone in the prevention of bone loss. Climacteric. 2016;19(5):471-7. PMID: CN-01208891. Ineligible treatment duration.

575. Kang J, H. Keller, J, J. Lin, H, C. A population-based 2-year follow-up study on the relationship between bisphosphonates and the risk of stroke. Osteoporosis International. 2012 Oct;23(10):2551-7. PMID: 22270858. No outcomes of interest.

576. Kang J, H. Keller, J, J. Lin, H, C. Bisphosphonates reduced the risk of acute myocardial infarction: a 2-year follow-up study. Osteoporosis International. 2013 Jan;24(1):271-7. PMID: 23152093. Ineligible study design.

577. Kanis J, A. Johnell, O, Black, D, M. Downs, R, W. Jr, Sarkar, S, Fuerst, T, Secrest, R, J. Pavo, I,. Effect of raloxifene on the risk of new vertebral fracture in postmenopausal women with osteopenia or osteoporosis: a reanalysis of the Multiple Outcomes of Raloxifene Evaluation trial. Bone. 2003 Sep;33(3):293-300. PMID: 13678769. Ineligible treatment duration.

578. Kanis J, A. Barton, I, P. Johnell, O, Risedronate decreases fracture risk in patients selected solely on the basis of prior vertebral fracture. Osteoporosis International. 2005 May;16(5):475-82. PMID: 15875093. Ineligible treatment duration.

579. Kanis J, A. Johansson, H, Oden, A, McCloskey, E, V. Bazedoxifene reduces vertebral and clinical fractures in postmenopausal women at high risk assessed with FRAX. Bone. 2009 Jun;44(6):1049-54. PMID: 19254788. No interventions of interest.

580. Karim R, Dell, R, M. Greene, D, F. Mack, W, J. Gallagher, J, C. Hodis, H, N. Hip fracture in postmenopausal women after cessation of hormone therapy: results from a prospective study in a large health management organization. Menopause. 2011 Nov;18(11):1172-7. PMID: 21775911. Ineligible population.

581. Karras D, Stoykov, I, Lems, W, F. Langdahl, B, L. Ljunggren, O, Barrett, A, Walsh, J, B. Fahrleitner, Pammer A, Rajzbaum, G, Jakob, F, Marin, F,. Effectiveness of teriparatide in postmenopausal women with osteoporosis and glucocorticoid use: 3-year results from the EFOS study. Journal of Rheumatology. 2012 Mar;39(3):600-9. PMID: 22247365. Ineligible study design.

582. Kasukawa Y, Miyakoshi, N, Ebina, T, Aizawa, T, Hongo, M, Nozaka, K, Ishikawa, Y, Saito, H, Chida, S, Shimada, Y,. Effects of risedronate alone or combined with vitamin K2 on serum undercarboxylated osteocalcin and osteocalcin levels in postmenopausal osteoporosis. Journal of Bone \& Mineral Metabolism. 2014 May;32(3):290-7. PMID: 23846118. No outcomes of interest.

583. Kaufman J, M. Orwoll, E, Goemaere, S, San, Martin J, Hossain, A, Dalsky, G, P. 
Lindsay, R, Mitlak, B, H. Teriparatide effects on vertebral fractures and bone mineral density in men with osteoporosis: treatment and discontinuation of therapy. Osteoporosis International. 2005 May;16(5):510-6. PMID: 15322742. Ineligible study design.

584. Kaufman J, M. Palacios, S, Silverman, S, Sutradhar, S, Chines, A,. An evaluation of the Fracture Risk Assessment Tool (FRAX) as an indicator of treatment efficacy: the effects of bazedoxifene and raloxifene on vertebral, nonvertebral, and all clinical fractures as a function of baseline fracture risk assessed by FRAX. Osteoporosis International. 2013 Oct;24(10):2561-9. PMID: 23595562. Ineligible treatment duration.

585. Kawate H, Takayanagi, R,. Efficacy and safety of bazedoxifene for postmenopausal osteoporosis. Clinical Interventions In Aging. 2011;6:151-60. PMID: 21753870. Ineligible study design.

586. Kayan K, Johansson, H, Oden, A, Vasireddy, S, Pande, K, Orgee, J, Kanis, J, A. McCloskey, E, V. Can fall risk be incorporated into fracture risk assessment algorithms: a pilot study of responsiveness to clodronate. Osteoporosis International. 2009 Dec;20(12):2055-61. PMID: 19436939. No interventions of interest.

587. Keaveny T, M. McClung, M, R. Wan, X, Kopperdahl, D, L. Mitlak, B, H. Krohn, $\mathrm{K}$,. Femoral strength in osteoporotic women treated with teriparatide or alendronate. Bone. 2012 Jan;50(1):165-70. PMID: 22015818. No outcomes of interest.

588. Keaveny T, M. McClung, M, R. Genant, H, K. Zanchetta, J, R. Kendler, D, Brown, J, P. Goemaere, S, Recknor, C, Brandi, M, L. Eastell, R, Kopperdahl, D, L. Engelke, K, Fuerst, T, Radcliffe, H, S. Libanati, C,. Femoral and vertebral strength improvements in postmenopausal women with osteoporosis treated with denosumab. Journal of Bone \& Mineral Research. 2014 Jan;29(1):158-65. PMID: 23794225. No outcomes of interest.
589. Keaveny T, M. Crittenden, D, B. Bolognese, M, A. Genant, H, K. Engelke, K, Oliveri, B, Brown, J, P. Langdahl, B, L. Yan, C, Grauer, A, Libanati, C, Greater Gains in Spine and Hip Strength for Romosozumab Compared With Teriparatide in Postmenopausal Women With Low Bone Mass. Journal of Bone and Mineral Research. 2017 September;32(9):1956-62. PMID: 617013862. No outcomes of interest.

590. Keech C, A. Sashegyi, A, Barrett, Connor E,. Year-by-year analysis of cardiovascular events in the Multiple Outcomes of Raloxifene Evaluation (MORE) trial. Current Medical Research \& Opinion. 2005 Jan;21(1):135-40. PMID: 15881485. No outcomes of interest.

591. Keegan T, H. Schwartz, A, V. Bauer, D, C. Sellmeyer, D, E. Kelsey, J, L. fracture, intervention trial,. Effect of alendronate on bone mineral density and biochemical markers of bone turnover in type 2 diabetic women: the fracture intervention trial. Diabetes Care. 2004 Jul;27(7):1547-53. PMID: 15220226. Ineligible treatment duration.

592. Kendler D, L. Ringe, J, D. Ste, Marie L, G. Vrijens, B, Taylor, E, B. Delmas, P, D. Risedronate dosing before breakfast compared with dosing later in the day in women with postmenopausal osteoporosis. Osteoporosis International. 2009

Nov;20(11):1895-902. PMID: 19296144. Ineligible study design.

593. Kendler D, L. Roux, C, Benhamou, C, L. Brown, J, P. Lillestol, M, Siddhanti, S, Man, H, S. San, Martin J, Bone, H, G. Effects of denosumab on bone mineral density and bone turnover in postmenopausal women transitioning from alendronate therapy. Journal of Bone \& Mineral Research. 2010 Jan;25(1):72-81. PMID: 19594293. No outcomes of interest.

594. Kendler D, L. McClung, M, R. Freemantle, N, Lillestol, M, Moffett, A, H. Borenstein, J, Satram, Hoang S, Yang, Y, C. Kaur, P, Macarios, D, Siddhanti, $\mathrm{S}$, Daps, Investigators. Adherence, preference, and satisfaction of postmenopausal women taking denosumab 
or alendronate. Osteoporosis International. 2011 Jun;22(6):1725-35. PMID: 20827547. No outcomes of interest.

595. Kendler D, L. Palacios, S, Cox, D, A. Stock, J, Alam, J, Dowsett, S, A.

Zanchetta, J,. Arzoxifene versus raloxifene: effect on bone and safety parameters in postmenopausal women with osteoporosis. Osteoporosis International. 2012 Mar;23(3):1091-101. PMID: 21374068. No outcomes of interest.

596. Kendler D, L. Marin, F, Zerbini, C. A, F. Russo, L, A. Greenspan, S, L. Zikan, V, Bagur, A, Malouf, Sierra J, Lakatos, P, Fahrleitner, Pammer A, Lespessailles, E, Minisola, S, Body, J, J. Geusens, P, Moricke, R, Lopez, Romero P,. Effects of teriparatide and risedronate on new fractures in post-menopausal women with severe osteoporosis (VERO): a multicentre, doubleblind, double-dummy, randomised controlled trial. Lancet. 2017 Nov 09;09:09. PMID: 29129436. No outcomes of interest.

597. Kerschan SK, Haschka, J, Obermayer, Pietsch B, Gasser, R, W. Dimai, H, P. Fahrleitner, Pammer A, Dobnig, H, Roschger, P, Preisinger, E, Klaushofer, K, Resch, H, Pietschmann, P,. How long should women with postmenopausal osteoporosis be treated with a bisphosphonate? Hormone and Metabolic Research. 2013;45(9):621-8. PMID: 52630294. Ineligible study design.

598. Khalili H, Huang, E, S. Ogino, S, Fuchs, C, S. Chan, A, T. A prospective study of bisphosphonate use and risk of colorectal cancer. Journal of Clinical Oncology. 2012 Sep 10;30(26):3229-33. PMID: 22649131. Ineligible treatment duration.

599. Khan A, Dubois, S, Khan, A, A. Rahman, M, Z. Khan, O, A. Syed, H, T. Derzko, C,. A randomized, double-blind, placebocontrolled study to evaluate the effects of alendronate on bone mineral density and bone remodelling in perimenopausal women with low bone mineral density. Journal of Obstetrics \& Gynaecology Canada: JOGC. 2014 Nov;36(11):976-82. PMID: 25574674. Ineligible population.
600. Kharazmi M, Hallberg, P, Schilcher, J, Aspenberg, P, Michaelsson, K,. Mortality After Atypical Femoral Fractures: A Cohort Study. Journal of Bone \& Mineral Research. 2016 Mar;31(3):491-7. PMID: 26676878. Ineligible treatment duration.

601. Khosla S. Update on estrogens and the skeleton. Journal of Clinical Endocrinology \& Metabolism. 2010 Aug;95(8):3569-77. PMID: 20685883. Ineligible study design.

602. Khosla S, Bilezikian, J, P. Dempster, D, W. Lewiecki, E, M. Miller, P, D. Neer, R, M. Recker, R, R. Shane, E, Shoback, D, Potts, J, T. Benefits and risks of bisphosphonate therapy for osteoporosis. Journal of Clinical Endocrinology and Metabolism. 2012 July;97(7):2272-82. PMID: 365192552. Ineligible study design.

603. Kika G, Izumi, S, Mori, A, Murano, T, Suzuki, T, Cai, L, Y. Nakamura, E, Goya, K, Maeda, H, Uchida, N, Shida, $\mathrm{M}$,. Beneficial aspect of oral estriol as hormone replacement therapy: consideration on bone and lipid metabolism. Tokai Journal of Experimental \& Clinical Medicine. 2009 Sep 20;34(3):92-8. PMID: 21319007. Ineligible treatment duration.

604. Kim D, Sung, Y, K. Cho, S, K. Han, M, Kim, Y, S. Factors associated with atypical femoral fracture. Rheumatology International. 2016 Jan;36(1):65-71. PMID: 26202894. Ineligible study design.

605. Kim S, W. Park, D, J. Park, K, S. Kim, S, Y. Cho, B, Y. Lee, H, K. Shin, C, S. Early changes in biochemical markers of bone turnover predict bone mineral density response to antiresorptive therapy in Korean postmenopausal women with osteoporosis. Endocrine Journal. 2005 Dec;52(6):667-74. PMID: 16410657. Ineligible study design.

606. Kim S, J. La, H, O. Kang, Y, S. The change of bone mineral density by bisphosphonates therapy with calciumantagonists in osteoporosis. Biomolecules and Therapeutics. 2008 June;16(2):141-6. PMID: 355189292. Ineligible study design. 
607. Kincse G, Varga, J, Somogyi, P, Szodoray, P, Suranyi, P, Gaal, J,. The impact of secondary hyperparathyroidism on the efficacy of antiresorptive therapy. BMC Musculoskeletal Disorders. 2012 Dec 10;13:244. PMID: 23227959. Ineligible study design.

608. Kinov P, Boyanov, M,. Clinical utility of risedronate in postmenopausal osteoporosis: Patient considerations with delayed-release formulation. International Journal of Women's Health. 2012;4(1):167-74. PMID: 365290683. Ineligible study design.

609. Kishimoto H, Fukunaga, M, Kushida, K, Shiraki, M, Itabashi, A, Nawata, H, Nakamura, T, Ohta, H, Takaoka, K, Ohashi, Y, Risedronate, Phase I. I. I. Research Group. Efficacy and tolerability of once-weekly administration of $17.5 \mathrm{mg}$ risedronate in Japanese patients with involutional osteoporosis: a comparison with 2.5-mg once-daily dosage regimen. Journal of Bone \& Mineral Metabolism. 2006;24(5):405-13. PMID: 16937274. Ineligible treatment duration.

610. Koh J, M. Chung, D, J. Chung, Y, S. Kang, M, I. Kim, I, J. Min, Y, K. Oh, H, J. Park, I, H. Lee, Y, S. Kravitz, B, Waterhouse, B, Nino, A, Fitzpatrick, L, A. Assessment of Denosumab in Korean Postmenopausal Women with Osteoporosis: Randomized, Double-Blind, PlaceboControlled Trial with Open-Label Extension. Yonsei Medical Journal. 2016 Jul;57(4):905-14. PMID: 27189284. Ineligible treatment duration.

611. Komm B, S. Chines, A, A. Bazedoxifene: the evolving role of third-generation selective estrogen-receptor modulators in the management of postmenopausal osteoporosis. Therapeutic Advances in Musculoskeletal Disease. 2012 Feb;4(1):2134. PMID: 22870492. Ineligible study design.

612. Komm B, S. Mirkin, S, Jenkins, S, N. Development of conjugated estrogens/bazedoxifene, the first tissue selective estrogen complex (TSEC) for management of menopausal hot flashes and postmenopausal bone loss. Steroids. 2014
Nov;90:71-81. PMID: 24929044. Ineligible study design.

613. Komulainen K, Tuppurainen, Mt, Heikkinen, Am, Honkanen, Saarikoski,. Identification of early postmenopausal women with no bone response to HRT: results of a five-year clinical trial. Osteoporosis international. 2000;11(3):2118. PMID: CN-00278081. Ineligible population.

614. Komulainen M, H. Kroger, H, Tuppurainen, M, T. Heikkinen, A, M. Alhava, E, Honkanen, R, Saarikoski, S,. HRT and Vit D in prevention of nonvertebral fractures in postmenopausal women a 5 year randomized trial.[Reprint in Maturitas. 2008 Sep-Oct 61(1-2):85-94 PMID: 19434882]. Maturitas. 1998 Nov 30;31(1):45-54. PMID: 10091204. Ineligible population.

615. Komulainen M, Kröger, Tuppurainen, Mt, Heikkinen, Am, Alhava, Honkanen, Saarikoski,. HRT and Vit D in prevention of non-vertebral fractures in postmenopausal women a 5 year randomized trial. Maturitas. 1998;31(1):45-54. PMID: CN00161089. Ineligible population.

616. Komulainen T, Mt, Kröger, Heikkinen, Am, Puntila, Alhava, Honkanen, Saarikoski,. Vitamin D and HRT: no benefit additional to that of HRT alone in prevention of bone loss in early postmenopausal women. A 2.5-year randomized placebo-controlled study. Osteoporosis international. 1997;7(2):12632. PMID: $\mathrm{CN}-00140037$. Ineligible treatment duration.

617. Kondo N, Fukuhara T, Watanabe Y, et al. Bone formation parameters of the biopsied Ilium differ between subtrochanteric and diaphyseal atypical femoral fractures in bisphosphonate-treated patients. Tohoku Journal of Experimental Medicine. 2017 December;243(4):247-54. PMID: 619950007. Ineligible study design.

618. Kosus A, Capar, M, Kosus, N,. Cyclical alendronate treatment in postmenopausal women with osteoporosis. International Journal of Gynaecology \& Obstetrics. 2005 
Nov;91(2):182-4. PMID: 16098978. No outcomes of interest.

619. Kraenzlin M, E. Meier, C,. Parathyroid hormone analogues in the treatment of osteoporosis. Nature Reviews

Endocrinology. 2011 Jul 12;7(11):647-56. PMID: 21750510. Ineligible study design.

620. Krege J, H. Wan, X,. Teriparatide and the risk of nonvertebral fractures in women with postmenopausal osteoporosis. Bone. 2012 Jan;50(1):161-4. PMID: 22036910. No outcomes of interest.

621. Kristensen S, Abrahamsen, Madsen, Js, Gram, Rejnmark, Rud, Jespersen,. Venous thrombosis is not increased in younger women on genuine oestrogen postmenopausal hormonal replacement therapy: results from the Danish Osteoporosis Prevention Study (DOPS). Thrombosis and haemostasis. 2006;95(5):915-6. PMID: CN-00565072. Ineligible population.

622. Kumagai H, Padhi,. A randomized, doubleblind, placebo-controlled, single-dose study to evaluate the safety, tolerability, pharmacokinetics and pharmacodynamics of denosumab administered subcutaneously to postmenopausal Japanese women. Bone. 2011;49(5):1101-7. PMID: CN-00843351. Ineligible treatment duration.

623. Kung A, W. Yeung, S, S. Chu, L, W. The efficacy and tolerability of alendronate in postmenopausal osteoporotic Chinese women: a randomized placebo-controlled study. Calcified Tissue International. 2000 Oct;67(4):286-90. PMID: 11000341. No outcomes of interest.

624. Kurland E, S. Cosman, F, McMahon, D, J. Rosen, C, J. Lindsay, R, Bilezikian, J, P. Parathyroid hormone as a therapy for idiopathic osteoporosis in men: effects on bone mineral density and bone markers. Journal of Clinical Endocrinology \& Metabolism. 2000 Sep;85(9):3069-76. PMID: 10999788. No outcomes of interest.

625. Kuroda T, Shiraki, M, Shiraki, Y, Tanaka, $\mathrm{S}$,. The importance of absolute bone mineral density in the assessment of antiresorptive agents used for the prevention of osteoporotic fractures. Journal of Clinical Densitometry. 2012 Oct-Dec;15(4):392-8. PMID: 22521539. No interventions of interest.

626. Kushida K, Shiraki, M, Nakamura, T, Kishimoto, H, Morii, H, Yamamoto, K, Kaneda, K, Fukunaga, M, Inoue, T, Nakashima, M, Orimo, H,. The efficacy of alendronate in reducing the risk for vertebral fracture in Japanese patients with osteoporosis: A randomized, double-blind, active-controlled, double-dummy trial. Current Therapeutic Research - Clinical and Experimental. 2002 September;63(9):60620. PMID: 35178341. Ineligible treatment duration.

627. Kushida K, Fukunaga, M, Kishimoto, H, Shiraki, M, Itabashi, A, Inoue, T, Kaneda, K, Morii, H, Nawata, H, Yamamoto, K, Ohashi, Y, Orimo, H,. A comparison of incidences of vertebral fracture in Japanese patients with involutional osteoporosis treated with risedronate and etidronate: a randomized, double-masked trial. Journal of Bone \& Mineral Metabolism. 2004;22(5):469-78. PMID: 15316868. No outcomes of interest.

628. Kushida K, Shiraki, M, Nakamura, T, Kishimoto, H, Morii, H, Yamamoto, K, Kaneda, K, Fukunaga, M, Inoue, T, Nakashima, M, Orimo, H,. Alendronate reduced vertebral fracture risk in postmenopausal Japanese women with osteoporosis: a 3-year follow-up study.[Erratum appears in J Bone Miner Metab. 2005 23(1):95]. Journal of Bone \& Mineral Metabolism. 2004;22(5):462-8. PMID: 15316867. No outcomes of interest.

629. Kwon J, W. Park, E, J. Jung, S, Y. Sohn, H, S. Ryu, H, Suh, H, S. A Large National Cohort Study of the Association between Bisphosphonates and Osteonecrosis of the Jaw in Patients with Osteoporosis: A Nested Case-control Study.[Erratum appears in J Dent Res. 2016 Jan 95(1):121 PMID: 26446937]. Journal of Dental Research. 2015 Sep;94(9 Suppl):212S-9S. PMID: 26001708. Ineligible treatment duration. 
630. LaFleur J, DuVall, S, L. Willson, T, Ginter, T, Patterson, O, Cheng, Y, Knippenberg, K, Haroldsen, C, Adler, R, A. Curtis, J, R. Agodoa, I, Nelson, R, E. Analysis of osteoporosis treatment patterns with bisphosphonates and outcomes among postmenopausal veterans. Bone. 2015 Sep;78:174-85. PMID: 25896952. Ineligible study design.

631. Lamberg A, L. Horvath, Puho E, Christensen, S, Sorensen, H, T. Use of oral bisphosphonates and risk of venous thromboembolism: a population-based casecontrol study. Osteoporosis International. 2010 Nov;21(11):1911-7. PMID: 19997902. Ineligible study design.

632. Landfeldt E, Lang, A, Robbins, S, Strom, O,. Gastrointestinal tolerability and patterns of switching in patients treated for primary osteoporosis: the Swedish Adherence Register Analysis (SARA). Calcified Tissue International. 2011 Sep;89(3):234-45. PMID: 21695544. No outcomes of interest.

633. Langdahl B, L. Teglbjaerg, C, S. Ho, P, R. Chapurlat, R, Czerwinski, E, Kendler, D, L. Reginster, J, Y. Kivitz, A, Lewiecki, E, M. Miller, P, D. Bolognese, M, A. McClung, M, R. Bone, H, G. Ljunggren, O, Abrahamsen, B, Gruntmanis, U, Yang, Y, C. Wagman, R, B. Mirza, F, Siddhanti, S, Orwoll, E,. A 24-month study evaluating the efficacy and safety of denosumab for the treatment of men with low bone mineral density: results from the ADAMO trial. Journal of Clinical Endocrinology \& Metabolism. 2015 Apr;100(4):1335-42. PMID: 25607608. Ineligible study design.

634. Langdahl B, L. Libanati, C, Crittenden, D, B. Bolognese, M, A. Brown, J, P. Daizadeh, N, S. Dokoupilova, E, Engelke, K, Finkelstein, J, S. Genant, H, K. Goemaere, S, Hyldstrup, L, Jodar, Gimeno E, Keaveny, T, M. Kendler, D, Lakatos, P, Maddox, J, Malouf, J, Massari, F, E. Molina, J, F. Ulla, M, R. Grauer, A,. Romosozumab (sclerostin monoclonal antibody) versus teriparatide in postmenopausal women with osteoporosis transitioning from oral bisphosphonate therapy: a randomised, open-label, phase 3 trial. The Lancet. 201730 September - 6 October;390(10102):1585-94. PMID: 617542976. No outcomes of interest.

635. Langenegger I, Q. Opazo, M, F. Garcia, A. M, Z. Therapeutic equivalence and adherence to treatment with ibandronate 150 $\mathrm{mg}$ and alendronate $70 \mathrm{mg}$ in postmenopausal women of concepcion city, Chile. Actualizaciones en Osteologia. 2011 September/December;7(3):175-83. Ineligible treatment duration.

636. Langer R, D. Landgren, B, M. Rymer, J, Helmond, F, A. Opal, Investigators. Effects of tibolone and continuous combined conjugated equine estrogen/medroxyprogesterone acetate on the endometrium and vaginal bleeding: results of the OPAL study. American Journal of Obstetrics \& Gynecology. 2006 Nov;195(5):1320-7. PMID: 16875644. Ineligible treatment duration.

637. Langsetmo L, A. Morin, S, Richards, J, B. Davison, K, S. Olszynski, W, P. Prior, J, C. Josse, R, Goltzman, D, CaMos, Research Group,. Effectiveness of antiresorptives for the prevention of nonvertebral low-trauma fractures in a population-based cohort of women. Osteoporosis International. 2009 Feb;20(2):283-90. PMID: 18581034. Ineligible study design.

638. Lanza F, Sahba, B, Schwartz, H, Winograd, S, Torosis, J, Quan, H, Reyes, R, Musliner, T, Daifotis, A, Leung, A,. The upper GI safety and tolerability of oral alendronate at a dose of 70 milligrams once weekly: a placebocontrolled endoscopy study. American Journal of Gastroenterology. 2002 Jan;97(1):58-64. PMID: 11808969. Ineligible treatment duration.

639. Lapi F, Cipriani, F, Caputi, A, P. Corrao, G, Vaccheri, A, Sturkenboom, M, C. Di, Bari M, Gregori, D, Carle, F, Staniscia, T, Vestri, A, Brandi, M, Fusco, V, Campisi, G, Mazzaglia, G, Bisphosphonates Efficacy-Safety Tradeoff, study group,. Assessing the risk of osteonecrosis of the jaw due to bisphosphonate therapy in the secondary 
prevention of osteoporotic fractures.

Osteoporosis International. 2013

Feb;24(2):697-705. PMID: 22618266.

Ineligible treatment duration.

640. Lau E, M. Woo, J, Chan, Y, H. Griffith, J,. Alendronate prevents bone loss in Chinese women with osteoporosis. Bone. 2000 Nov;27(5):677-80. PMID: 11062355. No outcomes of interest.

641. Leder B, Z. Tsai, J, N. Uihlein, A, V. Burnett, Bowie S, A. Zhu, Y, Foley, K, Lee, H, Neer, R, M. Two years of Denosumab and teriparatide administration in postmenopausal women with osteoporosis (The DATA Extension Study): a randomized controlled trial. Journal of Clinical Endocrinology \& Metabolism. 2014 May;99(5):1694-700. PMID: 24517156. No outcomes of interest.

642. Leder B, Z. O'Dea, L, S. Zanchetta, J, R. Kumar, P, Banks, K, McKay, K, Lyttle, C, R. Hattersley, G,. Effects of abaloparatide, a human parathyroid hormone-related peptide analog, on bone mineral density in postmenopausal women with osteoporosis. Journal of Clinical Endocrinology \& Metabolism. 2015 Feb;100(2):697-706. PMID: 25393645. Ineligible treatment duration.

643. Leder B, Z. Tsai, J, N. Jiang, L, A. Lee, $\mathrm{H}$,. Importance of prompt antiresorptive therapy in postmenopausal women discontinuing teriparatide or denosumab: The Denosumab and Teriparatide Follow-up study (DATA-Follow-up). Bone. 2017 May;98:54-8. PMID: 28286299. Ineligible study design.

644. Lee C, Suzuki, Jb,. CTX biochemical marker of bone metabolism. Is it a reliable predictor of bisphosphonate-associated osteonecrosis of the jaws after surgery? Part II: a prospective clinical study. Implant dentistry. 2010;19(1):29-38. PMID: CN00743084. Ineligible treatment duration.

645. Lee J, Youn, K, Choi, N, K. Lee, J, H. Kang, D, Song, H, J. Park, B, J. A population-based case-control study: proton pump inhibition and risk of hip fracture by use of bisphosphonate. Journal of
Gastroenterology. 2013 Sep;48(9):1016-22. PMID: 23307040. Ineligible study design.

646. Lee KJ, Yoo JJ, Oh KJ, et al. Surgical outcome of intramedullary nailing in patients with complete atypical femoral fracture: A multicenter retrospective study. Injury. 2017 Apr;48(4):941-5. PMID: 28274470. Ineligible study design.

647. Lee S, Yin, R, V. Hirpara, H, Lee, N, C. Lee, A, Llanos, S, Phung, O, J. Increased risk for atypical fractures associated with bisphosphonate use. Family Practice. 2015 Jun;32(3):276-81. PMID: 25846215. Ineligible study design.

648. Lee W, Y. Sun, L, M. Lin, M, C. Liang, J, A. Chang, S, N. Sung, F, C. Muo, C, H. Kao, C, H. A higher dosage of oral alendronate will increase the subsequent cancer risk of osteoporosis patients in Taiwan: a population-based cohort study. PLoS ONE [Electronic Resource]. 2012;7(12):e53032. PMID: 23300854. No outcomes of interest.

649. Lee Y, K. Ha, Y, C. Choi, H, J. Jang, S, Park, C, Lim, Y, T. Shin, C, S.

Bisphosphonate use and subsequent hip fracture in South Korea. Osteoporosis International. 2013 Nov;24(11):2887-92. PMID: 23681088. Ineligible study design.

650. Lee Y, K. Ha, Y, C. Park, C, Yoo, J, J. Shin, C, S. Koo, K, H. Bisphosphonate use and increased incidence of subtrochanteric fracture in South Korea: Results from the National Claim Registry. Osteoporosis International. 2013 February;24(2):707-11. PMID: 52024432. Ineligible study design.

651. Lee Y, K. Kim, H, Y. Kim, D, Y. Park, Hm, Ha, Y, C. Atypical subtrochanteric fractures in korean hip fracture study. Calcified tissue international. Conference: 44th annual european calcified tissue society congress, ECTS 2017. Austria. 2017;100(1 Supplement 1):S89-s90. PMID: CN01375488. Ineligible study design.

652. Lees B, Stevenson, J, C. The prevention of osteoporosis using sequential low-dose hormone replacement therapy with estradiol- 
17 beta and dydrogesterone. Osteoporosis International. 2001;12(4):251-8. PMID: 11420773. Ineligible treatment duration.

653. Lenart B, A. Neviaser, A, S. Lyman, S, Chang, C, C. Edobor, Osula F, Steele, B, van der, Meulen M, C. Lorich, D, G. Lane, J, M. Association of low-energy femoral fractures with prolonged bisphosphonate use: a case control study. Osteoporosis International. 2009 Aug;20(8):1353-62. PMID: 19066707. Ineligible study design.

654. Leo M, Morgante, Lanzetta, Setacci, Petraglia,. Randomized control study of the effects of raloxifene on serum lipids and homocysteine in older women. American journal of obstetrics and gynecology. 2001;184(3):350-3. PMID: CN-00328357. Ineligible treatment duration.

655. Leslie WD, Majumdar SR, Morin SN, et al. Change in Trabecular Bone Score (TBS) With Antiresorptive Therapy Does Not Predict Fracture in Women: The Manitoba BMD Cohort. Journal of Bone \& Mineral Research. 2017 Mar;32(3):618-23. PMID: 27933656. Ineligible study design.

656. Leung J, Y. Ho, A, Y. Ip, T, P. Lee, G, Kung, A, W. The efficacy and tolerability of risedronate on bone mineral density and bone turnover markers in osteoporotic Chinese women: a randomized placebocontrolled study. Bone. 2005 Feb;36(2):35864. PMID: 15780963. No outcomes of interest.

657. Leung K, Y. Lee, T, K. Lee, C, N. Sum, T, K. Chan, M. Y, M. Tong, C, M. The effects of different dosages of oestrogen on the bone mineral density of postmenopausal Hong Kong Chinese women: Randomised controlled trial. Hong Kong Medical Journal. 1999;5(1):9-14. PMID: 29318651. Ineligible population.

658. Levin VA, Jiang X, Kagan R. Estrogen therapy for osteoporosis in the modern era. Osteoporosis International. 201801 May;29(5):1049-55. PMID: 621191901. Ineligible study design.
659. Levine J, P. Zoledronic acid reduces osteoporotic fractures and ensures medication compliance for 1 year. Postgraduate Medicine. 2010 May;122(3):248-50. PMID: 361557323. Ineligible study design.

660. Levis S, Quandt, S, A. Thompson, D, Scott, J, Schneider, D, L. Ross, P, D. Black, D, Suryawanshi, S, Hochberg, M, Yates, J,. Alendronate reduces the risk of multiple symptomatic fractures: Results from the Fracture Intervention Trial. Journal of the American Geriatrics Society. 2002;50(3):409-15. PMID: 34229393. Ineligible treatment duration.

661. Lewiecki E, M. Denosumab: a promising drug for the prevention and treatment of osteoporosis. Women's health. 2006 Jul;2(4):517-25. PMID: 19803959. Ineligible study design.

662. Lewiecki E, M. Miller, P, D. McClung, M, R. Cohen, S, B. Bolognese, M, A. Liu, Y, Wang, A, Siddhanti, S, Fitzpatrick, L, A. A. M. G. Bone Loss Study Group. Two-year treatment with denosumab (AMG 162) in a randomized phase 2 study of postmenopausal women with low BMD. Journal of Bone \& Mineral Research. 2007 Dec;22(12):1832-41. PMID: 17708711. No outcomes of interest.

663. Lewiecki E, M. Zoledronic acid in the management of osteoporosis: The HORIZON trials. Therapy. 2008;5(6):82935. PMID: 355218907. Ineligible study design.

664. Lewiecki E, M. Denosumab for the treatment of postmenopausal osteoporosis. Women's health. 2009 Jan;5(1):15-22. PMID: 19274891. Ineligible study design.

665. Lewiecki E, M. Keaveny, T, M. Kopperdahl, D, L. Genant, H, K. Engelke, K, Fuerst, T, Kivitz, A, Davies, R, Y. Fitzpatrick, L, A. Once-monthly oral ibandronate improves biomechanical determinants of bone strength in women with postmenopausal osteoporosis. Journal of Clinical Endocrinology \& Metabolism. 2009 Jan;94(1):171-80. PMID: 18840641. No outcomes of interest. 
666. Lewiecki E, M. Bisphosphonates for the treatment of osteoporosis: insights for clinicians. Therapeutic Advances in Chronic Disease. 2010 May;1(3):115-28. PMID: 23251734. Ineligible study design.

667. Lewiecki E, M. Intravenous zoledronic acid for the treatment of osteoporosis: The evidence of its therapeutic effect. Core Evidence. 2010 Jun 15;4:13-23. PMID: 20694061. Ineligible study design.

668. Lewiecki E, M. Cooper, C, Thompson, E, Hartl, F, Mehta, D, Papapoulos, S, E. Ibandronate does not increase risk of atrial fibrillation in analysis of pivotal clinical trials. International Journal of Clinical Practice. 2010 May;64(6):821-6. PMID: 20337751. Ineligible treatment duration.

669. Lewiecki E, M. Clinical use of denosumab for the treatment for postmenopausal osteoporosis. Current Medical Research \& Opinion. 2010 Dec;26(12):2807-12. PMID: 21050058. Ineligible study design.

670. Lewiecki E, M. Treatment of osteoporosis with denosumab. Maturitas. 2010 Jun;66(2):182-6. PMID: 20236778. Ineligible study design.

671. Lewiecki E, M. Denosumab--an emerging treatment for postmenopausal osteoporosis. Expert Opinion on Biological Therapy. 2010 Mar;10(3):467-76. PMID: 20095877. Ineligible study design.

672. Lewiecki E, M. Safety and tolerability of denosumab for the treatment of postmenopausal osteoporosis. Drug Healthcare \& Patient Safety. 2011;3:79-91. PMID: 22279412. Ineligible study design.

673. Lewiecki E, M. Safety of long-term bisphosphonate therapy for the management of osteoporosis. Drugs. $2011 \mathrm{Apr}$ 16;71(6):791-814. PMID: 21504254. Ineligible study design.

674. Li C, Wang, H, R. Li, X, L. Zhou, X, G. Dong, J,. The relation between zoledronic acid infusion and interbody fusion in patients undergoing transforaminal lumbar interbody fusion surgery. Acta Neurochirurgica. 2012 Apr;154(4):731-8. PMID: 22297398. Ineligible treatment duration.

675. Li I. Does alendronate treatment prevent vertebral fractures in men with osteoporosis? Journal of Family Practice. 2000 Dec;49(12):1077. PMID: 11132054. Ineligible study design.

676. Li M, Xing, X, P. Zhang, Z, L. Liu, J, L. Zhang, Z, L. Liu, D, G. Xia, W, B. Meng, X, W. Infusion of ibandronate once every 3 months effectively decreases bone resorption markers and increases bone mineral density in Chinese postmenopausal osteoporotic women: a 1-year study. Journal of Bone \& Mineral Metabolism. 2010 May;28(3):299-305. PMID: 19855926. No outcomes of interest.

677. Li M, Zhang, Z, L. Liao, E, Y. Chen, D, C. Liu, J, Tao, T, Z. Wu, W, Xia, W, B. Lu, Y, J. Sheng, Z, F. Lu, C, Y. Meng, G, L. Xu, L, Zhang, W, J. Hu, Y, Y. Xu, L,. Effect of low-dose alendronate treatment on bone mineral density and bone turnover markers in Chinese postmenopausal women with osteopenia and osteoporosis. Menopause. 2013 Jan;20(1):72-8. PMID: 22968256. No outcomes of interest.

678. Li Y, Zhang, Z, Deng, X, Chen, L,. Efficacy and safety of risedronate sodium in treatment of postmenopausal osteoporosis. Journal of Huazhong University of Science and Technology. Medical Sciences. 2005;25(5):527-9. PMID: 16463664. No outcomes of interest.

679. Li Y, Zhao, W, B. Wang, D, L. He, Q, Li, Q, Pei, F, X. Liu, L,. Treatment of osteoporotic intertrochanteric fractures by zoledronic acid injection combined with proximal femoral nail anti-rotation. Chinese Journal of Traumatology. 2016 Oct 01;19(5):259-63. PMID: 27780504. Ineligible treatment duration.

680. Liang B, C. Shi, Z, Y. Wang, B, Wu, P, Kong, L, C. Yao, J, L. Li, C, W. Shi, X, L. Intravenous Zoledronic Acid $5 \mathrm{mg}$ on Bone Turnover Markers and Bone Mineral Density in East China Subjects with Newly 
Diagnosed Osteoporosis: A 24-month Clinical Study. Orthopaedic Audio-Synopsis Continuing Medical Education [Sound Recording]. 2017 Feb;9(1):103-9. PMID: 28276638. No outcomes of interest.

681. Liang L, Zhang, Shen,. Evaluation of multidimensional outcomes of chronic diseases: a clinical example from China. Archives of gerontology and geriatrics. 2011;52(3):e106e9. PMID: CN-00888831. No interventions of interest.

682. Liberman U, A. Weiss, S, R. Broll, J, Minne, H, W. Quan, H, Bell, N, H. Rodriguez, Portales J, Downs, R, W. Jr, Dequeker, J, Favus, M,. Effect of oral alendronate on bone mineral density and the incidence of fractures in postmenopausal osteoporosis. The Alendronate Phase III Osteoporosis Treatment Study Group. New England Journal of Medicine. 1995 Nov 30;333(22):1437-43. PMID: 7477143. Ineligible treatment duration.

683. Liberman U, Hirsch, Lj,. Esophagitis and alendronate. New England journal of medicine. 1996;335(14):1069-70. PMID: CN-00129086. Ineligible study design.

684. Lim P, Ong, Fb, Adeeb, Seri, Ss, Noor, Aini My, Shamsuddin, Hapizah, Mohamed, Al, Mokhtar, Wan, Hwh,. Bone health in urban midlife Malaysian women: risk factors and prevention. Osteoporosis international. 2005;16(12):2069-79. PMID: CN00557817. Ineligible treatment duration.

685. Lim V, Clarke, B, L. New therapeutic targets for osteoporosis: beyond denosumab. Maturitas. 2012 Nov;73(3):269-72. PMID: 22925430. Ineligible study design.

686. Lin D, Kramer, J, R. Ramsey, D, Alsarraj, A, Verstovsek, G, Rugge, M, Parente, P, Graham, D, Y. El, Serag H, B. Oral bisphosphonates and the risk of Barrett's esophagus: case-control analysis of US veterans. American Journal of Gastroenterology. 2013 Oct;108(10):157683. PMID: 23857477. Ineligible study design.
687. Lin T, C. Yang, C, Y. Yang, Y, H. Lin, $\mathrm{S}$, J. Comparative effectiveness of osteoporosis drugs in preventing secondary nonvertebral fractures in Taiwanese women. Journal of Clinical Endocrinology \& Metabolism. 2013 Dec;98(12):4717-26. PMID: 24081731. Ineligible study design.

688. Lin T, C. Lee, C, H. Yang, C, Y. Yang, $\mathrm{Y}, \mathrm{H}$. Lin, S, J. Incidence and risk of venous thromboembolism among Taiwan osteoporotic fracture population under osteoporosis pharmacological treatments. Journal of Clinical Endocrinology \& Metabolism. 2014 May;99(5):1599-607. PMID: 24606074. Ineligible treatment duration.

689. Lin T, Y. Yang, S, C. Tsai, T, T. Lai, P, L. Fu, T, S. Niu, C, C. Chen, L, H. Chen, W, J. Correlation between zoledronic acid infusion and repeat vertebroplasty surgery in osteoporotic patients. Current Medical Research \& Opinion. 2016 May;32(5):921-7. PMID: 26854688. Ineligible study design.

690. Lindsay R, Nieves, J, Formica, C, Henneman, E, Woelfert, L, Shen, V, Dempster, D, Cosman, F,. Randomised controlled study of effect of parathyroid hormone on vertebral-bone mass and fracture incidence among postmenopausal women on oestrogen with osteoporosis. Lancet. 1997 Aug 23;350(9077):550-5. PMID: 9284777. No outcomes of interest.

691. Lindsay R, Cosman, F, Lobo, R, A. Walsh, B, W. Harris, S, T. Reagan, J, E. Liss, C, L. Melton, M, E. Byrnes, C, A. Addition of alendronate to ongoing hormone replacement therapy in the treatment of osteoporosis: a randomized, controlled clinical trial. Journal of Clinical Endocrinology \& Metabolism. 1999 Sep;84(9):3076-81. PMID: 10487668. No outcomes of interest.

692. Lindsay R, Gallagher, J, C. Kleerekoper, M, Pickar, J, H. Effect of lower doses of conjugated equine estrogens with and without medroxyprogesterone acetate on bone in early postmenopausal women. JAMA. 2002 May 22-29;287(20):2668-76. 
PMID: 12020302. Ineligible treatment duration.

693. Lindsay R, Scheele, W, H. Neer, R, Pohl, G, Adami, S, Mautalen, C, Reginster, J, Y. Stepan, J, J. Myers, S, L. Mitlak, B, $H$. Sustained vertebral fracture risk reduction after withdrawal of teriparatide in postmenopausal women with osteoporosis. Archives of Internal Medicine. 2004 Oct 11;164(18):2024-30. PMID: 15477438. Ineligible study design.

694. Lindsay R, Gallagher, J, C. Kleerekoper, $\mathrm{M}$, Pickar, J, H. Bone response to treatment with lower doses of conjugated estrogens with and without medroxyprogesterone acetate in early postmenopausal women. Osteoporosis International. 2005 Apr;16(4):372-9. PMID: 15654581. Ineligible treatment duration.

695. Lindsay R, Miller, P, Pohl, G, Glass, E, V. Chen, P, Krege, J, H. Relationship between duration of teriparatide therapy and clinical outcomes in postmenopausal women with osteoporosis. Osteoporosis International. 2009 Jun;20(6):943-8. PMID: 18923884. No outcomes of interest.

696. Lindsay R, Gallagher, J, C. Kagan, R, Pickar, J, H. Constantine, G,. Efficacy of tissue-selective estrogen complex of bazedoxifene/conjugated estrogens for osteoporosis prevention in at-risk postmenopausal women. Fertility \& Sterility. 2009 Sep;92(3):1045-52. PMID: 19635616. Ineligible treatment duration.

697. Lindsay R. Preventing osteoporosis with a tissue selective estrogen complex (TSEC) containing bazedoxifene/conjugated estrogens (BZA/CE). Osteoporosis International. 2011 Feb;22(2):447-51. PMID: 21069294. Ineligible study design.

698. Lindsay R, Watts, N, B. Lange, J, L. Delmas, P, D. Silverman, S, L. Effectiveness of risedronate and alendronate on nonvertebral fractures: an observational study through 2 years of therapy.[Erratum appears in Osteoporos Int. 2013 Aug 24(8):2375-6]. Osteoporosis International. 2013 Aug;24(8):2345-52. PMID: 23612793. Ineligible study design.
699. Lippman M, E. Krueger, K, A. Eckert, S, Sashegyi, A, Walls, E, L. Jamal, S, Cauley, J, A. Cummings, S, R. Indicators of lifetime estrogen exposure: effect on breast cancer incidence and interaction with raloxifene therapy in the multiple outcomes of raloxifene evaluation study participants.[Erratum appears in J Clin Oncol 2002 Mar 1 20(5):1430]. Journal of Clinical Oncology. 2001 Jun 15;19(12):3111-6. PMID: 11408508. No outcomes of interest.

700. Lippman M, E. Cummings, S, R. Disch, D, P. Mershon, J, L. Dowsett, S, A. Cauley, J, A. Martino, S,. Effect of raloxifene on the incidence of invasive breast cancer in postmenopausal women with osteoporosis categorized by breast cancer risk. Clinical Cancer Research. 2006 Sep 01;12(17):5242-7. PMID: 16951244. No outcomes of interest.

701. Liu B, Gan, F, Ge, Y, Yu, H,. Clinical Efficacy Analysis of Percutaneous Kyphoplasty Combined with Zoledronic Acid in the Treatment and Prevention of Osteoporotic Vertebral Compression Fractures. Journal of Investigative Surgery. 201722 Aug:1-6. PMID: 618079533. Ineligible study design.

702. Liu J, Muse, Kn,. The effects of progestins on bone density and bone metabolism in postmenopausal women: a randomized controlled trial. American journal of obstetrics and gynecology. 2005;192(4):1316-23 discussion 23-4. PMID: CN-00512139. No interventions of interest.

703. Lo J, C. Hui, R, L. Grimsrud, C, D. Chandra, M, Neugebauer, R, S. Gonzalez, J, R. Budayr, A, Lau, G, Ettinger, B,. The association of race/ethnicity and risk of atypical femur fracture among older women receiving oral bisphosphonate therapy. Bone. 2016 April 01;85:142-7. PMID: 608960022. Ineligible treatment duration.

704. Lobo R, A. Pinkerton, J, V. Gass, M. L, S. Dorin, M, H. Ronkin, S, Pickar, J, H. Constantine, G,. Evaluation of bazedoxifene/conjugated estrogens for the 
treatment of menopausal symptoms and effects on metabolic parameters and overall safety profile. Fertility and Sterility. 2009 September;92(3):1025-38. PMID: 50591769. Ineligible treatment duration.

705. Lofthouse M. Denosumab improves low BMD in postmenopausal women. Nature Clinical Practice Endocrinology and Metabolism. 200619 May;2(5):249. PMID: 43714924. Ineligible study design.

706. Lopez DL, Riancho, Zarrabeitia L, Riancho, J, A. Genetic and acquired factors influencing the effectiveness and toxicity of drug therapy in osteoporosis. Expert Opinion On Drug Metabolism \& Toxicology. 2016;12(4):389-98. PMID: 26891809. Ineligible study design.

707. Lu P, Y. Hsieh, C, F. Tsai, Y, W. Huang, $\mathrm{W}, \mathrm{F}$. Alendronate and raloxifene use related to cardiovascular diseases: differentiation by different dosing regimens of alendronate. Clinical Therapeutics. 2011 Sep;33(9):11739. PMID: 21849210. Ineligible treatment duration.

708. Luckey M, Kagan, R, Greenspan, S, Bone, H, Kiel, R, D. Simon, J, Sackarowitz, J, Palmisano, J, Chen, E, Petruschke, R, A. de, Papp A, E. Onceweekly alendronate $70 \mathrm{mg}$ and raloxifene 60 mg daily in the treatment of postmenopausal osteoporosis. Menopause. 2004 JulAug;11(4):405-15. PMID: 15243278. No outcomes of interest.

709. Lufkin E, G. Whitaker, M, D. Nickelsen, T, Argueta, R, Caplan, R, H. Knickerbocker, R, K. Riggs, B, L. Treatment of established postmenopausal osteoporosis with raloxifene: a randomized trial. Journal of Bone \& Mineral Research. 1998 Nov;13(11):1747-54. PMID: 9797484. Ineligible treatment duration.

710. Luisetto B, Zangari, Tizian, Scalella, Pozzuoli,. Effects of three therapeutic regimens on postmenopausal bone loss in oophorectomized women. Current therapeutic research - clinical and experimental. 1996;57(11):839-48. PMID: CN-00171776. Ineligible population.
711. Lydeking OB, Jensen Je, Setchell, Kd, Holm, Jensen. Soymilk or progesterone for prevention of bone loss--a 2 year randomized, placebo-controlled trial. European journal of nutrition. 2004;43(4):246-57. PMID: CN-00490744. No interventions of interest.

712. Lyles K, W. Colon, Emeric C, S. Magaziner, J, S. Adachi, J, D. Pieper, C, F. Mautalen, C, Hyldstrup, L, Recknor, C, Nordsletten, L, Moore, K, A.

Lavecchia, C, Zhang, J, Mesenbrink, P, Hodgson, P, K. Abrams, K, Orloff, J, J. Horowitz, Z, Eriksen, E, F. Boonen, S, Horizon Recurrent Fracture, Trial. Zoledronic acid and clinical fractures and mortality after hip fracture. New England Journal of Medicine. 200711 01;357(18):1799-809. PMID: 17878149. No outcomes of interest.

713. Ma Y, L. Zeng, Q, Donley, D, W. Ste, Marie L, G. Gallagher, J, C. Dalsky, G, P. Marcus, R, Eriksen, E, F. Teriparatide increases bone formation in modeling and remodeling osteons and enhances IGF-II immunoreactivity in postmenopausal women with osteoporosis. Journal of Bone \& Mineral Research. 2006 Jun;21(6):85564. PMID: 16753016. No outcomes of interest.

714. Ma Z, Li, Y, Zhou, M, Huang, K, Hu, $\mathrm{H}, \mathrm{Liu}, \mathrm{X}, \mathrm{Xu}, \mathrm{X},$. Predictors of Ibandronate Efficacy for the Management of Osteoporosis: A Meta-Regression Analysis. PLoS ONE [Electronic Resource]. 2016;11(3):e0150203. PMID: 26930292. Ineligible study design.

715. Mackey D, C. Black, D, M. Bauer, D, C. McCloskey, E, V. Eastell, R, Mesenbrink, P, Thompson, J, R. Cummings, S, R. Effects of antiresorptive treatment on nonvertebral fracture outcomes. Journal of Bone \& Mineral Research. 2011 Oct;26(10):2411-8. PMID: 21710615. Ineligible study design.

716. Magaziner J, S. Orwig, D, L. Lyles, K, W. Nordsletten, L, Boonen, S, Adachi, J, D. Recknor, C, Colon, Emeric C, S. Mesenbrink, P, Bucci, Rechtweg C, Su, 
G, Johnson, R, Pieper, C, F. Subgroup variations in bone mineral density response to zoledronic acid after hip fracture. Journal of Bone \& Mineral Research. 2014

Dec;29(12):2545-51. PMID: 24839241. No outcomes of interest.

717. Magnusson C, Baron, J, A. Correia, N, Bergstrom, R, Adami, H, O. Persson, I,. Breast-cancer risk following long-term oestrogen-and oestrogen- progestinreplacement therapy. International Journal of Cancer. 1999;81(3):339-44. PMID: 29165190. Ineligible study design.

718. Majima T, Komatsu, Y, Shimatsu, A, Satoh, N, Fukao, A, Ninomiya, K, Matsumura, T, Nakao, K,. Efficacy of combined treatment with raloxifene and alfacalcidol on bone density and biochemical markers of bone turnover in postmenopausal osteoporosis. Endocrine Journal. 2008 Mar;55(1):127-34. PMID: 18219181. No interventions of interest.

719. Majima T, Shimatsu, A, Komatsu, Y, Satoh, N, Fukao, A, Ninomiya, K, Matsumura, T, Nakao, K,. Effects of risedronate or alfacalcidol on bone mineral density, bone turnover, back pain, and fractures in Japanese men with primary osteoporosis: results of a two-year strict observational study. Journal of Bone \& Mineral Metabolism. 2009;27(2):168-74. PMID: 19183836. Ineligible study design.

720. Makras P, Hamdy, N, A. Zwinderman, A, H. Ballieux, B, E. Papapoulos, S, E. Bisphosphonate dose and incidence of fractures in postmenopausal osteoporosis. Bone. 2009 May;44(5):766-71. PMID: 19442613. Ineligible study design.

721. Malouf SJ, Tarantino, U, Garcia, Hernandez P, A. Corradini, C, Overgaard, S, Stepan, J, J. Borris, L, Lespessailles, E, Frihagen, F, Papavasiliou, K, Petto, H, Aspenberg, P, Caeiro, J, R. Marin, F,. Effect of Teriparatide or Risedronate in Elderly Patients With a Recent Pertrochanteric Hip Fracture: Final Results of a 78-Week Randomized Clinical Trial. Journal of Bone and Mineral Research. 2017 May;32(5):1040-51. PMID: 615860628. Ineligible treatment duration.
722. Mamdani M, Kopp, A, Hawker, G,. Hip fractures in users of first- vs. secondgeneration bisphosphonates. Osteoporosis International. 2007 Dec;18(12):1595-600. PMID: 17767369. No outcomes of interest.

723. Maraka S, Kennel, K, A. Bisphosphonates for the prevention and treatment of Osteoporosis. BMJ (Online). 201502 Sep;351 (no pagination)(h3783) PMID: 606944253. Ineligible study design.

724. Marc J, Prezelj, J, Komel, R, Kocijancic, A,. VDR genotype and response to etidronate therapy in late postmenopausal women. Osteoporosis International. 1999;10(4):303-6. PMID: 10692979. No interventions of interest.

725. Marcus R, Holloway, L, Wells, B, Greendale, G, James, M, K. Wasilauskas, C, Kelaghan, J,. The relationship of biochemical markers of bone turnover to bone density changes in postmenopausal women: results from the Postmenopausal Estrogen/Progestin Interventions (PEPI) trial. Journal of Bone \& Mineral Research. 1999 Sep;14(9):1583-95. PMID: 10469288. Ineligible treatment duration.

726. Marcus R, Wang, O, Satterwhite, J, Mitlak, B,. The skeletal response to teriparatide is largely independent of age, initial bone mineral density, and prevalent vertebral fractures in postmenopausal women with osteoporosis. Journal of Bone \& Mineral Research. 2003 Jan;18(1):18-23. PMID: 12510801. Ineligible treatment duration.

727. Maricic M, Adachi, J, D. Sarkar, S, Wu, W, Wong, M, Harper, K, D. Early effects of raloxifene on clinical vertebral fractures at 12 months in postmenopausal women with osteoporosis. Archives of Internal Medicine. 2002 May 27;162(10):1140-3. PMID: 12020184. Ineligible treatment duration.

728. Maricic M. New and emerging treatments for osteoporosis. Current Opinion in Rheumatology. 2007 Jul;19(4):364-9. PMID: 17551368. Ineligible study design. 
729. Maricic M. The role of zoledronic acid in the management of osteoporosis. Clinical Rheumatology. 2010 Oct;29(10):1079-84. PMID: 20544246. Ineligible study design.

730. Maricic M. Intravenous zoledronic acid: what are the indications for male osteoporosis? Current Osteoporosis Reports. 2010 Mar;8(1):4-9. PMID: 20425084. Ineligible study design.

731. Marjoribanks J, Farquhar, Cindy, Roberts, Helen, Lethaby, Anne, Lee, Jasmine,. Long-term hormone therapy for perimenopausal and postmenopausal women. Cochrane Database of Systematic Reviews. 2017(1) PMID: CD004143. Ineligible study design.

732. Martin K, E. Yu, J, Campbell, H, E. Abarca, J, White, T, J. Analysis of the comparative effectiveness of 3 oral bisphosphonates in a large managed care organization: adherence, fracture rates, and all-cause cost. Journal of Managed Care Pharmacy. 2011 Oct;17(8):596-609. PMID: 21942301. No outcomes of interest.

733. Martin ME, Huerta, Alvarez C, Prieto, Alhambra D, Alvarez, Gutierrez A, Montero, Corominas D,. Secular trends of use of anti-osteoporotic treatments in Spain: A population-based cohort study including over 1.5 million people and more than 12 years of follow-up. Bone. 2017 December;105:292-8. PMID: 618880923. Ineligible study design.

734. Martinez-Laguna D, Reyes C, CarbonellAbella C, et al. Use of drugs for osteoporosis treatment in patients with type 2 diabetes mellitus: Population-based cohort study. Revista de Osteoporosis y

Metabolismo Mineral. 201701

Dec;9(4):107-12. PMID: 620866198.

Ineligible study design.

735. Masud T, McClung, M, Geusens, P,. Reducing hip fracture risk with risedronate in elderly women with established osteoporosis. Clinical Interventions In Aging. 2009;4:445-9. PMID: 19966913. No outcomes of interest.
736. Maugeri D, Mamazza, C, Lo, Giudice F, Puglisi, N, Muscoso, E, G. Rizzotto, M, Testai, M, Bennati, E, Lentini, A, Panebianco, P,. Interleukin-18 (IL-18) and matrix metalloproteinase-9 (MMP-9) in post-menopausal osteoporosis. Archives of Gerontology \& Geriatrics. 2005 MayJun;40(3):299-305. PMID: 15814163. Ineligible study design.

737. Maughan K, L. Preventing osteoporotic fractures with alendronate. Journal of Family Practice. 1997 Apr;44(4):336. PMID: 9148031. Ineligible study design.

738. McCloskey E, V. Johansson, H, Oden, A, Vasireddy, S, Kayan, K, Pande, K, Jalava, T, Kanis, J, A. Ten-year fracture probability identifies women who will benefit from clodronate therapy--additional results from a double-blind, placebocontrolled randomised study. Osteoporosis International. 2009 May;20(5):811-7. PMID: 19002369. No interventions of interest.

739. McCloskey E, V. Johansson, H, Oden, A, Austin, M, Siris, E, Wang, A, Lewiecki, E, M. Lorenc, R, Libanati, C, Kanis, J, A. Denosumab reduces the risk of osteoporotic fractures in postmenopausal women, particularly in those with moderate to high fracture risk as assessed with FRAX. Journal of Bone \& Mineral Research. 2012 Jul;27(7):1480-6. PMID: 22431426. Ineligible treatment duration.

740. McCloskey E, V. Johansson, H, Oden, A, Harvey, N, C. Jiang, H, Modin, S, Fitzpatrick, L, Kanis, J, A. The Effect of Abaloparatide-SC on Fracture Risk Is Independent of Baseline FRAX Fracture Probability: A Post Hoc Analysis of the ACTIVE Study. Journal of Bone and Mineral Research. 2017 August;32(8):162531. PMID: 617001726. Ineligible treatment duration.

741. McClung M, Clemmesen, B, Daifotis, A, Gilchrist, N, L. Eisman, J, Weinstein, R, S. Fuleihan, G. el- H Reda, C, Yates, A, J. Ravn, P,. Alendronate prevents postmenopausal bone loss in women without osteoporosis. A double-blind, randomized, 
controlled trial. Alendronate Osteoporosis Prevention Study Group. Annals of Internal Medicine. 1998 Feb 15;128(4):253-61. PMID: 9471927. Ineligible treatment duration.

742. McClung M, R. Geusens, P, Miller, P, D. Zippel, H, Bensen, W, G. Roux, C, Adami, S, Fogelman, I, Diamond, T, Eastell, R, Meunier, P, J. Reginster, J, Y. Hip Intervention Program, Study Group,. Effect of risedronate on the risk of hip fracture in elderly women. Hip Intervention Program Study Group. New England Journal of Medicine. 2001 Feb 01;344(5):333-40. PMID: 11172164. Ineligible treatment duration.

743. McClung M, R. Wasnich, R, D. Hosking, D, J. Christiansen, C, Ravn, P, Wu, M, Mantz, A, M. Yates, J, Ross, P, D. Santora, A, C. nd, Early Postmenopausal Intervention, Cohort Study,. Prevention of postmenopausal bone loss: six-year results from the Early Postmenopausal Intervention Cohort Study. Journal of Clinical Endocrinology \& Metabolism. 2004 Oct;89(10):4879-85. PMID: 15472179. Ineligible population.

744. McClung M, R. San, Martin J, Miller, P, D. Civitelli, R, Bandeira, F, Omizo, M, Donley, D, W. Dalsky, G, P. Eriksen, E, F. Opposite bone remodeling effects of teriparatide and alendronate in increasing bone mass.[Erratum appears in Arch Intern Med. 2005 Oct 10 165(18):2120]. Archives of Internal Medicine. 2005 Aug 822;165(15):1762-8. PMID: 16087825. No outcomes of interest.

745. McClung M, R. Siris, E, Cummings, S, Bolognese, M, Ettinger, M, Moffett, A, Emkey, R, Day, W, Somayaji, V, Lee, A,. Prevention of bone loss in postmenopausal women treated with lasofoxifene compared with raloxifene. Menopause. 2006 May-Jun;13(3):377-86. PMID: 16735934. No outcomes of interest.

746. McClung M, R. Lewiecki, E, M. Cohen, S, B. Bolognese, M, A. Woodson, G, C. Moffett, A, H. Peacock, M, Miller, P, D. Lederman, S, N. Chesnut, C, H. Lain, D, Kivitz, A, J. Holloway, D, L. Zhang, C,
Peterson, M, C. Bekker, P, J. A. M. G. Bone Loss Study Group. Denosumab in postmenopausal women with low bone mineral density. New England Journal of Medicine. 2006 Feb 23;354(8):821-31. PMID: 16495394. No outcomes of interest.

747. McClung M, Recker, R, Miller, P, Fiske, D, Minkoff, J, Kriegman, A, Zhou, W, Adera, M, Davis, J,. Intravenous zoledronic acid $5 \mathrm{mg}$ in the treatment of postmenopausal women with low bone density previously treated with alendronate. Bone. 2007 Jul;41(1):122-8. PMID: 17468062. No outcomes of interest.

748. McClung M, R. Bolognese, M, A. Sedarati, F, Recker, R, R. Miller, P, D. Efficacy and safety of monthly oral ibandronate in the prevention of postmenopausal bone loss. Bone. 2009 Mar;44(3):418-22. PMID: 18950736. Ineligible treatment duration.

749. McClung M, R. Miller, P, D. Brown, J, P. Zanchetta, J, Bolognese, M, A. Benhamou, C, L. Balske, A, Burgio, D, E. Sarley, J, McCullough, L, K. Recker, R, R. Efficacy and safety of a novel delayedrelease risedronate $35 \mathrm{mg}$ once-a-week tablet. Osteoporosis International. 2012 Jan;23(1):267-76. PMID: 21947137. No outcomes of interest.

750. McClung M, R. Boonen, S, Torring, O, Roux, C, Rizzoli, R, Bone, H, G. Benhamou, C, L. Lems, W, F. Minisola, S, Halse, J, Hoeck, H, C. Eastell, R, Wang, A, Siddhanti, S, Cummings, S, R. Effect of denosumab treatment on the risk of fractures in subgroups of women with postmenopausal osteoporosis. Journal of Bone \& Mineral Research. 2012 Jan;27(1):211-8. PMID: 21976367. Ineligible treatment duration.

751. McClung M, R. Benhamou, C, L. Man, Z, Tlustochowicz, W, Zanchetta, J, R. Eusebio, R, Balske, A, M. Matzkin, E, Olszynski, W, P. Recker, R, Delmas, P, D. A novel monthly dosing regimen of risedronate for the treatment of postmenopausal osteoporosis: 2-year data. Calcified Tissue International. 2013 
Jan;92(1):59-67. PMID: 23150144.

Ineligible treatment duration.

752. McClung M, R. Balske, A, Burgio, D, E. Wenderoth, D, Recker, R, R. Treatment of postmenopausal osteoporosis with delayedrelease risedronate $35 \mathrm{mg}$ weekly for 2 years. Osteoporosis International. 2013 Jan;24(1):301-10. PMID: 23079690. No outcomes of interest.

753. McClung M, R. Zanchetta, J, R. Racewicz, A, Roux, C, Benhamou, C, L. Man, Z, Eusebio, R, A. Beary, J, F. Burgio, D, E. Matzkin, E, Boonen, S, Delmas, P,. Efficacy and safety of risedronate $150-\mathrm{mg}$ once a month in the treatment of postmenopausal osteoporosis: 2-year data. Osteoporosis International. 2013 Jan;24(1):293-9. PMID: 22752050. Ineligible treatment duration.

754. McClung M, R. Grauer, A, Boonen, S, Bolognese, M, A. Brown, J, P. Diez, Perez A, Langdahl, B, L. Reginster, J, Y. Zanchetta, J, R. Wasserman, S, M. Katz, L, Maddox, J, Yang, Y, C. Libanati, C, Bone, H, G. Romosozumab in postmenopausal women with low bone mineral density. New England Journal of Medicine. 2014 Jan 30;370(5):412-20. PMID: 24382002. Ineligible treatment duration.

755. McClung M. Using Osteoporosis Therapies in Combination. Current osteoporosis reports. 2017:1-10. PMID: CN-01396645. Ineligible study design.

756. McClung MR. Romosozumab for the treatment of osteoporosis. Osteoporosis and Sarcopenia. 2018 March;4(1):11-5. PMID: 2000608377. Ineligible study design.

757. McClung MR, Brown JP, Diez-Perez A, et al. Effects of 24 Months of Treatment With Romosozumab Followed by 12 Months of Denosumab or Placebo in Postmenopausal Women With Low Bone Mineral Density: A Randomized, Double-Blind, Phase 2, Parallel Group Study. Journal of Bone and Mineral Research. 2018 PMID: 622333399. No interventions of interest.
758. McClung MR, Williams GC, Hattersley G, et al. Geography of Fracture Incidence in Postmenopausal Women with Osteoporosis Treated with Abaloparatide.[Erratum appears in Calcif Tissue Int. 2018 Jan 31;:; PMID: 29383409]. Calcified Tissue International. 2018 Jun;102(6):627-33. PMID: 29285549. Ineligible treatment duration.

759. Mebane S. Effects of estrogen or estrogen/progestin regimens on heart disease risk factors in postmenopausal women: The postmenopausal estrogen/progestin interventions (PEPI) trial. Journal of the American Medical Association. 199518 Jan;273(3):199-208. PMID: 25024741. Ineligible treatment duration.

760. Mebane SI. Effects of hormone therapy on bone mineral density: Results from the postmenopausal estrogen/progestin interventions (PEPI) trial. Journal of the American Medical Association. 199606 Nov;276(17):1389-96. PMID: 26365844. Ineligible treatment duration.

761. Melamed M, L. Blackwell, T, Neugarten, J, Arnsten, J, H. Ensrud, K, E. Ishani, A, Cummings, S, R. Silbiger, S, R.

Raloxifene, a selective estrogen receptor modulator, is renoprotective: a post-hoc analysis. Kidney International. 2011 Jan;79(2):241-9. PMID: 20927038. No outcomes of interest.

762. Mellstrom D, D. Sorensen, O, H. Goemaere, S, Roux, C, Johnson, T, D. Chines, A, A. Seven years of treatment with risedronate in women with postmenopausal osteoporosis. Calcified Tissue International. 2004 Dec;75(6):462-8. PMID: 15455188. Ineligible study design.

763. Mersereau J, E. Khouri, C, Jaffe, R, B. The role of alendronate in mitigating perimenopausal bone loss: a double-blind randomized controlled pilot study. Fertility \& Sterility. 2010 Aug;94(3):1138-40. PMID: 20056199. Ineligible population.

764. Messori A, Fadda, V, Maratea, D, Trippoli, S, Marinai, C,. Anti-reabsorptive agents in women with osteoporosis: determining statistical equivalence 
according to evidence-based methods. Journal of Endocrinological Investigation. 2014 Aug;37(8):769-73. PMID: 25008458. Ineligible study design.

765. Meunier P, J. Arlot, M, Chavassieux, P, Yates, A, J. The effects of alendronate on bone turnover and bone quality. International Journal of Clinical Practice. Supplement. 1999 Apr;101:14-7. PMID: 12669736. Ineligible study design.

766. Meunier P, J. Vignot, E, Garnero, P, Confavreux, E, Paris, E, Liu, Leage S, Sarkar, S, Liu, T, Wong, M, Draper, M, $\mathrm{W}$. Treatment of postmenopausal women with osteoporosis or low bone density with raloxifene. Raloxifene Study Group.[Erratum appears in Osteoporos Int 1999 10(5):433]. Osteoporosis International. 1999;10(4):330-6. PMID: 10692984. Ineligible treatment duration.

767. Michaelsson K, Baron, J, A. Farahmand, B, Y. Ljunghall, S,. Use of low potency estrogens does not reduce the risk of hip fracture. Bone. 2002 Apr;30(4):613-8. PMID: 11934654. Ineligible study design.

768. Michalska D, Stepan, J, J. Basson, B, R. Pavo, I,. The effect of raloxifene after discontinuation of long-term alendronate treatment of postmenopausal osteoporosis. Journal of Clinical Endocrinology \& Metabolism. 2006 Mar;91(3):870-7. PMID: 16352692. No outcomes of interest.

769. Michalska D, Luchavova, M, Zikan, V, Raska, I, Jr, Kubena, A, A. Stepan, J, J. Effects of morning vs. evening teriparatide injection on bone mineral density and bone turnover markers in postmenopausal osteoporosis. Osteoporosis International. 2012 Dec;23(12):2885-91. PMID: 22426952. No outcomes of interest.

770. Michalská S, Jj, Basson, Br, Pavo,. The effect of raloxifene after discontinuation of long-term alendronate treatment of postmenopausal osteoporosis. Journal of clinical endocrinology and metabolism. 2006;91(3):870-7. PMID: CN-00561717. No outcomes of interest.
771. Migliore A, Broccoli, S, Massafra, U, Bizzi, E, Frediani, B,. Mixed-treatment comparison of anabolic (teriparatide and PTH 1-84) therapies in women with severe osteoporosis. Current Medical Research \& Opinion. 2012 Mar;28(3):467-73. PMID: 22256908. Ineligible study design.

772. Mikulec A, A. Kowalczyk, K, A. Pfitzinger, M, E. Harris, D, A. Jackson, L, E. Negative association between treated osteoporosis and benign paroxysmal positional vertigo in women. Journal of Laryngology \& Otology. 2010 Apr;124(4):374-6. PMID: 19930786. Ineligible study design.

773. Miller B, E. De, Souza M, J. Slade, K, Luciano, A, A. Sublingual administration of micronized estradiol and progesterone, with and without micronized testosterone: effect on biochemical markers of bone metabolism and bone mineral density. Menopause. 2000 Sep-Oct;7(5):318-26. PMID: 10993031. Ineligible treatment duration.

774. Miller P, D. Schnitzer, T, Emkey, R, Orwoll, E, Rosen, C, Ettinger, M, Vandormael, K, Daifotis, A,. Weekly oral alendronic acid in male osteoporosis. Clinical Drug Investigation. 2004;24(6):33341. No outcomes of interest.

775. Miller P, D. Roux, C, Boonen, S, Barton, I, P. Dunlap, L, E. Burgio, D, E. Safety and efficacy of risedronate in patients with age-related reduced renal function as estimated by the Cockcroft and Gault method: a pooled analysis of nine clinical trials. Journal of Bone \& Mineral Research. 2005 Dec;20(12):2105-15. PMID: 16294264. Ineligible treatment duration.

776. Miller P, D. Shergy, W, J. Body, J, J. Chen, P, Rohe, M, E. Krege, J, H. Longterm reduction of back pain risk in women with osteoporosis treated with teriparatide compared with alendronate. Journal of Rheumatology. 2005 Aug;32(8):1556-62. PMID: 16078334. No outcomes of interest.

777. Miller P, D. McClung, M, R. Macovei, L, Stakkestad, J, A. Luckey, M, Bonvoisin, B, Reginster, J, Y. Recker, R, R. Hughes, 
C, Lewiecki, E, M. Felsenberg, D, Delmas, P, D. Kendler, D, L. Bolognese, M, A. Mairon, N, Cooper, C,. Monthly oral ibandronate therapy in postmenopausal osteoporosis: 1-year results from the MOBILE study. Journal of Bone \& Mineral Research. 2005 Aug;20(8):1315-22. PMID: 16007327. Ineligible treatment duration.

778. Miller P, D. Schwartz, E, N. Chen, P, Misurski, D, A. Krege, J, H. Teriparatide in postmenopausal women with osteoporosis and mild or moderate renal impairment. Osteoporosis International. 2007 Jan;18(1):59-68. PMID: 17013567. Ineligible treatment duration.

779. Miller P, D. Bilezikian, J, P. Diaz, Curiel M, Chen, P, Marin, F, Krege, J, H. Wong, M, Marcus, R,. Occurrence of hypercalciuria in patients with osteoporosis treated with teriparatide. Journal of Clinical Endocrinology \& Metabolism. 2007 Sep;92(9):3535-41. PMID: 17609307. No outcomes of interest.

780. Miller P, D. Chines, A, A. Christiansen, C, Hoeck, H, C. Kendler, D, L. Lewiecki, E, M. Woodson, G, Levine, A, B. Constantine, G, Delmas, P, D. Effects of bazedoxifene on BMD and bone turnover in postmenopausal women: 2-yr results of a randomized, double-blind, placebo-, and active-controlled study.[Erratum appears in J Bone Miner Res. 2008 Jun 23(6):972]. Journal of Bone \& Mineral Research. 2008 Apr;23(4):525-35. PMID: 18072873. Ineligible treatment duration.

781. Miller P, D. Epstein, S, Sedarati, F, Reginster, J, Y. Once-monthly oral ibandronate compared with weekly oral alendronate in postmenopausal osteoporosis: results from the head-to-head MOTION study. Current Medical Research \& Opinion. 2008 Jan;24(1):207-13. PMID: 18042311. No outcomes of interest.

782. Miller P, D. Derman, R, J. What is the best balance of benefits and risks among antiresorptive therapies for postmenopausal osteoporosis? Osteoporosis International. 2010 Nov;21(11):1793-802. PMID: 20309524. Ineligible study design.
783. Miller P, D. Delmas, P, D. Huss, H, Patel, K, M. Schimmer, R, C. Adami, S, Recker, R, R. Increases in hip and spine bone mineral density are predictive for vertebral antifracture efficacy with ibandronate. Calcified Tissue International. 2010 Oct;87(4):305-13. PMID: 20737140. Ineligible treatment duration.

784. Miller P, D. A review of the efficacy and safety of denosumab in postmenopausal women with osteoporosis. Therapeutic Advances in Musculoskeletal Disease. 2011 Dec;3(6):271-82. PMID: 22870485. Ineligible study design.

785. Miller P, D. Ragi, Eis S, Mautalen, C, Ramirez, F, Jonkanski, I,. Effects of intravenous ibandronate injection on renal function in women with postmenopausal osteoporosis at high risk for renal disease-the DIVINE study. Bone. 2011 Dec;49(6):1317-22. PMID: 21945737. Ineligible treatment duration.

786. Miller P, D. The kidney and bisphosphonates. Bone. 2011 Jul;49(1):7781. PMID: 21232648. Ineligible study design.

787. Miller P, D. Jamal, S, A. Evenepoel, P, Eastell, R, Boonen, S,. Renal safety in patients treated with bisphosphonates for osteoporosis: a review. Journal of Bone \& Mineral Research. 2013 Oct;28(10):204959. PMID: 23907861. Ineligible study design.

788. Miller P, D. Recker, R, R. Harris, S, Silverman, S, Felsenberg, D, Reginster, J, Day, B, M. Barr, C, Masanauskaite, D,. Long-term fracture rates seen with continued ibandronate treatment: pooled analysis of DIVA and MOBILE long-term extension studies. Osteoporosis International. 2014 Jan;25(1):349-57. PMID: 24136103. Ineligible study design.

789. Miller P, D. Long-term extension trials to prove the efficacy and safety of bisphosphonates. Clinical Investigation. 2014 January;4(1):35-43. PMID: 370567863. Ineligible study design. 
790. Miller P, D. Pannacciulli, N, Brown, J, P. Czerwinski, E, Nedergaard, B, S.

Bolognese, M, A. Malouf, J, Bone, H, G. Reginster, J, Y. Singer, A, Wang, C, Wagman, R, B. Cummings, S, R. Denosumab or Zoledronic Acid in Postmenopausal Women With Osteoporosis Previously Treated With Oral Bisphosphonates. Journal of Clinical Endocrinology \& Metabolism. 2016 Aug;101(8):3163-70. PMID: 27270237. No outcomes of interest.

791. Miller P, D. Hattersley, G, Riis, B, J. Williams, G, C. Lau, E, Russo, L, A. Alexandersen, P, Zerbini, C, A. Hu, M, Y. Harris, A, G. Fitzpatrick, L, A. Cosman, F, Christiansen, C, Active Study, Investigators. Effect of Abaloparatide vs Placebo on New Vertebral Fractures in Postmenopausal Women With Osteoporosis: A Randomized Clinical Trial.[Erratum appears in JAMA. 2017 Jan 24 317(4):442 PMID: 28118431]. JAMA. 2016 Aug 16;316(7):722-33. PMID: 27533157. No outcomes of interest.

792. Milliken L, A. Wilhelmy, J, Martin, C, J. Finkenthal, N, Cussler, E, Metcalfe, L, Guido, T, A. Going, S, B. Lohman, T, G. Depressive symptoms and changes in body weight exert independent and site-specific effects on bone in postmenopausal women exercising for 1 year. Journals of Gerontology Series A-Biological Sciences \& Medical Sciences. 2006 May;61(5):488-94. PMID: 16720746. Ineligible treatment duration.

793. Min Y, K. Lee, D, Y. Choi, S, J. Kim, J, H. Choi, D, Yoon, B, K. Effects of adding alendronate to ongoing hormone therapy on bone mineral density in postmenopausal Korean women: a randomized, double-blind, placebo-controlled clinical trial. Menopause. 2013 Jul;20(7):761-6. PMID: 23403498. No outcomes of interest.

794. Minne A, Simões, Me, Obermayer, Pietsch Sigurðsson, Marín, Dalsky, Gp, Nickelsen,. Bone density after teriparatide in patients with or without prior antiresorptive treatment: one-year results from the EUROFORS study. Current medical research and opinion. 2008;24(11):3117-28.
PMID: CN-00786923. Ineligible study design.

795. Mirkin S, Pickar, J, H. Management of osteoporosis and menopausal symptoms: focus on bazedoxifene/conjugated estrogen combination. International Journal of Women's Health. 2013;5:465-75. PMID: 23966802. Ineligible study design.

796. Mirkin S, Komm, B, S. Pan, K, Chines, A, A. Effects of bazedoxifene/conjugated estrogens on endometrial safety and bone in postmenopausal women. Climacteric. 2013 Jun;16(3):338-46. PMID: 23038989. Ineligible treatment duration.

797. Mirkin S, Pickar, J, H. Selective estrogen receptor modulators (SERMs): a review of clinical data. Maturitas. 2015 Jan;80(1):527. PMID: 25466304. Ineligible study design.

798. Miyauchi A, Matsumoto, T, Sugimoto, T, Tsujimoto, M, Warner, M, R. Nakamura, $\mathrm{T}$,. Effects of teriparatide on bone mineral density and bone turnover markers in Japanese subjects with osteoporosis at high risk of fracture in a 24-month clinical study: 12-month, randomized, placebo-controlled, double-blind and 12-month open-label phases. Bone. 2010 Sep;47(3):493-502. PMID: 20580870. No outcomes of interest.

799. Mizunuma H, Okano, H, Soda, M, Kagami, I, Miyamoto, S, Tokizawa, T, Honjo, S, Ibuki, Y,. Prevention of postmenopausal bone loss with minimal uterine bleeding using low dose continuous estrogen/progestin therapy: a 2-year prospective study. Maturitas. 1997 May;27(1):69-76. PMID: 9158080. Ineligible treatment duration.

800. Mizunuma H, Shiraki, M, Shintani, M, Gorai, I, Makita, K, Itoga, S, Mochizuki, Y, Mogi, H, Iwaoki, Y, Kosha, S, Yasui, T, Ishihara, O, Kurabayashi, T, Kasuga, Y, Hayashi, K,. Randomized trial comparing low-dose hormone replacement therapy and HRT plus 1alpha-OH-vitamin D3 (alfacalcidol) for treatment of postmenopausal bone loss. Journal of Bone \& Mineral Metabolism. 2006;24(1):11-5. PMID: 16369892. No outcomes of interest. 
801. Moen M, D. Keam, S, J. Spotlight on denosumab in postmenopausal osteoporosis. Biodrugs. 2011 Aug 01;25(4):261-4. PMID: 21815702. Ineligible study design.

802. Moen M, D. Keam, S, J. Denosumab: a review of its use in the treatment of postmenopausal osteoporosis. Drugs \& Aging. 2011 Jan 01;28(1):63-82. PMID: 21174488. Ineligible study design.

803. Montessori M, L. Scheele, W, H. Netelenbos, J, C. Kerkhoff, J, F. Bakker, $\mathrm{K}$,. The use of etidronate and calcium versus calcium alone in the treatment of postmenopausal osteopenia: results of three years of treatment. Osteoporosis International. 1997;7(1):52-8. PMID: 9102064. Ineligible treatment duration.

804. Morabito N, Crisafulli, A, Vergara, C, Gaudio, A, Lasco, A, Frisina, N, D'Anna, R, Corrado, F, Pizzoleo, M, A. Cincotta, M, Altavilla, D, Ientile, R, Squadrito, F,. Effects of genistein and hormone-replacement therapy on bone loss in early postmenopausal women: a randomized double-blind placebo-controlled study. Journal of Bone \& Mineral Research. 2002 Oct;17(10):1904-12. PMID: 12369794. Ineligible treatment duration.

805. Morii H, Ohashi, Y, Taketani, Y, Fukunaga, M, Nakamura, T, Itabashi, A, Sarkar, S, Harper, K,. Effect of raloxifene on bone mineral density and biochemical markers of bone turnover in Japanese postmenopausal women with osteoporosis: results from a randomized placebocontrolled trial. Osteoporosis International. 2003 Oct;14(10):793-800. PMID: 12955333. Ineligible treatment duration.

806. Morin S, Rahme, E, Behlouli, H, Tenenhouse, A, Goltzman, D, Pilote, L,. Effectiveness of antiresorptive agents in the prevention of recurrent hip fractures. Osteoporosis International. 2007

Dec;18(12):1625-32. PMID: 17634854. Ineligible study design.

807. Mortensen L, Charles, P, Bekker, P, J. Digennaro, J, Johnston, C, C. Jr,.
Risedronate increases bone mass in an early postmenopausal population: two years of treatment plus one year of follow-up. Journal of Clinical Endocrinology \& Metabolism. 1998 Feb;83(2):396-402. PMID: 9467547. No outcomes of interest.

808. Mosca G, Barrett, Connor Collins, Wenger, Abramson, Bl, Paganini, Hill Geiger, Mj, Dowsett, Sa, Amewou, Atisso Kornitzer,. Effect of raloxifene on stroke and venous thromboembolism according to subgroups in postmenopausal women at increased risk of coronary heart disease. Stroke a journal of cerebral circulation. 2009;40(1):147-55. PMID: CN-00667734. Ineligible population.

809. Mosekilde B, Nielsen Sørensen, Oh, Nielsen, Sp, Charles, Vestergaard, Hermann, Ap, Gram, Hansen, Tb, Abrahamsen, Ebbesen, En, Stilgren, Jensen, Lb, Brot, Hansen, Tofteng, Cl, Eiken, Kolthoff,. Hormonal replacement therapy reduces forearm fracture incidence in recent postmenopausal women - results of the Danish Osteoporosis Prevention Study. Maturitas. 2000;36(3):181-93. PMID: CN00330324. Ineligible population.

810. Mosekilde L, Vestergaard, P, Rejnmark, $\mathrm{L}$,. The pathogenesis, treatment and prevention of osteoporosis in men. Drugs. 2013 Jan;73(1):15-29. PMID: 23329464. Ineligible study design.

811. Mulgund M, Beattie, K, A. Anaspure, R, Matsos, M, Patel, A, Adachi, J, D. Atypical femoral fractures in patients taking longterm alendronate. Journal of Rheumatology. 2011 Dec;38(12):2686-7. PMID: 22134795. Ineligible study design.

812. Murphy M, G. Weiss, S, McClung, M, Schnitzer, T, Cerchio, K, Connor, J, Krupa, D, Gertz, B, J. M. K. Alendronate Study Group. Effect of alendronate and MK677 (a growth hormone secretagogue), individually and in combination, on markers of bone turnover and bone mineral density in postmenopausal osteoporotic women. Journal of Clinical Endocrinology \& Metabolism. 2001 Mar;86(3):1116-25. PMID: 11238495. No outcomes of interest. 
813. Murphy MM. Role of the Pharmacist in Medication Therapy Management Services in Patients With Osteoporosis. Clinical Therapeutics. 2015 Jul 01;37(7):1573-86. PMID: 25913920. Ineligible study design.

814. Muschitz C, Fahrleitner, Pammer A, Huber, J, Preisinger, E, Kudlacek, S, Resch, H,. Update on denosumab in postmenopausal osteoporosis--recent clinical data. Wiener Medizinische Wochenschrift. 2012 Sep;162(17-18):374-9. PMID: 22688622. Ineligible study design.

815. Muschitz C, Kocijan, R, Fahrleitner, Pammer A, Lung, S, Resch, H,. Antiresorptives overlapping ongoing teriparatide treatment result in additional increases in bone mineral density. Journal of Bone \& Mineral Research. 2013 Jan;28(1):196-205. PMID: 22836585. Ineligible treatment duration.

816. Muscoso E, Puglisi, N, Mamazza, C, Lo, Giudice F, Testai, M, Abbate, S, Santangelo, A, Panebianco, P, Maugeri, $\mathrm{D}$,. Antiresorption therapy and reduction in fracture susceptibility in the osteoporotic elderly patient: open study. European Review for Medical \& Pharmacological Sciences. 2004 Mar-Apr;8(2):97-102. PMID: 15267123. No outcomes of interest.

817. Nabhan A, F. Rabie, N, H. Isosorbide mononitrate versus alendronate for postmenopausal osteoporosis. International Journal of Gynaecology \& Obstetrics. 2008 Dec;103(3):213-6. PMID: 18805524. No outcomes of interest.

818. Nagahama K, Kanayama, M, Togawa, D, Hashimoto, T, Minami, A,. Does alendronate disturb the healing process of posterior lumbar interbody fusion? A prospective randomized trial. Journal of Neurosurgery: Spine. 2011 April;14(4):5007. PMID: 361584144. No outcomes of interest.

819. Nakamura T, Liu, J, L. Morii, H, Huang, Q, R. Zhu, H, M. Qu, Y, Hamaya, E, Thiebaud, D,. Effect of raloxifene on clinical fractures in Asian women with postmenopausal osteoporosis. Journal of Bone \& Mineral Metabolism.
2006;24(5):414-8. PMID: 16937275. Ineligible treatment duration.

820. Nakamura T, Matsumoto, T, Sugimoto, T, Shiraki, M,. Dose-response study of denosumab on bone mineral density and bone turnover markers in Japanese postmenopausal women with osteoporosis. Osteoporosis International. 2012 Mar;23(3):1131-40. PMID: 21927920. No outcomes of interest.

821. Nakamura T, Sugimoto, T, Nakano, T, Kishimoto, H, Ito, M, Fukunaga, M, Hagino, H, Sone, T, Yoshikawa, $\mathrm{H}$, Nishizawa, Y, Fujita, T, Shiraki, M,. Randomized Teriparatide [human parathyroid hormone (PTH) 1-34] OnceWeekly Efficacy Research (TOWER) trial for examining the reduction in new vertebral fractures in subjects with primary osteoporosis and high fracture risk. Journal of Clinical Endocrinology \& Metabolism. 2012 Sep;97(9):3097-106. PMID: 22723322. No outcomes of interest.

822. Nakamura T, Nakano, T, Ito, M, Hagino, H, Hashimoto, J, Tobinai, M, Mizunuma, H, Mover Study, Group. Clinical efficacy on fracture risk and safety of $0.5 \mathrm{mg}$ or 1 $\mathrm{mg} /$ month intravenous ibandronate versus $2.5 \mathrm{mg} /$ day oral risedronate in patients with primary osteoporosis. Calcified Tissue International. 2013 Aug;93(2):137-46. PMID: 23644930. No outcomes of interest.

823. Nakamura T, Matsumoto, T, Sugimoto, T, Hosoi, T, Miki, T, Gorai, I, Yoshikawa, H, Tanaka, Y, Tanaka, S, Sone, T, Nakano, T, Ito, M, Matsui, S, Yoneda, $\mathrm{T}$, Takami, H, Watanabe, $\mathrm{K}$, Osakabe, $\mathrm{T}$, Shiraki, M, Fukunaga, M,. Clinical Trials Express: fracture risk reduction with denosumab in Japanese postmenopausal women and men with osteoporosis: denosumab fracture intervention randomized placebo controlled trial (DIRECT). Journal of Clinical Endocrinology \& Metabolism. 2014 Jul;99(7):2599-607. PMID: 24646104. Ineligible treatment duration.

824. Nakamura T, Ito, M, Hashimoto, J, Shinomiya, K, Asao, Y, Katsumata, K, Hagino, H, Inoue, T, Nakano, T, Mizunuma, H, Movest Study, Group. 
Clinical efficacy and safety of monthly oral ibandronate $100 \mathrm{mg}$ versus monthly intravenous ibandronate $1 \mathrm{mg}$ in Japanese patients with primary osteoporosis. Osteoporosis International. 2015 Nov;26(11):2685-93. PMID: 26001561. Ineligible treatment duration.

825. Nakamura T, Fukunaga, M, Nakano, T, Kishimoto, H, Ito, M, Hagino, H, Sone, T, Taguchi, A, Tanaka, S, Ohashi, M, Ota, Y, Shiraki, M,. Efficacy and safety of once-yearly zoledronic acid in Japanese patients with primary osteoporosis: two-year results from a randomized placebocontrolled double-blind study (ZOledroNate treatment in Efficacy to osteoporosis ZONE study). Osteoporosis International. 2017 Jan;28(1):389-98. PMID: 27631091. Ineligible treatment duration.

826. Nakamura Y, Suzuki, T, Kamimura, M, Ikegami, S, Murakami, K, Uchiyama, S, Taguchi, A, Kato, H,. Two-year clinical outcome of denosumab treatment alone and in combination with teriparatide in Japanese treatment-naive postmenopausal osteoporotic women. Bone Research. 2017 13 Jun;5 (no pagination)(16055) PMID: 616749617. No outcomes of interest.

827. Nakano T, Shiraki, M, Sugimoto, T, Kishimoto, H, Ito, M, Fukunaga, M, Hagino, H, Sone, T, Kuroda, T, Nakamura, T,. Once-weekly teriparatide reduces the risk of vertebral fracture in patients with various fracture risks: subgroup analysis of the Teriparatide OnceWeekly Efficacy Research (TOWER) trial. Journal of Bone \& Mineral Metabolism. 2014 Jul;32(4):441-6. PMID: 24213216. Ineligible treatment duration.

828. Nakano T, Yamamoto, M, Hashimoto, J, Tobinai, M, Yoshida, S, Nakamura, T,. Higher response with bone mineral density increase with monthly injectable ibandronate $1 \mathrm{mg}$ compared with oral risedronate in the MOVER study. Journal of Bone \& Mineral Metabolism. 2016 Nov;34(6):678-84.

PMID: 26462480. No outcomes of interest.

829. Nakatoh S. Effect of osteoporosis medication on changes in bone mineral density and bone turnover markers after 24- month administration of daily teriparatide: comparison among minodronate, raloxifene, and eldecalcitol. Journal of Bone and Mineral Metabolism. 2017 14 Mar:1-8. PMID: 614823849. Ineligible treatment duration.

830. Namba R, S. Inacio, M, C. Cheetham, T, C. Dell, R, M. Paxton, E, W. Khatod, M, X. Lower Total Knee Arthroplasty Revision Risk Associated With Bisphosphonate Use, Even in Patients With Normal Bone Density. Journal of Arthroplasty. 2016 Feb;31(2):537-41. PMID: 26454569. Ineligible treatment duration.

831. Nand S, L. Webster, M, A. Wren, B, G. Continuous combined piperazine oestrone sulphate and medroxyprogesterone acetate hormone replacement therapy--a study of bleeding pattern, endometrial response, serum lipid and bone density changes. Australian \& New Zealand Journal of Obstetrics \& Gynaecology. 1995 Feb;35(1):92-6. PMID: 7772012. Ineligible treatment duration.

832. Nand S, L. Wren, B, G. Gross, B, A. Heller, G, Z. Bone density effects of continuous estrone sulfate and varying doses of medroxyprogesterone acetate. Ogen/Provera Study Group. Obstetrics \& Gynecology. 1999 Jun;93(6):1009-13. PMID: 10362172. Ineligible treatment duration.

833. Napoli N, Langdahl BL, Ljunggren O, et al. Effects of Teriparatide in Patients with Osteoporosis in Clinical Practice: 42-Month Results During and After Discontinuation of Treatment from the European Extended Forsteo Observational Study (ExFOS). Calcified Tissue International. 201816 Jun:1-13. PMID: 622613162. Ineligible study design.

834. Napoli N, Schwartz AV, Palermo L, et al. Risk factors for subtrochanteric and diaphyseal fractures: the study of osteoporotic fractures. The Journal of Clinical Endocrinology \& Metabolism. 2013;98(2):659-67. Ineligible treatment duration. 
835. Narula R, Mujtaba, T, Iraqi, A, A. Singh, $\mathrm{S}$,. Effect of risedronate and strontium therapy on bone mineral density in postmenopausal osteoporosis. International Journal of Research in Ayurveda and Pharmacy. 2012 July/August;3(4):543-7. PMID: 365972839. No outcomes of interest.

836. Navaneethan S, D. Palmer, Suetonia, C. Vecchio, Mariacristina, Craig, Jonathan, C. Elder, Grahame, J. Strippoli, Giovanni, Fm. Phosphate binders for preventing and treating bone disease in chronic kidney disease patients. Cochrane Database of Systematic Reviews. 2011(2) PMID: CD006023. Ineligible study design.

837. Naylor K, E. Clowes, J, A. Finigan, J, Paggiosi, M, A. Peel, N, F. Eastell, R,. The effect of cessation of raloxifene treatment on bone turnover in postmenopausal women. Bone. 2010 Mar;46(3):592-7. PMID: 19897063. Ineligible treatment duration.

838. Naylor KE, Bradburn M, Paggiosi MA, et al. Effects of discontinuing oral bisphosphonate treatments for postmenopausal osteoporosis on bone turnover markers and bone density. Osteoporosis International. 201810 Mar:111. PMID: 621191795. Ineligible treatment duration.

839. Neele S, J. Evertz, R, De Valk-De, Roo G, Roos, J, C. Netelenbos, J, C. Effect of 1 year of discontinuation of raloxifene or estrogen therapy on bone mineral density after 5 years of treatment in healthy postmenopausal women. Bone. 2002 Apr;30(4):599-603. PMID: 11934652. No outcomes of interest.

840. Neer R, M. Arnaud, C, D. Zanchetta, J, R. Prince, R, Gaich, G, A. Reginster, J, Y. Hodsman, A, B. Eriksen, E, F. Ish, Shalom S, Genant, H, K. Wang, O, Mitlak, B, H. Effect of parathyroid hormone (1-34) on fractures and bone mineral density in postmenopausal women with osteoporosis. New England Journal of Medicine. 2001 May 10;344(19):1434-41. PMID: 11346808. Ineligible treatment duration.
841. Negredo E, Bonjoch, A, Perez, Alvarez N, Ornelas, A, Puig, J, Herrero, C, Estany, C, del, Rio L, di, Gregorio S, Echeverria, P, Clotet, B,. Comparison of two different strategies of treatment with zoledronate in HIV-infected patients with low bone mineral density: single dose versus two doses in 2 years. HIV Medicine. 2015 Aug;16(7):4418. PMID: 25944411. Ineligible population.

842. Nelson H, D. Rizzo, J, Harris, E, Cauley, J, Ensrud, K, Bauer, D, C. Orwoll, E, Study of Osteoporotic Fractures, Research Group,. Osteoporosis and fractures in postmenopausal women using estrogen. Archives of Internal Medicine. 2002 Nov 11;162(20):2278-84. PMID: 12418942. Ineligible study design.

843. Nenonen A, Cheng, S, Ivaska, K, K. Alatalo, S, L. Lehtimaki, T, Schmidt, Gayk H, Uusi, Rasi K, Heinonen, A, Kannus, P, Sievanen, H, Vuori, I, Vaananen, H, K. Halleen, J, M. Serum TRACP $5 \mathrm{~b}$ is a useful marker for monitoring alendronate treatment: Comparison with other markers of bone turnover. Journal of Bone and Mineral Research. 2005 October;20(10):1804-12. PMID: 41361881. Ineligible treatment duration.

844. Neviaser A, S. Lane, J, M. Lenart, B, A. Edobor, Osula F, Lorich, D, G. Lowenergy femoral shaft fractures associated with alendronate use. Journal of Orthopaedic Trauma. 2008 May-Jun;22(5):346-50. PMID: 18448990. Ineligible study design.

845. Nevitt M, C. Thompson, D, E. Black, D, M. Rubin, S, R. Ensrud, K, Yates, A, J. Cummings, S, R. Effect of alendronate on limited-activity days and bed-disability days caused by back pain in postmenopausal women with existing vertebral fractures. Fracture Intervention Trial Research Group. Archives of Internal Medicine. 2000 Jan 10;160(1):77-85. PMID: 10632308. No outcomes of interest.

846. Newcomb P, A. Trentham, Dietz A, Hampton, J, M. Bisphosphonates for osteoporosis treatment are associated with reduced breast cancer risk. British Journal of Cancer. 2010 Mar 02;102(5):799-802. PMID: 20160722. Ineligible study design. 
847. Nguyen N, D. Eisman, J, A. Nguyen, T, V. Anti-hip fracture efficacy of biophosphonates: a Bayesian analysis of clinical trials. Journal of Bone \& Mineral Research. 2006 Feb;21(2):340-9. PMID: 16526127. Ineligible study design.

848. Nielsen M, Raundahl, J, Pettersen, P, C. Loog, M, Karemore, G, Karsdal, M, A. Christiansen, C,. Low-dose transdermal estradiol induces breast density and heterogeneity changes comparable to those of raloxifene. Menopause. 2009 JulAug;16(4):785-91. PMID: 19322115. No outcomes of interest.

849. Nordstrom P, Toots, A, Gustafson, Y, Thorngren, K, G. Hommel, A, Nordstrom, A,. Bisphosphonate Use After Hip Fracture in Older Adults: A Nationwide Retrospective Cohort Study. Journal of the American Medical Directors Association. 201701 Jun;18(6):515-21. PMID: 614544283. Ineligible population.

850. Oglesby A, K. Minshall, M, E. Shen, W, Xie, S, Silverman, S, L. The impact of incident vertebral and non-vertebral fragility fractures on health-related quality of life in established postmenopausal osteoporosis: results from the teriparatide randomized, placebo-controlled trial in postmenopausal women. Journal of Rheumatology. 2003 Jul;30(7):1579-83. PMID: 12858462. Ineligible study design.

851. Ohishi T, Fujita, T, Suzuki, D, Nishida, T, Okabayashi, R, Yamamoto, K, Ushirozako, H, Banno, T, Matsuyama, Y,. Initiation of Monthly Minodronate Therapy at an Early Stage After Hip Fracture. Journal of Clinical Densitometry. 2016;21 PMID: 609670788. Ineligible study design.

852. Ohta H, Solanki, J,. Incorporating bazedoxifene into the treatment paradigm for postmenopausal osteoporosis in Japan. Osteoporosis International. 2015 Mar;26(3):849-63. PMID: 25448837. Ineligible study design.
853. Ohtori S, Inoue, G, Orita, S, Yamauchi, K, Eguchi, Y, Ochiai, N, Kishida, S, Kuniyoshi, K, Aoki, Y, Nakamura, J, Ishikawa, T, Miyagi, M, Kamoda, H, Suzuki, M, Kubota, G, Sakuma, Y, Oikawa, Y, Inage, K, Sainoh, T, Takaso, M, Ozawa, T, Takahashi, K, Toyone, T,. Teriparatide accelerates lumbar posterolateral fusion in women with postmenopausal osteoporosis: prospective study. Spine. 2012 Nov 01;37(23):E1464-8. PMID: 22872218. Ineligible study design.

854. Oktem M, Atar, I, Zeyneloglu, H, B. Yildirir, A, Kuscu, E, Muderrisoglu, H,. Raloxifene has favourable effects on metabolic parameters but has no effect on left ventricular function in postmenopausal women. Pharmacological Research. 2008 May;57(5):364-8. PMID: 18467115. Ineligible treatment duration.

855. Ongphiphadhanakul B, Piaseu, N, Tung, S, S. Chailurkit, L, Rajatanavin, R,. Prevention of postmenopausal bone loss by low and conventional doses of calcitriol or conjugated equine estrogen. Maturitas. 2000 Feb 15;34(2):179-84. PMID: 10714913. Ineligible treatment duration.

856. Orimo H, Nakamura, T, Fukunaga, M, Ohta, H, Hosoi, T, Uemura, Y, Kuroda, T, Miyakawa, N, Ohashi, Y, Shiraki, M, A. Top research group. Effects of alendronate plus alfacalcidol in osteoporosis patients with a high risk of fracture: the Japanese Osteoporosis Intervention Trial (JOINT) - 02. Current Medical Research \& Opinion. 2011 Jun;27(6):1273-84. PMID: 21554143. No interventions of interest.

857. Orozco C, Maalouf, N, M. Safety of bisphosphonates. Rheumatic Diseases Clinics of North America. 2012 Nov;38(4):681-705. PMID: 23137577. Ineligible study design.

858. Orr WB, Wattie, D, J. Evans, M, C. Reid, I, R. Effects of prolonged bisphosphonate therapy and its discontinuation on bone mineral density in post-menopausal osteoporosis. Clinical Endocrinology. 1997 Jan;46(1):87-92. PMID: 9059563. Ineligible study design. 
859. Orwoll E, Ettinger, M, Weiss, S, Miller, P, Kendler, D, Graham, J, Adami, S, Weber, K, Lorenc, R, Pietschmann, P, Vandormael, K, Lombardi, A,. Alendronate for the treatment of osteoporosis in men. New England Journal of Medicine. 2000 Aug 31;343(9):604-10. PMID: 10979796. No outcomes of interest.

860. Orwoll E, S. Scheele, W, H. Paul, S, Adami, S, Syversen, U, Diez, Perez A, Kaufman, J, M. Clancy, A, D. Gaich, G, A. The effect of teriparatide [human parathyroid hormone (1-34)] therapy on bone density in men with osteoporosis. Journal of Bone \& Mineral Research. 2003 Jan;18(1):9-17. PMID: 12510800. Ineligible treatment duration.

861. Orwoll E, S. Miller, P, D. Adachi, J, D. Brown, J, Adler, R, A. Kendler, D, Bucci, Rechtweg C, Readie, A, Mesenbrink, P, Weinstein, R, S. Efficacy and safety of a once-yearly i.v. Infusion of zoledronic acid $5 \mathrm{mg}$ versus a once-weekly 70-mg oral alendronate in the treatment of male osteoporosis: a randomized, multicenter, double-blind, active-controlled study. Journal of Bone \& Mineral Research. 2010 Oct;25(10):2239-50. PMID: 20499357. No outcomes of interest.

862. Orwoll E, S. Binkley, N, C. Lewiecki, E, M. Gruntmanis, U, Fries, M, A. Dasic, G,. Efficacy and safety of monthly ibandronate in men with low bone density. Bone. 2010 Apr;46(4):970-6. PMID: 20060082. Ineligible treatment duration.

863. Orwoll E, Teglbjaerg, C, S. Langdahl, B, L. Chapurlat, R, Czerwinski, E, Kendler, D, L. Reginster, J, Y. Kivitz, A, Lewiecki, E, M. Miller, P, D. Bolognese, M, A. McClung, M, R. Bone, H, G. Ljunggren, O, Abrahamsen, B, Gruntmanis, U, Yang, Y, C. Wagman, R, B. Siddhanti, S, Grauer, A, Hall, J, W. Boonen, S,. A randomized, placebocontrolled study of the effects of denosumab for the treatment of men with low bone mineral density. Journal of Clinical Endocrinology \& Metabolism. 2012 Sep;97(9):3161-9. PMID: 22723310. No outcomes of interest.
864. Ott S, M. Oleksik, A, Lu, Y, Harper, K, Lips, P,. Bone histomorphometric and biochemical marker results of a 2-year placebo-controlled trial of raloxifene in postmenopausal women. Journal of Bone \& Mineral Research. 2002 Feb;17(2):341-8. PMID: 11811565. No outcomes of interest.

865. Paccou J, Confavreux, C, B. David, C, Leboime, A, Mehsen, N, Cortet, B,. Stabilized severe osteoporosis: should the treatment be stopped? Joint, Bone, Spine: Revue du Rhumatisme. 2010 Dec;77 Suppl 2:S120-7. PMID: 21211749. Ineligible study design.

866. Padhi D, Allison, M, Kivitz, A, J. Gutierrez, M, J. Stouch, B, Wang, C, Jang, G,. Multiple doses of sclerostin antibody romosozumab in healthy men and postmenopausal women with low bone mass: a randomized, double-blind, placebocontrolled study. Journal of Clinical Pharmacology. 2014 Feb;54(2):168-78. PMID: 24272917. Ineligible treatment duration.

867. Paganini HA. The benefits of estrogen replacement therapy on oral health: The leisure world cohort. Archives of Internal Medicine. 199527 Nov;155(21):2325-9. PMID: 25349480. No outcomes of interest.

868. Paggiosi M, A. Clowes, J, A. Finigan, J, Naylor, K, E. Peel, N, F. Eastell, R,. Performance of quantitative ultrasound measurements of bone for monitoring raloxifene therapy. Journal of Clinical Densitometry. 2010 Oct-Dec;13(4):441-50. PMID: 20850365. Ineligible treatment duration.

869. Paggiosi M, A. Peel, N, McCloskey, E, Walsh, J, S. Eastell, R,. Comparison of the effects of three oral bisphosphonate therapies on the peripheral skeleton in postmenopausal osteoporosis: the TRIO study. Osteoporosis International. 2014 Dec;25(12):2729-41. PMID: 25074351. No outcomes of interest.

870. Pal L, Hailpern, S, M. Santoro, N, F. Freeman, R, Barad, D, Kipersztok, S, Barnabei, V, M. Wassertheil, Smoller S,. Increased incident hip fractures in 
postmenopausal women with moderate to severe pelvic organ prolapse. Menopause. 2011 September;18(9):967-73. PMID: 51517467. Ineligible study design.

871. Palacios S. Efficacy and safety of bazedoxifene, a novel selective estrogen receptor modulator for the prevention and treatment of postmenopausal osteoporosis. Current Medical Research \& Opinion. 2010 Jul;26(7):1553-63. PMID: 20429824. Ineligible study design.

872. Palacios S. Bazedoxifene acetate for the management of postmenopausal osteoporosis. Drugs of Today. 2011 Mar;47(3):187-95. PMID: 21494696. Ineligible study design.

873. Palacios S, de, Villiers T, J. Nardone, Fde C, Levine, A, B. Williams, R, Hines, T, Mirkin, S, Chines, A, A. B. Z. A. Study Group. Assessment of the safety of longterm bazedoxifene treatment on the reproductive tract in postmenopausal women with osteoporosis: results of a 7-year, randomized, placebo-controlled, phase 3 study. Maturitas. 2013 Sep;76(1):81-7. PMID: 23871271. Ineligible treatment duration.

874. Palacios S, Kalouche, Khalil L, Rizzoli, R, Zapalowski, C, Resch, H, Adachi, J, D. Gallagher, J, C. Feldman, R, G. Kendler, D, L. Wang, A, Wagman, R, B. Adami, $\mathrm{S}$,. Treatment with denosumab reduces secondary fracture risk in women with postmenopausal osteoporosis. Climacteric. 2015;18(6):805-12. PMID: 26029985. Ineligible treatment duration.

875. Palacios S, Silverman, S, L. de, Villiers T, J. Levine, A, B. Goemaere, S, Brown, J, P. De Cicco, Nardone F, Williams, R, Hines, T, L. Mirkin, S, Chines, A, A. Bazedoxifene, Study Group,. A 7-year randomized, placebo-controlled trial assessing the long-term efficacy and safety of bazedoxifene in postmenopausal women with osteoporosis: effects on bone density and fracture. Menopause. 2015 Aug;22(8):806-13. PMID: 25668306. No interventions of interest.
876. Palacios S, Williams, R, Mirkin, S, Pan, K, Arias, L, Komm, B, S. Efficacy and tolerability of bazedoxifene in Mexican women with osteoporosis: a subgroup analysis of a randomized phase 3 trial. Menopause. 2016 Jul;23(7):771-7. PMID: 27116464. Ineligible treatment duration.

877. Palacios S, Arias, L, Lavenberg, J, Pan, K, Mirkin, S, Komm, B, S. Evaluation of efficacy and safety of conjugated estrogens/bazedoxifene in a Latin American population. Climacteric. 2016 Jun;19(3):261-7. PMID: 26940720. Ineligible treatment duration.

878. Palomba S, Orio, F, Jr, Colao, A, di, Carlo C, Sena, T, Lombardi, G, Zullo, F, Mastrantonio, P,. Effect of estrogen replacement plus low-dose alendronate treatment on bone density in surgically postmenopausal women with osteoporosis. Journal of Clinical Endocrinology \& Metabolism. 2002 Apr;87(4):1502-8. PMID: 11932272. No outcomes of interest.

879. Palomba S, Orio,, Jr. F, Russo, T, Colao, A, Lombardi, G, Zullo, F,. Dose reduction and discontinuation of alendronate in postmenopausal osteoporotic women who were receiving estrogen therapy. Fertility and Sterility. 2004 February;81(2):476-8. PMID: 38223941. No outcomes of interest.

880. Palomba S, Orio, F, Jr, Russo, T, Falbo, A, Tolino, A, Manguso, F, Nunziata, V, Mastrantonio, P, Lombardi, G, Zullo, F,. BsmI vitamin D receptor genotypes influence the efficacy of antiresorptive treatments in postmenopausal osteoporotic women. A 1-year multicenter, randomized and controlled trial. Osteoporosis International. 2005 Aug;16(8):943-52. PMID: 15739035. No outcomes of interest.

881. Panico A, Lupoli, G, A. Marciello, F, Lupoli, R, Cacciapuoti, M, Martinelli, A, Granieri, L, Iacono, D, Lupoli, G,. Teriparatide vs. alendronate as a treatment for osteoporosis: changes in biochemical markers of bone turnover, BMD and quality of life. Medical Science Monitor. 2011 Aug;17(8):CR442-8. PMID: 21804463. No outcomes of interest. 
882. Papapoulos S, Chapurlat, R, Libanati, C, Brandi, M, L. Brown, J, P. Czerwinski, E, Krieg, M, A. Man, Z, Mellstrom, D, Radominski, S, C. Reginster, J, Y. Resch, H, Roman, Ivorra J, A. Roux, C, Vittinghoff, E, Austin, M, Daizadeh, N, Bradley, M, N. Grauer, A, Cummings, S, R. Bone, H, G. Five years of denosumab exposure in women with postmenopausal osteoporosis: results from the first two years of the FREEDOM extension. Journal of Bone \& Mineral Research. 2012 Mar;27(3):694-701. PMID: 22113951. Ineligible study design.

883. Papapoulos S, E. Bone diseases: Bisphosphonates in osteoporosis - Beyond 5 years. Nature Reviews Rheumatology. 2013 May;9(5):263-4. PMID: 52541074.

Ineligible study design.

884. Papapoulos S, Lippuner, K, Roux, C, Lin, C, J. Kendler, D, L. Lewiecki, E, M. Brandi, M, L. Czerwinski, E, Franek, E, Lakatos, P, Mautalen, C, Minisola, S, Reginster, J, Y. Jensen, S, Daizadeh, N, S. Wang, A, Gavin, M, Libanati, C, Wagman, R, B. Bone, H, G. The effect of 8 or 5 years of denosumab treatment in postmenopausal women with osteoporosis: results from the FREEDOM Extension study. Osteoporosis International. 2015 Dec;26(12):2773-83. PMID: 26202488. Ineligible study design.

885. Park L, Evans, M, F. Raloxifene: designer estrogen for preventing postmenopausal osteoporosis. Canadian Family Physician. 1999 May;45:1191-3. PMID: 10349062. Ineligible study design.

886. Parviainen M, Jääskeläinen, Kröger, Arnala, Alhava,. Urinary bone resorption markers in monitoring treatment of symptomatic osteoporosis. Clinica chimica acta international journal of clinical chemistry. 1999;279(1-2):145-54. PMID: CN-00160304. No interventions of interest.

887. Passos J, S. Vianna, M, I. Gomes, Filho I, S. Cruz, S, S. Barreto, M, L. Adan, L, Rosing, C, K. Cerqueira, E, M. Trindade, S, C. Coelho, J, M.

Osteoporosis/osteopenia as an independent factor associated with periodontitis in postmenopausal women: a case-control study. Osteoporosis International. 2013 Apr;24(4):1275-83. PMID: 23001114. Ineligible study design.

888. Patel D, V. Horne, A, House, M, Reid, I, R. McGhee, C, N. The incidence of acute anterior uveitis after intravenous zoledronate. Ophthalmology. 2013 Apr;120(4):773-6. PMID: 23290982. Ineligible study design.

889. Patel D, V. Bolland, M, Nisa, Z, Al, Abuwsi F, Singh, M, Horne, A, Reid, I, R. McGhee, C, N. Incidence of ocular side effects with intravenous zoledronate: secondary analysis of a randomized controlled trial. Osteoporosis International. 2015 Feb;26(2):499-503. PMID: 25187119. Ineligible treatment duration.

890. Patrick A, R. Brookhart, M, A. Losina, E, Schousboe, J, T. Cadarette, S, M. Mogun, $\mathrm{H}$, Solomon, D, H. The complex relation between bisphosphonate adherence and fracture reduction. Journal of Clinical Endocrinology \& Metabolism. 2010 Jul;95(7):3251-9. PMID: 20444916. No outcomes of interest.

891. Pazianas M, Blumentals, W, A. Miller, P, D. Lack of association between oral bisphosphonates and osteonecrosis using jaw surgery as a surrogate marker. Osteoporosis International. 2008 Jun;19(6):773-9. PMID: 17999023. Ineligible study design.

892. Pazianas M, Cooper, C, Ebetino, F, H. Russell, R, G. Long-term treatment with bisphosphonates and their safety in postmenopausal osteoporosis. Therapeutics \& Clinical Risk Management. 2010 Jul 21;6:325-43. PMID: 20668715. Ineligible study design.

893. Pazianas M, Abrahamsen, B, Wang, Y, Russell, R, G. Incidence of fractures of the femur, including subtrochanteric, up to 8 years since initiation of oral bisphosphonate therapy: a register-based cohort study using the US MarketScan claims databases.

Osteoporosis International. 2012 Dec;23(12):2873-84. PMID: 22431012. Ineligible study design. 
894. Pazianas M, Abrahamsen, B, Eiken, P, A. Eastell, R, Russell, R, G. Reduced colon cancer incidence and mortality in postmenopausal women treated with an oral bisphosphonate--Danish National Register Based Cohort Study. Osteoporosis International. 2012 Nov;23(11):2693-701. PMID: 22392160. No outcomes of interest.

895. Pazianas M, Clark, E, M. Eiken, P, A. Brixen, K, Abrahamsen, B,. Inflammatory eye reactions in patients treated with bisphosphonates and other osteoporosis medications: cohort analysis using a national prescription database. Journal of Bone \& Mineral Research. 2013 Mar;28(3):455-63. PMID: 23044864. Ineligible treatment duration.

896. Pazianas M, Kim, S, M. Yuen, T, Sun, L, Epstein, S, Zaidi, M,. Questioning the association between bisphosphonates and atypical femoral fractures. Annals of the New York Academy of Sciences. 2015 Jan;1335:1-9. PMID: 25294742. Ineligible study design.

897. Pelayo I, Haya, J, De la, Cruz J, J. Seco, C, Bugella, J, I. Diaz, J, L. Bajo, J, M. Repolles, M,. Raloxifene plus osseinhydroxyapatite compound versus raloxifene plus calcium carbonate to control bone loss in postmenopausal women: a randomized trial. Menopause. 2008 NovDec;15(6):1132-8. PMID: 18791486. No interventions of interest.

898. Peng L, Luo Q, Lu H. Efficacy and safety of bazedoxifene in postmenopausal women with osteoporosis: A systematic review and meta-analysis. Medicine. 2017 Dec;96(49):e8659. PMID: 29245225. Ineligible study design.

899. Peng Y, L. Hu, H, Y. Luo, J, C. Hou, M, C. Lin, H, C. Lee, F, Y. Alendronate, a bisphosphonate, increased upper and lower gastrointestinal bleeding: risk factor analysis from a nationwide population-based study.

Osteoporosis International. 2014 May;25(5):1617-23. PMID: 24566586. Ineligible treatment duration.
900. Pentti K, Honkanen, R, Tuppurainen, M, T. Sandini, L, Kroger, H, Saarikoski, S,. Hormone replacement therapy and mortality in 52- to 70-year-old women: the Kuopio Osteoporosis Risk Factor and Prevention Study. European Journal of Endocrinology. 2006 Jan;154(1):101-7. PMID: 16381998. Ineligible population.

901. Pentti K, Tuppurainen, M, T. Honkanen, R, Sandini, L, Kroger, H, Alhava, E, Saarikoski, S,. Hormone therapy protects from diabetes: the Kuopio osteoporosis risk factor and prevention study. European Journal of Endocrinology. 2009 Jun;160(6):979-83. PMID: 19321660. Ineligible population.

902. Peretz A, Siderova, V, Body, J, J. Dumon, J, C. Rozenberg, S, Fellemans, C, Fuss, M, Bergmann, P, Interdisciplinary Group for the Study of, Bone Diseases, Calcium, metabolism,. Response to alendronate in osteoporotic women previously treated with pamidronate. Maturitas. $2003 \mathrm{Feb}$ 25;44(2):111-5. PMID: 12590006. No outcomes of interest.

903. Perkins RM, Kirchner HL, Matsushita K, et al. Bisphosphonates and mortality in women with CKD and the presence or absence of cardiovascular disease. Clin J Am Soc Nephrol. 2014 May;9(5):874-80. doi: 10.2215/CJN.07790713. PMID: 24578337. Ineligible population.

904. Phillips H, K. Harrison, S, J. Akrawi, H, Sidhom, S, A. Retrospective review of patients with atypical bisphosphonate related proximal femoral fractures. Injury. 2017 June;48(6):1159-64. PMID: 615346209. Ineligible study design.

905. Phillips L. HRT, BMD, and CA: boning up on estrogen. Hospital Technology Series. 1997 Apr;16(5):2-4. PMID: 10167460. Ineligible study design.

906. Pilipovi B, Vujasinovi, Stupar. Effects of Alendronate on bone mass in women with osteoporosis. Medicinski pregled. 2006;59(9-10):427-35. PMID: CN00579079. Ineligible study design. 
907. Pilipovic N, Brankovic, S, Vujasinovic, Stupar N,. Effects of Alendronate on bone mass in women with osteoporosis. Medicinski Pregled. 2006 Sep-Oct;59(910):427-35. PMID: 17345818. Ineligible study design.

908. Pines A, Katchman, H, Villa, Y, Mijatovic, V, Dotan, I, Levo, Y, Ayalon, $\mathrm{D}$,. The effect of various hormonal preparations and calcium supplementation on bone mass in early menopause. Is there a predictive value for the initial bone density and body weight? Journal of Internal Medicine. 1999 Oct;246(4):357-61. PMID: 10583706. Ineligible study design.

909. Pinkerton J, V. Pickar, J, H. Racketa, J, Mirkin, S,. Bazedoxifene/conjugated estrogens for menopausal symptom treatment and osteoporosis prevention. Climacteric. 2012 Oct;15(5):411-8. PMID: 22853444. Ineligible study design.

910. Pinkerton J, V. Harvey, J, A. Lindsay, R, Pan, K, Chines, A, A. Mirkin, S, Archer, D, F. Smart, Investigators. Effects of bazedoxifene/conjugated estrogens on the endometrium and bone: a randomized trial. Journal of Clinical Endocrinology \& Metabolism. 2014 Feb;99(2):E189-98. PMID: 24438370. Ineligible treatment duration.

911. Pinkerton J, V. Thomas, S,. Use of SERMs for treatment in postmenopausal women. Journal of Steroid Biochemistry \& Molecular Biology. 2014 Jul;142:142-54. PMID: 24373794. Ineligible study design.

912. Pinkerton J, Pickar, Jh, Ryan, Ka, Yu, C, R. Mirkin, Komm, Bs,. Conjugated estrogens and bazedoxifene in minority populations: pooled analysis of four phase 3 trials. Menopause (New York, N.Y.). 2016;23(6):611-20. PMID: CN-01166860. Ineligible treatment duration.

913. Piscitelli P, Auriemma, R, Neglia, C, Migliore, A,. Alendronate: new formulations of an old and effective drug to improve adherence avoiding upper gastrointestinal side effects. European Review for Medical \& Pharmacological
Sciences. 2014;18(24):3788-96. PMID: 25555868. Ineligible study design.

914. Pitale S, Thomas, M, Rathi, G, Deshmukh, V, Kumar, P, Reddy, S, Shetty, N, Kakar, A, Babhulkar, S, Mody, B, Chacko, J, Acharya, S, Joglekar, S, Halbe, V, Kravitz, B, G. Waterhouse, B, Nino, A, J. Fitzpatrick, L, A. A randomized placebo-controlled trial of the efficacy of denosumab in Indian postmenopausal women with osteoporosis. Indian Journal of Endocrinology and Metabolism. 2015 Jan-Feb;19(1):148-54. PMID: 25593843. Ineligible treatment duration.

915. Pittman C, B. Davis, L, A. Zeringue, A, L. Caplan, L, Wehmeier, K, R. Scherrer, J, F. Xian, H, Cunningham, F, E.

McDonald, J, R. Arnold, A, Eisen, S, A. Myocardial infarction risk among patients with fractures receiving bisphosphonates. Mayo Clinic Proceedings. 2014 Jan;89(1):43-51. PMID: 24388021. No outcomes of interest.

916. Pols H, A. Felsenberg, D, Hanley, D, A. Stepan, J, Munoz, Torres M, Wilkin, T, J. Qin, sheng G, Galich, A, M. Vandormael, K, Yates, A, J. Stych, B,. Multinational, placebo-controlled, randomized trial of the effects of alendronate on bone density and fracture risk in postmenopausal women with low bone mass: results of the FOSIT study. Fosamax International Trial Study Group. Osteoporosis International. 1999;9(5):461-8. PMID: 10550467. No outcomes of interest.

917. Poole K, E. Compston, J, E. Bisphosphonates in the treatment of osteoporosis. BMJ (Online). 201211 Aug;345 (7869) (no pagination)(e3211) PMID: 365420037. Ineligible study design.

918. Popp A, W. Bodmer, C, Senn, C, Fuchs, G, Kraenzlin, M, E. Wyss, H, Birkhaeuser, M, H. Lippuner, K,. Prevention of postmenopausal bone loss with long-cycle hormone replacement therapy. Maturitas. 2006 Jan 20;53(2):191200. PMID: 16368472. Ineligible treatment duration. 
919. Popp A, W. Guler, S, Lamy, O, Senn, C, Buffat, H, Perrelet, R, Hans, D, Lippuner, K,. Effects of zoledronate versus placebo on spine bone mineral density and microarchitecture assessed by the trabecular bone score in postmenopausal women with osteoporosis: a three-year study. Journal of Bone \& Mineral Research. 2013 Mar;28(3):449-54. PMID: 23018784. No outcomes of interest.

920. Popp A, W. Buffat, H, Cavelti, A, Windolf, M, Perrelet, R, Senn, C, Lippuner, K,. Cortical bone loss at the tibia in postmenopausal women with osteoporosis is associated with incident non-vertebral fractures: results of a randomized controlled ancillary study of HORIZON. Maturitas. 2014 Mar;77(3):287-93. PMID: 24485216. No outcomes of interest.

921. Popp AW, Varathan N, Buffat H, et al. Bone Mineral Density Changes After 1 Year of Denosumab Discontinuation in Postmenopausal Women with Long-Term Denosumab Treatment for Osteoporosis. Calcified Tissue International. 2018 Jul;103(1):50-4. PMID: 29380013. Ineligible study design.

922. Prelevic G, Bartram, Wood, Okolo, Ginsburg,. Comparative effects on bone mineral density of tibolone, transdermal estrogen and oral estrogen/progestogen therapy in postmenopausal women. Gynecological endocrinology. 1996;10(6):413-20. PMID: CN-00172742. Ineligible study design.

923. Prestwood K, M. Kenny, A, M. Kleppinger, A, Kulldorff, M,. UltralowDose Micronized 17beta-Estradiol and Bone Density and Bone Metabolism in Older Women: A Randomized Controlled Trial. Journal of the American Medical Association. 200327 Aug;290(8):1042-8. PMID: 37430444. Ineligible treatment duration.

924. Prevrhal S, Krege, J, H. Chen, P, Genant, $\mathrm{H}$, Black, D, M. Teriparatide vertebral fracture risk reduction determined by quantitative and qualitative radiographic assessment. Current Medical Research \&
Opinion. 2009 Apr;25(4):921-8. PMID: 19250060. No outcomes of interest.

925. Prieto AD, Judge, A, Arden, N, K. Cooper, C, Lyles, K, W. Javaid, M, K. Fracture prevention in patients with cognitive impairment presenting with a hip fracture: secondary analysis of data from the HORIZON Recurrent Fracture Trial. Osteoporosis International. 2014 Jan;25(1):77-83. PMID: 23812596. Ineligible treatment duration.

926. Prieto AD, Pages, Castella A, Wallace, G, Javaid, M, K. Judge, A, Nogues, X, Arden, N, K. Cooper, C, Diez, Perez A,. Predictors of fracture while on treatment with oral bisphosphonates: a populationbased cohort study. Journal of Bone \& Mineral Research. 2014 Jan;29(1):268-74. PMID: 23761350. Ineligible treatment duration.

927. Prince R, Sipos, A, Hossain, A, Syversen, U, Ish, Shalom S, Marcinowska, E, Halse, J, Lindsay, R, Dalsky, G, P. Mitlak, B, H. Sustained nonvertebral fragility fracture risk reduction after discontinuation of teriparatide treatment. Journal of Bone \& Mineral Research. 2005 Sep;20(9):1507-13. PMID: 16059622. Ineligible study design.

928. Prinsloo P, J. Hosking, D, J. Alendronate sodium in the management of osteoporosis. Therapeutics \& Clinical Risk Management. 2006 Sep;2(3):235-49. PMID: 18360599. Ineligible study design.

929. Prior J, C. Vigna, Y, M. Wark, J, D. Eyre, D, R. Lentle, B, C. Li, D, K. Ebeling, P, R. Atley, L,. Premenopausal ovariectomy-related bone loss: a randomized, double-blind, one-year trial of conjugated estrogen or medroxyprogesterone acetate. Journal of Bone \& Mineral Research. 1997 Nov;12(11):1851-63. PMID: 9383690. Ineligible population.

930. Purdie D, W. Rees, M,. Parathyroid hormone in osteoporosis. Journal of the British Menopause Society. 2003 Dec;9(4):175. PMID: 15107263. Ineligible study design. 
931. Qaseem A, Forciea, M, A. McLean, R, M. Denberg, T, D. Clinical Guidelines Committee of the American College, of Physicians,. Treatment of Low Bone Density or Osteoporosis to Prevent Fractures in Men and Women: A Clinical Practice Guideline Update From the American College of Physicians. Annals of Internal Medicine. 2017 Jun 06;166(11):818-39. PMID: 28492856. Ineligible study design.

932. Qin L, Choy, W, Au, S, Fan, M, Leung, $\mathrm{P}$, . Alendronate increases BMD at appendicular and axial skeletons in patients with established osteoporosis. Journal of Orthopaedic Surgery. 2007 May 21;2:9. PMID: 17511887. No outcomes of interest.

933. Rachner T, D. Khosla, S, Hofbauer, L, C. Osteoporosis: now and the future. Lancet. 2011 Apr 09;377(9773):1276-87. PMID: 21450337. Ineligible study design.

934. Ralston S, H. Binkley, N, Boonen, S, Kiel, D, P. Reginster, J, Y. Roux, C, Chen, L, Rosenberg, E, Santora, A, Focus, D. Trial. Randomized trial of alendronate plus vitamin D3 versus standard care in osteoporotic postmenopausal women with vitamin D insufficiency. Calcified Tissue International. 2011 Jun;88(6):485-94. PMID: 21479913. No outcomes of interest.

935. Randell K, M. Honkanen, R, J. Kroger, H, Saarikoski, S,. Does hormone-replacement therapy prevent fractures in early postmenopausal women? Journal of Bone \& Mineral Research. 2002 Mar;17(3):528-33. PMID: 11874244. Ineligible treatment duration.

936. Rapuri P, B. Gallagher, J, C. Haynatzki, $\mathrm{G}$, . Endogenous levels of serum estradiol and sex hormone binding globulin determine bone mineral density, bone remodeling, the rate of bone loss, and response to treatment with estrogen in elderly women.[Erratum appears in J Clin Endocrinol Metab. 2005 Mar 90(3):1711]. Journal of Clinical Endocrinology \& Metabolism. 2004 Oct;89(10):4954-62. PMID: 15472191. Ineligible study design.
937. Ravn P, Clemmesen, B, Riis, B, J. Christiansen, C,. The effect on bone mass and bone markers of different doses of ibandronate: a new bisphosphonate for prevention and treatment of postmenopausal osteoporosis: a 1-year, randomized, doubleblind, placebo-controlled dose-finding study. Bone. 1996 Nov;19(5):527-33. PMID: 8922653. Ineligible treatment duration.

938. Ravn P, Christensen, J, O. Baumann, M, Clemmesen, B,. Changes in biochemical markers and bone mass after withdrawal of ibandronate treatment: prediction of bone mass changes during treatment. Bone. 1998 May;22(5):559-64. PMID: 9600792. Ineligible study design.

939. Ravn P, Alexandersen, P, Mollgaard, A, Bone densitometry: a new, highly responsive region of interest in the distal forearm to monitor the effect of osteoporosis treatment. Osteoporosis International. 1999;9(4):277-83. PMID: 10550443. Ineligible study design.

940. Ravn P, Clemmesen, B, Christiansen, C,. Biochemical markers can predict the response in bone mass during alendronate treatment in early postmenopausal women. Alendronate Osteoporosis Prevention Study Group. Bone. 1999 Mar;24(3):237-44. PMID: 10071916. Ineligible treatment duration.

941. Ravn P, Weiss, S, R. Rodriguez, Portales J, A. McClung, M, R. Wasnich, R, D. Gilchrist, N, L. Sambrook, P, Fogelman, I, Krupa, D, Yates, A, J. Daifotis, A, Fuleihan, G, E. Alendronate in early postmenopausal women: effects on bone mass during long-term treatment and after withdrawal. Alendronate Osteoporosis Prevention Study Group. Journal of Clinical Endocrinology \& Metabolism. 2000 Apr;85(4):1492-7. PMID: 10770187. Ineligible population.

942. Ravn P, Neugebauer, G, Christiansen, C,. Association between pharmacokinetics of oral ibandronate and clinical response in bone mass and bone turnover in women with postmenopausal osteoporosis. Bone. 2002 
Jan;30(1):320-4. PMID: 11792604. No

outcomes of interest.

943. Ravn P, Thompson, D, E. Ross, P, D. Christiansen, C,. Biochemical markers for prediction of 4-year response in bone mass during bisphosphonate treatment for prevention of postmenopausal osteoporosis. Bone. 2003 Jul;33(1):150-8. PMID: 12919710. Ineligible population.

944. Rea T, D. Psaty, B, M. Heckbert, S, R. Cushman, M, Meilahn, E, Olson, J, L. Lemaitre, R, N. Smith, N, L. Sotoodehnia, $\mathrm{N}$, Chaves, P, H. Hormone replacement therapy and the risk of incident congestive heart failure: the Cardiovascular Health Study. Journal of Women's Health. 2003 May;12(4):341-50. PMID: 12804341. Ineligible population.

945. Real J, Galindo, G, Galvan, L, Lafarga, M, A. Rodrigo, M, D. Ortega, M,. Use of oral bisphosphonates in primary prevention of fractures in postmenopausal women: a population-based cohort study. PLoS ONE [Electronic Resource].

2015;10(4):e0118178. PMID: 25861000. Ineligible treatment duration.

946. Recker R, R. Davies, K, M. Dowd, R, M. Heaney, R, P. The effect of low-dose continuous estrogen and progesterone therapy with calcium and vitamin $\mathrm{D}$ on bone in elderly women. A randomized, controlled trial. Annals of Internal Medicine. 1999 Jun 01;130(11):897-904. PMID: 10375338. No outcomes of interest.

947. Recker R, R. Weinstein, R, S. Chesnut, C, H. rd, Schimmer, R, C. Mahoney, P, Hughes, C, Bonvoisin, B, Meunier, P, J. Histomorphometric evaluation of daily and intermittent oral ibandronate in women with postmenopausal osteoporosis: results from the BONE study. Osteoporosis International. 2004 Mar;15(3):231-7. PMID: 14727011. No outcomes of interest.

948. Recker R, Stakkestad, J, A. Chesnut, C, H. rd, Christiansen, C, Skag, A, Hoiseth, A, Ettinger, M, Mahoney, P, Schimmer, R, C. Delmas, P, D. Insufficiently dosed intravenous ibandronate injections are associated with suboptimal antifracture efficacy in postmenopausal osteoporosis. Bone. 2004 May;34(5):890-9. PMID: 15121021. Ineligible treatment duration.

949. Recker R, R. Kendler, D, Recknor, C, P. Rooney, T, W. Lewiecki, E, M. Utian, W, H. Cauley, J, A. Lorraine, J, Qu, Y, Kulkarni, P, M. Gaich, C, L. Wong, M, Plouffe, L, Jr, Stock, J, L. Comparative effects of raloxifene and alendronate on fracture outcomes in postmenopausal women with low bone mass. Bone. 2007 Apr;40(4):843-51. PMID: 17182297. Ineligible treatment duration.

950. Recker R, R. Delmas, P, D. Halse, J, Reid, I, R. Boonen, S, Garcia, Hernandez P, A. Supronik, J, Lewiecki, E, M. Ochoa, L, Miller, P, Hu, H, Mesenbrink, P, Hartl, F, Gasser, J, Eriksen, E, F. Effects of intravenous zoledronic acid once yearly on bone remodeling and bone structure. Journal of Bone \& Mineral Research. 2008 Jan;23(1):6-16. PMID: 17892374. No outcomes of interest.

951. Recker R, R. Ste, Marie L, G. Langdahl, B, Masanauskaite, D, Ethgen, D, Delmas, $\mathrm{P}, \mathrm{D}$. Oral ibandronate preserves trabecular microarchitecture: micro-computed tomography findings from the oral iBandronate Osteoporosis vertebral fracture trial in North America and Europe study. Journal of Clinical Densitometry. 2009 JanMar;12(1):71-6. PMID: 19084450. No outcomes of interest.

952. Recker R, R. Bare, S, P. Smith, S, Y. Varela, A, Miller, M, A. Morris, S, A. Fox, J,. Cancellous and cortical bone architecture and turnover at the iliac crest of postmenopausal osteoporotic women treated with parathyroid hormone 1-84. Bone. 2009 Jan;44(1):113-9. PMID: 18983947. No interventions of interest.

953. Recker R, R. Ste, Marie L, G. Langdahl, B, Czerwinski, E, Bonvoisin, B, Masanauskaite, D, Rowell, L, Felsenberg, D,. Effects of intermittent intravenous ibandronate injections on bone quality and micro-architecture in women with postmenopausal osteoporosis: the DIVA study. Bone. 2010 Mar;46(3):660-5. PMID: 19909829. No outcomes of interest. 
954. Recker R, R. Mitlak, B, H. Ni, X, Krege, $\mathrm{J}, \mathrm{H}$. Long-term raloxifene for postmenopausal osteoporosis. Current Medical Research \& Opinion. 2011 Sep;27(9):1755-61. PMID: 21787127. Ineligible study design.

955. Recknor C, Czerwinski, E, Bone, H, G. Bonnick, S, L. Binkley, N, Palacios, S, Moffett, A, Siddhanti, S, Ferreira, I, Ghelani, P, Wagman, R, B. Hall, J, W. Bolognese, M, A. Benhamou, C, L. Denosumab compared with ibandronate in postmenopausal women previously treated with bisphosphonate therapy: a randomized open-label trial. Obstetrics \& Gynecology. 2013 Jun;121(6):1291-9. PMID: 23812464. No outcomes of interest.

956. Recknor C, P. Recker, R, R. Benson, C, T. Robins, D, A. Chiang, A, Y. Alam, J, Hu, L, Matsumoto, T, Sowa, H, Sloan, J, H. Konrad, R, J. Mitlak, B, H. Sipos, A, A. The Effect of Discontinuing Treatment With Blosozumab: Follow-up Results of a Phase 2 Randomized Clinical Trial in Postmenopausal Women With Low Bone Mineral Density. Journal of Bone \& Mineral Research. 2015 Sep;30(9):1717-25. PMID: 25707611. No interventions of interest.

957. Reeve J, Mitchell, A, Tellez, M, Hulme, P, Green, J, R. Wardley, Smith B, Mitchell, R,. Treatment with parathyroid peptides and estrogen replacement for severe postmenopausal vertebral osteoporosis: prediction of long-term responses in spine and femur. Journal of Bone \& Mineral Metabolism. 2001;19(2):102-14. PMID: 11281158. No outcomes of interest.

958. Reginster J, Minne, H, W. Sorensen, O, H. Hooper, M, Roux, C, Brandi, M, L. Lund, B, Ethgen, D, Pack, S, Roumagnac, I, Eastell, R,. Randomized trial of the effects of risedronate on vertebral fractures in women with established postmenopausal osteoporosis. Vertebral Efficacy with Risedronate Therapy (VERT) Study Group. Osteoporosis International. 2000;11(1):83-91. PMID: 10663363. No outcomes of interest.
959. Reginster J, Y. Risedronate increases bone mineral density and reduces the vertebral fracture incidence in postmenopausal women. Clinical \& Experimental Rheumatology. 2001 Mar-Apr;19(2):121-2. PMID: 11326471. Ineligible study design.

960. Reginster J, Y. Felsenberg, D, Pavo, I, Stepan, J, Payer, J, Resch, H, Gluer, C, C. Muhlenbacher, D, Quail, D, Schmitt, $\mathrm{H}$, Nickelsen, T,. Effect of raloxifene combined with monofluorophosphate as compared with monofluorophosphate alone in postmenopausal women with low bone mass: a randomized, controlled trial. Osteoporosis International. 2003 Sep;14(9):741-9. PMID: 12827224. No outcomes of interest.

961. Reginster J, Y. Sarkar, S, Zegels, B, Henrotin, Y, Bruyere, O, Agnusdei, D, Collette, J,. Reduction in PINP, a marker of bone metabolism, with raloxifene treatment and its relationship with vertebral fracture risk. Bone. 2004 Feb;34(2):344-51. PMID: 14962813. Ineligible treatment duration.

962. Reginster J, Y. Adami, S, Lakatos, P, Greenwald, M, Stepan, J, J. Silverman, S, L. Christiansen, C, Rowell, L, Mairon, N, Bonvoisin, B, Drezner, M, K. Emkey, R, Felsenberg, D, Cooper, C, Delmas, P, D. Miller, P, D. Efficacy and tolerability of once-monthly oral ibandronate in postmenopausal osteoporosis: 2 year results from the MOBILE study.[Erratum appears in Ann Rheum Dis. 2008 Feb 67(2):280]. Annals of the Rheumatic Diseases. 2006 May;65(5):654-61. PMID: 16339289. Ineligible treatment duration.

963. Reginster J, Y. Antifracture efficacy of currently available therapies for postmenopausal osteoporosis. Drugs. 2011 Jan 01;71(1):65-78. PMID: 21175240. Ineligible study design.

964. Reginster J, Y. Neuprez, A, Beaudart, C, Lecart, M, P. Sarlet, N, Bernard, D, Disteche, S, Bruyere, O,. Antiresorptive drugs beyond bisphosphonates and selective oestrogen receptor modulators for the management of postmenopausal osteoporosis. Drugs \& Aging. 2014 
Jun;31(6):413-24. PMID: 24797286.

Ineligible study design.

965. Reginster J, Y. Ferrari, S, Hadji, P,. Current challenges in the treatment of osteoporosis: an opportunity for bazedoxifene. Current Medical Research \& Opinion. 2014 Jun;30(6):1165-76. PMID: 24495098. Ineligible study design.

966. Reginster J, Y. Al, Daghri N, M. Bruyere, O,. Abaloparatide Comparator Trial In Vertebral Endpoints (ACTIVE) confirms that abaloparatide is a valuable addition to the armamentarium against osteoporosis. Expert Opinion on Pharmacotherapy. 2017 Nov 14:1-3. PMID: 29048260. Ineligible study design.

967. Reid D, M. Hosking, D, Kendler, D, Brandi, M, L. Wark, J, D. Weryha, G, Marques, Neto J, F. Gaines, K, A. Verbruggen, N, Melton, M, E. Alendronic acid produces greater effects than risedronic acid on bone density and turnover in postmenopausal women with osteoporosis : results of FACTS -international. Clinical Drug Investigation. 2006;26(2):63-74. PMID: 17163237. No outcomes of interest.

968. Reid D, M. Hosking, D, Kendler, D, Brandi, M, L. Wark, J, D. Marques, Neto J, F. Weryha, G, Verbruggen, N, Hustad, C, M. Mahlis, E, M. Melton, M, E. A comparison of the effect of alendronate and risedronate on bone mineral density in postmenopausal women with osteoporosis: 24-month results from FACTS-International. International Journal of Clinical Practice. 2008 Apr;62(4):575-84. PMID: 18324951. No outcomes of interest.

969. Reid I, R. Brown, J, P. Burckhardt, P, Horowitz, Z, Richardson, P, Trechsel, U, Widmer, A, Devogelaer, J, P. Kaufman, J, M. Jaeger, P, Body, J, J. Brandi, M, L. Broell, J, Di, Micco R, Genazzani, A, R. Felsenberg, D, Happ, J, Hooper, M, J. Ittner, J, Leb, G, Mallmin, H, Murray, T, Ortolani, S, Rubinacci, A, Saaf, M, Samsioe, G, Verbruggen, L, Meunier, P, $\mathrm{J}$. Intravenous zoledronic acid in postmenopausal women with low bone mineral density. New England Journal of Medicine. 2002 Feb 28;346(9):653-61.
PMID: 11870242. Ineligible treatment duration.

970. Reid I, R. Eastell, R, Fogelman, I, Adachi, J, D. Rosen, A, Netelenbos, C, Watts, N, B. Seeman, E, Ciaccia, A, V. Draper, M, W. A comparison of the effects of raloxifene and conjugated equine estrogen on bone and lipids in healthy postmenopausal women. Archives of Internal Medicine. 2004 Apr 26;164(8):8719. PMID: 15111373. Ineligible treatment duration.

971. Reid I, R. Gamble, G, D. Mesenbrink, P, Lakatos, P, Black, D, M. Characterization of and risk factors for the acute-phase response after zoledronic acid. Journal of Clinical Endocrinology \& Metabolism. 2010 Sep;95(9):4380-7. PMID: 20554708. Ineligible treatment duration.

972. Reid I, R. Black, D, M. Eastell, R, Bucci, Rechtweg C, Su, G, Hue, T, F. Mesenbrink, P, Lyles, K, W. Boonen, S, Horizon Pivotal Fracture, Trial Horizon Recurrent Fracture Trial Steering, Committees. Reduction in the risk of clinical fractures after a single dose of zoledronic Acid 5 milligrams. Journal of Clinical Endocrinology \& Metabolism. 2013 Feb;98(2):557-63. PMID: 23293335. Ineligible treatment duration.

973. Revel L, Sorbera, L, A. Martin, L,. Teriparatide: Treatment of osteoporosis, parathyroid hormone analog. Drugs of the Future. 2000;25(8):803-8. PMID: 30768820. Ineligible study design.

974. Rhee C, W. Lee, J, Oh, S, Choi, N, K. Park, B, J. Use of bisphosphonate and risk of atrial fibrillation in older women with osteoporosis.[Erratum appears in Osteoporos Int. 2012 Jan 23(1):255]. Osteoporosis International. 2012 Jan;23(1):247-54. PMID: 21431993. Ineligible treatment duration.

975. Riis B, J. Overgaard, K, Christiansen, C,. Biochemical markers of bone turnover to monitor the bone response to postmenopausal hormone replacement therapy. Osteoporosis International. 
1995;5(4):276-80. PMID: 7492867.

Ineligible treatment duration.

976. Riis B, J. Ise, J, von, Stein T, Bagger, Y, Christiansen, C,. Ibandronate: a comparison of oral daily dosing versus intermittent dosing in postmenopausal osteoporosis. Journal of Bone \& Mineral Research. 2001 Oct;16(10):1871-8. PMID: 11585352. Ineligible treatment duration.

977. Ringe J, D. Faber, H, Dorst, A, . Alendronate treatment of established primary osteoporosis in men: results of a 2year prospective study. Journal of Clinical Endocrinology \& Metabolism. 2001 Nov;86(11):5252-5. PMID: 11701687. No outcomes of interest.

978. Ringe J, D. Dorst, A, Faber, H, Ibach, $\mathrm{K}$,. Alendronate treatment of established primary osteoporosis in men: 3-year results of a prospective, comparative, two-arm study. Rheumatology International. 2004 Mar;24(2):110-3. PMID: 13680141. No outcomes of interest.

979. Ringe J, D. Faber, H, Farahmand, P, Dorst, A,. Efficacy of risedronate in men with primary and secondary osteoporosis: results of a 1-year study. Rheumatology International. 2006 Mar;26(5):427-31. PMID: 16001181. No outcomes of interest.

980. Ringe J, D. Farahmand, P, Schacht, E, Rozehnal, A,. Superiority of a combined treatment of Alendronate and Alfacalcidol compared to the combination of Alendronate and plain vitamin $\mathrm{D}$ or Alfacalcidol alone in established postmenopausal or male osteoporosis (AAC-Trial). Rheumatology International. 2007 Mar;27(5):425-34. PMID: 17216477. No outcomes of interest.

981. Ringe J, D. Farahmand, P, Faber, H, Dorst, A,. Sustained efficacy of risedronate in men with primary and secondary osteoporosis: results of a 2-year study. Rheumatology International. 2009 Jan;29(3):311-5. PMID: 18762944. No outcomes of interest.

982. Ringe J, D. Development of clinical utility of zoledronic acid and patient considerations in the treatment of osteoporosis. Patient preference \& adherence. 2010 Jul 21;4:23145. PMID: 20694183. Ineligible study design.

983. Ringe J, D. Doherty, J, G. Absolute risk reduction in osteoporosis: assessing treatment efficacy by number needed to treat. Rheumatology International. 2010 May;30(7):863-9. PMID: 20035331. Ineligible study design.

984. Rittmaster R, S. Bolognese, M, Ettinger, M, P. Hanley, D, A. Hodsman, A, B. Kendler, D, L. Rosen, C, J. Enhancement of bone mass in osteoporotic women with parathyroid hormone followed by alendronate. Journal of Clinical Endocrinology \& Metabolism. 2000 Jun;85(6):2129-34. PMID: 10852440. No interventions of interest.

985. Ritzmann. Does alendronate lower the risk of fracture in reduced bone density? Benefit for women without previous fractures remains uncertain. Praxis. 1999;88(40):1631-2. PMID: CN-00891636. Not available in English.

986. Rizzoli R, Greenspan, S, L. Bone, G, rd, Schnitzer, T, J. Watts, N, B. Adami, S, Foldes, A, J. Roux, C, Levine, M, A. Uebelhart, B, Santora, A, C. nd, Kaur, A, Peverly, C, A. Orloff, J, J. Alendronate Once-Weekly, Study Group, Two-year results of once-weekly administration of alendronate $70 \mathrm{mg}$ for the treatment of postmenopausal osteoporosis. Journal of Bone \& Mineral Research. 2002 Nov;17(11):1988-96. PMID: 12412806. Ineligible treatment duration.

987. Rizzoli R, Reid, D, M. Ibandronate: An IV injection for the treatment for postmenopausal osteoporosis. Bone. 2007 November;41(5 SUPPL. 1):S24-S8. PMID: 47575999. Ineligible study design.

988. Rizzoli R. Zoledronic Acid for the treatment and prevention of primary and secondary osteoporosis. Therapeutic Advances in Musculoskeletal Disease. 2010 Feb;2(1):316. PMID: 22870433. Ineligible study design. 
989. Rizzoli R, Laroche, M, Krieg, M, A. Frieling, I, Thomas, T, Delmas, P, Felsenberg, D,. Strontium ranelate and alendronate have differing effects on distal tibia bone microstructure in women with osteoporosis. Rheumatology International. 2010 Aug;30(10):1341-8. PMID: 20512336. No outcomes of interest.

990. Rizzoli R, Reginster, J, Y. Boonen, S, Breart, G, Diez, Perez A, Felsenberg, D, Kaufman, J, M. Kanis, J, A. Cooper, C,. Adverse reactions and drug-drug interactions in the management of women with postmenopausal osteoporosis. Calcified Tissue International. 2011 Aug;89(2):91104. PMID: 21637997. Ineligible study design.

991. Rizzoli R, Akesson, K, Bouxsein, M, Kanis, J, A. Napoli, N, Papapoulos, S, Reginster, J, Y. Cooper, C,. Subtrochanteric fractures after long-term treatment with bisphosphonates: a European Society on Clinical and Economic Aspects of Osteoporosis and Osteoarthritis, and International Osteoporosis Foundation Working Group Report. Osteoporosis International. 2011 Feb;22(2):373-90. PMID: 21085935. Ineligible study design.

992. Rizzoli R. Bisphosphonates for postmenopausal osteoporosis: are they all the same? Qjm. 2011 Apr;104(4):281-300. PMID: 21258058. Ineligible study design.

993. Rizzoli R, Chapurlat, R, D. Laroche, J, M. Krieg, M, A. Thomas, T, Frieling, I, Boutroy, S, Laib, A, Bock, O, Felsenberg, D,. Effects of strontium ranelate and alendronate on bone microstructure in women with osteoporosis. Results of a 2-year study. Osteoporosis International. 2012 Jan;23(1):305-15. PMID: 21909729. No outcomes of interest.

994. Ro C, Cooper, O,. Bisphosphonate drug holiday: choosing appropriate candidates. Current Osteoporosis Reports. 2013 Mar;11(1):45-51. PMID: 23296595. Ineligible study design.
995. Robinson JD, Leighton, R, K. Trask, K, Bogdan, Y, Tornetta, P, rd,. Periprosthetic Atypical Femoral Fractures in Patients on Long-term Bisphosphonates: A Multicenter Retrospective Review. Journal of Orthopaedic Trauma. 2016 Apr;30(4):170-6. PMID: 27003029. Ineligible study design.

996. Roh Y, H. Noh, J, H. Gong, H, S. Baek, $\mathrm{G}, \mathrm{H}$. Comparative adherence to weekly oral and quarterly intravenous bisphosphonates among patients with limited heath literacy who sustained distal radius fractures. Journal of Bone and Mineral Metabolism. 201705 Oct:1-7. PMID: 618678198. No outcomes of interest.

997. Rollason V, Laverrière, Alexandra, MacDonald, Laura, Ci Walsh, Tanya, Tramèr, Martin, R. Vogt, Ferrier Nicole, B. Interventions for treating bisphosphonaterelated osteonecrosis of the jaw (BRONJ). Cochrane Database of Systematic Reviews. 2016(2) PMID: CD008455. Ineligible study design.

998. Rosen C, J. Chesnut, C, H. rd, Mallinak, $\mathrm{N}$, J. The predictive value of biochemical markers of bone turnover for bone mineral density in early postmenopausal women treated with hormone replacement or calcium supplementation. Journal of Clinical Endocrinology \& Metabolism. 1997 Jun;82(6):1904-10. PMID: 9177404. Ineligible treatment duration.

999. Rosen C, J. Hochberg, M, C. Bonnick, S, L. McClung, M, Miller, P, Broy, S, Kagan, R, Chen, E, Petruschke, R, A. Thompson, D, E. de, Papp A, E. Fosamax Actonel Comparison, Trial Investigators,. Treatment with once-weekly alendronate 70 mg compared with once-weekly risedronate $35 \mathrm{mg}$ in women with postmenopausal osteoporosis: a randomized double-blind study. Journal of Bone \& Mineral Research. 2005 Jan;20(1):141-51. PMID: 15619680. Ineligible treatment duration.

1000. Rosen C, J. Khosla, S,. Placebo-controlled trials in osteoporosis--proceeding with caution. New England Journal of Medicine. 2010 Sep 30;363(14):1365-7 discussion e22. PMID: 20879887. Ineligible study design. 
1001. Rosen H, N. Parker, R, A. Greenspan, S, L. Iloputaife, I, D. Bookman, L, Chapin, D, Perlmutter, I, Kessel, B, Qvist, P, Rosenblatt, M,. Evaluation of ability of biochemical markers of bone turnover to predict a response to increased doses of HRT. Calcified Tissue International. 2004 May;74(5):415-23. PMID: 14961215. No outcomes of interest.

1002. Rosenfeld J, A. Can the prophylactic use of raloxifene, a selective estrogen-receptor modulator, prevent bone mineral loss and fractures in women with diagnosed osteoporosis or vertebral fractures? Western Journal of Medicine. 2000 Sep;173(3):1868. PMID: 10986186. Ineligible study design.

1003. Rossini M, Gatti, D, Girardello, S, Braga, V, James, G, Adami, S,. Effects of two intermittent alendronate regimens in the prevention or treatment of postmenopausal osteoporosis. Bone. 2000 Jul;27(1):119-22. PMID: 10865218. Ineligible treatment duration.

1004. Rossini M, Lello, S, Sblendorio, I, Viapiana, O, Fracassi, E, Adami, S, Gatti, D,. Profile of bazedoxifene/conjugated estrogens for the treatment of estrogen deficiency symptoms and osteoporosis in women at risk of fracture. Drug design, development \& therapy. 2013;7:601-10. PMID: 23901263. Ineligible study design.

1005. Rossouw J, E. Anderson, G, L. Prentice, R, L. LaCroix, A, Z. Kooperberg, C, Stefanick, M, L. Jackson, R, D.

Beresford, S, A. Howard, B, V. Johnson, K, C. Kotchen, J, M. Ockene, J, Writing Group for the Women's Health, Initiative Investigators,. Risks and benefits of estrogen plus progestin in healthy postmenopausal women: principal results From the Women's Health Initiative randomized controlled trial. JAMA. 2002 Jul 17;288(3):321-33. PMID: 12117397. Ineligible population.

1006. Roux C, Seeman, E, Eastell, R, Adachi, J, Jackson, R, D. Felsenberg, D, Songcharoen, S, Rizzoli, R, Di, Munno O, Horlait, S, Valent, D, Watts, N, B.
Efficacy of risedronate on clinical vertebral fractures within six months. Current Medical Research \& Opinion. 2004 Apr;20(4):433-9. PMID: 15119979. Ineligible treatment duration.

1007. Roux C, Goldstein, J, L. Zhou, X, Klemes, A, Lindsay, R,. Vertebral fracture efficacy during risedronate therapy in patients using proton pump inhibitors. Osteoporosis International. 2012 Jan;23(1):277-84. PMID: 21365461. No outcomes of interest.

1008. Roux C, Hofbauer, L, C. Ho, P, R. Wark, J, D. Zillikens, M, C. Fahrleitner, Pammer A, Hawkins, F, Micaelo, M, Minisola, S, Papaioannou, N, Stone, M, Ferreira, I, Siddhanti, S, Wagman, R, B. Brown, J, P. Denosumab compared with risedronate in postmenopausal women suboptimally adherent to alendronate therapy: efficacy and safety results from a randomized openlabel study. Bone. 2014 Jan;58:48-54. PMID: 24141036. No outcomes of interest.

1009. Rubinacci A, Peruzzi, E, Modena, A, B. Zanardi, E, Andrei, B, De, Leo V, Pansini, F, S. Quebe, Fehling E, de, Palacios P, I. Effect of low-dose transdermal E2/NETA on the reduction of postmenopausal bone loss in women. Menopause. 2003 May-Jun;10(3):241-9. PMID: 12792297. No outcomes of interest.

1010. Ruza I, Mirfakhraee, S, Orwoll, E, Gruntmanis, U,. Clinical experience with intravenous zoledronic acid in the treatment of male osteoporosis: Evidence and opinions. Therapeutic Advances in Musculoskeletal Disease. 2013 November;5(4):182-98. PMID: 370250530. Ineligible study design.

1011. Saag K, G. Petersen, J, Brandi, M, L. Karaplis, A, C. Lorentzon, M, Thomas, T, Maddox, J, Fan, M, Meisner, P, D. Grauer, A,. Romosozumab or Alendronate for Fracture Prevention in Women with Osteoporosis. New England Journal of Medicine. 201710 12;377(15):1417-27. PMID: 28892457. No outcomes of interest.

1012. Saarelainen J, Hassi, S, Honkanen, R, Koivumaa, Honkanen H, Sirola, J, 
Kroger, H, Komulainen, $\mathrm{M}, \mathrm{H}$. Tuppurainen, M,. Bone loss and wrist fractures after withdrawal of hormone therapy: The 15-year follow-up of the OSTPRE cohort. Maturitas. 2016 Mar;85:49-55. PMID: 26857879. Ineligible population.

1013. Safford M, M. Barasch, A, Curtis, J, R. Outman, R, Saag, K,. Bisphosphonates and hip and nontraumatic subtrochanteric femoral fractures in the Veterans Health Administration. JCR: Journal of Clinical Rheumatology. 2014 Oct;20(7):357-62. PMID: 25275761. Ineligible treatment duration.

1014. Sahota O, Fowler, I, Blackwell, P, J. Lawson, N, Cawte, S, A. San, P, Masud, T, Hosking, D, J. A comparison of continuous alendronate, cyclical alendronate and cyclical etidronate with calcitriol in the treatment of postmenopausal vertebral osteoporosis: a randomized controlled trial. Osteoporosis International. 2000;11(11):959-66. PMID: 11193249. No outcomes of interest.

1015. Saita Y, Ishijima, M, Mogami, A, Kubota, M, Baba, T, Kaketa, T, Nagao, M, Sakamoto, Y, Sakai, K, Homma, Y, Kato, R, Nagura, N, Miyagawa, K, Wada, T, Liu, L, Matsuoka, J, Obayashi, $\mathrm{O}$, Shitoto, K, Nozawa, M, Kajihara, H, Gen, H, Kaneko, K,. The incidence of and risk factors for developing atypical femoral fractures in Japan. Journal of Bone \& Mineral Metabolism. 2015 May;33(3):3118. PMID: 24852205. Ineligible study design.

1016. Saito O, Saito, T, Asakura, S, Akimoto, $\mathrm{T}$, Inoue, M, Ando, Y, Muto, S, Kusano, E,. Effects of raloxifene on bone metabolism in hemodialysis patients with type 2 diabetes. International Journal of Endocrinology and Metabolism. 2012 April;10(2):464-9. PMID: 365275597. Ineligible treatment duration.

1017. Sakai A, Ito, M, Tomomitsu, T, Tsurukami, H, Ikeda, S, Fukuda, F, Mizunuma, $\mathrm{H}$, Inoue, $\mathrm{T}$, Saito, $\mathrm{H}$, Nakamura, T, Advanced Study, Group. Efficacy of combined treatment with alendronate (ALN) and eldecalcitol, a new active vitamin D analog, compared to that of concomitant ALN, vitamin D plus calcium treatment in Japanese patients with primary osteoporosis.[Erratum appears in Osteoporos Int. 2015 Apr 26(4):1453 PMID:

25656519]. Osteoporosis International. 2015 Mar;26(3):1193-202. PMID: 25592133. Ineligible treatment duration.

1018. Salari P, Abdollahi, M,. Long term bisphosphonate use in osteoporotic patients a step forward, two steps back. Journal of Pharmacy and Pharmaceutical Sciences. 201225 Apr;15(2):305-17. PMID: 364794626. Ineligible study design.

1019. Saleh A, Hegde, V, V. Potty, A, G. Lane, J, M. Bisphosphonate Therapy and Atypical Fractures. Orthopedic Clinics of North America. 2013 April;44(2):137-51. PMID: 368799857. Ineligible study design.

1020. Salmén H, Am, Mahonen, Kröger, Komulainen, Saarikoski, Honkanen, Mäenpää, Ph,. The protective effect of hormone-replacement therapy on fracture risk is modulated by estrogen receptor alpha genotype in early postmenopausal women. Journal of bone and mineral research. 2000;15(12):2479-86. PMID: CN00330176. Ineligible population.

1021. Salmén H, Am, Mahonen, Kröger, Komulainen, Saarikoski, Honkanen, Mäenpää, Ph,. Early postmenopausal bone loss is associated with PvuII estrogen receptor gene polymorphism in Finnish women: effect of hormone replacement therapy. Journal of bone and mineral research. 2000;15(2):315-21. PMID: CN00275813. Ineligible population.

1022. Salmen T, Heikkinen, A, M. Mahonen, A, Kroger, H, Komulainen, M, Saarikoski, S, Honkanen, R, Maenpaa, P, H. The protective effect of hormone-replacement therapy on fracture risk is modulated by estrogen receptor alpha genotype in early postmenopausal women. Journal of Bone \& Mineral Research. 2000 Dec;15(12):247986. PMID: 11127213. Ineligible population.

1023. Salmen T, Heikkinen, A, M. Mahonen, A, Kroger, H, Komulainen, M, Pallonen, H, 
Saarikoski, S, Honkanen, R, Maenpaa, P, $\mathrm{H}$. Relation of aromatase gene polymorphism and hormone replacement therapy to serum estradiol levels, bone mineral density, and fracture risk in early postmenopausal women. Annals of Medicine. 2003;35(4):282-8. PMID: 12846271. Ineligible population.

1024. Salmen T, Heikkinen, A, M. Mahonen, A, Kroger, H, Komulainen, M, Pallonen, H, Saarikoski, S, Honkanen, R, Maenpaa, P, $\mathrm{H}$. Relation of androgen receptor gene polymorphism to bone mineral density and fracture risk in early postmenopausal women during a 5-year randomized hormone replacement therapy trial. Journal of Bone \& Mineral Research. 2003 Feb;18(2):319-24. PMID: 12568409. Ineligible population.

1025. Salminen H, Sääf, Me, Johansson, Se, Ringertz, Strender, Le,. The effect of transvaginal estradiol on bone in aged women: a randomised controlled trial. Maturitas. 2007;57(4):370-81. PMID: CN00609740. No outcomes of interest.

1026. Sambrook P, N. Geusens, P, Ribot, C, Solimano, J, A. Ferrer, Barriendos J, Gaines, K, Verbruggen, N, Melton, M, E. Alendronate produces greater effects than raloxifene on bone density and bone turnover in postmenopausal women with low bone density: results of EFFECT (Efficacy of FOSAMAX versus EVISTA Comparison Trial) International. Journal of Internal Medicine. 2004 Apr;255(4):503-11. PMID: 15049885. No outcomes of interest.

1027. Sambrook P, Cranney, A, Adachi, J, D. Risk reduction of non-vertebral fractures with intravenous ibandronate: post-hoc analysis from DIVA. Current Medical Research \& Opinion. 2010 Mar;26(3):599604. PMID: 20055751. Ineligible treatment duration.

1028. Sambrook P, N. Cameron, I, D. Chen, J, S. March, L, M. Simpson, J, M. Cumming, R, G. Seibel, M, J. Oral bisphosphonates are associated with reduced mortality in frail older people: a prospective five-year study. Osteoporosis International.
2011 Sep;22(9):2551-6. PMID: 20959963. Ineligible treatment duration.

1029. Samelson E, J. Miller, P, D. Christiansen, C, Daizadeh, N, S. Grazette, L, Anthony, M, S. Egbuna, O, Wang, A, Siddhanti, S, R. Cheung, A, M. Franchimont, N, Kiel, D, P. RANKL inhibition with denosumab does not influence 3-year progression of aortic calcification or incidence of adverse cardiovascular events in postmenopausal women with osteoporosis and high cardiovascular risk. Journal of Bone \& Mineral Research. 2014 Feb;29(2):450-7. PMID: 23873632. No outcomes of interest.

1030. Sanad Z, Ellakwa, H, Desouky, B,. Comparison of alendronate and raloxifene in postmenopausal women with osteoporosis. Climacteric. 2011 Jun;14(3):369-77. PMID: 21254911. No outcomes of interest.

1031. Sarioglu M, Tuzun, C, Unlu, Z, Tikiz, C, Taneli, F, Uyanik, B, S. Comparison of the effects of alendronate and risedronate on bone mineral density and bone turnover markers in postmenopausal osteoporosis. Rheumatology International. 2006 Jan;26(3):195-200. PMID: 15580349. No outcomes of interest.

1032. Sarkar S, Mitlak, B, H. Wong, M, Stock, J, L. Black, D, M. Harper, K, D.

Relationships between bone mineral density and incident vertebral fracture risk with raloxifene therapy. Journal of Bone \& Mineral Research. 2002 Jan;17(1):1-10. PMID: 11771654. Ineligible treatment duration.

1033. Sarkar S, Reginster, J, Y. Crans, G, G. Diez, Perez A, Pinette, K, V. Delmas, P, D. Relationship between changes in biochemical markers of bone turnover and BMD to predict vertebral fracture risk. Journal of Bone \& Mineral Research. 2004 Mar;19(3):394-401. PMID: 15040827. Ineligible treatment duration.

1034. Sato M, Ye, W, Sugihara, T, Isaka, Y,. Fracture risk and healthcare resource utilization and costs among osteoporosis patients with type 2 diabetes mellitus and without diabetes mellitus in Japan: retrospective analysis of a hospital claims 
database. BMC Musculoskeletal Disorders. 2016 Nov 25;17(1):489. PMID: 27887655. Ineligible study design.

1035. Sattari M, Cauley, J, A. Garvan, C, Johnson, K, C. LaMonte, M, J. Li, W, Limacher, M, Manini, T, Sarto, G, E. Sullivan, S, D. Wactawski, Wende J, Beyth, R, J. Osteoporosis in the Women's Health Initiative: Another Treatment Gap? American Journal of Medicine. 2017 Aug;130(8):937-48. PMID: 28366425. Ineligible population.

1036. Sawada K, Morishige, K, Ohmichi, M, Nishio, Y, Yamamoto, T, Hayakawa, J, Mabuchi, S, Isobe, A, Sasaki, H, Sakata, M, Tasaka, K, Murata, Y,. Peripheral quantitative computed tomography (pQCT) is useful for monitoring bone mineral density of the patients who receive hormone replacement therapy. Maturitas. $2007 \mathrm{Apr}$ 20;56(4):343-9. PMID: 17010541. Ineligible population.

1037. Schaefers M, Muysers, C, Alexandersen, P, Christiansen, C,. Effect of microdose transdermal 17beta-estradiol compared with raloxifene in the prevention of bone loss in healthy postmenopausal women: a 2-year, randomized, double-blind trial. Menopause. 2009 May-Jun;16(3):559-65. PMID: 19423999. Ineligible treatment duration.

1038. Schafer A, L. Sellmeyer, D, E. Palermo, L, Hietpas, J, Eastell, R, Shoback, D, M. Black, D, M. Six months of parathyroid Hormone (1-84) administered concurrently versus sequentially with monthly ibandronate over two years: the PTH and ibandronate combination study (PICS) randomized trial. Journal of Clinical Endocrinology \& Metabolism. 2012 Oct;97(10):3522-9. PMID: 22791766. No outcomes of interest.

1039. Scheres LJJ, van Dijk FS, Harsevoort AJ, et al. Adults with osteogenesis imperfecta: Clinical characteristics of 151 patients with a focus on bisphosphonate use and bone density measurements. Bone Reports. 2018 June;8:168-72. PMID: 2000710582. Ineligible study design.
1040. Schilcher J, Michaelsson, K, Aspenberg, $\mathrm{P}$,. Bisphosphonate use and atypical fractures of the femoral shaft.[Erratum appears in N Engl J Med. 2011 Oct 20 365(16):1551], [Erratum appears in N Engl J Med. 2012 Aug 9 367(6):582]. New England Journal of Medicine. 2011 May 05;364(18):1728-37. PMID: 21542743. Ineligible treatment duration.

1041. Schmidt G, A. Horner, K, E. McDanel, D, L. Ross, M, B. Moores, K, G. Risks and benefits of long-term bisphosphonate therapy. American Journal of Health-System Pharmacy. 2010 Jun 15;67(12):994-1001. PMID: 20516469. Ineligible study design.

1042. Schneider P, F. Fischer, M, Allolio, B, Felsenberg, D, Schroder, U, Semler, J, Ittner, J, R. Alendronate increases bone density and bone strength at the distal radius in postmenopausal women. Journal of Bone \& Mineral Research. 1999 Aug;14(8):138793. PMID: 10457271. No outcomes of interest.

1043. Schnitzer T, Bone, H, G. Crepaldi, G, Adami, S, McClung, M, Kiel, D, Felsenberg, D, Recker, R, R. Tonino, R, P. Roux, C, Pinchera, A, Foldes, A, J. Greenspan, S, L. Levine, M, A. Emkey, R, Santora, A, C. nd, Kaur, A, Thompson, D, E. Yates, J, Orloff, J, J. Therapeutic equivalence of alendronate 70 mg once-weekly and alendronate $10 \mathrm{mg}$ daily in the treatment of osteoporosis. Alendronate Once-Weekly Study Group. Aging-Clinical \& Experimental Research. 2000 Feb;12(1):1-12. PMID: 10746426. Ineligible treatment duration.

1044. Schwartz A, V. Efficacy of Osteoporosis Therapies in Diabetic Patients. Calcified Tissue International. 201701 Feb;100(2):165-73. PMID: 611430954. Ineligible study design.

1045. Scotland G, Waugh, N, Royle, P, McNamee, P, Henderson, R, Hollick, R,. Denosumab for the prevention of osteoporotic fractures in post-menopausal women: a NICE single technology appraisal. Pharmacoeconomics. 2011 Nov;29(11):95161. PMID: 21854080. Ineligible study design. 
1046. Scott L, J. Denosumab: a review of its use in postmenopausal women with osteoporosis. Drugs \& Aging. 2014 Jul;31(7):555-76. PMID: 24935243. Ineligible study design.

1047. Sebba A, I. Bonnick, S, L. Kagan, R, Thompson, D, E. Skalky, C, S. Chen, E, de, Papp A, E. Fosamax Actonel Comparison, Trial investigators,. Response to therapy with once-weekly alendronate 70 mg compared to once-weekly risedronate 35 $\mathrm{mg}$ in the treatment of postmenopausal osteoporosis.[Erratum appears in Curr Med Res Opin. 2005 Feb 21(2):325]. Current Medical Research \& Opinion. 2004 Dec;20(12):2031-41. PMID: 15706659. No outcomes of interest.

1048. Sebba A, I. Significance of a decline in bone mineral density while receiving oral bisphosphonate treatment. Clinical Therapeutics. 2008 Mar;30(3):443-52. PMID: 18405784. Ineligible study design.

1049. Seeman E. The antifracture efficacy of alendronate. International Journal of Clinical Practice. Supplement. 1999 Apr;101:40-5. PMID: 12675021. Ineligible treatment duration.

1050. Seeman E, Delmas, P, D. Hanley, D, A. Sellmeyer, D, Cheung, A, M. Shane, E, Kearns, A, Thomas, T, Boyd, S, K. Boutroy, S, Bogado, C, Majumdar, S, Fan, M, Libanati, C, Zanchetta, J,. Microarchitectural deterioration of cortical and trabecular bone: differing effects of denosumab and alendronate. Journal of Bone \& Mineral Research. 2010 Aug;25(8):1886-94. PMID: 20222106. No outcomes of interest.

1051. Seibel M, J. Naganathan, V, Barton, I, Grauer, A,. Relationship between pretreatment bone resorption and vertebral fracture incidence in postmenopausal osteoporotic women treated with risedronate. Journal of Bone \& Mineral Research. 2004 Feb;19(2):323-9. PMID: 14969403. Ineligible treatment duration.

1052. Seki S, Hirano, N, Kawaguchi, Y, Nakano, M, Yasuda, T, Suzuki, K,
Watanabe, K, Makino, H, Kanamori, M, Kimura, T,. Teriparatide versus low-dose bisphosphonates before and after surgery for adult spinal deformity in female Japanese patients with osteoporosis. European Spine Journal. 201701 Aug;26(8):2121-7. PMID: 614228982. Ineligible study design.

1053. Sellmeyer D, E. Black, D, M. Palermo, L, Greenspan, S, Ensrud, K, Bilezikian, J, Rosen, C, J. Hetereogeneity in skeletal response to full-length parathyroid hormone in the treatment of osteoporosis.

Osteoporosis International. 2007

Jul;18(7):973-9. PMID: 17333451. No outcomes of interest.

1054. Sethi BCMMKKKMRU. Efficacy of Teriparatide in Increasing Bone Mineral Density in Postmenopausal Women with Osteoporosis ? An Indian Experience. Journal of the association of physicians of india. 2008;56:418-24. PMID: CN01093380. Ineligible treatment duration.

1055. Shand B, Gilchrist, N, Blackwell, T, March, R,. The hemorheological effects of raloxifene in postmenopausal women with osteoporosis. Results of a 3-year placebocontrolled clinical trial. Clinical Hemorheology \& Microcirculation. 2002;26(4):249-55. PMID: 12122230. No outcomes of interest.

1056. Sharifi M, Lewiecki, E, M. Conjugated estrogens combined with bazedoxifene: the first approved tissue selective estrogen complex therapy. Expert Review of Clinical Pharmacology. 2014 May;7(3):281-91. PMID: 24580081. Ineligible study design.

1057. Sharma A, Einstein, A, J. Vallakati, A, Arbab, Zadeh A, Walker, M, D. Mukherjee, D, Homel, P, Borer, J, S. Lichstein, E,. Risk of atrial fibrillation with use of oral and intravenous bisphosphonates. American Journal of Cardiology. 2014 Jun 01;113(11):1815-21. PMID: 24837258. Ineligible study design.

1058. Sharman MS, Yu, J, Goldshtein, I, Ish, Shalom S, Rouach, V, Shalev, V, Modi, A, Chodick, G,. Cost and Consequences of Nonadherence With Oral Bisphosphonate Therapy: Findings From a Real-World Data 
Analysis. Annals of Pharmacotherapy. 2016 Apr;50(4):262-9. PMID: 26783359.

Ineligible study design.

1059. Shigematsu T, Muraoka, R, Sugimoto, T, Nishizawa, Y,. Risedronate therapy in patients with mild-to-moderate chronic kidney disease with osteoporosis: post-hoc analysis of data from the risedronate phase III clinical trials. BMC Nephrology. 2017 Feb 15;18(1):66. PMID: 28201994. Ineligible treatment duration.

1060. Shih A, W. Weir, M, A. Clemens, K, K. Yao, Z, Gomes, T, Mamdani, M, M. Juurlink, D, N. Hird, A, Hodsman, A, Parikh, C, R. Wald, R, Cadarette, S, M. Garg, A, X. Oral bisphosphonate use in the elderly is not associated with acute kidney injury. Kidney International. 2012 Oct;82(8):903-8. PMID: 22695327. Ineligible treatment duration.

1061. Shilbayeh S, S. Zumeili A Hilow, H, M. The efficacy and safety of Calidron tablets for management of osteoporosis in Jordanian women: A randomised clinical trial. Saudi Pharmaceutical Journal. 2004 April/July;12(2-3):86-95. PMID: 39013821. No outcomes of interest.

1062. Shiota E, Tsuchiya, K, Yamaoka, K, Kawano, O,. Effect of intermittent cyclical treatment with etidronate disodium (HEBP) and calcium plus alphacalcidol in postmenopausal osteoporosis. Journal of Orthopaedic Science. 2001;6(2):133-6. PMID: 11484098. No interventions of interest.

1063. Shiraki M, Nakamura, T, Fukunaga, M, Sone, T, Usami, A, Inoue, T,. A multicenter randomized double-masked comparative study of different preparations of alendronate in osteoporosis - monthly (four weeks) intravenous versus once weekly oral administrations. Current Medical Research \& Opinion. 2012 Aug;28(8):1357-67. PMID: 22769235. No outcomes of interest.

1064. Shiraki M, Ueda, S, Sugimoto, T, Kuroda, T, Nakamura, T,. Treatment responses with once-weekly teriparatide therapy for osteoporosis. Osteoporosis International.
2016 Oct;27(10):3057-62. PMID: 27234671. No outcomes of interest.

1065. Shoback D. Update in osteoporosis and metabolic bone disorders. Journal of Clinical Endocrinology \& Metabolism. 2007 Mar;92(3):747-53. PMID: 17341572. Ineligible study design.

1066. Sidlauskas K, M. Sutton, E, E. Biddle, M, A. Osteoporosis in men: Epidemiology and treatment with denosumab. Clinical Interventions in Aging. 201408 Apr;9:593601. PMID: 372819653. Ineligible study design.

1067. Silva B, C. Bilezikian, J, P. New approaches to the treatment of osteoporosis. Annual Review of Medicine. 2011;62:30722. PMID: 21054170. Ineligible study design.

1068. Silva I, Branco, J, C. Denosumab: recent update in postmenopausal osteoporosis. Acta Reumatologica Portuguesa. 2012 OctDec;37(4):302-13. PMID: 24126422. Ineligible study design.

1069. Silverman S, L. Delmas, P, D. Kulkarni, P, M. Stock, J, L. Wong, M, Plouffe, L, $\mathrm{Jr}$,. Comparison of fracture, cardiovascular event, and breast cancer rates at 3 years in postmenopausal women with osteoporosis. Journal of the American Geriatrics Society. 2004 Sep;52(9):1543-8. PMID: 15341559. Ineligible study design.

1070. Silverman S, L. Maricic, M,. Recent developments in bisphosphonate therapy. Seminars in Arthritis \& Rheumatism. 2007 Aug;37(1):1-12. PMID: 17303219. Ineligible study design.

1071. Silverman S, L. Watts, N, B. Delmas, P, D. Lange, J, L. Lindsay, R,. Effectiveness of bisphosphonates on nonvertebral and hip fractures in the first year of therapy: the risedronate and alendronate (REAL) cohort study. Osteoporosis International. 2007 Jan;18(1):25-34. PMID: 17106785. Ineligible study design.

1072. Silverman S, L. Christiansen, C, Genant, H, K. Vukicevic, S, Zanchetta, J, R. de, 
Villiers T, J. Constantine, G, D. Chines, A, A. Efficacy of bazedoxifene in reducing new vertebral fracture risk in postmenopausal women with osteoporosis: results from a 3-year, randomized, placebo-, and active-controlled clinical trial. Journal of Bone \& Mineral Research. 2008 Dec;23(12):1923-34. PMID: 18665787. Ineligible treatment duration.

1073. Silverman S, L. Chines, A, A. Kendler, D, L. Kung, A, W. Teglbjaerg, C, S. Felsenberg, D, Mairon, N, Constantine, G, D. Adachi, J, D. Bazedoxifene, Study Group,. Sustained efficacy and safety of bazedoxifene in preventing fractures in postmenopausal women with osteoporosis: results of a 5-year, randomized, placebocontrolled study. Osteoporosis International. 2012 Jan;23(1):351-63. PMID: 21779819.

No interventions of interest.

1074. Sim IW, Ebeling, P, R. Treatment of osteoporosis in men with bisphosphonates: rationale and latest evidence. Therapeutic Advances in Musculoskeletal Disease. 2013 Oct;5(5):259-67. PMID: 24101947. Ineligible study design.

1075. Simon J, A. Recknor, C, Moffett, A, H. Jr, Adachi, J, D. Franek, E, Lewiecki, E, M. McClung, M, R. Mautalen, C, A. Ragi, Eis S, Nicholson, G, C. Muschitz, C, Nuti, R, Torring, O, Wang, A, Libanati, C,. Impact of denosumab on the peripheral skeleton of postmenopausal women with osteoporosis: bone density, mass, and strength of the radius, and wrist fracture. Menopause. 2013 Feb;20(2):130-7. PMID: 23010883. Ineligible treatment duration.

1076. Sing CW, Wong AY, Kiel DP, et al. Association of Alendronate and Risk of Cardiovascular Events in Patients With Hip Fracture. Journal of Bone and Mineral Research. 2018 PMID: 622092167. Ineligible treatment duration.

1077. Singer A, Grauer, A,. Denosumab for the management of postmenopausal osteoporosis. Postgraduate Medicine. 2010 Nov;122(6):176-87. PMID: 21084794. Ineligible study design.
1078. Siris E, Adachi, J, D. Lu, Y, Fuerst, T, Crans, G, G. Wong, M, Harper, K, D. Genant, H, K. Effects of raloxifene on fracture severity in postmenopausal women with osteoporosis: results from the MORE study. Multiple Outcomes of Raloxifene Evaluation. Osteoporosis International. 2002 Nov;13(11):907-13. PMID: 12415439. Ineligible treatment duration.

1079. Siris E, S. Simon, J, A. Barton, I, P. McClung, M, R. Grauer, A,. Effects of risedronate on fracture risk in postmenopausal women with osteopenia. Osteoporosis International. 2008 May;19(5):681-6. PMID: 17968610. Ineligible treatment duration.

1080. Skelton D, A. Phillips, S, K. Bruce, S, A. Naylor, C, H. Woledge, R, C. Hormone replacement therapy increases isometric muscle strength of adductor pollicis in postmenopausal women. Clinical Science. 1999 Apr;96(4):357-64. PMID: 10087242. Ineligible treatment duration.

1081. Smith B, J. Laslett, L, L. Pile, K, D. Phillips, P, J. Phillipov, G, Evans, S, M. Esterman, A, J. Berry, J, G. Randomized controlled trial of alendronate in airways disease and low bone mineral density. Chronic Respiratory Disease. 2004;1(3):131-7. PMID: 16281654. No outcomes of interest.

1082. Solomon D, H. Hochberg, M, C. Mogun, $\mathrm{H}$, Schneeweiss, S,. The relation between bisphosphonate use and non-union of fractures of the humerus in older adults. Osteoporosis International. 2009 Jun;20(6):895-901. PMID: 18843515. Ineligible study design.

1083. Solomon D, H. Mercer, E, Woo, S, B. Avorn, J, Schneeweiss, S, Treister, N,. Defining the epidemiology of bisphosphonate-associated osteonecrosis of the jaw: prior work and current challenges. Osteoporosis International. 2013 Jan;24(1):237-44. PMID: 22707065. Ineligible study design.

1084. Song J, Jin, Z, Chang, F, Li, L, Su, Y,. Single and combined use of human parathyroid hormone (PTH) (1-34) on areal 
bone mineral density (aBMD) in postmenopausal women with osteoporosis: evidence based on 9 RCTs. Medical Science Monitor. 2014 Dec 12;20:2624-32. PMID: 25503108. Ineligible study design.

1085. Sorensen H, T. Christensen, S, Mehnert, F, Pedersen, L, Chapurlat, R, D. Cummings, S, R. Baron, J, A. Use of bisphosphonates among women and risk of atrial fibrillation and flutter: population based case-control study. BMJ. $2008 \mathrm{Apr}$ 12;336(7648):813-6. PMID: 18334527. Ineligible treatment duration.

1086. Sornay RE, Garnero, P, Munoz, F, Duboeuf, F, Delmas, P, D. Effect of withdrawal of hormone replacement therapy on bone mass and bone turnover: the OFELY study. Bone. 2003 Jul;33(1):159-66. PMID: 12919711. Ineligible study design.

1087. Sosa H, Segarra, Mc, Gómez, Peña, Betancor,. Effect of two forms of alendronate administration upon bone mass after two years of treatment. Journal of clinical densitometry. 2002;5(1):27-34. PMID: CN-00388877. No outcomes of interest.

1088. Speroff L, Rowan, J, Symons, J, Genant, $\mathrm{H}$, Wilborn, W,. The comparative effect on bone density, endometrium, and lipids of continuous hormones as replacement therapy (CHART study). A randomized controlled trial. JAMA. 1996 Nov 06;276(17):1397-403. PMID: 8892714. Ineligible treatment duration.

1089. Spiegel R, Nawroth, P, P. Kasperk, C,. The effect of zoledronic acid on the fracture risk in men with osteoporosis. Journal of Endocrinological Investigation. 2014 Mar;37(3):229-32. PMID: 24474679. Ineligible study design.

1090. St?pán J, Vokrouhlická,. Comparison of biochemical markers of bone remodelling in the assessment of the effects of alendronate on bone in postmenopausal osteoporosis. Clinica chimica acta international journal of clinical chemistry. 1999;288(1-2):121-35. PMID: CN-00263801. No outcomes of interest.
1091. Stakkestad J, A. Benevolenskaya, L, I. Stepan, J, J. Skag, A, Nordby, A, Oefjord, E, Burdeska, A, Jonkanski, I, Mahoney, P, Ibandronate Intravenous, Study Group,. Intravenous ibandronate injections given every three months: a new treatment option to prevent bone loss in postmenopausal women. Annals of the Rheumatic Diseases. 2003 Oct;62(10):96975. PMID: 12972476. Ineligible treatment duration.

1092. Stakkestad J, A. Lakatos, P, Lorenc, R, Sedarati, F, Neate, C, Reginster, J, Y. Monthly oral ibandronate is effective and well tolerated after 3 years: the MOBILE long-term extension. Clinical Rheumatology. 2008 Aug;27(8):955-60. PMID: 18180976. No outcomes of interest.

1093. Stanosz S, Puchalski,. An assessment of the influence of fluoride, modified transdermal replacement hormone therapy and supplement hormone therapy on unmanageable osteoporosis in postmenopausal women. Journal of elementology. 2009;14(3):545-51. PMID: CN-00790610. No outcomes of interest.

1094. Ste ML, G. Schwartz, S, L. Hossain, A, Desaiah, D, Gaich, G, A. Effect of teriparatide [rhPTH(1-34)] on BMD when given to postmenopausal women receiving hormone replacement therapy. Journal of Bone \& Mineral Research. 2006 Feb;21(2):283-91. PMID: 16418784. Ineligible treatment duration.

1095. Steinbuch M, D'Agostino, R, B. Mandel, J, S. Gabrielson, E, McClung, M, R. Stemhagen, A, Hofman, A,. Assessment of mortality in patients enrolled in a risedronate clinical trial program: a retrospective cohort study. Regulatory Toxicology \& Pharmacology. 2002 Jun;35(3):320-6. PMID: 12202047. Ineligible treatment duration.

1096. Stepan J, J. Vokrouhlicka, J,. Comparison of biochemical markers of bone remodelling in the assessment of the effects of alendronate on bone in postmenopausal osteoporosis. Clinica Chimica Acta. 1999 
Oct;288(1-2):121-35. PMID: 10529464. No outcomes of interest.

1097. Stevenson J, C. Teter, P, Lees, B,. 17betaestradiol (1 mg/day) continuously combined with dydrogesterone ( 5,10 or $20 \mathrm{mg} /$ day) increases bone mineral density in postmenopausal women. Maturitas. 2001 Apr 20;38(2):197-203. PMID: 11306209. Ineligible treatment duration.

1098. Stevenson J, C. Prevention of osteoporosis: one step forward, two steps back. Menopause International. 2011 Dec;17(4):137-41. PMID: 22120943. Ineligible study design.

1099. Stevenson J, C. Panay, N, Pexman, Fieth C,. Oral estradiol and dydrogesterone combination therapy in postmenopausal women: Review of efficacy and safety. Maturitas. 2013 September;76(1):10-21. PMID: 52667176. Ineligible study design.

1100. Stock J, L. Bell, N, H. Chesnut, C, H. rd, Ensrud, K, E. Genant, H, K. Harris, S, T. McClung, M, R. Singer, F, R. Yood, R, A. Pryor, Tillotson S, Wei, L, Santora, A, C. nd,. Increments in bone mineral density of the lumbar spine and hip and suppression of bone turnover are maintained after discontinuation of alendronate in postmenopausal women. American Journal of Medicine. 1997 Oct;103(4):291-7. PMID: 9382121. No outcomes of interest.

1101. Stovall D, W. Beard, M, K. Barbier, S, Chen, E, Rosenberg, E, de, Papp A, E. Response to oral bisphosphonates in subgroups of younger and older postmenopausal women. Journal of Women's Health. 2010 Mar;19(3):491-7. PMID: 20141367. Ineligible treatment duration.

1102. Stovall D, W. Tanner, Kurtz K, Pinkerton, $\mathrm{J}, \mathrm{V}$. Tissue-selective estrogen complex bazedoxifene and conjugated estrogens for the treatment of menopausal vasomotor symptoms. Drugs. 2011 Sep

10;71(13):1649-57. PMID: 21902289.

Ineligible study design.
1103. Strampel W, Emkey, R, Civitelli, R,. Safety considerations with bisphosphonates for the treatment of osteoporosis. Drug Safety. 2007;30(9):755-63. PMID: 17722968. Ineligible study design.

1104. Stroup J, Kane, M, P. Abu, Baker A, M. Teriparatide in the treatment of osteoporosis. American Journal of Health-System Pharmacy. 2008 Mar 15;65(6):532-9. PMID: 18319498. Ineligible study design.

1105. Studd J, W. MacCarthy, K, Zamblera, D, Dain, M, P. Efficacy and safety of Menorest (50 mikrog/day) compared to Premarin $0.625 \mathrm{mg}$ in the treatment of menopausal symptoms and the prevention of bone loss, in menopausal women. A single-center, comparative, randomized, double-blind, double-dummy study. Scandinavian Journal of Rheumatology - Supplement. 1996;103:89-90. PMID: 8966497. Ineligible study design.

1106. Stump A, L. Kelley, K, W. Wensel, T, M. Bazedoxifene: a third-generation selective estrogen receptor modulator for treatment of postmenopausal osteoporosis. Annals of Pharmacotherapy. 2007 May;41(5):833-9. PMID: 17426077. Ineligible study design.

1107. Sugiyama T, Torio, T, Miyajima, T, Kim, Y, T. Oda, H,. Romosozumab and blosozumab: Alternative drugs of mechanical strain-related stimulus toward a cure for osteoporosis. Frontiers in Endocrinology. 2015;6 (APR) (no pagination)(54) PMID: 604705795. Ineligible study design.

1108. Sumino H, Ichikawa, S, Kasama, S, Takahashi, T, Sakamoto, H, Koizumi, A, Kanai, H, Araki, Y, Itoh, T, Iwasaki, T, Sawada, Y, Saito, Y, Kumakura, H, Takayama, Y, Kanda, T, Murakami, M, Sakamaki, T, Kurabayashi, M,. Effects of raloxifene on brachial arterial endothelial function, carotid wall thickness, and arterial stiffness in osteoporotic postmenopausal women. International Heart Journal. 2010 January;51(1):60-7. PMID: 358307262. Ineligible study design.

1109. Suresh E, Pazianas, M, Abrahamsen, B,. Safety issues with bisphosphonate therapy 
for osteoporosis. Rheumatology. 2014

Jan;53(1):19-31. PMID: 23838024.

Ineligible study design.

1110. Sutton E, E. Riche, D, M. Denosumab, a RANK ligand inhibitor, for postmenopausal women with osteoporosis. Annals of Pharmacotherapy. 2012 Jul-Aug;46(78):1000-9. PMID: 22837347. Ineligible study design.

1111. Tabatabaei MO, Salari, P, Khashayar, P, Larijani, B,. New horizons in treatment of osteoporosis. Daru: Journal of Faculty of Pharmacy, Tehran University of Medical Sciences. 2017 Feb 07;25(1):2. PMID: 28173850. Ineligible study design.

1112. Taechakraichana N, Limpaphayom, K, Ninlagarn, T, Panyakhamlerd, K, Chaikittisilpa, S, Dusitsin, N,. A randomized trial of oral contraceptive and hormone replacement therapy on bone mineral density and coronary heart disease risk factors in postmenopausal women. Obstetrics \& Gynecology. 2000 Jan;95(1):87-94. PMID: 10636509. No outcomes of interest.

1113. Tan W, Sun, J, Zhou, L, Li, Y, Wu, X, Randomized trial comparing efficacies of zoledronate and alendronate for improving bone mineral density and inhibiting bone remodelling in women with postmenopausal osteoporosis. Journal of Clinical Pharmacy \& Therapeutics. 2016 Oct;41(5):519-23. PMID: 27440710. No outcomes of interest.

1114. Tanaka S, Kuroda, T, Sugimoto, T, Nakamura, T, Shiraki, M,. Changes in bone mineral density, bone turnover markers, and vertebral fracture risk reduction with once weekly teriparatide. Current Medical Research \& Opinion. 2014 May;30(5):931-6. PMID: 24392946. Ineligible treatment duration.

1115. Tanaka S, Yamamoto, T, Oda, E, Nakamura, M, Fujiwara, S,. Real-world evidence of raloxifene versus alendronate in preventing non-vertebral fractures in Japanese women with osteoporosis: retrospective analysis of a hospital claims database. Journal of Bone \& Mineral
Metabolism. 2016 Dec 27;27:27. PMID: 28028633. Ineligible study design.

1116. Tanaka S, Miyazaki, T, Uemura, Y, Miyakawa, N, Gorai, I, Nakamura, T, Fukunaga, M, Ohashi, Y, Ohta, H, Mori, S, Hagino, H, Hosoi, T, Sugimoto, T, Itoi, E, Orimo, H, Shiraki, M, Comparison of concurrent treatment with vitamin $\mathrm{K}<\mathrm{inf}>2</ \mathrm{inf}>$ and risedronate compared with treatment with risedronate alone in patients with osteoporosis: Japanese Osteoporosis Intervention Trial-03. Journal of Bone and Mineral Metabolism. 201701 Jul;35(4):385-95. PMID: 611564346. No interventions of interest.

1117. Tanko L, B. Felsenberg, D, Czerwinski, E, Burdeska, A, Jonkanski, I, Hughes, C, Christiansen, C, Oral, Ibandronate Group,. Oral weekly ibandronate prevents bone loss in postmenopausal women. Journal of Internal Medicine. 2003 Aug;254(2):159-67. PMID: 12859697. Ineligible treatment duration.

1118. Tanko L, B. Qin, G, Alexandersen, P, Bagger, Y, Z. Christiansen, C,. Effective doses of ibandronate do not influence the 3year progression of aortic calcification in elderly osteoporotic women. Osteoporosis International. 2005 Feb;16(2):184-90. PMID: 15197541. No outcomes of interest.

1119. Tankó L, Felsenberg, Czerwi?ski, Burdeska, Jonkanski, Hughes, Christiansen,. Oral weekly ibandronate prevents bone loss in postmenopausal women. Journal of internal medicine. 2003;254(2):159-67. PMID: CN-00439337. Ineligible treatment duration.

1120. Taquet A, Clemmensen,. A three-year double-blind placebo-controlled study of risedronate in postmenopausal osteoporosis. Osteoporos-int. 1996;6 Suppl 1:262. PMID: $\mathrm{CN}-00260120$. Ineligible study design.

1121. Tarantino U, Celi, M, Feola, M, Liuni, F, M. Resmini, G, Iolascon, G,. A new antiresorptive approach to the treatment of fragility fractures: long-term efficacy and safety of denosumab. Aging-Clinical \& Experimental Research. 2013 Oct;25 Suppl 
1:S65-9. PMID: 24046047. Ineligible study design.

1122. Tella S, H. Gallagher, J, C. Biological agents in management of osteoporosis. European Journal of Clinical Pharmacology. 2014 Nov;70(11):1291-301. PMID: 25204309. Ineligible study design.

1123. Tennis P, Rothman, K, J. Bohn, R, L. Tan, H, Zavras, A, Laskarides, C, Calingaert, B, Anthony, M, S. Incidence of osteonecrosis of the jaw among users of bisphosphonates with selected cancers or osteoporosis. Pharmacoepidemiology \& Drug Safety. 2012 Aug;21(8):810-7. PMID: 22711458. Ineligible study design.

1124. Terauchi M, Obayashi, S, Aso, T,. Estriol, conjugated equine estrogens, and alendronate therapy for osteoporosis. International Journal of Gynaecology \& Obstetrics. 2006 Feb;92(2):141-2. PMID: 16305796. Ineligible study design.

1125. Thadani S, R. Ristow, B, Blackwell, T, Mehra, R, Stone, K, L. Marcus, G, M. Varosy, P, D. Cummings, S, R. Cawthon, P, M. Osteoporotic Fractures in Men Study, Research Group,. Relationship of Bisphosphonate Therapy and Atrial Fibrillation/Flutter: Outcomes of Sleep Disorders in Older Men (MrOS Sleep) Study. Chest. 2016 May;149(5):1173-80. PMID: 26836889. Ineligible treatment duration.

1126. Thiebaud D, Burckhardt, P, Kriegbaum, H, Huss, H, Mulder, H, Juttmann, J, R. Schoter, K, H. Three monthly intravenous injections of ibandronate in the treatment of postmenopausal osteoporosis. American Journal of Medicine. 1997 Oct;103(4):298307. PMID: 9382122. Ineligible treatment duration.

1127. Thiebaud D, Bigler, J, M. Renteria, S, Pache, T, Welti, H, J. Landry, M, Burckhardt, P,. A 3-year study of prevention of postmenopausal bone loss: conjugated equine estrogens plus medroxyprogesterone acetate versus tibolone. Climacteric. 1998 Sep;1(3):202-10. PMID: 11907945. Ineligible treatment duration.
1128. Thiebaud K, Huss, Christiansen, Burckhardt,. Intravenous injections of ibandronate in the treatment of postmenopausal osteoporosis. 1996 PMID: $\mathrm{CN}-00846245$. Ineligible study design.

1129. Thompson R, N. Phillips, J, R. McCauley, S, H. Elliott, J, R. Moran, C, G. Atypical femoral fractures and bisphosphonate treatment: experience in two large United Kingdom teaching hospitals. Journal of Bone \& Joint Surgery - British Volume. 2012 Mar;94(3):385-90. PMID: 22371548. Ineligible study design.

1130. Thorsteinsson A, L. Vestergaard, P, Eiken, $\mathrm{P}$, . External auditory canal and middle ear cholesteatoma and osteonecrosis in bisphosphonate-treated osteoporosis patients: a Danish national register-based cohort study and literature review. Osteoporosis International. 2014 Jul;25(7):1937-44. PMID: 24664275. No outcomes of interest.

1131. Thorsteinsson AL, Hansen L, Vestergaard P, et al. Long-term benefits and risks of parathyroid hormone treatment in compliant osteoporotic patients. A Danish national register based cohort study. Archives of Osteoporosis. 201801 Dec;13 (1) (no pagination)(50) PMID: 621938336. Ineligible treatment duration.

1132. Tira M, Noyan, Yildiz, Yildirim, Daya,. Effects of alendronate and hormone replacement therapy, alone or in combination, on bone mass in postmenopausal women with osteoporosis: a prospective, randomized study. Human reproduction (oxford, england). 2000;15(10):2087-92. PMID: CN00331106. No outcomes of interest.

1133. Tiras M, B. Noyan, V, Yildiz, A, Yildirim, M, Daya, S,. Effects of alendronate and hormone replacement therapy, alone or in combination, on bone mass in postmenopausal women with osteoporosis: a prospective, randomized study. Human Reproduction. 2000 Oct;15(10):2087-92. PMID: 11006178. No outcomes of interest. 
1134. Tripto SL, Segal, E, Jaffe, A, Ish, Shalom S, Bachrach, R, Nachtigal, A, Militianu, D,. Atypical Femoral Fractures: Radiological Evaluation and Bisphosphonate Exposure. Israel Medical Association Journal: Imaj. 2015 Sep;17(9):554-8. PMID: 26625545. Ineligible study design.

1135. Tsai J, N. Uihlein, A, V. Lee, H, Kumbhani, R, Siwila, Sackman E, McKay, E, A. Burnett, Bowie S, A. Neer, R, M. Leder, B, Z. Teriparatide and denosumab, alone or combined, in women with postmenopausal osteoporosis: the DATA study randomised trial. Lancet. 2013 Jul 06;382(9886):50-6. PMID: 23683600. No outcomes of interest.

1136. Tsai J, N. Uihlein, A, V. Burnett, Bowie S, M. Neer, R, M. Derrico, N, P. Lee, H, Bouxsein, M, L. Leder, B, Z. Effects of Two Years of Teriparatide, Denosumab, or Both on Bone Microarchitecture and Strength (DATA-HRpQCT study). Journal of Clinical Endocrinology \& Metabolism. 2016 May;101(5):2023-30. PMID: 26964731. No outcomes of interest.

1137. Tsai J, N. Jiang, L, A. Lee, H, Hans, D, Leder, B, Z. Effects of Teriparatide, Denosumab, or Both on Spine Trabecular Microarchitecture in DATA-Switch: a Randomized Controlled Trial. Journal of Clinical Densitometry. 2017 October;20(4):507-12. PMID: 616793935. No outcomes of interest.

1138. Tsai J, N. Nishiyama, K, K. Lin, D, Yuan, A, Lee, H, Bouxsein, M, L. Leder, B, Z. Effects of Denosumab and Teriparatide Transitions on Bone Microarchitecture and Estimated Strength: the DATA-Switch HR-pQCT study. Journal of Bone and Mineral Research. 2017 October;32(10):2001-9. PMID: 618758916. No outcomes of interest.

1139. Tsai J, N. Burnett, Bowie S, M. Lee, H, Leder, B, Z. Relationship between bone turnover and density with teriparatide, denosumab or both in women in the DATA study. Bone. 201701 Feb;95:20-5. PMID: 613170230. No outcomes of interest.
1140. Tsai K, S. Yen, M, L. Pan, H, A. Wu, M, H. Cheng, W, C. Hsu, S, H. Yen, B, L. Huang, K, E. Raloxifene versus continuous combined estrogen/progestin therapy: densitometric and biochemical effects in healthy postmenopausal Taiwanese women. Osteoporosis International. 2001 Dec;12(12):1020-5. PMID: 11846327. Ineligible treatment duration.

1141. Tseng L, N. Sheu, W, H. Ho, E, S. Lan, H, H. Hu, C, C. Kao, C, H. Effects of alendronate combined with hormone replacement therapy on osteoporotic postmenopausal Chinese women. Metabolism: Clinical \& Experimental. 2006 Jun;55(6):741-7. PMID: 16713432. No outcomes of interest.

1142. Tsujimoto M, Chen, P, Miyauchi, A, Sowa, H, Krege, J, H. PINP as an aid for monitoring patients treated with teriparatide. Bone. 2011 Apr 01;48(4):798-803. PMID: 21168536. No outcomes of interest.

1143. Tsujimoto M, Uenaka, K, Iwata, A, Higashiuchi, Y, Sowa, H,. Effects of teriparatide in Japanese and non-Japanese populations: bridging findings on pharmacokinetics and efficacy. Journal of Bone \& Mineral Metabolism. 2012 May;30(3):326-37. PMID: 21938381. Ineligible study design.

1144. Tucci J, R. Tonino, R, P. Emkey, R, D. Peverly, C, A. Kher, U, Santora, A, C. nd,. Effect of three years of oral alendronate treatment in postmenopausal women with osteoporosis. American Journal of Medicine. 1996 Nov;101(5):488-501. PMID: 8948272. Ineligible treatment duration.

1145. Tuppurainen H, Komulainen, Kiviniemi, Kröger, Honkanen, Alhava, Jurvelin, Saarikoski,. Effects of continuous combined hormone replacement therapy and clodronate on bone mineral density in osteoporotic postmenopausal women: a 5year follow-up. Maturitas. 2010;66(4):42330. PMID: CN-00763120. No interventions of interest. 
1146. Tuppurainen M, Harma, K, Komulainen, M, Kiviniemi, V, Kroger, H, Honkanen, R, Alhava, E, Jurvelin, J, Saarikoski, S, Effects of continuous combined hormone replacement therapy and clodronate on bone mineral density in osteoporotic postmenopausal women: a 5-year follow-up. Maturitas. 2010 Aug;66(4):423-30. PMID: 20547017. No outcomes of interest.

1147. Uchida S, Taniguchi, T, Shimizu, T, Kakikawa, T, Okuyama, K, Okaniwa, M, Arizono, H, Nagata, K, Santora, A, C. Shiraki, M, Fukunaga, M, Tomomitsu, T, Ohashi, Y, Nakamura, T,. Therapeutic effects of alendronate $35 \mathrm{mg}$ once weekly and $5 \mathrm{mg}$ once daily in Japanese patients with osteoporosis: a double-blind, randomized study. Journal of Bone \& Mineral Metabolism. 2005;23(5):382-8. PMID: 16133688. Ineligible treatment duration.

1148. Um M, J. Cho, E, A. Jung, H,. Combination Therapy of Raloxifene and Alendronate for Treatment of Osteoporosis in Elderly Women. Journal of menopausal medicine. 2017 Apr;23(1):56-62. PMID: 28523260. No outcomes of interest.

1149. Unnanuntana A, Jarusriwanna, A, Songcharoen, P,. Randomized clinical trial comparing efficacy and safety of brand versus generic alendronate (Bonmax) for osteoporosis treatment. PLoS ONE [Electronic Resource]. 2017;12(7):e0180325. PMID: 28678853. No outcomes of interest.

1150. Urushihara H, Kikuchi, N, Yamada, M, Yoshiki, F, Miyauchi, A,. Raloxifene and stroke risks in Japanese postmenopausal women with osteoporosis on postmarketing surveillance. Menopause. 2009 SepOct;16(5):971-7. PMID: 19357545. Ineligible treatment duration.

1151. Utian W, Gass, Ml, Pickar, Jh,. Body mass index does not influence response to treatment, nor does body weight change with lower doses of conjugated estrogens and medroxyprogesterone acetate in early postmenopausal women. Menopause (new york, N.Y.). 2004;11(3):306-14. PMID:
CN-00482115. Ineligible treatment duration.

1152. Uusi RK, Kannus, P, Cheng, S, Sievanen, $\mathrm{H}$, Pasanen, M, Heinonen, A, Nenonen, A, Halleen, J, Fuerst, T, Genant, H, Vuori, I,. Effect of alendronate and exercise on bone and physical performance of postmenopausal women: a randomized controlled trial. Bone. 2003 Jul;33(1):13243. PMID: 12919708. No outcomes of interest.

1153. Uusi RK, Sievanen, H, Heinonen, A, Kannus, P, Vuori, I,. Effect of discontinuation of alendronate treatment and exercise on bone mass and physical fitness: 15-month follow-up of a randomized, controlled trial. Bone. 2004 Sep;35(3):799805. PMID: 15336619. No outcomes of interest.

1154. Uusi RK, Semanick, L, M. Zanchetta, J, R. Bogado, C, E. Eriksen, E, F. Sato, M, Beck, T, J. Effects of teriparatide [rhPTH (1-34)] treatment on structural geometry of the proximal femur in elderly osteoporotic women. Bone. 2005 Jun;36(6):948-58. PMID: 15878318. No outcomes of interest.

1155. Valimaki M, J. Laitinen, K, A. Tahtela, R, K. Hirvonen, E, J. Risteli, J, P. The effects of transdermal estrogen therapy on bone mass and turnover in early postmenopausal smokers: a prospective, controlled study. American Journal of Obstetrics \& Gynecology. 2003 Nov;189(5):1213-20. PMID: 14634543. Ineligible treatment duration.

1156. Valimaki M, J. Tahtela, R,. Serum tartrateresistant acid phosphatase 5b or aminoterminal propeptide of type I procollagen for monitoring bisphosphonate therapy in postmenopausal osteoporosis? Clinical Chemistry. 2005 Dec;51(12):2382-5. PMID: 16306104. Ineligible study design.

1157. Valimaki M, J. Farrerons, Minguella J, Halse, J, Kroger, H, Maroni, M, Mulder, H, Munoz, Torres M, Saaf, M, Snorre, Ofjord E,. Effects of risedronate $5 \mathrm{mg} / \mathrm{d}$ on bone mineral density and bone turnover markers in late-postmenopausal women with osteopenia: a multinational, 24-month, 
randomized, double-blind, placebocontrolled, parallel-group, phase III trial. Clinical Therapeutics. 2007 Sep;29(9):193749. PMID: 18035193. Ineligible treatment duration.

1158. Välimäki M, Laitinen, Ka, Tähtelä, Rk, Hirvonen, Ej, Risteli, Jp,. The effects of transdermal estrogen therapy on bone mass and turnover in early postmenopausal smokers: a prospective, controlled study. American journal of obstetrics and gynecology. 2003;189(5):1213-20. PMID: $\mathrm{CN}-00459109$. Ineligible treatment duration.

1159. Valverde P. Pharmacotherapies to manage bone loss-associated diseases: a quest for the perfect benefit-to-risk ratio. Current Medicinal Chemistry. 2008;15(3):284-304. PMID: 18288984. Ineligible study design.

1160. van Geel TACM, Bliuc D, Geusens PPM, et al. Reduced mortality and subsequent fracture risk associated with oral bisphosphonate recommendation in a fracture liaison service setting: A prospective cohort study. PLoS ONE. 2018 June;13 (6) (no pagination)(e0198006) PMID: 622416712. Ineligible treatment duration.

1161. Veerus P, Hovi, S, L. Fischer, K, Rahu, M, Hakama, M, Hemminki, E,. Results from the Estonian postmenopausal hormone therapy trial [ISRCTN35338757]. Maturitas. 2006 Sep 20;55(2):162-73. PMID: 16504428. Ineligible population.

1162. Vestergaard P, Thomsen, S, V. Treating postmenopausal osteoporosis in women at increased risk of fracture - critical appraisal of bazedoxifene: a review. International Journal of Women's Health. 2010 Aug 09;1:97-103. PMID: 21072279. Ineligible study design.

1163. Vestergaard P, Schwartz, K, Pinholt, E, M. Rejnmark, L, Mosekilde, L,. Use of bisphosphonates and raloxifene and risk of deep venous thromboembolism and pulmonary embolism. Osteoporosis International. 2010 Sep;21(9):1591-7. PMID: 19859641. No outcomes of interest.
1164. Vestergaard P, Schwartz, K, Pinholt, E, M. Rejnmark, L, Mosekilde, L,. Gastric and esophagus events before and during treatment of osteoporosis. Calcified Tissue International. 2010 Feb;86(2):110-5. PMID: 19957165. No outcomes of interest.

1165. Vestergaard P, Schwartz, K, Pinholt, E, M. Rejnmark, L, Mosekilde, L,. Stroke in relation to use of raloxifene and other drugs against osteoporosis. Osteoporosis International. 2011 Apr;22(4):1037-45. PMID: 20449570. No outcomes of interest.

1166. Vestergaard P. Occurrence of gastrointestinal cancer in users of bisphosphonates and other antiresorptive drugs against osteoporosis. Calcified Tissue International. 2011 Dec;89(6):434-41. PMID: 22002678. No outcomes of interest.

1167. Vestergaard P. Risk of newly diagnosed type 2 diabetes is reduced in users of alendronate. Calcified Tissue International. 2011 Oct;89(4):265-70. PMID: 21710315. No outcomes of interest.

1168. Vestergaard P, Mosekilde, L, Langdahl, B,. Fracture prevention in postmenopausal women. Clinical Evidence. 2011 May 03;03:03. PMID: 21542947. Ineligible study design.

1169. Vestergaard P, Fischer, L, Mele, M, Mosekilde, L, Christiansen, P,. Use of bisphosphonates and risk of breast cancer. Calcified Tissue International. 2011 Apr;88(4):255-62. PMID: 21253712. No outcomes of interest.

1170. Vestergaard P, Rejnmark, L, Mosekilde, $\mathrm{L}$,. Are antiresorptive drugs effective against fractures in patients with diabetes? Calcified Tissue International. 2011 Mar;88(3):20914. PMID: 21161194. Ineligible study design.

1171. Vestergaard P. Acute myocardial infarction and atherosclerosis of the coronary arteries in patients treated with drugs against osteoporosis: calcium in the vessels and not the bones? Calcified Tissue International. 
2012 Jan;90(1):22-9. PMID: 22120197. No outcomes of interest.

1172. Vickers M, R. MacLennan, A, H. Lawton, B, Ford, D, Martin, J, Meredith, S, K. DeStavola, B, L. Rose, S, Dowell, A, Wilkes, H, C. Darbyshire, J, H. Meade, $\mathrm{T}, \mathrm{W}$. Wisdom, group. Main morbidities recorded in the women's international study of long duration oestrogen after menopause (WISDOM): a randomised controlled trial of hormone replacement therapy in postmenopausal women. BMJ. 2007 Aug 04;335(7613):239. PMID: 17626056. Ineligible treatment duration.

1173. Vickers M, Martin, Meade, Tw, Arasaratnum, Collins, Furness, Ghali, Gordon, Huppert, Islam, Knott, Meredith, Prince, Purdon, Richards, Taylor, Welton, Zhu, C, Q. Zuhrie, Dowell, Lawton, MacLennon,. The Women's International Study of Long-duration Oestrogen after Menopause (WISDOM): a randomised controlled trial. BMC women's health. 2007;7:2. PMID: CN-00641636. Ineligible study design.

1174. Vignot $\mathrm{M}, \mathrm{Pj}$,. Effects of raloxifene on bone loss and fracture risk in the menopausal woman. Contraception, fertilite, sexualite (1992). 1999;27(12):858-60. PMID: CN00275161. Not available in English.

1175. Villa J, C. Gianakos, A, Lane, J, M. Bisphosphonate Treatment in Osteoporosis: Optimal Duration of Therapy and the Incorporation of a Drug Holiday. HSS Journal. 2016 Feb;12(1):66-73. PMID: 26855630. Ineligible study design.

1176. Vogel V, G. Costantino, J, P. Wickerham, D, L. Cronin, W, M. Cecchini, R, S. Atkins, J, N. Bevers, T, B. Fehrenbacher, L, Pajon, E, R. Jr, Wade, J, L. rd, Robidoux, A, Margolese, R, G. James, J, Lippman, S, M. Runowicz, C, D. Ganz, P, A. Reis, S, E. McCaskill, Stevens W, Ford, L, G. Jordan, V, C. Wolmark, N, National Surgical, Adjuvant Breast, Bowel, Project,. Effects of tamoxifen vs raloxifene on the risk of developing invasive breast cancer and other disease outcomes: the NSABP Study of Tamoxifen and Raloxifene (STAR) P-2 trial.[Erratum appears in
JAMA. 2006 Dec 27 296(24):2926], [Erratum appears in JAMA. 2007 Sep 5 298(9):973]. JAMA. 2006 Jun 21;295(23):2727-41. PMID: 16754727. Ineligible population.

1177. Vogel V, Qu, Wong, Mitchell, Mershon, $\mathrm{Jl}$,. Incidence of invasive breast cancer in postmenopausal women after discontinuation of long-term raloxifene administration. Clinical breast cancer. 2009;9(1):45-50. PMID: CN-00680257. No outcomes of interest.

1178. Walker M, D. Cusano, N, E. Sliney, J, Jr, Romano, M, Zhang, C, McMahon, D, J. Bilezikian, J, P. Combination therapy with risedronate and teriparatide in male osteoporosis. Endocrine. 2013 Aug;44(1):237-46. PMID: 23099796. No outcomes of interest.

1179. Walsh B, W. Cox, D, A. Sashegyi, A, Dean, R, A. Tracy, R, P. Anderson, P, W. Role of tumor necrosis factor-alpha and interleukin-6 in the effects of hormone replacement therapy and raloxifene on Creactive protein in postmenopausal women. American Journal of Cardiology. 2001 Oct 01;88(7):825-8. PMID: 11589863. Ineligible treatment duration.

1180. Walsh J, M. Cheung, A, M. Yang, D, Litwack, S, Grady, D,. Raloxifene and colorectal cancer. Journal of Women's Health. 2005 May;14(4):299-305. PMID: 15916502. No outcomes of interest.

1181. Walsh J, B. Lems, W, F. Karras, D, Langdahl, B, L. Ljunggren, O, Fahrleitner, Pammer A, Barrett, A, Rajzbaum, G, Jakob, F, Marin, F,. Effectiveness of teriparatide in women over 75 years of age with severe osteoporosis: 36-month results from the European Forsteo Observational Study (EFOS). Calcified Tissue International. 2012 May;90(5):37383. PMID: 51940011. Ineligible study design.

1182. Wan S, L. Review: Teriparatide reduces fractures in postmenopausal women with osteoporosis. Annals of Internal Medicine. 201218 Sep;157(6):JC3-4. PMID: 365978017. Ineligible study design. 
1183. Wang J, C. Chien, W, C. Chung, C, H. Liao, W, I. Tsai, S, H. Adverse cardiovascular effects of nitrogen-containing bisphosphonates in patients with osteoporosis: A nationwide populationbased retrospective study. International Journal of Cardiology. 2016 Jul 15;215:2327. PMID: 27128537. No outcomes of interest.

1184. Warming L, Christoffersen, C, Riis, B, J. Stakkestad, J, A. Delmas, P, D. Christiansen, C,. Adverse effects of a SERM (Levormeloxifene). Safety parameters and bone mineral density 12 months after treatment withdrawal. Maturitas. 2003 Mar 28;44(3):189-99. PMID: 12648882. Ineligible treatment duration.

1185. Warming L, Ravn, P, Nielsen, T, Christiansen, C,. Safety and efficacy of drospirenone used in a continuous combination with 17beta-estradiol for prevention of postmenopausal osteoporosis. Climacteric. 2004 Mar;7(1):103-11. PMID: 15259289. Ineligible treatment duration.

1186. Warming L, Ravn, P, Spielman, D, Delmas, P, Christiansen, C,. Trimegestone in a low-dose, continuous-combined hormone therapy regimen prevents bone loss in osteopenic postmenopausal women. Menopause. 2004 May-Jun;11(3):337-42. PMID: 15167314. Ineligible treatment duration.

1187. Wasnich R, D. Ross, P, D. Thompson, D, E. Cizza, G, Yates, A, J. Skeletal benefits of two years of alendronate treatment are similar for early postmenopausal Asian and Caucasian women. Osteoporosis International. 1999;9(5):455-60. PMID: 10550466. Ineligible treatment duration.

1188. Wasnich R, D. Bagger, Y, Z. Hosking, D, J. McClung, M, R. Wu, M, Mantz, A, M. Yates, J, J. Ross, P, D. Alexandersen, P, Ravn, P, Christiansen, C, Santora, A, C. nd, Early Postmenopausal Intervention Cohort, Study Group,. Changes in bone density and turnover after alendronate or estrogen withdrawal. Menopause. 2004
Nov-Dec;11(6 Pt 1):622-30. PMID: 15545790. Ineligible population.

1189. Watts N, B. Notelovitz, M, Timmons, M, C. Addison, W, A. Wiita, B, Downey, L, J. Comparison of oral estrogens and estrogens plus androgen on bone mineral density, menopausal symptoms, and lipidlipoprotein profiles in surgical menopause.[Erratum appears in Obstet Gynecol 1995 May 85(5 Pt 1):668]. Obstetrics \& Gynecology. 1995 Apr;85(4):529-37. PMID: 7898828. Ineligible population.

1190. Watts N, B. Nolan, J, C. Brennan, J, J. Yang, H, M. E. Stratab Osteoporosis Study Group. Esterified estrogen therapy in postmenopausal women. Relationships of bone marker changes and plasma estradiol to BMD changes: a two-year study. Menopause. 2000 Nov-Dec;7(6):375-82. PMID: 11127759. Ineligible treatment duration.

1191. Watts N, B. Josse, R, G. Hamdy, R, C. Hughes, R, A. Manhart, M, D. Barton, I, Calligeros, D, Felsenberg, D,. Risedronate prevents new vertebral fractures in postmenopausal women at high risk. Journal of Clinical Endocrinology \& Metabolism. 2003 Feb;88(2):542-9. PMID: 12574177. Ineligible treatment duration.

1192. Watts N, B. Cooper, C, Lindsay, R, Eastell, R, Manhart, M, D. Barton, I, P. van, Staa T, P. Adachi, J, D. Relationship between changes in bone mineral density and vertebral fracture risk associated with risedronate: greater increases in bone mineral density do not relate to greater decreases in fracture risk. Journal of Clinical Densitometry. 2004 Fall;7(3):255-61. PMID: 15319494. Ineligible treatment duration.

1193. Watts N, B. Worley, K, Solis, A, Doyle, J, Sheer, R,. Comparison of risedronate to alendronate and calcitonin for early reduction of nonvertebral fracture risk: results from a managed care administrative claims database. Journal of Managed Care Pharmacy. 2004 Mar-Apr;10(2):142-51. PMID: 15032563. Ineligible study design. 
1194. Watts N, B. Geusens, P, Barton, I, P. Felsenberg, D,. Relationship between changes in BMD and nonvertebral fracture incidence associated with risedronate: reduction in risk of nonvertebral fracture is not related to change in BMD. Journal of Bone \& Mineral Research. 2005 Dec;20(12):2097-104. PMID: 16294263. Ineligible treatment duration.

1195. Watts N, B. Chines, A, Olszynski, W, P. McKeever, C, D. McClung, M, R. Zhou, $\mathrm{X}$, Grauer, A,. Fracture risk remains reduced one year after discontinuation of risedronate. Osteoporosis International. 2008 Mar;19(3):365-72. PMID: 17938986. Ineligible study design.

1196. Watts N, B. Lewiecki, E, M. Bonnick, S, L. Laster, A, J. Binkley, N, Blank, R, D. Geusens, P, P. Miller, P, D. Petak, S, M. Recker, R, R. Saag, K, G. Schousboe, J, Siris, E, S. Bilezikian, J, P. Clinical value of monitoring BMD in patients treated with bisphosphonates for osteoporosis. Journal of Bone \& Mineral Research. 2009 Oct;24(10):1643-6. PMID: 19712042. Ineligible study design.

1197. Watts N, B. Miller, P, D. Kohlmeier, L, A. Sebba, A, Chen, P, Wong, M, Krohn, $\mathrm{K}$,. Vertebral fracture risk is reduced in women who lose femoral neck BMD with teriparatide treatment. Journal of Bone \& Mineral Research. 2009 Jun;24(6):1125-31. PMID: 19113918. Ineligible treatment duration.

1198. Watts N, B. Roux, C, Modlin, J, F. Brown, J, P. Daniels, A, Jackson, S, Smith, S, Zack, D, J. Zhou, L, Grauer, A, Ferrari, S,. Infections in postmenopausal women with osteoporosis treated with denosumab or placebo: coincidence or causal association? Osteoporosis International. 2012 Jan;23(1):327-37. PMID: 21892677. No outcomes of interest.

1199. Watts N, B. Long-term risks of bisphosphonate therapy. Arquivos Brasileiros de Endocrinologia e Metabologia. 2014 Jul;58(5):523-9. PMID: 25166043. Ineligible study design.
1200. Watts N, B. Bilezikian, J, P. Advances in target-specific therapy for osteoporosis. Journal of Clinical Endocrinology \& Metabolism. 2014 Apr;99(4):1149-51. PMID: 24446660. Ineligible study design.

1201. Watts N, Brown, Jp, Papapoulos, Lewiecki, Em, Kendler, Dl, Dakin, Wagman, Rb, Wang, Daizadeh, Ns, Smith, Bone, Hg,. Safety Observations With 3 Years of Denosumab Exposure: comparison Between Subjects Who Received Denosumab During the Randomized FREEDOM Trial and Subjects Who Crossed Over to Denosumab During the FREEDOM Extension. Journal of bone and mineral research. 2017;(no pagination) PMID: CN-01366126. No outcomes of interest.

1202. Waugh N, Royle, P, Scotland, G, Henderson, R, Hollick, R, McNamee, P,. Denosumab for the prevention of osteoporotic fractures in postmenopausal women. Health Technology Assessment (Winchester, England). 2011 May;15 Suppl 1:51-9. PMID: 21609653. Ineligible study design.

1203. Weber T, J. Drezner, M, K. Effect of alendronate on bone mineral density in male idiopathic osteoporosis. Metabolism: Clinical and Experimental. 2001;50(8):9125. PMID: 32755007. Ineligible study design.

1204. Weinstein R, S. Parfitt, A, M. Marcus, R, Greenwald, M, Crans, G, Muchmore, D, B. Effects of raloxifene, hormone replacement therapy, and placebo on bone turnover in postmenopausal women. Osteoporosis International. 2003 Oct;14(10):814-22. PMID: 14610642. Ineligible treatment duration.

1205. Weiss S, Ellman, Dolker,. A randomized controlled trial of four doses of transdermal estradiol for preventing postmenopausal bone loss. Obstetrics and gynecology. 1999;94(3):330-6. PMID: CN-00414338. Ineligible treatment duration.

1206. Wells G, Cranney, A, Peterson, J, Boucher, M, Shea, B, Robinson, V, Coyle, D, Tugwell, P,. Risedronate for the 
primary and secondary prevention of osteoporotic fractures in postmenopausal women. Cochrane Database of Systematic Reviews. 2008 Jan 23(1):CD004523. PMID: 18254053. Ineligible study design.

1207. Wells G, A. Cranney, A, Peterson, J, Boucher, M, Shea, B, Robinson, V, Coyle, D, Tugwell, P,. Alendronate for the primary and secondary prevention of osteoporotic fractures in postmenopausal women. Cochrane Database of Systematic Reviews. 2008 Jan 23(1):CD001155. PMID: 18253985. Ineligible study design.

1208. Wensel T, M. Iranikhah, M, M. Wilborn, $\mathrm{T}$, W. Effects of denosumab on bone mineral density and bone turnover in postmenopausal women.

Pharmacotherapy:The Journal of Human Pharmacology \& Drug Therapy. 2011 May;31(5):510-23. PMID: 21923432. Ineligible study design.

1209. Westenfeld R, Ketteler, M, Brandenburg, $\mathrm{V}, \mathrm{M}$. Anti-RANKL therapy - Implications for the bone-vascular-axis in CKD? Denosumab in post-menopausal women with low bone mineral density. Nephrology Dialysis Transplantation. 2006 August;21(8):2075-7. PMID: 44295031. Ineligible study design.

1210. Whelan T, Goss, Pe, Ingle, Jn, Pater, Jl, Tu, Pritchard, Liu, Shepherd, Le, Palmer, Robert, $\mathrm{Nj}$, Martino, Muss, $\mathrm{Hb}$,. Assessment of quality of life in MA.17: a randomized, placebo-controlled trial of letrozole after 5 years of tamoxifen in postmenopausal women. Journal of clinical oncology. 2005;23(28):6931-40. PMID: $\mathrm{CN}-00530120$. Ineligible population.

1211. Whitmarsh T, Treece, G, M. Gee, A, H. Poole, K. E, S. Mapping Bone Changes at the Proximal Femoral Cortex of Postmenopausal Women in Response to Alendronate and Teriparatide Alone, Combined or Sequentially. Journal of Bone and Mineral Research. 201501 Jul;30(7):1309-18. PMID: 604952731. No outcomes of interest.

1212. Whitmarsh T, Treece, G, M. Gee, A, H. Poole, K, E. The Effects on the Femoral
Cortex of a 24 Month Treatment Compared to an 18 Month Treatment with Teriparatide: A Multi-Trial Retrospective Analysis. PLoS ONE [Electronic Resource]. 2016;11(2):e0147722. PMID: 26859142. No outcomes of interest.

1213. Wilkes M, M. Navickis, R, J. Chan, W, W. Lewiecki, E, M. Bisphosphonates and osteoporotic fractures: a cross-design synthesis of results among compliant/persistent postmenopausal women in clinical practice versus randomized controlled trials. Osteoporosis International. 2010 Apr;21(4):679-88. PMID: 19572092. Ineligible study design.

1214. Wimalawansa S, J. Combined therapy with estrogen and etidronate has an additive effect on bone mineral density in the hip and vertebrae: four-year randomized study. American Journal of Medicine. 1995 Jul;99(1):36-42. PMID: 7598140. Ineligible population.

1215. Wimalawansa S, J. Nitroglycerin therapy is as efficacious as standard estrogen replacement therapy (Premarin) in prevention of oophorectomy-induced bone loss: a human pilot clinical study. Journal of Bone \& Mineral Research. 2000 Nov;15(11):2240-4. PMID: 11092405. Ineligible population.

1216. Winzenberg T, Jones, G,. When do bisphosphonates make the most sense? Journal of Family Practice. 2011 Jan;60(1):18-28. PMID: 21209974. Ineligible study design.

1217. Woo C, Gao, G, Wade, S, Hochberg, M, C. Gastrointestinal side effects in postmenopausal women using osteoporosis therapy: 1-year findings in the POSSIBLE US study. Current Medical Research \& Opinion. 2010 Apr;26(4):1003-9. PMID: 20201623. No outcomes of interest.

1218. Woodis C, B. Once-yearly administered intravenous zoledronic acid for postmenopausal osteoporosis. Annals of Pharmacotherapy. 2008 Jul;42(7):1085-9. PMID: 18505912. Ineligible study design. 
1219. Worsfold M, Powell, D, E. Jones, T, J. Davie, M, W. Assessment of urinary bone markers for monitoring treatment of osteoporosis. Clinical Chemistry. 2004 Dec;50(12):2263-70. PMID: 15472034. Ineligible study design.

1220. Xu D, Zhi, Zhang, Wang, Wu,. Therapeutic effect of zoledronic acid on primary osteoporosis in elderly patients. Nan fang yi ke da xue xue bao [Journal of Southern Medical University]. 2012;32(9):1287-9. PMID: CN-00919911. Not available in English.

1221. Xu W, Xiang, C, Wang, H, Yuan, H, Zhao, X, Xiao, X,. Effect of zoledronic acid therapy on postmenopausal osteoporosis between the Uighur and Han population in Xinjiang: An open-label, longterm safety and efficacy study. Journal of Clinical Pharmacy and Therapeutics. 2017 PMID: 619158938. No outcomes of interest.

1222. Yaffe K, Krueger, K, Sarkar, S, Grady, D, Barrett, Connor E, Cox, D, A. Nickelsen, T, Multiple Outcomes of Raloxifene, Evaluation Investigators,. Cognitive function in postmenopausal women treated with raloxifene. New England Journal of Medicine. 2001 Apr 19;344(16):1207-13. PMID: 11309635. No outcomes of interest.

1223. Yaffe K, Krueger, K, Cummings, S, R. Blackwell, T, Henderson, V, W. Sarkar, S, Ensrud, K, Grady, D,. Effect of raloxifene on prevention of dementia and cognitive impairment in older women: the Multiple Outcomes of Raloxifene Evaluation (MORE) randomized trial. American Journal of Psychiatry. 2005 Apr;162(4):683-90. PMID: 15800139. Ineligible treatment duration.

1224. Yamamoto T, Tsujimoto, M, Hamaya, E, Sowa, H,. Assessing the effect of baseline status of serum bone turnover markers and vitamin D levels on efficacy of teriparatide 20 mug/day administered subcutaneously in Japanese patients with osteoporosis. Journal of Bone \& Mineral Metabolism. 2013 Mar;31(2):199-205. PMID: 23135345. No outcomes of interest.
1225. Yamamoto T, Tsujimoto, M, Sowa, H,. Safety of daily teriparatide treatment: a post hoc analysis of a Phase III study to investigate the possible association of teriparatide treatment with calcium homeostasis in patients with serum procollagen type I N-terminal propeptide elevation. Clinical Interventions In Aging. 2015;10:1101-9. PMID: 26185429. No outcomes of interest.

1226. Yamazaki T, Yamori, M, Yamamoto, K, Saito, K, Asai, K, Sumi, E, Goto, K, Takahashi, K, Nakayama, T, Bessho, K,. Risk of osteomyelitis of the jaw induced by oral bisphosphonates in patients taking medications for osteoporosis: a hospitalbased cohort study in Japan. Bone. 2012 Nov;51(5):882-7. PMID: 22917933. No outcomes of interest.

1227. Yan Y, Wang, W, Zhu, H, Li, M, Liu, J, Luo, B, Xie, H, Zhang, G, Li, F,. The efficacy and tolerability of once-weekly alendronate $70 \mathrm{mg}$ on bone mineral density and bone turnover markers in postmenopausal Chinese women with osteoporosis. Journal of Bone \& Mineral Metabolism. 2009;27(4):471-8. PMID: 19343272. No outcomes of interest.

1228. Yang T, S. Chen, Y, J. Liang, W, H. Chang, C, Y. Tai, L, C. Chang, S, P. Ng, $\mathrm{H}, \mathrm{T}$. A clinical trial of 3 doses of transdermal 17beta-estradiol for preventing postmenopausal bone loss: a preliminary study. Journal of the Chinese Medical Association: JCMA. 2007 May;70(5):200-6. PMID: 17524997. Ineligible treatment duration.

1229. Yang Y, Luo, X, Yan, F, Jiang, Z, Li, Y, Fang, C, Shen, J,. Effect of zoledronic acid on vertebral marrow adiposity in postmenopausal osteoporosis assessed by MR spectroscopy. Skeletal Radiology. 2015 Oct;44(10):1499-505. PMID: 26130070. No outcomes of interest.

1230. Yang Z, X, J. Zhu, X, J. Zhang, Bao, M, J. Xian, Wu, J, C. Liu, L, M. Li, P, Q. Comparison between recombinant human parathyroid hormone (1-34) and elcatonin in treatment of primary osteoporosis. Asian Pacific journal of tropical medicine. 
2015;8(1):79-84. PMID: CN-01099213. No outcomes of interest.

1231. Yanik B, Bavbek, N, Yanik, T, Inegol, I, Kanbay, M, Turgut, F, H. Uz, E, Akcay, A,. The effect of alendronate, risedronate, and raloxifene on renal functions, based on the Cockcroft and Gault method, in postmenopausal women. Renal Failure. 2007;29(4):471-6. PMID: 17497471. No outcomes of interest.

1232. Yasui T, Uemura, H, Umino, Y, Takikawa, M, Kuwahara, A, Saito, S, Matsuzaki, T, Maegawa, M, Furumoto, H, Miura, M, Irahara, M,. Serum estradiol concentration as measured by HPLC-RIA and bone mineral density in postmenopausal women during hormone replacement therapy. Hormone Research. 2004;61(3):117-25. PMID: 14676459. Ineligible treatment duration.

1233. Yavropoulou M, P. Giusti, A, Ramautar, S, R. Dijkstra, S, Hamdy, N, A. Papapoulos, S, E. Low-energy fractures of the humeral shaft and bisphosphonate use. Journal of Bone \& Mineral Research. 2012 Jun;27(6):1425-31. PMID: 22407939. Ineligible study design.

1234. Yavropoulou M, P. Pikilidou, M, Yovos, J, G. Anti-osteoporotic drugs and vascular calcification: The bidirectional calcium traffic. Journal of Vascular Research. 2014;51(1):37-49. PMID: 604054822. Ineligible study design.

1235. Yeap S, S. Hew, F, L. Lee, J, K. Goh, E, M. Chee, W, Mumtaz, M, Damodaran, P, Lim, H, H. Chan, S, P. Malaysian Osteoporosis Society Committee Working Group for the Clinical Guidance on the Management, of Osteoporosis,. The Malaysian Clinical Guidance on the management of postmenopausal osteoporosis, 2012: a summary. International Journal of Rheumatic Diseases. 2013 Feb;16(1):30-40. PMID: 23441770. Ineligible study design.

1236. Yen M, L. Yen, B, L. Jang, M, H. Hsu, S, H. Cheng, W, C. Tsai, K, S. Effects of alendronate on osteopenic postmenopausal Chinese women. Bone. 2000
Nov;27(5):681-5. PMID: 11062356. Ineligible treatment duration.

1237. Yeter K, Evcik, Fd, Demirdal, Us, Subasi, Cakir,. Evalution the effects of raloxifene and risedronate on serum biochemical markers. Turk osteoporoz dergisi. 2014;20(3):110-6. PMID: CN-01040592. Not available in English.

1238. Yildirim K, Gureser, G, Karatay, S, Melikoglu, M, A. Ugur, M, Erdal, A, Senel, K, Billen, H,. Comparison of the effects of alendronate, risedronate and calcitonin treatment in postmenopausal osteoporosis. Journal of Back and Musculoskeletal Rehabilitation. 2005;18(34):85-9. PMID: 43124604. No outcomes of interest.

1239. YildirimK G, KaratayS, MelikogluMa, UgurM, ErdalA,. Comparison of the effects of alendronate, risedronate and calcitonin treatment in postmenopausal osteoporosis. Journal of back and musculoskeletal rehabilitation. 2005;18(3-4):85-9. PMID: CN-00990917. No outcomes of interest.

1240. Ylikorkala O, Evio, S, Valimaki, M, Tiitinen, A,. Effects of hormone therapy and alendronate on C-reactive protein, Eselectin, and sex hormone-binding globulin in osteoporotic women. Fertility \& Sterility. 2003 Sep;80(3):541-5. PMID: 12969695. No outcomes of interest.

1241. Yoshiki F, Nishikawa, A, Taketsuna, M, Kajimoto, K, Enomoto, H,. Efficacy and safety of teriparatide in bisphosphonatepretreated and treatment-naive patients with osteoporosis at high risk of fracture: Post hoc analysis of a prospective observational study. Journal of Orthopaedic Science. 2017 01 Mar;22(2):330-8. PMID: 614559121. Ineligible study design.

1242. You L, Sheng, Z, Y. Chen, J, Y. Pan, L, Chen, L,. The safety and efficacy of earlystage bi-weekly alendronate to improve bone mineral density and bone turnover in chinese post-menopausal women at risk of osteoporosis. Journal of International Medical Research. 2011;39(1):302-10. PMID: 21672334. No outcomes of interest. 
1243. Young R, May, H, Murphy, S, Grey, C, Compston, J, E. Rates of bone loss in periand postmenopausal women: a 4 year, prospective, population-based study. Clinical Science. 1996 Sep;91(3):307-12. PMID: 8869413. Ineligible treatment duration.

1244. Yu E, W. Neer, R, M. Lee, H, Wyland, J, J. de la, Paz A, V. Davis, M, C. Okazaki, M, Finkelstein, J, S. Timedependent changes in skeletal response to teriparatide: escalating vs. constant dose teriparatide (PTH 1-34) in osteoporotic women. Bone. 2011 Apr 01;48(4):713-9. PMID: 21111078. No outcomes of interest.

1245. Yu J, Goldshtein, I, Shalev, V, Chodick, G, Ish, Shalom S, Sharon, O, Modi, A, Association of gastrointestinal events and osteoporosis treatment initiation in newly diagnosed osteoporotic Israeli women. International Journal of Clinical Practice. 2015 Sep;69(9):1007-14. PMID: 26278464. Ineligible study design.

1246. Yuh D, Y. Chang, T, H. Huang, R, Y. Chien, W, C. Lin, F, G. Fu, E,. The national-scale cohort study on bisphosphonate-related osteonecrosis of the jaw in Taiwan. Journal of Dentistry. 2014 Oct;42(10):1343-52. PMID: 24907558. Ineligible treatment duration.

1247. Yukizawa I, Kobayashi, Choe, Kubota, Saito,. Efficacy of Alendronate for the Prevention of Bone Loss in Calcar Region Following Total Hip Arthroplasty. Journal of arthroplasty. (no pagination), 2017. 2017;Date of Publication: September 10 PMID: CN-01370633. Ineligible treatment duration.

1248. Zaheer S, LeBoff, M, Lewiecki, E, M. Denosumab for the treatment of osteoporosis. Expert Opinion On Drug Metabolism \& Toxicology. 2015 Mar;11(3):461-70. PMID: 25614274. Ineligible study design.

1249. Zambon A, Baio, G, Mazzaglia, G, Merlino, L, Corrao, G,. Discontinuity and failures of therapy with bisphosphonates: joint assessment of predictors with multistate models. Pharmacoepidemiology \& Drug Safety. 2008 Mar;17(3):260-9. PMID: 18240162. Ineligible study design.

1250. Zanchetta J, R. Bogado, C, E. Cisari, C, Aslanidis, S, Greisen, H, Fox, J, Lems, $\mathrm{W}$,. Treatment of postmenopausal women with osteoporosis with $\mathrm{PTH}(1-84)$ for 36 months: treatment extension study. Current Medical Research \& Opinion. 2010 Nov;26(11):2627-33. PMID: 20923256. Ineligible study design.

1251. Zanchetta M, B. Boailchuk, J, Massari, F, Silveira, F, Bogado, C, Zanchetta, J, R. Significant bone loss after stopping longterm denosumab treatment: a post FREEDOM study. Osteoporosis International. 201703 Oct:1-7. PMID: 618543675. Ineligible study design.

1252. Zavras A, I. Shanmugham, J, R. Bisphosphonates, Osteoporosis, and Osteonecrosis of the Jaw: A Critical Review of a Large Nested Case-Control Study. The Journal of Evidencebased Dental Practice. 2016 Jun;16(2):136-8. PMID: 27449847. Ineligible study design.

1253. Zebaze R, Libanati, Austin, Ghasem, Zadeh Hanley, Da, Zanchetta, Jr, Thomas, Boutroy, Bogado, Ce, Bilezikian, Jp, Seeman,. Differing effects of denosumab and alendronate on cortical and trabecular bone. Bone. 2014;59:173-9. PMID: CN-00960286. No outcomes of interest.

1254. Zebaze R, Libanati, C, McClung, M, R. Zanchetta, J, R. Kendler, D, L. Hoiseth, A, Wang, A, Ghasem, Zadeh A, Seeman, E,. Denosumab Reduces Cortical Porosity of the Proximal Femoral Shaft in Postmenopausal Women With Osteoporosis. Journal of Bone \& Mineral Research. 2016 Oct;31(10):1827-34. PMID: 27082709. No outcomes of interest.

1255. Zhang J, Saag, K, G. Curtis, J, R. Longterm safety concerns of antiresorptive therapy. Rheumatic Diseases Clinics of North America. 2011 Aug;37(3):387-400, vi. PMID: 22023898. Ineligible study design. 
1256. Zhang L, Yang, M, Liu, D, Guo, C, Li, L, Yang, G,. The rhPTH (1-34), but not elcatonin, increases bone anabolic efficacy in postmenopausal women with osteoporosis. Experimental \& Clinical Endocrinology \& Diabetes. 2012 Jun;120(6):361-6. PMID: 22639400. No outcomes of interest.

1257. Zhang L, Pang, Y, Shi, Y, Xu, M, Xu, $\mathrm{X}$, Zhang, J, Ji, L, Zhao, D,. Indirect comparison of teriparatide, denosumab, and oral bisphosphonates for the prevention of vertebral and nonvertebral fractures in postmenopausal women with osteoporosis. Menopause. 2015 Sep;22(9):1021-5. PMID: 25944523. Ineligible study design.

1258. Zhang Z, Z, L. Liu, J, L. Changes of bone metabolic biochemical markers one year after applying raloxifene hydrochloride in healthy postmenopausal women. Chinese journal of clinical rehabilitation. 2004;8(27):5904-6. PMID: CN-00516668. Not available in English.

1259. Zhang Z, L. Liao, E, Y. Xia, W, B. Lin, H, Cheng, Q, Wang, L, Hao, Y, Q. Chen, D, C. Tang, H, De, Peng Y, You, L, He, L, Hu, Z, H. Song, C, L. Wei, F, Wang, J, Zhang, L, Santora, A, C. Alendronate sodium/vitamin D3 combination tablet versus calcitriol for osteoporosis in Chinese postmenopausal women: a 6-month, randomized, open-label, active-comparator-controlled study with a 6month extension.[Erratum appears in Osteoporos Int. 2015 Nov 26(11):2719-20 PMID: 26377424]. Osteoporosis International. 2015 Sep;26(9):2365-74. PMID: 25929192. No outcomes of interest.

1260. Zhao Y, Xue, R, Shi, N, Xue, Y, Zong, Y, Lin, W, Pei, B, Sun, C, Fan, R, Jiang, Y,. Aggravation of spinal cord compromise following new osteoporotic vertebral compression fracture prevented by teriparatide in patients with surgical contraindications. Osteoporosis International. 201601 Nov;27(11):3309-17. PMID: 610648251. Ineligible treatment duration.
1261. Zoehrer R, Roschger, P, Paschalis, E, P. Hofstaetter, J, G. Durchschlag, E, Fratzl, P, Phipps, R, Klaushofer, K,. Effects of 3and 5-year treatment with risedronate on bone mineralization density distribution in triple biopsies of the iliac crest in postmenopausal women.[Erratum appears in J Bone Miner Res. 2006 Dec 21(12):1968]. Journal of Bone \& Mineral Research. 2006 Jul;21(7):1106-12. PMID: 16813531. No outcomes of interest.

1262. Zysset P, Pahr, D, Engelke, K, Genant, H, K. McClung, M, R. Kendler, D, L. Recknor, C, Kinzl, M, Schwiedrzik, J, Museyko, O, Wang, A, Libanati, C,. Comparison of proximal femur and vertebral body strength improvements in the FREEDOM trial using an alternative finite element methodology. Bone. 2015 Dec;81:122-30. PMID: 26141837. No outcomes of interest.

1263. Bone HG, Wagman RB, Brandi ML, et al. 10 years of denosumab treatment in postmenopausal women with osteoporosis: results from the phase 3 randomised FREEDOM trial and open-label extension. The lancet. Diabetes \& endocrinology. 2017;5(7):513-23. doi: 10.1016/S22138587(17)30138-9. PMID: CN-01458543. Ineligible study design.

1264. Deeks ED. Denosumab: A Review in Postmenopausal Osteoporosis. Drugs \& Aging. 2018 02;35(2):163-73. doi: https://dx.doi.org/10.1007/s40266-0180525-7. PMID: 29435849. Ineligible study design.

1265. Gamboa A, Duaso E, Marimon P, et al. Oral bisphosphonate prescription and nonadherence at 12 months in patients with hip fractures treated in an acute geriatric unit. Osteoporosis International. 201801 Oct;29(10):2309-14. doi: http://dx.doi.org/10.1007/s00198-018-46226. PMID: 623528038. Ineligible treatment duration.

1266. Hagino H, Endo N, Yamamoto T, et al. Treatment status and radiographic features of patients with atypical femoral fractures. Journal of Orthopaedic Science. 2018 Mar;23(2):316-20. doi: 
https://dx.doi.org/10.1016/j.jos.2017.10.008. PMID: 29146093. Ineligible study design.

1267. Jung SM, Han S, Kwon HY. Dose-Intensity of Bisphosphonates and the Risk of Osteonecrosis of the Jaw in Osteoporosis Patients. Frontiers in Pharmacology. 2018;9:796. doi: https://dx.doi.org/10.3389/fphar.2018.00796 . PMID: 30079024. Ineligible treatment duration.

1268. Kendler DL, Chines A, Brandi ML, et al. The risk of subsequent osteoporotic fractures is decreased in subjects experiencing fracture while on denosumab: results from the FREEDOM and FREEDOM Extension studies. Osteoporosis International. 2018doi: http://dx.doi.org/10.1007/s00198-018-46872. PMID: 624004991. Ineligible study design.

1269. Miyagi M, Fujimaki H, Naruse K, et al. The impact of switching once-weekly teriparatide to denosumab in osteoporosis patients. Journal of Orthopaedic Science. 2018doi:

http://dx.doi.org/10.1016/j.jos.2018.08.001. PMID: 2001047051. Ineligible study design.

1270. Murphy PZ, Iranikhah M, Deas CM, et al. Fracture Risk Following Discontinuation of Teriparatide: A Review of the Literature. Consultant Pharmacist. 2018 Jul 01;33(7):365-75. doi: https://dx.doi.org/10.4140/TCP.n.2018.365. PMID: 29996965. Ineligible study design.

1271. Sing CW, Wong AYS, Kiel DP, et al. Association of Alendronate and Risk of Cardiovascular Events in Patients With Hip Fracture. Journal of Bone and Mineral Research. 2018 August;33(8):1422-34. doi: http://dx.doi.org/10.1002/jbmr.3448. PMID: 622092167. Ineligible treatment duration.

1272. Tripto-Shkolnik L, Rouach V, Marcus Y, et al. Vertebral Fractures Following Denosumab Discontinuation in Patients with Prolonged Exposure to Bisphosphonates.

Calcified Tissue International. 201801 Jul;103(1):44-9. doi: http://dx.doi.org/10.1007/s00223-018-0389-
1. PMID: 621563803. Ineligible study design.

1273. Wolverton D, Elliott DP. Evaluating the Evidence Behind Treating Osteoporosis in the Oldest Adults. Consultant Pharmacist. 2018 Jun 01;33(6):308-16. doi: https://dx.doi.org/10.4140/TCP.n.2018.308. PMID: 29880092. Ineligible study design.

1274. Wu J, Zhang Q, Yan G, et al. Denosumab compared to bisphosphonates to treat postmenopausal osteoporosis: a metaanalysis. Journal of Orthopaedic Surgery. 2018 Aug 02;13(1):194. doi: https://dx.doi.org/10.1186/s13018-0180865-3. PMID: 30071889. Ineligible study design. 